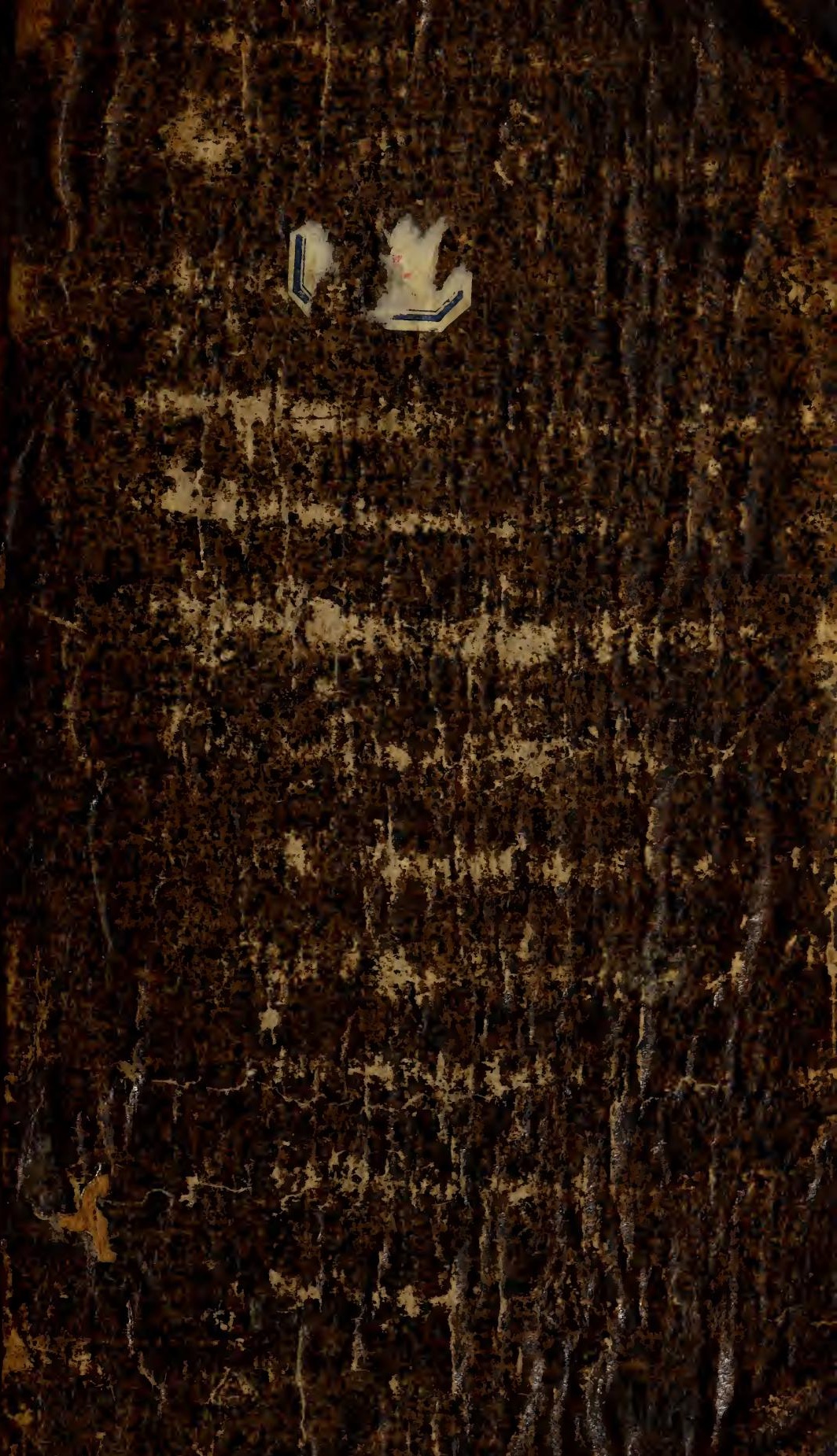




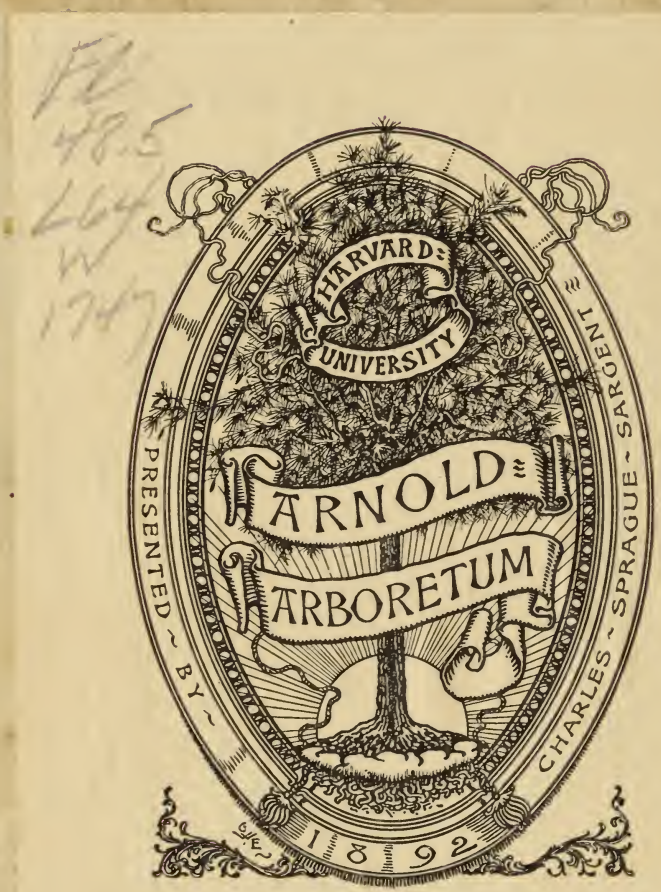


A กT I INTT AT T

1

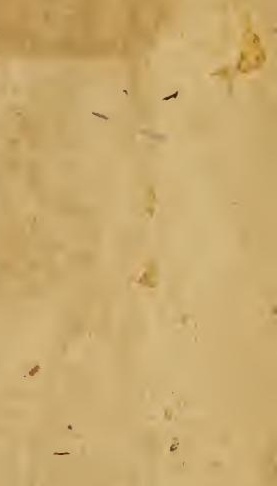





\section{CARL LINNÆI,}
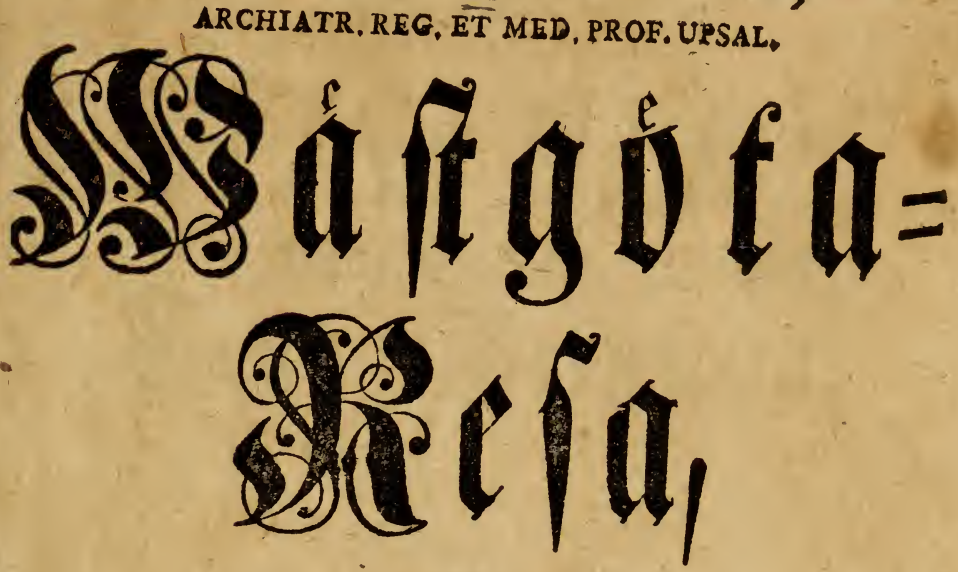

Эฺม

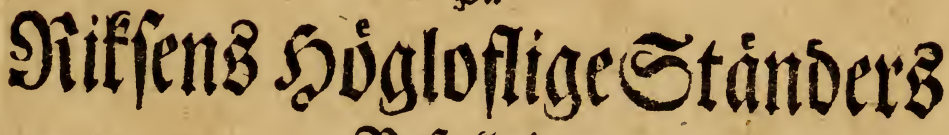 Sefallning}

Fíráttad \&ir 1746.

Med

\section{Plnmáfนningat}

\section{uti}

Oeconomien, Ratuffunnogbeten, Anriquiteter, Intwainarnes Seder od)

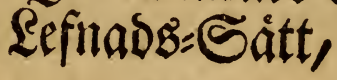

\section{SDRet}

Tilbơrige Figurer.

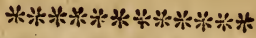

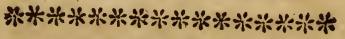

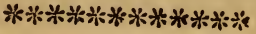

Stodkbolm,

Uplago på LARS SALVII Eoftmab. 1747. 



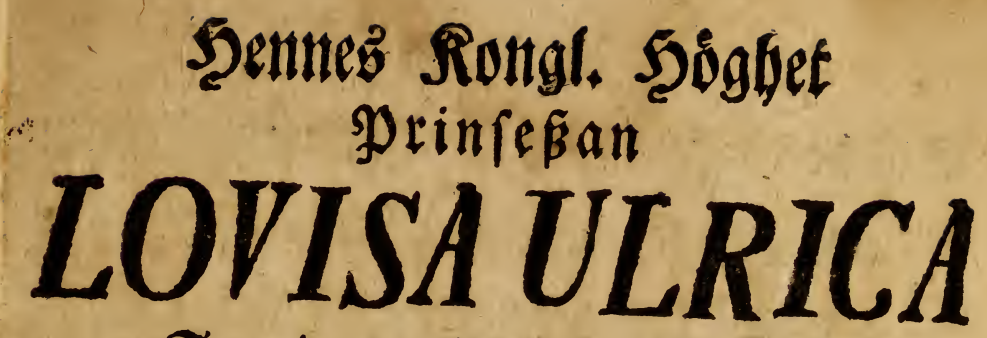

Siweriges, B̧ottbes, $23 e n d e s$

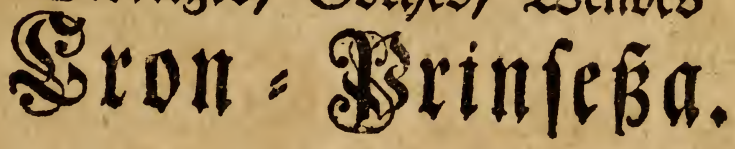

(6. ifens upfom(t) od) aftaganbe, folfes D fälfiet odh olyctá, wetemftapers tilwaizt od) unbergång, wainsas af 2Ulwisbeten genom Dofiwerbets=per= foners bänder. 


\section{)}

Silrdes hafwa 2Barjan, Pennan, Slogen od) Epolen baft fina osen, afiuen fom Bofern, faft Demna bod fâllan uti an nan, ån ben lycteligafte Pregering blif= wit opnas.

Ifrion wair Iflernåbigfte Sonungs antrábe pá Sbronen bafma nuttiga metenfe= per upivuxit och blomifrat; Deras mo. gnade frufter wointa wi fâ modiet mif= fare, at mes unserbaning fägnad ods bjup Sadfiajelie fà bảmta utur

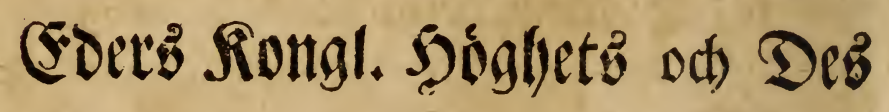
Gemailb, wår Raidigfte Slvf Jut

ftę̧ bóga bánoer, fom De mes fä ftor ombet od) fa muden nas bebagat Deras bafo od) forfwar fig antaga. 


\section{䋓}

Eocts Siongl. Sobghet lever fit ur fprung af Sionunga blos; en frrona, brwartil Edra ftora esemleaper mer, ain fóbfen, gjort Eser mársig, waintar

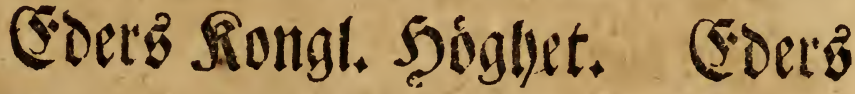
Rongl. ŞGigbet ár blefwen en Ma: Der til en פring, fom feal gifiwa Swea Sionunga Stol en ni) od) fórnyad ftrtfá.

STivad fällbet fór et Siife! brwab förmon fór $\mathfrak{B o f e f l i g e ~ f o n f t e r ! ~}$

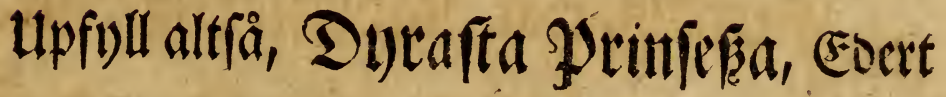
ftora ámme odh waint bopp; $\mathfrak{e f f}$ lánge od) ố Indéflig; Gryna wetenfléperne odh anfe mes milloa digon en Deras is:

$$
\text { ) } 3 \text { fare, }
$$




\section{(5) \\ fare, fom nu umberftär fig at frambaira Detta fả wàlmenta, fom ringa offer}

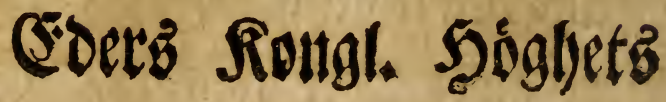

unberbanigfte tienare

Carl Linneus.

50 


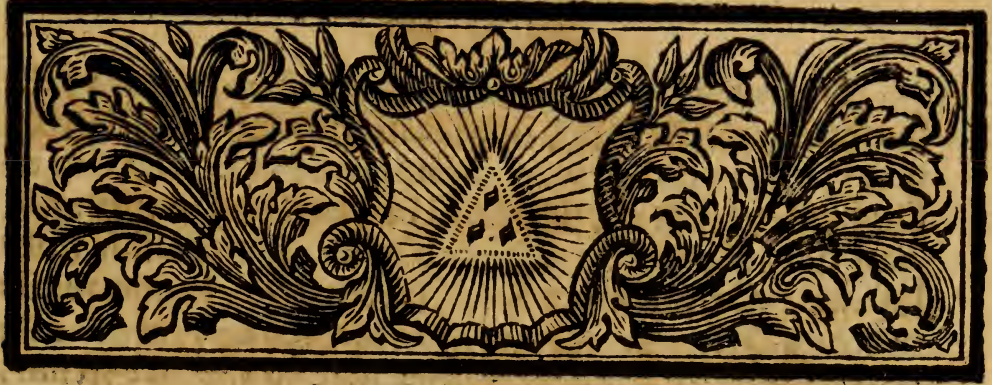

\section{Foretal.}

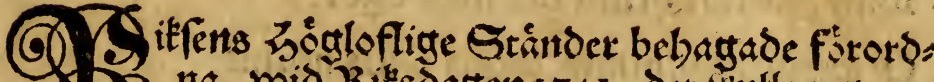

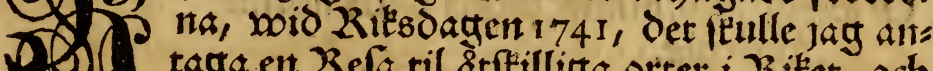

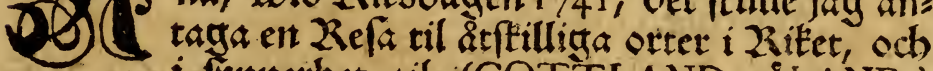
$i$ ivnnethet til (GOTTLAND, OLAND) WASTERGóTLAND wois Kinnekulle, Billingen, Mỏffebärg, Ollebarrg, Hunnebårg, Hallebårg.

Sanma ar fortåattade jay refant til SLAND od GOTTLAND, ocb gaf ut den famma forledit \&r. *

Tu atetfó WASTERGöTLAND, bxoarfore jag forled it ar 1746 , om fonmatriden, tefte ocb belag Des noakra orter: MARIEstan, Kinnekulle, LidróPING, SKARA, Billingen, sjófBE, Móffebårg, FaLKóPING, Ollebärg, BorÅs, Alings Ås, GỏTEBorG, Bohus, MARstrand, Wäftra Skirgården, UdDEWALLA, Trollhăttan, Hallebårg, W ENNERSBORG, och n\&got afDAL ocb WERMELAND.

Zroad jay p\& defie refor anmiatte af Antiquiteter, Phyfique, Oeconomie, Manufacturer, Medicine, Seder och maner, bar jag bár deit átan med noóronad fram= gifooa til mit Eara Sádernesland.

$$
x_{3} \text { main }
$$

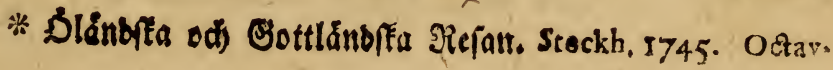




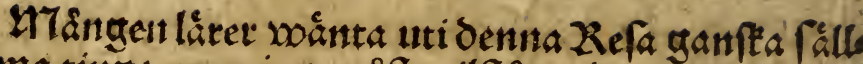
famma ting; men jag mafte tilfta, at jag p\& bela 200 bs gent ej n\&got annat feot, ät nagra STENAR, ORTER odb DJuR, oem jag upteknat, dair jag dem igenfuntit.

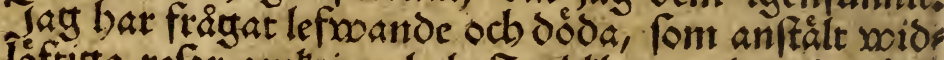
Ioftiga refor onterint bela Jordklotet, booad márt" wårbiga faeter de fèr uti fiartan lánder; men alle hafioca inftamt, at be icle cller teot annat an Stenat, Dárter och Djur, ocm Inwooanatena, dår fom liát, anwoinda til all fint nodrotft och uppebälle, fa at jag fäkett tror, och Kan formoízo utgifiooa, at Skaparen intet annat forlant männiferna, utom 延lementerne, och torde noaka nog tilrácleligit, allenaft man woál woifte, at rått brulea oet man átgoe.

O fortunati nimium, bona fi fua norint Agricolx.

2lit: hat jay itted flit upteknat i denta Refa, bxoad füllamma Stenarter, brodo márkooároiga Doixter, hxoad befynterliga Djur jay fedt p\& refan, och beftrifooit f\& mantga fátt, fom jag funnit brukas af Indoonarena; befinterligen, om de ej conarit alls mánt ófxoer Riket antagne.

Srodingheterna, font woid denna Refas genem feende motade máftentine Láfare, lára befta uti Lams

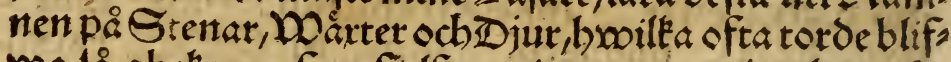
no lis obcḱante, fon fielfooa tingen; men lag kan icke báfwo defie hinder, ty den fom woil tala med nagon, mâfe nédfakeligen fôfte fproket: Om jag námuter óga, Zjork, 2uborte, eller Orre, och Lafaren ej

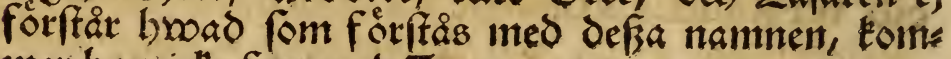
mer ban icke fort med Texten.

Nomina fi pereunt, perit \& cognitio rerum.

Detenfeapen, Hiftoria eller Scientia Naturalis lärer namnen odb naturen af alla Stenar, Doarter ocb 
Diut, bon åt altf̊̊ grunden til all den nytta woi fí af Defißa ting, och altíc til alt brooad mánniftan aflas med, faftät denna xoetenfapen for detta woid Latos bufent icle bounnit det burfeap, font många anore nytrige DOetenfeaper; bock fom denna át oumgeanges lig for booat och ent, torde den boartio firart gronfta, fom woarder oragande flere finnen til begatelfe at

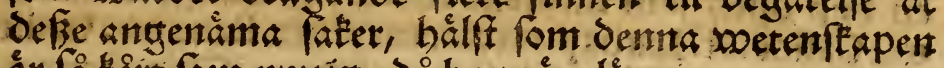

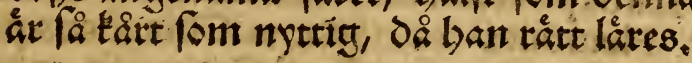

Tammen átoras ofta uti xo\&et Sxoenfea fprese pa Orter ocb Djur, efter årfillitge nåra Provincier, áfoosen fom Latinfte namnen bos Auctorerna: Ja,

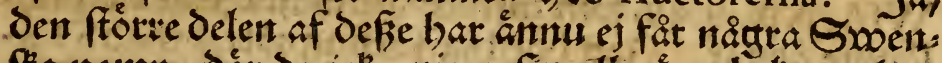
fia namn, dăr de icke gjort fig allmànt bekante bos 2allmogen gensm någon fynnetlig nytta, fárg, lulte,

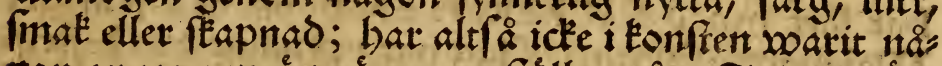

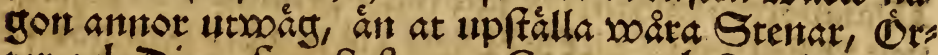
ter och Diur efter Syfteme: Genera ocb Species med Latinfée odb Sxoenfite Synonyma: De fóffe bar jag uptetnat i SYSTEMET *; De andre uti FLORA**, och be

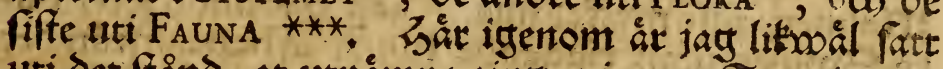
uti det ftånd, at utnámua tingen intom Sxoerige ty Deligate, án nagon annan uti fin Refes beftrifintro; bxoarfore jag woid Doexters ocb Diutrs namm altio citerat numren uti Flora och Fauna, fout băgge p\& Denna Refa fár anfentliga recruter.

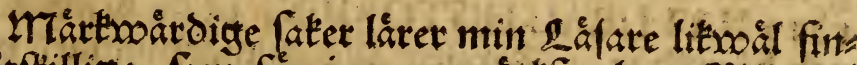

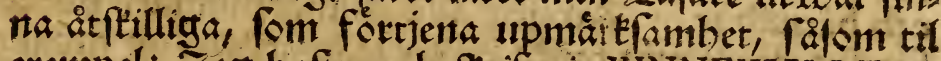
exempel: Jag hafiower beftrifwoit KINNEKULLE p. 20. Htt bxoilken Faturen forelagt of anatomien of jordfeorpan, p. 77, och woifat buru STRATA TERR blifuoit upftaplade i forna tider. Jag bar letat igen

$$
x 4
$$

- Syftema natura, Stockh, 1740. oca.

** Flora Svecica. Stockh. 1745. oa.

** Fauna Svecica, Stockb. 1746. oft 
en bel hop WAXTER, fom tilfornte i Spoctitue icfe 20: irit fofoin Sxoenfte betante. e. gr. Panicum p.14 $\mathrm{r}$ : Ruppia p. 186. Cerafus p. 25.32. 38. Cratægus p. I61. Potentilla p. 63.76.84. Lathyrus p.75. Pifum p.I0-. Senecio p. 142. Coreopfis p. 160. Quercus p.214. Ulva p. 169. Tremella p.I93. Byffus p.5 3. Jag bar beftrifiooit en hop ojut, fom tilforente watit i Swoerige okuntos ga: Caftor I6I. Larus p.I 5 G. Sternap.182. Rana 261. Gadii p.176.177.178. Pleuronedtes p. 178.179. Coregonus p. 231. Itom en hop insoch utande fiftar p. 137.138. 339, fant inlánofía Mratkar p. 173.174, od) Krä́t p. $169=172$. Jag hat frammoifat Oen ityetige KASSEWIA p.p. 44.48, forn gifnocr oet méfta fober pa fios lândte antgat. Jag bat bár forft uptát, broad ST A-

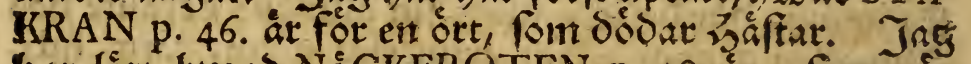
bar late bwad NACKEROTEN p. 98. ar, fom tyou Dalabortgs:fiukan. Jag bar upfuntit och beftrifs woit SKEPPSHWARFS-FLUGAN p. I53, fom gor f mycten fitada p\& Skeppobocarfwent Jay bar ups funnit ZINNGRAUPER p. 256 , eller ratta Certns Malmeit, faft mycket inta. Jag bar underlokt, bus ru Swoinen utan ringanbe kunna afbollas iftan bet Radeliga Rotandet pay. 95. Jag bar fett Getter, fom p\& ufelt bete p. 162 , mjelteat et belt fiop om malet, broilket torde Komma betaf, at be ci tunna trata tráben, emedan all bark adftringerar, ech alt adifringerande hámmal nijólkent. Jag bar gjort åtfillitta cón i NATURAL.HISTORIEN, til De Létrdas nóje odb batnab zc. B̉noilka alla 2limititkningat, broat for fit, jag i min tanka boller ia boctroige, at byat bera Eunde rikeligen betala min móda ocb Publici on: toftinad.

fortegat bar jay getna ELAKA EXEMPEL, fom jaty marit ftickad at gagna, och icke baft luft at no: goit ftada; derfore jag ock atfilligt ranfakat, mtent alorig oemt oet, fom soarit of ofoer mit Fortum. Jag bat wål undertioen infpàd nágea toliga ech 
menlófa uptog, at gifuo láfaten nojje, båk jag cj Eunde góra gagn.

Gfulle alla Sboctiges Provincier po dettat litt blifixo gentonletade, fer jag wól, at en Swoenfte PLI-

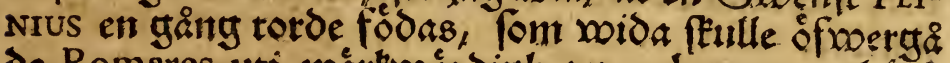
de Romares uti márkboároigheter och nytta, baift ware fábertesland kan firyta of bundrade taintor meta, an alt bet pral, fom utlániningarnas induftrie framalfrat, eburu Jeras lánber oraga naira sgotr, o\& no i tomma at befoka bem; ment jag lemnar gerna dentia tanka at efterkommante dagar och man; jag fout fjelf fatitar tilfálle, tio ocb krafter der til.

Dentra befwótrliga refan, gtenom et datgeligit och ftadigt rátgn, brot nog luften, ocb hintrabe mig, at fe alt, fom fes Eunnat; ment befynterligen fortryter mig, at jag berfore mâfe bolla Landswaitten och lánnth åt antora bet táck a Dal och Dermelaito, font gómmer fo mainga rlaturents Skatter. Refan babe ănnu blifuovit obrageligare, on ej Philofophix Candidaten ERIC Gustaf LmDEEC, Wermelandus, form i flete ar woid Upfala Academie woarit nin beftind

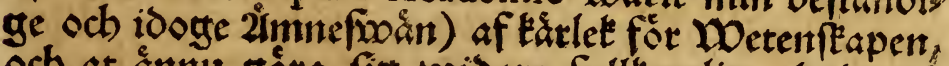
och at innu goto fig boibare fulltomlig, babe pa etgeit koftrab giort mig et nojfant fallftap, odb bars til láttat min moda noid Protocollets forande ocb ren: ifrifboande.

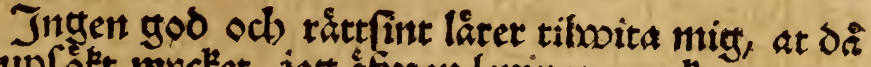
jau up fokt mycket, jag âfooen lemitat mycket; ty eut enda Socken Kulle ofta fordra en hel mants lefinad.

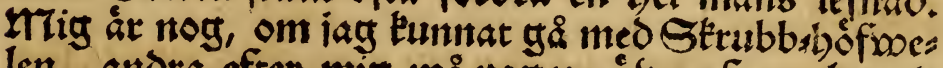
len, andra efter mig ma nogate ófloctfara alt nica Slättsbófixolen.

Bat jag Eumnat med betta mit lâp och dactis gen trotta tropp wointa mine formuftige ocb rétitin 


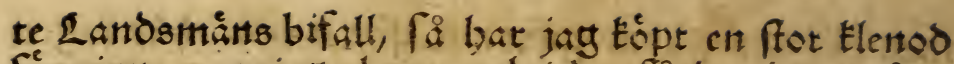
for ringa penting, broar ocb icte, fa bar jag woifers ligen mift bade nat och fift; xointer jug den famma, feal jag gerna rékena nig for beder, at tadlas of ants ora, howilleas beróm fitulle woara mig mer til laft ât finicker.

Upfala 7247.d.8.Aprilis.

\section{CARL LINNÆUS.}

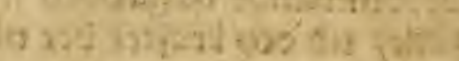
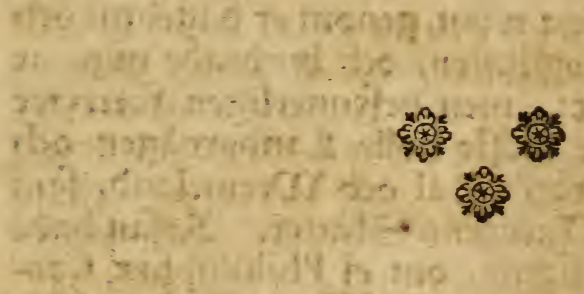


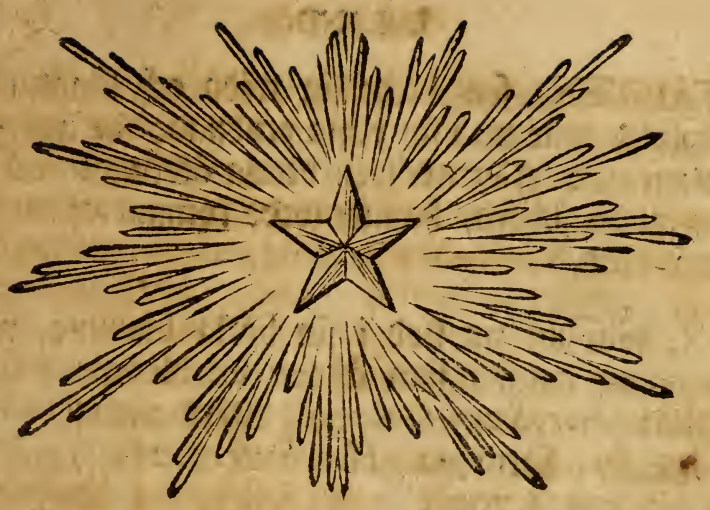

\section{UPLAND.}

1746. Jünii 12 .

T PSALA ACADEMIA Gade is ate fart of beftunts

oigt arbete, ifran Den tio óland od) Gottland lemmabes; Grwarken wille bàlfan eller Siiffenz

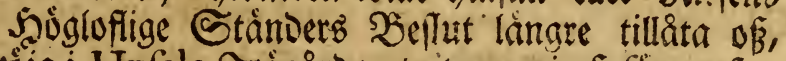
at Dróia i Upfala Srägaro; to togo mi of fore refan juft Dagarna efter Det Academie Srigarden frist

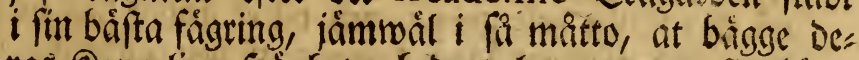
rab fongelige Sabgheter hedrat hunom med fin boga nårwarelfe.

SLOTTET i UPSAIA, fom inemot 200:De \&r warit fơr Sionungarna af Den Gustavianska Familien et liufligit refidence, intil Def Den fórifécteliga wadels Den 1702 lave Det, meo bela Uplala itad $i$ affa, bes. gynte mu ater fofom en Phonix refa fig utur fit grus,

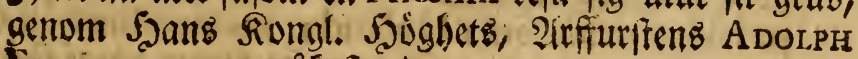
Friéprics egen pafoltmad. 
AKERFALTEN, font brede fig soiba of länt omfritg Upfala, lufte nu $i$ fin báfta prodnao; de aro allmánt betante för Det De rifeligen betala âfermannens mida, od) fillan Ma felt, bivileet torde fomma af Deras uráb origa bitio.

RAGEN, fom juft mu frod i fim béfta blomma, mar full of nảgra ogriś, fom áfwen mu blomftrade, fâfom:

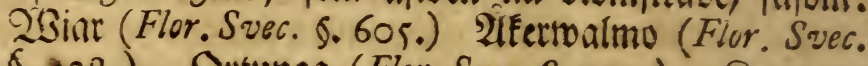
S. 428.), Ortunga (Flor. Svec. S. 253.), Simappar (Flor. Svec. §.709).

ANGARNA woro fulla af nigra wåkter, fom icfe allmaint fintua $i$ Somerige, fafom:

MEDICAGO MINIMA (Flor. 621), fom 2:the år gifะ wer barligt fooer, men forfininner merendel's bet treoje.

THLASPI INC.ANUM (Flor. 53I.), toidte ifiwerflis

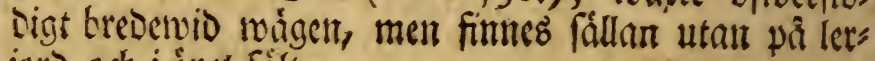
jord och) $i$ ípet fält.

CUMMIN (Flor. 245.), fom bår, od) på åtfillige ftíllen iUpland, waiper i frot mucfenbet, náftan fafom 1 Wes fertotten.

GÁSüRTEN (Flor. 4IY.) fant mycket wis waigarna meo fúfincrbmita blad; Det îr icfe fî allmánt befant, hruars füre blaben ya denua ôt ofta áro $i$ en odf famma âng,

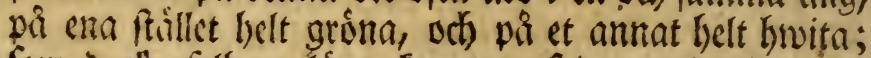
foun boct ofelbart lírer Eomma of Den underliggande jortmonten, hwilfett Etêparen melat bárigenom sifioa mânuifertua tiltánna.

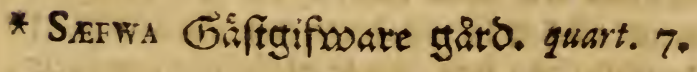

SWENSKA HỏFRỎET (Flor. 620), wåtte pra bioa fiodr um woigen wid Alnicke $i$ frờrre mustenhet, ât pân nâoun numor ort $i$ Upland; ia, langt mer ôn ume

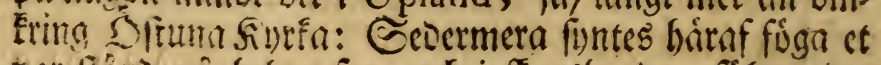

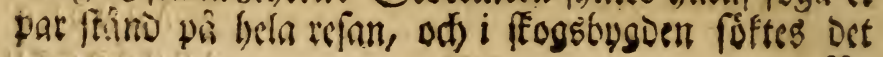




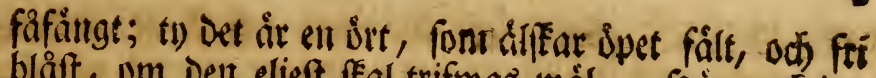
blåft, om Dent eljeft ffal trifruas wál. Şăr omfring Alnicke ár Det báfta tilfállet, at famla fróen af Detta bárliga fooret, blwars mittá och egenféaper tilfórene âro omtalte uti Gottlåndfka refán p. 242. Wetenkk. Acad. 1742. p. 191 , Kalm Bahus, p. z.

HUNDLOKA (Flor. 243) giorde de fitolánote ángar emels tan Alnicke och S Sujja, pá wainftra banden om wágen, helt broita, med fina blommor, fom waite $i$ fförte muses fenbet, àn man någonfin feot tilfourene; moo debe bloms

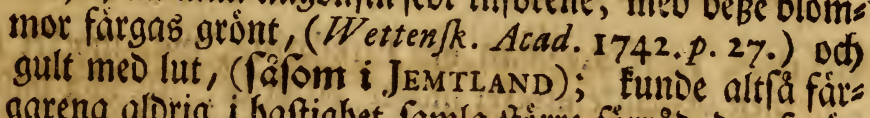

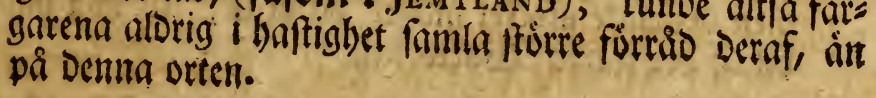

* LisLENa. quart. 6.

JOLSTER (Flor. 792.) JALSTER, JUSTER, HIL.

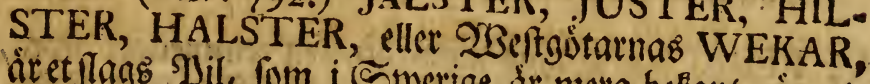

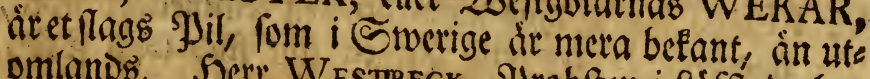
omlands. Syerr WESTBECK, Jurobiten i siffitad, bar for et par ar feoan, genom en fpeciel Tractat lät nation, et of Detta JOLSTERS och ELGESTJELCKENS, ciller GETSTABBENS (Flor. 304) frulft baimta be fiun, ellet pappus, meo hivilfen fróch flis ga odh Dem anmónton $i$ fátlct för Bomull. Stulle Demna Xomull fumna blifwa begărlig, boroe bent tras eten, fom hatr ling remte waigen at ENKópING, finutigas in; ti) hotr waitte.JOLSTER i fturfta mucfenthet, tillifa

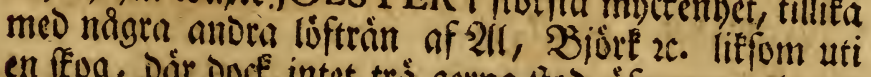
en féog, Dár boef intet trá gerna ftoo sofiver 2 glitarb Gogo: Orfafen, at alla trin pa Detta widuftign fait tet, eller matén, woro lifa fma, lârer ofelbart fomma af Den torra fanoen, fom lág ftrar under matioroen, octa gor hate Defe trioen fima, fom flifan pa oland * gür De
fma Shaflar.

* It. Òland, 37. 
* ENKÓPING quart 4 .

KALL war p'anterad wio ENxÓpING pa De fidlände Gials

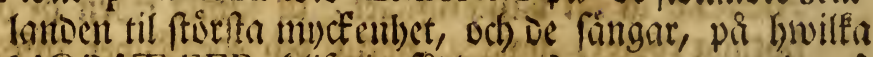
MORÓT IER blifwit FaDde, 17000 garnerade $\beta a$ fidorina mes Sount. Denna itas gür ned fina fiefs: Srryodors planteratide odf forfartantoc til frimgliggam De urter icte ringa antta; broantuti ban efterfótier jaok linosrnas intrittming, fom riftat fig af oyliea plantager, od, wore soil, om andra fima ftaber barutiman of ternpade ENKóping.

ANGEN, pif foota fioan om Enkóping, war mucfet fror, men berjemte nincfet maser wif grab; th) hon bave lis Dit of ben ftarka 23 arturkan.

UPLANIDS SITUATION beftod uti widlyftige afeerfält, hmilfa liffom med langa wifar woro utferticte emellan fmí med fren bef Burttrín belngde Sullar.

\&T TEBACKAR rigo nâgra fuscen temmeligen ftora, od hisa nid Nyqwarn, oirr en four flod lopp fortl, oct) jeifoe Upland ifrän Weftmanland.

\section{WESTMANLAND. Junii 13 .}

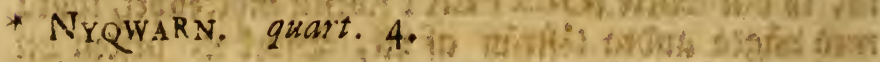

LUSORTEN (Flor. SOs.), fom af Uplanningen Eollas K ILLGR AS, encoan bon gifwer til anma Den unoers ligguitive Fallioroct, frod nu ixit brum meo fina mins gir rido blommor, hivarigenom oe nata angar pî flere Thillen bepryodes mico brun och ridor Sapeter.

* Wester.ås, quatz. 8. 
AREPORTEN, form ivar bugo $i$ Wefteràs for Derab fions geliga Saojgheter, ftod änm qwar nteo fólianoe inleription; pá cna firan:

Auguftis.Neanymphis. ADOLPHO.FRIDERICO.ET. LUDOVICA. ULRICA. Orbis. ArCtoi. Principibus. Hxreditariis. Sereniffimis. Pandor. Gratulabunda. Pandorque. FRIEDERICUS, Eat. Cum. Conjuge. Yrofper. ADOLPHUS. Mett pri anda:

En ego Arofiacx foetus pietatis, \& index. Principibus patuique meis porroque patebo. Transvectis tellus Dúcibus fanruletur, \& xther.

3iftepen i Wefterås Sgert Doctor KXILSENrus habe bât uid Scholan och Gymmafum berommeligen füran fraltat, at Lector Matbefeos feulle bảde mifa fitu difciplar om nâttertita conféllationerne, yả hims melen, odh âfiven läfa Gazetterne för Denn z:me gångor

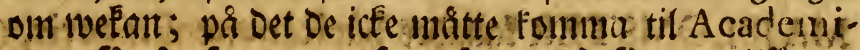
erne fia $x a$, form man ofta erfar, od fiowne tifforete intet mera lárt, ân nigon Latin, od) en bop utanlecor.

BOT ANIOUEN har hatrtil Dangs nlorig harit nagou courant 233 ara for Ecfolepiltante, churu ben lìgger fors ffa grunden til all privat-cecononie, fon lifieal â

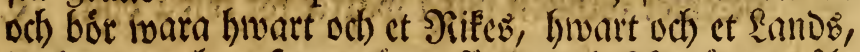

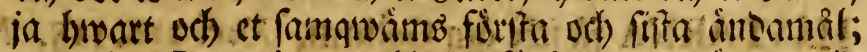
utan bar Botaniquen bittila fortit begunt litrib wio Academierne, fcom unimet begnut sininingoin ofte

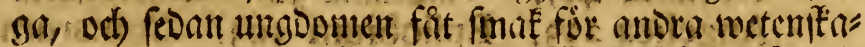
per. Scroarigenone feed, at alt for fa bituti funnit fit noie; men Biffup Kaldsenius til forctummande baraf, har formast Provincial-Medicum, at twenne

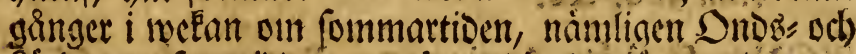
Súroags eftermitidagatne botanicera for ungoomen. Den tio lerret fommn, om elieft thiggurin acconomi-

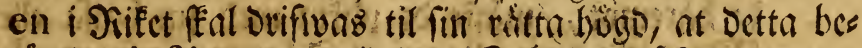
rónda inftitütun nio alli Sdjofar misfife autagns, 
til ett etvig heder for $\mathfrak{B i f t o p ~ K a l l s e n i u s , ~ f o m ~ b a ́ r u t i ~}$ warit Den forfta.

* Kolbecr. quart. $7 \frac{z}{2}$.

SITUATIONEN af WESTMANLAND beftos bár pa orten uti wiot utftricfte ÅKERFÅLT af tyggade actrar, * mes en lós, od) ej finnerlig liog flog, form betnefte KULLARNE, bivaremellan ANGARNA

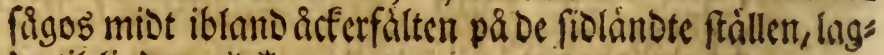
De till limbar. **

- Kalm bahus 7.

* Kalm Bahus. 10.

\&ingarna woro Denna tiben pryboe of SKALLLRGR ASET (Flor. 503. B.), fom giorde Dem gula; af \&NGUtLEN (Flor. 44.), fom giorde Dem bruita, ocf of LUS. ÓRTEN (Flor. s05.), fom bár od Dár gjorde Dem bruna.

MAJBLOMSTER fallades hár Ranunculus acris, (Flor.

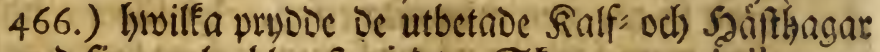
med fina gula blomfter, Dem Staparen privilegerat fơr bâfar och fánaocn. Det ăr merfivàroigt, at fe Den allmife Géaparens mifa inrittning hár pir jordelo: tet, huru fom han gifivet wifa orter àt roifa bjur, och Deremot forbudit wifa waiter for wifa djur. e. g.

HÅSTARNA fá icfé áta Galium, Flor. I 16. Ir8. Myofotis 149. Agrimonia 394. Hypericum 624.625. Ulmaria 405. Valeriana 30. Convallaria 273. Angelica 233. Epilobium 303. Comarum 422. Pteris 843. Geranium 572. Trollius 474. Aconitum 441. Arnica 684. Phellandrium 238. Alifina 300. \&c.

KONA fa ej âta Arnica 684. Ranunculus 466. Caltha 473. Aconitum 442. Cicuta 239. \&c. Synda De baremot, måte de plifte meo fin hálfa eller lif. ZBore iffe Denna Sngen af Gioparen gifwen, ffulle ct Hags 
fagb Sireatur få áta ut mifa tracter, at anota dár fifulle od af bunger. Sairaf hafiva Sollimbarne en regel, at Dár 8 Durt utbetat, Dâr fumna 2 Sajtar fóa fig,

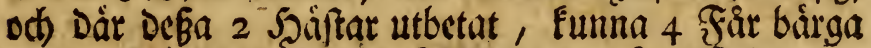
fig. : Saartil Eommer ocf Den lagen, fom Sfaparen gifmit wifa Diur, at gnaga nirmme intil joroen, aut andra, pá but Delt cha fFal lemma nigot for Den an bra. Detta Argument on ceconomia divina leumnts rvibare for dem at underfofa, fom bo pa lantoct, od) Gafwa tilrricfeligare tio; oc Eumna láttaft fe pa ojuren, hwilla toaiter brwarbera llaget farara at andra, belft dá be icfe tro fá finmertigen hungrige; Detto boller ing fa mucfet mertwárbigare, fom Det liggge grumben til Boféps ffofilen, bivilfen ar ben fompignite delen af œconomien.

LöFTRÁD woto unet Gír planterade omfrimg gardorma, an tilforenn; buatof tommer at Weftmanland fer altgenámare ut, ân Upland. Jyir fomliga ftällen refte fig ftora Lumbar i hogoen, fafont oe fisiffa cafteller meo perpendiculaire fioor, od morffgiona woiggar af

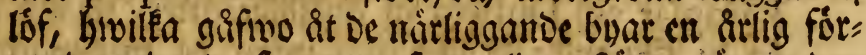
niyad pryoutao, fom war fammerligen fforre, an at ocn meo penningar funde betalas. Det wote ci ringa probnao for Silfet, om 2lmogen sfincralt i Smcrige funde formas, at woid fina garoar plantera loftrint, hailf af De 4 higgftammane flagen: ALM, LIND, LóN eller ASK, famt wid fine giâtocsgarrder KORG.

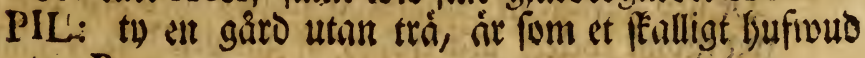
utan Peruque.

* Kóprngs ftao uppehólt of icfe lárge. Ji el ârevort ftodo forljande Inferiptioner, nántigen pá elta fidan:

Nune ULRIC A redit, redeunt $A D O L P H I A$ regna, Queis ego nec metas rerum, nec tempora pono.

Эa antora fiban:

Regia Virgo venit Regali fede, Toroque, Digno Gotbis, Cerolisque, Guftavisque datüra eft.

$$
\mathfrak{3} 4
$$


* óstuna quart. 8.

Sm natten Grwilade woi i BrórKSKoo.

\section{Jun, I 4 .}

Drobften BREDENBERG i Biơrffég war ben curieufafte iblaito alla Paftores, fom roi ráfade pa maigen; ty ban bade fatt fin ro nid lesiga timmar, odi dä ban war fri frân fithlanderoen, uti oet, fom Slaparen giont til mannilfenz nuje: San bade anlagt en liten trás gâto, od et litet orangerie, uti hroilka ban fórféaf:

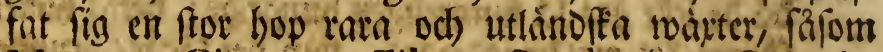
Myrten, Citroner, Fikon, Stockroler, Gerania Africana, Gnaphatiun Americanum, Bellis \&c. S bufen woro hela weiggarne betácfte meo nat of ftâls trî, imnom fywillea en nustenbet CANARIE-FOGLAR

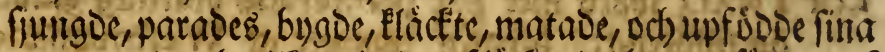
ungar, under hwoilfen tio Den elacfande honan föDsez af fin man, och be utflactete ungar af fin moder, til aferie Darenns nije och tideffurorif. San bave slimmande Pafoglar och Dufwor af atteillige arter, ibland by wils

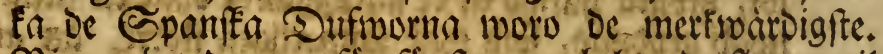
SBien arbetade utan för fónftren, od) ligade fig in til froitmnits.

SPANSKA DUFWOR, fom fór Detta warit nog fâlle funte i Swerige, furtianta fornemtigalt upmerefambet. De aro náten af enabano ftortet med Rarpar, haf: wa luona fótter, gra fropy/ af gulody Lazur glanfaus

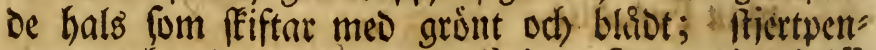
norne och) wimgpennorne (rectrices \& remiges) th wal, fom de fma fiaborar, fom talfte benen od) nafivet wio bes bafis, woró bimita.

HWITA JKORNEN meD róDa igon od fander, famt

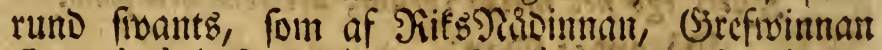
Cronttedt blifwit ffänt til Mufeum Academicum Upfalienfe, baje bar warit fangno iffogen. 
BRÓMSARNE (Faìna Svecica @ ro47) fitiobe med ó par woigentit Arboga, at wi meo fiourta móon funs De bolla Dem ifrän anjittet, broilfet bunderna fade bes tho a regn, faft bimimelen roar antua belt Elar, od eos len feetr miyctet bett.

ANGARNA, befinnertigen De fiolándte, frodo då fót tident -... formligftádes helt bruna af BRUNHWENEN ( Flor.

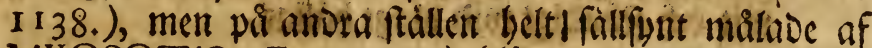
MYOSOTIS (Flor. 149.) bla, od af LYCHNIS (Flor. 3\%84) rióda.

* Arboga. quart. 4 . * a

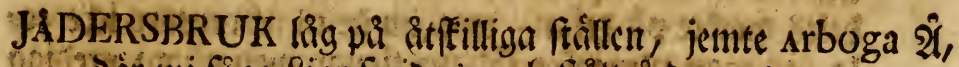
Dár wi fago siar fmidas, ved ftâttá Dragab.

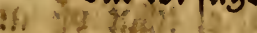

ÅLSANGSBRO ffilbe Weftmanland ifran Nerike, wio

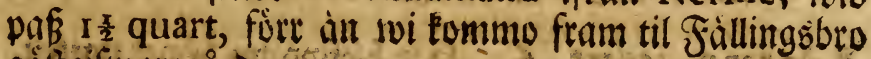
giftsifiwaregirio.

\section{NERIKE.}

* FALLi

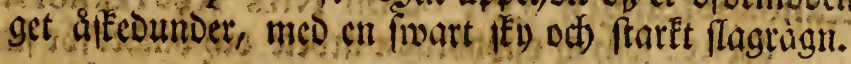

KAGLAN, emfor feog af ntager fanofed, off boga Sallar, lâs cmellan Fállingsbro uch Glantshanmar.

VIOLSTENARNA , fon mátites genon fin rósu förg,

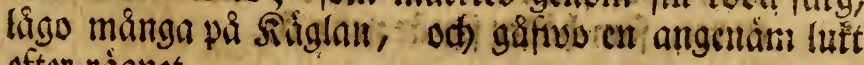
efter ràgnet.

- ceter

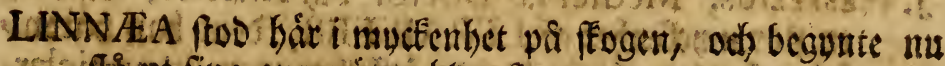
- scis ifir ut fira angentima blomifer.

WESTGOSTAR motte OB trente fruceen, fon orefino 2 I lofe bajtar, laftade med. Weffgóte goos: bivar ed) in baft bade en pába of groft baifnit bunden on mulen, Ris: 
at han iffe pa refan feulle uppebarla fig med betande toio woigen, ods) lita wail ága fri andeoriggt.

BAHL lägo âtffillige pí úmfe fibor om maigen pá Kàglan, til siminnelfe of atfeillige birt pí féogen forrofyctade

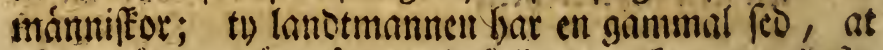

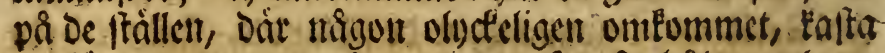
en fipp; gren cller ften, broataf befé biten upiomo nit od ftänoigt mio nugt hállab.

* Glantshammar. quart. 7 .

RUNSTENEN, fom ftoo iente toigett wio NARSTABr, lemnas åt Antiquariis, tillita med slla dem wi frams Deles mertte pa refan, emedan de til áfinenturs icfe tos Ia, at man utmarfer fadoune ftenar meo nagra oreciga bofftáfiver, Dá roar tio och calloamial cj tillit of at berwio lánge blifiva uppebâlne.

* Órebro. quart. 6. Jjír blefivo wi ófiver matter.

$$
\text { Jun. } 15 \text {. }
$$

SREBRO ftad uppebúlt of ófiwer Sündager.

Landshoffoingen Sycre Baron Nizs ReUTERHOLM, eir

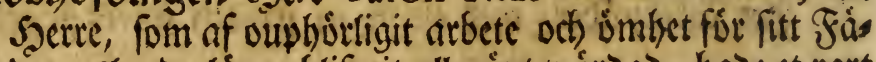

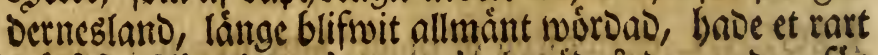
trá från Siberien planteradt i trípgároen neDan fợ Elottet. Det Eallab Caraguana, odf ât et fpecies Robiniz, famt et Siberinkt situ nied bla blonmmor, od en rot, fom baller fig år iftån år.

J. Hessenuus, Medicinx Doctor och Affeffor, wiffe of cn ftor mydenhet of LERARTER, Dent baut batromis Ering famlat, buaribland atffillige sooro til particlarne fá fina, at be táfinbe meo bolis. Saan hade off âffillige lorter of ASKA, Dem han brint of torf, af Giwilfa criber woro hivita fon frita, ody anora fivatta, fom blecf. Soan hade látit mala RENMOS- 
SA (Flor. 980.) til miol, od) gionde nu forfol, at hair of bata broo, fait mijulet gaf en nifgot woidrig odj langs fam rmat $i$ munnen.

von Aken, 2lpothefaren, anftâlte for of atifillige experimenter meD De phofphoris han fielf tillagat. "

CANARIE-FOGLAR funnos los famma sfpotbetare til ftorfta mucfenfict. Sumliga hade emot naturliga lagar beblandelfer med andra foglar; ty ban bade bops patat en fteglitfe zan med en Canarie zona; Defic forenade lade rigg, men äggen labeb under ätr Canarie-foglat, fom dem uttlictete, odf ungarna upfoode: Defe blanoningar blefivo til fárgen gula miot pa wingar: ne, famt bruna pa froorna oct) broftet. F(potbetaren, fom uti 16 âns tio, genom Canarie-foglar: lucfeliga fét: fer blifwit fa roan wio Dem, foin nigon Tyroler, gaf nig fóljamoe beráttelfe om oefe Baftarder:

"Seilnaben emellan en Baftard vel) äfta Canarie-fogel "Dines resan ifrän ungoomen uti oefs bo, at battarden "ar ftorre odf ftartare af ? aturen, odf nat en faban "ar fullwuren, Ean man fifa bonom med bara Cana"rie-fió. Gूngen âfta Canarie Saane ftấ Şonan "mest; Därtil trifrwez San foun en Stegfitfa uti ftart "Élo pa en Eammare, lowilfet en céta Canarie-fogel "iffe gerna tol. Firngen ât liufare, ẩ en Steglitfa; "och dar Steglitfan fer robo ut um hufwudet, fer Denna "branogul; bants lat, nair ban fiuger, àt náftan fom "ch Steglitfas, iblano font en Canarie-fogels, quits "trar falta fom en Canarie Sonn, men fjunger icfe "fardelez.

"Şwad parningen meo defa rátta Baftarder wibfont= "mct, bar jag Deraf 2:lle baft, vef) fom jag miritte, "at en af Dem mafte wara ban och bont Derfore fatte. "ing Dem anora siet ibop at paras, men ber. blef intet "utaf. Se patade fig icfe.

"Treoje aret fatte jag Dem ihop, iblans mes en affa has "na odf ibland meo en afta hona af Canarie-foglar, "o\& on of Defe Baftarder(fom jag forut tinfte frulle was 
"ra en Gona parabe fig meo en áftu Canarie bane; fes "Dan flapte jag Dem uti bacfan til fiere ciftu Canaric-fos "glar, Da trängoe Baltard honan fig uti ct bo, font of cu "annan âtal anarie-fogel fornt wor giorot, och Baftard

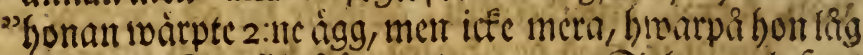
"rodugn. Tag feiriftádade ocm emst Eolen, od) fant "Dem allenaft til fieroe Delen fulla vith torri, limilfa jag "frray tog bart i Dent tanfan, at Defe fratie foglar fful "Le twárpa några igen, fafom de áfta, byilfa om aret "warpa 4 fullar, men Det blef ej mera af Det aret. 2(n: - ira Baftarden parade fig broareen meo baman eller "Konan af if ta linget.

"2ícet Derpá parade jag en tuacker áfta hane meo famma "Battard, fom aret tilforene bade wuipet, ftilte Dem "i en fartétio bur, da dar wal blef pcituing af, men ins gen natpning; to licupte las Detta paret i tid iblanto "De aEta, men Baitard bonan ftal fig igen uti en af De "anora âta Canarie-foglars bo od) lág Derpai i 8 Das gag, lifa fom hon haft àgg, Dár hon boct aldeles icfe "rorpt, utan lág i oct tomma boet Detta itet, til bef ing forftorbe Det. Defe Baftarder Doga altfî fonars "Een i back eller til afivel, utan mera at holla Dem par "couriolité, emedan de elieft tàmmeligen wal trifs "wis.

"Canarie-frỏ bar iag off fad på mina sfrat, och funnit, "at Det måte bittion om waren fas, fú blir Det moget "Fent om boften, och fin af 2 feilputo fro befommas " Sumna igen, men aftren minte ci roara for fet, els "Ier for mucet fiwart ioro, th Dir sifwer Det woil ftora "ap odf grof halm, men Fommer foga til fin mognao, "utan blir grónt ánoa til October manno.

$$
\text { Jun. } 16
$$

Siefan fálbes ifrån órcbro til Weftergỏthland. MosÅs. quart. 4 
BIORNUNGAR frambades 2:me fiucfen af et halft at: ith Der, mio y osas, a $2 B i$ figo med formudran Deras meofísode nighet, at fa fima ve moro, fá bade twa kars

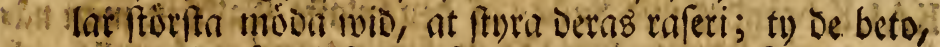
(bo rutte od refroo pil alla fiour, od) taftade ofta meo en crin farbeles wighet oct) fturfic omfull farlen, fom fËulle les ia Dem.

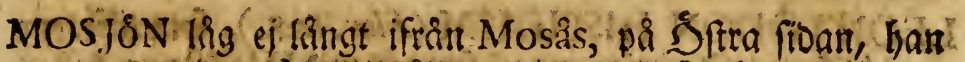
wat trio pris $\frac{1}{2}$. inil ling, timmelig fimal, od ar be faltot for fina fora ruoor.

en MOSSA, tummclig ftor, låg et ftude ifrån Mosås, p\& hogra banoen wio wagen, dà han medelft en bro un Del landsirigen bade utlopp i Moljón. Wratnet i - SMofan froo $i$ perpendiculair bogo med fión, funoe Moşun fále ocs ei Denma tioen görns frutitbar; men ef ter roD:De ar, eller nar twatnet med tioen forminffats i fiôn, och od cultur tiltommit, larer Den Eunna blifroa en barlig ang.

PLANTELAFWAR woro mid Stendby anlagde pà 6 alnars böga ftenelippor; De fago ut fifom grumben til et litet for ontigt bus, meo z:tle floos omlager of frocs far, hivilta afwen fom ftenarme lutade \& fiver, at De= fo bittre emottaga Golbadect. 2irtia invention at byga Srmosetappor pa ftenbillor. Slll ting Ean blif: na fiuttbort, allenalt man fan ffafta goo my 1 a, od atbija blobu.

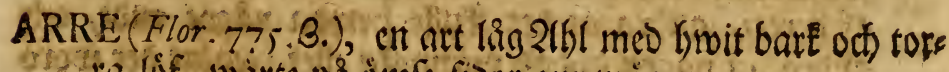
lia lof, warte pa inife fidor om toigen.

* Schyberga. quart. 5 .

HALMASEN ftrifte fig langa ftuctet mio landstoigent

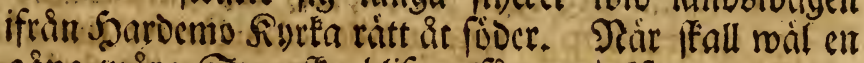
gâng wira Grwenffa blifma fa upmàrefamme, at De beftrifiua alla Siverigez afar, och Derigenom lägga grunoen for Phyficis, til Éweriges förlta landande (d) origine. EKAR- 
EKARNE ftodo blablsfe bogft up i topparma af mafent, fom átit up lófiven; men iffe fá pá neore grenaena.

KALK brándes wid Sko̊rs af Den fulafte Salffiten man nàs It: gonfin feot, th han fâg ut, font coagulerad mulla, oct) 4. gaf mucfet grả Sinle; i bruf emottogo I2 tumnor brino Salf, 2:ne tumnor fano.

LEONTODON HISPIDUM (Flor. 628.), for tilforene bliffuit $i$ Siverige endaft funnen mio Wadftena, ${ }^{*}$ fagg

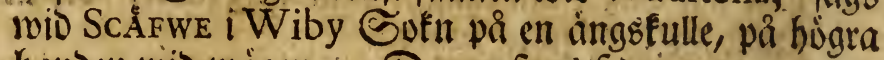
handen nit norigen. Denm fanb febermera nog alls Wt mänt of fruer liela Weftergótland.

* 4 It. Gottl. 339 .

* WREDSTORP quart. 6 .

BULLERBLOMSIER, Heleborus Trollius di-

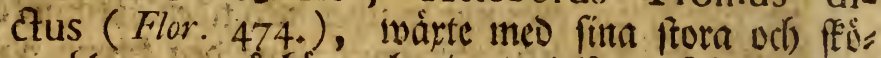

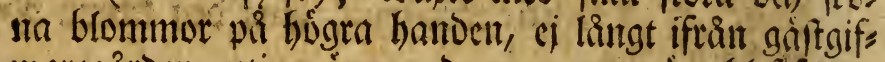
waregẩben, utti en ảng; Denna rara wât blef feoes: - mictu allmaini Weftergótland.

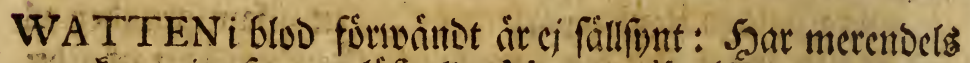
Eommit af mattulsíf eller Monoculis (Fauna I I 82.); mett wio ónnaby fants et blooröot watten uti elt Mio: graf eller Difé mid waigen, fom icfe war fürorfakadet af infecter. Nair man bölt pai, at utreca betta phxnomenon, ftantade böndarna, forn fároabes par woigen, $i$ formutoran bärisfiner; Dá fomliga mente, at bet blitwet af hereri, anora at bet feulle betyoa nágot onot, ofth anora, at någon disdad minniffa eller freatur Der noro

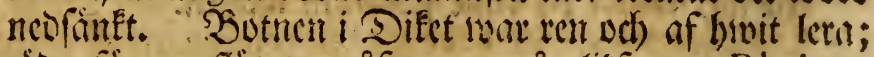
rüsa fïrgen flut torr affwan uppi, liffome et Rhabarber palfiver, ofh blandade fig iefe getra med watnet;

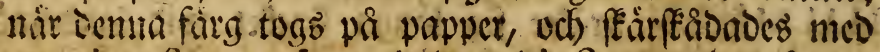
et microfcop, fom giorse objecterne tio gingor fuivre, nooro particlarne rumoa, men fa fma, nt de

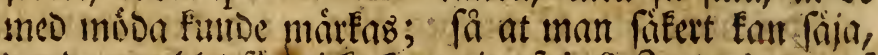
Det Denta bloofurg icfe fommit of infecter, ej eller af 
niggot unocrioroift mineralifkt todfenoe, uten tycfes lieaft marit ot lags Byflus. Et par grodor lopade up af Dettu matnet med rida bufiousen, roi fiffade efter Dem forgafinces, od trodoe belre, at De blifroet rivde firgade of Denna bloo, an at oc Eumnat utiputa en fo roio, tort oct) lós materia.

TIFWEDEN, en for ffog meo fanomo, utan fontrerliga barg ellex bogar, begyntés bar, och ftrdfte fig bela 3 milen. Denna bar $i$ forna tider warit namnitunnig of Sivfroare, afroen fom Sidiglan, Sismorden, SeDen od) Bonnfog; men mu ar bela Etoerige fá fätert at senomefa, fom nagot land i Europa.

STRUTHIOPTERIS (Flor. $84 \mathrm{r}$ ), ir et ratt flig af Osmunda, fom tilforene î funnit allenaft pa fá ftúllen i werben; námligen i Poorige, Sark, Sttrocis, ody for nigra à frocin pa Sivffa fícan. Jag bar allenaft funnit bet famma wio Sar. Prafid. Cronftedts Bars

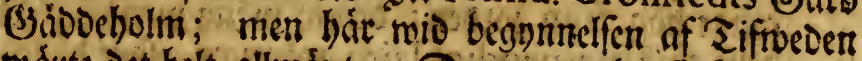
rodite det belt allmant. Senna ormbunes bade moll icfe fät fina or, men funde dodf' latteligen fanmas, fa af oe fiolgamle aren, fom befiunerligen af fina blas, broilla woro ftâlte $i$ ring, afwen fom ficorarna $i$ en volant.

ARNICA (Flor. 684.), *et Ipecies Doronici od Centaurium, fopas batgge ifran Suffano til mara 2(pott)e

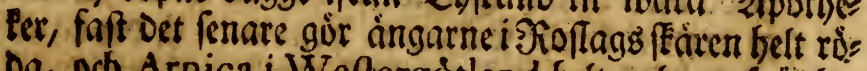
Da, och A rnica i Weftergótland bcit gula. Sotir be gunte Arnica forft finas meo fina gula blominor, fom ftadigt toinde fig efter Eolen, beftimbigare in nigon Golblommn; bun bade fat privilegium of Stapas ren, at rara fri fór for od oprar.

* Kazm Bahus. 14.

MISSE cller Calla (Flor. 744.), wdate i alla Edrr pa Tif weben, tibillig til brio i nopfall. * * Flor, lapp. 320.

* Eodarne quart. 8. Sgár Glef rokint nattligg. 


\section{WESTERGO̊TLAND. Jun. 17.}

Sefan fortfattes i bag ifrạı Bodarne til Marixftad.

WESTERGótLAND tog fin begunnelfe ftraft miD Bodarne, Dar in liten buck, lfilde Det ifrim Nerike.

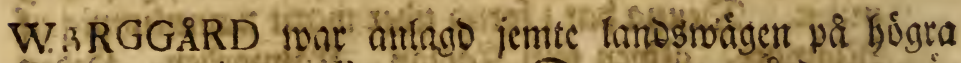
A1. hamben inemot BRoten. Demt toarggard war nis

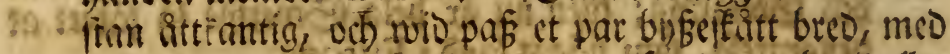
tall och gran beft titt betwikent. Shan war bugo eller

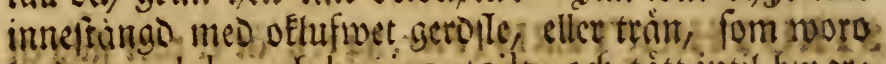
lagoe paralelt, of horizontailt, ofl) tatt intil hyvats anota til 6 almurs laingo i hógoen; Denua fá bógoe serocrigaro futwoe intit ad angulum rectum, fo at

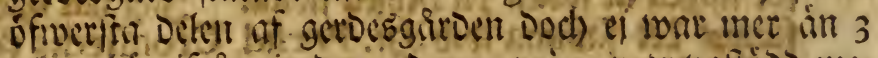
afinar hos ifran jorden; Denna woigg war spltiogo mes

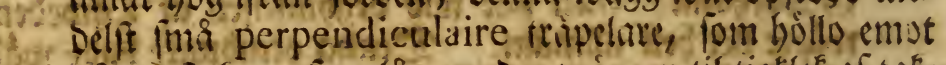

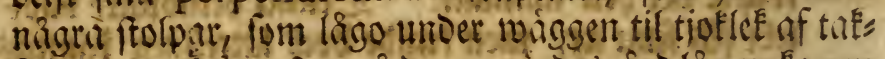

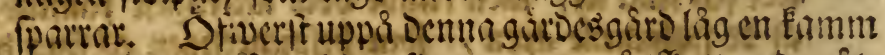
of tribinnar, font finte fina thggar bis fitut neder at, ellere itint. SGwarbera of oefe tripinnar cller taggue

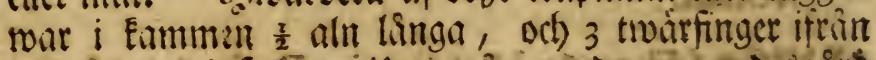

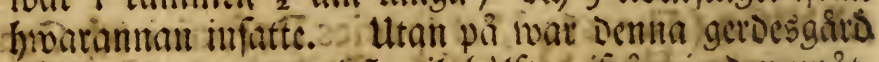
ftós mes antrat getojle til halffen ifrân jorocr upart,

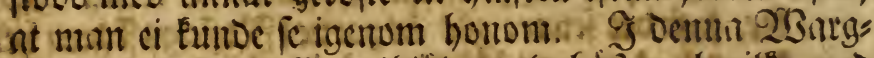

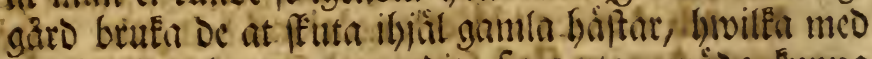

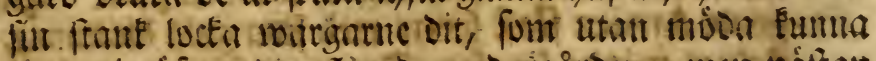

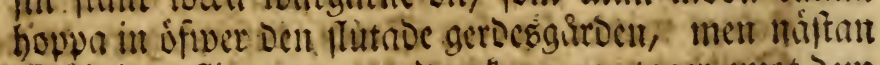
oinujeligen fippa ut, enceinn tanumen tager emot Den i uthoppringell.

* BRoTEN. quast $4 \frac{\text { T. }}{2}$.

SNARREFWER Enllloes Cufcula Flór. 138.), fom maips te par bumlen. 
* Hofva. quart. $4 \frac{x}{2}$.

Soir ar uti September, affwen fom i Scheninge, Den an= fonligafte Sremartuno.

LANDSWTAGEN mar nyligen omlagnd; grulet, fom war fort fram pir woigen, beftod merenoelo of en fattig jernmaliu, lif s)rom cllcr solumalm, fom coagulerat mia fano til frorre fenar.

* SkARpa. quart. $4 \frac{x}{2}$.

LANDET bconnte, ifrin Skarpa alt til Marialtad, of blif:

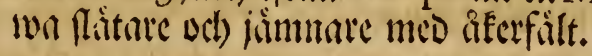

SMULTRON woro mu mogita, odl) funtros alleftitoes til ftorfta buntoghet fơr en ringa penning.

TIGGARE bave feocketal: famlat fig wo lanbswaigen, at man Enapt foot flere i Brab nnt.

ÅKRARNA, font allit woil ryggabe, hioe pa fitourna wis foren, foun feilier ruggar!te, milf fin gromfte of wintermatnct; men lambtmmmen bade. i Det fiället fiot forn, eller butror jente forett.

* Mariåstad. quart. 5. Sait anlinde mi mot aftonert.

\section{Jun. 18.}

MARIÁSTAD án liten, men behagelia, ligger pia Sftra fioun af fora fiun WENERN, bugd med fima, Dode ticke tribur, gaturna áro läta od) ljufa; bair refide. rade InnDshofoingen Falckenberg, odh hă bodde Den endafte Provincial Medicus bă $i$ lamber Doctor Guftaf Zimmerman.

MARIÅmolm ấ Sanobofoingeffatet, ligget ha fiblvaiftra fioun om traben, fringflutet of fluoen' Tide, fom Dcs lar fig och formerar Denna lilla $\$$, farf án ban inflys ter $i$ Wenern. 
KYRKAN mar wackert utprobo immantil. Def grumo har blifivit lago 1593 of F.C. ARL IX. od fullbordond I6 I s, men brann tillifa med bela fraden 1693. Soun blef Eallat Maria Furfa, Etnoen Marieftad, odh Refidencet Marizbolm of Giong C ARL IX. fürfta Sicmill Maria ifrän jocioclberg.

KroN-PRINCESSAN LOVISA ULRICA hade $i$ gar forlta gangen anlanot bit til fradon med fin Bemil Scans

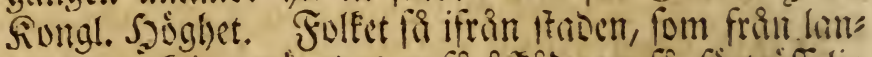

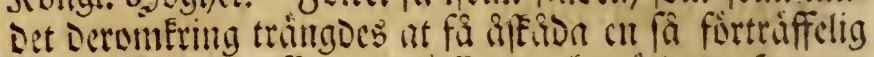
Princeffa. De fingo, De illende oct) wo broade Sacnucs feinthet, wishet od milobet.

WEXTER fring ftabon fingos inga merfwoiroige, fórntan PYROLA minor (Flor. $33 \mathrm{I}$.), fom tillifa mio Jafione (Flor. 713.) wijte woilter om Marizholm.

\section{Jun. 19.}

- RESAN fiñltes ifrîn Marixfad til Kinnekulle.

WAGEN ling it fóder igenom en feog, lif meo den, fom to at jer i Upland.

* Bjỏrksíter. quart. 5 .

WAGEN nict at waffer igentom oc barrlignte angar, war

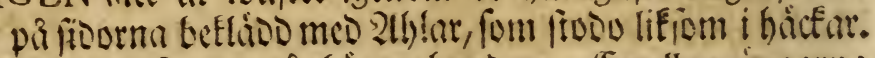
Venern fintes pâ bögra bamden. Emellan angarma mar niggon liten féog.

ANDORN cller Marrubium (Flor.485.) Ibixte bir lym liogt.

BONDSTUGORNE Gaic ofta pir firbra.fioan af taEet fit liue od) fonfiter, * od) fipiellet ofiman pa feoritenen, fom upratidice utan for bufet. **

* Kallir Bahus. 17.

- Kaim Bahus. 253.

ORR. 
ORRNAS, et Şäteri, lagg wio ftranden af Venern quart. $3 \frac{1}{2}$ ifrän Bjórk lätter. Denna gåro mar bugo pá faut: ma fitille, fom ARANÁs gamla flott * fordum ftảtt,

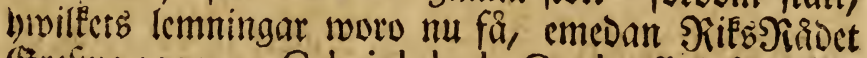
(Errefive agnus Gabriel de la Gardıe feal látit fórá muctet birraf til Lechó flott. Rudera efter murarna of A ranás flott finteb p\& ct uboe, fom p\& 3 fibor war Eringfluten, odh med atfeillige grafiwar feito ifrän lans Det; broilfa Docf nu til ffotrte Delen igenfallit, och) uttors Eat, fooan matnet tio efter anman mimfEatz $i$ Venern, fom alt tyDeligen Deraf merÉtes, at matnet, fom gict omering Denna foltmen, war nu fơga Diupare, än at

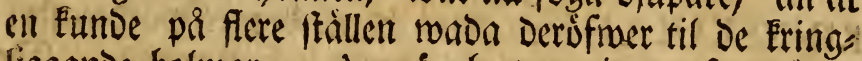
liagande bolmar. Aranås bar warit et af be alfita Sivngafäten i Wältergóthland, o̊ hărligare buggnad ef warit $i$ landet, hălft mio frong Olof Etotteonung: tio, Di det ftod i fin fisurfta hatrlighet. ** Omfring A ranås mar mucfen E⿺廴, och) jemte wờgen de bárligafte Socf wiloe alléer, mâlt utaf 2 lhl, fom gioroe wägen fis nigfonn, at ber Fnapt $i$ Emerige finnes fesinare.

- Messenil Sveopentaprotopolis p. I3. 24 .

** TUNELD. Geograph. I86.

* ForShem. quart. 7 .

FORSHEMS KYRKA war bel och batlen upbugs af groas

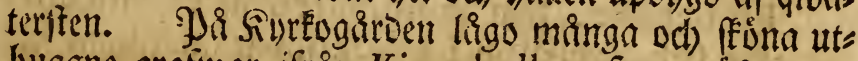
buggne grafrwar ifran Kinnekulle, fom mâft woro bindoer tillyorige.

9 Fivrken hade furnits benpipor af 6 quarters lángo: $\mathfrak{W i}$ fötte at fâ fe Dem, oct) utrơna, om De rooro mảnniffos ber; men de funmos icfe mer.

LANDET begunte mu at befta af fiffa, fom ftod naftan upraitt, od) fififrabe fís perpendiculairt.

TAX eller BARLIND (Flor. 825.) woipte et elloa trá, fom Docf war nog plundradt til fina qmijtar, mid paß quart.

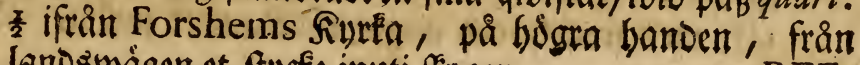
landormágen et fitycfe imati fengen. 
REFGRÁS hallubes bât Lycopcdiun (Flor. 858).

FOLAFÓTTER Eallades bît Tuffilago (Flor.680).

SHOGSVIOLER (Flor.716.), brulftes af butmerne at bick pa miblen, ná bon om fommartioen alt for baftigt lopnat odb felmat, eller formentes wara förgjord; od be taga blawen af ocfe Sfogsvioler od) fila fin miole derpá.

PYROLA minor (Flor. 331.) mixte affuen bair i feos gartic.

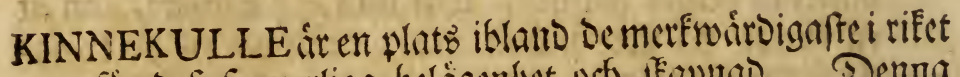
for befi finturliga beligenlet och) Feapmad. Denna Kinnekulle atr et baig meo bresa od) mislinftiga af: futtuingar, eillet trappewoio lagoe fill, fom fumma immes hâlla hela Gofnarne, bmartil fommer, at Debe nflatts ningat aro betafte meo féna singar, mactra aferfailt, fog cller loftrit; Defutan beftir bmartsera for fig of foriffilota barbarter, bevilfar ifwen fom affittnimgarna gî nfiftan horizontailt omfring birgets meielpunct; Dertilmed ato befe affottmingur atfeifoe med perpendiculaire afterbinimsar cller Elefivor, fom lienta de als

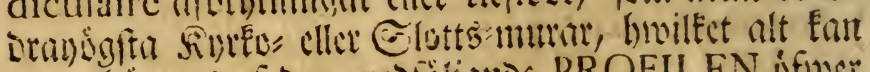

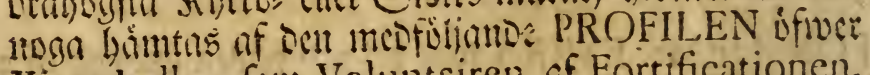
Himmekulle, fom Voluntairen of Fortificationen, Sjr. Joh. Svensfon Lidholm, wair nngentime led lagas re bfouce benma fillie, belt accurat afmitte o(t) aftog.

Strata of Kinnekulle. fom uyftiga i hogocu, langt bogre in Falu grufwa pá Diupst, cller 397 nlitar bogt i perpen" diculair linea ifrin wattu=horizonten i Wener:?, blefiog rifinto od) examinerade - uti luperficie, fâ at

3) SANDSTENS-KLEFW'AN* wat 450 alnar bres, metr i perpendikel meo bela flutuingen $4 \mathrm{r}$ nimar, bejtoo of bmit SANDSTEN, fom fiutte migot pia gult, hes

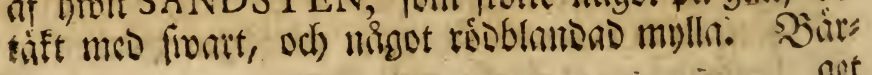
get 
get lơver längt uti Wenern, med famma flutande, lom Det antaget pfwam watrict.

* Kalir Bahus Iij.

b) LIMSTENS-W ALLEN* år i längDen 800 almar, men i perpendikel 36. Ditta ftratum ât of KALK. STEN. fom bair pa orten blifiver brind til brif, och

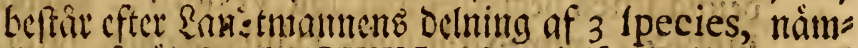
liget of raitt KALKSTEN, fom blifivet til goo lalk genom brimning; af LEFWERSTEN, fom i eloen, ved) Dit ban blifiver acnomwarmo, furinger i fucfen, med farte feott eller fmall; od of ORSTEN, brvils feit ait parallelt cryftallicerad fafom et falt: of fuan pa Denna simften ligger KRAKEBERG i hâtoa od fofita ftrata; Detta bivarf ai talft meo rod jord, uti bwilfen firnes ofta fivarta flintor.

* Ralm Bahus 22.

c) RóDSTENS KLEFWAN * ftiger i lángòn til 800 almar, beftar of ólands ftet, och orr of 3 Hag: GRON GRIFFELSTEN wid paf et par almartiock. Ofroan pá Denna griffelen ligger den fâ tallade gra TÁL.JSTENEN, of brilken gulfiten furffiudigab, fâm paobland, vel) änteligen ligger vfiwan Derpa Den tóba TáLJSTE-

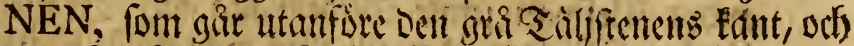

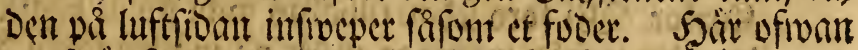

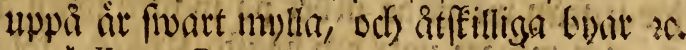

* Kalm Bahus 24.

d) GORSTENSKLEF WAN ât i lintigoen 600 alnăr, ment i perpendikel iftin c. til e. 77 almat. Brtmen, eller birget beftâr of en grof enulig falefter, fom lowar: fen ticnat til Falfbrinteri eller Etemliperi, ody Fallos af bonderma GORSTEN.

c) HöGA BAC KAR oni 600 altuars lingo, af iocl tull, loga grâftenat upfilolo.

f) KRÅKB̊̊RGET af fiunt Gdjiffer, ftaré uds fjocf, til 500 alitars longs.

g) HóGSTA KULLEN, af grof odb hatr fortoften, twiet ifluer ifran g til $g$, af 862 almars lango; men i per-

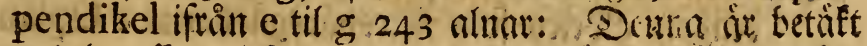

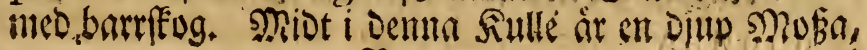


uti broiltens medelpunct fark filnadoftenen encllan s)ics oclplana od) Siterplima Sotnat eller Stogar.

Denna Profil frambifar Kinnekulle ifrån 2̧áftet, dir ban går i Målaren, til Ŝfter ât Fullóla marfert, fâ at Denna linea beguntes mio fiơn Venern, fisomâf un

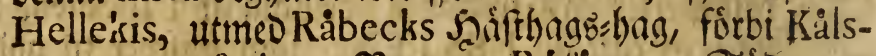
torp, Jutegarden. Noir om Rolatter, Gooer ont Kullatorpet, twert ¿fiver bredoen på Hógkullen, neder pa Sitra fidan emellan Norrffagerne, utmeo Skalltomten, glort om Waffatter, ned uti Käringe-

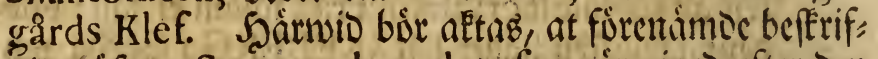
ning offuer Strata a. b. c d.e.f. g. ir giord efter Den fio an pa Kinnekulle, fom wetter at $2 B_{3}$ ifter, cller ât Venern.

LANDSWAGEN gicf up ait Kinnekulle wio Gólätter, pá Deb Trovoofter fita, Dar ban war mindic brant, broariftån han fólgDes pá Norra fioali om Kinnekulle,forbi Hónslatter od) Hellekis Eitteggirder, Dire ban widare fróf pa waiftia fioan at foder, upfór ocit andra Silefiwan iemte Nedelplama och) 2Bifferplana Sivitor. 2Si foro fedan tilbakas miot up at higlta fiut lett, broarifrán wi gingo theder pa woiftra fiban ât Rófatter, od) wibare norr om bogfta fiulleit, folgoe fe" Dermer sftra lambswägen at Siferpland, Siletwa od) Hufaby Rurla, fom ligga langft i fooer af Kinnekulle. SSwad wi pa Denna wágen obferverat, utom Det Sert KaLM hat anforot i fin Bahufinka les fa, ffal hă med fá ord námutas.

JORDMONEN blef :óbaftig, fa frart ini fommo til Gófatter, od) full meo los tunn Gdsiffer, fom blifwit pulve riferad med leran.

PLOMMONGÅRDAR funnę fring Gólâtter $i$ for mul: Eenbet, aldeles fom Sersbargaroarna $i$ Upland, til 
et Elart tecferr, at JJlommonttri bait Gitttre trifivas, ăn pia migot nutat fitlle $i$ Siverige.

SKOGEN på norra fidan om Kinnekulle beftod af (Siran, och) Enapt ch emia Sall.

ENEBUSK +RNA wirte alla hogga od's raka, fom Cippref fer, och) wet jag antmu ei hworfoure (Encbuffarna altio pá branta bógoer Dragn fig ibop pia finorma, ods ftrice

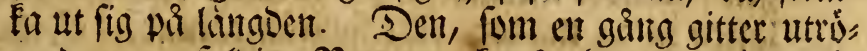
na Demna cufaloiga Paturens Eomit, ban Ean lóra wă ta Srigarosmajtare, bet be án icte weta, och prosa

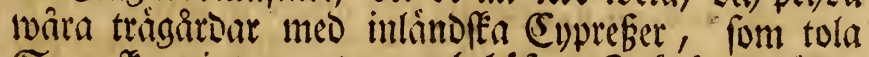
Swenffa mintrar, utan at bebsofiva Srígårosmäita. rent arliga tuftan.

ANGARNA fring Gơfätter frooo bärliga af frima lôftráu od) artiga wàkter, fâfom of

2(pel, (Flor.402.) Sind, (Flor.432.) \{lff́, (Flor.830.) $2(i j)$, 819. 2llm, = 219. (EE, $=484$. Bsetbart = 193. Sgagtorm, = 399. Frtsbir " 195 . Actza = 43r. Vifcaria $=364$. Sanicula 222 . Anthyllis = 594. Melica " 57. Aftragalus = $59 \mathrm{r}$. Mercurialis 823. Cufcuta $=\mathrm{I} 38$. Hieraceum m.637. Scorzonera 647. Heracleum 23 $\mathrm{I}$. Melampyrum $\mathrm{II}_{3}$. 5I4. Vicia Sylvatica 647.

SCORZONERA 647. 1witte i mucfenthet til $\frac{2}{2}$ alms. bogd. SANICULA, 222. tilrácteclig for 21 potbefer. KRUSBÅR, 195. waipte broe 1 íngarma od) i féogen yms nugt.

KALKUNGNAR * fagob kring Góf fatter atffillige i ffos gen, De woro anlagde Der bacten lluttade pai cta fidont, at fom de til en ftoi del blifiwit neografue i bacfen, fá

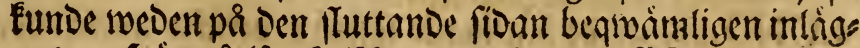

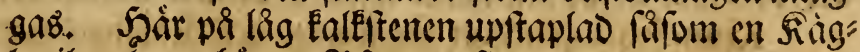
la til 8 aluars 6ọgo. Sja yttea fídan war ingen upmurno ßื 4 ugn, 
ngn, utin Den war til ent Dell Eringlago nted grofiva

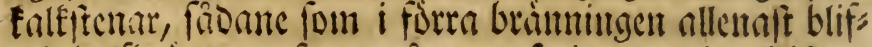

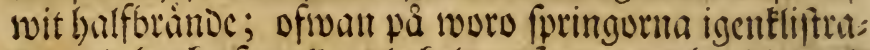
De med brue af mislln ocl) falte, fom wort hopblandoro

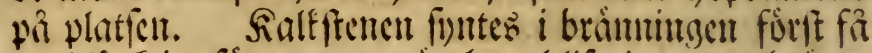
en blinattig fírg, neen nite boun blifwit genombrime, mard ban helt gul. Salfugnen brimbes i 3 Duglt,

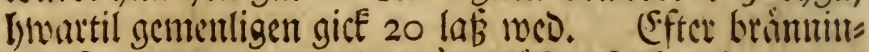
get famlades merendelä 7 à 8 liffter fonle, limarbera lisfect til 24 tumnor, eller fipiltrint riffnad. Eecormes ta fälies lowar läft til $4 \frac{x}{2}$ Dall. S:mut.

- Kalar bauus $4^{1}$.

RALKSTENEN mar grâaftig, och upgrafiucit i Gö-

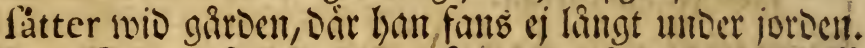
Petrificater fiuntes $i$ alla furinger pá Denna fent fí

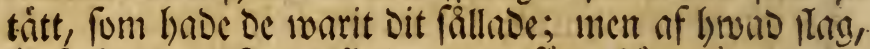

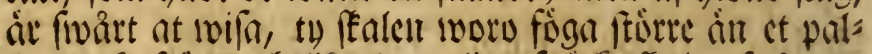

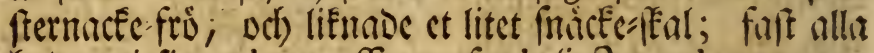
have uti lig crimpreffion of ct infecto coleoptrato.

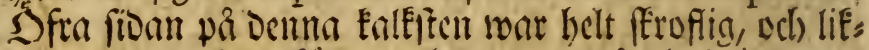
inde et uttorkadt Éarr, ty hon war grii, beftrobo med fina perpendiculaire trubbiga Lameller, fom fiutes

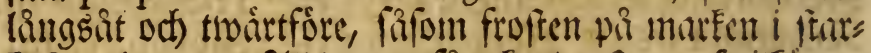

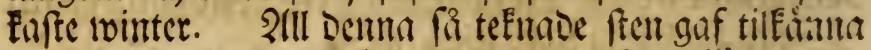
med fitt fitinfande, nt ban war en Lapis fiillus, efler Drfent. Ofta líg Drfen fom runda billar imeti Bent

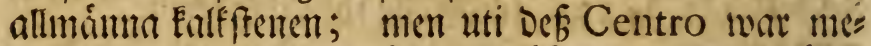
rendels en Cavitet, upfis/D med härd lera cller Litho-

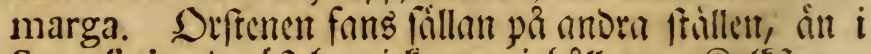
fuperficie, Deráft han iffe war $i$ billar. Falfifenten

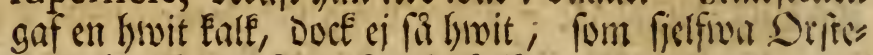
netl, bwilfen gaf ben finalte falt?

LUNDAR af De histligaite lofftrin betáfte notra fiban of Kinnekulle, nconn for féogen och siósfrens flefwam. De lifnnoc meta triginatoar, än wila parfer, och) gior= De Dema orten ljufligate än nagon annan $i$ Swerige, 
fi nt Cättericutue Hönslätter, Hellekis ock Rabeck, fom liggn pa fiesfioan at Venern, mippeligen tilfille if

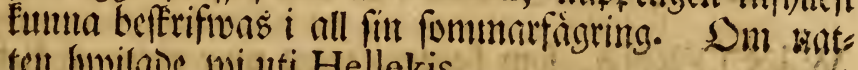
ten buvilnde wi uti Hellekis.

\section{Jun. 20 .}

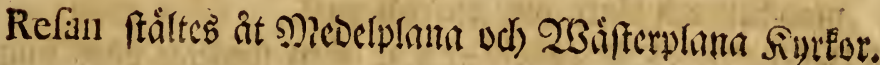

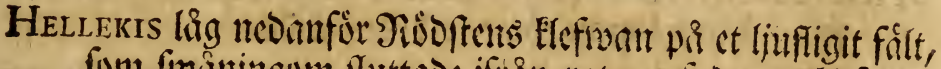

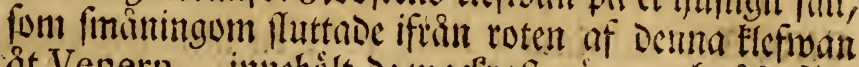
at Venern, immebolt De wactiafte inirg = och ẩerfält,

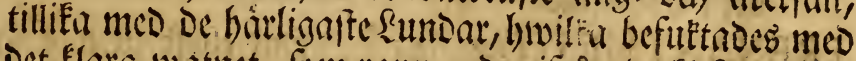
Det flara watuet, foum ramu neoer ifrän be Elefinor eller precipitier, fom lágo Der ofivanfüre. Detta Hellekis formentes fât fit namm af en refe, fon betet $\mathrm{Hcl}$ -

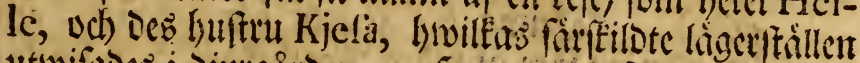
utwifades $i$ sjurgiroct. Sactites genf war mid pá

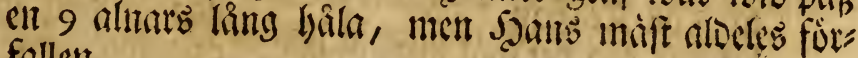
fallen.

WALNÓTETRA wákte många iHellekis trigaño planterade; mcu alla figgo mu ut, fom ftora bulear, fcoan birroa minterent 1740 . Doclagt Deras ftammar. STHr=

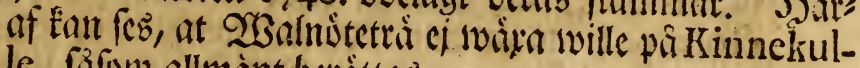
le, fâfon allmànt betîttas.

KERSEBSRSTRA waite nog, faft nin icfe fa manga, fom anira wilon trán. De woro fá bogga, fom boürêtar, Del) alldelcos nilloa, fafom antora feogstrint, odh ingá lumoa planterade: alla tooro af Fogetbirts Mlagen,

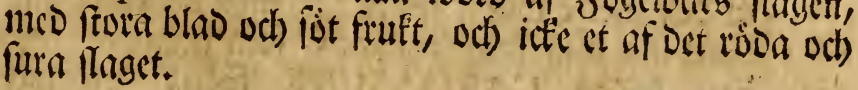

ONONIS, (Flor. 622.) prisobe singarma med fite purpurs fingade blommor.

ASTER (Flor. 696), fom fillan feB, utan inis hafïftonn,

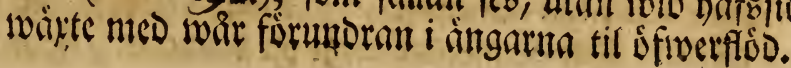


THALICTRUM minus (Flor. 454.), fom cj pligat wifa fig fă langt i norden, waite afiven bir sfiwernit.

WAXTERNA, fom fommo fram utur roten af Elefimant, eller off utur fielfiod des af brytning, woto nog fillfant: me i Stoerige, odb fadane allematt, fom forbrade en

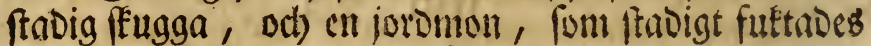
mes Ealt bets flart watten, fafom:

SPRINGKORNET (Flor. 722.), fom foritter bart fitta fro, fa fnart man rorer wio des fetioa.

CIRCAA minima (Flor. 5.), en litch od) ganffa rat ort i Smerige.

PRENANTHES (Flor.645.), form elieft âr nog falliunt utom ffogarna.

CARDAMINE, Nafturtium amarum dicta (Flor. 560.). Denna ort wayte hat ymnigt, od hols leb allmant fore af wara 21 pothefure, at wara Den rats

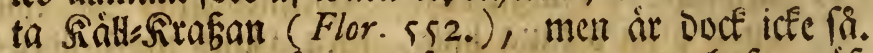
Rifwift kan Det bal meogifwas, at Demna hafwer âfs well fa goD od) fark toerfan cmot férbiugg, fom nas gonfin Den ratta Gálllerafen; men Deremot da de i ftâls

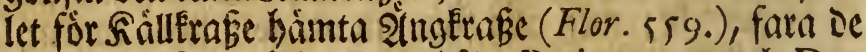
miscfet wille, otf) beoraga bide Patienterne od) Doctorerne; to à mit rio, at 2lpothefarne plantera

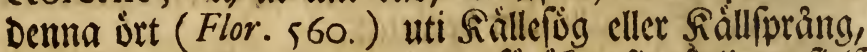
Der bon en gång infommen, fơrofer fig årligen fielf til Deras tjenft; bälft fom Den rätta Sällfrafen pa De

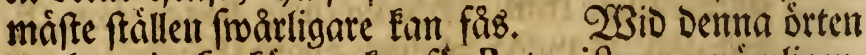

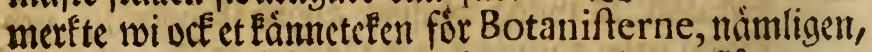
at Dé itielfe roäer fuller raf, men Deriemte llar ut ex fummis alis fma ftolones, fom taga rot; Limarfore hon fallag CARDAMINE foliis pinnatis ex funmis alis flolonibus reptans.

CARDAMINE apetala fam Den urten fallas, fom under namm af CARDAMINE foliis pinnatis, pinnis laciniatis (Flor.561.) at fram= ftílo. Set âr merEwarbigt pá Denne ort, fom til ut= toâs 
wairtes ffapnno har mucén lieflet med Sophia Chirurgorum, at bon if al frambringn flores petalis dettitutos, brvilfet ir swant $i$ bela claffen, om man envait undantager LEPIDIUM (Flar. 534.) Sloms morna befti af en fortolt blombsole, eller Calyx, fom

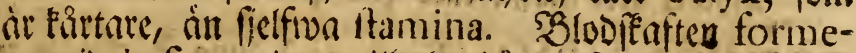
ra wio bafin twimme tilbaten bogoe fyetfat, hivaraf pijelten fer ut, fom moro bon fipulis inftructus, Det man ej eller martet uti nâgon nunor af famma art.

STACHYS Maxima (Flor. 489.) frinfte mio goroebs gârdarna.

CAMPANULA Maxima (Flor. 180.), fom piffi frits

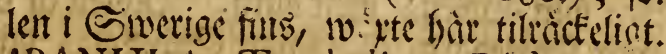

CAMPANULA Trachelium Dicta (Flor.181.) aff toen bair allmint.

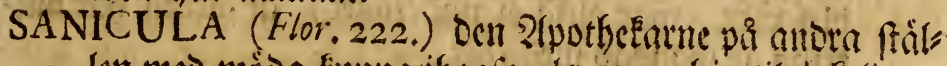
len meo moda Euma ifoplamila, wat bar tilracterig.

LUNARIA (Flor: 529.) fon endaft tilforene ar fumen i Ekine pia fa ftallet, wiltes of of Sat. Lidholm i Hellekis Djurghib, brollas blommor lutta angentimt om nattterna.

RAMSLÖK (Flor. 263.) fallabeb batr en ơt, forn witte afwen wid Den furra, od) at utom Gläre, Sivolagen od) Sottlano * fállam feod i Evocrige.

* It. Gottl. 169.

COCHLEA fpira aupta (Funa shecica 1298.) fants acuita nog i Elefivan. Deśf fabl loar firgad, fom et fétos padoe feabl, men fielfwa biuret war bleft med fwat ftrimma, fom pa hwardera fidan frutite fig ifrân det fitsre bormet eller ógat ut ât ruggen.

PHALIENA viridi-cærulea (Faun. Svec, 838.) fanb i antren, DCB born eller antennx wora ef pectinatr, utan prismaticx, eller fubclivatx, áfwen fom PHAL/ENA (Faun. 814). Denna blighotra Fiatilen Ents las PAPILIONOIDES pratenfis viridis nuinor. Pet. Muf. 35. n. 329.

NATTSKÁRRAN (Faux. 248.) hade byod fit naffe $i$ Den feogrifa Elefiona af ren fwart mull, lagot Det pa hori- 
zontaile flippar, och tif fom fivarfivad set pa inte fi: Dan til feapuno, fom en lmornft, blvilfers bredo mat ct quarter, men Djupet 4 tumm, wiggarme woro alt: fa runoa, odb helt bars, men botnen war betieft mes mucfet litet mof́t cller quisquilier.

DOFHJORTARNA (Faun. 40.) lups frâcetetals uti ca privat ojurgario wio Hellekis.

ANDRA KLEFWAN wat naftan perpendiculairt af: bruten, Deb horizontaile hatluar befoso bitr woid Hellekis af roonftig grof fal", fom habe atteillige fnular ifig, frora fom ayplen; ment olifa. Sioten af Demna

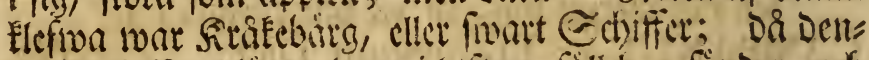
na Edyiffrem limac legat i luften, füll ban fonder, och blef rod; wat altia ef unserliget, at jorben bat oms fring ar blamdad mco robt.

HONUNGSDAGG, af Det howita llaget, (Flor. II 24.)

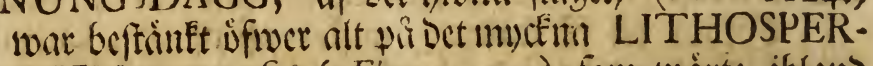
MUM arvenfe (Flor. I s2.) form warte iblanto

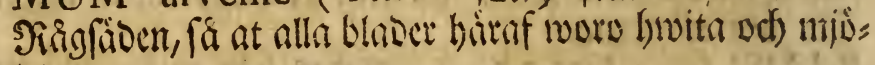
labe.

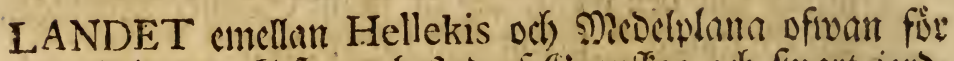

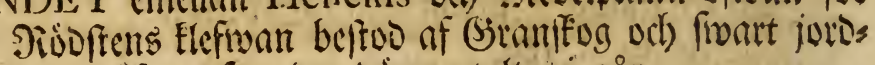
mon, âfiven fom Den Dirum taltes i gart.

STENEN, fom Per Stenhammar upreft til imninnelfe of wair nidigfte Somungo miftambe hili pai sortelt, Den 24. Sept, 1728. ftod toid wigeh, fe Kalm Bahus 39.

* Medelplana Kyrka. quart. 2, ifirin Hellekis.

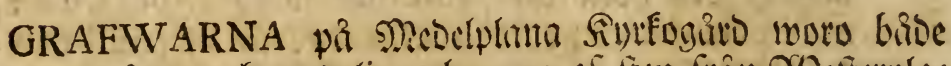

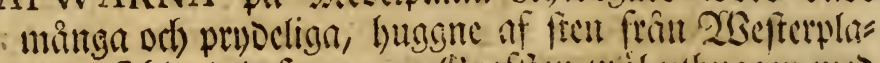
na. Golano Debe war en (Sirnfiren wal utlauggen med

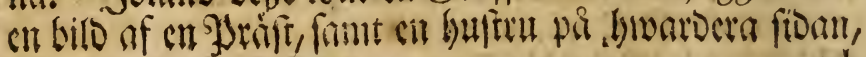


Qch) 14 barn frôlde unser füráloranta nti miniateur. IJriffen war fliddo efter modet i fin tio med Callot,

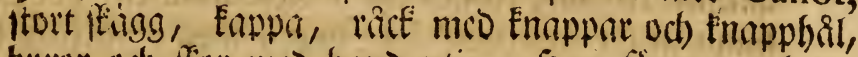
burer od feror mo bano uti: SJan furmencs hetat Martinus Zacharix, fom fétat warit bair $i 30$ in Pattor. Füljande verfer lîfteb pấ fanma fen:

Eximius Praco facrorum conditur bicce, Prapofitus clarus fouvi gravitate verendus; Qui fatu: annorum reddidit ofla folo.

Has focias binas, bis feptem bec pignora lecti Fidit, quâ fenii dulce levamen erant.

O venerancie Senex falve, falve inclyte myfies, 2ui in coelis velut ftella corrifca micas.

En LIKSTEN ftod i Choret jemte Sacriftigan imfatt $i$ wingen, uti hivilfen Harald :Ericksion (Raj.) til Selmfitter med Deß Fru Ingeborg Geta moro i fin bela frow: ftorlet uthuggme 1635 .

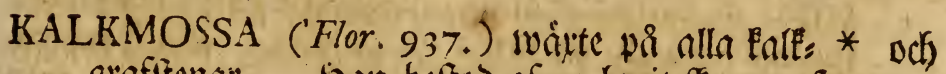
grafitenar. Scan beftos af en hwit feorpa, fom mes rendels war runot utwiognot, och hade midt uti fig âtifillige fivarta puncter, foni woro omgjorsnde meo bivit tant; men bela s?lokans vettra fant war fioart: aftig, lowilfat Docf föga mitreas fumbe, dirt icfe $2:$ me

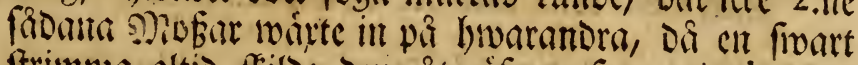
ftrimma altio feiloc Den at, sffoen forn uti chartznofan (Flor.940.)

* It. Gottl. 183.

LANDET blef wio Q Z Bafterplata inew flatt, och bart at fodra fioan, odh jorimonentog bar igen fin roba firg.

AKRARNE fâ băr 2:me fr, Det tredje binila be. Sadoen at gemenligen 2 Binteriag od) fextandigt Siort, t) gumring fas bar fallam. Sorben it ofta el sfroer quarters Diuy, hâraf feer, at affrminn fôllan fumma Difns, bivarfore aften blifwer ofta fur vd) fEammer maiten, Da wata or infolla: Deremot om jorben âr 
fuetrig, Dá fibent fis ut oun miren, och oet froctmera

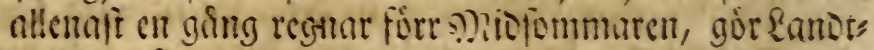
mantuen fig merchoeló gout bowp, men i fiartare torfa brimmes faoen birt. Ateratme goves buart trede ar,

xo - Dir oc fá fomma it.

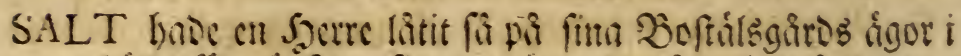
frisllet for goofect: fitoen mirte Derefter belt frodigt et âr; men Det follianse forfiunn fruttbarlseten.

HWETE ̊IRRARNA figgo ut, fom rîg of) buete blans Daot tiffamman worit fast pa aften; itmimfone trat treoje belen rág; men lamotmanen forfaftude, at rent buete blifinit foot, och troboe at hroctet fúrbuttes $i$ righ, th od banbabe haim at Det renafte buete ifrin andra orter, blef wâl ock fórita arioga tämmelinen ren, men Deš anora mírt eller utfabe fí rágblandadt, fom Dẹt bat figge, bwaraf buctemiolet atr mera griot bir pas

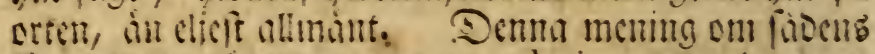
formwanthing it en error popularis, eller bontotto, fom allmint at antagen sifiver bela eswerige, men frâr mot Strućturen, Phyfiquen od Efapelfes lagar: demintrote fom oe mu of Den lítoa merloen begripas, th) bor en fisoun fots antingen benifas genom oförliencs ligen flatii róth, eller oct nldelis lemmas.

SWINKÅL Eallades hír Pीferfennen (Flor. 548.$)$, fom gictoe ofta forni rama belt gula, men i riggisframa

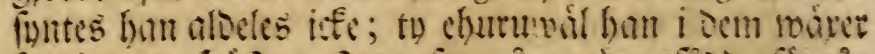

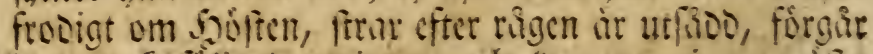

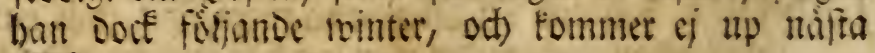
gBat.

FEM FLISOR figgo ut fom liefterar, od) moro fralte jems

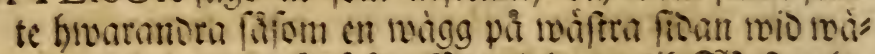
gen, oit man giff ifrion smedelplana til 2 Zeefterplas

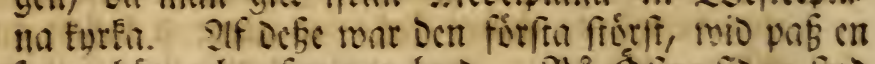
famn big od 2 fommar broo. Mli Sitra from troo artalet 1589 , od treme wapin uthaganc, of hwilfa 
Det roserfia war et Trolle wapen, Det medlerfta et Poffe mapen, oub Det norierita et of obifant, fom bas De liffom troinute cuneos hinc dentatos.

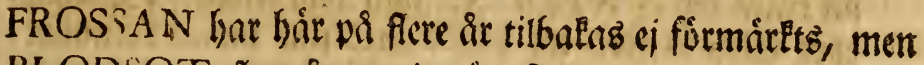

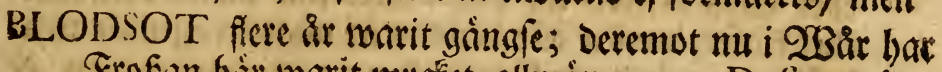
Frofan fir warit mudfet allmăt, men Dylenterien alocles uphórdt.

GARDESGARDARNE moro maif antingen of róda Faleflifor, fefom enfla murar upfaplade, ellex ocf af titt tilfamman pactide Enbuf́ar boplagde.

STORAN GEN kig neDanfor Siobftenb flefiuan, od) ftrate to fig alt in til Venern, wio paf I quart $i$ bredoen, och 2 quart i längen: Denna war maif of wermu ven meo mane boga Sabelbuffar, fá at emellan Dem funtes liefom fma torg, famrar od) labyrinter, in, hade de allenafi pia fidorma maxit Elipte af en

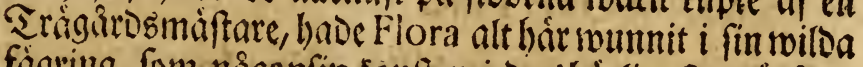
fägring, fom nagonfia fonften $i$ Den bărligafte tràgaro. (starne uplyfte allema fina bigg Pronor ofroer Defe. låga Sanflar, frfom Eapreber iblano Viburnum. Dian fag Defutom i ienna liulign aing en for bop roa fra blomfter, fom nu ftodo $i$ fin bâfta făgring, fafom: Paris (Flor. 325. Pinguicula 21. Ophrys 738. Melica \$5\%. Lythrum 393. Valeriana 30. Clinopodium $4 \times 79$. Carduus paluftis 659. Campanula minor 176. Heracleum 231. Xylofteum 192. Rhamnus 193. WUillapel 402. Oxvachantha 399. Sorbus 400. Jiofer 406. Hieracium 637. After 696

WESPELTEN Eallader VISCUM (F/rr. 8 r6.) fom fings bầ pa Kinnekulle mer àn pa andra ftallen, bălft $i$ Aplar. Gemene man trosie, at Da denne 2 Befpeltes nen bongdes i bufet, ffulle oet roara fafert forr, tosis
clo. 
EKNAS fallader VIRGA. SANGVINEA (Flor. I; I.), cul buffi, fom utom Sfine vd) (Sottlano it nog fill: fint $i$ Smatige, men bat pa frolingen nllman. Sin= ma ritta BENWEDEN, fom han fallab $i$ EFinte,

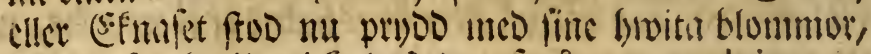
eller flafor, huvilta iffe bejtodo of migon regulair umbella, litin boide en Corymbum varie ramolum, \& fubdivilum absque involucro, broarföre ban dif fan fallas CORNUS corymbis nudis, emcoan alla anora ! pecies Corni bafion flores umbelıatos cum involucro.

SCORZONERA (Flor. 647.), at giDDn fivit

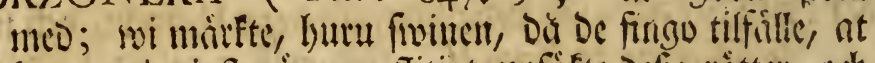
fomma in i ftoringen, flitigt upfofte Defe rotter, cdl) Serfore bornoe diupt nid if jorden, fa at Dait Semma ros

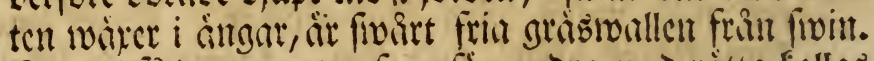
Fant altia Demin roten frumfót andra med ritta fallas SWINBLOMSTER.

H.GGEN war pâ flere ftallen liefon hopivafiucn of fpint ncltra, imom livilfen fans cn muctenlset of Fripular. (Faun. 89r.)

HIERACIUM cichorii folio (Flor. 638.), wiitte

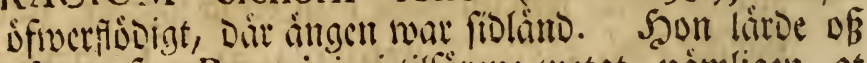
nîgot, form Botanici ci tilfureme metat, matmligen, at

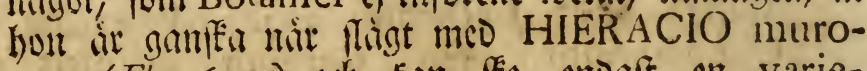
rum (Flor.637.) Did) fan fEe enoafi en varietet, foum genom ben fuftiga födelfe orten blifwet ant

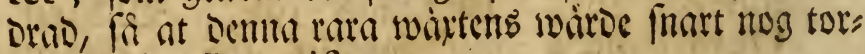
De falla los Botanifterne.

KERSBAR cller FOGELBÅR whipte åfroen $i$ fturingen misctet, nedre mot Venerns framb.

ÀKERLEJA (Flor. 443.) waipte pa atffilliga ftillen swid fiviftranden altio med bla blommor.

ASTRAGALUS procumbens (Flor. 59I, ) waite åfimen nuycet mot fiofitori. 
LITHOSPERMUM (Flor. I 5i.), bWars fri biutirs pi Ipotbefen, watte pif fremoen.

CXNOSURUS criftatus (Flor.8r.), winte ofwen for Sijoftcio Slefwan uti angen.

STRUTHIOPTERIS (Flor. 84r.), Den rakafe ormbun= fen iblamb alla Groufta, waite mer an allmin wio Eanoftens Silcfiont $i$ fturángen.

MYSKA (Flor. II4.) Dd' MAJGRAS (Flor. 55.) tointe bisge ifturficoigt emellan Venern odh Ganditens Siletwon, bigge willuftande fâfom en Molchus.

HEDER, (Flor. 190.) cller Hedera, waixte, faft fällan, wio Eumbltub filciman.

TORNEROSOR me fritfwita Glommor funtmos jemte grsilferplama inmio Slefwan.

ABROTANUM campeftre (Flor. 668.), fom fiflom fins fir allman i Everige, fom fring Uplala, maty te frusigt $i$ abafrerplana Sintfogard.

SANDSTENS KLEFWAN lig Enapt ot ftenteaft ifran Venem, hon wat mucfer brant, ifwerwute med

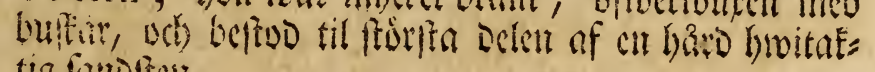

- tisfomofter.

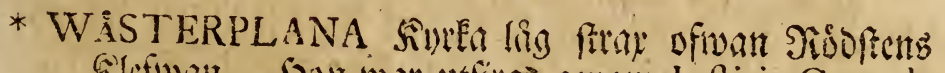
Silcfuan. Soun war utfino genon Juftixix Cancel-

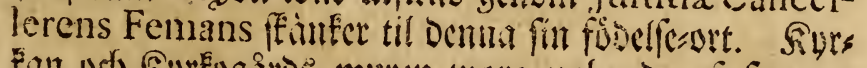
fan Dd) Surkogitros muren woro upbigde a famma flagis ften, fom fieffina bet fältet beftod utaf, bivarpa De waro bugde, nemligen af rod finleficn.

STENBROTTEN moro anlagoe dmEring 2 Bifferpioma

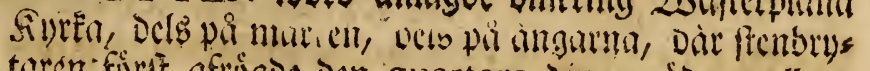
taren firlit afrogde Den quarters ojupa rida mullam, fou cl anmat toar, an cu mulla med rodt bleke beblank

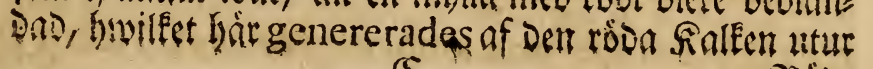


Soivoftens flifan, på famma fătt, fom hinitt blefe genereras pá Gottland, * och brunt pa óland. Ees Dan Dem iorden war afiogo, fólgoe ftentrotaren fneds ren och twerfucoden i birget: afrogoe Den ifra filan,

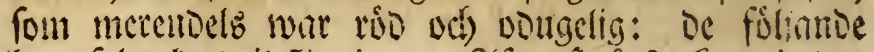
hivarf bruttes til Ripnina. Zif De fmi litycten gjo:tes

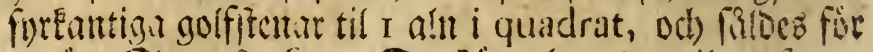

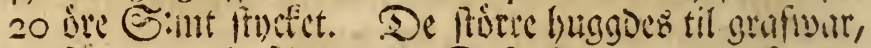
arafitenar, milpâlar ze. Defe fenar moro of tregs

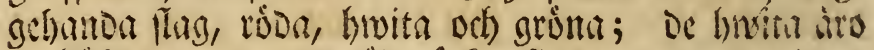

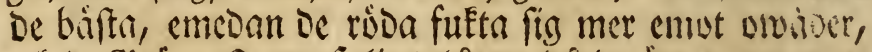

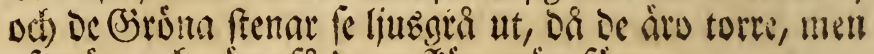

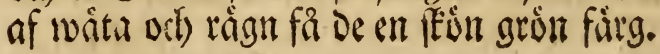

* It. Gottl. 179.

SKADA îr, at Sambmannen mucfet féfmmer fin ang oitfier meo Defie fienbrotten, utom Det han birigenom forfummar fit aferbrute; bivaraf feer, at sen bonoen gemenligen àr fattigaft, fom mâf hármeo arbetar. Set borse ei tilftiojias, at ängarna fa mefect uptrys taz til ferm, och ewimmerligen forbantfivas, balft da be fa litet gia pa diupet, ci eller borbe hwar och ell utan steffirna fis bruka Denma nüring. Set woro bittre, om wifa perfoner, fom ei féta garosbrue, togos birs til, buarigenom man finge bittre misfare i fonften od) bittte arbete. Sifruen borbe alla be platfer, fom til fenbrott feulle anmandoas; Dem tillinnas, meo forbs"

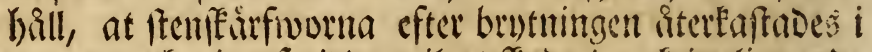
gropen, vit) ef utipridoes til at féada den fringliggande gribsuallen; ja, afiven at Den afrógoe mullin lades

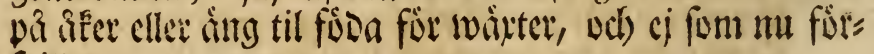
fwilleb.

SKURQWARNARNA for fremflipmingen woro batr aloe: les pi famma fitt inrittabe, four pir Oland * orb fi= posco bir 3 ferme tillifa, nomiigen en, fom gicf fors ut, ofl) 2 efter.

*It. Sland. $5^{2}$. 
SIENHUGGAREN Stenhammar, fom iftin Det han warit 6 \&rig, ballit fig wid ftenisuggningen, fâg a an mu wid 82 stret $i$ god vigeur, od) meo fafta lun gor, fifum ct tart crempel at $i$ fá láng tio utbaroa ften Dammet.

* NAT TEN fom pa, od) wi hivilade i Redelplana.

\section{Junii $2 I$.}

RESAN frifloce ifran Medelplana it ifrerffa Kinnekulle, fobermera norr om Sullen at' Siterplana ods Silcfiva.

KULLATORP lig pa wriftrn fiban on fullen (t.I f.F.) pir en liten plan of Rifwerten, clier firitibarg, hats

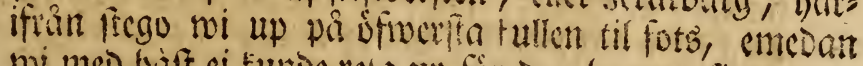
wi meo baft ef tuito reta up for ben beanta fioan.

OFWERSTA KULLEN beftoo bel ob billent of grafberg

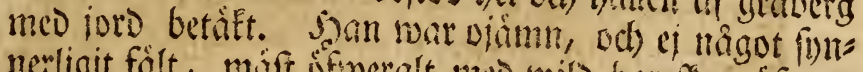
nesligit fält, maft of weralt med wils barmitog of

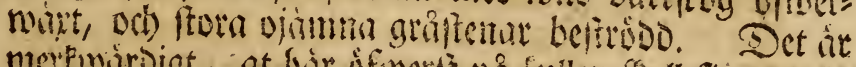
methuardigt, at bir ufinerfit pa fullen fatl finnar che

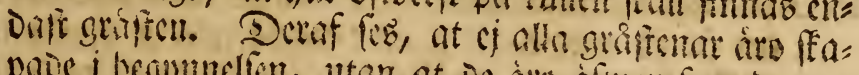

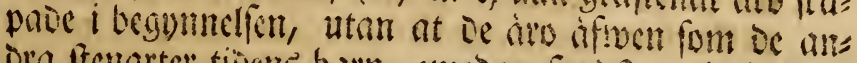
ora fenarter tivens burn, entodn fansfens bruarfivet are blifivit af fand; Enlfitens buarfwet of tera, med fina petrificater; ffifwerftenten of mulla, od minilan af maiter; ifwer alla befe ligga grafictnarte, fom alt fa bair icfe funna warit feapade. Jlia waftra fion. om Fullen fintes en ling horizont ifiner Venern. Hellekis fintes fom neo for fotterna; Lechó ling pá en fmal od) ling Jolme, bivifen utitriftes bela 2 mis len, fafom et hant litoe ifrán L idkóping ut ât Venern; bir utanför funtes jarte (Een och) Eute. $23 i$ bade hin funnat fe ftaden Åhmal pa Dal, fom ligger twart ofwer Venern, om bimmeien warit Elarare. जja 


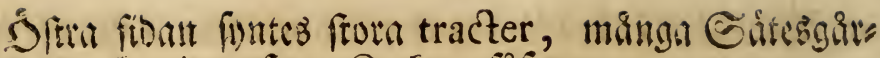
Dar, od) wiD patis 40 Silteve, fafom:

Bjórklitter, Berg,

Fullólia, Gótene,

Hufaby, Hangelófa,

Kellby, Lixberg,

Lecho, Ljung,

Mellby, Råda,

Skalenc, Scheby,

Saleby, Trellsberg,

Ziel,

órslóna,

Broby, Forshem, Górslunda, Halmentad, Kjeftad, K'efwa, Let/jo, Lidkóping, Liungenäs, Mariaitad, Rackeby, Sjelfwom, Stró, Scara, Wedum, Waittelöra, Zunnerberg, ̊̊laka,

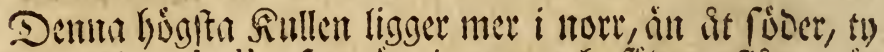
hela Kinnekulle, fon ait $i$ nore och fooce aflang, it

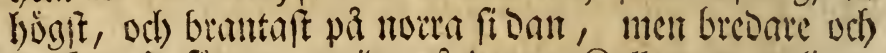

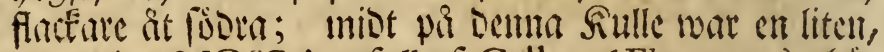
meet Ditup MOiS A, full of Calla, (Flor. 744.): lyit ofiwnitpa witte Trientalis (Flor. 302.) Oxalis 385. Pyrola uniftora 334. Linnxa 522. Munflur $\mathrm{I}_{\mathrm{O}} \mathrm{2}$. Ranunculus 465, fom Eallndes SO LóGON, Dit) Lycopodium dicothomum 857 , fom fallaber LUS. BLOMMOR.

INGEFÅRA formuntes allmint mîna pî Kinnekulle; met

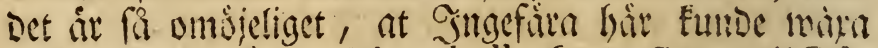
wildt, font oet ar, at Kinnekulle funde finttas tilOnindien, bilfe ingen tract it $i$ Europil fis warm, at

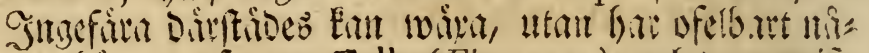

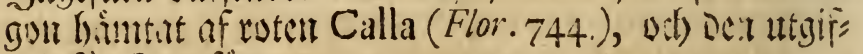
wit för Sultgefarir.

TRYFFEL cllet Tubera (Flor. II I6.), wirte mituce

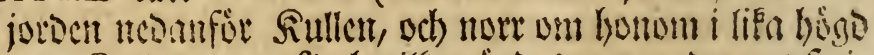
meo Sullaturp, (f) builfa rügocs genom set, at fivis

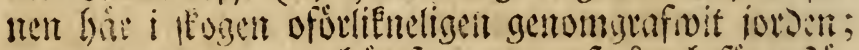

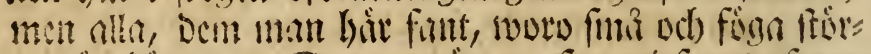

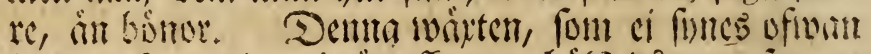

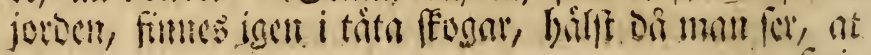


fivinen flitigt grafivit, bivilfa altio fatta bemma delicenf from for all amor mat, od) getrom fir godu luft lâtt mitika Den.

ADOXA (Flor. 326.) Wâte nuycet ringa notr om Sillth torp.

FALTET, fon ligger neDanfor Sitifbuitget cller feifivers

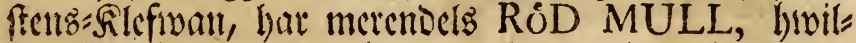
fen fî wall fom (Sidibairget, och Den rioda tälgftenen, (golffienen) tickes wara färgad at Det boatnet, fom rumut utur Gfifwerftens= Silefwan pa oe neonnfót liggande Strata, cmedan Den uplofte fifiverifenen ât als tio ros, fa at troligt kan wara, Det róda myllan âe ct Silefe af oen bruma golfitenen, odf) at Den bruma golfs frenen blifivit färgat of fifiverftenen, od) feifroerfenent of fin egen Victriol.

GORSTEN Eallades firt ben Tüloften, (ỏlands-ften cller (Golfiten), fom war brumattig, oct) af luften eller bryt: ningen fonderföll i runda od sipple forntiga ftucter, fas fom (bior, eller barda excrementer.

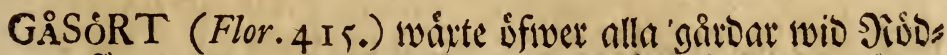
fitter, cller pa (Sorflefivan, mer ín pa andra ffillen i Giverige, od) lifa fâ mucfen, fom Stampergrib (Flor. 322.) pa andra garbar i Sillet.

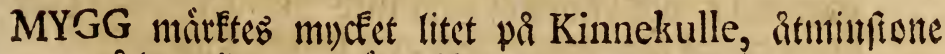
pâ den tiden wi dầ wiltades.

MUSCA nigra, oculis bruneis, abdomine fubcinereo, alarum bali fubflava. Demna in ch liten flut ga, fom firapt at Dubbelt fa frov, fon en Diffingar (Faun. I 109.) od's ei tiffirene of mig befferifwen. Sgon flog tufendtals, od liffom fima bifivarmar, omfring bufioubet pâ bâftarma, sá wi moro nirmuft Eullen,

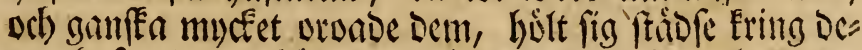
ras bufioud, at baftarna naftan alorig fingo balla det rìtt filla.

$$
\mathcal{E}_{3} \text { SPATT }
$$


SRATT woto De máfe baftar băr plägade af, fâ at bats

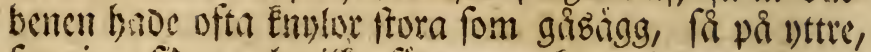
fom inte froan, burifet furmentes fommit at en binglt from He lekis, fom haft Don famma fiuloom, hivals

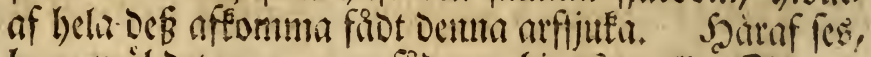
bum wal Det wore, om fäbana bingitat cller. Sto at Deles fourboides, pá det hela lamoet ei ma blifma upfulot mo odugeliget bafteflig.

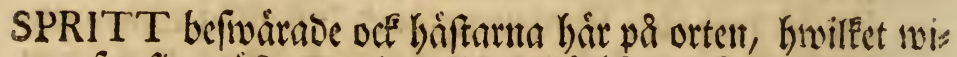
far fig mait om wintertioen, oa haren pa benen begun: na fparta ut, od) refas, bivarpa en ful peabb boviar up/riga ifrán lopfoen up à benet, at Det ànteligen blifs soer ffallott.

BLCDSOT fingo merendelib fáboffenen, ba de ifrän ansta

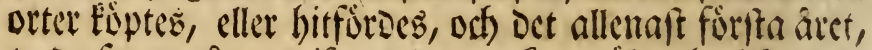
t) De fentre aren trifwas de mucfet wâl; hivilfet tor: De forcuriakas af EalEwatnet, emeoan mant fer, at folf, fom forfit Eomma til Paris, od) Dricfa Elitwatnct ui Sen, fá antingen Febrar, DurElop, ellex bloofot forfta sret; men feocrmera icfe.

KERSEBÅRSTRÅN wåte wilba od) ftora fow (SElt ont fring Jivofâtter.

* Ósterplana Sivrta.lag pa Śltía fioun af Kinnekulle, sent emot, och) i famma plait ned 2 bifterplana, Doce fî, at bàgge liggo mer i fóder, ân böglta fiullen.

STICKE.TAPETER * beflitboe wriggarna; ent enfaloig Docf artig invention; De woro liffom worfue, belt titt of fticfor. Eticfor cller partor fallos tumna, icmus bresa eller et par tums bresa flangor flufne utif Furu, broilea Finnat odh Smalcinoniwant bruka i ftillet fơr ljus.

- kalm bahus 51.

LANDET fring Sீfterwlana war utan tri, med anthar, sterfält och betesmat?. 
ANGEN cmellan Śfterplana odh Filefiva bade mingn ars tiga waikter, fafom:

Cornus (Flor.13.1.) Mespilus 403. Agrimonia 394. Ononis 622. Orobus 196. Silene $; 64.366$ Origanum 480. After 696, Aftragalus 590. Filipendula 404. Fimpinella 24\%. Vicia 605. Trollius 474. Centaurea $>09$. Heracleum 23 I. Comarum 422. Cardamine 560 . A elampyrum 5:0. 513. 4rt. Veronica 10, 11. Lyfimachia 166. Anthyllis 594. Carduus paluftris 659. Leontodon 628. Lathyrus 599. Galium 116. 118 I19. Hieracium 637.638 . Mercurialis 823 . Hipochoe. ris 63T. Paris 325. Lychnis 384. Biftorta 321. Menyanthes 163. Parnaflia 252. Arnica 684. Scorzonera 647. Hypericum 624. Prunella 498. Geranium uniflor: $57 \mathrm{I}$. Orchis 728. Carex 768. Cryfanthemum 700. (eraftium 379. Linum 235. Cynofurus 81. Nardus 47. Feltuca 90.910 Agroftis 61. I1 38. Aira 63. Pedicularis 504. 505.

PEDICULARIS! waijer $\mathrm{i}$ Sw werige allmaint af 2:me flag, fom fuller áro af Rotanicis attfilbe, Dock ei fór Detta fá

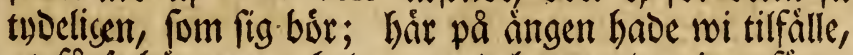
at fa fi bảgge, och Dem med hworantora jemnffira:

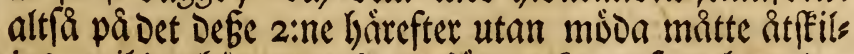

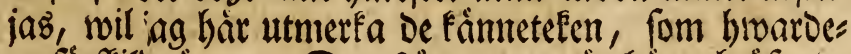

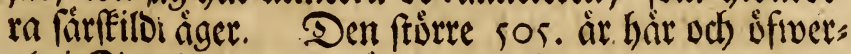
alt $i$ Siverige mer allman; Den mitrore Deremot utom:

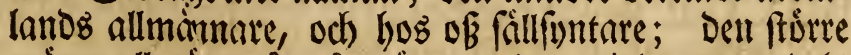
woiker allmánt' pá wasta ängar, ien minore merendels iblano granffog. Caly x eller Blombâlfen pa Den ftót: re âr cigglie, rand utan fanter, beftródo med fmi ups boggoe puncter, neen of fwantil merendelb fördelt $i$ 2:ne imféturne láppar. Deremot ảr Den mindres Caly $x$ mer aflíng, muscete fantig, helt flàt od utan puncter, of wantil fórdeld i fem ima fliéar, af howilfa Den inre ấ minft. Den ftistre tar i fin B Blomerona, eller Corolla, pà bágge fioor af jfre läppen en neobigo tand, od 
neore lóppen î ivino utan ungon bjertformig flic mis balin; men sen minders blomfrema hat wis fit

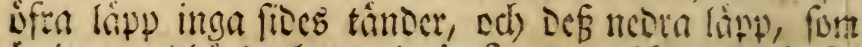
ar jent neotoigd, hat wio bafin-en ungligo flact, fas fom et bicrta, Ean altfia Den ftorre Pedicularis (Flor. 505.) Fallas Pedicularis caule ramo/o, calycibus tuberculato-punctatis, corolle labio obliquo; men icn nindre Pedicularis (Flor. 504.) má Eallno Pedicularis caule ramofo, calycibus angulatis glabris, corolle labio macula cordiformi notato.

MELAMPYRUM criftatum (Flor. 510.) fâg bit ifter mucfenbet, med ljufa at od) brvita blommor.

BRATTEFORS futade Demna frora ningen miot for flef:

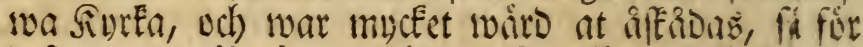

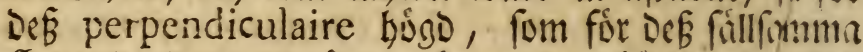

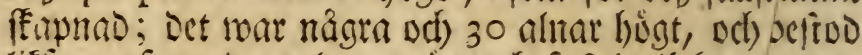
liffom of runda pelare, titt odf faft inti! fowarandera

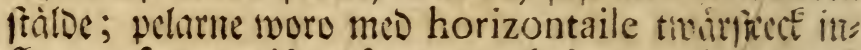

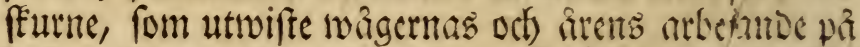
Denna fidan, Den tioen watnet beticfte bet ncoanfior liggande landet, och arrligen aftog. Barrgat beftir af

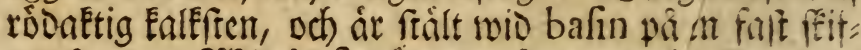
werften. 2Bio bafin ár en grâtta, uti EnvilEen. Fere perfoner funna fitta torra imunder fielfiwo watneí, ou Det brufar the fur benma anfenliga hoos. fe from Brattefors tab. I.

WAXTER fimma wois betta fall âtffillige fom pả antra

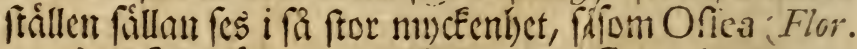
131.) Aftragalus 59.. Vicia 603 Geranium $57 \mathrm{r}$, Origanum 480. After 696. Rof 406 . Rubus 410. Tuffilago $€ 80$.

MELILOTUS (Flor.619.) waxte hạm wilst mes gula blomfer, Inttig for Zfpotsefarn!, ien utom Gottland, òland och) Skâne ej ar fumer of of $i$ Emerige toilst wápande.

* Klefwa Givifa blef wait nattcuarter efter 5 quarts tefa. junii 


\section{Jun. 22.}

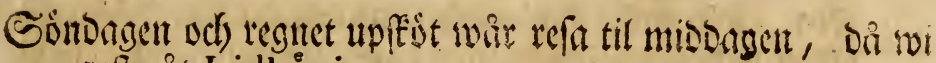
refte it Lidkóping.

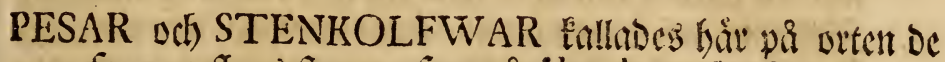

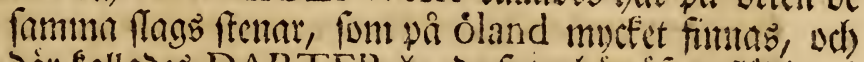

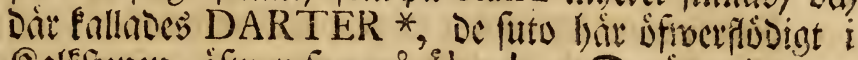
Sinffferten, áfiven fom ya oland. Se aro ci numat ant petrificationer of et itugs fractio, fom tallhs Nautilus rectus, Gmilfens petrificerade ffel atro be rate

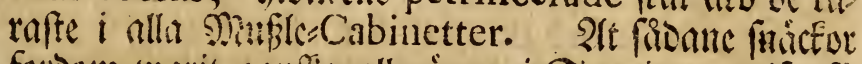
fordom warit ganffia allmàne $i$ Swerige, wifa fa oland, foin Kinnekulle; men hwareft be nu tonit svigen, wet man icfe; fommlige Manflor abl Snictur bolla fig uaren intil fremberte, nubra forter Lemma als

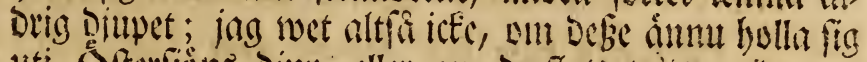

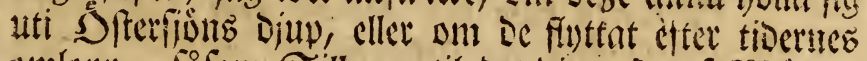
onlopy, fafom Sillen, til bet bilipalte of $2 \mathrm{Biffe}=$ bafivet.

$$
\text { * It. Sland. 40. 14r. }
$$

KALKUGNAR fagos $i$ byantma fring Flefiva, fom tigo wio famma Gơgo med Dem, font umtalab Dén 20. Jun.

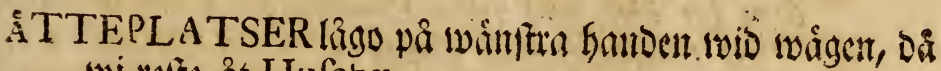
wi refte ât Hufaby.

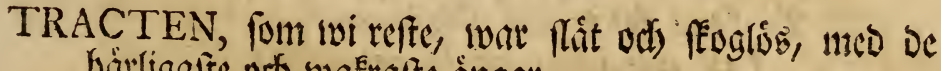
barlligafte veth wakralte ángar.

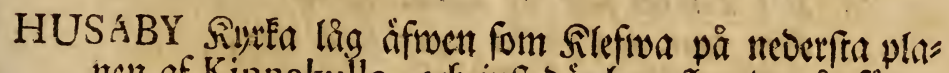
Incu of Kinnekulle, och juft dår ban flutader at foocr, bevaraf Detta Hulaby babe ber biarligafte profpect ât foser och) effer sfiner Det nedanfor liggntoe hintiga lantiet. Sieffiva Siurtan war huggen of fait fen, fia Dinn fom brutes pa Kinnekulle; i hentue fágos nágra

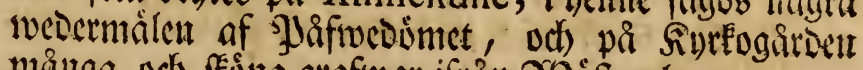

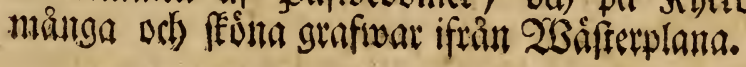


SANCT SIGFRIDS KÅLA, uti huilfen Tonung Oro Giotffonutg folat r 497. blifmit bout af Sanct Sig. frid, fom ifran \&urgetano fortt inforde Den Plafinifea

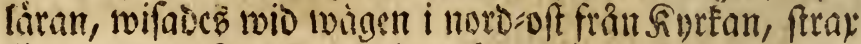
forr an man framfom; boul las wio toten af nederfta Flefioan, och wat nu wio henne en RóNN upmuten, fa at bon nâfran tactte Sirlian, och) latteligen utwifte bente, men alorig bar senna ftat butorabe ar, minore 350 ar. Sf fielfiva llippan waitte Acrofticum (Flor. 856.), Trichomanes (Flor. 854.) v(t) Abrotanum campeftre (Flor.668.). Sente lanosmaigen of fmat for Siallan frod en upreft fren, fom war of ara Ealeften faft meo rod firg ofimeritrufen; bofffafiwerne, fom i bonom woro utl)uggne, funtez hel hwita, til Def De af regnet blefioo mal witta, ba de fingo en artig bog grin farg. Infcription pa denna ften war fótiande:

$P$ i thetia rum fick Sverige boot $I$ bedendom mot Jjála- - Soot,

At mórkeras makt mind' bisna,

Nair Erichs Son Kong Olof from

Hir antog dop och Cbrifterdom,

Ocb làt fig gårna Cbriftna

Af Sancti Sigfrids taicka band,

Som bit blef fend frin Engeland,

At Cbriftendomen planta:

Af plantan ung blef ymnig fid,

Ty Svea folck thet fólgde med,

Faft morckfens mackt det danta.

Ett Tempel ftar pa denna Rebn,

Som war den fórfta wairckftad klen,

Ther Gud mand' bwilo finna.

Sen ban ingådt $i$ Giótha land

Thet watna med fin milda band,

At wi til naden binna.

Betinck thet wail, o Wandringsman!

Gif Gudi tack med bwad Iu kan

För ftora nider fina, 
Som ban med thetta wairck bar giordt

$\mathrm{Ocb}$ annu gior alt tid, ocb fort

Ocb daglig laitsr Jkina.

\section{Jas allora fidan:}

Thenna aminnelfe-ften bar Guds barmbertighet til pris, på det fjubundrad tjugu forgla aret, fedan Olof Skottkonung en bedring med bela fitt bof, antog dop och cbriflendom, uti kjaillan, fom flyter nedanför denna klippa, uprátta latit anno Cbrifti $172 \mathrm{I}$.

Fob. Aling.

RUDERA efter at gammalt ftenlyus, fom warit någon $\mathfrak{B}$ is feopo Refidence, ftodo sinmu quvar et par bysiffott if fail Sivréan åt wâfter. Det war en mur oin 10 fammars breso, ock niften liea bogo: wio biviffen waitte Cufcuta (Flor. 138.) Prenanthes (Flor.645.) od) Libbefticka.

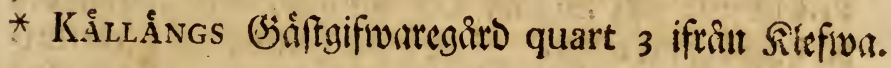

RUNSTENAR, twäme fincfen, itodo en pá hroartoera fioan sun waigen wio fállbu; Den cta of Dem toat wio pấ 6 aluar bog, och Den anora 9 alnat.

BI odh GASS Rigor i mucfentoet wid alla de ghroar bir $i$ Weltergótland, fom wi bartil feot.

RESAN ling ofmer ftora fillt af aErar odb angar, bwilta fe: nare pa Denua lanviord wooro nog magre. Detta fät tog of med en affottning, od lantoct blef liggre inemot fión Venern.

STRANDERNA wio Venern, Dem wi fulave hela 3 ficrn Delb nilen intil Lidkóping, beftodo af fin fanto, och) woro mucfet grumon, at clt wall funde rioa $\frac{x}{2}$ quart uti fion. Ja atfitiliga ftällen babe fión up altat ov, fi at lanoet tiltager arligen mer od mer, utom Det, at fion wid ftranderna upblaft ftora fanobogar, fom woro bogre ân Det innanfor liggande lano. 
FLODER, âffillige fincen gingo ut i Venem forr ät toi fonmo fram til Lidkóping.

POA panicula diffufa, fpiculis fexfloris linearibus

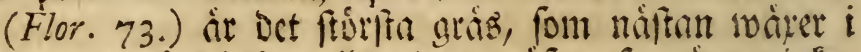

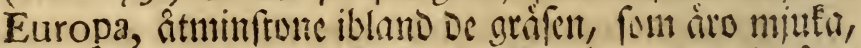

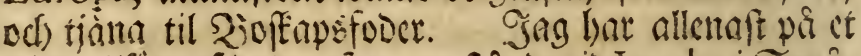
ftalle tilforme foot Dernf ct pat frano wio husby i@ma

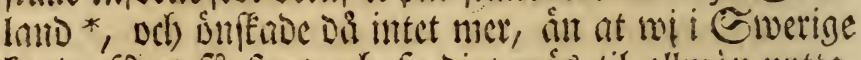
fande fâ et fâ fort eds) frodiegt grás til allmin mutta. Da ing refre forbi sefe fooct wio Venern, fig jag et

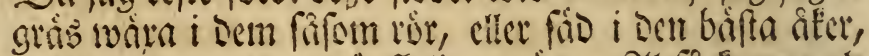
bivilfet alt war betta sulfeliga gris. Sfltfia fumma als

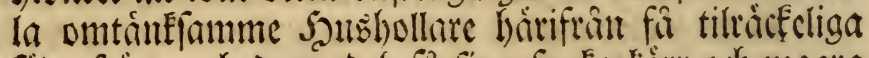

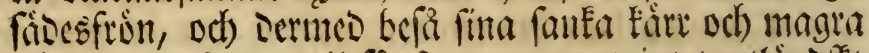
bictar cller floocr, til fa fror nusta, at intet utlänolet Sosfró háremot i nagor maitto forlifune fan. Sagh foller ietta cmoa fâ warbint, at Det Ean betala bisie Publicum od) inig all Den foftund, fom anmuandeb bã bela benna refan. Gag fonn fanma griós federmes ra pa atfélliga ftällen bà $i$ lanoct, ber set fallabcs Sanfervia.

* It. Gottl. 32 I.

PHRYGANEA cinerea, alis fecundariis pallidioribus: margine interiore pilofo albido. Demma fuga, form war subbelt fiorre in en mugga, flóg million tals, eller fom en bifivarm, langs ît bela franden af Venern. Def Antennx woro balfanmn ginng fa lint ga, fom wingarna, och) grả meo brvita ringar.

STRANDEN wio Venern, ifwer hwilfen wi refte, wat wall til ct bufeferts breod af idel fand.

* Lidkóping. quart. 5 .

\section{Jun. 23.}

RESAN wintocs $i$ bag frân Lidkỏping til Lechó, od foran âtet tilbấns til Lidkóping. 
JORDSKÅTT åro mucfet fállfinnte; men wio Gunnergs

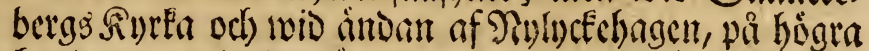

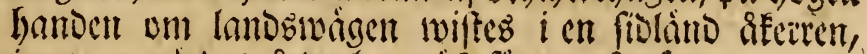
jemte en girbesgind, lywarift für 10 are feoan en torf

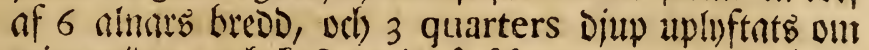
twintertioen, vd) faftats impa \& \& fren til 4 alnur sängo

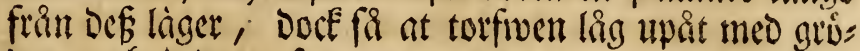
Dan, och indoan af Den quantemnade grop war liffom af ell plog upriftao med en fora. Paftor Sunborg beristtabe belt trugt, at $i$ ivatnet, fom famlat fig efter Den upenftrade torfiven, feolat om wärtioen funmitb en litent givooa, ocl) troose at Detta Phanomenon fơr:

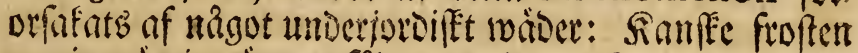
warit măgtig göra et fádont underwere. Om Dylifá fam man lif $\mathfrak{a}$ Hjernes fract.

STOHLA Catteggâro, fom af urminnes tiber bört til ent Def) famma familie, nuimligen Ekebladrka, odf mu

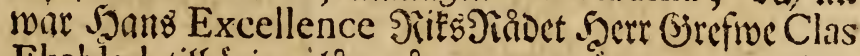
Ekeblad tillyơrig, låg pả en ongenàm ort eller liten bỏgo, 5 quart ifrün Lidkóping, omgifnen meo ant=

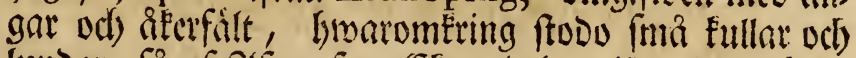

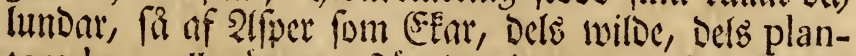
terade; alla igorma frámgocs imnon en Canal cller graf, buvilfer meo bägge cindar hade fit utlopw i Ve. nern, fom frutte til pa waifra fioan.

GAMLA EKEN, fom frod norr stm gitroen, in $i$ aingen,

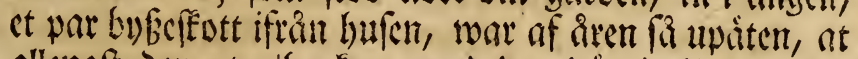
allenaft ben ytre Gorken, med Den bảr intil ligganse i)ta af tribet, hisgit til et par truerffirtgers tjoflef, uppebjolt hennes gamnla lif; bennes ftam ling at ena

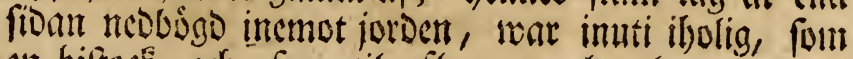
etr biffoce, och) ofiwantil aflurggen; bon bade utflaget 2:tre ftura grenar, foum formerade can warfere grön-

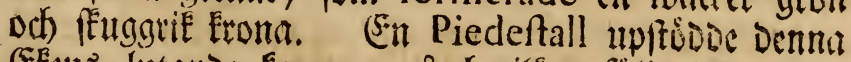

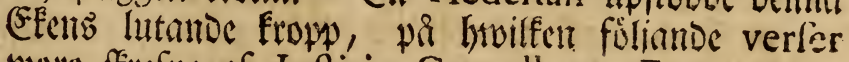
woro fitefue of Juftitix Cancelleren Feman: 
Nar jag for bundrad tio ir Si gammal war och fwager; At jeg tyckis fidt mitt banefir, Ocb jedt min fifta dager, Di ftódde mig en bielp am man, Din Faders Fader Fader, $P$ i Stobla fjelfwer bodde ban, Bar namn of EKEBLADER.

Nit kommer Sonens SoneSon $N$ air ftoden börjar Jljelpa,

Ocb ar derom ej mindre mon, Hur' ban mig ock mi bjelpa; $S i$ at cinnu min gamla rygg Kan tid och aren picka, Mot kjoild ocb betta reara trygg Ocb ej för ftormen bäcka

Jag fall frambira EKEBLAD

Til tacksambet och offer, $P$ i Stobla lefwe altid glad Bid' CLAS ocb CLAS Cbriftopber.

WAT TUWAXTER fannos icfe allenaft de mifte, utun

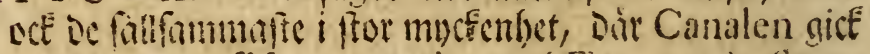
ut $i$ Venern, falom: Stratiotes (Flor. 444.), Sparganium 77\% Sagittaria 80 . Myriophylum 785. Butomus 328. Alifina os. Herba Kritannica 292. Sium 235. Phellandriumi 28. Cicuta 239. Potamogeton 139. Perficaria amphibia 318. 3. Nymphæa 426.427. Equiletum fuviatile 836 . Ranunculus longifolius 417. Utricularia 24. P'Oa 73.

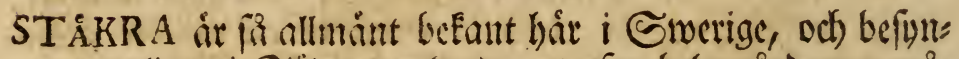
nerligen $i$ Gobermantimo, at ofta bela gároarna mis fre fist bemes leulo blifiva soe; emedan da baftarte fo tita boet batuf, fom ar ocrais ftarfalte gift, do de bait, la at bonown milter fima baftar, Den ena efteb 
Den anori, blit beraf alocles uturmad, od mâte

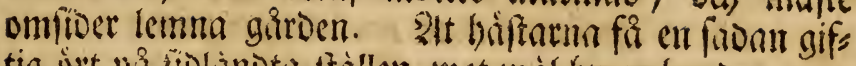
tig ort pa fiolimota frôllen, bet wall bmar bonde; men bwas ort bet ar, bar ingen annu swetat. Sallmant two De, at Derma Etrifra ar Alisma, fom off fả pá flere frillen tallas Etitra; jing àger en gammal edition af Matthiolus in Diofcoridem, uti bmilfen en Defi fors Dom ingure ffrifwit meo sammal ftyl wid Alifma (Flor. 3co.) at istma more ien râtta Gtâfran, fom oidade hatfar, odi) Antido um Deremot wore Imperatorix radix. Gaig fin iffe $m^{\varepsilon} a$, at jog in altio turifat, om Alima wore ben farffoloige Etäfran,

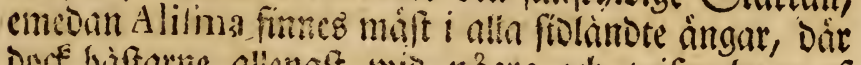
Duce baftartie allenaft wio nagra vd) mifa byar af Gtiterffufoomen arligen dosas. Songl. Thetten= fenps Acad. communicerade med mig et rön frôn Dannemora, $i$ broiffet Ranunculus flammula dictus (Flur. 458.) debiteras force at wara Don ritta Étifran, bwilfet ing ia mucfet mindre funde under= ffrifua, fom intet fiere $i$ Smerige finneb, fom ide ar fult af Flanimula: men bat po orten larbe en bonde mig Den famftyloiga od) ratta erten, namligen PHELLANDRIUM (Flor. 238.). S) Ran bür barpa iffe twifla; to Den fom lifit Botaniquen, twet at alla plantx umbellatx, fom waja i matnet, aro siftige; * fifom Cicuta: om bivilien et mitrfouroigt rosn frome ocles gifines i Denna refa; Oenanthe, af broiffen 2:ne perfoner mis Hag forte lifiot til, för Det De allenalt fimafade risterna of benne od) $P$ hellandrium. Sactil fommer, at Phellandrium itfe finnes allnant $i$ Erose rige, utan allenaft pa fa frällen i mucfenhet. Srten

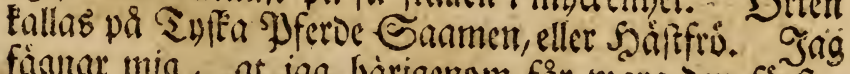
fägnar mig, at jag birigenom făr mara ben forfte, fom allmint fan läa mina lanbsmän, at farme Den ràtta Striftan, bwarigenom frittermannen mucfet lát fan wata i ftand, at afta fig for en fa for eller tioffitummig och tilliéa fFadelig ort, Den ban ganfera list tan unowila med lian, eller uprucfa meo bander:

na. 
11a. Sag ffulle tro, at om an enda gâro i Comeriges

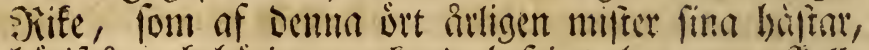

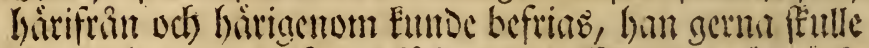

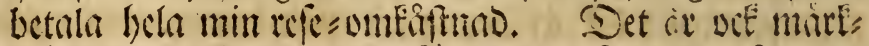
worbsigt, at Soctma cller fümosen ei fara illa of Demmat mott, chum fenselig ban ai for bafram, afwen fom baftame cifendas af Gtormbattoblomman, cliet Aconitum (Flor. 442.) dhum Belt bobnt Fit od) (Gitter:

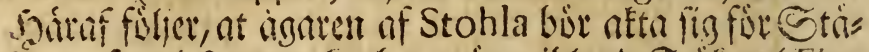
Fum, fom i ftor myctenlet warer iblano Frifen (Flor. 836.), at Det ei gifues briftema, od) Det fa muctet mer,

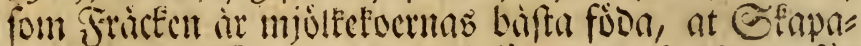
reit tuctes liffom welat privilegerat Srafenet för sivertin.

* Fund. Botan. 342.

SPRANGORTEN, Cicuta (Flor. 239.) witte bit i

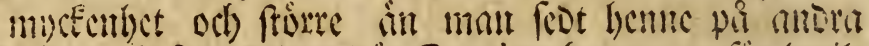
fiallen, befinnerlinen dar Canalen begunteg, for bswils tom man bai bor fia noga afta Socrma, fom for fielfe wa Dósert.

KASSEWTIA fallnoes biat of Bonberna loa maxima (Flor.73.) Dent nil i gite (p. 44.) füft pis Demen refont

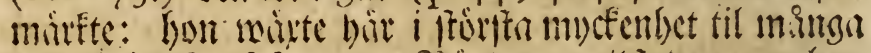
laf forofele of fooret: Somberma tillitoso, at bout gaf et mintt od belangeligt foser, boide for fior od)

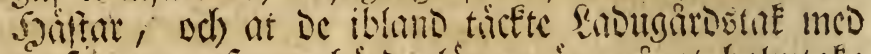

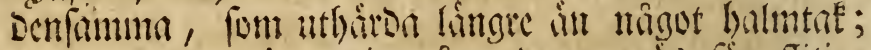
t) recommenderar ing aiter Detta grits für fitige

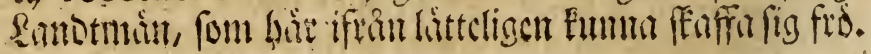

RANUNCULUS foliis lanceolatis, caule crecto (Flor.

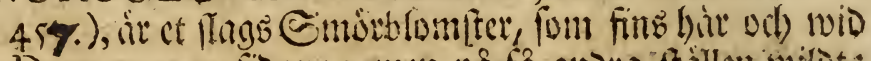

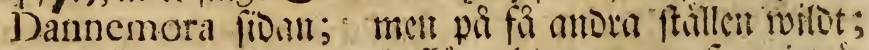

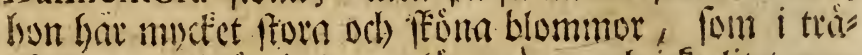
garonana uti los joromon litt màn, od isfe litet prys= Da Dem. 
UTRICULARIA nectario conico (Flor. 24.) wápte bàr i Canalen ftórte, in man benne fedt på nảgot ftälle.

STRATIOTES (Flor. 444.) ftod bat ynutogt, odf meo många blommor, fom eljeft ej ăr myctet allmânt.

COCHLEA tefta membranacea fubflava ovata, mucrone obtufo, anfractibus tribus ( Faun I 302.), 1302 fanz i muctenlyet pa bladen af Stratiotes, til ffapnad, fiorle od) Elarbet med parlor.

SEDUM caule erecto folitario annuo, foliis ovatis feffilibus gibbis alternis (Flor. 39i), incipte pa flip: porna wid Stohla allmán; en liten surt, fom àt obes Eant för De miffe. Botanicis.

KUMMIN wajkte $i$ muctenliset pa ången at Lindholms fidon.

STENSQWATTT-UNGAR togos pi ingen: Caput, una cum dorfo, ex cinereo \& fulco varium. Rectrices a medio bafin verfus albx, apicem verfus nigræ; fummis apicibus pallidis: harum rectrices intermedix, tantummodo bafi albx. Remiges fufcx, at fecundarix una cum tectricibus margine exteriore ferruginex. Abdomen pallidum. Pectus flavefcens, fufco undulatum. Det ât froât Hăma alla foglar, då de ánmu áro ungar, entedan de metendels ândra fin fá̀g, fedan de blifiva fullwurne, th bafwer jag altio warit angeliggen, at noga befirifina pennorne i wingarme (remiges), odb

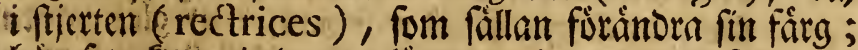
bath tyctes mig Denna lâter roarit en unge af Motacilla (Forin. 219.)

LINDHOLMEN å en prifftig o(t) lringfinten Gátesgarro, fom ligger ifrain Stohla twert ifwer en liten wit of Venern. Syart wat et mucfet wail anlagot Palais, jomi blifwit upbugot af (Birefive Bengt Oxentjerna, out) ar aftagit uti Dalbergs Svecia antiqua \& nova. Den barliga buggnaben, de féma gipsmitantme, de gamle 
Imperatorum Romanorum Bufter, fom ftodo i fine Nicher i fürtugan, Den behageliga lituation,

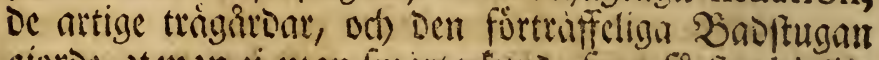
gorde, at mine ci utan finarta funde fe en fa fisor barligs bet, meo fa ftor fuftutas, od) eftertanfa anlagd, nu fia tom od) utan inbuggare.

BADSTUGAN, fom war anlago i Lindholms trägard, war et máfterftucte, och Det príttigafte jag feot i bela Weftergótland, ja, hwars life til Baofruga man fâfángt lärer fóta $i$ Éwerige. Demna war gjord ad imitationem Balneorum veterum Romanorum, och forbelt $i$ trente rum; nåmlligen i Frigidarium, Caldarium oth Tepidarium. Frigidarium war en fal meo liufa fónfter och lateral pelare, bumiffa pelare, tillif a meo fielfioa waiggarme, woro incrufterade meo grub och) ffinmrande Mica, af atffillige couleurer, til anfeende of mallningar, font fóreftâlte Palatfer od Profpecter; tafet war innantil giordt, fom et bwalf, Dd) áfiwent incrufterat lifa fom waiggarne. Baslfwet war lagot med Grwit och frwart Miarmorften. Detta rummet war bet follafte. Caldarium eller fielfroa boiset od hetafte rummet, war en Eummare innanfor Frigidarium, mycfet hijg, od) inuti \&ttfantig. Founs ftren lyfte endaft fram ufmerft pa fitourna under fielfwa bronffiwet. De 8 fioorta af rummet beftood broardes $\mathfrak{r a}$ liffon af en garderobe, af hivilfen bruardera

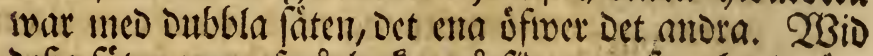
defa fáten looro fma luctor pa fidortta, fon funde ips nas, at flippa fram woirmant. Mich bronaranor of Defe 8 fioor twors incrufterade of glanfanoe ffenar:

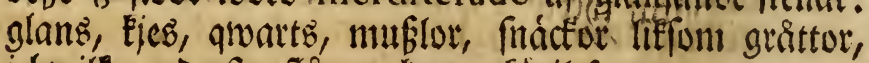
i hwilfa neoerft affaunga fopparfintil fattes, at emottis ga marmare od, fallare watten. Salet twar bugot

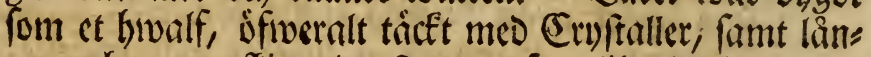
ga och) gerromffinande ffentur, fout lifuade istappar, od) Stalactiter. (5silfivet, fom roar lagot meo fivart od) biwit Maarmor, hade midt uti fig en atteantig affätts ning, fom gice neset, Denua war omgifreen meo 8 pes 
lare od) et lingt Efpaille, immom bivilfet de nafne bes gutes och affísilgoes meo watten, fom pa Detta lảga 5)? armols galf famlades til centrum, od) forfinan uns Dee fotterna; ment be liage pelarne, fom inftangde betta fílgerum, flutades ineo runda bufiouden eller Enaps nuiv, bivilsa aftrone, inblisfte Den toirman i rummet, of hinilfen Det upwarmtoes. Tepidarium war ent Samminte innom Frigidarium, och iemte Caldarium, fom emottog de warme gaffer utur Caldario, at

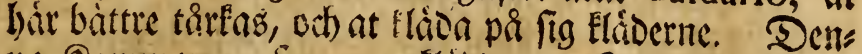
na faumare, fom war flido med surfiffa guldene ffuggstapeter, babe allenuft et choa attfantigt fónfer pis ella fioan, briffet imlippte bagen; pa Den miot entot

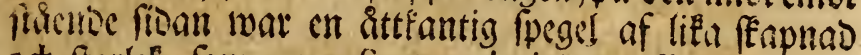
od) frot!cE, fom reprefenterade $i$ Detta fEumma rum alt, brow fom leceoc utan fore. S neora bainingen mar et bivalf meo en reverbere ugn, fom utan four bufet clondes, men feoctmera tilfuten, inflappte all fin mirma i rummet, bwatifran moirnen genom tór int Ripptes efter behag til oet ofman uppá bugoe Caldarium, od) genom ietta unorn rummet neolops canaler. fom afforoe but watnet, fom uti Caldario wat uts gutit.

LECHo̊ Glott ling s quart frîn Lindholmen $i$ notr, vtterfit pa deuna länga trooe, KALLAND fallao, fom gict ifrản Lidkóping, hela 2 milen $i$ norr, ut át Venern. Detta matra Glott war altfî pâ 3 fioor om= sifioct med fio, odb frilot ifrun falta landet med en graf, fom genoin fieffiva bällebirget war utfprango. Jja ytterfa fioan af Elottsmuten war en hojg odf tring grotta, Flâfegrafroen fallad, utfprängo i fiels, wa bàllebirget til 20 almars längo orti s almarz hógo, bivilfen bade et Ealt fóllewatten, fom Dör EumDe uppum= pas in $i$ Slottet. Elottet bade warit upbugot $i$ Jar piffifé tiden for $\mathfrak{B}$ iffoparne i Scara, federmera inora git til Fronan, ênteligen blifroit fentet ril Srefive Jacob De la Gardie, off af Def Gun (Srefrue Magnus Gavriel de la Gardie arfenligen forbuttrour; men

$$
\text { D } 2 \text { ater }
$$


Siter til fronan reduceradt. Det war ganféa ftort, web) froces befta of 365 rum. Uti Sittsfalent woro hirs liga flifoerict od målningar, fom fơ âfesoarne wifte:

Gustaf Adolphs antomft til Pomern 1630. Bataillen wio Leipzig 163I.

Scatts gång ufiner. Renftrómmen 1632.

Bataillen wid (Sibftiommen $16_{32}$.

Bataillen wio Jancovitz 1645 .

Bataillen fammaftíbes eftermidoagen.

Bataillen twis Lützen 1632 .

Bataillen wid Wittfock 1636 .

Bataillen wid Leipzig.

Änteligen wiffe fiomung Gustaf AdOLPH Erónt med en gion sagererunts, upluftad af en froart outh, lemnandes Sceptren och fironan at Dots tien CHRistina, med ifmerférift: Curfum confumnavi, fidem fervavi, in repofito mihi eft corona juftitix.

De formante Generalerne wio Syfer Siriget woro bar woil aftagne, fâfom:
Joh. Baner,
Nils Brahe, Axel Lilje,
C. G. Wrangell,
Hans Wachmeifter. Arwid Forbutz,
Lars Linde,
Jacob Duvalt,
Joh. Liljehók,
Robert Duglas,
Chriftcph.Kọnigsmark,
Leon. Torftenfon, GuftafHorn.

WALNóTS-TRÁNA ftoDu mucfet ftora i Slottbagåroen, fom tillifa med RUTA packat emot De farfa wintrar ry 40, emedan de af Slottet pa notia fidan woto be tôfte.

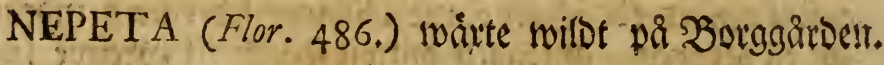

ROSER (Flor. 406.) meo hivita blommor, màte fa woil miloe fring Slottet, fom pir anora ftaflen bar omering. 


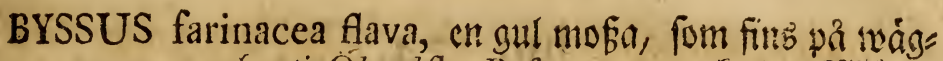
garua, vé) uti Olandfka Refan pag. 30. Kallab 23üggs

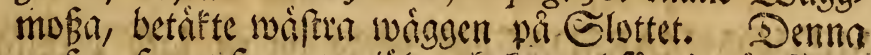

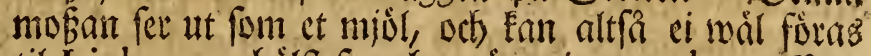
til Lichenes, bälft fom ban aiger inga pelras: Nieo bonom plaiga De färsa gult.

WINTER-KRASSE, eller Barbarea (Flor. 557.) wainte fa fring Sluttet, foun bela lambet wilbt.

QWARTSÅDROR fágo nuyctet ftora, odf bóllo litet jern. De woro pa bágge fitbor befládoe meo fina fultbanto, liffom hade De warit rena Netallidoror; Deke gingo

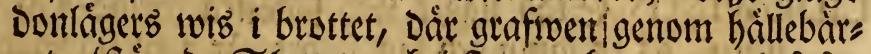
get affitángoe Slottet, och i famma brutt woro pi its

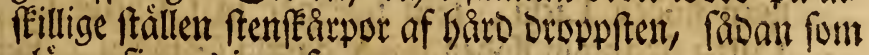
plägar finnas i grufwor.

RUNSTENEN lemmadeb at Antiquariis, fon ling th en Sulle nár intil Slottsbrutr, och pă bougra hanuen nầ wi tefte frán Slottet.

WADERQWARNAR * ftoDo mid De máfte gardom, DC,

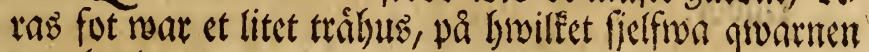
war bugd.

* KaLM BahUs. 286. tab. 286 ,

MIDSOMMARS-AFTON Fommo ini efter 9 quarts 2 efa, tilbaka ifrån Lechó til Lidkóping, Elactan i 2 om natten, då mi hela mágen feot ungoomen, if fin illbers Midofonmar, figna fig af arets belbagecligafte tib.

MAJST ANGER moro pá många ftîllen uprattade o(t) bes

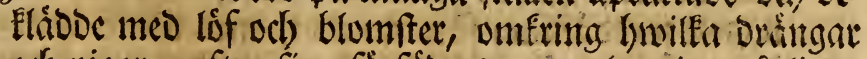
och) pigot, efter fina fürfóders' urgamla wib̆, àlligell Danfade natten för of(t) efter Mnid fommarsongen. Dets ta mentlofa nojet nefades futler Dem af Siâleforijarena;

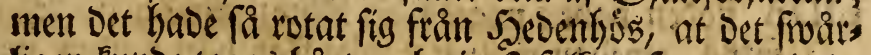
ligen Eumbe tagas bärt, och tien/tefolfet, fom nu giovoe

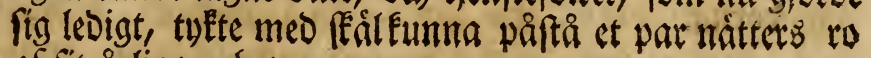
af jit âtliga arbete.

$D_{3}$ Jun. 


\section{Jun. 24.}

MIDSOMMARSDAGEN hơroe ni en líro predifant i

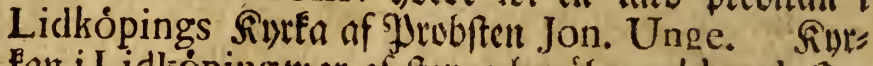
tan i Lidkóping war af ften, och wál meublerad. Ors gelwerfet, fom blifwit giorot odl fitantt of foriom $\mathrm{Pa}$ tore Rubero, Cederbjelkarnes fitrmfader, waf mivctet wacfert.

RENFANAN (Flor, 666i) waitte i mycfentet pia fiyts Eogaroen ibland De många oct) huggne ftengrafiwar.

LIDKóPINGS Stad ligger mid fodositte firanoen af Venern, pi en iämm och fandig ont, ir $i$ tu fórbelad ges nom flooen Lida, fom bair gấ in i Venern. Jja S/tra fioan om floden ligger gamla Stnden, form át minore odf irregulairare. Jja maitra fitan ât nua Stnden af Birefwe Magnus Gabriel de la Gardie anlago, meo belt regulaire gator, mîngn twoirgntor,

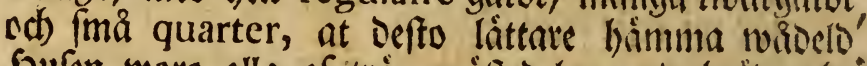
Saufen woro alla of tria, máftedelen meo britier be Hlídode.

TORGET i mya Gtaben wat bet anfenligafte i Mifst.

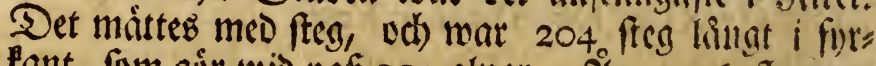
fant, fom gưr wio pá́ 250 alnar. 2ir 1736 . log $\mathrm{cn}$

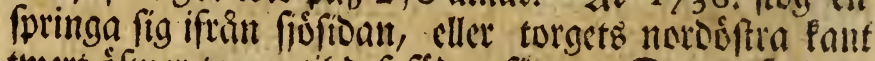
tweer sfriber torget til Dés fódra fioa. Denna foringa war wid pas et par tumim bred, ods serem Det bour firóf under et fturt trô pó torget, flufoe hon bet frim: ma, at Det fórgicfs: Mainga troobe, at Detta warit af

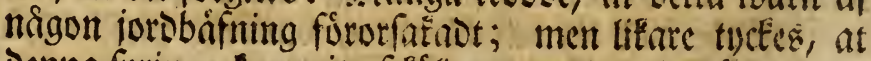
Denna foringa fommit af EślDen, emedan Det fécode om winteren.

LIDA floden hade i nyare tiber blifivit mucfet iristre, in i forna, emedar watmet fadigt féar bart fanden wio fis Dorna. 
IVATNET i Venern gian wiba â higre up, od atet wifa ar faller mera ut, fient âfiven ofta birt fanden af ftraitiorna.

SANDEN omering ftaden, fom fonmer ifrån fiom, laftas af blaftert, foin en flugfano, och gor ofta nagon liten finda, medelfi bet ban blâfer yả grảstwallen. Denna fano beftir af en belt hivit och) flar quartz med mudfet fa fivarta inblandade fina grun.

MUSCA alarum punctis duobus nigricantibus (Faun. Svec. Io53.), fanb hundrndetals på fandftranden, Dár matnet ftod qloat innom fanden. Scon flog mucfet finált, at man meo ftorfta móda Eunde fanga en enda: Son fatte fig altio pi watnet torrffodo: Sennes fropp lypte mot Solen af Fopparfärg, odf hennes fötter wo: ro nog langa, $i$ anfecnde til bennes frorlef: Shanen war náftan bálften minore, än Sonan; ment bade finart Dubbelt langre ben.

\section{Jun. 25 .}

RESAN lảg ifrån Lidköping til Skara.

FóLJESLAGARNE Doctor Juris Jor. E. Fick, odj) Politie Borgmáftaren i Upfala C. Prints, fom bágge for fin barla lful bade warit med of i fillfiap alt ifran Uphala, begáfwo fig mu tilbala. Eannerligen on bwar od) en wifte, hivad ogemen nuttr ribambe om commartioen gor for hilfau, ffulle flere numftilla ficouna refor hat i Swerige, od) minore folf famlas til bruts narne, Gálft mant fan meo lifa depence refa, form at ligga wio butunen; Rtminftone in Detta risandet for Dem, fom hafwa andetippa, begunncle til lungefot, mielt fot eller obftruction, et botemedel, fom ófivergar alla bade brumar och medicamenter.

LANDET emellan Lidko̊ping od S Skara mar mucfet jemt, od) nâfan aloeles utan ften; Kinnekulle fags

$$
\text { (2) } 4
$$




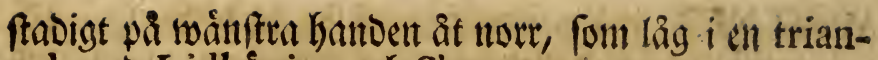
gel med Lidkóping och Skara.

MILSTOLPARNE woro af huggen ffen, hwarfore be oce

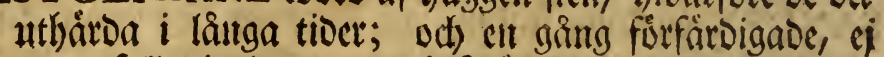
mera falla lanotmaunen befivartlige meo mua anläggs ningar, fimarfüre famma brué $i$ Deß̧e fentare âren res Dan ár antagit i Nerike.

CYANUS (Flor. 710. ) giorbe untoertiben aferatne belt bla med fina blommor.

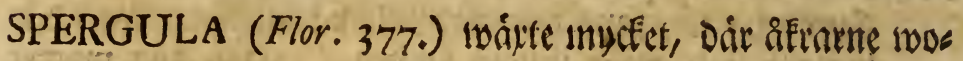
ro magre.

SURBRUNNEN, fom ligg irmio Skara, war af fơga wåroe.

SKARA Crtaot låg 2 mil ifrån Lidko̊ping i ơfter, han air mu en liten ftao, ehuru lan i fürta Dagar warit manga

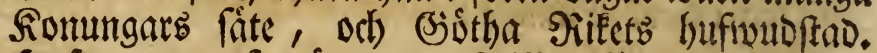
Şufen woto af trá, utom SBiffepsfätet, Scholan, Gymnafium oct) Bibliotheket. (Sstorne woro vienme, fimmige odh Erofige, at man pơ hontom funto fe, hats

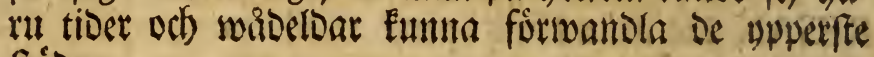
ftíder.

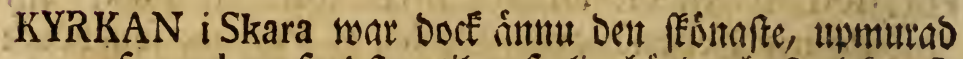
af quadrat fambfen til anfentig hogo och) ftorlef. S henme beivarabcs elt gutlofnele, af niagra uch so ducater, befatt med Diamanter. General Sops graf frod $i$ Choret of fruart Marmor utfuggen, Dir 5 an od Sanz Fru ofiwan upwa laggo i hivit Mintmor afbiloas De. Jja fitorna fúrefitiltes de actioner ban gjordt til fijo:.

DULCAMARA (Flor. I89.) wiatte hoggt up i fovrfomus ren på norra fibar utite furingurte; wi unorabe, at en wattusirt funde waika $i$ tortafte muret.

SKARABORGS Slott babe allemaft leminat miagrt rudera efter fig páfódra fiban imwio friden. Det bade twarit 


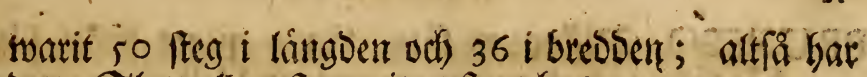
Detta Slott allenaft wanit et frort bub.

GÁLLAQWIST Glott bade legat ftrant utanfor bet för

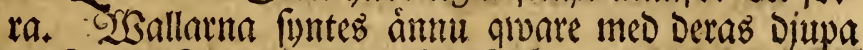
grafiwar, famt en Sultle efter fieffiva Elottet. Det

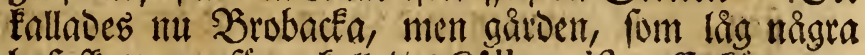

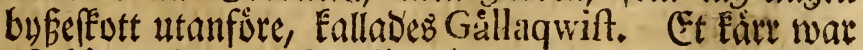

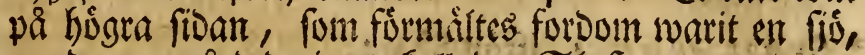
emedan en gåro Derjemte Eallabes Silusen.

DJURGARDEN lig i foDoft ifrain ftaden, war mu for

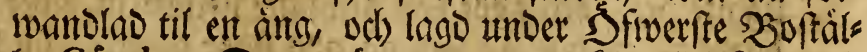
let Gótala. Dentra íngen iwar mucfet wiolyftig, och

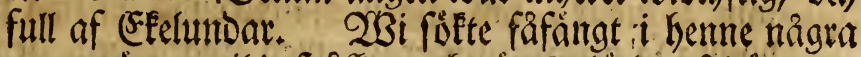

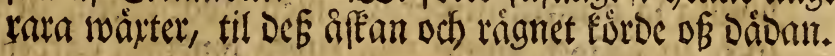

HASSELBRODD EAllaber AIRA (Flor. 70.) odf wointe

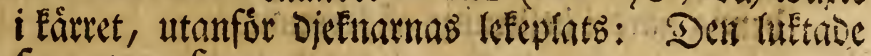
fom et perfume.

CORNUS herbacea (Flor, 132.) weitte pa uDDert, fom giff ut i famma fintr, utom Den fortra.

Magifter Sven Hor, Då Rector Schola, nu Lector Mathefeos, quaftionerades utt Botanicis; man famt

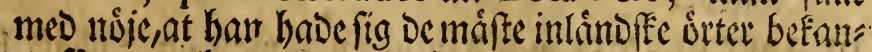
te, fáat um faan til bent applicerar De muare Botanifter-

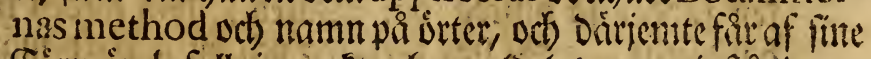
Sơrman befallning, Fant ban nldeles toara i ftäno, at iffiven baruti banoleon Den fuderande ungoomen soid Skara, odó) fáledeb inplanta meo unga âten i Schola piltarme de frón, fom frimbeles tunna sifroa Fă Dernestandet mycten frutt.

\section{Junii 26.}

RESAN tugg ifrain Skara åt Hójentorp od fórbi Billingen $i$ nurr ât Berg. 
BRUNSBO, Biffopents fáte, lisg I quart ifthint Skara; hár fôtte jag at recommendera Hiftoriam naturalem hos Sherr Biffopen Doćtor Juslenius, at fór Den fluderande ungoomen láfab.

TRACTEN war flat utan fomnerliga backar, of) utan fien, Doct berwuren med Stran, Dár isfe gároar woro anlagbe.

* Skerf quart. 4. ifrån Skara.

HóJENTORP, r quart ifråı Skärf, lịg på den behages ligafte ort, ock agbe den artigaffe fituation, Det war bugot pâ en backa, foum pâ alla fioor omfring fig hade minore Bullat, och áfiven en muctentet of ima fioar, hiwilfa fâ communicerade med hivaranora, at maar med en seffroce Eunde refa rund oinfrimg gárben och Des ígor. Commercie Piádet Ahlftrom hade nit Denna grito $i$ befittning af fironam.

TRÁGÅRDEN låg på en flat aflång Fulle, od) uti fontom

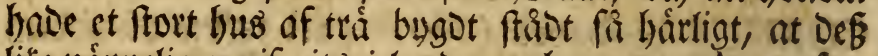
life náppeligen gifwits i lanoct, bwars rudera efter branden 1722. ámmu funtes, fajt Det federmera icfe blifwit ontbugot. Traiden meo Alleerne woro fơt: nutune.

PLANTERING war hăr ingen amman ản Tobak, hrwils Een fadoes fa bittion om 2ssaren, fom jorden funde bambterab. En fror târfhida od) en à part máftare war hastil, broartigenom man bar fict bride mucken od) feìn TobaE. Denna ivettenfeapen hade affiven finittat fielfron Sorparma, at oe antagt for fig fielfioa Sobak, bivarigenom De bade tilräcfecligit, atminftone til husbehof.

SCHEFFERIET beftod inemot of 300 far, biwilfa alla woro of utwalot 2ingelift sller Epanift flag.

SPANSKA FÅREN hade miufare ull, och bornen pi Des ras gumblar woro nec worcone of utorngme på froorna, 
at man lätt Eutoe feitia bem, allenaft af Deras utfipertar

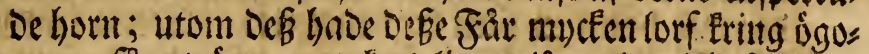
nen, fa at ógonen toftes ligga lantgt inuti hufwuder. Foliet berittande, at mat far i 2 Diark ull pá Gwardera af De trórita Fären. Fären worto flippte Dagen förut, och ullen inlóft, at mici finge fe en fia ónfeelig fanting; to om

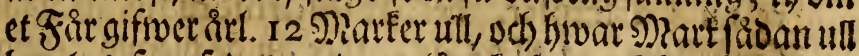
betalab, fom fades, med en plat, Ealitar et Fart af fig arlis gen I2 plâtar, foin int mucfet anfentigt; man hade baft luft, at utroina en fa twitivelaftrig, metr angelitgen faE; men $\mathfrak{Z}$ efallningsmanuet, Infpectoren, Siâffer:

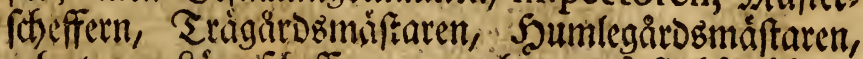

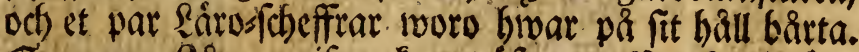
Gentente Säten gifiva Entapt ófioer I Mare ull, fom

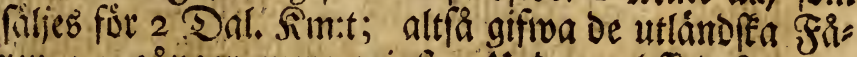
vell 70 gaingor mera winft. Uunorar altfa befto mer, bwond bair berittadez af Scheffer-Eleverne om Tuts

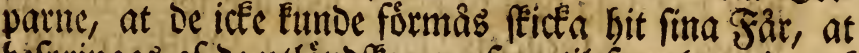
befpringas of be utlänoffe gumfar, til fortplantning af ent battte ait.

FåRLOSS aro wál af flere hag, men det fturtfa flaget fict mart hár tilfälle at befferifwa, brviteret Eallab Pediculus Ovinus, (Frifch. Germ. 5.pag. 40.t. 18.) odf ât

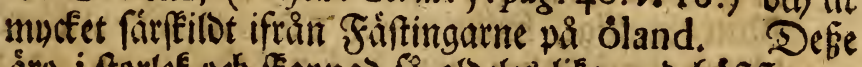

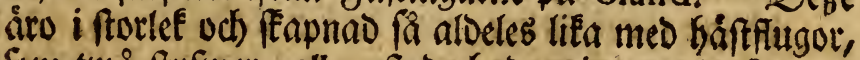
fom troa ftufiwer, allenaft be hade noingar; De funnos inucfenthet emellan ullen pả de oftippte Sammen: Táfrocet war utrî́ft cylindrilkt, trubbigt, od til bafin flufot, Groaremellan en liang tunga utríftes, fom en borft. Antenne furte och pipitige. Kroppen mes fótter, hufwuto ods bróft gulattig od befténet meo bar. MTagen oval, grå meo tumma bav, runde oms Pting trubbig, mibt pa nigot flatare : pa hivardera fis Dan om Miagen woto 4 gula uphógoe, langain ftálde, od) liea ifrán liwarandra feiloe puneter. Nagetiz bafis roar of wan uppa gul; men pa ryggen af $\$$ Ragen woro niggra forte mờtle flacefar. Anus prominens, cylindricus, Alavefcens, bivalvis. Klotrta på 
Gharbera fotelt wor wálo 2, men bwardera hade inums Der mid bafin en liten frof éler Elo. Detta Erä hade hrwarfen wingar eller halteres, war Docf ofelbart et f́pecies af häjtfluga, odb bir Eallab Hippobofca aptera.

SCHEFFERIE-Scholan war hair aulago, oct) uti Deri wos ro mu 4 difciplar, De Dair informerades af Maffer: Edheffern, 2 til $2 \frac{x}{2}$ ard, fúrr ân De blefwo tienlige til provincial-Sdseffrat. En artig inráttning, alletraft Maffer:Cedefifern net, at gơra Den upbuggelfe med fina difciplar, form af honom funde fordras: ti) ban borde I) lâra Dem, at noga anatomicera Făren, at Deraf fe Deras inwairtes buguna, och âfwen ipma be Faren, fom sogo bart, at wifa, bwatuti fiutoomen beftod. 2) Stwilfa irter af Farren átab, ved biwilfa

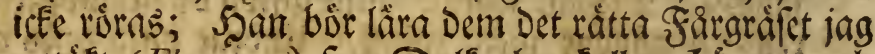

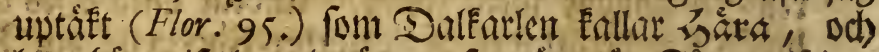

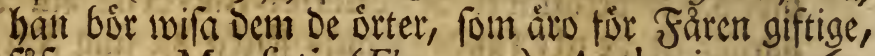
fifom: Myolotis (Flor. I 49.) Anthericum 26\%. Juncus 287. Drofera 257. Pinguicula 21. Mer9458. curialis 2823 . Flammula $45 \%$ Equiletum 833.

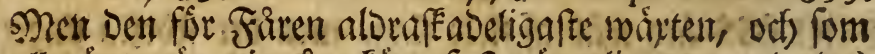
allmånt ivsiver i toåra Eärre (faft náppeligen utemland

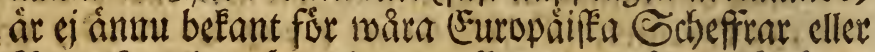

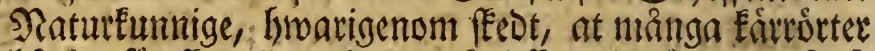

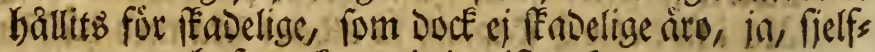
wa matnet bafwer Eommit i mistanta, at wata mera farliget, ån Det fó Dem roerfligen âr. 3) Borbe Echeffern låra Dem, blowo climat befommer Färent baift. 4) Samiler fiuteomar oc máft plaggas af, meo

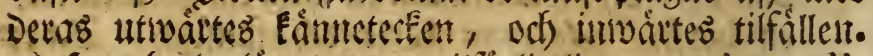
5) San borbe lára sent en tiffưrlitelig materia medica utaf purgerande, vomerande, fruettorifinamoe od) Urinorifrontioe mediçamenter, fom genom rơn pá Fareetr fabane airo befunone, uch iffe argumentera ifrám manuiféans medicine. 6) SJoroe han lára Dem curer utt hmarjebanda fiufiomar, fom endaft

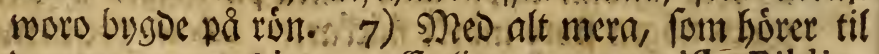
Denna ceconomien. Et litet ceconomifkt Biblio- 
theque hade Sherr Commercie Rilidet Ahlftróm ant lagt für be bár ftuderande Schefirar, men fuapt tils rîcéeligit.

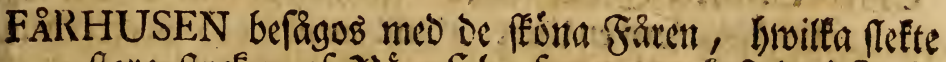

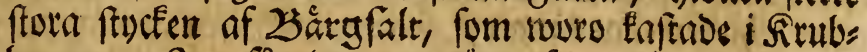
borma. Jas fäg lammer gứa, fom nuiorarme, meo

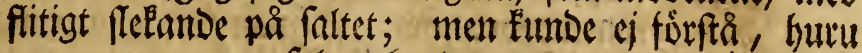

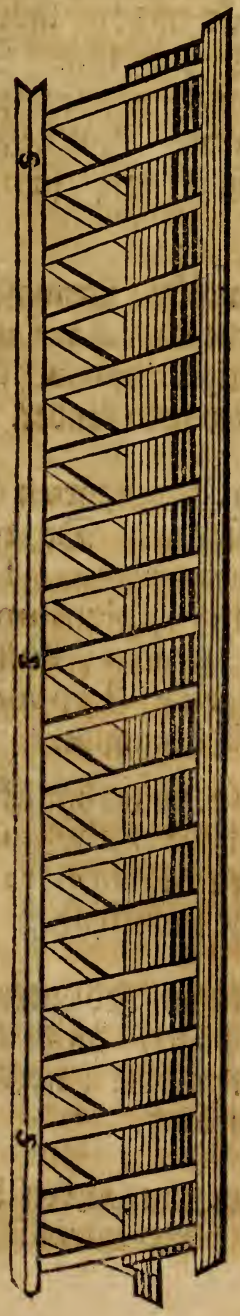
faltet funde wara Den baiffofamt, fa linge de lefoe af mjuste. zóce Earne $i$ fäarbufet nouro artigt bugs De, oct) ftăloe mitot pâ gâlfwet, fís lebeb at troa brảber lágo st quarter parallelt ifrán bwatanora långåât, och) på Eaut worto flutas De tilfammanz ad angulum acutum, odh falebeg giorbe en lång rygg. Sedan labes en háce vå luwarbeta fitoan om Denna rugg, fom war en alin bojg, odf med 3 twairfingers diftance emellan broat

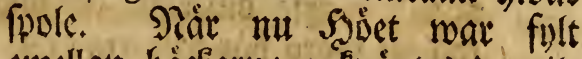
emellan hấctarna, entiptes be til fammans ofwantil, lyarigenom ernabdes Det, at Faren ef tunde foilla 520 , Doce noál Eumma at Det, emcoan ryggen emellan bớctarna fórbe bet at fioorna. fe fig.

FÁHUSET war pa Sadlindffa in rîttaot, Dár utom fiocoggângurna er: rätt gång gice midt igenom rum met, meo bifar pá omfe fitor om gången; hyout bås war en famn breot, och allenaft 2 alnar langt $\mathfrak{u t}=$ oun frubban, fom lăg nármaft ât gintrgen; hroar bas tog emot et par opar cller For ; fáledes Eunde Enutgarospign alorabequaimligaft gifina 
sifima Boffapen fit fader, oct) renfa derab ipilt.

CHINESISKA Givin făgob: Fargalten war mucfet liag,

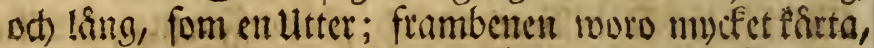
Galfen mar längre, fetare, och tiecture an pis anora

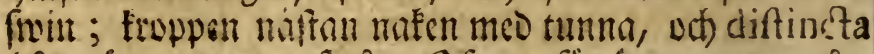
bâr; ovrotien moro fmả: Sfivan för úguntent woro ats ferllige djupa ffervulur: Mofer war nimore utoringen,

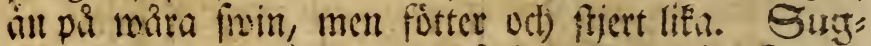

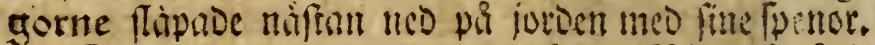

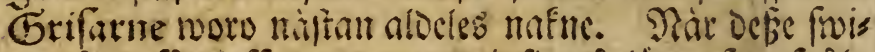
nen site, fia glofface de muten i fig pa fast, form finila humoar.

WA TTNET wib Daummen eller lifla fiơn, nirmaft Soj: jentorp, ivar fia farpt, at Det giorde Hlabeena, fom dars inti twoittabes, icfe ullenalt gulattige, utan ocf fá fés re od brdéflige, at oe ftrijt Derefter fóllo fonter.

ÖRTERNE Ctákan (Flor, 238.) vc) Sprángơrten (Flor. 239.) wajte omining be fina fibur. De rarafte warter woro bár Alliaria 558 . Barbarea 557 . Leontodon 628. Dentaria 56 . Dd) Chrylolplenium 317. Sair Eallades Menyanthes (163.) Siriefot; Pulfatilla 446 Essefnure; Paris 325 Sojgon; Higopodium 247 Firs, oct) Oxycoccus 3 Is Srangintor.

MELAMPYRUM cxrulea coma 512 . finntes bir iffe, foif Iunoarne woro bebageligare, an bioe Brahelund od Walfătra, ja, folt alla De andora Melampyra pratenfia 5 10. 5 I3. 514. Wuitte biar $\mathrm{i}$ muctenbet.

LUSTHUSBACKEN, fom ligg efinellnn Sajjentorp oft) T3arnbemb Rlafter, war migot lougre, än oc antore.

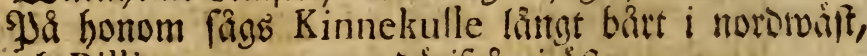
och) Billingen I quart Darrifrin $i$ ofter.

FÁRGRASET (Flor. 9r.) wadrte pa sufthubacten hógre, men tunmere àn på univa frillen. Mercurialis 823. 
meo Equiletum 833. watte brigg? i mucfenlyet, mint Dre tienlign forr Faren; men Den aloruffadeligalte Far irten wayte i farren tillifía meo Andromeda.

SKIFWERSTEN od) ftifrig Drpten fägos pa atffillige ftâls len; inemot Dár ffifuerjetenen féulle finnas, màrtte man en rodaftig mylla $i$ joroen.

POTENTILLA foliis pinnatis alternis: foliolis quinis ovatis crenatis, caule erecto. Hort. Clif. I93. Pentaphylloides erectum. Baub. Hift. 2.p. 2, p. 398. Quinquefolium fragiferum, Baub.Pin.326. Clus. bift. $2 . p$. 107.n. 5. ait en ort, fom alorig tilforene

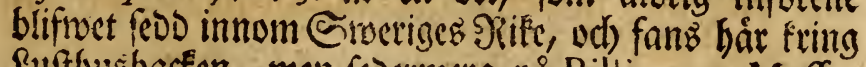
quftubbacfen, men feocrmera pa Billingen, Myffeberg. Olleberg od wio Gótheborg. Radix perennis. Caulis femipedalis, teres, purpurafcens, definens in Corymbum faftigiatum. Folia pinnata: pinnis trium parjum cum impari; foliolis fub. ovatis, incifis, fubfefflibus, petiolo longo communi infidentibus; adeoque folia Gei, aut Poterii. Stipula membranacex acutx. Flores albi, magni.

BILLINGEN refte roi fơrbi pa máptra fidan ifrân Şojen= torp, at - Bergs Siurta i nort; Da Billingen fontes of:

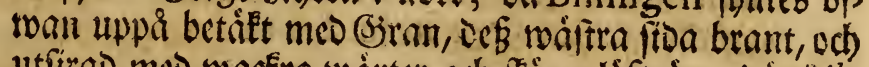

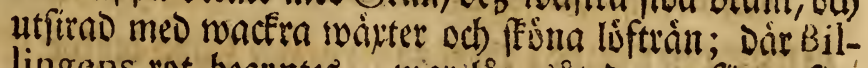
lingens rot beguntes, war langbit Denna fitan fio: lino, ftraj utanfơre mar lanoct langsit fátt; men quart iftan Billingen til i à 2 quart mar lanbet langos at pi Denna fidan alt bactigt, fullrigt, gropigt, of pa alt fâtt ojemt. man icte Detta mande wara baf: wets egen werfan, da Det fordom frod sfroer Detta land, at Billingen wat en 5 , od todgorna tilbafars ftó teg af Billingens måggar? Siffer Det ân i Dag, od fá licrer Det furem feot.

* Bergs fivelfa quart. 6 ifria Sosjentorp. 


\section{Jun. 27.}

Jiefan ling i Dag ifrån Berg, forbi norta fitoun, odj jemte Uittro Enaten of Billingen til sko̊fde. Billingen gicf meo fin norowáftra Eant intil Bergs âgor. Billingen war bât pá norra fidan I quart bied, ment Des läng i nort odb fooer freg wid pas til $3 \frac{\pi}{4}$ mil.

BERGS ângat woro bâtligen utfitade med mucfen (EF,

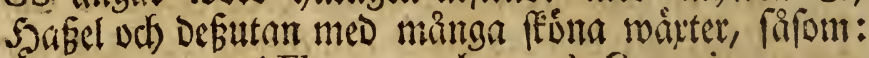
Melampyrum (Flor. 513. Ddh 5 I 4.) Geranium 572 . Biftorta 321. Scorzonera 647. Hypochæris 63 $\mathrm{I}_{\text {。 }}$ Trollius 474. vd) Defutom:

ANGELICA Silveftris 234. mid baicfarme. CIRSIUM 657. med finibruita blab pá untore fitoan. HIERACIUM paluftre 638. i mucfenthet.

JUNCUS foliis nodofo-articulatis 285 . i alla Eáre. CYPERUS gramineus 38. waikte helt allmain, Der ängen war fỉlänt.

SPERGULA 377, i alla åFrar.

EKARNE, af Det be pa fitoouna blifiwit Kamlade, bade ut: Inagit Ima qwiftar efter hela laingoen fring om tribet,

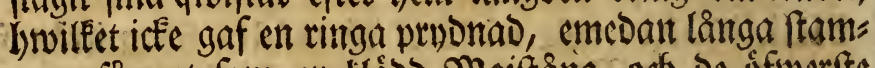
men fing ut fom en tléos Manjtang, od be sfruerfie oríroc gruifar flogu ut fin Frona.

MERCURIALIS (Flor. 823.) wâtte i alla bulfar.

FÅBOSKAP, Litfórbe frän náfrgrinnfande orter, fingo me= rendelb altio furtha aret bloofot, śfwen fom pa Kinnekulle. 23 io Kinnekulle (pag. 38.) giśabe wi, at Det torde fomma af falfivatnet; men fom bầ icfe

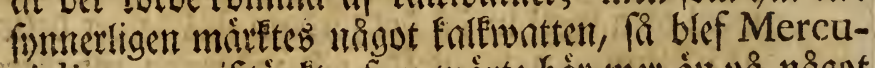
rialis 823 . mif́tänft, fom wầte bàr met át på något

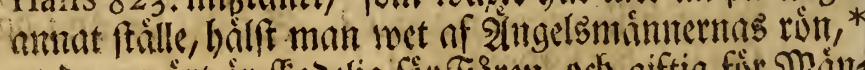

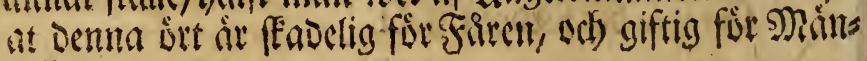
unfer.

* Raj. fyn. $3 \cdot p_{\text {. }}$.

ORET 


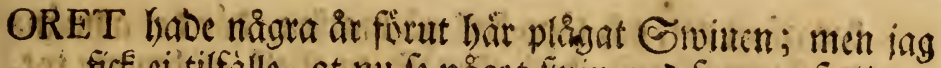
ficf ej tilfälle, at na fe náget froin med fomma fur Don allfatt En beffedeclig 90 ara bonde, foin oce fintoe Denna fiu bomen, berittanoe, at han war fimittofam på

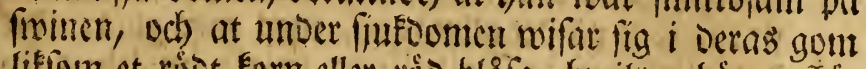

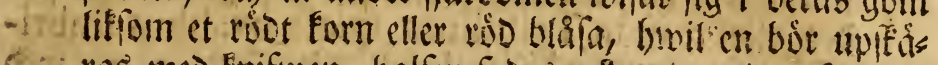
tas med frifiven, balfen fades of under tioen firuina igen. Syan berittade mill om et aunat Mago Dret, men fá otijoeligen, at man oarpa ingen rcoa fumbe fấ.

ORKORN war medicin boz bónocrma hä pa orten emot Sret; Srkorren famlases, od) gofiwes froinen inj, met

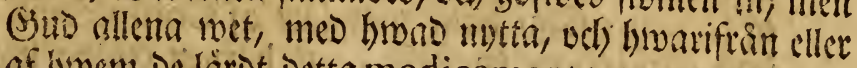
af brem De larot Detta medicament; ti) genene man

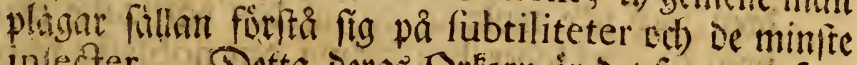
intecter. Detta Derns Drforn ár Det fanma, fom Emathinoninger Eallar Siftborr, och brufar für fina barn, Dá de hafiwa Siehoftan, brilfet wakere yeterelít

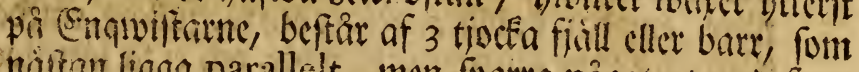
nitran ligga parallelt, men faarra nâgut ut mes fipets farta; innom befe ligga 3 fma fiàl, fom bect nuga fluta fig tilfammans, och innebolla en liten ros mafe, brulEen forbiter fig uti en Tipula (Faun. II 50,1$)$

PHALENA feticornis fpirilinguis nafuta viridis (Faun. 895.) fumb $i$ angen. Tungan war ganfía Fist: Yisingarne woro iboplagoe horizontailt, bas til mucfet emarginatx.

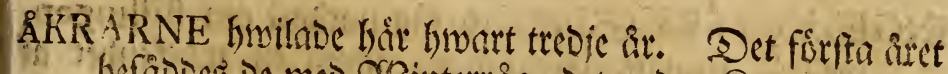

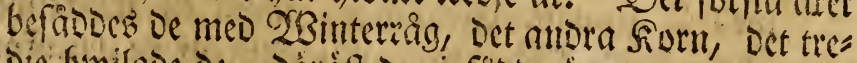

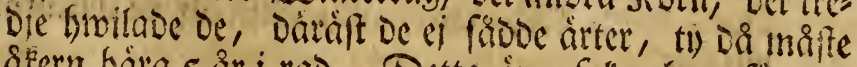
kîern baira 5 ăr $i$ rad. Detta are erfaten brarfoure all âfer bâr ât fürdeld i 3 garrocr.

TRIFOLIUM lupulinum (Flor. 6I8.) waìte i ängarna

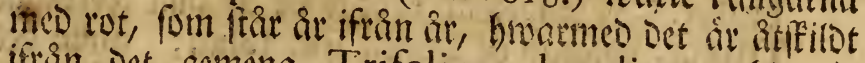
ifran Det semena Trifolium lupulinum (617.),

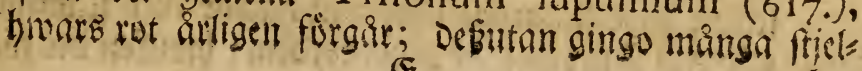


lat af ell rot, ott) ligo fall pả matêt, Dair Det alimåne na fliget (617.) bar allenaft en ftiele af brwaric tot, od) Den rak, eller upftiende; ftelearne moro lingre ods ftorre, ân pa den allminnta; blommorna woro toaí fa frora, fom pa Det allmánna, men mer gula; Det ffils Des mintore ifrá Trifolium Ipicis ovalibus imbricatis, foum fantẻ pa Gotcland, (it. Gottl. 257.) med ftorre blommor od ftielfar.

FAREN bir pa ortent woro af gemena llaget; ty) frigabes Bónderna, lowarfore de icese läta fima fir befpringes

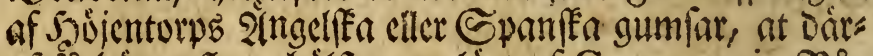
af fâ boittre llag, billf mun lint of Commercie Siis Detả Ahlitróms theorie, at barnen blifima lifa fas Dren, odh icfe modren, od) of Den Dartignfte mur ges nom en abel fader kan blifwa Den noblafte familie.

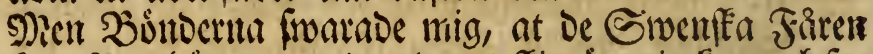
âto langt bättre, emedan Derab ffin áro tiocfare och fos fare, emedan De blifina fetare odb Evittfullare, emedan de

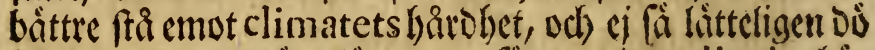
bait, emeona de aro lattare at fóda med ordinairt ho.

GETTER hallas băr mucket fă, emeban be for flogarnes feul âro forbunone; men om Jjrobften Weftbecks los ra famnas, fom beftrioer, at (Sictterne icfe rota ofmers fta tappen pa barrffogen, flulle fülia, at Gettertta toos ro offyloige odf fü Derab fora untta, áfwen fom $\mathfrak{F}$ : ren, borbe mes premier upmunttab.

INGESATTTERS Sittưgarto lag nutboft om Billingen,

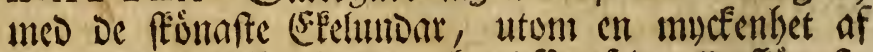

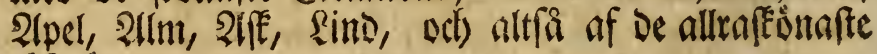
loftrin.

ALMEN aifiver forttreffeliga bróden, befinnerligent Den Sinenfera, foil ungefar ga up cmot walnote tribets

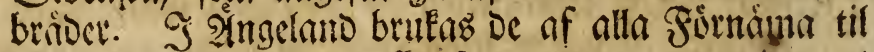
lifeiftor, emenan de gonfera fent rotura; utom sct, at

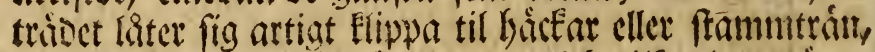

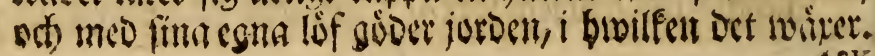


ASK áx et nuttigt tráb til Rlancarder, Mufical Inftumenter, 2(rlar och) anora fega aleerreofeap: Defutom

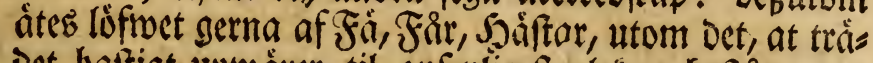
Det baftigt uproaker til anfenlig ftorlet, och ftär nuys fet grunt, faft Det nagot fent fare ut fina lóf.

APEL bar oce fin untta, faft frulten ir fiti, hat tientar til Cider, od) âfivent til fiminenb fóda; barfen fárgar gult; men trábet är befiunnerligen tienligit fór fruarf: wate,

LIND ầ Det angenám/ta trå $i$ ángar, Dem hou gibet med fina blad cller liff, act) redernmecter meo fim feugga, utom Det at löfinen aro gooa til lófflingningat, oct bats fen til baft.

EKENS mitta ấ allmånt betant, men bon Liatab på åfer

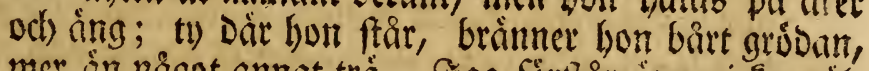

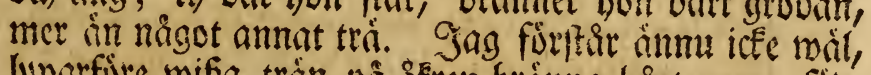

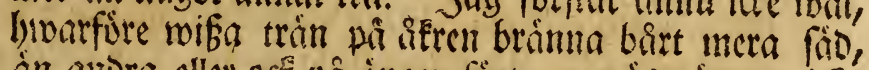
in ambra, cller sce pa angen fürtaga gräubartern; icfé Feer Detta Deraf, at folfrrsianna fruta ifrän trábets fros no per reflexionem pa grioan, ti) oa fifulle tríbet icte peroo warterna pa norra fioor, fom doce feer. Gefe heller feer setta of righnet; form faller pai trabens blao, oil) feoermetra pa marter, til man fer, at trabets ferdocliga werłan ftrâcfer fig mangn fammar wioare, in wattuoroppen. Ei eller feer Det of rítter na, form

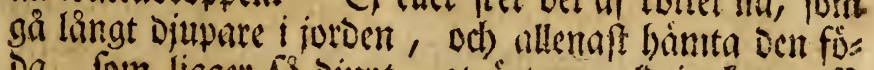
Da, fom ligger fo Djupt, at irterta nlorig euma fa nytta Deraf. Det funes Derfure troligaft, at wátfan, fom upfupes af trivens rutter, od gar up genom ftamm, gremer, lof, neen aldrig ncder tilba fa it roten, Dageligent uttounftar fafom et ofingliget rönn pa be Ering"

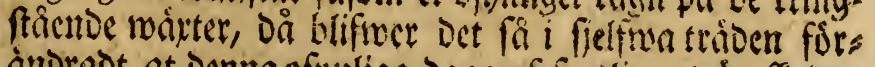

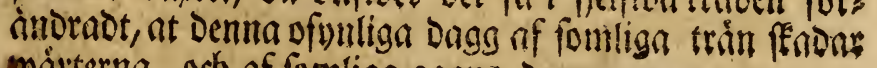
wàtterna, ocb af formliga gagna Dem.

BỏKAR ftoso a:me ftucfen wid whigen i en Elelund, jentte Inc, satter, och boro Defe De nuteerfta iblano alla 
Boffar jag feot, on icfe De, fom whir pa Smbirget i Sitergotland, tâfla med Dem.

ALUNBRUKET wio MULLTORP lig ph sitra fioan Din Billingen, at befi norra anoa. 2Nlumfifren brotb

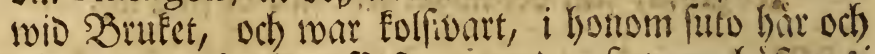

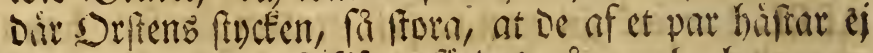
Dragas funde. EFifren fortocb hâ gamla liwarpen,

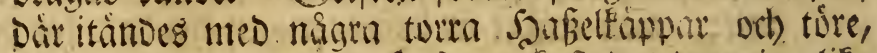

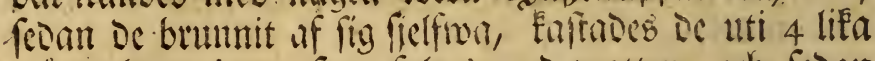
trit = refervoirer, fom foltes mes watten, oil) feoun wattnet utoragit faltet, tappades luten i- en brumit; biwarifran Den ran genom bwatpen, neDer at Fofbulet,

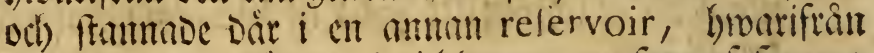
Denl fesermeta flipptes in i blipsannan, fom of famma Sllumbatten efter band, odb) fa fort Det funde af sota,

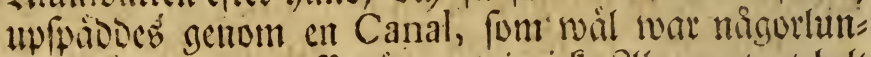
ba tiltipt, men ei fa töt, at ju icfe slumbatnet belt

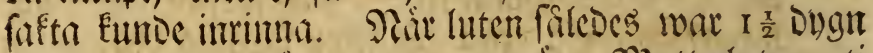
evaporerad, Mlapptes Denna grina D) Dutterluten uti

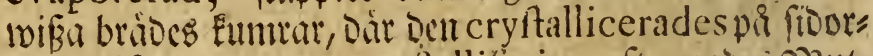
ria. (sfter 8 Dagars cryitallifering aftappaber s)?

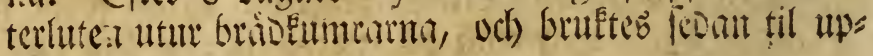
fporoning i blypankorna.

ALUN-CRYST ALLERNE woro míl en teffera octoëdra, men Defi anguli laterales tworo liffom affilade, och meo hwardera fpeter wife et planum rhombi, aifivenfom anguli verticales, meo firta afmatte focts firc, wifte et planum quadrangulum oblongum.

RoDFARGA blef af flemmet, fom ftannabe quar i blupans nan efter fo ninget, biwil"et Dorrutut uptaget, toréaies och brandes, da det fiet fin ridon färg.

ORSTEN, for fats ibluno Sfifwaritenen, brinoes un= Der tioen til fale; men himarken blef Dent fa hwit, eller

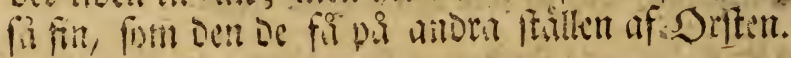

SK\&FDE Etan quar. 6. iftuin Berg, war en musfet lis 
ten flact, beligen pa ofitra fioan om Billingen, utan fii, cller nangen firtoeles fituation, hufen woro fma, gatorne irregulaire. od) Sivt ogarden tringfatt mid feima 2lfeat. Denna fad har fordom marit Sinctx Helenx fate.

\section{Jun, 28 .}

RESAN ifrån Skótde til Falkóping.

HAT TAR buros allmaint om fommartiden af qwinte sett, at be ci matte blifwa folbránde, hwarigenom Szonte Deqminfolfen werkeligen berwarat fin by, battre

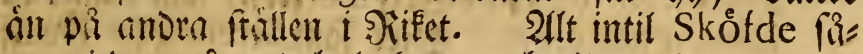
go wi Dem gá meo halmibattar, hwilkab Dinpa fulle ci war efter nuafte modet; men feoan man pafferat Skơfde, brufabes af quininfolfent froatta hattar, ordinairt lifea med manfoletris, fajt Djupare Eulle, och ei uphâftade pa fidorme.

FRIERI Kar off fina mode: Nár Bonbeoringen fáal fria til pigan, lâter han bâlfa herne genom nágon alfivar:

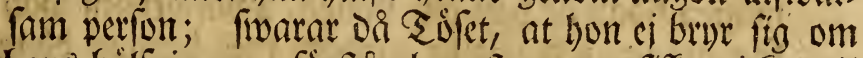
bans balliningat, forftar han ftrat, at fifeen icfe wil nappa pa Den utlagoe Erofen; men firarar lyon: Şälfa

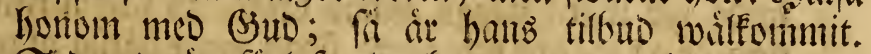
Scoan de aro fortlofivade, bar bet warit, brue, at bait

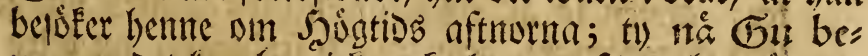
xoars, Det fan hon iffe nefá honom, feoan han art tros lofwad; th ja Eors Det hafiwa fíderme bru' at fort hent= ne. Detta ár det famma, fom Bosnobrangante, fes Dan be àro trolofiwnde, pá Gottland falla gå ocb

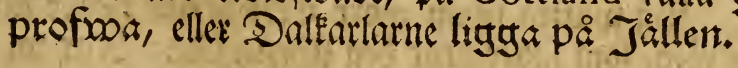

JASIONE (Flor, 713.) wätte på marken omering Segerstorp.

RAPHANUS filiquis teretibus articulatis unilocularibus (Flor. 568.) 'wâkte allmaint i ẫ̂tarna med gula De) ej buvita blommer, mâftedeló ibland fortnet.

$$
\text { E. } 3 \text { \% HENE. }
$$


* HENE. quart. 2.

BLLLINGEN, font twi i dang fólgde hela bagen på offtra fitoan, bade wi luft at fe ofiwan uppå; wi frego up mid Sfulturp, Doir mi mórtte, at roten af Billingen war âfwen fom Kinnekulle af Sand fren (Tab. I. fig. a.). Den medlerfite Delen of Billingens perpendikel beftoo af hwita och rósa Kaleflifor, sifwen fom pa Kinnekulle, (Tab. I. fig. b.c. d.e.) och) Det Ufinerfta af Gr\&ffen, affiven fom pa Kinnekulle (Tab. . fig.g.), fia at Det nederfta Sambitens fältet ăr mera flatt, Det medlerfta Fial fáltet mera Eullrigt; ment Det śfwerfita Burniftens fültet mera perpendiculairt. Emellan aila

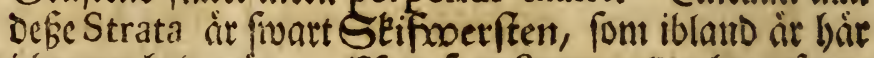
$i$ biottet belt grin. Effer fom Strata eller bworfiven iBillingen aro alseles de famme meo Kinnekulle,

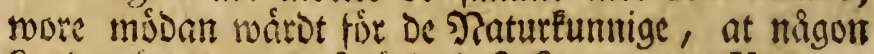
Samitmatare hoga afivigde Defe Strata emot Venern, och) fage efter, om de icfe ligga i fammia boggo, ocb wattupas meo Kinnekulle, ty fá fórefomma De efter ỏgnamáttet. Sâ angentum fom Billingen war på

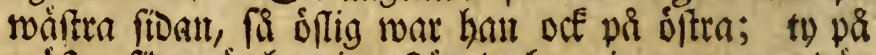

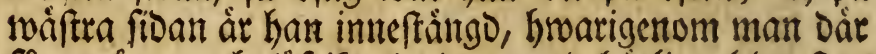
fág gróna, och) losfrifa luttoar, meo hảrliga blomfter, Daremot âr siftra fitoan gifiven til fpilld ât fámaden; torbe off wara nagon autuan orfake, ti) fáfom Billin-

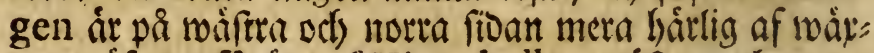
ter, éfiwent fí ât off Kinnekulles toáftra och norra

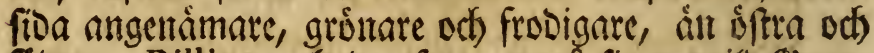
fobra. Billingen hade of want uppa fig ent wild Sran ffeg mes inager mar , vel) 3 fisar: Seminjón, Swart:

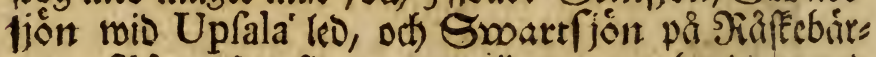

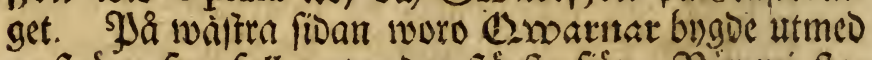
en ftrom, fom faller utur Den fteserfa fion: Nate ni fros

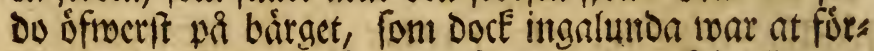
lifna emot Kinnekulle, $i$ anfectnoe til Det fringliggans

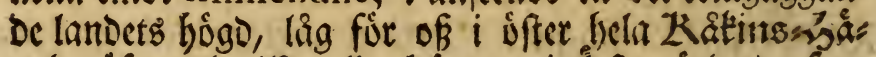
rad, öfiver bmilfect Ombärget i Softergitland, fom ligger wid sfter friban af Vettern, funtes i fuilt siter, 
Dod Eunde fielfiva Vettern ci funas, fuft ban lig pả ben na fiban. Eöber ut finntes ftora fîtter oct) rumber, Gruaruppa marttes twaime hoga och ofivan pa llàta bărg , Barnabårget od) Kongslenabárget Eallabe; ttom mingr. Surfor odf en wioloftig horizont.

POLYPODIUM ilvenfe (Flor. 8 50.) waxte til ett mues fenlet $i$ filefwan of ofiverfta grabarget, fom war perpendiculairt.

RóDLERA fallabes en rós mylla, fom lảg nebanfót flips porna, odi) fororfintadeb af Den uploffe Gfifwerfenen. Sisnderme berittade om Denna Siobleran, at hon $i$ wata ar wore goo for âten, men $i$ torra Deremot ut torfade bonom.

SANDSTEN los, eller foroun fom tienar til bryne och fân nor, fans ftycfetals i ăfrarna neoanfơ billingen.

KÁLLAN, af bwilfen Spultorpb bu fice fit watten, tank utur Billings fioan, Dár feifwerftencu flutade fig wio Sialeflifan. Detta rena od) Elata mattuet ledoes fes Dermela sentom rámor til byn, fom låg băr ttednnför, at folfet funde Daraf taga watten til mat odb Drucf, famt bolfapen. Sarr i 2un woro twäne Qwinfole barit

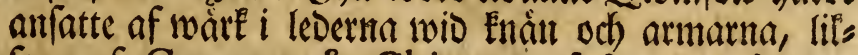
fom af Gonagra \& Chiragra, faft utan frillar, hivarmed De oragitz i flete ar. Denna waire torde

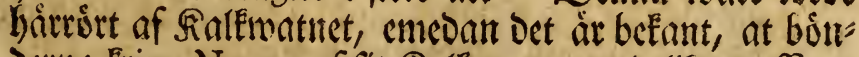
Derma Ering Narva of fit Falewuatten med lifa paffion allmânt áro pliggabe. Sag raboe Dem, at fórit en tio bruffa infufum ftipitum Dulcamars, od) foralt wais fen woro barta, pulverem radicis herbæ Britannicæ \& Gentianæ. Folfet hờ $i \mathfrak{B}_{\text {y) }}$ pligndes fällan cller alorig af Fruban.

MATLEDSBÅR fallades Gár på orten Dulcamara. (Flor 189.)

RAGNET, fom un fơll, siorbe bubblar pi̊ watmet, bivat: 


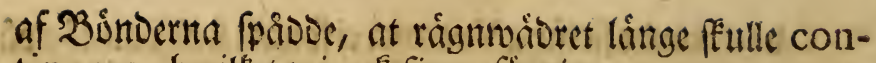
tinuera, broilfet wi off fingo förninmma.

LANDSWÁGARNE woro pá fleta ftitllen lagade meo ioel feifinerften.

BLAKLINTEN (Flor. 7 Jo.) gjorve Sirigåfrarme, fom ftodo tumna af 23 artovifan, belt bla.

SWINKALEN (Flor. 548.) gjorde Fornafrarna ofta belt sula.

FALBYGDEN begnnteg Găar, fom ấ et flát lono utan fFog, nog lift Flandern, med ftora ingar och) wios

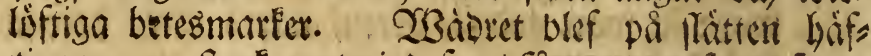
tigare, en frare motwind fatte fa an, at frart frgot sgonen wille blifa utur fenllen, biftent raglade od hids ftan utur wadgen. 233 blefivo oct geromblotta if flagrignets băftiga feurar. Nu nuaftes, broto belias gelighet feogfulla lanter aga for be llata.

* Ramstad. quart. 4.

BARGA låg på hỏgra handen om wigen, ofí war under Commercie gisiots' A hlftróms difpofition, Dar ban habe et Echefferie of 1300 Firit, foum vet fabcs.

WAKTHOJAN, uti bmilen Finherdarma funde bivila, Da de waitnde fint hioro, war of 2 alnars bogge, $I_{2}^{\frac{x}{2}}$ altis breos, 3 almars lingo, rullandes pa 3 lgjul ute pà quvierna om fommintmâtterna.

MASTER-SCHEFFERN rôEDocs hair pio otten, fom tuEtes behơfwa en goo influction, meo mera. Jaa tiffias

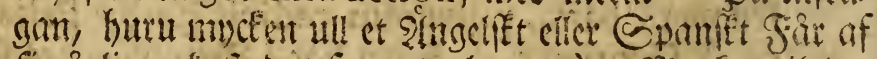

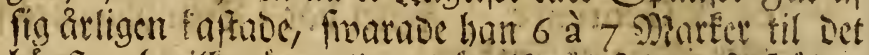

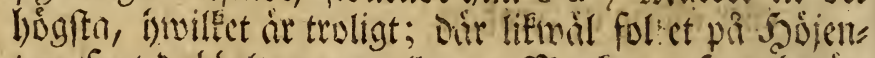
torp fagt Dubbelt meri, eller 12 Marfer. Som berats tade, at alla Torparme pa Sjojentorp, od tring Det famma, bave genom blandning ffaffat fig fa goo ant af. Fart, lonilfet friboe emot Det Edbefferne base fogt 17. ó pá fielfía Sajienttorp. 
* TORBjóRnSTORP. quart. 6 .

SILEX cinereus, at en ften, fom hómtabes utifuat mats fen, od) rone wàl utainda bovit, foin elt fintiten, men ci los til fétpan; Di han flogs fontock, war ban inusi balft genomftinande odh liuggra; han feiftade fig med nigorluntou convexa, och concava brott, fiñm $\mathrm{en}$ flinta, men war Docf mera fontig, naftan fom en

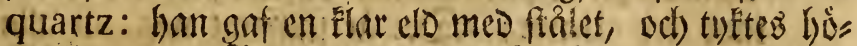
ra til genus filicis, faft han icfe af Erita war gene. rerad, Daraft ban icfe immediate af blefe fram= bragts.

MARMOR rude, lamellis undulatis, efler en broit siales fien, fom wastalb ffifrade fig. Denna war bato, balf gengunfimanoe, od) fEulle ofelbart gifwa et ganilea bivit SialEmisl; men icfe Gisps, fom Det fơnenentes. Denne fans fuscetals ibland ierarna.

KLACKSTEN * fins afiven bár iblano ftora boce lofa ftenar, Da Dente fatloes pă 3 fitter, gaf han flang, fom en s)ietall; ban war fiorrtgria, mucfet litet lern bunden, od) beftod af ftrott feimmer meo nagon fí firt ifprango quariz, at Den nappligen med blotta bgonen funde marfas, och hade darjente uti ftg många dumfla Siranater.

* KaLM BaHUS. I29.

MÓSSEBÅRG lág pa mófrra fionn $\frac{r}{8}$ mil ifran Falkóping. Sarget wât ofwamuppa tammeligen flatt od) bart, wio paf i vifer ud) waifer 2 quart língt, men i norr oc) fober I quare Dema flate Fulle beftod of Siras bori, meo perpendiculaire fibor, bwors fot feoermes in utfrifte fig til fere buscffotts diameter; fullrig neDer at fâltet; buvilfet gict ut til en falbugd, lintt els ler alfiwar; ef lamb, hrwilfet iffe hade diup jord, fort: an man fom neber til tód fifan eller Saltécillen, fon fivaraje emot Sélgftens bällen pa Kinnekulle, fa at Móffebărg boftit of famma Strata od ftenar, fom 
Kinnekulle, allenaft Eringliggande lantet om Mỏffebảrg âr boigie, ản omf ring Kinnekulle.

WAXTERNE giorbe of upmirefanme, emeoan Der fans neder on Móffebårg ve aloratuafrafte órter, och jâms toíl De fällfammafte; niór wi Deremot fommo uppa fielfiwa siullen, woro warterna belt anora, tiffom bas de man pa et par mimuter blifivit forto $i$ et belt ammat Iand ocl) climat: Det âr fiflfiamt nog, at alla Defe birgen, fafom Kinnekulle, Billingen, Mơffebårg oct) ̊llebårg, sfwerft uppa dár bárgen befta nt Bras

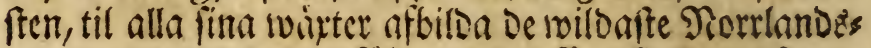
maticr, men beremot fälten nedonfore Siutharne froms britiga ej annat, án De wafknfte majter ifrân Skảne, ólands od) Gottlands (unoar, od) at Detta fia muceet tyoeligare mâ fésujne, wil jag upráfnn de fórnäm/ta.

KULLENS ÓRTER moro De allmámmafte:

fiung (Flor. 309.) श̂́renpriz 8. Bllăbår 313. Sattefótter 672. Doon 312. En 824. Singon 3 14.

WALLENS O̊RTER, eller Denconvexitet, fom li̊g nes Danfót Sillem, habe fótiande wáter:

Scanfel (Flor. 787.) Campanula vulg. 1 $; 6$. Origa" num 480. Draba alpina 526. Camp. perficifolia 179. Viola hirfuta 718. Sienfama 666. Camp maxima 180. Anthyllis 594 . Epilobium 304. Camp, hirfut. 181. Solidago 68r. Tormentilla 42I. Melampyrum vulg. \{13. Arnica 684. Leuchanthemum 700 . Melamp. coroll. hiant. 514. Galium luteum r16. Geranium fanguin. 57ז. Melamp. criftat. 5'0. Gal.alb.118. Geran. aconiti fol. $\tau^{2}$. Orchis palm. macul. 129. Orchis alba bifolia 723 . Orchis longis calcar. 727 .

SCORZONERA 647. war bär iftortre mucfentlist, in pa nigon amman ort $i$ Smeriae, fa at hou giorde nlla ein gar gula omering Mơfebärg. Behofion altfá 2(po: 
thefarne ci at farn sfiner 2irm efter watten, the en parte

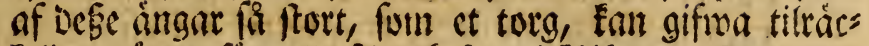

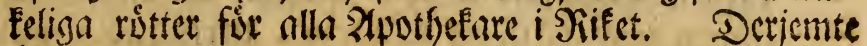
ât off intet twifwel, at roten, fom tages af oemma irt, ju ar langt tienligare til medicine, än Den, fom wirs rer $i$ trigintoarne, eller Gämta utumlandos.

CONVALLARIA foliis verticillatis (Flor.275.) maj"s

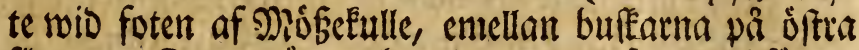
fitan. Denta orten bar jag entoaft fumnit tifforene

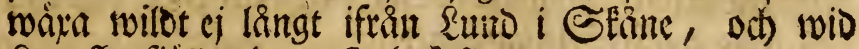

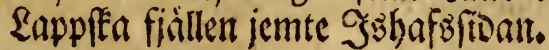

HUMLE (Flor. 818.) wipte willot wid foten of Misceluls le emellan rafet, flitgrnoe fiut refivor iblano buffétrma, pa siftra fidan.

LATHYRUS pedunculis multifloris, cirrhis diphyllis tetraphyllisque: foliolis lanceolatis. Lathyrus major narbonenfis anguftifolius. Baub. bift. 2. p. 264. Demta wayten hat ing alorig tifföretna funnit wära willot, men nut ráfabe jag Dent icke allenaft bär wio Mớffebảrg, utan off mio Ållebärg. Şerr P.KALM har funnit Denfanmma wio Kinnekulle. (It.Ba-

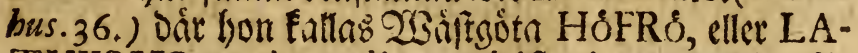
THYRUS pedunculis multifloris, cirrhis diphyllis: foliolis lanceolatis internodiis membranaceis. Gag mil iefe hir uppelyolla mig med Synonyma, fom cmbaft hôta Botanifterna til, utait

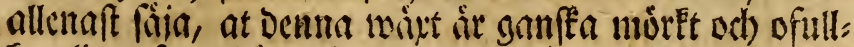
founligen framftálo af allo, clutru listt och flart bou elo

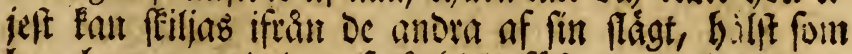
bout har merendels 4 fimí biab fijta wio bivarbera blad Faftect. Sgå set Botanifterne ci frambeles maitte famla bürmio, wil iag sifiva Dem fóliande befferifning: Radix perennis. Caulis quadripedalis, quadrangu. laris: angulis oppofitis in membranam dilatatis, \& hinc anceps, margine tenuiffme denticulatus. Stipula conjugats, utrinque acuminatx, e regione bafe- 
baleos acute angulatx. Petioli apice in cirrhum ramofum delinentes, triquetri, a bafi ad inlerticnem prioris paris foliorum utrinque membranacei, dein membrana deftituti. Foliola quatuor, duplici conjugatione feu duo paria in fingulo petiolo, lanceolata, trinervia, mucrone inermi acuminata, flexilia, minusque rigida, po licem lata. Pedunculi foliis longiores, ftriati? multiflori. Flores quoad vexilluni fubtus \& alas incarnati, carina vero exalbidi, \& vexillo extus pallide incarnati.

PRIMULA minor (Flor. I62.), fom elieft war mucfet rar hât $i$ landet, waite wio Mólfebärg.

HELIANTHEMUM (Flor. 433.), fom clieft ár allmån $i$ Giwerige, fingo icfe forr ân wi fommo hit.

POTENTILLA foliis pinnatis alternis, foliolis quinis ovatis crenatis, caule erecto, (Hort. Cliff. 193.) Den man forit uptrikte wio Sjuientorp, maite hàr uffweralt $i$ Silefinan, dá wi ftego up pá Móffebảrg.

SPARRE-LOSTA (Flor. 89.) wipte bart i mucfenlyet.

CARDAMINE foliis pinnatis: foliolis fubrotundo angulofis (Flor. 560.) war almant i Dilloerna.

FALENS ant odh natur fumbe tyocligaft infämtab af Siandt ocf) Ballerltens âng, fom lágo pa ojtra fioan om Mólfebårg, och hurbe til Commercie Siadet A hlftroms Sdhefferie; hon finarnoe emot Den frota Setesmats

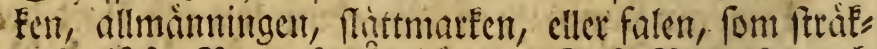
te fig ifrán Derga st sillebutig. Defe Banlerften och) Siambt hade warit twanme gardoar, efter hanilfa allenaft fifaut ftod qwar, pa bwilfen de warit bugbe, od) utur Den famma fiprang cin filla, fom formerade en bact. Gamma flifa ligg flat af rós Sialeften horizontail med laitset, och) belt bat, fâ at bet gruna gráfet allenalt funte rota fig i deb foringor. En fadan Falferifa bor man forreftalla fig unber bela Falbogoen, Djupare inum= 


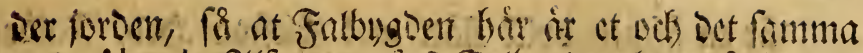
med ólands ellfwor, fafi fallygoen bar nígot mer Mulla uppirfits.

STRATA TERRE aro ofmeralt omfring Móftebirg, Allebärg, Billingen alt lifa meo Kinnekulle, fir at Da en har fig ftenh searfiden i Kinnekulle tisderigen bes fante, met man, bwas fom alleftäbes fan foftas bai pia Djupet. Hinneliulle beftir iffwerft of Graften, Darrnät Fommer et tunt hroarf Stifioser, feoan et Dlupt

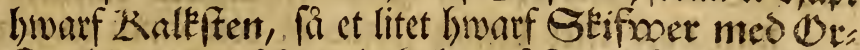
Iten blandond, fift et tioct hwarf Sandfen. Enles Dez à Det affuen beftaffast med Billingen, Mófebårg, Ollebärg, Hunnebairg, Hall, fom alla ós, werl hefta af Grabirty meo en mager joromort, lift Porrland: Efogatm; unver Denna bärgarten liggee en Stifoser, feonn Fommer iet roba Kalebärtyet, fom gât ut $i$ filt, afwen fom mio 2Bâfterplana pa

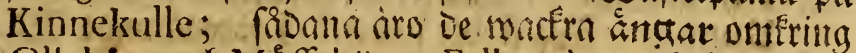
Ollebärg od) Mo̊febärg, Falbygden, Klefwa-Hed 2c. bivilta delo aro fruftammare, ba de hafma uppa fig mera froartmulln, Delo torte och fFarpare, da de hafwa mindere tilurectig Noblla. Sa oefe ftrata ga

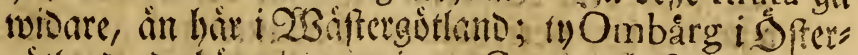
guttans, De hisga burgen iemtc Grenna hafioa fanma Atrata, ia indetigen A lfwaren wi Ślano, Carlsónrua, Torsburgen ocb Hoburgen fí (s)urtiani airo lita

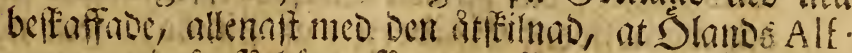
war $x$. ci gir fal hogt, fa at bet far be ofmertite ftrata of (Sirabitg vd) Etifmer, utan ban betrar of Den ro: Da flifan eller faltbiriget, fom lifnar Miedelplana on

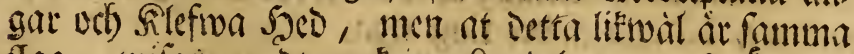
flag, wilas rundt omtring Santotborgen pá Ślano, broars rot ät en Etifner mes Orften, font befiunterli

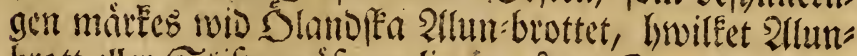
brott eller Siffwer âfmen ligger pa en Sanoften, Den Der froarar emot iet laga Ganoften: ftenbrottet wio Burswiken pa Bottlond. Det âr mig berattad of Sof Jintto Sianot Baron Sten Bjelke, nt GelaEftand 


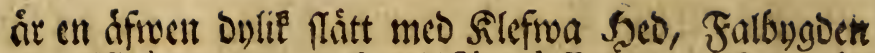

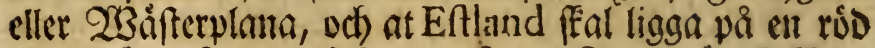
Salffilia, fomt at filfinergrufivan Rongshoirg i Nods

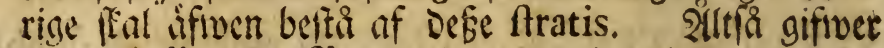
Kinnekulies Profil tab. 1. anleoning til Strata terre cller jordferorpans Anatomie, icfe allemaft bát i agafferguttano, utan tör bända til fture Delen af ivertbett.

LITHOGENESIEN ir fufler en muctet enfaldig faf, men Douf efter Dema tioeng fă rón ámu nog móré. 2Si wete, at af 万afefanto blifiwer Sandfen, Cos. of Bufwets fediment Leta, of Sera Ziale, af Siale Z3lete, of sBlete Zitita, of Sirita Silex cller flinta.

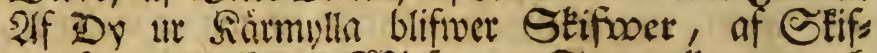
ren \&ter Mrylla. 2Bi le, at Spat, Outurs ved Bornften mo Mica waita Dár bárgen fpruffit, od

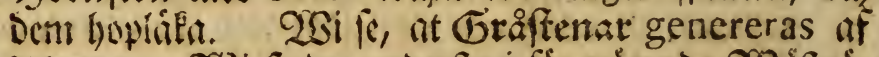

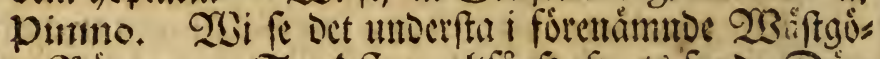

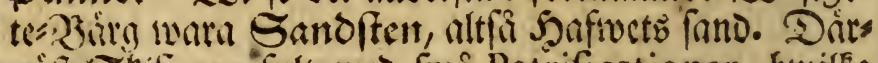
najt Skifiner fult meo fmá Petrificationer, hroilfa áfivent fig infiumn i Dent med Elifien blandade orften.

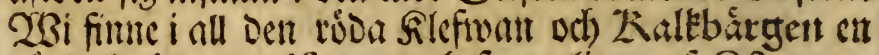
- aintoelig bop petrificater, befinunerfigen af Oftrocodemata, foun nlla fortoum wateit til frota fick. Sháe ofivan uppa ligger Żlappur, four finarfinats of Sals wets wigor; Dit pa stet Seifioer, fom toarit Mrulla, och alderasfiverft Graberg (of glinmo), foum nub fos feligen måfe morn temporis filia. Salt Detta fátter Den, fom fer fig tilbafa, i fturyta fórumoran sfroce Dent

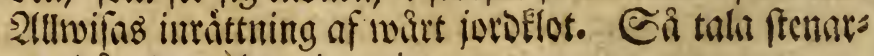
ue, Dá slla aitore ting tiga.

* FALKÓPING.

\section{Jun. 29.}

SÓNDAGEN hơlt of frilla i Falko̊ping, vel eftêt nibode gen moro wi pia ct bróllop i girnen TWIK. 
FALKóPINGS ftad raar râtt liten, ban matteb ned fteg, Da Gan fans $i$ bredoen wara 200 , men $i$ längoen 400 fteg. Gatorna iftnoen woro linge efter halfannor,

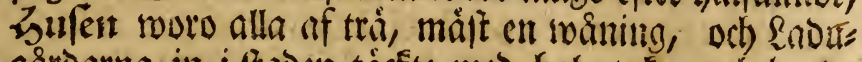
girboarna in i fraben tácfte meo balntaé, ocl bugbe med fiffert. Cortget war $i$ proportion mut frauen

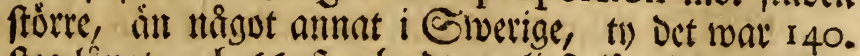
fteg taingt, och 66. fieg breot; elieft ling fraven pa cit bebagelig dech rlat ort, emellan de wacetra bärgen Mớfe- odj Alleberg.

GARDESGARDER hảromering woro uprefte meo et attigt páfund, til gárosilets fparande. De taga 4 aे 5 nls narb langa fistrax, fròta Dem perpendiculairt $i$ joroen, 3 tumm ifrân bmaranora, bảra Dem ofivantil igenom,

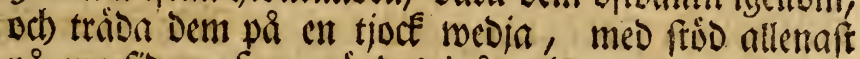
pá cna fidom, fom woindes inat, hwarigetom be iffe allemalt fia et titt od') tâft ftängfer, utum oef Det meo minfta smesftnas.

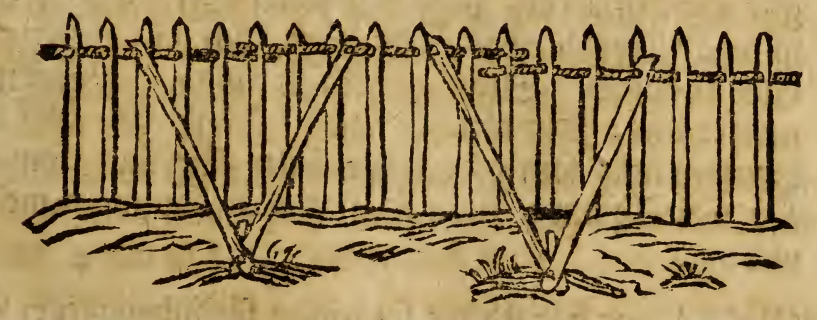

RIGN fommer altio af moln, moltr af bimma, och bim, man of watnet $i$ jorden; men bôt one nagot mer i natu ren, fom fiwarligare begripes, nimligen at bärgen Dras ga molnen st fig, hwillet oce nilla \&appar sueta berista

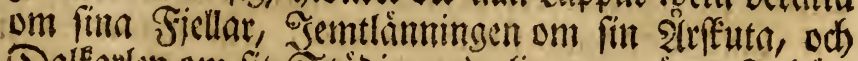
Dalfatlen om fit Stitoja, nuimligen at neir Debe bärs gent tran på fis liefom en bufwa, fom de fäja, Det ár, Da Deras ufiverfta hufivuden liefom omgifivas meo en tîfn eller rí, fá féal rágn fumma; Det famma utrôts tes i Dag pa Móffebarry, fom liefom rófte utur fis futs 


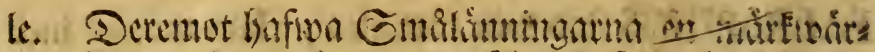

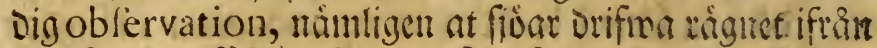

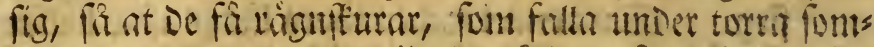
naren, merctiocls undwifa de gerrour, fom liggat nars

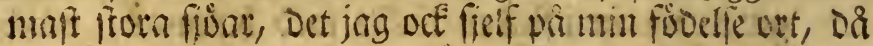
ing anmu toar ynofms, oitnd cliga gangot meo firtum Dran fág fantmos. 2iltha foller bataf, at meta righ faller pa birgen od) biga lander, an wa fidtanote, ellir

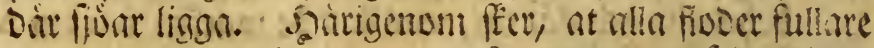
rimn, emeonn be alla taga fit batten utuf Det riagn,

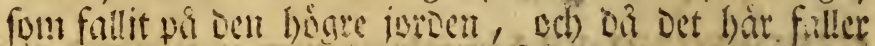
mer genom Denm of Genparen fir unberligen imrattade las, fia circulerar vit loatnet mer, od) jorben befüe tes incr, imnan iet framar i bafibet.

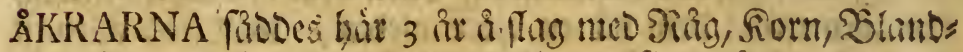
fio, bd) broilade mafted ds bwart fierde ar.

BETESMARKEN fring Falkóping befroo af twibar oct) bim fill, at alorig en bule fintes pia Dem, utom mas gra biggt quarters bjga uch breda isinebufear, med mânga finat en treatiband bogh, od pa fioornt rum base tufmor. Stenar woro hat inga. 2jetertwar

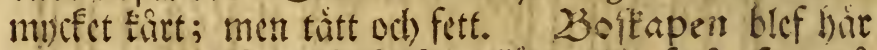
bivarfen fror, fom pa Eefine. llitt, cller Inn, fom pa

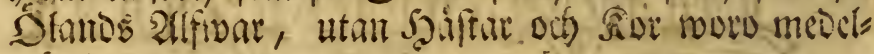
mattige.

SKOGLOSA war bir ftorfita fwaright for inbuggarne, fom ufta mafre gifma 3 à + Dall. Simt fert meoln bet, och oct med glabt bjerta, allenafe be Eunde fia det for fen= rimgnr.

GARDESGÅRDARNA woro merendels af trix, ef utan

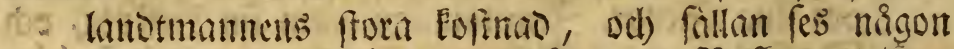
(Sarosgaro of roda fialeflifor, th) Sogfapent rifida Detl neoct.

HACKAR feulle alorabeqwamlignt boir funna anliggno

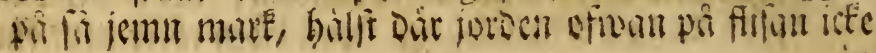




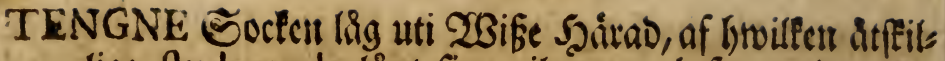
lige ftuderande länt fima tilmamn, befinumerligen De, fom af wilba ftammar blifivit cultiverade, och haraf tan man fe, på buru manga fätt tilnamn Eumn fä af an odh faimma fafe, firfom:

\begin{tabular}{|c|c|c|}
\hline $\begin{array}{l}\text { Tengnander, } \\
\text { Tengner, } \\
\text { Tengneeus, } \\
\text { Tenglin, } \\
\text { Tenglind, } \\
\text { Tengdin, } \\
\text { Tengden, } \\
\text { Tengzelius, } \\
\text { Tengftad, }\end{array}$ & $\begin{array}{l}\text { Tengborg. } \\
\text { Tenghem, } \\
\text { Tengmarck, } \\
\text { Tenglund, } \\
\text { Tengdal, } \\
\text { Tengvall, } \\
\text { Tengberg, } \\
\text { Tengbolim, } \\
\text { Tengfors, }\end{array}$ & $\begin{array}{l}\text { Tengftroim, } \\
\text { Tengbeck, } \\
\text { Tengbom, } \\
\text { Tengrot, } \\
\text { Tenggrem, } \\
\text { Tengquif, } \\
\text { Tengblad, } \\
\text { Tenglof, } \\
\text { Tengblom. }\end{array}$ \\
\hline
\end{tabular}

\section{Jun. 30.}

RESAN feetobe ifråı Falkỏping tillallebärg, Mơffebärg, Odens Kulle od) Klefwa.

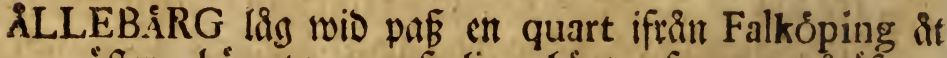
offer; bárget war anfenligen bogt, ofwan uppil aifiwent fom De andora Wäftgóta bàrgen, meo betešmark, Docf utun fFog; fiDorne eller Elefwoorne fring bárget musfet brante, och ofta of flippor perpendiculaire, fon bet? hogifta fottämur. . Sedan omgafs det med ct uteorigt fált, fom feoermera med en Eullrig wall fluttabe ut ât,

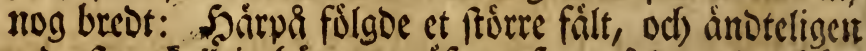
nederft en fullitis bárgstot, áfroen fom pá De amber bite gen i Wäftergótland, naimligen Kinnekulle, Billıngen och) Móffebàrg, fa at hair mat sfmerfta lutlent millomark, men neoanfơr fullen Sfmeralt angar, fom ndermaft tullen moro fulltwuine mẹo buffar och bóga wäkter.

KULLENS WAXTER pa Ållebårg woro utom of́ndes lige mannge tufwor, alt milda Norrlanoswákter, frfom: En, 'Bläbir, Singon, Sinttfitter, Sjiottron, Sloorot. 
CORNUS herbacea (Flor. I32.) waitte băr $i$ ftoc mucfenthet och) belt allmant, Den wi tifourene pa refan endaft foot wio Skara.

PYROLA floribus racemofis difperfis, Ptaminibus piftillisque rectis (Flor.331.) fon clieft warit fâfint, od) fort 6 in feocit ef $i$ Grocrige feod, wayte bar belt allmint.

PROS!ECTEN ofmerft på Ållebärg war artig; bầ fou tes pa moromáfra jion Kinnekulle, Mófebärg od Bifkopsklacken wio SBrunsbo; pa vifta Wartofta Saurad, pa fodra Falbygden, pa waiftra Nyckelängarna. Sblano manga andra Furkor fage Carlabylânga, namntunnig af Det, at 3 Sionungar Dâr fEstat blifwit begrafne. Et artigt fpectakel martte jag bidglt uppa bourget, da man fäg neder pa De ftora odb flatta ingat Deromtring, fom af Eslenes feen woro uplyfte, bura molnen med fora froarta flicefor woro pa fame ma fâtt, liefom frtubde pá angen, od) flogge undan, fom se clieft pläga gora pa himmelen, liniffet fom af

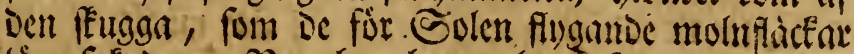
fororfatiod. Sag bar hroart baril jeot jenggain af moln niuga of wer joroen, men alorig pa nagot fitt at forlitna emot Detta, ty De fagos fma odi) morta fom ea natt.

STRATA woro juft de famma $i$ fllebárg, fom i Billingen, ods be andra bägen, meo bejuunerlig mucfen ffifiver.

RODLERA war på atffilliọa ftållen omfring Allebårg mucfenlset, lie en robbrun lera, fa at rottupuferna, på ben rodgen fom gick bir forbi, woro efter rignet brunfärgave.

KÁLLOR meD Det aldraljufligafte twatten vanno nebcr pi foooftra fidan af birget.

WAXTERNE pa fórfta convexiteten nedan fär bairget $\mathfrak{F}_{2} 2 \ldots$ hit 
St Hotra fibon woro befunmerligen: Aftragalus (Flor. 591.) Ulmus 219. Cepa 265. Scrophularia szo. Stachys 489. Trifolium montanum 6ı. Vicia fylvatica 603 . Cirfum 657. Veronica fpicata7. Tulfilago 68C. Ononis 622 .

CONVALLARIA foliis verticillatis mirte miyctet mid fioorma of Allebàrg.

POTENTILLA foliis pinnatis alternis, foliolis qui nis ovatis crenatis, caule crecto. Hoit. Ciiff. 195. witte iffucit miD firoth of Elefixat.

RHAMNUS (Flor. 193.) whitte for bit fom ammarficites

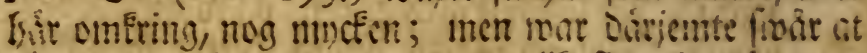
fintula; to ban soar ingalumba lif lit enit tro, utan wirte ut lon cu Exyanoc ort, mo fingers longa fict:ar.

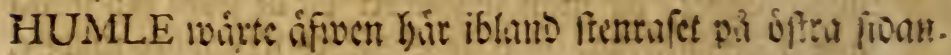

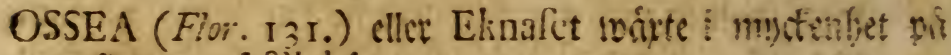
liovene of slliebarg.

GRODOR (Fan. 250.) boptrite $i$ fror mucforbct up ent

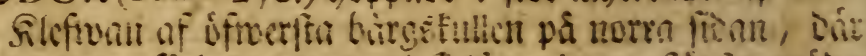

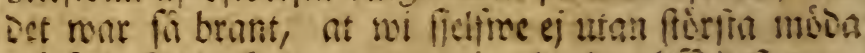
od) furn funos Fomma up; mi umbrade alffir scfomer,

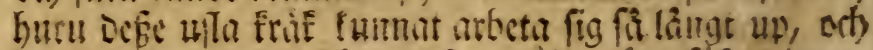
hwab Defe wille at hogect, fom altid pliga fóta biupe.

COCHLEA tefta pellucida, It. Ölard. 155 . fans ifmer bitr i Elcioun utinu firg.

MUSCA thoracis apice bicorni, abdominis margine acuto nigro: maculis feptem flavis funs but wil Allebire, fom fillan cliefi forefonmer, to bar male aftat nodigt, at bejerifixa bene nware: Bufwoubet

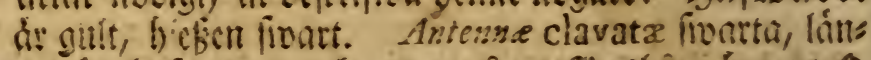

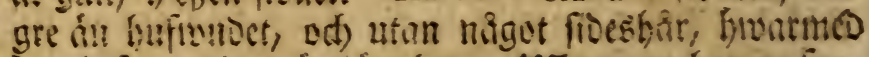

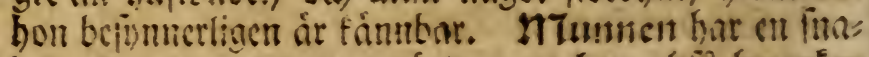

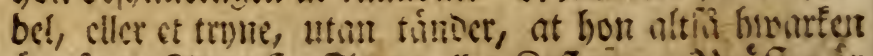

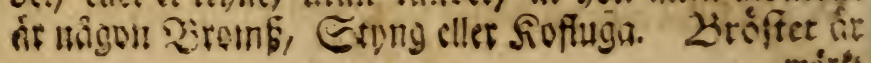




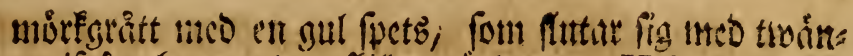

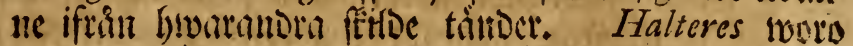

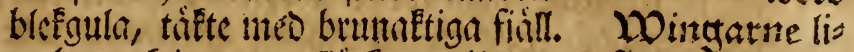

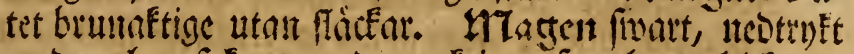
meD an buab fant rund onfring, fou bar 7 blckgula

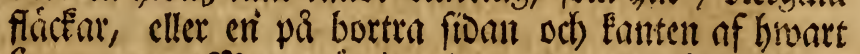
fegment. Diaget ât imumber gul, mec \& brunaftiga mảocr. foetterne grabruma. Det maitEvaroignfte

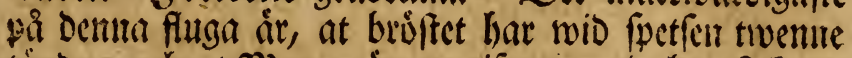
tänocr, och) at 2) iagen ât omgifiven meo lowa k kant. Deima ăt elt odf) fammin meo Mufca. (Faun. Saiecico ro83.) ment togate bar beftrefiven och) unocrioft.

GRÅSWRXTEN war fá hăr fom på Kinnekulle oç Bil-

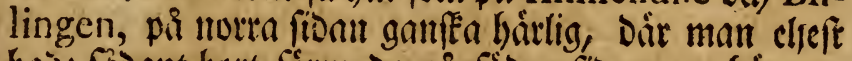
bave fibont butt formooa pa fisora fían om bargen, b) wilfa ligga liffom oreforntar emot Eolet ; ty wares

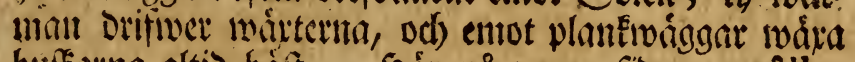
buffirna altio báf. Sẩ på norra fionan om Âllebärg war ingen fis, fon funte limbu bet bâronte climatet, fafoum pa Kinnekulle, lifafult wörte orterne bât frobigate. Sglvad wattet angât, fa rant bet pa

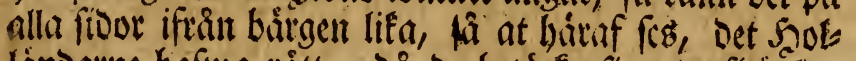

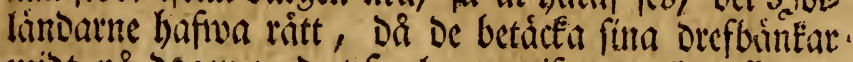
mibt pir sagen nico et fegel, at gifitr en fiten feugga for miodags Solell, äfiven foum ocke bärgens miodogs ffugga bjelper be orter, fors waja pi Derne notea fioa.

NYCKELÁNGARNE lâgo intil Allebårg, fom fî̉gas fät

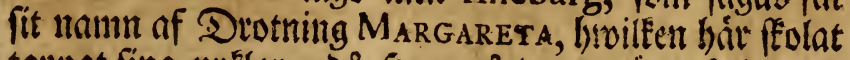
tappat fina nyElar, da Syon pi dema aim ftoo meo fiit Frigghait emot fiong A LBRECHT. Jag bnt alorig feot fa litita angar, pa bwilfa fnapt en entoa tufroa funde fintas, de woro defaten nuycfet ftora, fa horizontaile, fom en plancras trígáro, mes sitfilliga af =

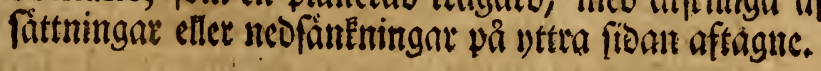

MöSSEB ÅRG, fom wi lenumade i forrgait afton, befigs

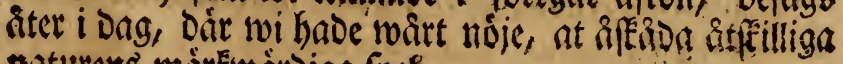
mitturets márEtaciroiga fpek

$$
{ }_{3} 3 \mathrm{CA} \text {. }
$$




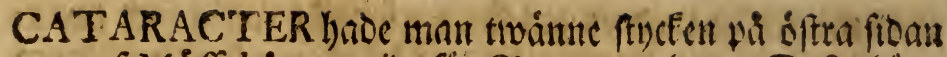

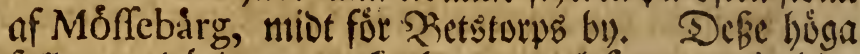
fath woro Dirriente mucfet brante, oct) formerade hars liga Cafcader, fom Diupt af watnets ftabiga arbete

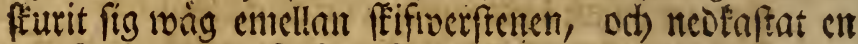
nucfentliet iten i fisffiva fallet. Uti Den nurra Cafcaden fintes, huru wattet gice bela byfecfottet imunder jorden eller ftenarma, oth âter lom ut isen med Elart lopy, brvilfet iffe allenaft gaf et priggtigt utfecride, utan

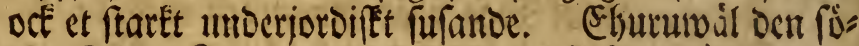
Dra Cataracten war Denna tiben på âet náftan torr, ods Den notra fiste ci beiler nu muctet watten, funbe

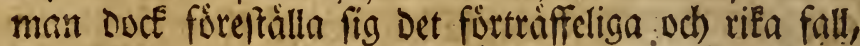

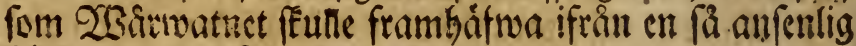
hógo, emellan fá många od ftora ftenar.

SKIFWERSTENEN, fom på fioorna af fallet war afbrus telt, Deltes med perpendiculaire fétriningar eller finitt, ad angulos rectos, vel acutos, utom Det, at han horizontailt fififrade fig, fom blad $i$ en boe. 2if Dent na flifiverfeten war Den, fom lág ófwereft, och inuti bárs get, hâro och frwart; men Den, fom ilaig nedantil, ether

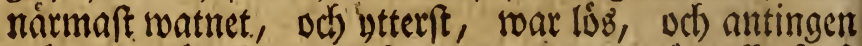
grión eller ródalttig. Då utan med Degn gróna féref på Den fivarta, blefivo bofftáfmerna brita, ment Da man fferef med Den rỏda, blef feriften ròd. Där Gol od tígn länge badat pâ fwarta feifwerftenen, blef han folverad til chl lós jorviften, och) Då máftedel'b utanpå

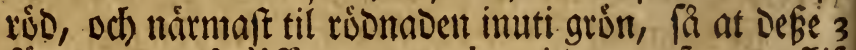
fárgar allenaft differera gradu, $i$ Det at jowatta flif reir fürft blifwer grosn, fä ród, fedan füufaller ban til

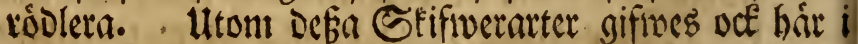

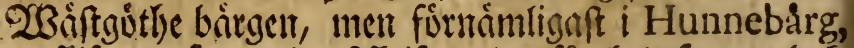
en fififwer, fom wio paffrifiwandet fär helt frwarta boes

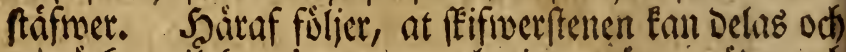
utmàrfas til fpecies getrom brwita, grưna, rída od fuarta Characterer, 'fom ban lómmar wid pâfertifs ningen. 
KALKSTEN fants til mucfenbet i Detta fall, af ett gaulfa fällfam art, Dánutimun, at dả feifiverfenen med fitta blad eller lameller gifnat, liffon cu balf open bop, hafiver ban blifwit upfislo emellan lamellerna med et fin lera, font nindteligen girot til en brvit EalE; Derfore (j)nteg ffifiverbladen ita ut med fina fiwarta Eanter, til en twáffingers längo utom feenen aloeles ofórcinotade. Nár Dentu ften flogs fonder, och ell mente fig fiuma i bonum diverfe ftrata of feifwer ort laleften, farom en fivart planbot med hwitt papper interfolierad, cller alternerande lameller af fiwart ffifiwer od bivit Ealf, ficf man emot all formobant $\mathcal{C}_{\text {, }}$ at feifiverftenen iffe als lemaft inuti bytt fiut fivarta färg til liusgra, utan ocf riEtigt fơrninorat fin egen art, od) blifwit aloeles til Eale, juft af formmu art meo Den Eale, font fommit af leran emellan lamellerna. SJatraf fluter et oemotfajeligit tón, icfe allenaft, at leran gấr til fale, utan ocf, at falfen fan fórbota feifren til fin egen natur.

\section{PETRIFICATIONER futo nog mainga uti De af fallet neds} Eaftade falflidlar, od) beftooo merenoels af Conchis ftriatis, befinnerligen of Concha (Faun. 1341.) och) af Pectine (Faun. I 343.), hivariblano act funnos ate tige Dentriter, fom liêtabe en mofa eller trá, of lune Dar uti mignature afritabc. Gblano nnoro Petrificata fans bár cul muctenbet af Det fơr mis obefanta Petrificatum, foun ing afritat uti olandnka Siefart pag. 147. hwilfet bat genom flcen och àtfeilliga impreffioner gaf mera anleoning at utforffa, of broa jut Det mátte bafwa lommit.

Se figuren pa fólianie fida.

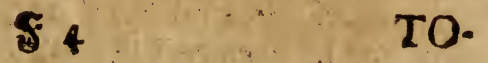



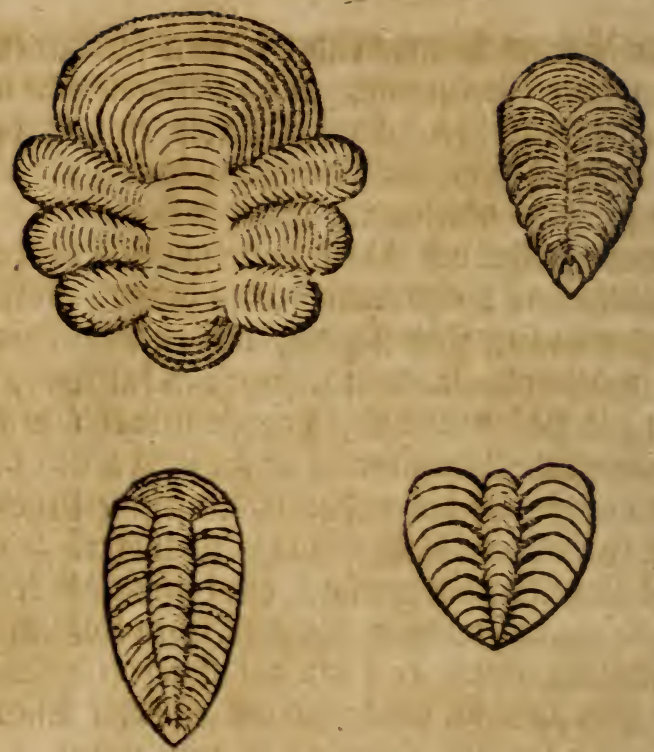

TOPHI af fivefefulors cller fora muflors feapnas ods trios, funmos imuti fafineritenten, od) beftoso ficlfue af froart feifwer, men babe blifinit batbare af et jerts watten, fom lig i Dem infupit, och Dem meo ch ftals fearpa fargat, fâ at oe funde bollus for en fivag jerts malm.

BARCBEK frottade ut af feifren pả atffilliga fraflen, fom

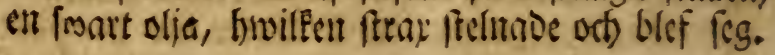

CARDUUS acaulis, calyce glabro (Flor. 656.) waite bar twio Cataracterna, befunnerligen wio Dent utorta; ban át fitlan feos of of $i$ Eiverige, utom Gottland od) óland.

ATTEPLATSAR lịgo ảtffilliga på fluta intmatna, nås got litet offucr jorden Eullrige, Eringlagoe med ftes nar, tiffte med jord och gris. Sila hade mid uti fig et ruin, Eringlatt af fififrig, od́) pá fant ftâenoe Slfivars=ffert, ofwan uppa meo ftora frenar täte, als Deles lifa Hjelmars rór, faft minore; trár man ff́ar

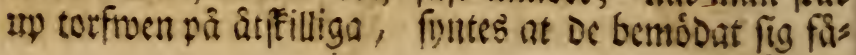


fâtugt, fom af:froutmulllans hogo formodat fig tumu Dobmat om attplatfarmes alber; ty Där, foull ell fooan àtteplatţ legat fere ar inmeftảngo, eller $i$ en sing, far ban Dubbelt, ja furoubbelt mera mullin, an sent, fout legat pa betesmarken, ty finartmullan blifiver of fors multmabe rotter och toilter, font altio waxa fiptoare o(t) minove pâ beteğmat'etr, án $i$ ängar; Defutan gầr

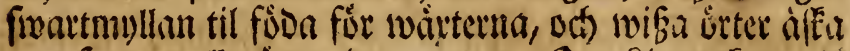
mer fivartmilla ồ amora: e.g. Dm \&jung Eommet at waja pi cn utlago ätg, fom bade 3 quarters

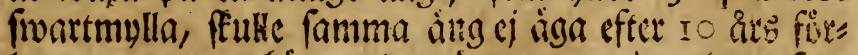
lopy I quarters bog myila; an mer, ent itteplats, fom ligger pa et hogt lant, fan alorig witita yi rooo:de

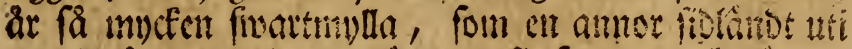
soo:De ar ; to Dels tota ofterthe fig fmarare od latture

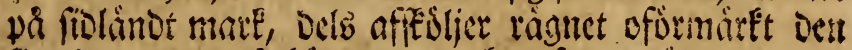

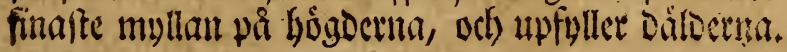

SOLEN gice meder forfta gången $i$ bag, dá wi moro I quart iftån Falkóping.

GARDESGARDARNE beftodo Delb af gátrble, Delb af Sillerftenar. De fenate woro of 3 . Tlag: I) uplagbe ftenar, fom ftodoes pa ftoan mes frorar, livilfa par od) par ofroantil woro hopbumine. 2) af tipftaplave ftenar, ibland bwilta de minfta lingo of fuerit od bes táfteb med torf, at de ei fa latt mitte feingrab.

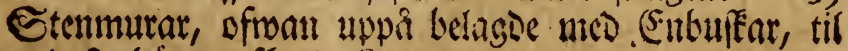
at defto battre afbolln sircoturent.

ODENS KULLE lág wio paß̧ 2 quart iftran Falkơping, pa mân(tra banden wis wrigen; baul wat nátan fí big fom Ållebärg, mett cj wio, od) utan trá, betraft meo grỉzivall, ock) ej pî fitoorna afbruten, utain gioro meo fmâ âtffitlige fólt od) fmâ bơgar. Stroan uppa soat et flïtt fălt, fiffom et ftort torg, pá brwilfet man i forbma pefttioer gifivit De Doba at Oden, och Dem be: grafivit: Det berittades, at hà warit tilfórene arlig

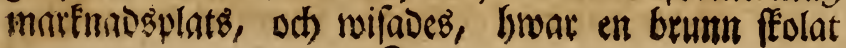


warit grafiselt, fon doct net lienaoc rudera cfter $\mathrm{ch}$ graf uted fter.

HAMANS GALGE baoe alorig enunat loft tionde Des Ien fá längt, fom Den galgs, hyvilfer war upreft pá fös Dra Eantelr af Det bóga Móffebårg, Dáe bärnet givorde

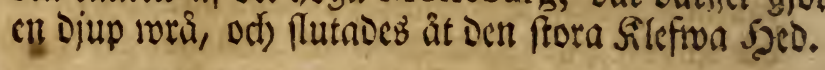

KLEFWA HED, på hroilfen Drottning Margareta flog Ronunt ALBRECHT, lìg cmicllan Odens fulle od Silefina Sunta; hon frambar De alrabárligafte ángar

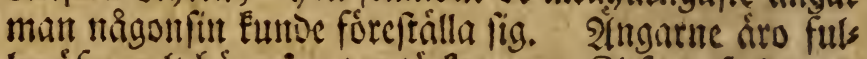

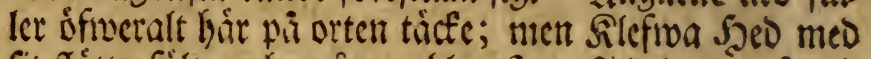
fit flítta fält, oct mânga blomifcr, froo Denna sirstic Den fat tice och riuffig, at han of fwergict all Den fome

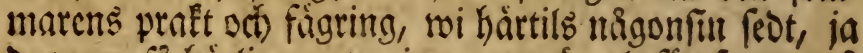
be woro fis birtiga, at min perma âr alt fơr fivag, på

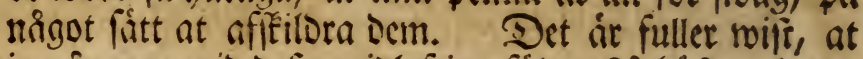

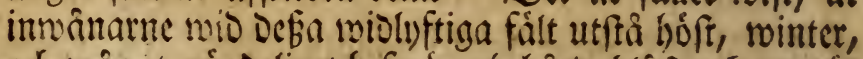

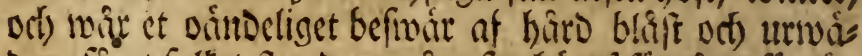

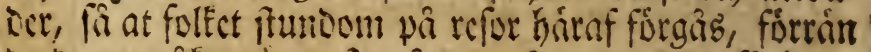

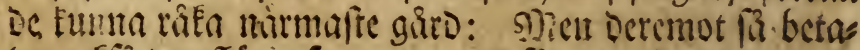

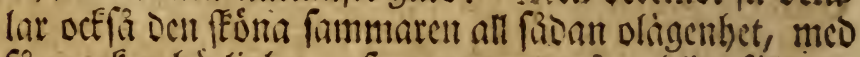
fá mucfent bartighet, four naturent pá et bliot fält uts

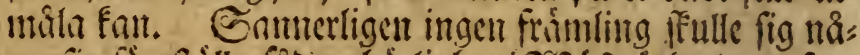

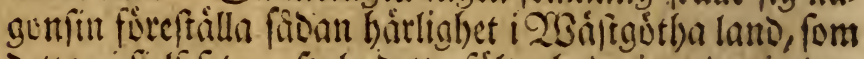
Detta ei fielf fet. Sacla Detta fáltet bave inumber jorben

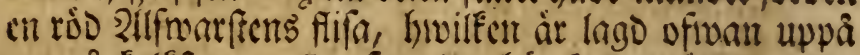
cn gri Ealfften, eller fom oct hár Eallas tilloftent eller golffitem, hiwillet tyderligen at at fe wio filefiva Gurefa,

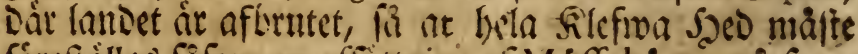

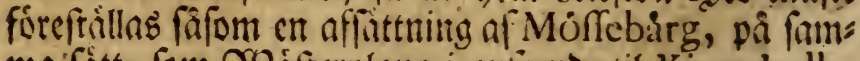
maifitt, fom 23 âferplana i anjeende til Kinnekulle. Zjlomiften, fom Denna tioen mâlade Silefiva Saco, moro befiunterligen Det róda Trifolium (Flor. 6I s.) De bla Filactor 176, oen broita Jy Den gule Arnica 684, Lotus 609, od Anthyllis 594 . 


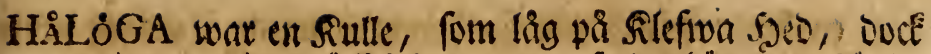

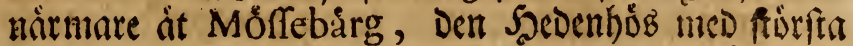
mosa upreft, til ffapma od) hojgs med en af gamla Upfala hogar, hroilten iffe ringa fägnade $D c m$, fom

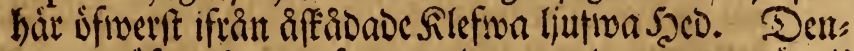
na war of fwerft utgrafiwen med en rund grop, mer án til ell mans Djup, od) altfa längt fâll fammate, àn Jung" fru żolan utan fưr Falkópings port.

WEDBRIST war hằ på orten Snndtmaunnens ftónfta fwärighet. Sann flagabe altamaft sfiwer 2llunbrufen, fom redan woro 'nullagoc, eller Dâr anlágga tánEas, fá at han fruftade fig med tiden ànmu fiärmare nobgas feaffa fig bránosle, odt) troobe, at den fürmon, fom fâ àgoe, med tioen fétle idelingga hela Falbugoen.

BRASSICA perfoliata flore luteo (Flor. 546.) Iwaitte bấ ófuerflobigt $i$ Siornet, fom icte fintỏ $i$ norra ore terna of 'Sáftergithland.

SOLEN gicf anbra gången neder, Då wi efter 4 quarts re: fa fommo til Silefiva; to da wi lemnade Falkóping utur ógonent pá óftra fitoan om Möffebärg, war So: len Dar neogângen, emeban Falkóping ligger fa lågt, at bela ftaben cir lingre, an ftora Falen, ia, fa at ftas

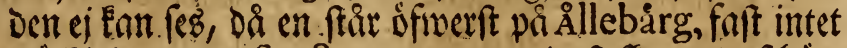
trie binorar profpecten, utan cmoaft fisgges af bats

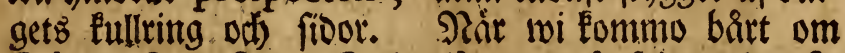
Odens Sulle funtes Solen lingt uppa Sgimmelen, $f e$

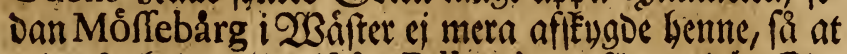
wi refte hela milen ifrán Falköpings fioan, Dár $S_{0=}$ len redan nar neoganmen, fortan wi fommo til Silefs na, of Golen giet neber.

\section{Julii $\mathbf{~}$.}

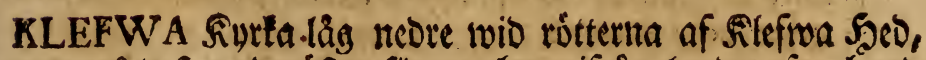

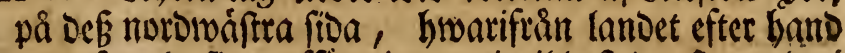
tog af med fere affáttningar, intil Deß Det ftammad i 


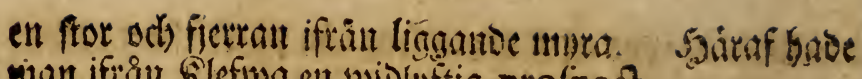
man iftin Silefiwa en wislinfig profpect.

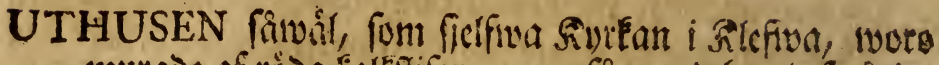
murade af rüda fallefifor; man fäg meo hinao fiveritgs het leret baftade nio dema rido a talefternen, emedar

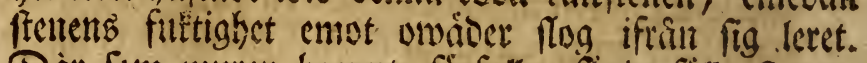

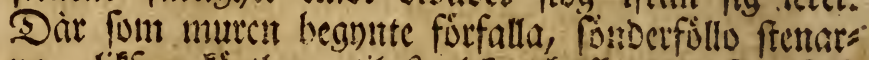

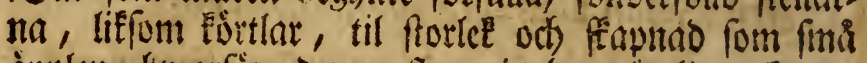

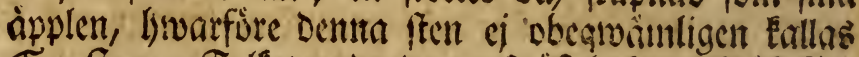
Gorfter. Folfer untorade at s)hafebafar, Medífefäs for, famt 2Iltarciufar multmabe bart i Sacriftigan;

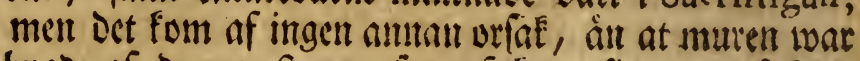
bugd af Dema fren, fout futtar fig mot. fultigt roiber.

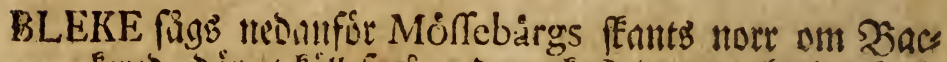

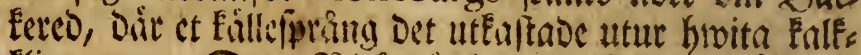
Elippornt. Detta BleEct ar brooitt, fifoum pi óland,

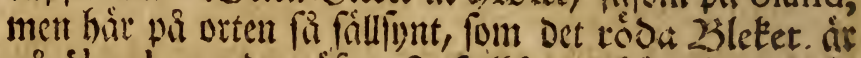

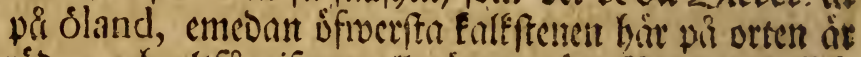
xío, och) altfa gifinct allmuint et róot SBlcke mes fififs swer/fert, foum af râgn, luft, froft, oct) Eol, fürfaller

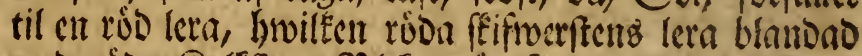

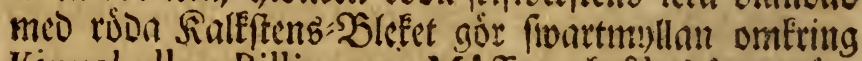
Kinnekulle, Billingen, Mófe- och) Ål ebårg, róds aftig. Det bowita 3j!eker nuttjaves pá 2 fitt af

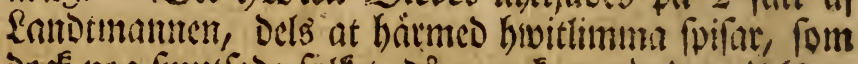
ioce nog fimut fade folfect, Di man Eom nar Dem, DClE at barmeo utputfa tringulfiven, feoan de forrut twättatz, b)wataf de wäl blifiva finfore od ivactrare; men Dárs

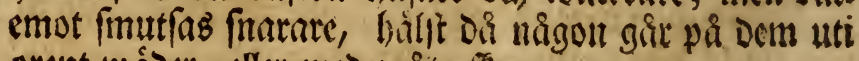
orent wíder, eller med waita fror.

ORTERNE fring ß̧acferoobgąaro, (natale Prof. Aboënf. Dni Haffelbaum) fom ligg notr om Rlefroa Sigrla, woro befuntuerligen:

Circaa (Flor. 5.a.) Biftorta 32 r: Vicia fylv, 603. Leontodon hirl. 628. Scorzonera 647. Trol- 


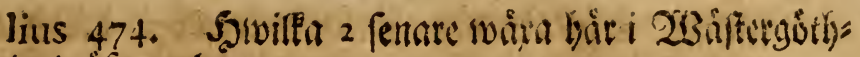
land ifwertalt.

SRTENAMNEN aro afiven fat har, fon $i$ anora farthito ta Provincier, olife; ty Lấr folllades

Trifolium rubrum 615. Róbskalla, Ulmaria. Mrutragtás, Filix mas 846. Oenngris, Trollius 474 . Smórboltat.

ASKEN 83c. war iblatio atta triit, fom mant hitintil feot

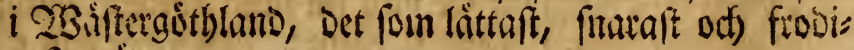
gaft waike.

POLYTRICHUM Scoparium 868. a bruftes hár til fos per eller ngnawaftar, med bwilfa affi utur ugnet fos pares.

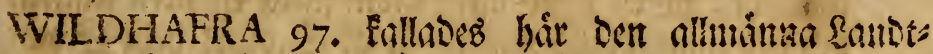

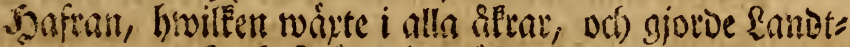
sannten naycet befwir; ban sfiwerwants med Det, at

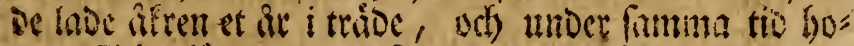
toun fitigh fivede; ment fom geantens setar ligga mes

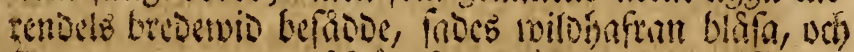
tinge utur genument \& \& til milit in befmitta.

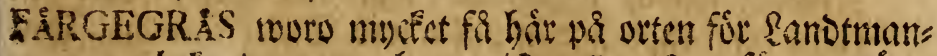
nen befanite; th ban wife alleneft ne fütga grónt meo Xoippa eller Glommorita of Sist, (Flor.99.) od)

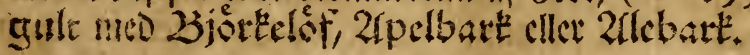

STENGaRD A R figgos bar Fring Flefwa bittere nnlagbe, sut tilfórene; ti) De moro uningec of fluta, od) i fortant

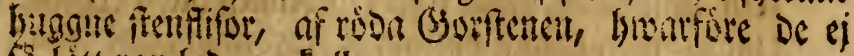
fa latt tomlade omend.

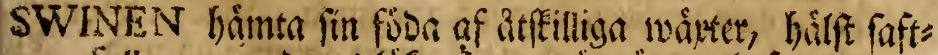

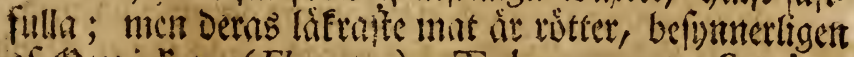
af (Oxoictrot (Flor. IOS), Tubera I116, Stachys paluftris 490 , Scorzonera $64 \%$, Cumin $245 \& \mathrm{c}$. biwarigenom Stanaren, uttsm affigt pa Cwiteng fö:

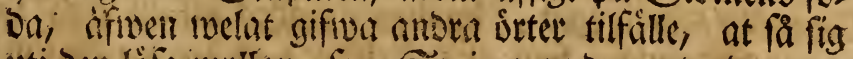

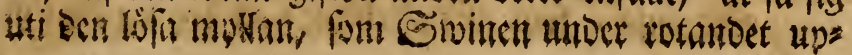

taptat. 


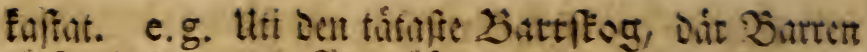
tiffer jorben, od firgubifiver alla tourter, bar ber wilajte Eefaparen gifiwit bemlvift for Tubera, Tryf-

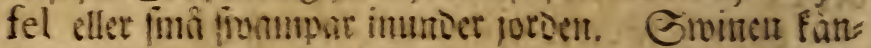
ma tem mes fin goon iuft, sob upploja Dem meo fit linga funte, fâ at bivariebanou waiter bir litteligen Eumin fälig, for brvilta Det sliejt finle mara omsielis git. Sanomanuent bar belt aumat atmoamâl meo fia ang, Den ban ofta fer meo fin frota forluft upgrafioas od) Dismmas af Defe Fireatur; th bafwer han pifun nit, at hroar $2 B$ ar ringa eller ligga" full Strinet, fróta ihop mumen med ivrlen, at hat ma bària des: nen rôt Emincts oliocliga ferif, feoun med en Eul ge: nombora nojen, at Dirigenom fatta en liten fialtra, od) Den tilfammans wrion, fâ at Ervinet bela Som: maren feal unma meo nojen, od) altfa ingaiumda iro: th; hroarigenom Ewinen ifroen nuafet oroag, unver Det ie fig fobu feola af anora wirter. Den féaba, foun be oringabe Sminen tilfoga angen, gor iffe alles naft igaren, utan ouf grammarme fror vlàgentet, bwars fore Sfiverheten mis plift och roite forstalats tilfolla Eamotmannen, brvart ât ringa fina Eivin, ellet saír ban Det forfummar at plifta tvio Singsio iatten. Det= ta arfiga ringandet bar icfe litet befimir meo fig, ej als lenaft for Sandmannen, utan od for Sivineu; th bar fonfen pifunnit, at feira Swimen iffwer nojen, pia Det oe alorig ner féta rota, brwilfet Gal. Sanos: bifsingen Strómfeldt genom en allmán publication latit befalla alla fima sanotmin bair i 23 affergetbs Iano; men Dentra myttiga Fortoronitig buse redan fia af

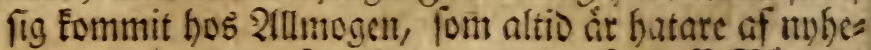
ter, at nappligen funnos mu mera några fá Bo noer, fon formo ibog och) eftetfommo fammu paffuno. Sif ht fe huru Detta lister fig góras, tog jag ct Givinthufs muo at anatomicera, Da jag afflád buden ofwan ya trontet, fann jag en mulcel, fom lág frammanfor broarocra ogat, froilfen llutades uti en ling Tendo eller fena, fom af tioflef med en fogelpenna giof bet 
fmal outh lång in til fpitfen af nofent, Dîn ban fafte fig uti cll, trubbig Siruff, fom gir Den Del, brvilken ligger i nofen ofiwant fờ nâfeburorma; Dir man orager pa Defe fe fenor, fer man, at nofen upluftes, at altfá fürenamm. De fena ár fáfert Denfanmua, fum endort gòr, at Sivis net fan rota, bwoilect alt tyocligen fan funas uti bos: gâende figur:

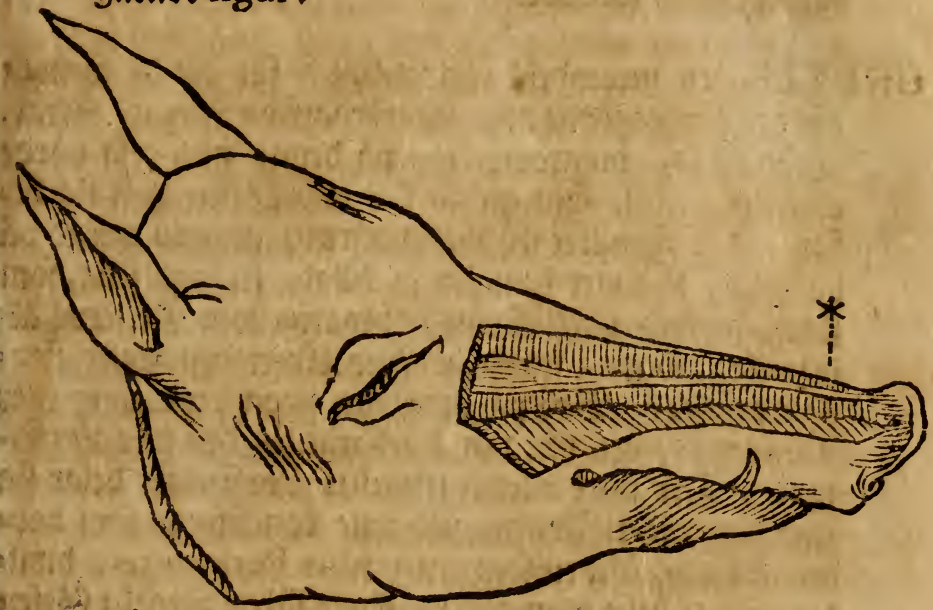

Salir of folier, at om man tager grifen, Da han ât 3 cller

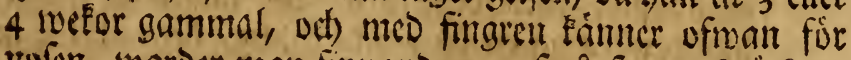

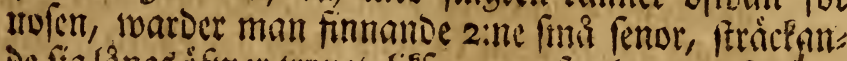
De fig lings ófwer tronet, liffom zille fegelgarns trån:

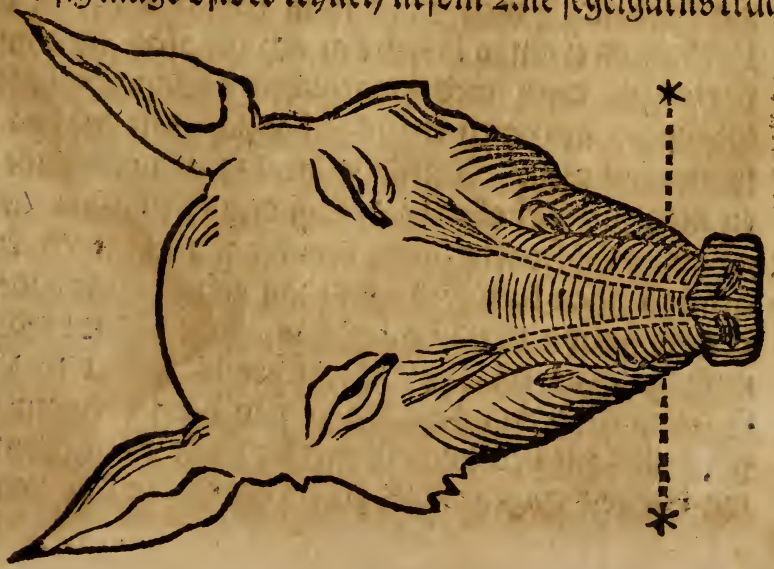


Sefe triar fér man af mes cu hrwaf peutetuif, wio martet *, feoan man forrut litet opmat buden, brwarige" nom tiontet fa blifiner firopt, at thet fesan alorig, fa linge Gwinet lefwer, fan rota; bar igenom fipper

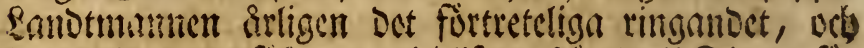

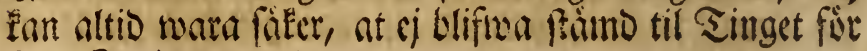
fina Swinz rotande.

GETTER iro metendels fortretelige, for bet be fpringa

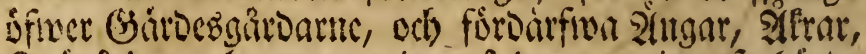
Ergigardai, bwaremot jog pa denna min refa boroe et artigt model, fom anora for mig áfiwen baft fig bes Eant. * Somillet féal befta Dáruti, at man afféti pa

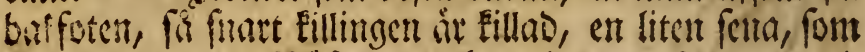
feal fitta wio batefloftuan, lowarigenom Getternte als

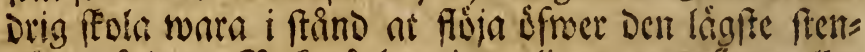

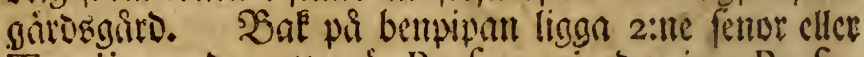
Tendines, icu yttra ar Perforantis, Den inte Perfo. rati. Den yttre cller mulculus Perforans Delar fig inmoer fmatlofivarme uti 2:me tendines, fom bops Drage foten, iffoell fom mulculus Perfaratus; hroils

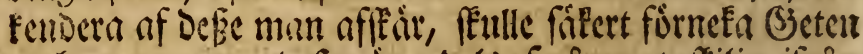
at lyoppa; men Defic aro delo fimire at feilia ifrim firouranora pa en lefwanoe Eilling, Dels alt för grofioa at afbugga, utan Betens men, fon fiffert bäraf blefs wo finfoent; men feulle inan funtma gora, at tendo Perforans ci gingo loš, fram od) tilbatas, inctrom Perforatum, utan mes bmarmora hopivule, (i)tes nog tyodigen, at Sicten ci funde boypa, faft wall gi utan funnerligit men. Folfet berattace, at man feulle féara an liten mufcel ellet fent, foun orager tilfommans boigs ge ftora flofionrme, om bwilfet ing cinnu icfe funnat ầnå fá noga tunoffap, fom jag gerna sifuntoade, wil

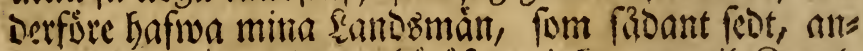
mosase, at tyocligen birofiver intomma til fiongl. orsettenferaps Acadenien, fom Det fcocumera fumbe Butra allmant befant. (3)etterna ffulle härigenom blifs wa bubbelt angenimare for Sanotmannen, om bat 
funte nign fadone Sitentur, foum ei furftodo fig pa at volticera.

- Kalm bahus $z$.

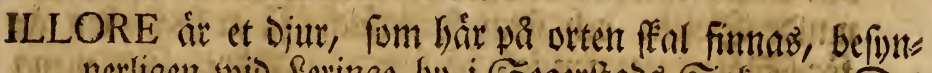
nerligen wio feringe bu $i$ Eegeritads Solen. Iet

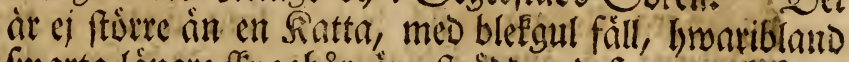
fratta lángre fingghar aro frridoe, Dé nuun ocf oron aro hmita: Saan hade helt frocirt at sifa, bwad oetta mainoe wara fort et Djur, om iefe Drabanten Scerr Mag. Tengmark feocrmera Det famma feutit, odf finmet inpoifat, Da man lätteligen fäg, at Det noor en Iller eller MUSTELA Putorius dicta (Faun.8.).

Go̊DA ÅKRN âr angelógit fờ Santotmannen, allenaft gúbslen icfe foftar for mucfet. Itt fürófa góosten, ty)ftes bơnderna Eving Slefwa oct anora orter ftumit pa ct hàrligit husháidos grep, nánligen at upefiga torf, lägga Den i bügar pa atren til toreambe, Den torfade turfiven federmera brinma, DCh faicoes góda after med affan, bivilfen fora invention gar up emet Finnars nas Sutteiand. Den fina mullan, fom bliffere of firmultrtade rotter od) warter, ir Den ritta givsslen,

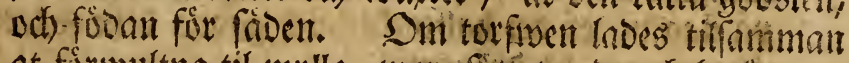
at formultna til mylla, worc fattet goot och berìmmes

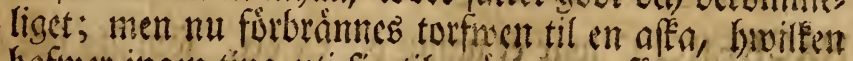
bafiver ingen ting uti fig til waeternas food, utoum ct choa falt, fom haftigt med ràgnet orager utur áferens mylla be findfe particlar af fivartmullan, fwarigenent

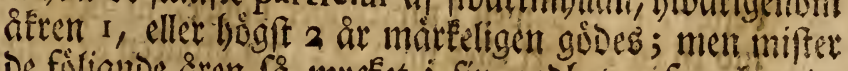
De fortiande iren fa muctet $i$ fin goobet, fom ban oe

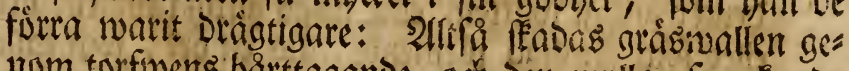
num torfivens barttagande, oci) Den mulla, fom fundoe fás af torfwen, blifiver genom bianningen aloeles do : Dad, utan nt båtu áEren; ty wigar jag ráfna Dema invention iblamo de féadeligafte.

SLÅTTERMANNEN wat mu $i$ fin anletes fivett, oft)

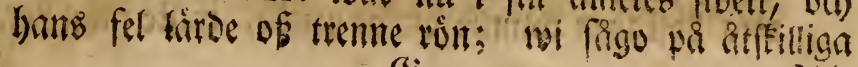


ftillen, Dár Den iDige Sanbtmantent unber faendet gict

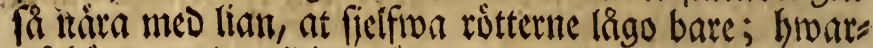
af hände, at Den joigas ängar wors långt magrare, án Den Latab, emedan De bare rostterite torkas af Den brin

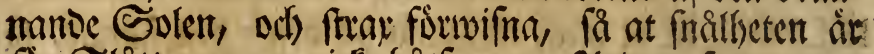
for Slättermannen icfe batfam. 2(more, fom mena fig wara flofare, fla bittioa fina ângar, medar wartene fta anna $i$ blumma; ty De hafwa ofelbart lint af lä́farena, at ôrterne under blomftertioen bafioa fin angencimfte lult, fmak och) Eraft, liswarfore oft De oa feola fa Det baifta touct; ment de fonma ej ifog, at trebingen of alln wayter aro annuelle, eller feola ups Fomma of arliget fro; Debe fa hárigenum ej mogna $i$ fit fró; ei fá fig, bivaraf De fútiande a aecen mifta en tres oing af hoet, fom Den fenfäroige fär utan notfago boivs ga i fin lada. 2lnore läta quinfolfen fülia fig unver

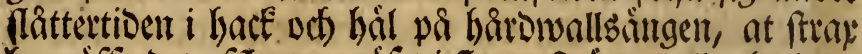

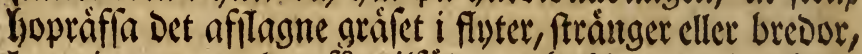
howarigenum torfan fär tilfálle, at haftigt angripa ftuts: ben, fom bela fonmmaren fürnt ftát fuetig od) Daifiven af finggan, Da grăstoten idfe litet af Denma haftis

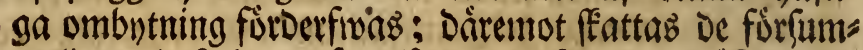
meligare lucfelige, fom lista Det afilagna gräfet ligga

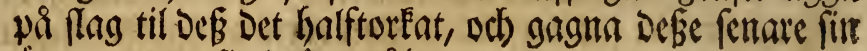
sing genom fit srógşmal.

NÁCKEROT war et namn på en brt, fom iag icfe hade mig befont, brwilfet forrefom under Det, at binderna

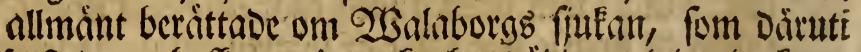
beftud, at boffapen i nulefenget Düboe, af Det De Eums

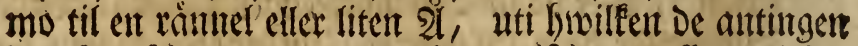
Drudfo giftigt watten, eller fingo giftiga maffar uti fig, eller fom fomliga formente, ato ent unt, fom Eallades SRäcfartot. 2it blifwa underràttad och fürni Bad om Denna fae, refte wi til Trimbtorp i Giöflents Sofen, fom lág fosoroift ifrân Odens Fullc. Dá ivi fommo til Trimstorp, wifades of af bönderma en rimtande

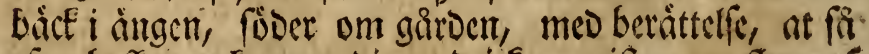
ofta bofeapen foummo dit at Dricfa, mifte nuan fera of 
Dent, fom ftrar tiffatte lifwet. Lieutenant Lundberg, fom tiffurene bodt bât fern air, od) miftat wis famma bidef en fror mucfenlict bajftar, of undertioest Firr, wifte beratta of, at ocf noanniferor ofta farit illa af wotnet, eller af Det de bitit af virter, Da De watnat

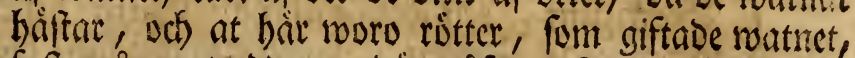
faft mánga troooe, at oair gâfruos fina nuafear, fom gjorde alt Detta onda. Mien $i$ ietta moro was intet

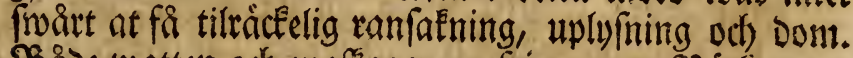
SBàe watten och) mafíar woro fric; men Piáitaroten

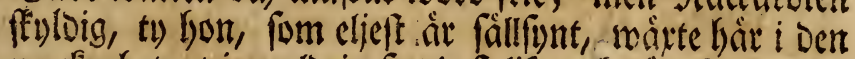

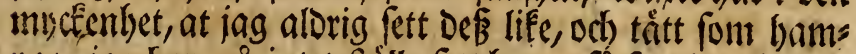
pa; lag bar pa intet ftâlle fett henne fá frodig oct) ftor.

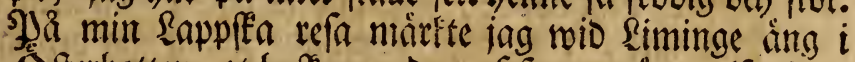
Sifterbotten, at boffapen oog af famma int, (fe Flor. Lappon. 103.) oct et ánnu mer sfivertygande ejempel bade iag 1744 , Dả nàgra rótter af Denna irt upEaftas

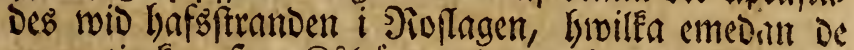
woro tiocta, fom Sailrutter, burub ben of $2 \mathfrak{B a l i ́ b j o s}$

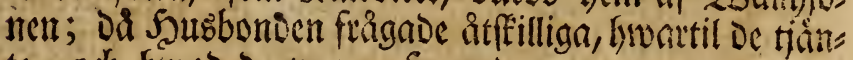
te, Deh hivad de woro, fiwarade elt summa, at ic

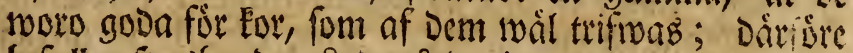

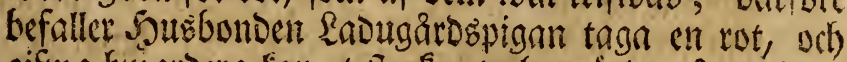

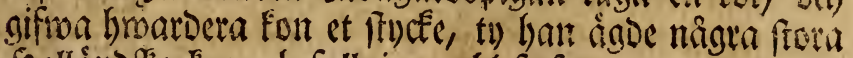
Sgollámoffa For: befallningent blef efrertonmmen, forna begnnte raima, falla liffom $i$ brott, blifwa ftimue, oi

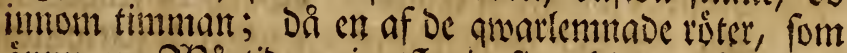

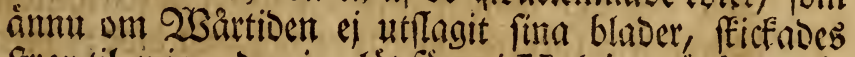
firar til mig, Den jag lát fôtta i Uplala triggaro, Dâr bon efter 8 Dagar fig wifade mara Den famma, fon

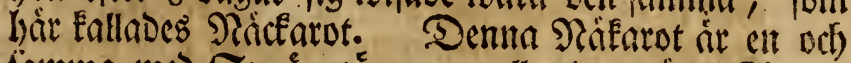
famma mes Eprángótent eller Den râtta Cicuta Flor. 239.), om hroillens gift Wepferus de Cicuta aquatica ferrifinit en hel tractat, och här befanna at hon icfe allenaft àr böbelig fưr. minniffor, utan uch

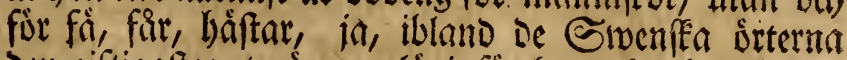

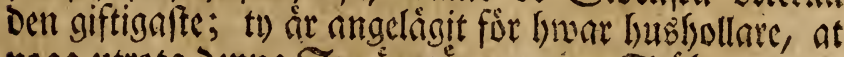
noga utrota Denma Springortent 239. Stâtean 238. 
Stormbattsblommant 442, fo fant ban ât angelá gell om fitm Sircatur.

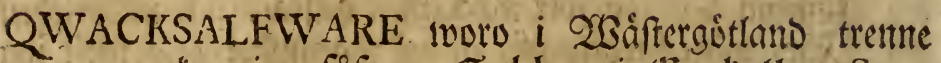
namntunnige, fafom: Gobben i senckulla, Sven i 3ractnum, veb Dés difcipel Valtin Sträng $i$ Zinntexoalds Soten. Folfet $i$ landet fofte bot bos Dem cmot fita finfoomar, ef atuturledes in af De frovs:

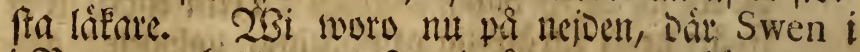
i Siragnum boboc, ty refe wi pai I quart at Gora han wishet.

SVEN i BRAGNUM habe fopt fig en liten gîro, på

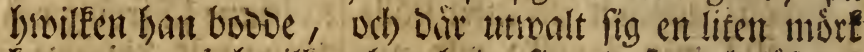

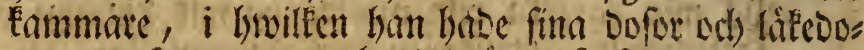

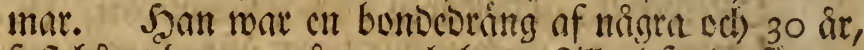

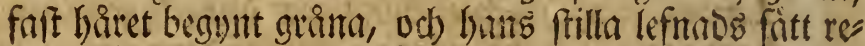
Dan gifwit honom en anfenlig ifferbuf. Slatr roi fom mo iit, och helfinoe pa Gonum, ftoo han orourlig, utan

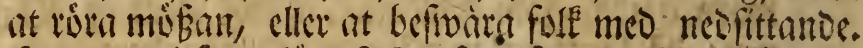
Saun war i finar litt, foft alfwarfam. Shina fólietlas gare funve ei affolla fig, at fingera fiuktomiat, od) risofura fig meo honom. Den cate $M$. T. füregaf, at

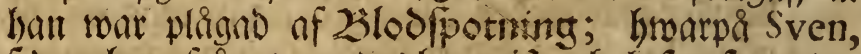
feoan ban frisgat un bes hemmitt cch fofa, fivarabe: 2tt hait fittle Fspa pa Siputhetet $i$ Gótheborg

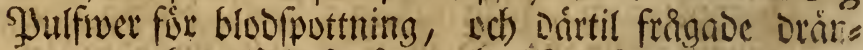
gen, om han lastit fig hocer, hwilleet of patienten nes eades, foum fade, at han worr ritod fur blod; men blef Docf riob, at ánoteligen ipma isoerm pi armen. Dem andre E. G L. frigane rifo fúr et erromfufande; Drán gen fivarabe: 2ft patienten ingen timg feulle kruefa; ty) ju mer ment brufáa, ju woirte blifwer bet, utan alle maft öpna abern under sirefinippen, Dár fave han, at 3

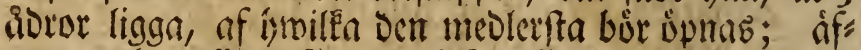
wen at han feulle fatta en filfwerffeco pa globs; vel) Da

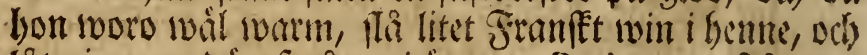
latta immman dirraf gâ up i urat. Patienten fràgnoe, bronaf ocuna fiufoumen fommit? Drängen fromade: 
Rt bet wore bloo, fom ned fallit for itut. Patienten

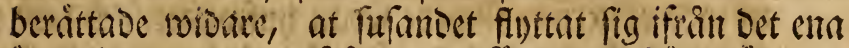
orat til Det andra, frigabe serföt, om bigge bronen have niggon communication? Drongen fivarade: Sa hifla tie má $\Im$ bet tro. Den ente begarte at fá tala mev Dringen enffils, Di Dringen od) Patienten gingo in $i$ Eammaren allena. Patienten furegaf, at kan tas git ufafta fieg, och onirigenom riffat ut for et venerilkt taEDropp; Drangen lafivade, at Det ffulle blifiva goot, gaf Derfore ett groft ortespulfiwer, fom feulle ins

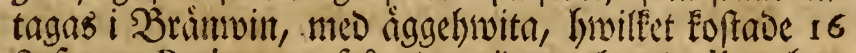
frifiner: Patienten frigade widare, lywa diat hant feulle forra? Drangen fwarnde: Alt Det wory lifén muses fet, allenaft man faftar en tima for Souffiorets inta gande. Patienten friagabe soidare, om inm far fupa SBrămerwin? Dringen froatade: Sa, må S tro Det, Det

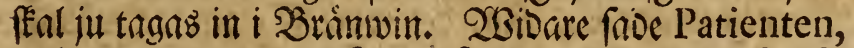
at ban wore wan, at fupa 3 fupar om Dagen, oct) fras gabe, om han fär belsolla famma dixt? lowarpa drä= gen firarabe, at 3 fupar woro ingen ting. 2Bi bade ta= git meo of irten af Cicuta, lowarfore oringen frign: De, bmad wi falle gora med Stritterna? 2Bi fiva: rade, at be tagits wid S rimbtory odf at de woro feas Deliga; Dringen mente nei, och log Derrst, faijandes, at De hiwarken woro feabeliga eller mettiga, utan at hat wille àtn up altfammanb, od) tog ôtten i mening at bita pa heme; men jang formekave honom betta alf:

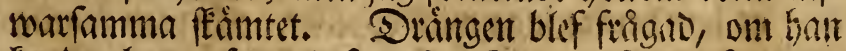
Eutbe bota failandefoten? Fallanoefoten fibarave ban, jo Den har jag curerat naanga litmorabe ganigor, od) wil altio bota hente for 2 Sal. S:mt. Salt bolt Denna fiufoomenfore en of be littafte: Jing frigha De bonom: Sm han trodoe fig funna bota all fallanoe

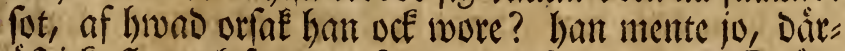
âf icfe fing od fallande fot woro tilfammans. Drans gen frägnoeb., om han kumbe bota alla fiufoomar? Sowartil han froarade ia, Dâräft bet icfe wore til döden lagot. Drangen fatte fig utanfor Dơrren, at placfa en 


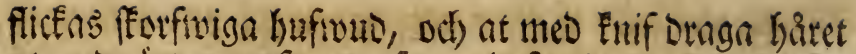
ut mes rutterna, fmoros fesan hufivudet, ods labe birs Sfruar et Sarborrtblad; hivilfet nit ban lagaoe for en Siffödlet, cller $4 \frac{1}{2}$ Dal. Fi:mt. (En bonto fom od) begarte ljielp för ca finte Diarrhée: Dá Sven frigabe honom, limarfure han ci lagat fig ut fon, utim foms

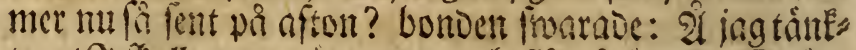

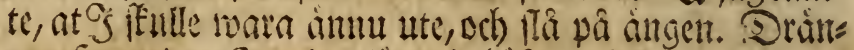
gen fimaraie: Ja, jag läter beliófiva tifece, jag; mis

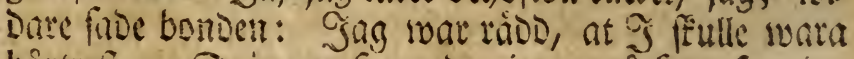
birtreft. Srangen fwarabe, jü, en tâcen, foun fig

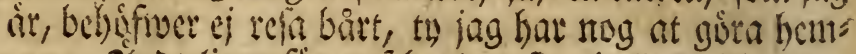
ma. 2indetigen forregaf bonden fig ej eller willa foms

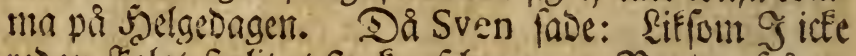
resant feolat fulit et fincefe af honom. Sonden friga:

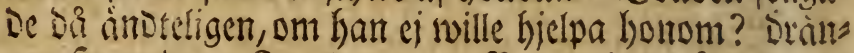
gen frurabe: Sa, bet vore fa, om jag wålade mig fiâ med eDer i natt. 2Bi fago nágra medicamenter liggr pâ boroct, námligen Calmušrot, Şohannis ort od) 23 allorten eller Confolida. 231 funno nogfant, at Drangen bave ingen infigt bmarten uti Anatomicis, Phyfiologicis, Semioticis, Diateticis, eller Botanicis, utan at lecla bans funffap beftod i niggra fá medicamenters fraft uti fiufoomat, libataf allmo: gen argumenterade a minori ad majus, bâlft bi De fago, at han under cr alfwarfam mine Dólgoe tis: got Djupare; men Daruti war han Docf at berómma, at han brufte inga misfécppelfer, ob) war fogelig meo betaluingen. En fiuf mannifita ma wál lienas wis et ffepp, hwillet ffal fegla mânga farliga orl) wiblofti= ga feir fôrbi, uti en fticfande ftorm; Dent Geppare ät

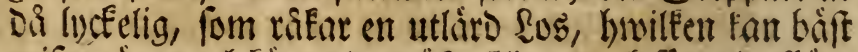
wifa wägen, och Eunma de mafta Flippor odh fordolde ffear; men Den, fom lemnat fit ffeps i en ofumnig farlb băts bei, fom fnapt bar fig cit eller anman Elippa befant,

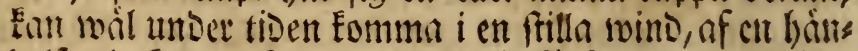
Sclie, ly ifeligen fram; men bock oforfiwarligen, at han wignt all fin ágentom uti fỉ ofafra bantoer. 
ASEN blef wånt tattquatter, feoalt no reft 8 quarts frot= refa ifrån Klefwa,

\section{Junii 2.}

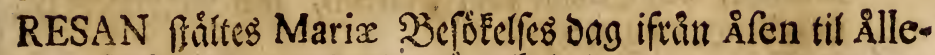
ftad, unoer et fendigt rägn licla bagen.

FóLJESLAGARNE Dorgmåftaren i Falko̊ping Sert Rudberg, och) Drabanten Mag. Tengmarck, fom 3 bagar beleofagat of pa de angelágnafte ftällen, - togo Iili afifed.

MJÁLLERUNGA Simrta lág imwio Venern, Dár wi bes wiftade Siudstiänften. SGär figo wi, huru Den, fom gict med hafwern, då han făt af bonden I vere fioppars munt i pungen, tillifa med en nicf, tog ftrax utur bafs seet en penning, och lemnabe ben frax pa andra froalt at famma bonocs huftru, fia at âfiven bon Eunde bafwa Den âram at gifwa $i$ pungen, o\& bafiwen fom at befo: fa benne; făledes fprang Dett, fom bar bafivent, ftadigt ifran 'mansfolarna, til qwimnoftolama, of orfaf, at

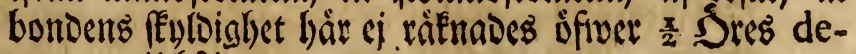
pence til hâfwent.

QWINFOLKEN brufte mu mera inga battar, font tils fürene.

LANDMONEN begynte blifwa mera bacfig, od $i$ âns garna fäg ent tâmmelig bop loftrân; men Betesmar ten war lifallig och f foglos, full of siung, ocb) I alns hoga Enbuffai. De fora bedarna woro afbrimbe, at

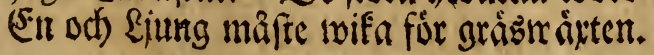

KNIPA BRO laig ífiver en liten flod, emellan Sieoabo ods Selgorp, fom giorde ffilmaden emellan Scaraborgs od Elfsborgs \&añ.

* Hrlgorp. quart. 7 . 


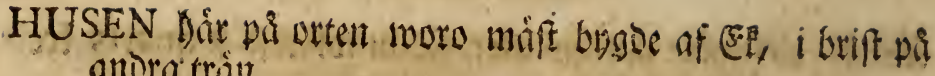
anotra trint.

PLANTER-HAGAR woro moift i alla Comar anlagoe, naimligen cht i bwar Goten; De woro fma af fen up: mutabe, od) (Starne waxte Dormti, fajt merendels illa phunbrave of getter.

EKEN bơlt Sandtmamten fơre bâft Eunna fẩ i mofia un: Der (Enebuffar, od) at ben cj upwerite utan mofa, ci athet eller blefinc wal berwarno ifrân Sireaturen, utan (E),

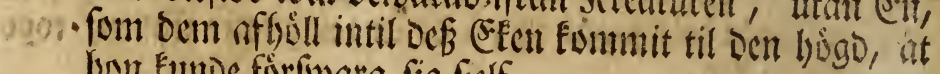
lon Eunde forfiwata fig fielf.

* LJUNG. quart. 3.

TORF hade man bår begunt uptaga i mucterkst, mes fesc

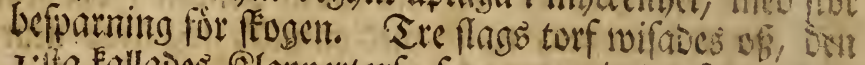
1: th Eallabes Silappertorf, fom loar gioro of Das, Dest= ni, utair ritter, brann fenare och langfamare: ment gaf ftarkafte worman. 2:Dra giottertorf, beftod of

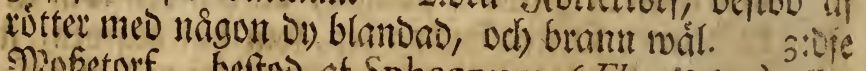
Mo betorf, beftod of Sphagnum (Flor. 864.) cllet Siod mo of bar, Denna brann bafrigt, odf gaf misteta toirman. ziféar efter torfivent, bief bà pis orten robagtig; men

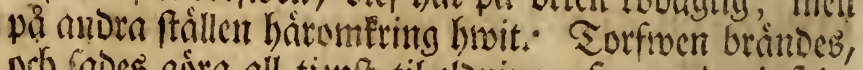
od) fabes gora all tiunt til cloning, fom meo, i fois, fofelugnar, bryeg, fmibe zc. ment ei fa til bak, icfe

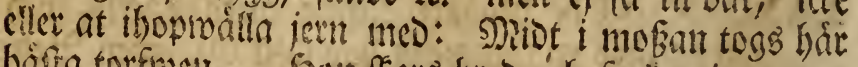

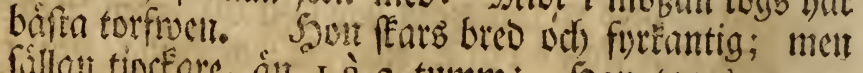
fiflon tiocforce, ont i à 2 tumm: Saon tages up om 23 aren, fa firat man lagt neo froken, cller luEtat afers biutet; upfeuren neo en fpaba, lagges i bogar, clict roteri, at tortiob sifwer fointmaren.

MJoLK oftes af bonoqwinnturna muctet litet $i$ limarbera

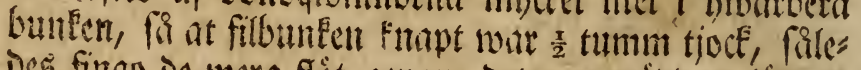
Des fingo de inera flot senom Det, at grioban lattare futhe upfista. 


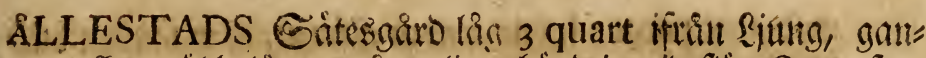
fea wäl beligen, pit en liten bigg inmois fion Sem, fom gice intil bufen. Syufen woro bugDe pa gammalt fít,

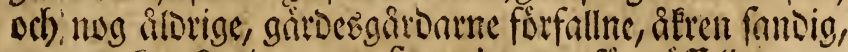
(c) marten ftenig; men fituationen fortroffeclig.

\section{Julii 3.}

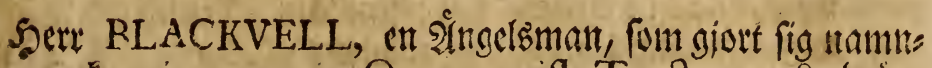
funnig genom en Oeconomink Tractat om serbocin

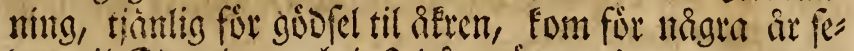
Dan til Giverige, och iuft oa, noir nationen med all ifwer beaunte tünEa pa Oeconomiens upeomit i Sic fet. Shan, fom lat fig formatio riga en ogemen fun= fap uti Oeconomien, blef med manga formoner be gafiund, od) Dint I fice betta Fiongs: Eiteriet Sllleftad

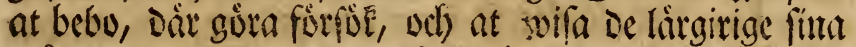
många arcana aconomica, buwatont ban lît afgả en wiolnftig fpecification til alla \&nnd sho foingeosmen i Siifet. 2 Bi bave reft bit, at fe od lirta be bogftons féliga rin, fom funde gagna bảoe of od) publicum. Rif:Medicus Blackvell war ej obencigen, at mifa of frax alt hwas ban härtil: utrittat. I) J̧a Caracters buggningen bade han niot emot iorren at fiofit: Dan, latit utlyugga en luftoorr. 2) Thara humbrode

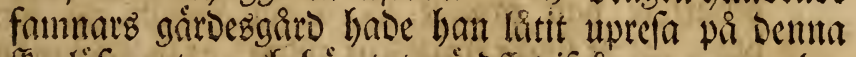

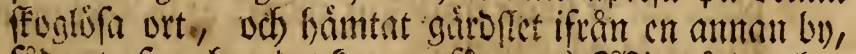

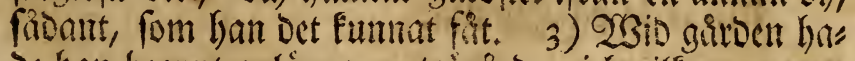
be ban begunt anligga cut trighro, i builten, utom Siósfafer, han bave fatt nigna frano Galium album Ir 7 . Lotus fylveftris $60 \%$. Melilotus luteus 6:9. Trifolium bituminofum. Phafeolus humilis, huvilfa ftodo bront pa fin fang. 4) Sil Şum= legâro hade ban látit upgrafiwa en bow fingar wal a nlms hose, vd) vågot mera brede, uti bwilfa ban tänfte plantera Şumle; imen $i$ imeslertio uti ritffilliga fatt Potatos, bd) binter. 5) S(Eken, fom war fattig pa fwartmulla, baoe ban litit plogn til it tumnts bjup, 
med 4 par ofiar, hwarigenom alfiwent, eller fanten blifs wit blandad ineo myllan, biwilfen fano hait formente

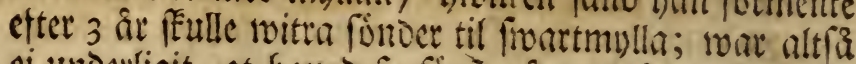

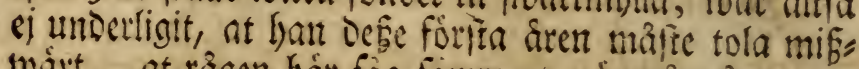

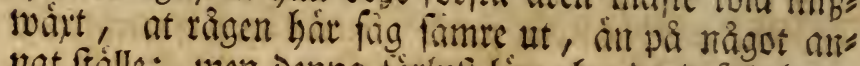

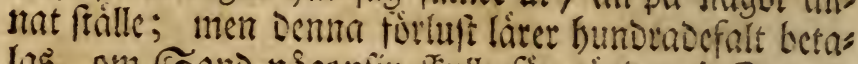

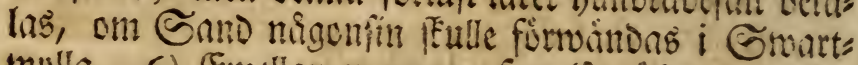
mylla. 6) Emellate ch mura, fom ling efter om gars:

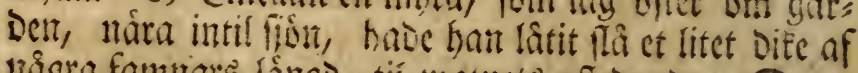
några famtiats lânge, tii toatmets afleounte. Detta alt hafwer ban bittils utrittat, mucfet mera hoppas tman framieles.

Ro̊D-HUMLE Gơlt sif-Medicus Blackwell fóre at mara berttre, in Den hmite Syumle, fom hafwer lofare tups pur, Dir sen róda har Dem biabe ymitogare och faftare; âfwen at Den Soumlen, fom féal brufas til utwalot orics Ea ná lingre groar, blifwer ban bälate od) oangends marc.

HONUNGSDAGGEN hade redan bcount at fafta fig pa Saumlen wid bondegärdarna; hwars oifat \&if:Medicus Blackwell rátteligent faftabe pâ Det, at S.umler ftâr fá tâtt, fâ at Dent Dang, fom om natten faller, ej fan afblafa, utan torfas mid blaben of follectan, Guarfore ban àrnade lägga fin Scumle pà fängar, byar rot et fincefe ifrån Den anora, at woidoret mátte fá fritt fyela pá Şumlen.

FEMJORD Enllabes Går på otten wår gábiort, om hivils fen wi frågade sif=Med. Blackwell, buru Den formuna

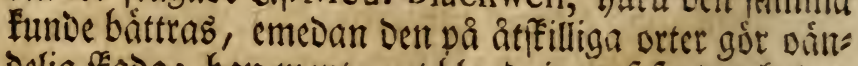
Delig fFada; ban mente, nt blanoning af fano och lera funde bielpa henne; men fom wi fett faoan gasioro bas De pá fond od lergruno, foreflog ban affe, brvilfet foms mer un pá fórfóe.

SIGILLI Salomonis (Flor. 274.) birr, fade Saert Blackwell af eget forfó, faba baide orat od talfroar, ja, 
Dem ofta ooda, od) ânoteligen, at han bem ittgifivit ât hunoar, fom háraf oobt, brvilfet ej toarit mig tilforente befant.

THEEBLOYMOR ifün China fagos hăr uptorḱabe, De gafiro Théet en ftark perfumerad luet od) (maḱ, fâ at man icfe fitê lágga mer ăn I eller 2 blommor til bet ambra Théet $i$ Thée-fannan. SOlommans Caly wai $\mathfrak{e n}$ Periantbium minimum, pentaphyllum: foliolis orbiculatis. Corolla magna, rofacea, regularis, enneapetala: petalis tribus exterioribus dimidio minoribus, reliquis fex interioribus in orbem politis.

WAXTERNE woro befintmetligen hât pa orten: EMPETRUM (Flor. 832.) Vaccinium maximum 312. Tetralix 310. iblano \&iungen wio SJâlatts: torp. Raphaniftrun 568 . meo gula blonmor i seluarna.

PISUM petiolis tetraphyllis, ftipulis crenatis, pedunculis unifloris, wáte bà nllmánt i âkarna,

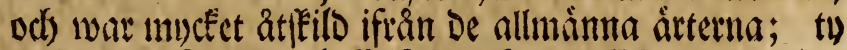
soil jag gifma Des beffrifning fort: Planta annua, $P$ ifi arvenfis \& fatici facie, fed longe minor. Petioli tetraphylli, foliolis ovatis, oblongis. Sti . pule, majufculx, crenatx. Pedunculi uniflori. Flores vexillo cærulefcente, venis purpureis; Alis obfcure purpureis; Carina alba.

GALLAPLEN futo pa blaoen af Glechoma 483. De bas De uti fig mánga ima cellulas.

VERONICA pratenfis (Flor. 12.) hade wid flutet af fiter Earna fima luona bufmudocm, fom woro ibopfatte of et par runoade lubna blroce, inuom broilfa lago rode larvæ mulcarum.

RESAN ftâltç ifrån Ållentad åt Borâs.

HÅFSHED låg wio wägen, mucfet ftor, jemı od angenim. CHAMOMILLA romana 701 . wirte mucfet wio Flabårg. 
* FLABÁRG. quart. 3.

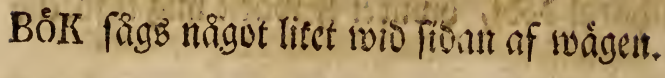

ORTEN mar liufig meo beoar, wattent, loffrita ingar, od) wägen frmoig. Dimman frod, fom fmá moln of wer bärgetr.

* BORSTENA. quart. 4. Si. I2. om natter.

\section{Julii 4.}

RESAN iftån Borftena til Borås.

NOSSE-SJÓN lág intil Borftena, han war mucfet liten, Docf fabes gifina beguntelfen til floden Lida.

FLOTBIORKER (Flor. 776.) twoinme ftucfen, (mâ, oct)

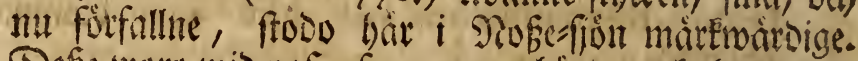

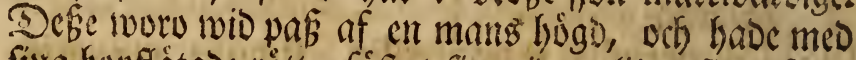
firm bopflitade rötter fáftat fig wio en liten flottufiwa, fom fist nu til Dern ena, mu til Derr andora fteanden, ods witte ofta ifop meo oet land hon rórie, intil Des nas gon braft, froft, eller floo låbade beme. Biörkerna

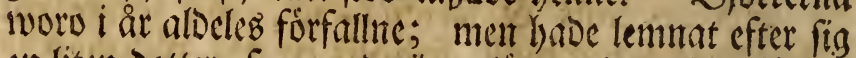

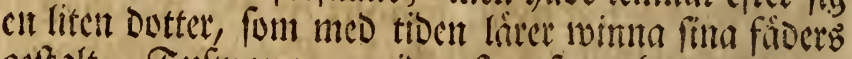
geftalt. Suffivon warer wio pas en fanta bred, et quarter Diup, och have ingen ting minore uti fig, ein aghs viotter (Flor. 35.) af broillea Phyfici troot, at fivetloos

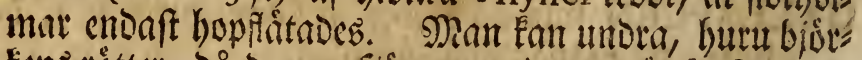
fens rơtter, od benna fisit on twinteren âr frufert, ods rôtterne sf fweralt mes is omgifue, lietwil Eunna uthats: Da wio lifivet, faft icfe en ctroa tră af Demt ât fri fràn

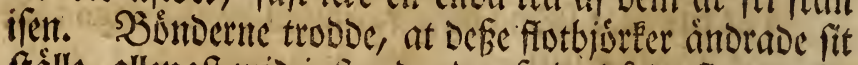
fétlle, allenaft mid imfturtoanoe ofred, ba be fisttadefig

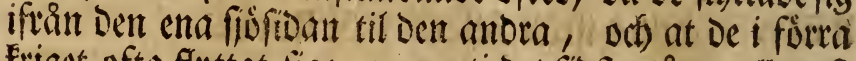
friget ofta fluttat fig; neen uti bet fir fta tảget allenaft

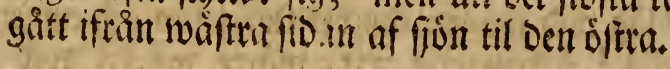

WÁGEN at Boras sick up, meofore, och pa froorna af bronk 


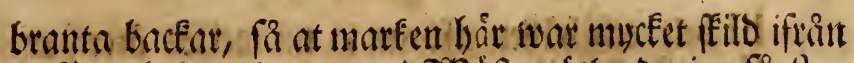
De furru belagelign orter $i 23$ áftergiotland, ia, fi tteril, granfull oct) viọnur, fom fjelfwa 2ingermanland.

óRE-S JơN ligg på hujgra bamien alt ifrån Påtorp in til Borás fitad.

* BoRÅs. quart. 8.

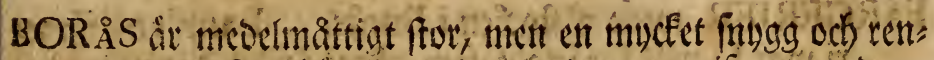

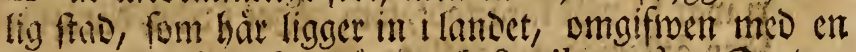
bactig, frogfull, flipplanot odb fteril tract? Strooen futtiar sit en frilla floo 2 siféa Eallad, fom fluser utur

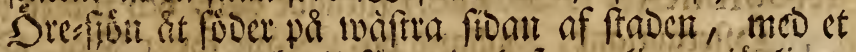
govt watten, och til färgerier befinntertigen tiämliget. Siv (Sa) tweetfore, alla regulaire, $\mathrm{BCb}$ wid pás 12 almat bres De; Debe woro nul, foft riginet fradigt continuerat, fieta mector alderes torre; emedan alle flutta, och aro will fentagee. Demna torra fituation af fraben

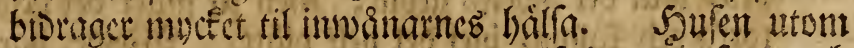

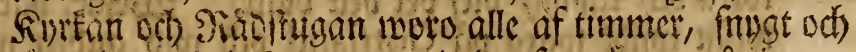
jumt bygoe, bettedo metenvel's af twönne wäningar,

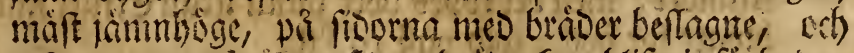
tafen meo torf tâtte, feDan briidtafen blifivit fortbusme

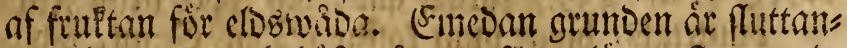
De, hade man bebiff pa ena fiban lágga fengruno, hwilfen nui, éfwen fom wäggarne, blifwit tä́t meo britoer, De Dir Doct gingu futt ifrän woiggen neDant til ut at; Detta bioreg, at gaterne woro fingsare. Epis farte i hufen woro af en fritllfen, eller Saxo micaceo quadammodo fiffili, Den inbyggarne wifte artigt hutgs ga i furfant efter mintt: Debe fpifar federmera affliftus

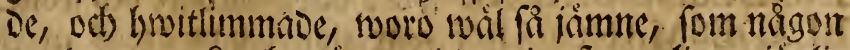

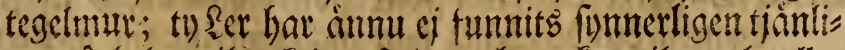
get bi kela milen Ering fraben, hwarken til tegel, eller lerkinit; imati noro bufou fingt meublerade, faft ef fonnerligen fofformt, och tomterne thitt inbugoc, fa at ocma fint, fom â 1727. emellnn Den 21. oct) 22. Ju. 
lii belt oct) ballen afbran, nu tyctes attertwunnit fit förra ffict. Lltan fơr bmar port ftoo en tanna natter med an grumrufea für wådoclo féll.

\section{Jul. 5 .}

BORÅS uppehólt of til eftermidengen.

FÅRGERIER woro 3 ftucten i Borâs. Langlets fírge

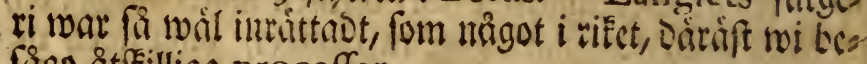
figgo atffilliga proceffer.

ANGSKÁRAN (Flor. 660.) fárgabe gult, oct) Eéptes ifrin - Sitergótlant, Saallano och Eiante für I Dal. E:mt sifpundet.

WEJDE (Flor. 543.) brnftes til blåeupen, fom upblanons Des med indigo, brvilfen flist och ftasigt feunnmader wid fiborna, fant roifade fig blandas liffom adoror,

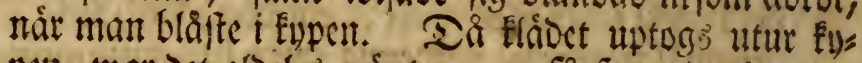
pelt, war Det albeles grunt; men fa fmart set begunte torfa od fallna, blef Det helt blatt. 23 ejoct har iag

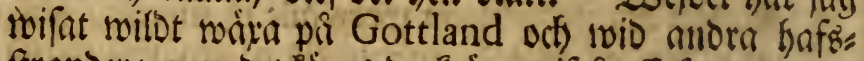
ftrander; men Det füpes Doce ännu ifrän Erfurt fattals, bwart fat holler wio pas 16 \&

WAU (Flor. 439.) fárgar gult, od) Écpes ifrån Franfrile buntetals, broar bunt foltar i Dal. E:mt, od) beftai af 10 , til 12 Marfer. San Füpes ucfia utifran, faft han lánge wákt willot Ering Sund i Ekante, och) till gan= 1'a lvál wånt climat.

MJóLONRIS (Flor. 439.) med Victriol tírgnide fiwart. GLANSLÁROFT giordes med en flipad finta, fom fis

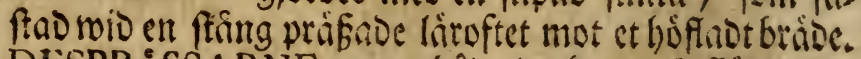
KLADDESPRASSARNE noro bise Dyrbare odh feime. KLADESMANGELEN war fror; ban Drogg of $\mathrm{cm}$ for igonen bunoen bâf, fom gicf omering fiit axin perpendicularem. Shâten wifie of fig fieff to.indor um, lopa fortare eller oct ftanna, alt efter fom ban meo or: Deil commenderades. 
TRYCKLAROFTETS ftrimplar woro ffurna i parontró, Livilfa Doppades uti en howit materia, fom fradigt frrofs iñmm, fon en tunn grot, af en annan gofe; fe= Dan ftrmpelen war biruti boppas od) tryst pa lároftet, ftrobses en bivit mo eller fin fanto pa de wâta characterer, och) fafmade Dairwid; Demma materien afbollt

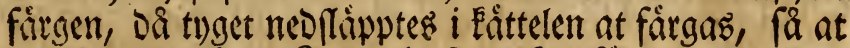
De patryite Characterer blefivo efter fargningen bivis to, eller ofargnoe, feoan Den patrofte materien blifivit aftruittio.

CASSIANS-TRYCKERIET beftos of 3 falfar eller Cylindrer, iblano hwilfa Den medlerfte war af metall, pringtigt utgrafiven, men imuti ilgolig; en ftor upeldad jernbult funde lăggas uti bonom, od bärmed upmắ:

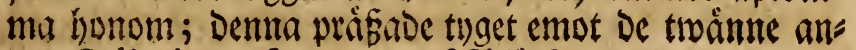
Irr Cylindrar, fom woro af Sijore.

MANUFACTURERNE of garn od) fpanab habe ganffa muctet intotat fig pa Denma ort, ty quinfolfen ipumno,

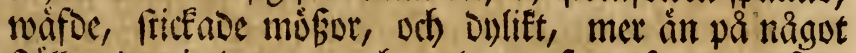
fiälle; De giorde tuger och) malmar, fom oftade trotfade et tơmmelig goot tlabe. Sonbuggarne fupte fig. Defs

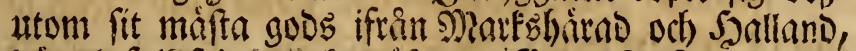

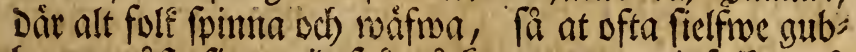
batme mafte fitta wio foanrâcen; men quinfolen ga Dír fillan ifran waifitolen, utan lemna mannen mes rendels omforg om sadugarden.

SMIDET far Borås-boen ifran atfeilliga fringliggande foes nar, befinnerligen ifran Sanolsult focten, bair de mits fte bonder aro meser, od) tilmerta enifwar, liar, fpas Der, ullfajer, mungigor meo meta.

FATKNALLAR, eller sandimain, fom fwarfwaferarat, tap: par, after, finmes mâfi $i$ orpa och alfpere fofner. Htom Det Defe fielfwa refa omfring med fina wator, fer, at Drangar, fom hos Dem taga furfiruar, bufa famma banoel. 
BOR.S-boertue bafima iftin urmintes tiber warit thames Eummige for fin funnertiga io shet, at fiba fig genom egma producter odt) imriés banoer, ocm de afiven uts

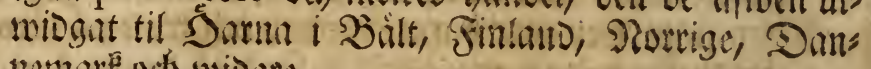
- b nemare dit) wisare.

CAPITALS-DRANGAR, eller fídone, fon fingo til Credit of fin Guzbondoe 600 til 1000 . 2nl. E:mit, woro moligen bit affituffine, of erfies, at en Capitals-oring,

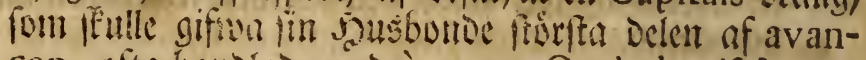
cen, ufta bandinde meo à parte Capitaler ifrän ans

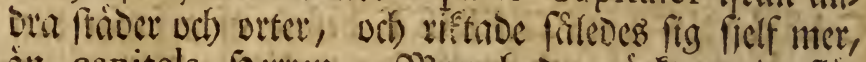
ant capitals Soertert. Man hade mátet, at De flà ite Borgare faledes lisit pa Defe oringar, utom Det, at Dringarme ufta tyme birt mes bela capitalet, cller forlleple bet, fafom oof ofta fatt fig neo i anora finder, til Derma fradems fíada.

STUDERANDE moro hat fi, eller inga fom bollos wid Academien; th Den iooge Sorgaren frod feet be ftuderandes tringfer $i$ Stiftelt, och Dirfüre lât fina barn arbeta och handa, fom aldrig gưr nagot traings: mail.

APOTHEKET war litet, men nog frott $i$ anfeende tif Det bruk, fom imminntme girn of medicinen, brouts till Dent torra fituation of itaven myefer biornger; th Sorgarme weta finapt of nigon froba, Den be ej eller naigonfin hafiva, bir de Den famuma ef fiot under fima refor.

SURBRUNNEN lảg $\frac{x}{8}$ mil ifrân ftaben, pir nuoffra fit ant

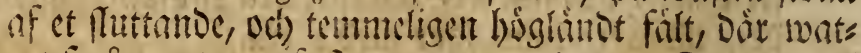
net foring utur et falf glub, utan lerio. Det war til

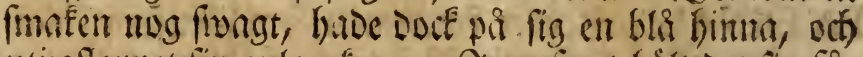
ittiafloppet fin gula ofra. S Sprofivet bolt Det fig falever: 2if

Saccharo faturni blef bet lowit.

Pulv. Gallarum \& Thex brunt, men fivigt.

Minio icfe fottare. 
Oleo Tartari ofortinbrat.

Spirit, vitrioli ofürnindrat.

Syrup. violarum?

Succ. heliotropii flift ordinairtSutbrumb watten

Pulv. coccinellx J

Alt is wat Denne liE De ordinaire Gutbuns watnet, merr Dock af De fwagafte.

KALEN i Borås wat en finnertig art, af bwit bufwudesal, Den man intet gerna fett pa andra ftâllen; ty) bufwus Dent wors aflange, fometägg; ment Däriemte nuyefet fas fie, odf funde wăl bewaras in pa anora aret.

JORDMONEN ontring Boras, fom funte cultiveras, mar mucfet lifen, mafte bade goons och longgas i lin=

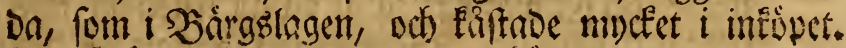
Sin, fmuly, od matwarer woto bar nog Dura.

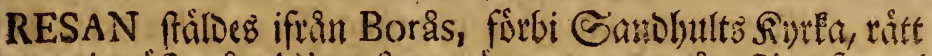
i wafter at Alingsas: wrigen mar en gangftig, for ef

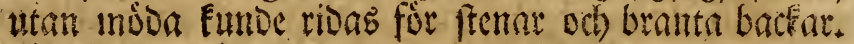
2Bi hnilade sfruer natten utiHEARE, quart 6 ifrin Borås.

\section{Julii 6.}

WESTGÓTA FJALLAR fár jing lof at Falla bet lamb, fom ligger cmellan Borås odf) Alingsås; cmedan Det

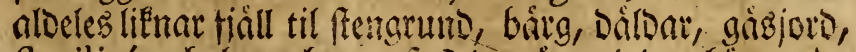
fterilité och) brantbeter, falt Det án minore hosgt, ant antre fiáliar, ej eller refer fig fá langt up, meo minore

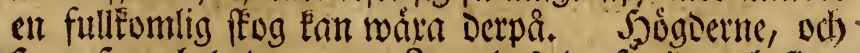
finart fagt, hela Denna tracten, beftod af wida od) flac: fa bairg. 23argen beftoov af en grâften, font woat hopfatt af en fmâfiâllig fimmer (Mica), blandad med fmå quarts gru)n. 2)(lla Deß̧e bărgen fluttade st offer meo et fafta affallanoe; men Dercmot woro de pa wă ftra fioan frucferwis afbutte til flere almars bogo; be flifrade fig uti tioffa flifor magorlunoa horizontailt, S Dock 
Doce mera futtande ait offer. Wtan, eller sfte fidan

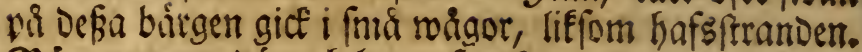
Şärgen rooro Dár och broar fprufne med perpendiculaire fpringor ifrân offer til maifter, och) fprictorne ibops láfte meu en grof och feráficis fpat, fom merendels leme nnoc et ârt af en tumb hơgo hojgre in bärget. Mian fer pâ flipporna nid Bohus, Dá de friga up utur hafs

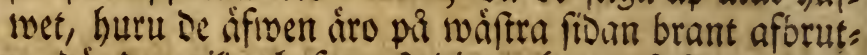
ne, Dár Det twilda bafiwet findigt arbetat pá fidan; men flutta fig fmâningom pí Den óftra efter lungnet emot lans

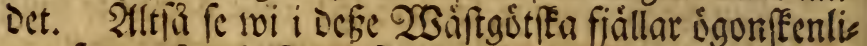
gen fport efter hafivets fortta arbete, ocb huru Det eftes hano affiumfit, famt pa í sitra fidan lemnat Elara märs

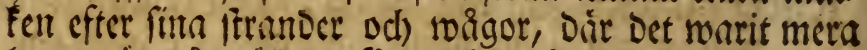

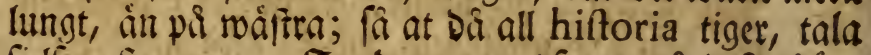
fielf we fenarne. Ieromentelt of wan pa Deß̧a bârg war merendelo en rúd $\mathbb{M}_{0}$, fom ingalunea frigonde

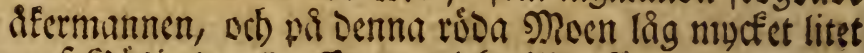
grof SGisisoro, eller \$nulla, i bivilfen föga annat fans śn Ljung 309, hrwarb blommor ănn ef frameoms mit, Dáriff icfe Tetralix (Flor. 310.) hăr ach Dă framlyy med fina täcta blomifer.

SWOGEN, fom ftod emellan birgen i diupafte Dílder, twat

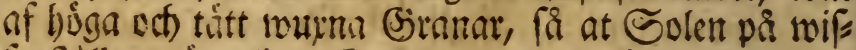
fis ftallen nappeligen funde en enda minut fora in $i$

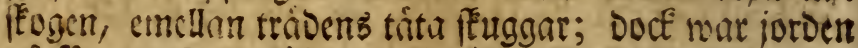
pia ficoma frillen ifrocralt betáft meo $\mathrm{en}$ Miofa, utom broilfen icfe en enoa dort funde bérga fig i Dentma dages

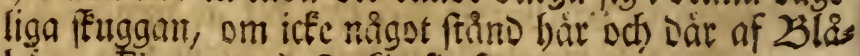

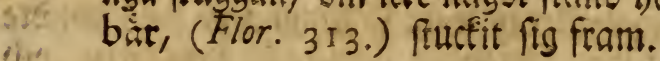

HYPNUM proliferum (Flor. 872.) mar Dent Noba a, fom tîfte iorden i Den féugga, fom Solen alorig borts oref. Den famma swarte bà rátt frodigt, ock lemmas De ci en enda plats, form ben ci tagit in, fí at man beiraf font fe, buru Göaparen forroronat waikter, at tácfia alia orter, Dd) at Denna D?oßan Ean wára utan Eol.

ÁNGAR 
LNGAR fagas några fá inftingoe umfring gårdarna, pa Defa 23 afftgota fiăllar, bwilla rooro beroume med 23jork, Ljung 309. Arnica 689, Melampyrum album 513. Cornus herbacea 131 . Trientalis 302. Vaccinium maximum 312 . odf Parnaffia 252, fom $i$ Dag wifnoe fína förfta blommor i dálierna af Deça ängar. (Sirâfen moro måft De uflafte; Zaacks tåtel 68, färgråfet eller Sirran, 95 , od) Untgborft

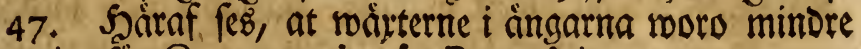
artige for Oeconomis, ån Botanicis.

KARREN biar pa fiállarna moro torfmobar af Sphagnum

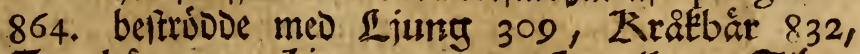

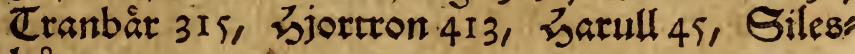
bar 257.

SJOARNE woro mucfet fă, miD bmilEas ftrander mákte Lobelia 714, Plantago monanthos I28, och) Ranunculus minimus 459 .

LYCOPODIA 857. 858. 859. funnos bấ od Dár wib bargsitioorna.

SENECIO fylvatica, for af roar tios ftirfta Medico od nammEtumigafte Botanico Şerr. Archiater HALLER musfet tàct blifiwit afritao unoer namin of JACOBÆA Tenecionis folio incano, (Flor.Fen.177.t.4.) woitte $i$ alla be frocojor, fom for et ar feoan burit rag, och) innu woto inftangoe til grástoât.

CAMPANULA foliis Echii 183 . wåte minore allmån wid àngarna omfring Detta fiall.

QWARNSTENAR lägo mis maigen huggne af Detta fiạls lets baraart, hwarfure de of nirmaft fommo til Dals ffa nurEqmarnarna, i Det, at quarts-grunen, fom toos to birbe och enagglige, bophaftabes of ffimret, och altfá giorde Dem tiannligare til qwarnftenar, emeban de fállan bebofoe hacfas, och) Derfore lemnaoe minore ften pulfiver i miolst.

$$
\text { S } 2 \text { SPIS- }
$$


SPIS-STENAR huggoces af Denna fiálffetr, af Groiffa fâ bár omfring, fom i Borås fpifar upmurnoes i ftâllet für tegel. De wors Därtil fa muctet tiánligare; emedant

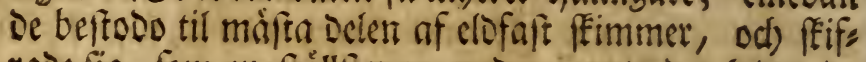
rade fig, fom en ftállfien; under muranbet lndes De horizontailt, at fanten fom in at e(Dftedert, och) fis ledes lángre utháronde bettan.

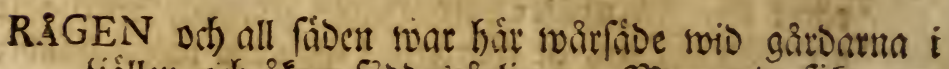

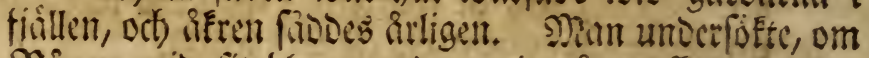
Siâgen wid fit blommande trait năgon feada at Det mucena rignet: man fint, at bwart ar bade twesinte fivor, bivar fida 8 glumas eller blomfoder, biwart blomfocer trainte folculos eller fmá blomifert, od)

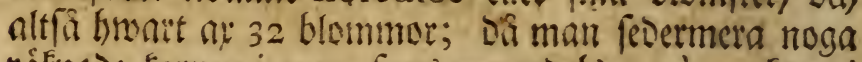

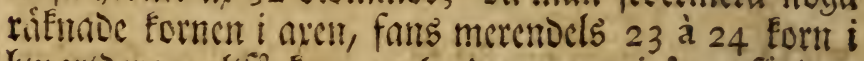
biwartoern; alffa fommer lanotmannen $i$ ar at forlora ent ficricoel of fiut Biág, entiaft for Det, at rignet Eoms mit i blonmman.

LANDET blef, efter ro quarts tefa ifrin Boras, fiolines tare vch mitbare, meo buar, ingar; aEerfált odd bârlis

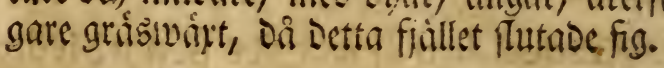

PTARMICA (Flor. 7 66.) Jyars blommor oct int brufas

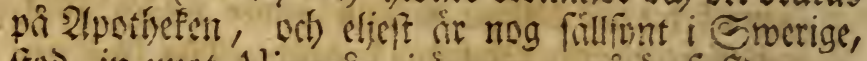
foo, in emot Alingsass, i Engarna, pir ome foor on moigen.

* Alingsis. quart. i g. ifran Borås.

ÁLINGSÅS \&́r en flicf, fom tilfótene nagt frads:privilegier, intil ar 1723 ; har mu fin Juftitiarius, och bals

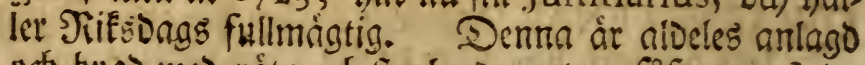
Def bugo meo rita ach) frenlagon gator, fafour en ftad; 4 gatur gå längsiat ftaden i ófter och mäter, af frwilla Den treoje ligger pá simfe fibor om foocm, fom fluter igenom ftaden: uttem Defe cir ringgatan, fom inmelus ter fraden pâ alla 4 fibor, of lien lángD; men deutre

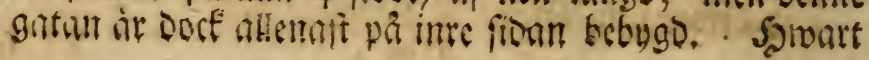


quarter af ftaben åt oalt uti 4 lifa tomter, pâ hivilfa l)u fen áro bugoe wib hosmen, men i centro af quarteret, Dât temterme fista tilfammants; àr en commun Siunn fơr alla 4 tomterna. Demua fitaden är belàs sen pì et llătt fâlt, form beftár of fir fano eller mo; akerfált omaifwa ftaoen pa alla fioor, och) utom Dem

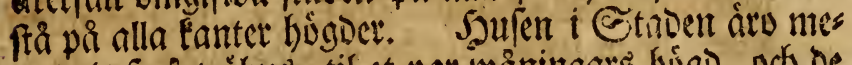

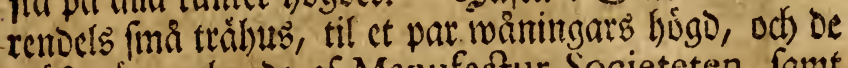
máita iro upbugoe of Manufactur-Societeten, famt unberkollab til Laboratorier od) Şandtwerfare.

MANUFACTUR-SOCIETET wât hạ $\mathfrak{i}$ Alingsås anlago, fom fa intagit alt, at man fogga anmat fag eller borde, ân Manufacturer, Manufacturier odh Manufacturifter, bmilket alt blef ob wifat af Jat. Juftitiarien Ekman.

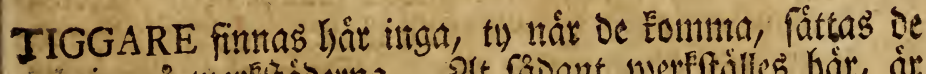

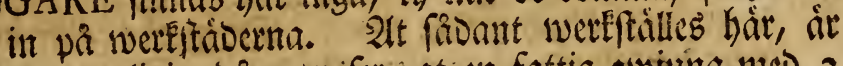
e) underlicit, Da man fer, at en fattig qurima med 3 barn Ean fortiäna fig pa pipbrufet 27 . Dal. Si:mt om weEnn, fom gứ ârligen 1402 Dal. Si:mt.

ORIENTALISKE BACKARNE woro myligen flipte, of

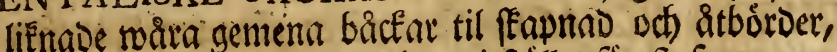
men Daruti atfeilde, at De buto i ftîllet for fruf ragg cn

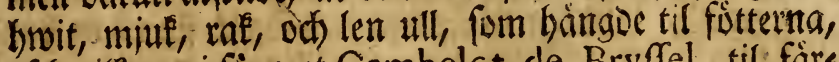
af bwillen soi fägo ct Cambelct de Bryffel, til färs gen fivart, odi fa fént, fom nigonfin migot utlänoffet, boullet ofelbart wat set forfta, fom $i$ Emerige giorts af eget Eamelgarn.

GOTTLÁNDSKA GUMSAR gingo bir meo 4 frotit born.

SPANSK HUND fägz; han war liten od flitt pa ftoppert, men oronen ffilde bonom nogfamt ifrán antora; ti) be

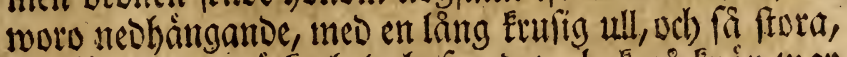

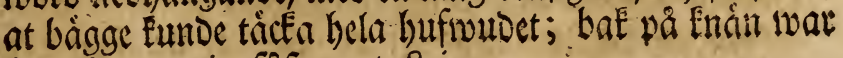
ban langraggig fáfom et . Eejon.

$$
\text { S? } 3
$$

TUR. 
TURKISKA KATTORNE fFiloes daitmed iftån wåra, at be bade baide längre och) mjutare bar, famt at Deras frouns war hărigare, năftan fom på ent mâro.

SWENSKE RÁFWARNE, fom hait woro bundm, habe Grwita làpyar, rafa óton, lâitg rât firang, meo hroit fpits, robaftig fropd, DCs) fótter, fom pas framitoan woro fiwattaftige. De gapade midare, án någon

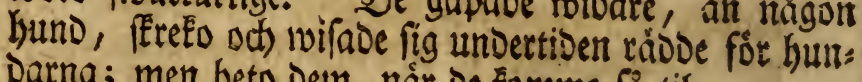
Darna; men beto Denn, nát de Émmo fâ til.

SPINA VENTOSA war hair en paffio endemica, fom plågade átfitilliga barn, och Det mer, án på något an nat ftállle: Zaarnen fingo Enullar pâ benen, báatoer, ars mar, anfigte, áfruen ocf pá fieffiva bróftet: Entrlarne tooro frote, fom fimả applen, oef futo ofta i bullet, Doce maift báftade fig faft nid underliggande ber. Sieren Dgls lvoro de hároe, od) utan wäk. Sallan óprade De fig odf) gáfruo et litet war. De giorde contracturer, och fémbe underliggande ben. Denne paffion

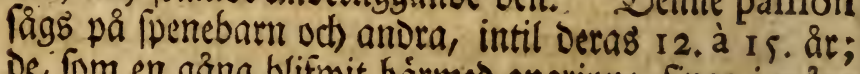
De; fom en gång blifwit bármeo angripne, fingo i måns ga ấ bebolla Denna froarighet.

GUTTA ROSACEA fâgs på en giftruuren Sung fru, fom miot på finden fórft baoc fâtt en liten. Puftula, eller qwefa, Grwilfen feocrmera utwiogat fig til en bandolofs rees mibo. Folfet hade rảot henne, at utivártes brus Ea quvitunomisle, hivaraf paffionen blifwit wórte: De bave ofelbart lifitit i Medicorum bofecer, at Lac virginale (eller Sumgfrumjósie) moro goot emot Guttam rofaceam; men de hade fórtagit fig, dá de tagit quvin: nosmiơle för Gutugfulumióle, ty Den förea prepareras $i$ bivart torp, men Den fenare cillenaft pá 2lpotbefen.

DRAG eller FLUSS, in en paffion, fom nog brue de fors númare i Stwerige, fárdeles Fruerma, ja, ofe of ta fielfs wa Doctorerne, Eefinmerligem da de lät fin practik wid utlindifa Academier, eller af utlinolén bocter,

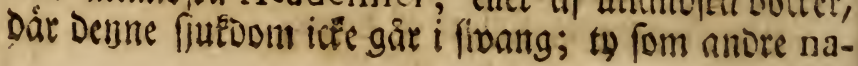


tioner ei brufa fijill , at Dörmed giva fantrarna til baoftugor, fi aro de ocf fric ifrän Denun oligentlet, emedan man fíllan bơrer nägon fir Drag, for Det han refer $i$ frare bláf. Drag, med et ord, ate et fpecies catarrhi, od) fororfafab, Då en ftaut Fall pult Eummiex på en Dáfwig fropy, hivilfenz porer of hettan fta mycs fet opme, hivarigettum fmetten ftetnar i porerna, fom baftigt fig iboporaga, och faledes fororfata, at tranfpirstionen aldeles blifwer forrtoppas pa set fiallet; bivilfet alt fá tyoeligen lägges fơr ögunen uti füljnmos cafu, at en blins fan derpa taga med fin faipp. En Patient begarte mit rád; han hade for 12 ar feoan boot uti en liten Eammare, dár en Eactelugn war ftillo iffiljewriggen, emellan en for fal oib fammaren, pa Det, at uni: Eacfelugnen upeldades, Gan tillifa muâte upuarma bioe faleir ofg Eammaren. Folfet, fom boos De $i$ falcit, mósgabes Dngeligen gúra Eactelugnen imucet warm, dá Det De màte fororifiva fóloen, lywarigenom Den lilla fammaten blef alt for mucfet warm, fi at wår Patient nobgaves opna fónftict pa glänt, fuft fallafte

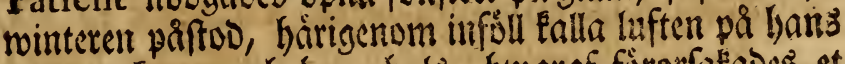
swarma fropp och bara halb, hwaraf forotfatadez et ftarft orag eller flußs, at halfiva hufwubet meo ben ies len af halfen, fom toanies at fonftret, upfwulnabs, fi at Patienten $i$ foritone brourfen funde fivalga unatt cls let tort. Saaremot brutade ban gamfera mainga wás oli= fomedicamenter, fom for honom at Medicis bifis: wit ordinerade; men utan werfan, intil Deb ban blef raso, at twoitta Den fiuka Delen meo warm cittifa, uti hwileen twal wat rifiven; hatraf forgict paffionen til majfa Delen, utom Det, at tungfoenet fom oftait up: fwulnade med en balibranna, font alienaft bolt fig up uti fielfioa froalget, ocl) tillifa forrorfatade en tioppnits i nifan, eller en tort catharr, famt et ftimbigt fufanoe for óronen, liwilket alt feoan oroat Patienten, Docf utan fel pá bölflen. 


\section{Jul, 7 .}

MANUFACTURERNE i Alingsâs meo alla bât imåt tade weelffáder befigs i dag.

ULLSORTERINGEN, Dár ullet med alla fina flag plåcs Eades ifrain Givaranman med fingrarna. Soàr făgo no

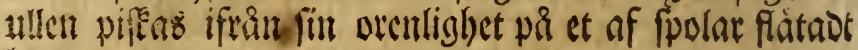
bord.

ULLKAMMERIET med Dubbla jernfammar, forrffrefne ifran (sienact); Eammarte warmbes uti et par fimå

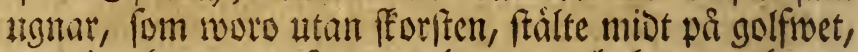
umering lywaroera futo 4 arbetare, och blowa arbetare Eammade uflen med 2:lte Eamutuar; Eammarte wärmbeg fränisgt genom horizontaile foringor på ugttet. Ulgnarne gafivo et ftabigt $0 B$, befintnerligen om winters tioeli, fa lainge fönftren ei fingo opunas: wi märfte Doce icfe, at arbetaute hade fâtt amuan oligenlet, esin Det allema, at De miffat luften, och) at $D e$ af mimita quantitet $\mathrm{i}$ ftarka bricten finatt blefivo betagne.

SPINNHUS fagos tilliea âtffillige.

SPINNERIET til fina Elâdent, Dăr alla tullantme fpunnos til féapnad af en figigla.

WARP-MACHIN, eller Geertan, fom fielf finttade tri?

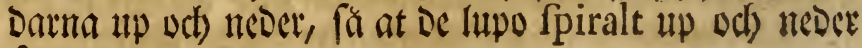
at warpen.

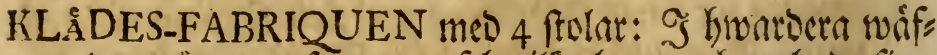
be twointe perforter, of hivilen bwar od en bave fitta

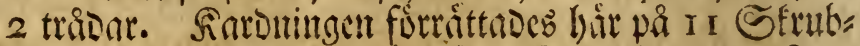
bor. Htilen gice ifrian De grofinn Strubbor til de firas

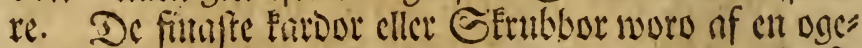

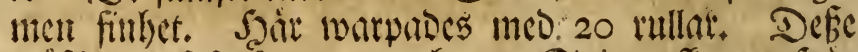

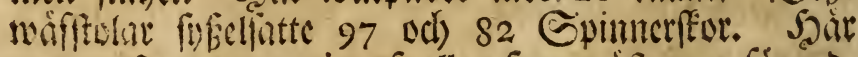
ware et frott magazin of ull, forn meifit war fárgan, odl) et aumat magazin of färgast od ufargnot garm. Demn Fabrique bnoe fit egit fárgeti. KLLÁ- 
KLÅDESWÅFWERI fơr fina Hláben, med 2:me ftolar. Jàr fags, at ju lofare ullen war fpunnen til inflag, ju fajtare od) tátare blef Elídet.

KLÅDESWÅFWERI fơr grofrua flíden mç ullens faroning odf) Dȩ́, magazin.

SFWERSKÅRERI, Dår 2:me Farlat med frotr oft ofantes

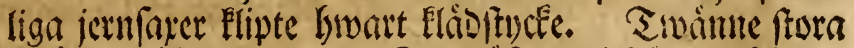

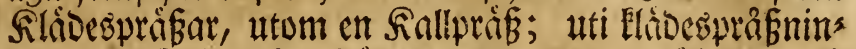

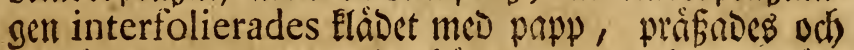

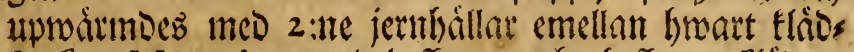
ftycfe. Magazinet mes beffurna och obeffuma Elíden.

RUGGHUSET Dair Hläbet, fom formt blifivit fultabt, rug: sades cller fardades af 2:ne Enrlar, Givilfas Eardor twos ro ibopfatte of 8 capitulis Dipfaci. Magazinet, $i$ frwillet farbor, eller capitula Dipfaci, berwarates in futurum ufum; hạr torfades ocef e eläbct, faunt filis: Desmangelen brogs af en båt.

RAMGARDEN war ftor och wiolyftig, meo manga ram: mar, pa bivilf́a Eläben uphángdes til torkatbe.

FÅRGERIET fơrfeot med fora od) Féna Eattlar, iblamo brilfa war en for od furkentig Sentếttel för Charlacans fárgeriet, tillié med typhufet.

TWINN-MACHINEN trar oval meo 40 rullar, eller 20 pa bivardera fídan: Denma twinnabe tilfammnans Det garn, fom fơrut mar duppleradt pa en liten rick, od allenaft en liten flicfa brog omÉning Sivinremachinen. Saút förfátoigadez beffa Floretts-band.

GARN-MAGAZINET, meD garn af alla forter, fouts net af Bondespigorna; Det finafte have 59 ha fiwor pa bwar mare ull. Somull figs băr fpannen til ben fis naffe trá.

STRUMPWAFWERI werkftaden, uti bivilfen făgb I4 Strumpftolar, fom forfäroigade fit arbete fa enfaldigt S? 5 
fom be Eneffigt rooro ihey fatte. Tiu woro allenaf 7 ftolar i gång, af orfal, at frumpwoiffwerie i De fettare aren pá anora ock fiere ftállen blifmit anlagoe, fî at confumtionen lyăr aftagit. QBio biwat wáfitul hämide et loort glas: Elut fullt med flart watten, fon

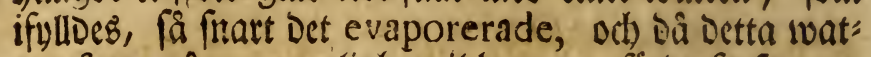
ten fatte någon orentighet til botten, fórorefz fanma floccus meo nagra oroppars tilgjuttintg of fécivatten;

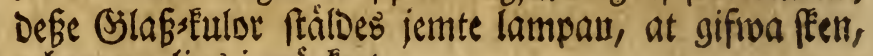
och) mera lius i mórteet.

STRUMPKARDERIET, Dâr ftrumporne fardades med 3:me hopfatte bufwuder af: Dipfaco, hrwilfa woro ftáls de pả et iern, lift en treudoig gaffel: taggarne på Deß̧a hufwuden af Dipfaco troro muctet ftufroa; men kuk De Docf ei längre nyttjas, ân ent Dag.

FRISWAFWERIET; bâr woro 3 máfitolar, 6 ulstaro:

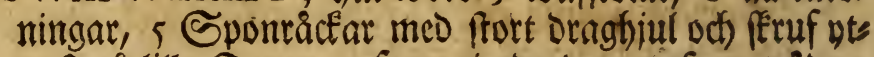
terft pá lilla Tenen, fom gjorde den grofraa trảden: Shar futo pa hivardera wififtolen 2:me Earlar, Gmilla Groar hade 4 tráder, od) De Eaftade Den ftora féttelek genom wáfiwen til byaramora. Saár arbetades $1 \frac{\pi}{4}$ SE:po. ull hroar reecta.

ESTOFTSWÁFWERIET med i 4 wafftolar, fom noro lifa med De allmánna wáfítolar; men fätet, pá brwik Fet arbetarne futo, bade wis ándorna en apel, at Det funde bojjas fram och tilbafa.

DAMASCERAD SAT TIN wáfoes pà famma fätt, fom Damank od) Triumphant; många Súlf bängie pa fitoan af wâfmen, Dât Det ena Sülfiwet nedorugä efter Det andra at en goBe, til Des alle Sólfwent ivoro neDs

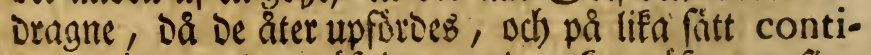
nuerades; under wáfningen nedtryefte loáfwaren fina ordinaire ङólf med fútterna, form giorbe en ordinair miff, men lateral @olfiven gioroe rituingen.

RASKWÁFWERIET: Magazinet bártil mar mucfet 
anfentigit. Sarn fing bàr fint fom Sille; fa at man af et ferlyumb hande 9.6 barfivor, hniar barfwa of 5 flicipp, och broar fnàp af 80 omgingar, altia lywar Dera barfwan af 60 almat. Dupler- od) Twinns Machin.

BOMULLSWÁFWERIET: Såt fảgs Parcum wâfs maż meo 4 Sólf och träbar. Slefplatien läg bậs jemte utbreos, od , full meD atffitliga Somullstyger.

NOPPKINS WÄFERIET, Dît roaffitolen beftod af \& Solf od) 4 trioor.

BAND-WAFWERIET af 6 ftolar, pi hivilfa floretts

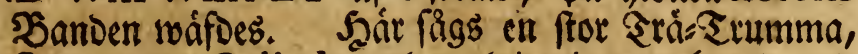
suma eller Cylinder; alt omfring igenom borao meo tấta bål, genom lowilka figurerna på banoen utftafas Des; på et Eonftigt uträfmadt fätt. Magazinet med elt mocfenbet band itti.

BANDSTOLAR 3 fucfen, Da bivilla 24 à 25 band tillis fa toafines, od ofta broardera banoet af olila coleur; alle Defe banden wäfoe fig fielfwe, når allenaft et litet barn brog wefrwen. Saăr wáfoes 60 ftycfen banto pa en wecfa, broardera ftyctet 50 alnat langt.

SILKES-BANDS-WAFWERIET: Shir woro , Folar $i$ full gang, fom alle wáfoe fina band, af bivilfa fomlis ge rooro utzitrade med rofor, fomlige med boeftáfwer, och fomlige med andra sitater.

RA-SILKES-BODAR, Dár râtt Gilfe fpant, imman bet blef floretts bano. Magazinet af Eilte.

SILKES-REDERIET, Dár Lachagie Fofabez, lardades

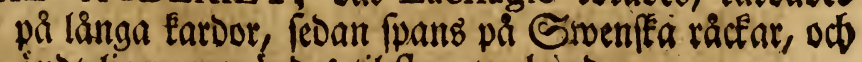
ânbteligen antoandeb til floretts banb.

NALMAKERI-WERKSTADEN.

STRUMPSTOLSMAKERIET, uti brillet frtump:machi- 
chiner af iern fintiboes, od fortárbigndes lonfigare, ån något Utr. Utur bemura werefftao woro atffilliga Strumpsmachiner fürfürdigade, ods falde til andra Manufacturers tienft. Sadir fägs âfwert ent Strumps machin, af Commercie-giádet PolHeim invente-

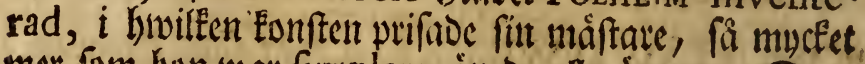
mer, fom hon swar fimplare, än be allmánnate. Dene nte nusttiades Docf iffe, emedan han mentes wora fók

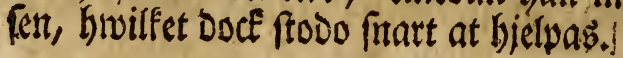

GULDSLAGERIET: Saảr fingo wi Gutu gulbet lades cmellan furtantiga lappar, fora fom et par tort. Def fe lappar woro alle togne. utur Gimnan pá Giątarmen i: opat, och ferdeles praparerade. S5asn lapp beftod af troanne bopfatte binnor; 500 fidoane bimnor giorde elt bof, Gwars ytterfta blab war of pergament; emele

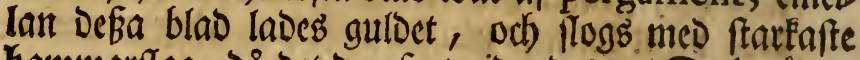
Gammarflag, Dả Det Deraf utmiogabes. De berátta De, at man af broarie ole ci fict mer ant en enda lapp, ty allenaft en liten flacte af tarment tienar til en fadoan

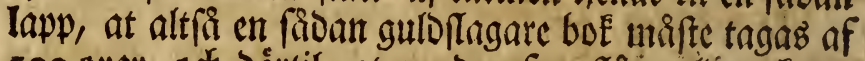
500 opat, och Dórtil, at om Dent, fom fliri gulbet, Eoms mer til at fla et enda orieftigt flag, fpringer ofta bela bofert, fom feocrmera blifiver onyttig. Nan tog nas gra bimmor eller blad af gamla guldilagare boceer, at .

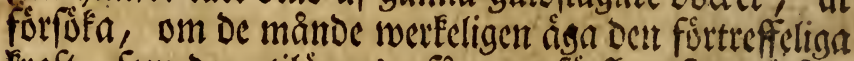

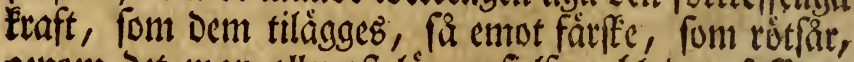
getrom bet man allenaft lágner fielfiwa blabet pả firet, och fedant bet ár pálagot, allenaft fticfer igenom det et Dar hăl med ent nắ.

METALL-ARBETERIET hos Petter Wirgman: Sadir fảg man twánne artiga machiner, af oell ftora PoLHEIM inventerade, Den ena at igenombara iert, Den andira at prába fnapyar och) Duliet' mer. Soir mifades of en Fàrniba, fom lięnade guld, od bơlt fig faft pa

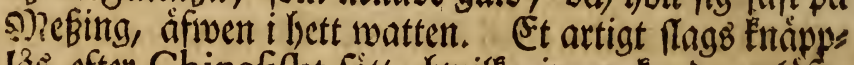
lab efter Chinefifkt fitt, hwwilea ingen kunde upläa, fom zif feot uplăfningen tilfơrene, faft än ban babe nus: 


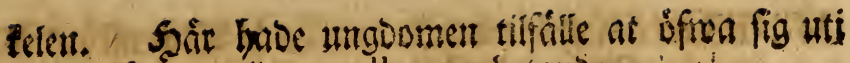
handaflogiver mid metallers arbctamice.

MINUT-BODEN, Dár allebanoa bair tifrwerffade tnger, Rlaben, Fris, Etrumpor, Nopptin, Parcum, Sats $\mathfrak{t a r}$, Cardufer $\mathfrak{i c}$. faldes $i$ minut.

STRUMPGARNS-BODEN, i hmilfen ftrumpgarn figs

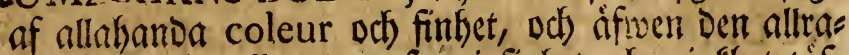

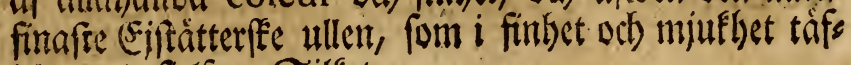
lade meo fielfiva Silfet.

MATERIAL-MAGAZINET ivar et ftort ftenthus, utt: Der hiwilferis botten war en frot och warm fíllare, i bivilen hivarjebanda wata faker, och cn far hop far sur bewaraber. S Detta Magazinet figgo ofantelige ulifficfar, fonftiga Cattuns former, Dd) allabanoa

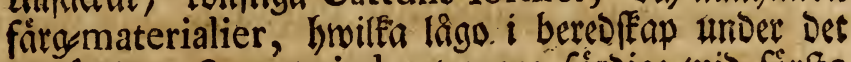
De gumbe et ftort capital, at mara fároige wio furrfta wink. 2ff De materialier ing bâr fág, wil fag allenaft fpecificera De fórnímfta :

LIGNA: Brefilia, Brefiletta, Fernbock, Gelbholts, Blauholts, Werfertholts, Japanhults, Sandel, Buxbaum, Lignum vitz, Spanffrù

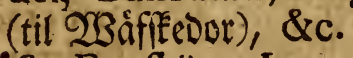

FARGGRÁS: Engfkära, La vau (Jranff oct) Smenff́),

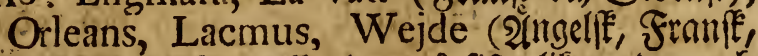

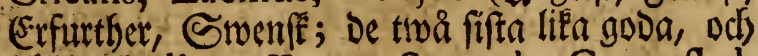
bifit), Indigo, Krapp, Sumack, Granathal, Lagerbảr, Gallaspple, Cochinell, Knoppergall (gallæ fpecies), Spannk Peppar (Capficum), Sennetsblad, Cocus-nkal, Angelicxrot, Calmus-rot, Fœnum gracum, Floefamen (Pfyllium), Janingo-fró (trilocularis), Karborre,fró, Coloquint (at rófa mot infe. cter), Karder.

PINGUIA: Gummi Arabicum, Tragacant, Hartz,

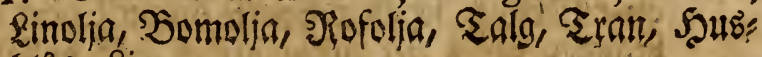

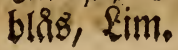




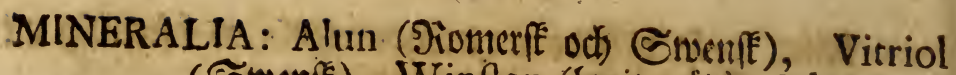
(Swenfif), Winften (hivit, rio), Sal tartari, Potanka (Simenff́, Jienff́, Juálí), Souda, Salmiac, Efcowatten, A rlenicum, Auripigment. Lithargyrium, Minia, Brunocker, Gulocker, Kólnifk Erde, Sittgelb, Spanfkgróna, Ten, Bly, Jern, Meffings-latun, Aurum rubr.

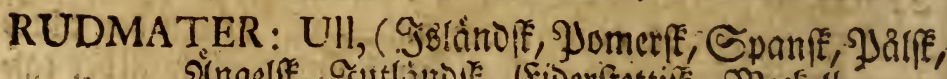

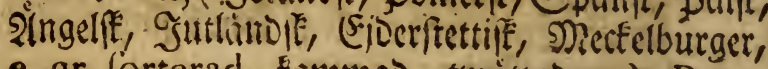
e. gr. forterad, fimmmo, twattad zc.), Bomull fin, ordinair, grof), Camelgarn, Hampa, Kohår (til Filidecs siftor), Florett (A. B, Kolnifk), Silke (trom-ordofetta, Lauage), Flock af Frifen til Tapeter, Klådesfkaf, Lin, häckladt, Garn, blekt, fortPolementeurbanio 6 flag, Bảfwerhár til battar.

PIPQRUKET mar antagot utem ftaden; fár tworo 60 pers

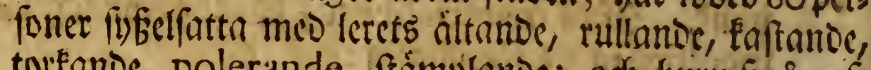
tortande, polerande, ffimplanoe; od buru fina goós for har anmandes til famma tienft fom frore: SHipors ne formerades uti ent Dicfings: form of troanne ftye=

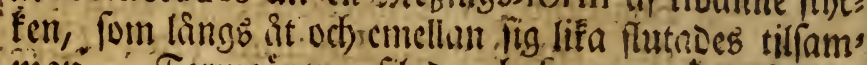

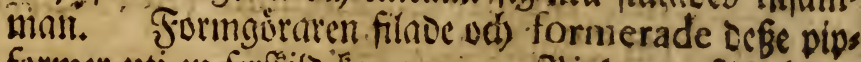
former uti ent ferftillo Enmmare. Jipleran, fom hims: tabes ifrian Holland, war liwit, od ruomabe idfe uti brimmingen; Dả maun ferapade par herne med nagel els ler Enif, slänfade bon, fon en thwit Falf, ob) miölet mar emellan fingrarna fott, lipprigt, Elibbigt, niffait fom en ₹nlg eller ₹alfmiôt. Fïllare moto amlagoe, i hrwilfa de obrinte pirver betwarsies for froft od) ftare torfa. Summorne, ibmiles piporne brindeb, tovero af Ier, lienade nog Eucferformer, faft Defe babe en flat botten. Nár piporne fattes in i Sumant, tâftes houn med et papper, fom inor bog got in formam coni, ed

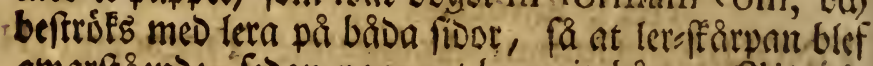
gwarftenende, feoan papperet biannit bârt. - J Siportic bxándes: 24 a 26 timar uti en bis balf Cylindrifk 
ugin; Is à 16 Sunnor brandes tillita, 10 à 20 grofs $i$ bwar tumna, I 44 Jipor i Groart grols.

PLANTAGER moro anlagde pa norra od) fodra fiona om ftaben, fordelte uti mif̧a quarter eller torg genom lefs wande Sachefar, eller ocf boiga affuggne Enebuffar, fom ei under torkandet lemmade fina blad, eller bart, brvilfa alla giffro et beljageligit lungn fơr Plantagerne, De bár sooro fa af Färgegrás, fom Tobaf.

FARGEGRÅSEN moro pa norra fiban af ftaden, och bes ftooo of Wejde, Wau, Krapp.

KRAPPEN eller Rubia Tinctorum, raar planterad titi ritt for mucfenbet, och wäte ganffa frodigt utam at

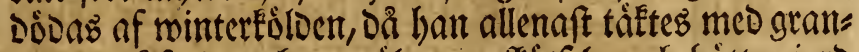
ris, Doce fororar bon wăl mera ffótfel, och bättre jord bos ob, án utomlands cller $i$ Glane; henmes roba rits fir wo:o reban tămmeligen ftore.

LA VAU, cller Luteola (Flor. 439.) fås hâr arligen uti fror musfenket; i ât war bon twingas af niartor: an, ometring Sund i Stane félle hon aldrabeqwamligaf́t fomma fort, Dâr bon fielf wáter wildt.

WEIDE, Ifatis (Fler. 543.) twat áfwen faoo i ftor mues fenbet; Det warar allenaft tu ait, th) feoan Det burit fruet, formifnar merendels roten; Det wáte wâl od) frooigt; men jag fifulle tro, at Det änu blefwe frodi= gare, om Det fadoes pa ipet fált utan hảdfar, emedan Des hemmift ar mid ftrander, dair blaften altid riftar soirterna. Blaben afíkires til plantan, od) prapareras til ballar, efter Det fätt, fom allmaint brutas. (Raj. bif. 842.)

WEJDEQWARNEN beftod af et flatt runot golf, uti briffet: centro upgicf en vertical atel, bogke ân ett farl, utur hwilkens fidon en arm utftrátteb med et frens bjul, lift en gwarnften: Dir en hăft Drog omering han= Deit af Denna qwarn, och forang rumbt omfritg, ruls Iandes fenen pa solforet, fom efter fentens woig wat 
beflagit med tiveriatrt, foun frodo r quarterifrån bivats andera, at bistmed fortivermala Wejdet.

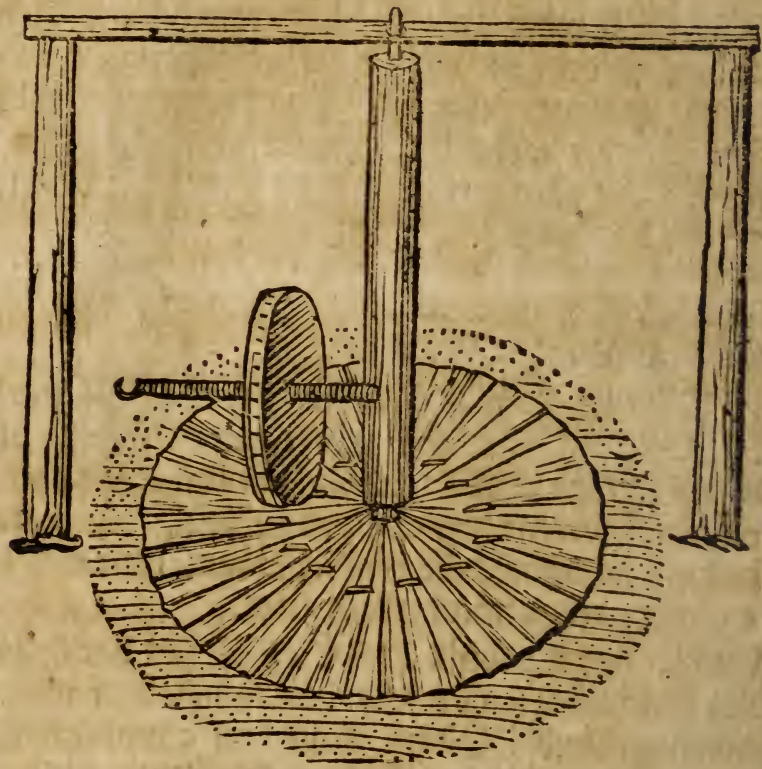

TOBARSHAGARNE beftodo of langa, od) met ait altrs

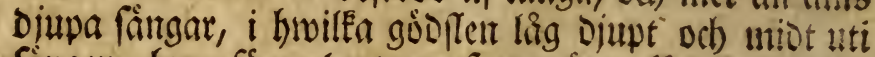
fangen, bivarfore planturne fent woikn, fór àn de med fina rótter unfumma, da de feoermera baftigt blifiva ftore. Siśd, watter och) waider áro Tobafen fiender.

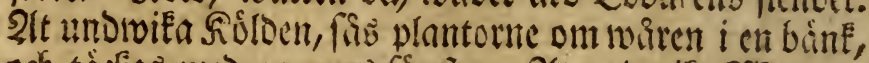

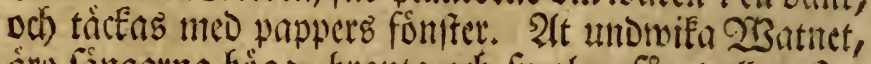
aru fungauna böga, branta och) fimala; fa at allenalt 2

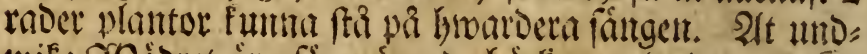

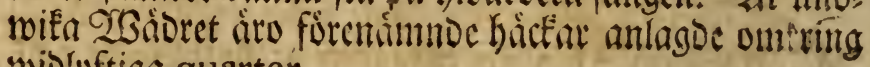
ividhfigign quarter.

TORKHUSEN woro ftore, och et uti hwarbera Tobaf:

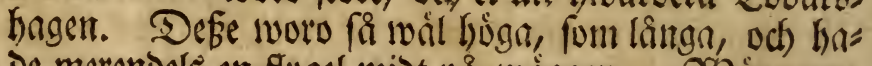
De merendels en flugel midt pa wäggen. 2ritiggarne

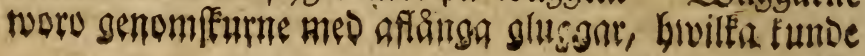


tillutab funde med nedrängande luctor, genom bwilfa luften imlliptes unoer torketioen.

TOBAKS-SPINNERIET af 12 rullat: Sुăr fảg Siulls tobakb ffaringen, furfifurne Carducers forrfäbigande,

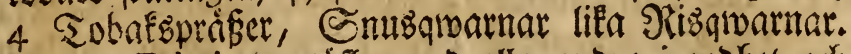
Denna Fabrique tîflar med alla andra $i$ goblet od quantitet of Emenfe sobak; ty man bar baar fors ledit ar ffulat fpumnit Sobak for 40000 Dal. S:mit. od) 39000 \&Et. af De blaber, fom bår wuthit.

TOBAKS-MAGAZINET for bladen befagg: Filappsmachinen, Dår ftielf́arte (petioli) planerades emellan et par jemfalfat.

COMMERCIE-R̊̊DET JONAS ALSTRÓM Gade alt Defta imrattat, falt musfet mes publici biclp; ban hade for"s feafiat machiner, furferrifroit arbetare, fom alt war fa muctet, at ini funde trottma twid endaft at fe bet med flygande ogon. Den, fom bar ice ficlf warit, Eon als orig forreftalla fig alt octsa. Scar fes buru wioa dent fan gi, fom anmonber fin tanke pa nagot witit, ocl) birtil ager en bitmande luft at tiena publicum; En fádan giv oftr mer, ân manga tufende obognde. Fòt Soert ALSTK OMS tid tooro Manufacturerne hos of $i$ et biuwt morter, sfiver mafta Sifet, fom un Eliod ods foon fi manga tufenbe innom landet. De mâfe arós tate wid Alingsâs woro nu Sruenfet fole, fom lîrot af De hit utifuan forferefne Sonfmérev, fa at De mälse vereftioer mu Drefwos of wara egna sandsmän, fom

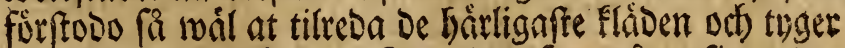
med Swenffa händer $i$ Smerige, fom nagonfin andore nationer utomlanos. Shä hafroa atficillige Manufaçurifter fât fonften, Sandtwerkare anleoning til machiner, od) mange Gtandsperfone blifwit upmuntra: De, at bereda fig tyger til busbebof, ja, fielfwe sandts mannen bar bait omering blifwet begifwen pa fpanas oc) wafnad. 
RESANDE ynglingar, fom tånfa befoptr utrilez orter, bors De forft befe Alingsås ocd) Eahlu grufioa, at be icfe ma fofa utomlanos, Det de agga hemma, och be ftuderande mid Academien, fon bofiva någorlumba tilfálle, borde átmimftome I a 8 dagar bar anroantod, uti broars ie werfitad, at fe buru de flàder gúras, meo builfa De fielfoce prutera.

\section{Jul. 8.}

TOBAK planterabes af atffilliga Bustber omering Alingsâs, til egit bebof, od) mar Dem unog, om De allenaft ing De 150 plantor. Sönderne torḱa fina Tobalšblaioer, od) hafiwa lárot at nigot litet fivetta Dem; nio Guls tioen beftänén de bladerna med litet roúrt, eller färféd,

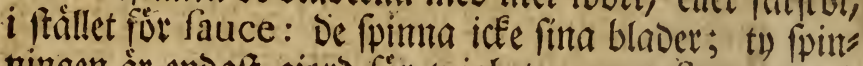
ningen ar endalt giord fost migheten, tranfporten och afmatningen. Sa nottigt fom det â för sBownecuta,

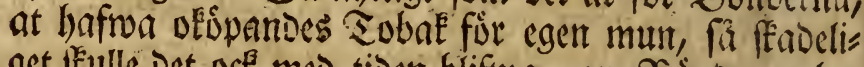
get faulle Det oce med tiden blifion, on soindertie be= gunte anlägga ftora plantager, och Derigenom ftiâla

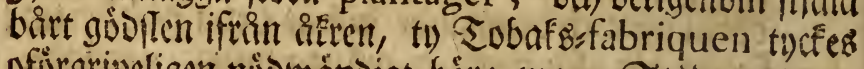
ofürgripeligen nubwaindigt bưra wara Etadernes ent peliote.

SURBRUNNEN låg ftrax nedant om Alingsås, Des wats ten prófiogdes, fom war längt ftartare, an mid Eur brumen i. Borqs.

cum Gallis tingebatur colore atropurpureo. Pulv. Thee dilutiore. Succ.heliotrop. immutata. Syrup. vioiar virefcens. Cocsinella obfcurius rubra.

NORRBY WATTEN, fom ufgafs för Surbrumm, prófs tonoes pa fúmma fâtt; men bölt intęt mineral, utan war et rent notten.

ROT ANIQUEN, cher âtminftone wanen at fánna órter, 
Dif) at weta pa dem nigra namn, lárbes Jiftâne of Scholxmâtaren Hultén, genom fmå premiers uts gifwande til Den af Dilciplerne, fom baruti wifade fia faroigaft; biwarigentom en otrolig bog rotade fig i bes rab prido it, for en gagnelig toettenftap, Den feoerme: ra frointigen utrotab.

HEDERA (Flor. 190.) twat funnen af Serr Hultén toid Alingsăs.

LATHRIEA Squamaria dicta (518.) war feob bá om Ering $i$ Siơrelumbarna neDanfúr bárgen.

CALCEOLUS marix (735) mav upletad af fammi Hultén wí Timsdala pá Billingen.

VAC INIUM maximum ( 32 ) white pa sitfelliga

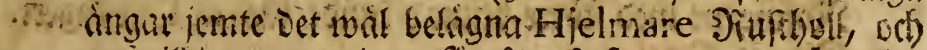
gat tilfimma Det ringa forrao pa froartmulla, fon an gen imncholt, ty fomar fom halft cenne buffe wajer i nágon quantitet, âr altio en ufel iocomont.

PUNAROT tollades of 230 frgutarna Artemifa (667.). WEKAR tallsoer Salix (792.), eller Hlplänningen Julfter.

POTATOS, eller Solanum tuberolum war planterad af arbetsfol et wio glipbrutet, ba fielfioa bärget emels lon filpporna, dit mulla war forto och lago; Det swapte wal, od) rotterna woro on hoffen ife froira at igenfinma, ty oe bade ej tilfálle at gî pa djupet.

JET TEGRYTAN of I alng Diup od) lifa breod fägr uti en ricofalten Elippa moid Jjipbrufet.

DYNGEST ACKEN wio Jipbrufet base iemte fig et fort

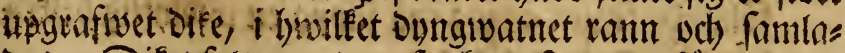
Des. Diket fulter med torf och mokar utar kirren, fum infopo Dyngmatnet, och falebes blefwo fórwandlade uti mucten oflafen gódel.

GETSTALLET war Eringfatt med SBantar, pa Kwilka (Setterne Eunde ligga, fom gerna ligga turt odj boggt. .... \$2 


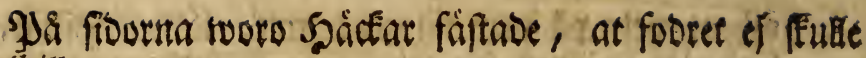
Tjillab.

TEGELBRUK war anlagit ut wib Jipfrulet pa cit ant genám out, mäfter oum ftaden, Dár watnet tringffuret oe fiśn blomfterfältent.

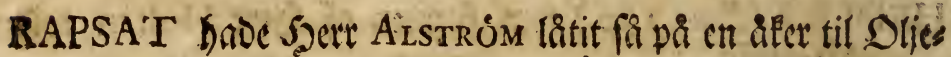
Nageri. Demme Brafice calvx war femipatens. Gaulis lavis, ramofus. Folia fuperiora longa, fubdentata, bafi rotundata, amplexicaulia; inferiora lyrata, glabra uti tota planta. Silique longx, craffx, fubtorofx. Denne luctabes ci funnerlis gen wäl $i$ profwet; Gag fifulle holla fore, at Cottlands Siofinorne imed ftürte bătnad Eunde fias til Dlieflageti. Se. It. Gottl, 240.

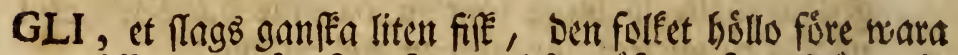
fifitungav, for fom folgran $i$ ant, âfroen fom short mid ftranderna om leketiden. De woro ingalunda ftérte sin mugg; twi upfilfabe några, och funno, at de icfé woro fifFungar, utan et ferfifitot flagte eller PHOXINUS leu Cobitis Cyprini fpecies. (Faun. 33r.) Deras rugg war med vändeligt många fimã fivarta pricfar beftridod, od) Deras bue bwit.

KÁRRORNE bár pia orten beftodo af en ftega, fom laber pa en nel meb et par bjul efter ollmint bruf; meit bacfen formerades of furit, ciler Eappar, fom neobigig: Des unver fecgans fpol i for's, DCh indarme bögde fig

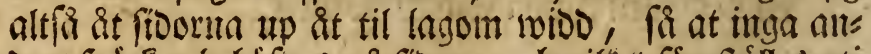

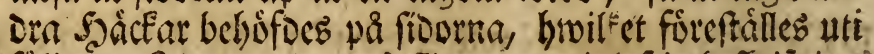

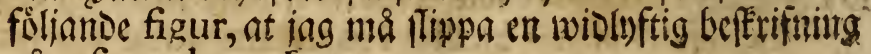
pa et fimpelt werettyg:

Ge figuren pa fúlianto fido: 


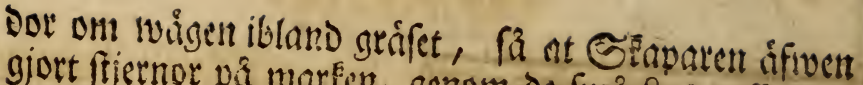
giort fitiernor pá marken, senom de fma susmafêt.

LYSMASKARNE (Faun. 584.) habe under fijerten 3 (b) fande ringar, of buvilea ben fifta beftos of 2 puncter; Sonorne af Defa Susmaffar aro de, fom bar ligsap? marfen utan wingar; Inen Samnarne báremot fola âga babe ivingar od) wingffal: Nan har allmaint troot, at Syonorne gingo meo isefa lisf tor, nt wifa fira măn (wảgen, och) at läna Dem elo; then wár ftore Entymologus Saerr Eammarlerent CARI de GEER bat

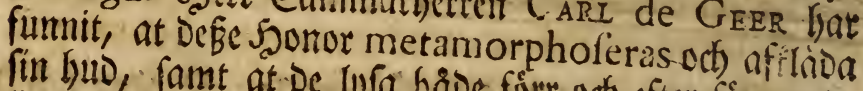

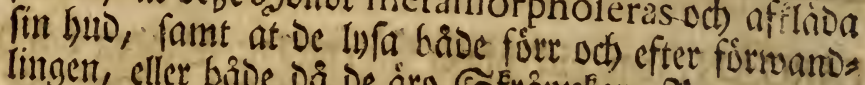
lingen, eller báde dá de áro SErâpufar, Jjouppor och Siráf; hroaraf tocfes flutab, at berme elo icfe ar nigon aúntefs eld, then favan brinner alorig bos barm, fom

* Lerum, quart. $5 \frac{\mathrm{L}}{2}$.

\section{Julii 9.}

RESAN ifrån Lerum til Götheborg.

LANDET ánorabe fig, fa fitart man infommit uti Gótheborgs fän, Dât fantostwigen I mil ifrain Lerum gicf never fồ et liỏst bärg. Etrat śptaves Góthe-

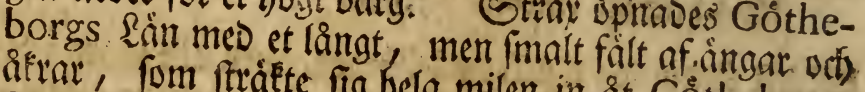
sfrar, fom ftráfte fig hela milen in at Gótheborg. Detta lienade nog Tems floven, (allemnit Det ftadot un= Der.watten) Do man ifrän bafiwet feglar in at London, foft be pa bágge fibor liggande bairg noro bire meer ffals lote och Elipwfulle. Da Denta tract foroum fistt th: Der watten, bar ofetbart Detta warit inloppet och hams: nent for 23 aiftgitarna, under Den tiden de foro $i$

SKOGEN tog alt mer ods mer af, fâ at man fallaut fig nis gra trán, utom bem fom blifwit planterade wio git:= GÁRDS. 
GARDSGARDARNE woro láge och finale, uymurade of Fullerftenar, fom wid forfta piftutandet ramlade ontill.

ONONIS inermis (Flor. 622.) wakte sfroctalt uti ans garna.

PTARMICA 706, ftod allmån på ímle fioor om woigen. RUBUS maximus, fructu nigro 409 wairte lif $\mathfrak{B}_{\text {ijumbar }}$ iblant buffarna, och jemte gitroeggarbarna, men muses fet bogre od) faftare an Siürnbären. Flores corymbofi. Petala, ftamina, \& piftilla alba. Calyces quinquepartiti foliolis ovatis, concavis, acuminatis. Senna bar jag tilforene icte funnit $i$ Swerige

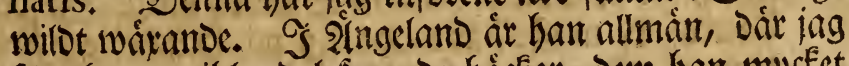
fedt honom iblano lefwande bäcfar, Dem han mycfet ifopilatar och gor faftare:

* Gótheborg. quart. 8.

GóTHEBORG war den tícfafte ftad iblans alla i Silfet, til ftorlet något minore ăn Upfala; náftan rumo, om= gifiven od) befäftad med 2 (sallat od) (jirifivar; afoeld meo regulaira, râta od jemina (3ator ; gemomfeuren

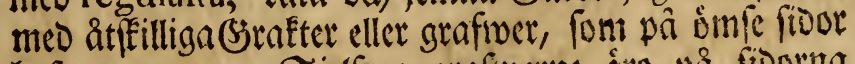
baftua gator. Eielfiva grafwerme áro ba fiooma perpendiculairt mutade meo giafiten, fa at gatant til

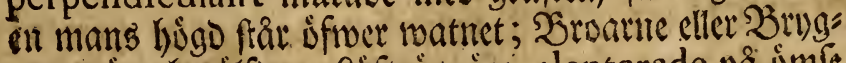

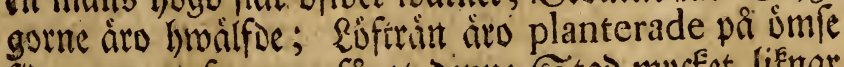
fidor om grafiocuna, fá at Denne Stad mucfet lifmar de Sollindfea fádoer. Saufen aro til frorita delen ftos ve off froara Trabyggningar, af twa roaningar, ock nára intil Gwarandera ftílde, utanpa bellagne mes brós Der, famt málade meo rơd eller gul fărg, men Enutar och) fơnfterkarmar meo hivit eller bla; fa at de på nas gon diftance mucfet liena ftenhus: grunden eller mus rarne aro äfwen fom wäggarme beflagne med brider.

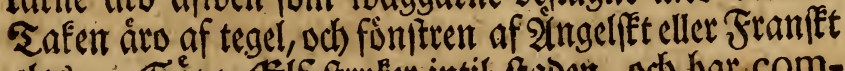
glab. Gota SElf fruler intil faben, ocb bar com-

$$
\text { \$े } 4
$$


munication med glifterta; mett pi wiffta fibnt fivs ter Syafsmifent til fraden, fo at frora fecpp Eumta mo

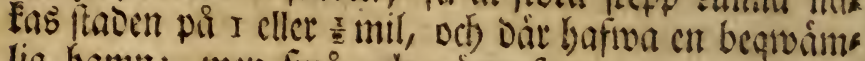
lig bamm; men fma sch natta fartug fumba ga im i fielfwa ftrocen; elieft feilia et par boga birig bafwet ifran fraben. Soun ligger usg fiolandt, fir at bujelt of"

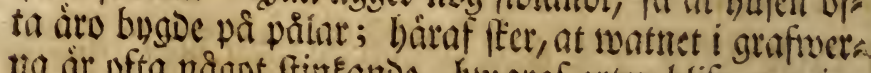
na ar ofta magot fimeande, brwaraf orten blifwer minore funt. Jar i fadon ar Eanosbofoingefite, Gymnafium, Guarnizon, Ammiralitet, Fortification, Artillerie; twoine Sorgnaftare, clt ffón Silio ftuga meo Söirf; trente Surfor och O/findiffa Compagniet. Den beqwämliga Seglation ifran frotit ro ftra bafivet, utan at paffera Sumbet, láttar Sandelin; hrvataf be máfte hár lâgga fig par Scandel och Seglation. Eiraden ar folfrie af Santolante, Eegianoc, Milice, Frummanto, oct Fols, fom ftota til ifrin bes la lambet; hivaraf bát àr mucter roullance, fî at ins buggarne ma wad, och nga fisma famt wactert meublerade hus. Caltellerne Gota Lejon i morbolt, odf Zitortant i foser ligga pa hivar fit büto, laga, cylindrifka, inio bafin furtantige, pa alla fioor meo ftucfoluggar fortigode.

\section{Julii 10.}

CURIEUSITETER famlabes i oag bos be Syerrar i Gótheborg, fom af lifin fafer woro âlfêre. Directeuren ifrwer Sfrimbiffi Compagniet Satr SAHLGREN finte mig en P.ARADIS FOGEL; Gtab:shyficus Sherr Affeffor BOETIUS en CAPRISCUS, Setr Doctor LECHE en bop INSECTER od) ett CAPRISCUS; F(pothefaren LUT en GVAPERVA och en CANCER : ?lpotheforen BAUCH en P'SCIS QUADRANGULARIS, "n NAUTILUS, en Tefta teftudinis, od) nigra Oftrocodemata; Affeffor FERBER, mamntumnig of fin Horto agerumenfi, wifade of bruatichamon, odb Lestor Exstróm leis 
- fagabe of at fe Bibliothequet, Dar en Sturio, en Anguilla marina maxima, en Vitulus biceps, en Teltudo maxima, et Titanokeratophyton od) ell DJapeguia betoarades, tillifa med en bop watra bocfet.

PSITTACUS ruber, alis viridibus, remigibus rectricibusque introrfum purpureis, war fror fom en Dufs wa, offweralt blodrio, utom pennorme i ficerten och iningartre, fom woro grina pá Den fioan fom filtteb; men fa mucetet af Dem, fom táltes af anora ficorar, war affwen robt.

CANCER cantonenfis, Cancer marinus minimus quadratus, Sloan.bift.2.p.270.t.245.f. I. Thorax quadratus, convexus, lavis, lituris obfcure ferrugineis adfperfus, margine acuto cinctus. Oculi prominuli, pallidi, finu oculos excipiente poftice acuto. Cibele obtule dentatx, brachio acute angulato. Pedes, prater chelas, 8, lanceolati, ciliati. Cauda fub abdomine tota inflexa, ovata, obtufa: articulis 6, latis, depreffis, ciliatis. Pinnæ fub cauda 4 utrinque, anguftiffimx, ciliatx, \& præterea Racemi ovorum itidem 4 utrinque. In Abdomine fubtus punctorum par eminentium \& offeorum. Magnitudo cancri ad Arenas noftrates fylveftres maximas accedit. Figuren fan feb Tab. 3. fig. I, Dâr a pa sfra fían, b pa undre wifes $i$ naturlig frotlek.

BALISTES, quæ Gvaperva Chinenfis. Corpus acutum, compreffum. Dorfume antice gibbum.' $P e$ ¿tus compreffum, pinnis pectoralibus terminatum, prominulumque. Latera corporis picta lituris lacteis, annularibus, interruptis, puncto centrali lacteo notatis. Caput magnum, thoraci immerfum, dentibus minutiffimis inftructum. Filamentum fubulatum, erectum, loco narium. Pinna dorfalis anterior retrorfum falcato-fubulata, mollis, alta, e gibbere dorfi juxta caput enata,

$$
\text { Эิ } 5
$$


radio r. Pinna dorfalis pofterior radiis I2. Pinne pectorales brachiorum inftar manubrio infructa, $\&$ antrorfum manum inftar inflexx, (quod in pifcibus fingulare) radiis 10. Pinnæ ventrales approximatx, radiis 5 . Pinna caude radiis 9. Pinna ani radiis 7. Iris oculorum alba. Magnitudo totius pifcis vix extimum pollicis articulum fuperat. Diverfifima 'fpecies a Gvaperva Jonf. Tab. 36. f. 6. quam ex America poffideo. Figuren ya Denna Fanffes uti noturlig Rorlet $T a b .3 . f .5$. broaraft ban pá fioan at aftagen lit. a, men pa ryggen lit. $b$, at armarne pa pinnis pectoralibus ma tyoes ligen inifas fort soguten.

OSTRACION quadrangulus, maculis variis plurimis. Art. J $n$. 85. gen. 56. eller Pificis triangularis major. Cluf. exot. 142. Firoppen war táft meo bruffeltig ferpa, til formen furfantig; fieffiva fíorpan war lie: fom tâft meD fertantige figurer, fom bade fma uphoggs De puncter, liffom chagrin; men Stierten war et Dar twirfinger framfor itiertfenan bar, och tâft med en tun fud. Radii Pinnx Pectoralis 10. Dorfalis 8. P. ani e regione dorfalis 9. P. caude 10, integrx.

OSTRACION compreffus, fafciis nigris, cauda lateribus muricata. Corpus compreffum, figura Percx. Dentes in utraque maxilla 8 , lati, parum acuti: intermediis fenfim longioribus. Squaree corpus tegentes, rhombex, punctis callofis fcabris. Latera corporis cinerelcentia cum Fafcia nigra, a vertice per oculos, pone pinnas pectorales, ad pinnam ani ducta. Fafcia altera acuminata, nigra margine albo, $a b$ ano antrorfum porrecta. Linea alba a fine pinnx dorfalis pofterioris,llad medium pilcis latus, \& alia ad marginem pofteriorem fafciæ nigræ prioris, in angulum acutum coiens cum priori. Abdomen fubtus cartilaginolo-fcabrum. Cauda latera quadruplici ordine retrorlum aculeata funt (ordine:fuperiore bre- 
viore), quod in hoc pilce fingulare. Pinna dor. falis anterior radiis aliquot: quorum anticus cralfiffimus \& antrorfum fcaber. P. dorfalis pofterior radiis 24 , ramofis, P. pectorales radiis 13 . P.ani radiis $2 \mathrm{I}, \quad \mathrm{P}$. cauda integra, radiis $I 2$, a D. D. LECHE.

OSTRACION compreffus rufefcens, pinnæ dorfalis radio longo retrorfum dentato. Monoceras. Clus. exot. 142. Corpus rufefcens, compreflum: fquamis rhombxis, elevato-angulatis, punctatis. Os utrinque dentibus plurimis. Caude latera levia, nec aculeata. Pinna dorfalis anterior radiis 3, quorum r:mus maximus, longiffimus, craffiffimus, antrorfum reflexo-dentatus. 2 :dus brevis, acutus, primo adhrens. 3:us remotiffimus, diftinetiffimus, feparatus in foffa dorfali, intra quam integra pinna complicari poteft. P. dorfalis pofterior radiis 30 circiter, mollibus, inermibus, diftinctiflumis. P. pectorales radiis 10 circiter. P. ani radiis 26 circiter, mollibus, diftinctiffimis. $P$. Caucle integra radiis 8 vel 10. Apex pectoris prominet acumine fcabro, a D.D. Boerio.

PARADISÆA lutea, cauda pectore abdomineque brunneis, gula azurea. Reftram picx, aut fternæ, cultratum. Caput fupra fulvumi. Dor fam flavum. Gula viridi-cærulefcens, anea. Pectus, \& abdomen brunneum. Margo roftri bafin cingens, ater tum lupra, quam infra. Cauda longitudine corporis: rectricibus brunneis: 10, quarum 8 xquales; at intermedix duxlongiffimx, corpore cum cauda triplo longiores, filiformes, brunnex, radiis lateralibus, qux pennam conftituerent, omnino deftitutx, hinc quafi ferratx. Uropygion $\mathrm{r}$. pennx caudx bafin fupra tegentes, breves, caudx concolores, Hypocbondrii pennx, \& qux caudam fubtus tegunt longiffimx, (attingentes longitudinem rectricum intermediarum filiformium:) albx, radiis diftinctis, fimpliciter radia- 
tis, ut hx penna clare, \& diftincte decompolitx conlpiciantur: funt ha pennæ verfus bafin davefcentes. Femora \& pedes, ut $\&$ alx defunt.

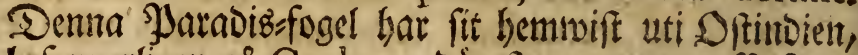
befiumertigen pá Ceylon, Dầ ftora nuyror, fá fitart

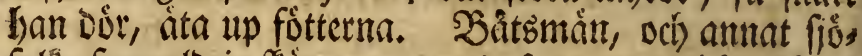

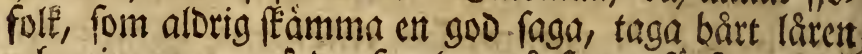
vet) wingarna, pá bet fogelen ma fortas fílfanmmare, vch at Europeerne, fom fortunora fig sfruer alt liwa frammande ât, má láttare tro, at Denne fogel wiftas

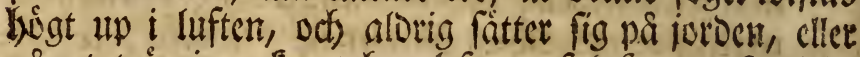
nnagut tró, ja océ, at han lefwer af luften: Syivillet alt boet ât en fägen; ty han bar fotter, fom en Sirita veb) tager ofta minore foglar til rof. Deuma fogel ficte

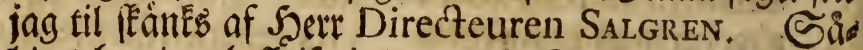

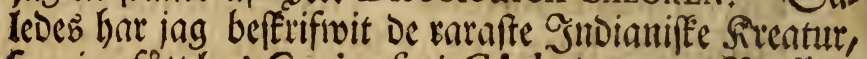
fom jag fátt hos Curieufe i Gótheborg. Tiu fólia

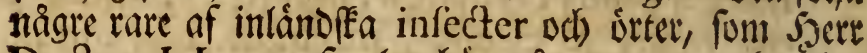
Doctor J. LeCHE famlat hâr pá orten, Dem ban bes bagat medocla mig, til at fúrúfa Fádernélandeț Natural-hiftoria.

PHALANA pectinicornis elinguis, alis incumbentibus albidis: lituris fulvo-fuliginolis: angulis po:fticis fufcis. Magnitudo media. Ale albx: lituris transverfis fulvo-fuliginofis, obfoletis; angulis alarum omnium ano proximis fufco-purpurafcentibus. Ale omnes fubtus poftice flavo purpurafcentes.

PHAL/ENA pectinicornis fpirilinguis, alis patentibus flavis: fuperioribus fafciis duabus coloratioribus. Magnitudo Tabani majoris. Ale infe. riores fupra flavx, fuperiores flavx, fupra falciis duabus, quarum anterior prope bafin ala, pofterior ante apicem transveria ; fubtus fafcia pofterior omnes alas fecat; margo pofticus alarum itidem coloratior eft. Fafciz fupra alas in mare fu$f \mathrm{c} x$, in fómina coccinex. 
PHALFNA feticornis Jpirilinguis, alis deflexis: exterioribus albo-maculatis, inferioribus nigro fulvoque variis. Magnitudo media. Ale exteriores cinereo-fuliginofx, leu nigro albo cinereoque varix; inferiores nigro fulvoque varix: omnes ala fubtus lutex margine poftico \& fafcia media, nigrx.

RHAL住NA feticornis fpirilinguis', alis incumbentibus: fuperioribus cinereo-nebulofis maculis duabus ferrugineis. Magnitudo Tabani. Ale ferruginex. Corpus cinereum; in fingulx alx fuperioris medio ad marginem craffiorem macula dux ferruginex, quarum anterior minor rotunda; poftericr major didyma.

PHALANA nafuta nigra, alis fuperioribus lunula alba: linea media nigra. Tota parva nigra. Ala exterior prope maryinem interiorem, in medio alx lunila alba retrorfum fpectante quam lutiulam linea nigra transverfim fecat; hinc ex duabus lunulis quafi compolita.

RADIOLA (Flor. 256.) wifades af D. LEChe wiD Kallebeck.

SATYRIUM (Flor. 733.) Pfeudo-orchis Micbelii hade D. LeCHE funtit pa torta od) ffallota bacfar wio Kallebeck I mil ifrån Alingsâs.

PANICUM fpicis alternis remotis laxis, Hort.Cliff. 27. Gramen paniceum fica divila. Baub Pin.8. Gamme Doctor bave fumnit Denta wio Silen pá wåta afrer, befunnerligen wio Sianlofa od sund $i$ Ekane.

CRYST ALLER gioroe af Alun od) et of affa extraherat fal alcali, bwillax Ealt:Cryftaller woto til formen

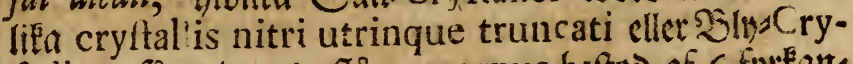
ftaller, fi at Deras afange corpus beftod of 6 fortants 
- tiga langa fioor cller plana, men árbarne moro trúncati stler twart iffitume med et fertantigt planum.

LECHE, M. D. fom tof fä wara liffom födo til hiftoriam naturalem, har tiffuienc meo of communicerat et frort antal af orter, foglar od) inlecter $i$ Gine, mes bmilfa ban fơtú te wẩ Faunam och Floram svecix. Syan funde wara i frâno, at wid Gymnafium i Gö-

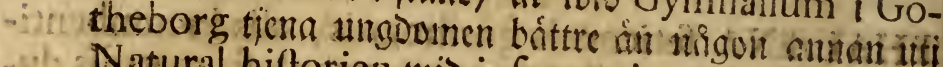
-nl. Natural hiftorien mid informations imerfet, Diriif

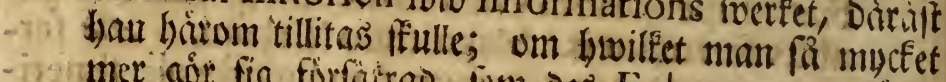

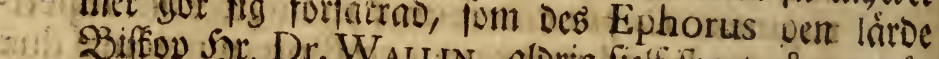
Wuffep Sar. Dr. WaLLiN, alorig fielfipart någon mús Da for whttige odf curieufe metemitapers upfomit i
Ditet.

\section{Jul. II.}

SENECIO foliis pinnatifidis fefflibus: lacinulis angulofis, fuperioribus latioribus, forum radiis difco brevioribus; Roy: liggd.164. Senecio foliis pinguibus pinnatis, pinnis alterne breviflinis, radiis perexiguis. Hall. Helv. -32 . Senecio hirfutus vifeidus graveolens. Dill elth. $347 . t .2\{8 \cdot f \cdot 336$. ki en liter ort, fom gllumant fans pis (siotheborgs ontor, befunnerligen pa fiuftion jemte börgent. Denne at ganffa liê meo Den allmuina Senecio 690: men har

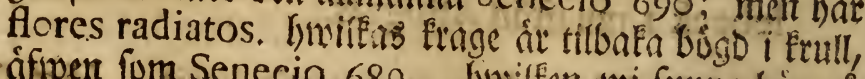

- âfiven fom Senecio 689, hwilten wi fumo băr wá refan Den 5, Julii; Doce aro Deffe twá finb emellom nog seffitiloe; tn Denne Götheborglke ar fiten, Den andre ftor. Denne ár offmeralt beftruod med fmí bar, fom utrila en feg faft; Den anore bar ic Senne bar pá fine pedunculis 2 à 3 blommor mid fitoan af fticleen; Den anderc wadrer in corymbum faftigiatum; Denure air lie Senecio minor 690, Dent ants Dre lifnar Jacobra vulgaris 688 . wh Deme ato Calycis fquamx inferiores laxa, spice fphacelatx, lon- 
gitudine fere calycis; men pa Dent allora áro defỉe fquamx infimx minimx \& arctx. Jå bàgge iro blommorna (ma, od) fragen, eller radius revolutus,

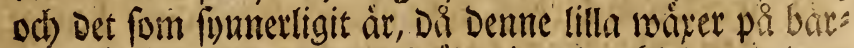
gett, är fon merendels beftrudo inumber bladen med en lepra, cller et boggult miol, bwilket àr falliunt iblans wivterna, om wi hos of undantage Rubum 4 II. Alchemillam 135 . Tragapogon 648 . Efter Debe twa fomma of foerens in corollis, odf ctro brigge befprings De mei et befonnerligit gult miól, dá be woita pá bogs Din ellee taf, od) efter Deme lilla blifmer pa bärgen bis: gie, lemnas at cfterfommanie Botanicis, at fiantfés Da, om Débe twa ăro narmare fins emellan liagt, än man allmant formodat.

NISSENS TRÁGARD låg utom ffabet, artigt pá Holländrka fattet anlago, med grafroar, broaruti rimnams De watten lop genom trigaroen, meo mafra bàctar af broit ${ }^{2}$ of eller Charme 786. famt med et litet oran. gerie.

HWITA BOKENS 786. blaber woro máft bîr i backars na iboprullade, eller meo fanterna inbogoe, fa at afs wiga foran blef runoad; men med fina uphogge nerver ganfea mndet frufad. GXi formodade, at A phides ihoprullat blaben; men ber fans icfe en enoa, ehu= ru wifúte Derefter; matt wifte el eller, om ftarta wars toefan Det fororfatat, cller um Det war et nutt mode af trufade blab. :-

SWANOR (Fain, 88.) lago uti wattugrakten frit = hwi ta, med fina langa balfar, fom på bufwubet bade jerns

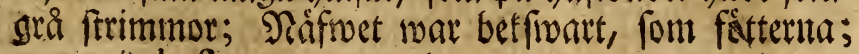
men wio bafin war det gult.

ANAS facie nuda papillofa (Faun. 98.) fans i Trigare Den til for nuceenbet; alle woro be bivita, alle fmá, alle lutitade fom molchus, at dese altra matte wava en minore varietet, oin ic fe nigot fontnerligit lpecies. 
BROTTFALLING läg wio wigct, under Det twi tefte ifrín forentimbe Trigaño it Carlebeck: Deme elàn

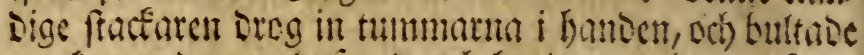

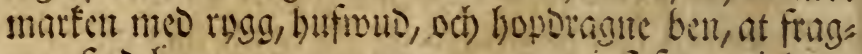
gan frod fonom om munten; mot juft fom wi toms no intil bonom, frante paroxyfmen, utur nigon pis fólanoe fonm, fom bet plignt fie i titt fallande fot, och han begunte tiggn, hmarof man forltod, at paffionen ei war riftig, web) beangte barot tiltah bonom, fom anoteligen mafie tiffte, feoan man befingit bonom $i$ talet, at ban fingerat Dema fiufoom, til at beweka wirt wh anoras nelibante; th ban wat en forfingen fari, fom utan paf froe setom landet.

KONGSGRAFWEN låg i fooft ifrin Carlebeck, uti en liten atteplats med uptittude fienar; bon war $2 \frac{r}{2}$

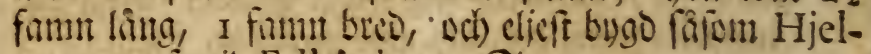
mars graf wio Falkóping. Ee pag. 81.

RUNSTENAR weto bir ittge, men Binderne beráttabe, at $i$ ofter ifrian Denta graf pis anora froan om cn fror

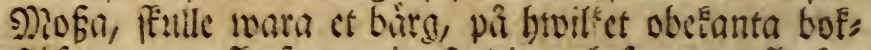
ftafmer woro ferefne; wi refte sit, och fumb 3 ferefne raser, fom frrifte fig wio pab ico:be alnar $i$ langectr; Soffiffwerne fisco intom linict, ofioan od) nedat, Guvilfa linice freifte fig lantgeit en flat od) fluttande bargseflippa; buar bofitaf nat 2 a 3 quarter lints,

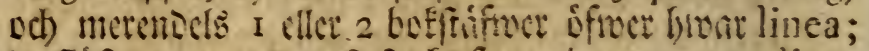
De flifte moto nllenaft ftref, fom gingo perpendiculairt ellet oblique till bigat ellet wànfter; gag â fis Eer, at seme Siunfen si warit lifen pá roo:De at, th toriven lig pa flera fraflen ifiver feriten til 2 à 3 fins gers tjoctleE. Sm Bemta ferift ef mitte twan $2 B$ ills

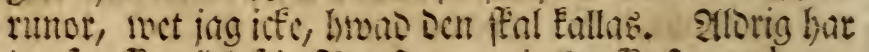
jas lett fa mioluftio Siunjert, ej eller fa frora characterer ferefne iften.

SILENE floribus erectis, petalis emarginatis, calycibus teretibus, foliis lanceolatis (Flor. $36 \%$ ) $\mathrm{ctr}$ litett 
liten wacer $\mathfrak{N e g l i e a , ~ f o m ~ a ̊ r ~ n o g ~ f o ́ l l f u n t ~ i ~ G w e r i g e , ~}$ ftod icfe allenaft pa Defa SiunElippor, utan oce på alla tringligganoe baig. Radix biennis. Caulis Ipithamrus, dichotomus. Folia oppofita, lanceolata, feffilia, glabra. Periantbium clavatum, decagonum. Petala s, obtufa, emarginata, alba; limbo bafi aucto duobus denticulis, ungue latiufculo. Stamina 10, alba. Styli 3.

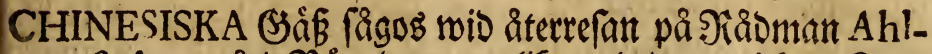

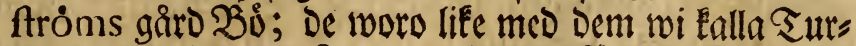

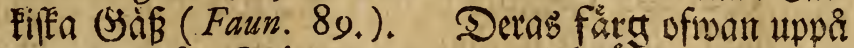
war jerngra. Stjertpentaturte od) bróftet grånftige; matgen och omfring ftierten broite, of wan pa balfen nagot brumaftige (fubgrifea); men halfen pa fiborna hiwitaftig; forterne blekgule; naffoct beffivart med en ftor fivart flunf wid nafruets bafin i pannan, lswils fer war i proportion fturte, än pi de Surtife; Gals fen war ganféa ling, och) proportionerad emot eat Givanthals; Palearia eller fammarne under hafant kunde nàppeligen märkas; Defe Ssäfens låte war olita meo de Surfiléta.

AMURGRÅS kallabé AIRA, qux Gramen marix borulforum 70 , Den wi fago wara biumtad af Bondepis gorna, oct fálias i Gótheborg i fina quaftor, fờ I ore fi:mt fisctet, at binga up i hufen, forr bergagelig luft, eller ocf fúr banoqwaftar, at lufta pi.

KOPPER berittades of J aldrig marit feode pa infula fanctx Helenx; men Dentre Śens Snbuggare få Dem, fom andre månuiftor, fa fitart be Fomma pa anora orter, Groaraft fioppet áto gângle.

FÅREN bade fơr nagon tio feoan baft utflag pa lroppen, Det be Erllade Foppor; men at elt, fom om winteren gifwit fina Fara Cardebenedict aloeles præferverat Dem for en fadon fiufa, faft bant Far sabt i bet mes andra fmittade Farar. 


\section{Jul, I2.}

9 Dag lemmade Sacre Directeur SALGREN of fin fum, af fegla ult at hafB̈wifen til mua od) gamla hwarfwet, quart 2, at fe, braräif Eeeppen hammade, meo meta.

CAPRIFOLIUM germanicum (Flor. 19r.) twajte twio

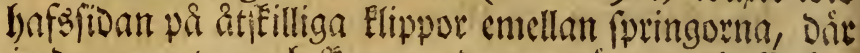
jurden war tore weh featper; Deme warte eller buffe has De jag ailenaft tilfúrente fett $i$ Swerige wid Siamlófa Surbun i @esane; ban meriterar upmareffambet, haiff sa mam fer Gonom tila ivara wintrar; ty ingen

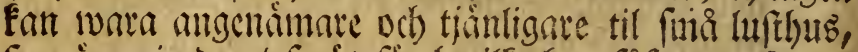

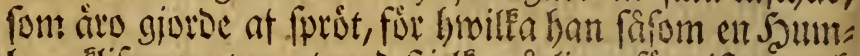
Ie upelifwer, utan at med frislen artigen formisua, och fáledes bela bufet betäcfer med fita mactra blader,

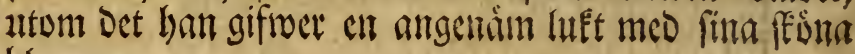
blommor.

SEDUM rupeftre (Flor. 388.) hade iag allenaft feot phis

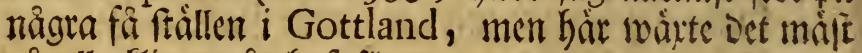
par alla flippor ît hafsition.

SILENE 367 , fom i gire omtaltes, waxte på alla llippor.

ORMAR fumos un entellan De brantefta flippor, of Gyile

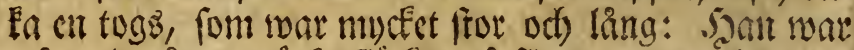

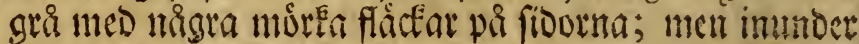
brofig at broitt och fiwart; bans fcuta abdominalia eller twoirtifiall cmellan buffubdet wech) afgängen woro $\mathrm{I}_{73}$ til antalet; men frierten .war imutoer betift meo 63 war fiäll. Jua brigge fioor on halfen war ban hiwit, oth i mumen hase ban ingen for eller giftig trond, fon Eunse inbsjas fom en Eattello; howaraf man fífert tuns De futn, at ban war en NATRIX 259, eller Tomtorm.

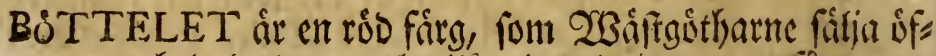
wer hela landect, om buvilfen iag alorig rummat fia genons

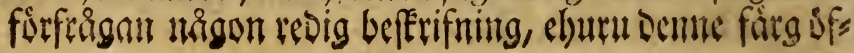




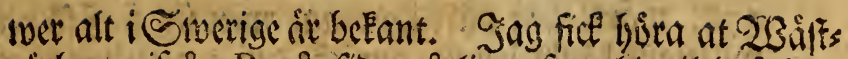
gottherte ifrat Borås fidan arligen fara bit til hafselips porna, men befumerligen til Hiffingen, fom pa nor: ra fidan fruter hit in , hwarát de famla en \$loba, af hwilten De federmera fórfäroiga fin Bubttelet : YBio tilfuigan wifte hroat man at wifa of Demna fargemo os fai, fom bar tointe bfroer alla flippor, oct wat LICHEN leprofus candidus, fcutellis fufcis : marsine albo; (Flor. 942.) en No Storige ei à fiunnerlig allmân, stminftone batiag mig icfe bekant, at nagon utomlanos toetat anwänoa

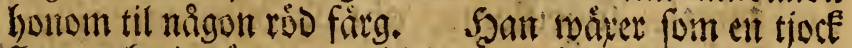
ferpa, hwitgrion, utan blader; Dés particlar áro ofs lange edf trubbige; man finner fállan dés Scutellas, fom aro muctet wior, flata, sulaltiga, mes ent bivit twubbig Eant. Mooban afferavas horfe efter fuftigt was Der, turtas och mules funter, ligges i ivatten, ontos: les, at all joro od grus ma fiunte, torkas, lägges $i$ en futúa, begiutes med urin, ftar fáleoes 4 à 5 wees for. Meo vetta fárgas feocuneta ród gautr. Shatuf fulier, at Sitteleten blifmer Den famma, form beferifs wito af Sert Kalm Babuf: 145 .

JETTEGRYTOR fagos et par ej langt ifrain brourfwet, In $i$ bargs tlipporna, fnapt et par buseffott ifran hmars andra: De woro baler fwarfroade neo i barget, fafom enCylinder, af en almb Diup, och liEa breod; of Defia grutor war ben ena pa fioan af barget, Dár Det ni̊got litet fluttabe, men balan bolt itg perpendiculairt, utan affígt pá fluttringen.

ELFSBORGS FÁSTNING låg I quart ifrin lanbet pa en liten bolme, naifan midt $i$ froalget af ingangen at Gótheborg, wio paf 3 quart iftan ftaven. Det

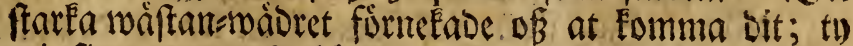

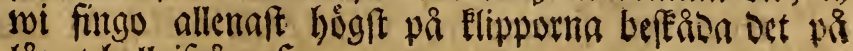
längt boll ifrån ok.

SPERGULA foliis oppofitis, pedunculis fimplicibus if 2 378, 
378. wåkte hár på Elipporna, men fig fielf fâ olie, at wi Enapt Eăndt henne, on icfe Dés 5 piftiller rógt bents ne; tis bladen noro breoe, lancett-liee, fticlEarne beit rafe, och) Eronbladen ei frürre, än fielfió blomfooret.

BILLINGEN war en liten bolme, meo ct Batterie, fom

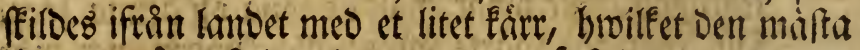
tiDen om aret ftod under matten, faft Det nu war uts torkadt.

ARENARIA maritima 376. $\beta$. ivåkte i Detta uttorlade Firre śfiwerflodigt; fitelffarne noro frupande; Folia fubteretia, fucculenta, obtufa cum acumine. Stipulx oppofitx, membranacex, breviffimx, latiflimx, acutz, infra folia coadunatx f. unitx. $\mathrm{Ca}$ lyces monophylli, quinquepartiti, pilis adfperfi, puncto fulco ad finum calycis. Flores fub florefcentia erecti, poft florefcentiam penduli, \& ma. turo femine iterum erecti.

CHAMÆMELUM maritimam waikte ummigt pa haf fs ftranden, Det jag ej tiffurence examinerat. Caules ramofi, diffufi, angulati, inferne purpurei. Folie duplicato-pinnata, vel multifida, glabra, fubcarnola, obtufiufcula, fupra convexa, fubtus carinata, margine reflexo marginata. Pedunculi fulcati, uniflori, Flores Leucanthemi, Radio albo, ampliffimo: petalis tridentatis; difco convexo, Glavo; Squamis calycinis margine membranaceo fufco cinctis. Semina Radii triangularia, tridentata; Semina Difci tetragona, quadridentata. Receptaculum henifphrricum, aut conicum, nudum. 2lttfâ air förft Denne et fpecies Matricaria, fom nầmaft fommer śfiverenb meo Chamæmelum vulgare 702 , faffän DeÉ caly nog liénar Cotulx fcetidx. Gag fentle off fannerligen tro, at Denna suten woro den famme mes CHAMEMELUM maritimum perenne humilius, foliis brevibus craffis obfcure virentibus, Raj. Jin. 3.p. 186, t. 7.f. 1. om allenaft petala radii maxit odelfe. 


\section{CUCUBALUS maritimus $360, \beta$, wâkte på Billingen.}

GAMLA HWARFWET lig pa foora fidin om miletr, utan fór Saga och) 刃afthugget (en liten fơrftad), I quart ifrån ftaden; Sa ar war Ammiralitets fécppgs buggetiet, hywaráft man fảg pả Ammiralitets gârcen

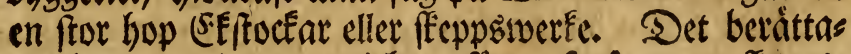
Des hemligen, iag wet ei Giwarfore, af nagon, at feepps: werket eller focfarne moro nuycfet fonderf restme af mafe, fom pa Dem giont fror fFada. Sag wardt picthogad at weta lywa maffar Detta giort, och hwad ffaban

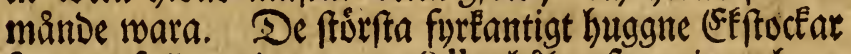
funnos fulle med tranverfelle hal, fom gingo hum orabetalb in $i$ frocten, od máft pâ fitoorna af ftocten;

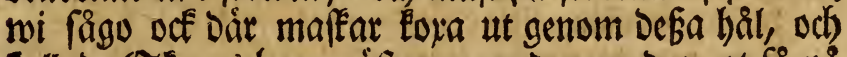

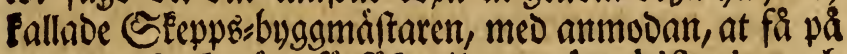
en enoa ftoce gưra forrfóe, til at utróna hiftorien och egenffapen af De maffar, fom gjorde all Denna féaban, på Det något medel emot fá féabeliga letáe mâtte kumna utfótás; jag wifade min Inftruction och mit Promotorial, men alla fóreftíllningar dalttade, ficte man Doct

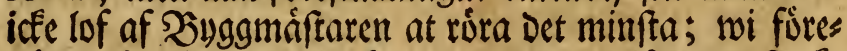
ftálde, brwad nutta Det Eunoe wara, at wigga en ftocf, nár famffé Dárigenom många roo:De fumbe conferveras; men Sleppasbuggaren lát fóta til protocolls, at ban hấlbre fäge mid mónftringen, at 100:De ftucfar

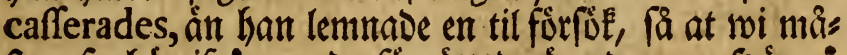
fte refa hăr ifrản med ofürrâtttade årender. Şấ pa gingo mi til Niva Sawarfivet, Dár mi rảáade et par ars tiga Sherrar af Ammiralitet, fom förfäfrerade fig fóda

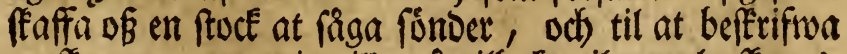
maffarna, om wi mille gå tilba brwarfivet. WBi Eommo tillbafa , od) med ftor âtra examinerade maffiftungen; th wi woro fätre, om mi allenaft fommo if fanto, at utróna maffenz art od) nas tur, wi bå látteligen ffutule Eumna profervera Denna olágenthet, hrwarigenom wi med et enda rón funde gas gna Publicum til många rooo:De Silezblr; ta) hrwad feada mátte idfe Det wara, at fabane Efeftoctar, fom 


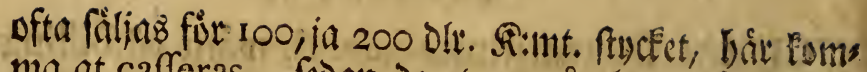
ma at cafferas, fedan De et par ar legat pai gároen.

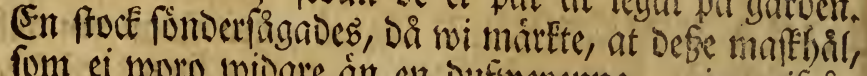
fom ei noro midare än ch bufinepenta, gingo iftin btan in i focfen, ofta til innerfita finmant tweet fure, ods) at De woro tomme, $T a b .2$. f. r. eneellan A. B. $233 i$

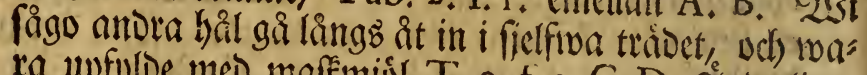
ra upfulde meo maffmiól, T. 2. f. I. C. D. Siltoteligent

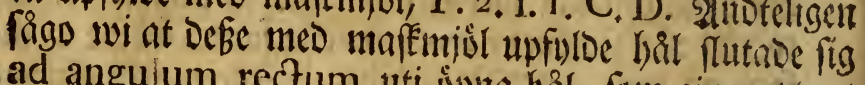
ad anguium rectum uti spina bal, foin gingo troert

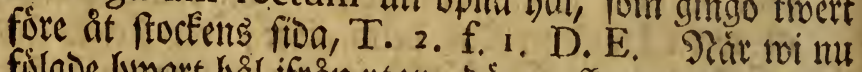

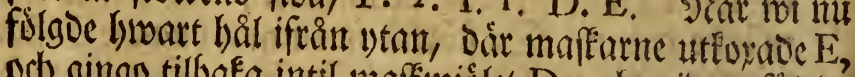
od) gingo tillbaka intil naffemislet $D$, ods midare fülgoe

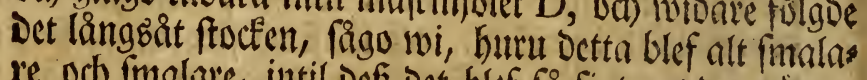
re odh fmalare, intill Dec Det blef fo fint, at Det noipyes ligen med blotta ugon funde mâreas C, lywarnio als

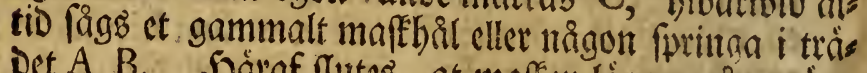

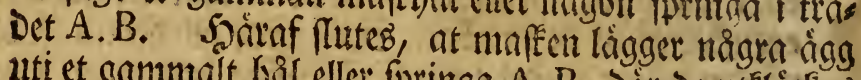

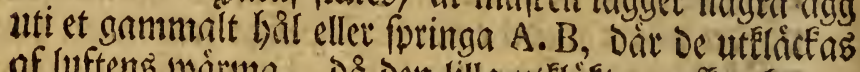
af luftens wairma, ba den lilla utElifte mafeer frupes mer eller mindre in at ftocfen, feberntera ater odj ars betar fig fram läng effer ftocfen i râtt linea C. D, ocl) fom han imedlertid warker, oct) blifwer frórre, frìter han

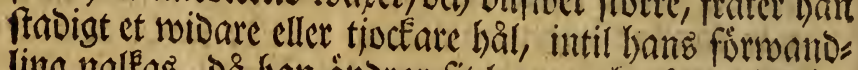

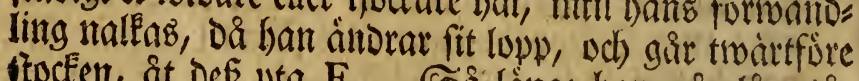
ftocken, at be ftocfen, fuller han ballet efter fig, men da han wa flutet sår tweetffire uttat, lemmat han ct opet hal, at han efs

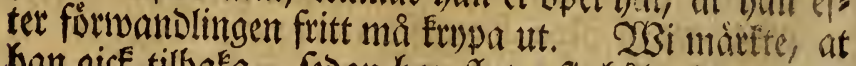
han gice tilbafa, feona han flutat fit hal wio ytan $\mathrm{E}$, til begunnelfen af tweerbilen D, Dár han lave fig, ods

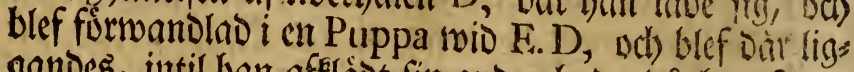
gandes, intil han affladot fint andra bub, da han feocrs

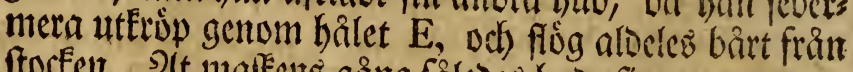

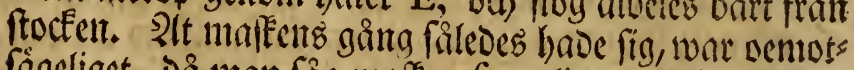

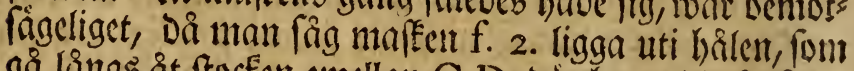

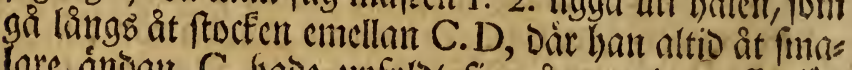
lare ánoan $C$ bade upfulot fin gaing med mafemió, 


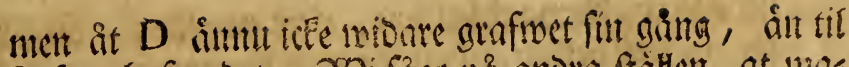

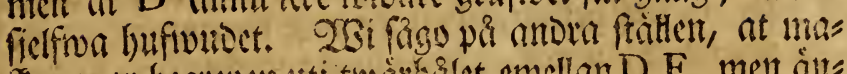
fen mar begrepen uti twoirhallet emellan D. E, men äs nu ej fa langt bumit, at ban Eunnat spma ytterfta fis Dan; Da wi figo lonom luta twärbálet med en open

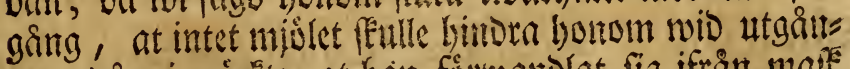
gen, Dat wi maitte, at hint furmantolat fig ifrain male

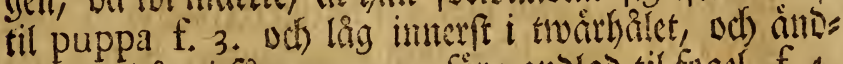
teligen, di wi figo puppan formandala til fogel, $\mathrm{f} .4$,

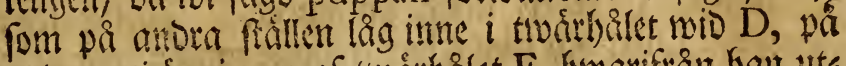
anora uti opningen af twoithalet $\mathrm{E}$, Gwariftan hat uts Erop, w(t) andetefigen flog, juft wio Denna tioen wi bets ta underfofte. Gedon maftarme uttommit, fiuga be od) parns, fafom andore infecter; Den hafivande Sos: narts enoafte traftan àt bos alla inlecter, at lígga ft: na fró pá fỉdana frâllen, binareft de uttilâte til ungat frav Eumnta fá fin fosa; altfí ligger Detta inlectet fit

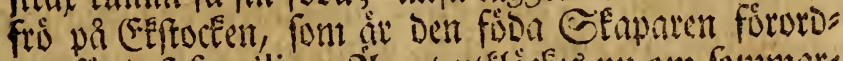
nat for dec familie. Ílgget utflickes mu om fommars tioen of luftens warma; Den nus utEläte lilla mafeen, fom ât fin, foin Den minftrighet, Exyper uti hålighete"

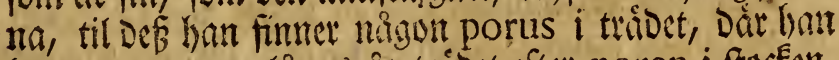
begunnet snaga lang: at tribet efter poren i ftocken, od) fradigt fisller gangent med Det mafemiol, fom ma" ffen nfgnaget. Jja dettr fättet arbetar ban längs ait ftocen, waiker til, gove efter fiu proportion alt wibas

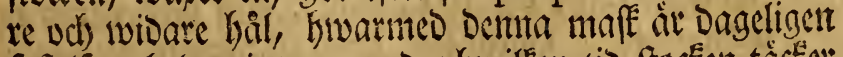

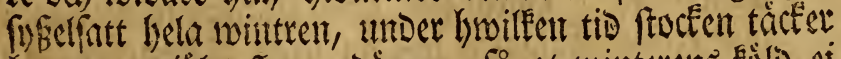
bonom, mislet flutar Dơrren, fa at winterenb Eúlo ef lint fabon honoun; andteligen of bet lider til fommaren, od) mafen blifwit fullwuren, frentar ban, cfter ber honom of Efaparen foreffuifne lag, at lingre ga langä: ît, litan cimburit fit lopp twert for frocten, at oen mäls mate fibass, pa bet ban ma Eomnta nit, od) feoan ban

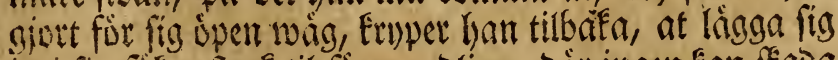
in i fin follu froce til forwanding, bair ingen fan feroa

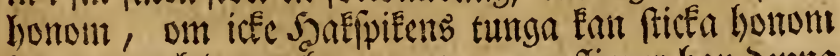
igenom, och stagn bonom ut; men lipper ban semma 政 4 
fin olucfa, fortuandas han, efter ett måntobs lywila uti fin puppæ geltalt, til et flugambe freatur, fedan far ban ut, finger, paraz, lágger fina ágg efter fina fübers mas ner, od) fom ailla andera infecter ftrar Dárefter oúr, Då ággen multiplicera $\mathrm{d} \mathfrak{c}$ ) confervera familien.

SKRÅPUKEN tab. 2. f. 2. (Larva) eller Denne Sleppss

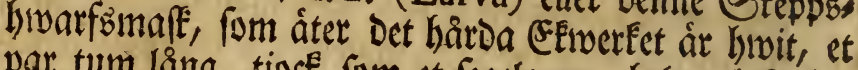
par tum lang, tjoce fom ct fegelgarts, helt mije, bes

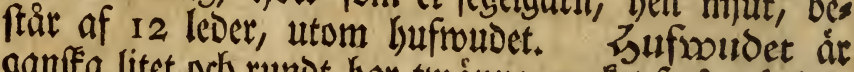

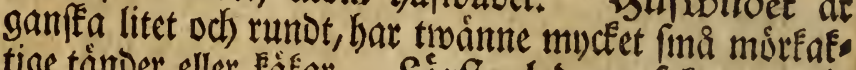
tige tämber eller káfar. fortfa ledent af Eroppen ár Den tiocfafte, ofivan til trubbig odf) uphosgo, at bufiwus

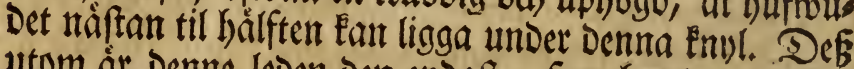
utom ár Denme leden Den endafte, fom har Den tiocfles fen, at han kan upfislla hela halet. De 3:Dic of 4 t:De lederne áro de fártafte; mett de andre áro fins emellan liêa länga. 3 par fótter fitta under de fürfta lederna af Eropyen ganléa farte, fram àt bógoe, meo fma hå fürfedoe, utan fintliga ellor. Gejertent eller ytterfta leden pa froppen ar trubbig med en tjock framifảende Enyl imunder, bivilfen fnyl ár nigot litet flyfo, och) alts fâ fielfiwa anus. Şáraf fes med fórumbran, buru et

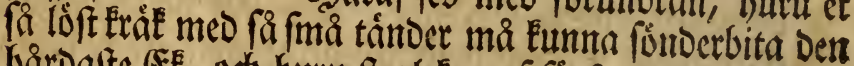

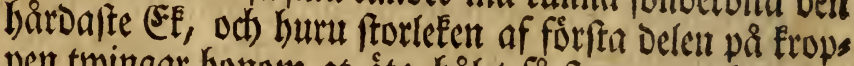
pen twingar honom at ata balet fa ftort, at han Ean tomma fram, Det âr fa niot, fom Slaparen weiat

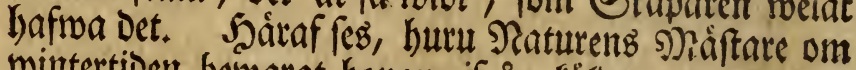

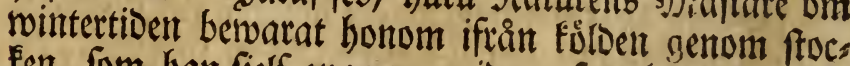
fen, fom han fielf gnager; widare feb, butu ban icfe âter alt Det miól, utan laftar fäbarma tilbaka; och ános teligen, huru en fâ ufel mafe Ean góra årligen til mån ga tufetto D:Irs ffáa.

PUPPAN tab. 2. fig. 3. (Propolis) blifwer, di mafeen boporager fig, och mifter fin sttta had; Denna ár neds trufter, afláng, funct mot ánbarna, gulaftig, på rugs: gen morte, od) utan fótter orórlig. 
SKEPPSHWARFS-FLUGANGlifwoc of fơremåmoc puppa, Då bon afflidot fin buo, och ât en CANTHARIS ferruginea thorace rotundato, elytrorum margine exteriore apiceque nigricante. Den ne Cantharis år fâ lâng, fom en ftugeffuga; ment mucfet (mal od) rundad. Zufwowbet är helt froart, áfwen fom sgonten, men Den lilla munnen á̀ nagot brun. Antenne ellet bornen áro Enapt balfparten fá lange, fom froppen, fơroelte $i$ io lántar, af hwilla Den ytterfta ầ ovalt afiang. Saela Zroppen med

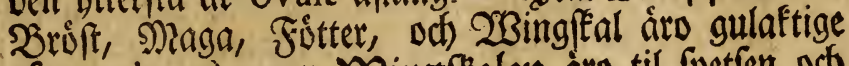
(ferruginex), men Dointfiealen aro til fpetfen od) meo yttra fioan langb at froarte. De underliggande Dingar áro belt od bållne morrfe. Mlageit ẩ guls $\mathfrak{a}^{\xi}$ tig (pallide ferrugineum), mett ofwan, eller bair wingarme betåcéa, ár magen mơrê. Sơtterne áro 6 . Zroffet har inumber, emellan Det andra och treoje pas ret af fotterna, en flat, ftor, något Fullrig lamina, eller broufben. Etjerten fan utoragas til cul tumb längo, fafom et perfpectiv, meo Den ena Cylindren imnom Den anora, fa at da benna fluga féal lágga fima ágg, hon då må fumma fticka ftierten långt in $i$ et mafes hall eller fpringa pa trábet. Son bar ingen tagg $i$ ftiers

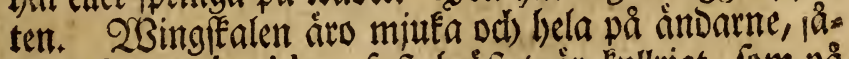
fom pa Cantharides, faft bróftet âr fullrigt, fom på en Leptura. Soúrat făr man efter en tyoelig befferifs ning pa infectet, et Elart lius uti alt buad tifforene on Demta warit i twifwelsmål. Sáraf fölier, at Dens ne maffen bar ingen fligt eller befantiffap meo Szollänos ffa Siómaften (Faun. 1 329.), font inficierar ffeppen eller ftocfarna uti fielfiwa watnet; ei eller ár Denna maffen flágt mes Det Erälet, fom upáter gamla tríbus (Faun. 366.); utan ár Denne maffen belt min, od) fá wis Da mig witterligit, alorig tilfưrene nirmo eller befferefs twen, faftăn FrISCH i I 3 :De Delen af Tyffa infecterna p. 24. f, 20. afritat et infect, fom mucfet lifmat Detta. Det war mucfen twift bák pá feepps=hivarfiwet, om maf́tartie ftungit trádet, medan Det ftått $\mathcal{}$ á fin S 5 
fiam och wirt? ellet om ie gencrerats i thitiet, les Don ict begynt antaga forfta graden af forrutnefe od) uggiemed? eller om traven blifroit feimse of malers: lia bie pa broarfioet? cller om De, fom firit traien, afs Iemuat maffungen meo frocfen? cller om malfarne les gat i trádect, feian det wuret? Rlle Defe trirwelemial uplofa fig lieifwa, feoan man bat fig foregácmo hiftoria om mofeens metamorphofi eller fortunneling och inatur flart bofont. SMan Ean pofitive fuia, at alla se maffar, fom mu funtos i frocten, eller $i$ ar utfoms met, blifivit forlesit ar lagoe igenom fron, fâ at frocs

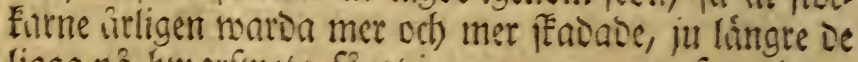
ligga pa hwarfwet; fa at inge genererats af ugglewe= Den. Débutan hase Den fom Épt foctearna borot fe pâ afóarfase froctell, om han roarit mucfet mafeftum: gent, bwaraf han fumat fluta, bum ban bade fig ins uti; ty for hroarie fioos hall, mâfte noofuleligen warit

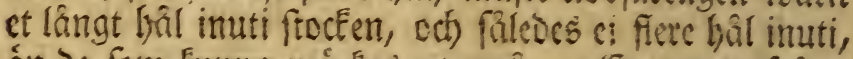
ám be fom Fumna márên utampa. En antror frảga toar, om en Eeftoce, fom af Defe maitar wat genom: bárad, fumbe nntoanons e. g. til Eól uti et ffepp $2 c$ ? Byggmaftaren bolt fore, at cr mafefungen froce feulie Draga matnet til fig, och) falleses taga rita. Det ár fuller ej utan gruno, at en maiffrungen ftocf ean fupa watnet, Doce forbinorns Det mycfet genom et aftramt

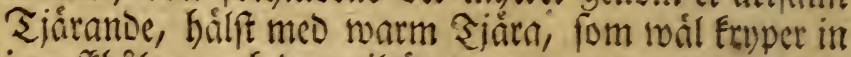
i maffeclent, oct) Dem tiltipper, utom Det, at Den lufs ten, fom ár imfluten i porerna, fielf ftâr emot roatnct, fom en Dyfarestlacfa; Doct fommer Det måfita an pá mucfenlyeten of halen; ty fupes soatmet in pa flera ftals len, fa tager Det haftigare rota, an om Det pa fâ ftits Ien blifwer Dafiwigt. Nen at rúra Det angelígnafte,

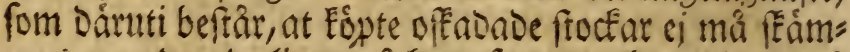
maś, medan de ligga pa frwarfivet, och) alt intil DeE de warda bruḱtade, fa fommer nit Derpa an, nt Defe

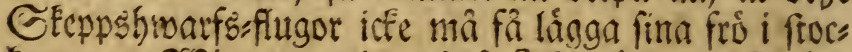
farna. $2 B i$ wete at intet infect fam ía fig, fá lint

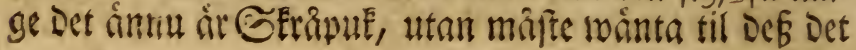
unders 
imbergått fitt fiffa forrwantoling; od) parats; Gworaf fútjer, at maffarme hát i ftoctarna ci Eunna multiplicera fig, untan måfte forft blifwa fingonde, fes

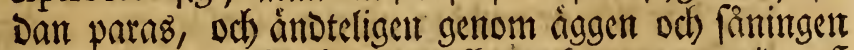
blifiua flere. Trsi fe, at mafferme fomma ut twio pas

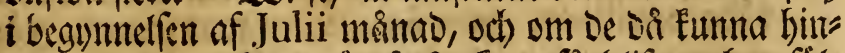
Drab, at lä̉gga fina tró pá froctert, fá blifwer han fóls

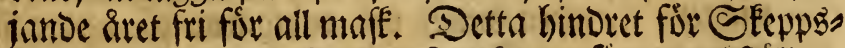
bwort

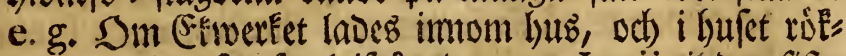
tes med litet fimafivel ifrän Den 16. Junii tii Den firta Julii, fétlle flugorna Del's os af fruafweltrófen, Dels hafwa affíi), at lägga fina fró pá de rófta ftoctar; men fom Detta torbe falla något be froúrligit, feulle jag rảda at ftocfarna allenaft midt $i$ Junii nånad sfrucroroges med en tunn Tiâra, fưr hwwiffen alla infecter hafwa

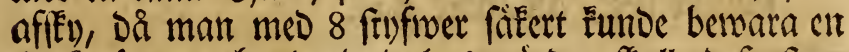
ftoef af et par hunorade Dalers wäroe; ffulle Defe ftoc: far allemaft ligga under take, Eunde De i flera ar, en ginng tiấrnoe, wara frie ifrån all mafḱ, utan at artligen ater beftrulab: tus fá lánge tiáran warade, fâ länge woro De oce fáfert frie. 2undtcligen funde ocf frocfarm bes waras ifrán Depe flugor, om de aldeles neofintetes $\mathfrak{i}$ watnet fa Djupt;, at icfe Den sofee fiban fär hojia fig up

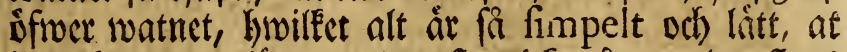
intet Eam wara láttare, allenaft at icfe nâgre andre flagb fiómafiear fomma at infinma fig, of set froctarne labes

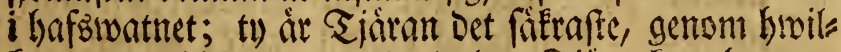
fen man altio med roo:de Dalers Siñra fan berwara

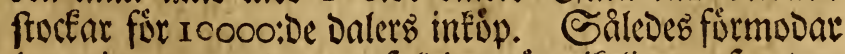
jag mig, genom Detta enfaldiga rün riecligen erfatt Dent omeáftnas, fom blifwit gioro pá refan, och at nuttan für Fádermeslandet blifwer ftor, Då betta ammindes

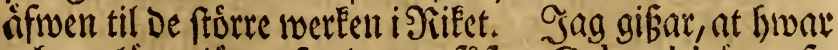
och en lârer fơrut fett Detta, fáfom Columbi ágg, fos Dan jag Det fatt i liufet, elsurtu ingen Det mairfte, Dis vet ànu låg i mustret. Så fan en liten fluga gưra for

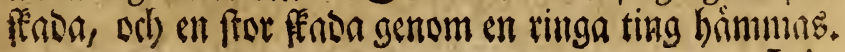


LARUS maximus elier en af be ftorfta fiffmeifar, peots

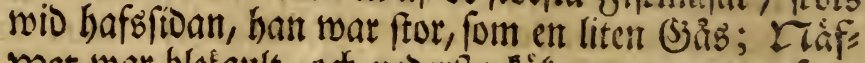
tooet war blef gult, och nederita fáferen soar utenot fpets fert, Dár Den àr trubbia, aldeles bloofárgad. Égon" lecken woro rund onetring pá fanten bare, meo bloos: rúda papillier; men ógonftenen war fivart; fortters ne woro blefe; hufwudet, ballen, ftijerten, broffeter magen, fitertpennorne, Def) swingarne pá unore fitoan woro bxoita. Sxoattaktige woro riggen, wingars ne ofiwan uppa ffrifpennornc, cller Remiges i wingen, byilfa fenare doce bade bivita fipetfar.

THEE - KOPPAR ifrån Limmare glabbruk, fägos giors De af himitt glas, fâ artigt, at be iife allenta jt táflade meo De Chinefíka til formen, utan ivioa offuergint go Dem i finket, uthárbade bett watten, och blefwo mindre hete, ân andre glas.

OSTINDISKA COMPAGNIET Off Des Shandel omtate tes myset; men fom fảdant ei hourde of til, lemma wi alt Detta, ei eller angare Det of, Givilfendera af Dem, ans timgen de, fom refa utomlanos at furefofra fig $i$ wettene féaper, eller de fom fara pa Spltindien, mer gagna els Ier IFaDa Siifect; Docf war artiat at fe, huru Det nuscena filfret, fom meo fa många rooo:de mánnifeors frectt utpinades utur Silfwergrufworna i Peru, lángft fós Der $i$ America, meo muden wisia transporteras åt Europa, Dár De máft cultiverade, ock fórmuftigafte nationer, fom Dáraf blifwit ágare, genum Det be fig bemágtigat Deras lano, Det famma til en annor werlo $i$ Dftinoien meo ftétre móoa od fara affíra ifrán Europa til oftuderade nationer, och) Dar buta bairt Det fór några blader af en bufke, fom lâtteligen Eunde wo ws ra hos of fieffiwa, om man allenaft féstte om, at för feaffa friféa frón; Depe blader brufa wi at oricfa in infulo, fom fielfrua âga 100:De flags bade fimakeligas re och batlofammare waikter; och ent Del af fiffret, fom

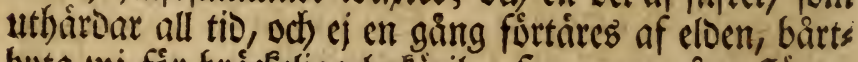
byta toi fồ brácefeliga lertáril, fom, en gàng Nhapte, alorig 
albrig fta at laga. Si fí commenderar wib̆heten penningen, od) modet wishleten.

$$
\text { Jul. } 13 \text {. }
$$

SURBRUNNEN låg utanfưt Go̊theborg, på wåftra fis Dan jemte wallarna; watnet waur ftartet af mineral, nêftan fom mio Medevi eller Fahlun; månge fiufe Dructo Detta Denna tiden med ftor lifa.

QWINFOLKEN brufte allmaint Káa $i$ ftaber, at Eafta ct fwart tug, Hlábe eller Eåpa sfiwer fig, Da be gingo ut pá gatorta, áfwen fom det brufás i Tylfa ftäberna.

RESAN frílocs efter 2iftonfängen ifrain Gótheborg it Bohus.

SALTKÅLLAN låg übe på en foblånot áng, 40 fteg ifrrån SBotha Eif, emellan ftaden ded Siotha Sejon. $223 i$ Imaknde Des falta wouttert. Soon. war grafwen uti en

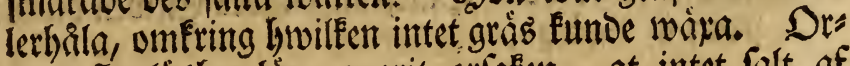
tens féoglissbet lâner warit orfaten, at intet falt af benne fotats.

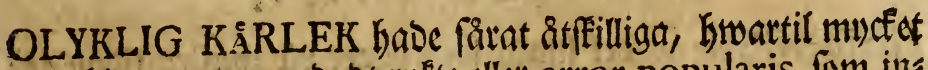
biorog et ogrumbabt rylfte eller error popularis, fom itr gen god utfipriot, naimligen at Den oluctade Ean blifwa fri, om han fár fimitta en amnan. En enda fioma Ean med Dema elo bränna och fóroerfioa humbrade i fin

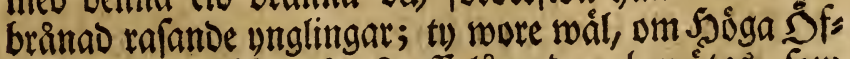
werheten med harbafte freaff lato Dem bemutas, fom âfroen pá Detta fâttet feadad en annor, at idfe fá wos Derftuggeligit ogriiz alt four mycetet finge vota fig til Nationens fíada.

HOSPIT ALET, fom fódoe 78 olydfl.perfoner, lagg inio wais

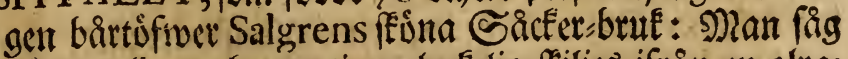

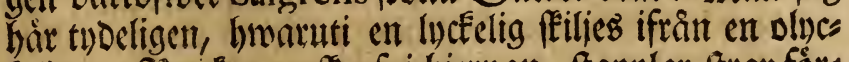
Eelig. Siuntar en feruf $i$ bjernan, ftapplar ftrax fors" nuftet; lucfellig Den, fom lärot täné lifa meo de máfe. 


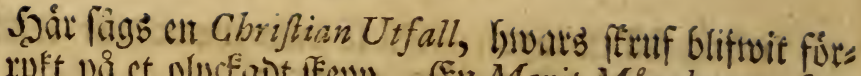
ry) ft pia ct oluctirot fepp. En Marit Minsdotter, fom för giftermill i 2 ât warit melancholink. En Ceder-

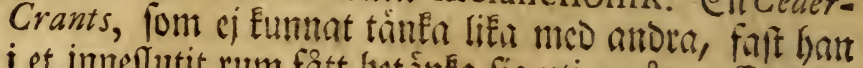
$i$ et inneflutit rum fâtt betsinfa fig uti 32 å. En Ma. giffer Winterkorn, fon gerna welat bafiva bros, ment e) fottet, od) altia mift bágge, brvilfet ban i 23 lit be: Elagat; Denne bave forgàtit werloen, mer in fin Latin, blvilfen ban talabe, fom en Tullii Sáriunge. En Eric Engelbrektfon, hade fom en Siger ofwer 20 air legat naten, imterluten $i$ en Frubba pa trágaller, war boit Eittfull, od) ftart; faft ban $i$ fa manga ât warit utan motion.

GóTHA ELF fólgbes prífitra fioan til $4 \frac{x}{2}$ quarts lóngo, alt intil शrubron. Man fag fmá frumbátar fara faes ta up och neD fort Denna (EIf meD goDs odh) jern.

NYBRON gicf ifwer (Jijtlya EIf, Dair hon war fmalaf, ef ler 250 fteg breo. Sarrifrän fólgoes Elfwen par wailtra fidan at Bohus.

\section{BOHUS-LÅN}

HISSINGEN ât Det lanto, fom ligger på lvâfre fiban ont Demra (sibtla (sif, ocf) ffiljes ifran Det ansra of Bohus sein, mebelfe en anman gren, fom ifran Bohus

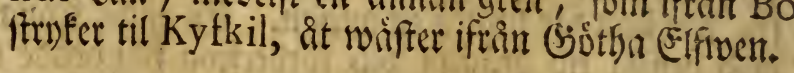

GóTHA ELF war pi omfe fitoor bepruso meo ingar, och Debe angar woto utan til intängde of hóga lluttande bargsflippor, äfiven fom ingângen ifrän bafiwet at Gotheborg, fi at ingen twitivel à, meo mintore be gamle (Sisther fatt feglat vd fifent, Dart fion mu fprins ger für Siromen, od) Dir Dren puytar unoer ofet.

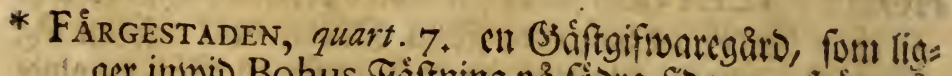

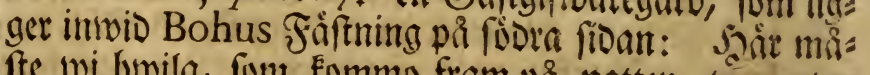
fte wi brila, fom fommo fram pa natten, ti) porten wio Fäfningen watiluten. 


\section{Julii I4.}

BOHUS FASTNING clle Glott ligg pa en liten bolme,

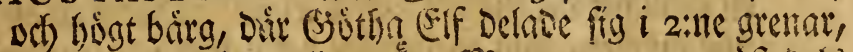

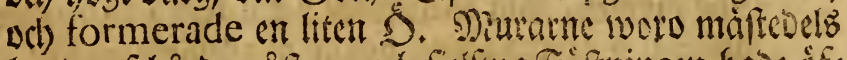

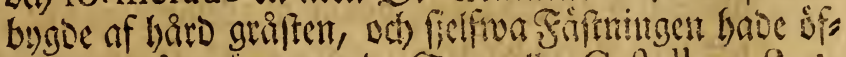
werfit uppa twäne rumba soun etler Cafteller, far's 3.ate vel) Mors Hry

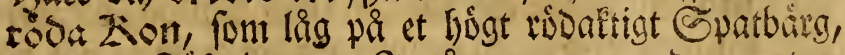
nort om Fesftningen. Gngängen war med 7 pertar,

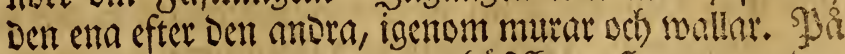
Den innerfa porten war en baffifo uplagen, mo 8 fóm, fom fajess fallit ifrân băften, dả Drotning CHRIsTiNa furlle refa up i Detta branta Glottet. SMlot pia

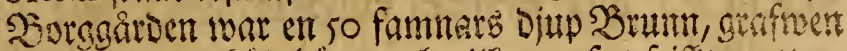

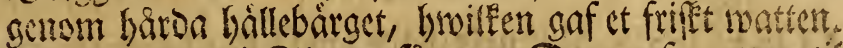

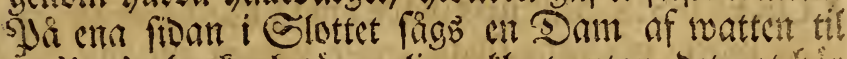
ordinairt bruf oci) timmeligen flart, fummos mainga MONOCULI conchaformes (Faun.

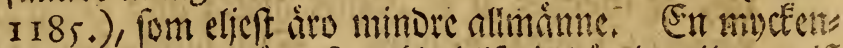
bet Ogerningbman, fom hit blifinit Dönoe til en wi eller erwig tid, angrabe gair at be fá litet wetat warba fit forthn fribet.

STALACTITES calcareus natrofus baingoe fom is:

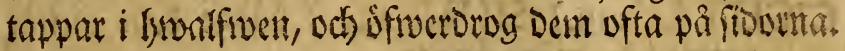
LICHEN fulvus $(96 \%$ ) ofli BYSSUS flava giorie de fiobra Elippor umoer Fáftningen gula.

BALLOTA (484) fom clief ar fälfisnt, wajte ometing Slottct.

MALVA major ( $58 \mathrm{r}$.) fom buteas, eller ifminftone bors

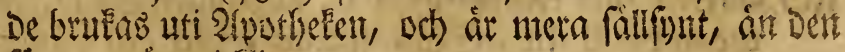
forre, loantre i tlipparta.

CONIUM (226), fom fomlige Botanici hollit fore suara

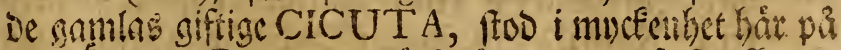

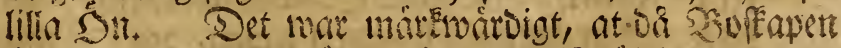

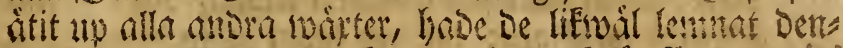

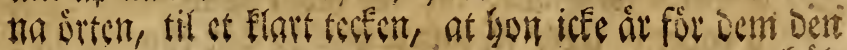


Gallofanmafte; bănaf fluter matt, at be icfe fara wils fe, fom bolla fore at hon ár giftig.

- ERYSIMUM foliis integris lanceolatis (Flor. 55..) ate

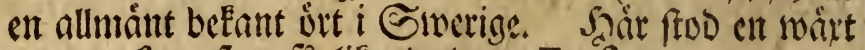
nog mucfen, fom fa lifnade Detta Eryfinum, at man fivarligen funse fäga, om bon allenaft war en varietet med ftorre blomma, eller oce et diftinct lpecies; ty) Folia tworo lanceolata, acuminata, parum fcabra, obfolete dentata, naftan fom ph Hefperis eller Cheiranthus. SBlommorma moro gule, odi) på foms lige ftanto belt fmâ, pa anorn medelmaittige och pá ans Draftore. Gefioorme woro furfantige.

SUBULARIA ( 527$)$, ent mucfet liten oanfenlig ort, waits te pâ frtandelt.

SENECIO, qux Jacobæa vulgaris (688.), wat hấ alls matr.

COREOPSIS bidentis bave ing fâ niar Eallat en ort, fom wairte pa sftra froan jemte waigen emellan Firgeftas Den od) Färinningen, iblano Callam, om ing icte wes tat, at fpecies af at genus albrig afla of fig genom varieteter anora genera. Denl fimife HALLER har uptifft fór Den lâroa werloen, at Denna ort ât en Dots ter af Bidens (664), od) Denna ürten lärer alla Botanifter, buru nå Coreopfis od) Bidens mandoe was ra llágt.

CICUTA (239.) SIUM (235.) baigge Defe giftige ortee wâkte $i$ De waita ängar.

KASSEWIA, Poa maxima (73.) witete fom lywa pí sult: fe ftoor on Glottet i floocit, hrwarifran aft famma sand: brufare kunde fornfoffa fig fró.

KONGELF eller KONGHÅLL, ât en liten Gtab, fom lig: ger innid Bohus Elott utftrift i s/fer od) waffer ients te buirgsfoten, fom pă norrafioan inftänger Stroden, afs wen fom (Sjota EIf (med fin minote gren, hwilken frrys fer at Kytkil) inmeluter Etaben pá foora. Sorget beftod af en Eullrig och oiämn Elippn, odf i Strocen at

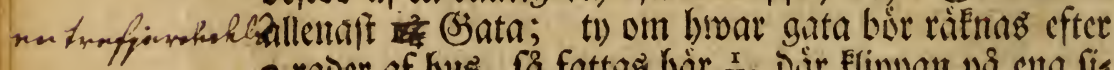
2 raver of bus, fa fattas băr $\frac{x}{4}$, Dir Elippan pá cua fis 
Dan af gatan gör tiơnft fơr lous; altfiu ár Denna ftaden tômmeligen lăng; men ganffa fmal; pa norra fiban utfiras meo S ràgairdar och Rålgároar, fom frtäcta fíg up ât băiggroten.

FLÁDER 250. war planteras hár i Srágårben til ftórte mucfenflet, ăn påanora ftällen, tillifa meo många fultbärande trin.

DESMANS-ROTTAN (Faun. 24.) fagb, lywatg lugg på ruggen war mucfet gra od) more, miuk od) tảt, mot fpitfarma bett more; lywariblano längre och froartare

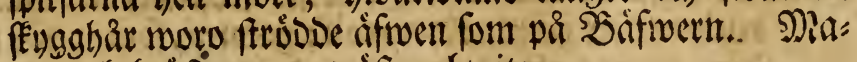
gen od) brojfet woto náftan bivite.

RHABARBER bave Comminiftren Kritlod forlenffiat fig i fin Srágåro, fom bonom war gifwen for áfta llaget, men war Doce en gemen Rhaponticum; ef eller bar någonfin Den veritable Rhabarber wurit $i$ Giverige, forrtin jag, fơr et par ar feoan, forrfafinoc bonom til Academie Stagarben; Dăr bant iffe fallentaft frodigt waiker, oaftandes wara kalla wintrar, utan oce fa fors bear fig, at manlei bebifiwer Eopa honom af Shimerer, Surkar, eller Srisßar.

CRATEGUS folio fubrotundo ferrato fubtus incano. Turnefort. inft. 633.eller ARIA, Dalech. Hif. 202. mar

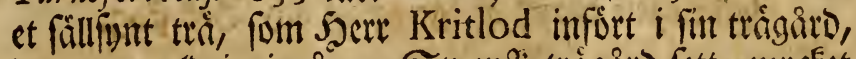
Det man alorig i nagon Gounf trigaro fett, nuycfet minore formodat wara Sivenfï Snbugaate. Det has De blifwit hitfortot ifran Torsby Sofen, Dar bet wait wilot uti et bângéflog; jag fice fe Det federmera pa et war ftállen i Elipporna jemte hafäfían. Bladen äro life meo Diel; men pa undra fían mera luore, och frublyite; Slabenb fanter gå icfe $i$ wagor, fom pa Oyeln; utan aro jämt fagabe fafom ct 2llmblao: elieft år Detta tríbet fa lift med Siel, fom det är med Dielm jiagt.

CALMUS 277. war bitforo ifran Sarby, Dár ban was fer wildt.

$$
\text { RESAN }
$$


RESAN ifrin BoHUs it MARSTRAND.

JEŔNỎRT Eallades bár Ononis 622, fom allmaint twajs: te $i$ ángarna.

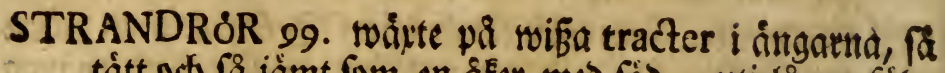

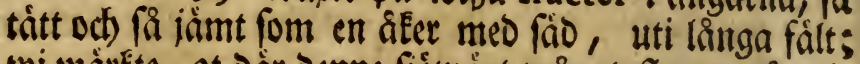
twi märfte, at Dair Denne fiơweint wargat fig up på wis Da ved) bóga ánsent, altio war et liéa ftort fällefeg uns Der jorden.

CASTELLEGÅRD war Śfiverfte Bofftâlle, bár man făg rudera efter gamla Kongself; gatorne, fom tzarit $i$ fta: Den, funnob ofta ámiu ofüránorade, men merendels en alt sjupt unver \&Eeren; En liten Fulle ftod qtwar, fom nifaoe hrwarafif Siloftret warit belígit. De ftora od bárlige ângar erfatte nágorlunoa Detr ftora briften på tweo, fom ofta måfre baimtas bela 4 milen bárifrản.

NATTEN bios of at hroila i Gullby, quart 2 ifrar

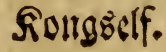

\section{Jul. 15.}

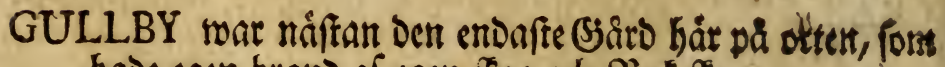
bade egen brand af egen flog od) Bobe-Fog.

SKOGSBRISTEN ât bir Den fivarafte; hela tracten ât

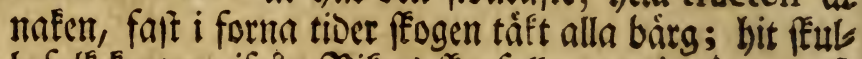

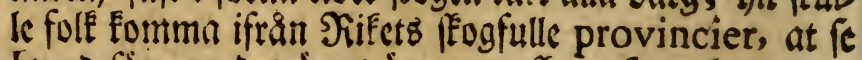
bwad furmon Det år at äga egen fég, fant buru ontt tweden hair fópes, Da Sounden germa gifroer 5 Miart E:mt fort brwar fpada om Dagen, med bivilfen ban fielf

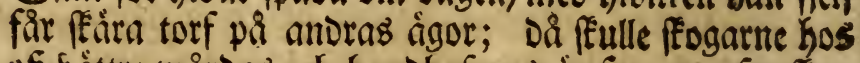

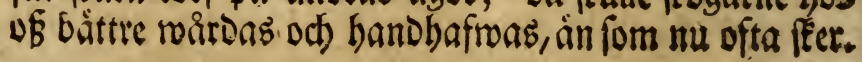

GETTER hafiwa biade fint ftora formon od frota oldagers het, biwarfiure man fóft (B)etteslorter laingft ifrän Ori-

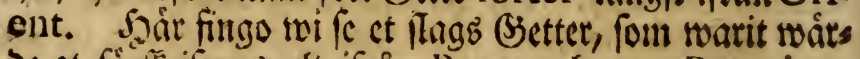
De at fóritriffuas alt ifrán Peru, ode emot Præmium 
planteras bos de mâfe Sweriges intwantare. De funnob idfe allenaft hár på Gullby bob Cornetten Memfen i ftort antal, utan woro off bos be mafte

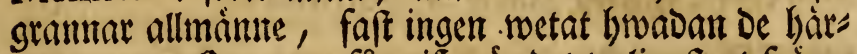

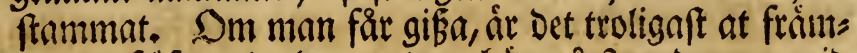
ruande fiofarande lemnat Dem hair pa ftrenderma, wio fin aterrefa. Ellorig har jag tilforene fett findane (Sictter pa allmánt och elándigt bete, ej eller pa bättre. Bsetterne soto ról aldeleb famma fpecies med waira allmain: na, od) fosga fturre, àn De. Bahcfarne base en ling bwit ragg, Docf ei fa lang och mite, at man funtoe tro Det Detta fligtet wore en degeneration of Ditindifea (Setter, fom furdom blifwit hitbragte. SODE Defa Get= ter woro 2 - ting, fom máf Drogo min upmárefambet; Det ena, fom almant berittades, nòmligen at be alurig gnagie, cller fealade feogen; Det andra, fom ing firg, wat at de nijolfade fullt et ftop $i$ målet. Jag itante i fórumbran, dá jag fảg Juret mes fpenarna, fom war s aln langt, fa at fpenarme räEte jult neber til leoen pa

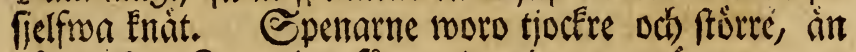
pa ell for fo; jag fảg med mina egna ogon, at en Siet, fom miolender, gaf I frop fa fullt meo mjols, at bon ram sufwet alla brabsar. Settr Fommer ingas lumba of betet, fom åx fämre ail hos of pâ marten, mucfet minore af ffogen, roio bwilEen Betterne brift trifwas; ty Den wat fa godt, fom ingen, od) fielfwa buffarne fattades. Galledes tiscfes mig funma med alt fog recommendera Defe (Sietter hos iooge SHubs: hollare, pa det et flag, fon miolfar fa mucfet, ach als orig gnager trän, má blifwa til allmán nutta, oct) ei fóramafo uti en aflågfen Someriges torả.

LANDET hạr i Bohus-s̊an inemot bafivet beftod of fulls rige bactar, fom woro ligna flippor, emellan hivilfa lago fma angar, fma byar odj fárt, utan nas gon feog. Det ác otroligt, lywas atfeilmad et lano fär, Dá Dé̉ bactar blifwa, utan fág, nafne flippor, Grwar af landet fer ut, fom wore bet far nagra sr foom of watnets afgruno upftiget. 
* Kyfkrls BSafftgifivaregâro. quart. 6 ifran Kongself.

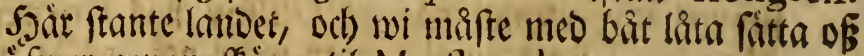
sifwer genum feären til Maritrand.

SKÁREN, emellan Kyf kil odb) Marttrand, figo ut fom lams Det pá anora fiban Kyfkil, oa oet babe warit neofunft umber bafinet, fa at allemaft bärgs tullarme uptopat ofs wer watnet; th) Demta ffär beftod af fmà rumbade och fulltiga flippor, nafna bolmar, od) flere grumoer, fa

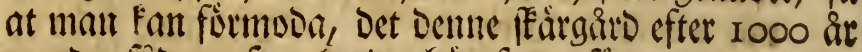
warder findan, fom lambet bitrofman füre.

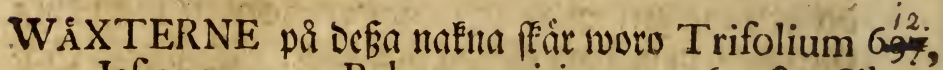
Jafione 713 , Behen maritimum $360, \beta$. Silene faxatilis 367 .

* Marstrand. quart. 4. ifrin Kyfkil.

MARSTRAND år en Féfitning od ftas, fom ligger på en liten Scolme eller Silippa, lie De andora bär i féren, Ducf iblanto de ytterita, I nil ifirin lambet.

CARLSTEN Eallaื̉ Fâftningen, bwilken år anlago på Marftrand, uppa ct hoigt od mulycet fluttande bärg, fom air en fanning for be lijofarande. Gieifwa Elottet fa wäl fom wallarne ăto bugde med famms breda gras ftensmurar od) bomfria bivalf. Ofroan uppa ait ent ftor Piundel cller Caftell, lywaraf bon forar bogt up $i$ luften. Den fom feot Demna Fafining, unorar icfe, at bon bolles for en af De fâfrafte $i$ Siitet, stminftone fá linge en Sfiwerfte Tungelfelts life Där âr Commendant.

FANGARNE, fom bit blifivit Dơmbe, fór fiut obetanefam bet och sfmerilning, singo mu i fin ofucfia, od) mafte betuga, at firftningen war faft. Den fom fer Defe ofalle, unorar ej, at Marftrands namm refer bâten på Dem, fon bơra bet nammab.

GUARNIZONEN eller Golonterme med DCE miferable flíoma furtjänar upmàrfambat. 
STADEN i Marftrand war mucfet liten, ock läg på nors ta fioan om Fâfiningen, utitraft efter liafsifranden $i$ offer ods wafter. Saufen áro fima, alle bugde af trí. Statorne ftenlagoc, merendels med en flat fififrig fálls ften, af bivilfen mâft alle báng befta hä pa orten, hwils fenb filfiverglänfande Mica gớr, at alla gator feina fom filfiver emot Solen. Saar woro näppligen iffwer 30. Borgare, och De mafte af Dem fattige, fom lietwal tyce eb funna blifwa de rifafte. Scăr pâ Son år ingen äng, seer eller betesmark. Folket ligga frängde ifrản landet, fa at fa af 2fllmogen Eonma bit med fina wa: vor, eller harifran hamta bet De behofiwa. Âtefillige mente et Porto Franco bair mes batnad od nutta for Sii et unse intartas, fafom boit war ben baffa hamn

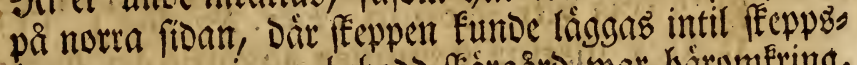
bryggnn; th) ingen bebood ffärgairo war bâromfring, genom bivil:en \&urenorigeri funnerligen funde befaras;

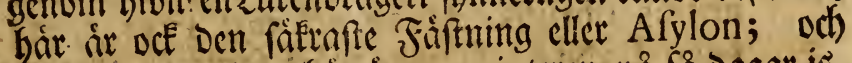
andteligen emeoan hâr ât om winteren på få dagar is, fom fent lágger fig, men bittioa gair up om waren, at fispmantmer fricoes bejto báttre Eumbe nytja tiben. Men jag lemnar alt Detta, fom jag utan tilráckelig int figt beffesoar, odf forfogar mig at tala om Naturalier, ibland hroilfa jag boppas funna toga fáfrare ftes.

\section{Jul. 16 .}

NATURALIER gafrwos bir i 23 afferbafwet fa minge, fallivnte, obctante, atminfone fór of $i$ Enerige, fom bo ifran Detta bafmet langt feifide, at wi borrofiver blefwo fatte i frorfita formonom; frenarne i hafiwet wos ro tafte med Enatfor, botnen med Ejonoapter af Confervis, Fucis, Ulvis, Algis; Gland hwilka )?anietter eller Medurx, Gioffientur eller Afterix; Salf: : froftor eller Cancri, utom átfleilliga amora Giös frík frupo; hwartil fom en mucfenl)et Şaféfiff, fom fiffadeb wio Defa ftramber. 2 ZBi botanicerade på

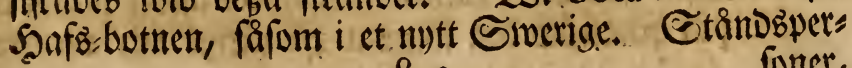




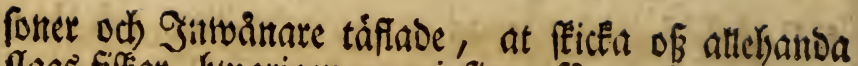

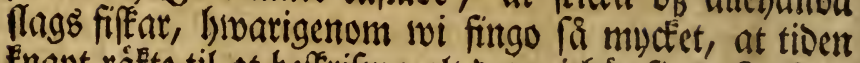

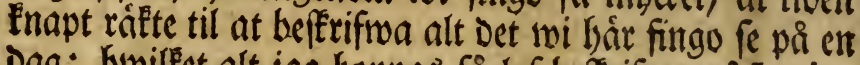

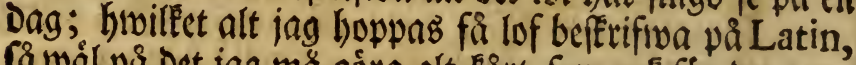
fá wál pí Det jag mả góra olt fárt, fom oce fór Det mait

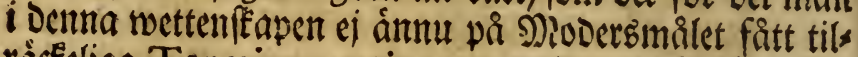
răcéliga Terminos artis; utom Det, at fá lára lấfa Detta meo upmantefambet, fom ei fielfiva fuderat $i$ Derna metenffapen tilfoseent; hivartil Fommer, at uts fómningar, fom máft pläga fógna fig af fabant from, bàr, utur en Šmenfé bol, tmâ fe buad fom finnes wid en Eivenfé frtand.

TANG Eallidoez i Marftrand ZOSTERA, ALGA anguftifolia vitriariorum (Flor. Sv. 364.) $^{4}$.) FUCUS 1. Alga marina graminea anguftifolia feminifera ramofior. Raj.Syn.3.p.52. RUPPIA foliis linearibus obtulis. Moerb. aZt. angl. 1741. p.21 7. Ba.

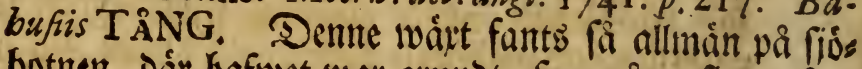
botnen, Dár hafivet war gruthot, fom nágunfin mogan

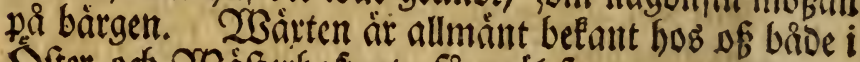

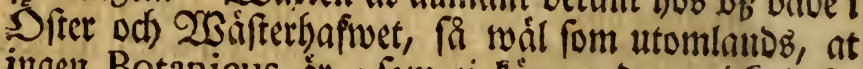
inger Botanicus âx, fom ej fänner Den; icfe Defto minore fattas beffrifning offiver Denfamma, stminftone pẩ blommant, fá at Denura waint albrig af någon Botanico Syftematico blifivit fatt $i$ Det lius bon bir; th ingen bărtilz bafwer gifivit pá Detta genus tilricefelig Character; lywillet Daraf tommit, at man antingen fälan rảafat da be ftảno, fom hafwa blommor, eller bả mout fâr fádana plantor, har man fivart fór at fe fjelfs wa blomftren, foum ligga fórooloe intron et blao; in gen Botanicus har gifrwit någon goo figur på Denna ort, od) Def blommor aro alorig aftagne. Det itr fuller wift, at Det finnes en Eårt befertifning pa herines blommor utt Raj. Synopsi tertia; Dode ar Dent ci fi ty: Delig, at mant băraf Ean fluta någon Character genericus. Dent lärbe och curieufe Botaniften MoEHRING har uti Transact. Anglic: unoer namm af Ruppia forreftấf Den famma pà artigt och) accurat fâtt; 
men jing habe alorig wetat, at ben warit famma woipt, om jag ej fielf rifat blomman. Nu fan jag truggt gơta hanz int til Synonyna meo min; jag blef häs igenom fatt i ftáno, at determinera Charactern pa et nytt genus, och) altfa upfylla ben brift, jag måfte Fánnas'nio, da jas utgaf Floram Svecicam, dár jag formadoe fora alla mis befanta Smenffa wáter til fina genera, utom Denna enda, bivilfen fommer efter Denna Dagens rín at transporteras til Gynandriam Polyandram. Siten fâg ut aldeles fom ct Flotgrís (77 I.), hroarz figuri iag gifiver Tab. IV. Caulis teretiufculus, articulatus fig. I. Folia folitaria, linearia, longiffima, glabra, fructificantia. fig. C. Fruetificatio fit ex folio, quod fupra bafin, aliquot pollicum Ipatio, articulo inftruitur; mox dilatatur utrinque margine latiufculo, utrinque obtufo, longitudinaliter connivente. fig. I. fia at Den fom har blaben, oct) ấ ofunnig, fulle tro, at en Vermiculus fubcutaneus bade átit Det inuti, od) allenaft flilt of fra buben eller fiban ifrån ben unore; men náe wajeten påkg, Dár han i hafwet wuyit, lifnar han gans ffa muctet worte befanta Flotgrib (771.), th) be itwers fte ámbarne af blaben flyta efter winden odj wågarna. Characterem genericum ffal jag gifiwa:

\section{ZOSTERA. Tab.IV.f. 2. 3.4. 5 .}

CAL. Spatba e folio fupra bafin longitudinaliter connivente.

Spadix linearis, planus, latere altero ftaminibus COR. nulla.

piftillisque, alternis, deflexis. fig.2.3.4.

STAM. Filamenta plura, breviffima, alterna. Antbere ovato-oblingx, nutantes, obtufa, pone furfum fubulato-incurva. fig. 15 .

PIST. Germina pauca, alterna, ovata, compreffa, ancipitia, fubpedicellata, nutantia, apice affixa. Stylus fubulatus, longitudine germinis, femibifidus. Stigmata capillaria, fimplicia. fig. 6 . 
PER, membranaceum, comprefum, ovale, longitu.

SEM dinaliter angulo dehifcens.

$$
\text { dinaliter angulo }
$$

SUDARE incolis FUCUS filiformis, fimplex (icog),

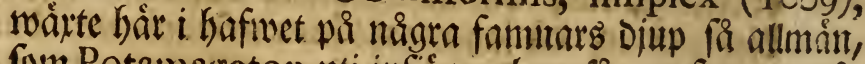
fom Potamageton utti infióar; ban fing ut, fom en tris, tjock med fegelgarn, grơn til färgen, bade ingen gren pá fig, war mije, och vfta nảgra fannar lång; flere fas Dane tráar uplwaxte af cin rot, och om De blefivo längre, an matnets Djup, floto de font Flotgráfet, eller fon Den fortre; meo Den atfeilnad allemaft, at Denna ei ling of worn pa watnet mes en torr fida, utan imunocr ớ: werita brunen af watmet, fâ at hon altio war wart.

KLOT ÅG odh SWINTANG, FUCUS folio dichotomo integro,; veficulis verrucofis terminantibus (Flor, 1002.) wápte wid frtanderna pả Flipportie odj)

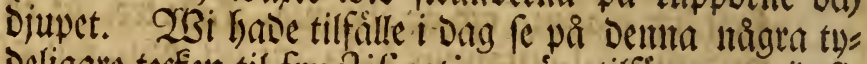
Deligare tecten til fructification, in tilfurene: noid is Dan af Glabet up cmot flutet, futto Flotrunde, fipantoe, bare oft) glatte bläfor, brwilfa inuti woro ibålige, oct) ftróboc med hår, fom ftálde fis inàt Centrum; men lìngre neo pá grenarna fígos par af blafor, fom woro apfylde med en gelatineus faft; debe blafor worv bes frtoboce med mainga fima puncter, bivilla fitto iblas fans himna fiffont grys, od) alle beje puneter woto

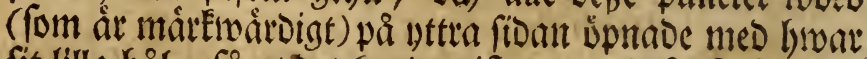

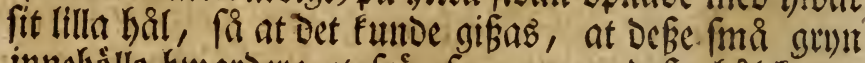
innelgöllo biwardera et fró, fon genom deça hál Eumbe utEaftab. Så at jag tror, at befe fentare blafor worto

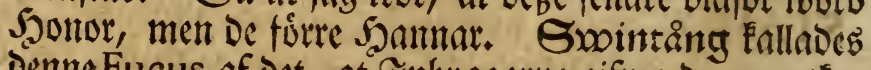
Denne Fucus af Det, at Ynbuggarme gifiva Demma utfos

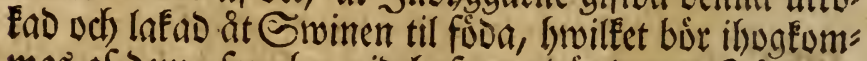
masiaf Dem, fom bo wib hafivet, sair Denna. Sang als orig plígar fattas; Guwarigenum be Eumna gưra ftor berparning für Smin=Ereaturen. Zlotang falladeb bon af fina fima blaffor cller Hlot, at bermed feilias ifrin Zoftera, fom bât allmănt nâtunes fpecifice Santg. 
FUCUS caule tereti breviflimo, folio maximo oblongo indivilo (Flor. roro.) taffe alleftíbes hafs? botnen wid Marftrands brugga; ban fåg ut, fom ef tunt líber, war oval eller afling, ofta of 2 alitars längo, vef i alns bredo; han gicf i waigor. 2 Bio bafin war han fmal, eller hnde en färt petiolus; deß̉ rot hâftade fig utan pa fieraina liefom meo fingrar.

ULEVA oblonga plana undata menibranacea viridis. Fucus longiffimo latiffino, tenuique folio. Baub. prodr. 154 . wajte rîtt mucfet pa hafäbotten, Där hout lag nog oamfull, liffom i morka wagor, at hon el wă

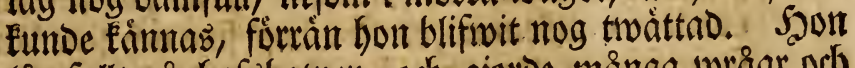
lag fullt pa hafsbotnen, od) gjorbe manga wråar od) mintlar, uti od) umber bwilfa atffillige hafübut fingo beqwåmliga gómmor, fafom Afterix, Medufx, Gamari. Denna å̀ tilfutene ci upfórd ibland Florz Svecicx rollar.

KUPUNGE fallades Cocblea tefta ovata: fpiris quinque friatis fafciatis, apertura margine poftico dilatato rotundato, Cocblea, fulca, falciis crebris anguftis pradita. Lift.Angl,162, I, 3. f.9. ent Snåcka, fom ej tilforeme blifwit inrollerad uti Swen: fí Faunx Armée, bodoe pa alla frander, fa wio Marftrand, fom Den andra féren bár omfring; bout tafte alla ftenar, och) Éftades up til franderna, fom gráz, fa at bet alleftäbes frafade unoer fóttren, da woi

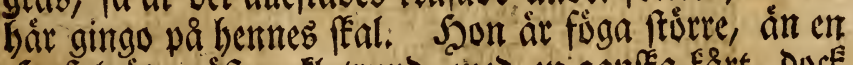

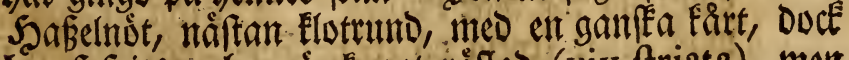

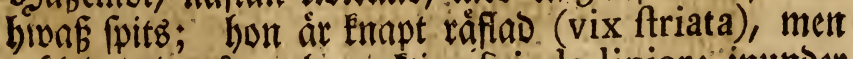
málao meo många biunattiga fpirale linier; imunoer ắ féalet ei genombaraot (ninime umbilicata), off) Des opning tåcfes meo et litet brunt tunt lack eller tumn umbilicus marinus, fom midt pa har en fuiral cha-

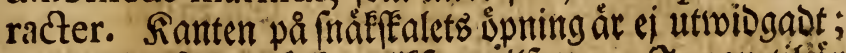
nen meo något mórkare liefom tilfatt. Gnnan tif ä̀ fálct brunt. Figuren har jag utfatt $T a b$. V. fig. 4:

COCHLEA tefta craffa ovata utrinque producta, fpiris quinque fulcatis, aperturx labro interne den-

$$
5 \text { ticu- }
$$


ticulato. BUCCINUM minus ex albo fubviride, ore dentato eoque ex flavo leviter rufelcente. Lift. Angl. $259 . t .3 \cdot f .6$. exercit.2.p. 85. fang octs fi mid ftranden, faft $i$ minore antal ât bent forta, âfs went tilfürene für of obefont. Tefta ovata, ftriis fpiralibus copiofis fulcata : apertura ovata, pone canaliculo terminata, labro minime expanfo, interne obfolete dentato, \& operculo claula.

CONCHA tefta oblonga lavi fubviolacea (Faun.1333) fang allmánt på alla frenar och Fucis $i$ hafwet, wio bwilfa hon altio war faftad. $23 i d$ gangiernen eller cardines utlommo en fror hop fine od fege bar, lies fom ifrän et centro, hyvilfa utividgade fig lafom en

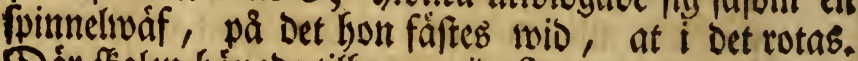
Dár ffalen băngoe tillfopa wio futuren, wat fiban

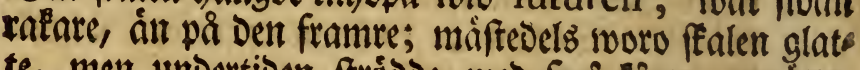
te, ment undertioen frtódoc med fimá fårta, upraitta, finfina băr, fom giorde Dem fearpa eller hifpidx.

LEPAS tefta conica fulcata, operculo acuminato: (Faun. 1349.) Demta Balanus fatt bár och Där pa

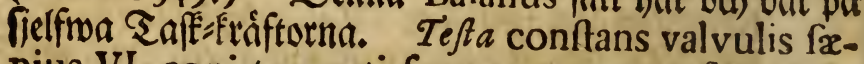
pius VI, coni truncati forma; apertura fuperiore tecta valvulis IV, acuminatis rhombi forma, quarum 2 inferiores majores.

DENTALIUM tefta adhrente flexuofa triquetra,

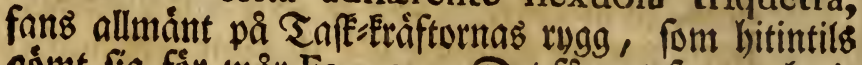
gómt fig fơr war Fauna. Det fág ut fom en bwit

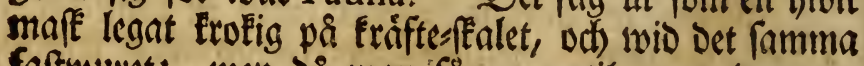
faftnuluet; ment dà man fäg noga til, war detta eat

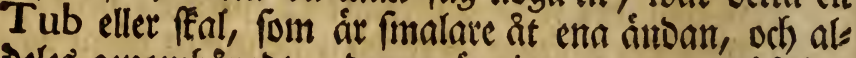
oeles genombáradt; Denna suning eller genombaring

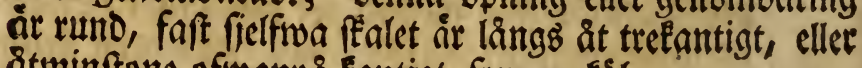
âtminftone ofivanpá Eantigt, fom en fór.

DENTALIUM tefta fpirali plana adhærente. VER: MICULUS in littore veneto foliis algx adh $x$. rens. Planc. conch, p. I 3,n 3. ár oif en obefant Recrute fir wår Fauna. Swintángen (Flor. 1002.), fom 
Gỏgtals upfaftades til frtanderna, twat bels betâlt med Efchara, Dels beftróso med hwita gri)n, fom mången i forftone fummat tro wara ङnäcfegryn eller = frỏ. Neen oa man tilfag, war hronet odf et of Defa gruyn en fulls fommen Snàcfa eller Dentalium, fom aloeles lifinas De en Concham tefta plana. J⿹entes ffal gicf uti en flat fpiral rumo rimg of 4 omgingar, eller ringar; at centrum woro Defe ringar altio nagot mindre; hwar: af Den śfra fiban blef năgot concav; men unore fidan war belt flat, och fo faftroutecen wio saingbladen, at hon mes móda funde fíliab Dárifràn.

PATELLA tefta orbiculata altero margine gibba.

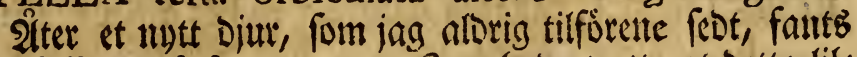
wa ffalet af Saumrarme. Gag broe trott, at Detta lits Ia ffal warit et ffal eller valvula of ent nutblit, som jag ei fielf feot annat: Gag trodoe i fourftone icfe niina egna sgon; ty) jas ty)tte, at jag fing et féal af en Concha, mer máfe finta, at Det war en Patella. Şas tror aldrig, at naggon Saturfummig, font finge fe Detta $i$ et Cabinet, naggonfin Eunde lâta intbilla fig, nt féalet icfe novro of nagon Concha. SEletet fíg ut fom ct musleffal of en Concha lavi rotunda; ment have intet nagoon Eamrad; Det war ftort, fom et fiffeffiäll, od) tálte et litet gult frák, fom lefoe och rưvocs. Tefta fuborbiculata, planiufcula, vix convexa, lavis, alba, pellucida, verfusialterum latus feu narginem parum gibba, uti in Conchis juxta cardinem.

LERNEA cauda duplici tereti, áfiwer et nuft Diurt iffe allenaft fór mig, uttan fä wióa jag wet, âfiwen fór hela wertDent. Sag fant Det i De miufáa gálen pả Snotent od) Sorfent, Dâr Det fugt jig faft fom en Sget. Det ât fuller wift, at Det icfe änu $i$ alla Delar tommer ifs mercnz meo Dent fúrta Lernea (Faun. 1283.), Doff ar Det nărmare flăgt til Det, ån något annat; imeolertio fât Det Dár ftå, til beß̉ werlden begunner nogare at mónftra GrấÁrméen. Thorax feu corpus molle, album, fubrotundum, convexum, carnofum, 
antice obtufum, feu potius retulum, latere utroque notatum denticulo molli antrorfum fpectante. Collum tenue, longiufculum. Caput trilo. bum, dilatatum; lobo intermedio obverle cordato. Cauda pone thoracem ex duobus corpufculis, pallidis, cylindricis, longitudine thoracis. Brachia fub thorace ad latera colli, utrinque duo, quorum par exterius bilobum, interius ovatum.

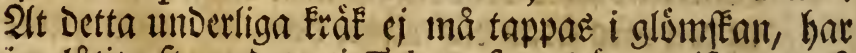
jag lattit aftagn Det uti $T_{a b} .3 . f \cdot 4$. Dir a wifar Det på ryggen, men b pa undia fiontr.

KORSTROLL Eallader Afterias radiis quinis latiufculis alperis (Faun. 1284.), hun wáltrabe fig til muse Eenget iblanto forentambe Ulva. Denna Sioftierna beftod af 5 frralar, war blirios, cller undertiden bivit, betâft pá alla foor med fma miufa taggar, men imuns Der betaft meo papiller, fom woro utifaloe i 4 rader, meo en liten opning eller utgrafiven fpits $i$ änoan: Mamen war $i$ fielfioa centro pa Detta Djutets unore fida.

MANIETT, fallades MEDUSA orbiculi margine fedecies emarginato (Faun. 1286 .), fiot ifiveralt $i$ watnet til for mucfenthet, Dock ej fa ymnigt, fon uti Sghafwet (1t. Lapp. Ful. I 2.). Defe finta i watnet, fom Golar, od fatta ut ifrain centro af unori fioan en bop tranar, af I eller 2 famnars lango, hivillea wal

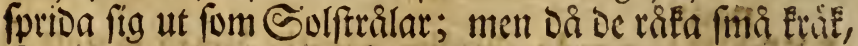
faftna De wid Dem, od Draga Dem til Dcuma Golens mun, fom Dem upater. Beffrifning pa Detta Diuret bar jag upfatt i Fauna, men fom figuren ef â râtt

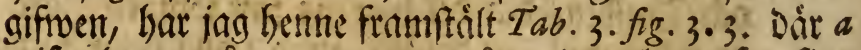
wifar henne pa ruggen utan triar, Dem hon ofta fins aldeles utan; men $b$ aftriger benne pá utbra fidan.

MEDUSA orbiculo fubtus quatuor cavitatibus notato (Faun. I 287. It. Lapp. Ful. I2.) ât nog allmán băr i fiơn, och) âfwent i Śfterbufivet, Dár jag bente bes 
ffrifinit iỏlåndfka refan (It.Oel. 160.); ment hår woil jag gifwa hennes figur, fadon, fom hon ảe på undra fitoan, th) Den ófre air helt plat, litet convex, od) utat naggon fällfam färg. Tab. 3. fig. 2.

TASK-KRÅFTA eller CANCER Pagurus dictus (Faun.

1244.) fanz hob filéare ibland de andra filfar. Sant war ftor fom en STummer, til färgen blek; ment ytters fte fpetferne af Elona woro frontte. Thorax latiffimus, marginatus, obtufus, crenatus plicis utrinque 8 , obtufis, excavatis. Inter cculos denticuli 3 , obtufi prominent, \& totidem denticuli fub fingulo oculo obtufiffimi, inæquales. Superficies thoracis convexa, levis, punctis pallidis. Antenne breviffimx, ruffx. Sinus fub antennis, in quibus Tentacula folitaria duorum articulorum. Bracbia manuum lavia. Mamusleves, pallidx, digitis maxima parte nigris. Pedes utrinque 4 , pilis grifeis, hifpidi: unguibus glabris, rectis, obtufiufculis, validis. Cauda inflexa, quatuor articulorum, exceptis primis duobus breviffimis. Balani odf) 2:me Dentalia woro fáfte pa Denner rygg.

KRABBA. CANCER brachyurus, manibus ventricofis lavibus unicoloribus. Deme frup iblano Ulvas, Fucos och Afterias. Thorax fubrotundus, ferraturis utrinque quinque pone oculos: quarum poftica acutiffima; antice thorax inter oculos denticulis tribus obtufis. Supra convexus eft thorax, inxqualis, levis; mamus lives, unicolores, ventricolx, articulo brachiorum manibus proximo introrfum acuminato. Pedes praterea 4 utrinque, apice fubulati. Cauda inflexa, açuminata, fimpliciffima, tribus articulis exceptis duobus primis linearibus, caudx fubjacet corpufculorum fubluteorum par loco genitalium. Antenne minutiffimx, fetacex. STon bade mucfen lifhet meo Den fúrra, Docet ei firatt pâ Elorna, och) naggot 
i anora belar olif, at hon âtminftone ôt ett varietet, om ei ferffilotleun.

HUMMER. CANCER gammarus dictus, (Faun. 1248. niger; Therax oblongus, punctis Iparfis excavatis, linea cxcavata longitudinaliter exaratus, \& transverfim alia linea exaratus. Roftrum acuminatum denticulis utrinque tribus, prater denticulum fupra oculos ad roftri latera. Antenne lumbriciformes, validx longirudine fere corporis, conftantes articulis brevifimis, nigro rufoque variis, tuberculis minutiffimis. Bracbia anteriora parum dentata. Mamus laves, latere anteriore dentatx; digiti extrorium obtufe dentati; in pollice cxtrorlum fupra bafin denticulus validus. Pedles praterea 4 parium utrinque, hifpidi fafciculis pilorum grifeis: horum paria duo priora biungulata feu digitis duobus terminata; Pofteriora duo paria digitis folitariis, extrorfum pilofis. Cauda longitudine corporis, pilis grifeis ciliata, 6 articulorum excepto ultimo: horum primus ad latera brevior. Ipfius caudx extremitas conftat valvulis 5 , quarum 3 ovata, utrinque denticulo obfcuro inftructa; valvulx 2. 4. obtufiffimx, valvulx vero 1,5 . rotundatx transverfim futura flexiles, futura margine corpori propiore acute denticulato. Sub cauda appendiculorum quinque paria (excepto primo), bipartita, ciliata, quibus ova adhærent. Inter antennas antenmule 2, breviores: fingulx binatx. Fxmina ferat. JJą ruggen woro Balani, Dentalia repentia, \& Spiralia; bigge wiowultte.

Hå fallatecs SQUALUS pinna ani nulla, corpore tereti (Faun. 269.) fants hair nog; en glupfé fife, fort ât en Tiger i fisin, lywilent od han ffal gripa rofwet, malfe lígga fig pá rnggen. Corpus longum, teretiufculum, fupra canum, infra argenteum. $R \cdot$. frum prominers, ovatum, tholle, lubdiaphanum. 
Oculi albo-cxrulefcentes, Narium foramina duplicata. Apertura magna, utrinque pone oculos, \& præterea aperturx s, transverfales, lineares, utrinque ad latera colli ante pinnas pectorales. Pinnx dorfales dux: angulo fuperiore obtufiore, inferiore, vero auctiore: Singulx antice armatz fpina acuta, valida. Pinnx peciorales obtufx: angulo exteriore paulo longiore. Pinnx ventrales remotiffi$m x$ a pectoralibus, femirhombra: fingulx ad marginem interiorem inftucte papilla lpinzformi. Canda teretiufcula, fubtus planior. Pinna caude oblique verticalis, bifida: lobo fuperiore longiore. Vulva magna, ovata. Folfet beråttade, at om nigon iticter fig af taggarne uti ryggfenam, fFal Det uprócta fafelig wäre. Demma fiffens buo ár Chagrin.

RÅCKA eller RAJA (Faun.268.). Corpus planum, rhombeum, fibtus leve, album, fupra cinereum fufcomaculatum. Oculi finguli antice f́pina unica, poftice duabus armati. Foramina folitaria magna pone oculos: \& praterea foramina brachiorum utrinque 5, transverfa. Os transverfum, cartilaginofum, fcabrum. Cartilago transverfa abdominis tulla, fi demas arcum diftinguentem abdomen a pectore. Pinnx ventrales radiis 24 , quo. rum 4 vel 5 longx in angulum acuminatum craffufculum $f$. lobum exeuntes. Spinx aliquot fecundum longitudinem dorfi. Cauda corpore longior, fupra convexa, aculeata, verfus apicem. Pinnas dorfales 2 , rotundatas gerens prater pinnam caudx minimam.

FJÅRSING EnIllaDeg TRACHINUS maxilla inferiore longiore cirrhis deftituta (Faun. 282.) fint hât minore allmán. Deß membrana brancbioftega beftoo af 6 -eller fiere frralar. Dm Denna berittar Bartbolinus in actis bafro. 3.p. 5 4. at $i$ Des ruggfenta, Da Des

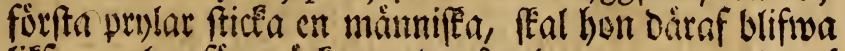
riffom gnlen fúr märË; anore âterigen mena, at bet ei 
âr sumat ån ordinairt ftick, om Det ci fticfer någon fena. Men Affeffor Boetrưs i Gótheborg beräts tnde mig, at han haft Patienter, fom haraf med en olibelig wáre fátt famma fom punctura tendinum,

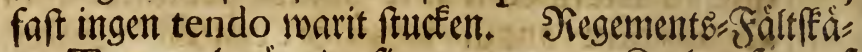
ren TEN AN beráttade fig curerat en Faul, fom af Dema file warit frungen i fingren, fwaraf hela banben och) armen fivulnabe med en slidelig wáre, fom năftan frammad e lit" Gangren, odl) efter 2 à 3 ípningar med

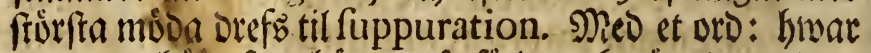

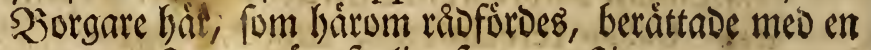
mun, at ftungen áro farlige fom ormfting.

KNOT Eallabé bian TRIGLA capite aculeato appendicibus utrinque tribus ad pinnas pectorales. (Faun.28 1.) Characteres maxillx inferiori infculpti. Opercula branchiarum pone in aculeum definentia. Pinna dorfalis prior radiis 7 acutis; Pinna dorf. pofferior radiis i 9 . Pinnx pectorales radiis 9. Pinnx ventrales radiis 6 , primo fimplici. $\mathrm{P}$. ani radiis 18. P. caude radiis $10, \mathcal{C}$ II. $\mathfrak{U t i}$ Dennes gel fants: afwen Den underlige Lernea med 2:He fitertar, form jag upritat T. 3. f. $4 .^{\circ}$

HWITLING fallabez GADUS dorfo tripterygio, ore imberbi, corpore albo, maxilla fuperiore lon-

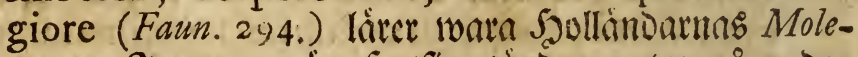

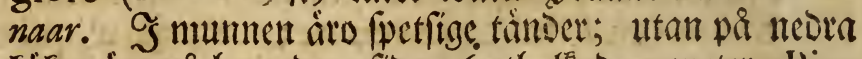

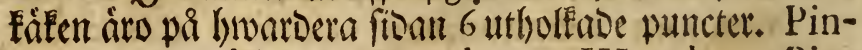
na dorfi $I$. radiis $14 . I I$. rad. 20. III. rad. 20 . Pinnæ pectorales rad. 17. Pinnze ventrales rad.6. Pinna ani anterior radiis 30 . Pinna ani polterior rad. 22. Pinna caude integra radiis plurimis.

KABBELJÅ war GADUS dorfo tripterygio, ore cirrato, cauda fubrquali: radio primo fpinofo (Faun. 295.) Cirrus fubulatus fub apice labii inferioris. Membrana brancbioftega radiis $\%$ Pinna dor $/ 2 I$. radiis 15. roturdata, II. $\ldots 20$. III, - 16 . Pin- 
næ pectorales rad, 20. Pinnx ventrales - -6. P. ani I. rad. $21 . I I$. . I6. Finna caude integra.

LANGA war GADUS dorfo dipterygio, ore cirrhato, maxilla fuperiore longiore. Art. gen. 22. $\int .{ }_{3} 6$. ASELLUS longus. Scbonev. icht. 18 . Will. IEt. 175 . Raj. Pifc. 55. ât iffe hitiititib inrollerad ibland

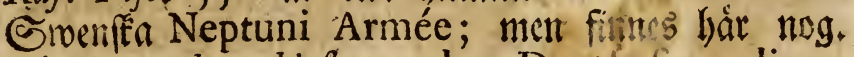
Membrana brancbioftega rad 7. Dentes, fecundi ordinis leu intra maxillas confit, i in ipfo palato, validi. Cirrbus fimplex fub apice naxilla inferioris. Pinna dorfi anterior - 15 . P. dorfi pofterior -63 . Pinne pectorales - 20. P. ventrales - 6 . Pinna ani - 60 .

LYRBLECKK Eallabcr GADUS, dorfo tripterygio, imberbis, maxilla inferiore longiore, linea laterali curva. Art.gen. 20. $y \mathrm{yn}$. 35. îr cn ftor adt blef att af Sorff. Pinna dorgalis $I_{0}--13$. II. - I I. IIL. - 19. P. pectorales - I6. P. ventrales - -6. P. ani prior- 27. ani pofterior - . 18. P. caude bifurca. Linea lateralis curva. Maxilla inferior longior absque cirrho.

TORSK kalladez GADUS dorfo tripterygio, ore cirrhato, longitudine ad latitudinem tripla,pinna ani prina officulorum triginta. Art. gen. 21. Spec. $6 r$. Corpus cinereum, lividum, punctatum. Cirrbus fubulatus fub mento. Oculorum Iris rubra. $\mathrm{Li}$ nea lateralis obliqua, articulata. Dentes acuti. Membrana brancbioffega radiis 6 . P. dor/ $I_{0} \ldots-12$, acutior: quorum primus brevior fecundo; $I I_{0}-20$; III. - - 20. Pinnx peEz orales radiis I8. Pinnx ventrales rad. 6: quorum duo primores longiores, molliores, rubicundiores. P. ani prior - -19 , ani fecunda dorfali oppofita. P. ani pofterior - - I9, Pinnx dorfali poftremx oppofita, P. caude integra rad. 40 . circiter. 
GADUS dorfo tripterygo, ore cirrhofo, cauda integra, linea pone caput excavata. Huic pinna dorfi $I$. radiis $13 ; I I .--17 ; 111 .-$ - 19. P. pe Etorales rad. 18; P. ventrales - -6: prinis duobus mollibus. P. ani prior - . 18; pofterior - - 17. P. caude integra radiis plurimis. Differt a pracedente colore cinereo, minime ruffo. Linca utrinque pone capnt excavata; hinc forte fola varietas.

GADUS dorfo tripterysio, ore papillofo, cauda bifurca, linea laterali recta. Corpus fupra cinereum, infra album. Linea lateralis integra. Oculi magni ; maxilla fuperior longior; fub inferiore papilla minutiftima in cirrhum minime elongata. Pinna dorf $/$. radiis $16 ; I I .--18 ; I I I .--20$. P. pectorales - 17. P. ventrales - - 5 . P. ani anterior --22 ; pofterior - 21 . P. caude bifurca.

TENGA. PLEURONECTES oblongus, maxilla fuperiore longiore, lquanis utrinque afperis. (Fann. 299:) Corpus ovato-oblongum, fupra cinereum fulco-maçulatum, fubtus album; utrinque vero fcabrum. Caput fubtus villofum. Os leporinum f. inferne ringens. Oculi a dextris. Pinna dorfalis radiis 71 , mollibus, fupra callofo punctatis. P. pectoralis exterior - -8 ; inferior -. 7. P. ventrales - 5. P. ani --40 . P. caude $-4^{\circ}$ circiter, rotunda.

PIGGHWARF. PLEURONECTES lavis: latere inferiore albo quatuor maculis aqualibus. Corpus ovale, lave, lipra cinereum, fubtus album maculis quatuor diftinctiffmis æqualiter oppofitis. Membrana brancbioftega rad. 7. Oculi a finiftris. Pinna dorfi rad. 7 , mollibus, a capite ad caudam extenlia. P. peEtoralis fuzperior and ra: prinio breviffimo cun decundo integris, reliquis bifidis. P. peitoralis inferior --12 . Piventrales --6 . $P$. ani_.-6r. P. saude _. - 8. rotundata. 
RóDSPOTTA falladé PLEURONECTES utrinque glaber, tuberculis VI. a dextra capitis( Faun. 30r.). Corpus rhombeum, fupra cinereum maculis amœene fulvis in latere \& pinnis. Roftrum acuminatum. Membrana brancbioftega radiis 7. Oculi a dextris prominentes. Caruncula in fronte parva, mollis, ovata ; Puncta 5 vel 6 offea, longitudinalia pone oculos. Pinna dorfalis rad. 14. P. pectoralis - -6 aut II, (variat lenim). P. ventralis -6. P. ani - -54. I. 57. P. cauda - - 20. circiter. Spina ad bafin ani antrorfum lpectans. $P$. dorfalis \& ani in medio latiores, hinc quafi rhombeus pilcis.

BÁRGSNULTRA (SPARI fpecies) membrana brancbioftega rad. s; opercula branchiarum !quamofa. Dentes in ore acuti: primoribus majoribus; Iris - culorum rubicunda: Corporis color fubvireicens, linea lateralis juxta dorfum, ad caudam curva. Facies Parcx. Pinna dor $\sqrt{2}$ conftans radiis primoribus i 9 acutis, fecundariis 8 longioribus, mollioribus; -ubi radii rigidi, ibi membrana connectens poft fingulos aculeos exit in acumen molle. P. pectorales radiis 13 : primo integro molli. $P$. ventrales fquama lata connexx, radiis $6:$ primo acuto. P. ani rad. 10: tribus primis craflioribus acutis. P. caude integra radiis 14. Macula nigra ad finem dorfi, ubi cauda incipit.

MARSTRANDS ARBETE war at befrifina od) examinera fi manga atffillige oct) owanlige bjur innom et Dugit. Man fitger allmüt $i$ Groerige um et mucfet traffamt arbete, at Det ár Marfrands arbete, bivilfen methaphora lierer fonmit af fangarmas fáp wid fúfrningen, Da de ifran fraden eller fivfidoorna feola trans. portera nagot til ben ftura od hioga faftningen, up fúc Den langa ock brauta bacten, meo Dragande cller flá panie; Det luftar pa fơr en fri lathuno at gá up ifrän ftaden til fäftningen, mucét mer för en Dómo. 
SJUKDOMMAR áro ci nter bâr gangle, fin på antora

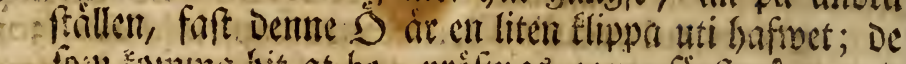

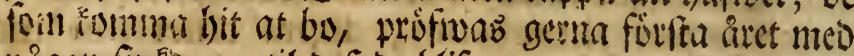

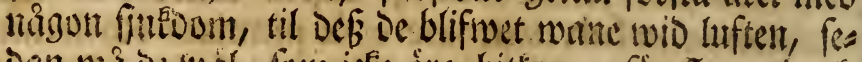

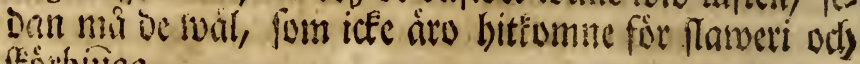
fétbiugs.

VOMITUS chronicus virginem 20 annorum diu detinucrat, qua affumtum mox rejecit cibun, unde debilis vix e lecto furgere valuit. Ventriculas lub thorace, in finiftro latere, inter cartilaginem enfiformem \& Lienem, durus, \& dolorificus. Pulius debilis; artus inferiores uti femora, \& tibix laxæ, cachecticx; Somnus mediocris. Alvus obfitipata folvebatur i 4 . quovis die; vomitu rejecta acidifima erant; menitrua, qux ante legtima, per 2 annos ceffartunt. Urina parca. Corpus tanien non exfuccum; vafa llanguinea in latere interiore palpebrarum rubra, florida. Fruftra ufa eft enematibus, abforbentibus, edulcorantibus, olcofis. Svafi ufun lactis recentis a vacca calentis, parva copia, fed frpe repetita.

HUSMEDEL cmot FROSSAN berittadez fafum ot fpe-

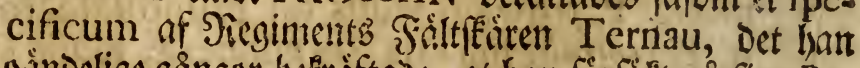

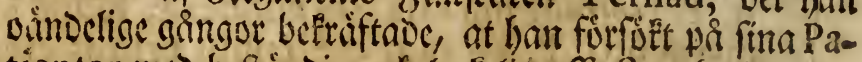
tienter nico befiandig och luctelig effect. Invilfet bes ftoo Däruti, at en niffwn of PALLGRASET tageB dCh

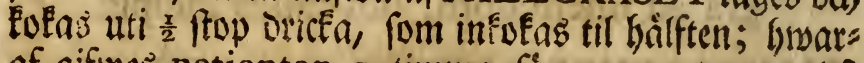
af gifine: patienten x timma fór paroxysmen, då

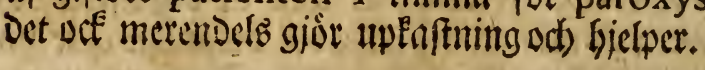

PALLGRASET wärte på Ellipporna, odf war SEDUM acre (F/or. 389.), brwilfet utom Det, at Des Eraft ar

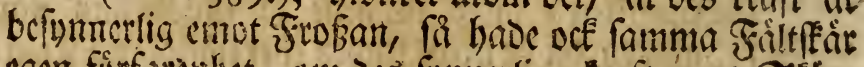

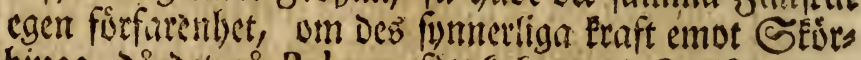
biugg, Dá Der pá Belows fátt Eokno med ¿allftruntar, Jeppartot, ofb litet Rhabarber. 
RESAN frabes cmot aftonen iftin Marftrand it Uddewalla fióledes. TBi bade sumat at gâ utan for Kóm orb Oroft: men wi nurs icfe toil ktfomme pif bafmet, forrin et fartet effeounoci, meo mulen oct) mort Gim: mel, ftom off flagrign, forbe of at tinga ledet innom peitio.

PATER NOSTER begInntes frrax i mafter, utonfor Marftands bammen, meo manga bionmingar ocb) Elippor, et farligit rabano for de ficfarande.

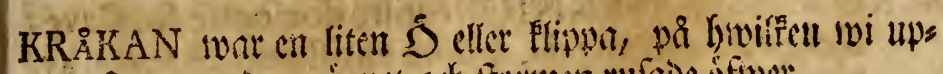

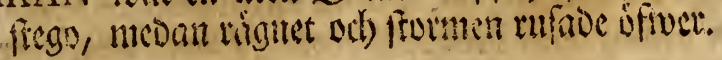

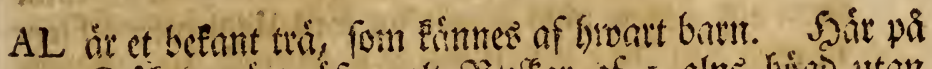

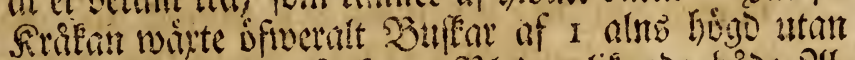
blomma od utan fruft. Blaben lifnabe base sll,

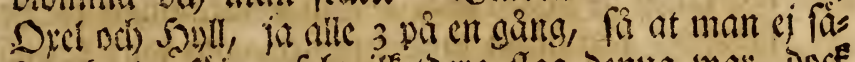

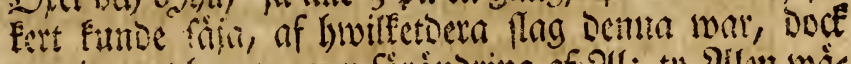

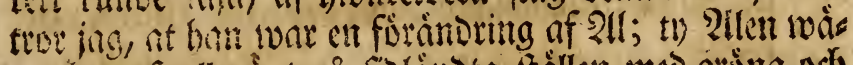
xer hos of ollmint pa fiolantota fällen mco arouna od)

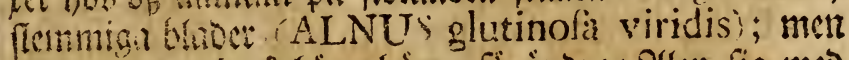
i stortano sci pá louga bairg, fortanorar allen fig med

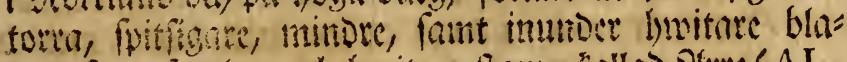

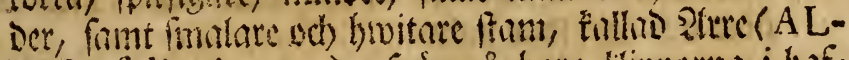
NUS folio incano). SThir pa bara tlipporma i baf:

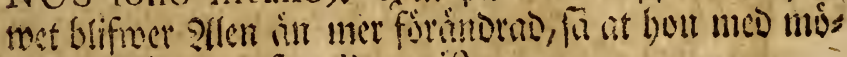
Q.? Ean Eumes of en Botanilt.

KRUTLASRARE Eatloos folfet ef obethimligen ben bes fante TYPHA 772 . ty lyan lifucit ist int trument,

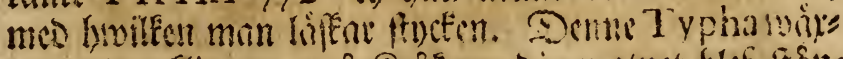

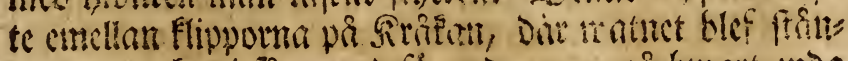

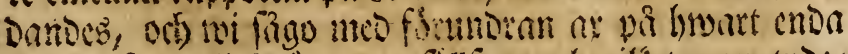

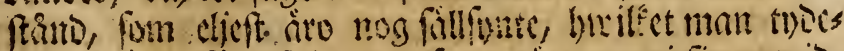

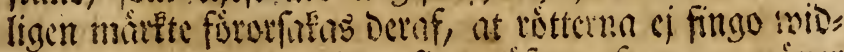
liftigt Eripe eller ttwiogn fig, afinen fon man rower

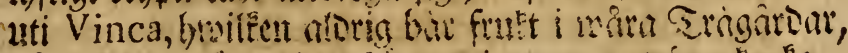

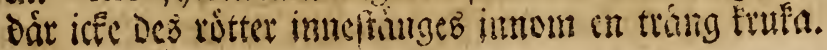


LIGUSTICUM foliis duplicato-ternatis (Flor. 232.) âr en rar ort i Swerige; men bár pá holmarna alls mán. Radix perennis. Caulis erectus, pedalis, rubicundus, friatus f. fulcatus. Folia duplicatoternata: foliolis feffilibus, glabris, nitidis, incifo-ferratis. Umbella involucro univerfali \& partialibus. Fructus fulcis $\&$ exaratus, \& Atylis duobus terminatus. Semen fingulum fulcis quatuor acutis, angulisque eminentibus acutis.

ALKUSORNE. BLENNIUS capite dorfoque fubflavefcente lituris nigris, pinna ani flava. Art. Jin. 45 . hade åfwen fom ?rábbgádeorme (Faun. 305.) gruna ben, od) benen lyfte $i$ murfret fom Hgglewed (Flor. II 30. )

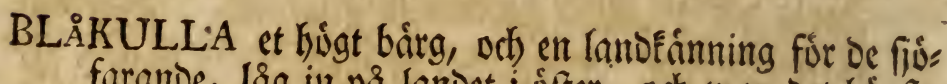
farande, lág in pa lambet i offer, och war bet bógfta ibland De fringliggande.

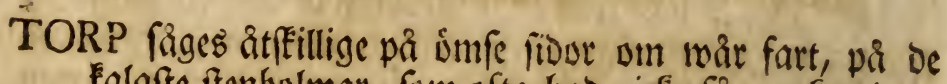
Ealafte ftenbolmar, fom ofta babe icfe fa mucfet joro, at De funde foida et enda fáll)ufivud, utan beftoo bela Şnoårarenas fromma uti eı liten Eálla, men Deras åker od) áng war hafwet.

ELOF (Faun. I29.) Fallabes Gạ ben froartaftige fiffmas fen, fom icfe fielf Earr na ned i fión, at fánga fiế, utan endaft ác féapas til rófware iblano fiffemafarme. Nian fág meo noje, huru Denna Cofaten forfólgoe de andre

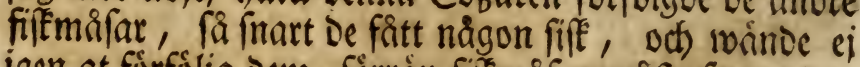
igen at forrfolia Dem, fơrran filtmafen mâfe fiv ut Den fife han fiffat och) redan impactat. Gag bar feot med forunoran pa en fiffmâfe, fom jag flere år baft i Academie Srigairoen hemtamo, at om han fâtt alorig fâ litet mat, och nảgon feoermera litet jagat efter honom, bar han oa firne utfpyt det han befommit. Denna egenfeapen at litt vomera hat SFaparen anmoinot at uppebolla war Elofite fanilie; ti) fom fifemafarme of: 


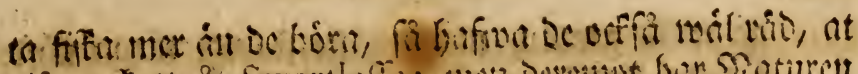

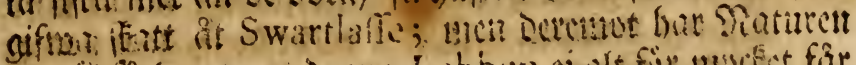

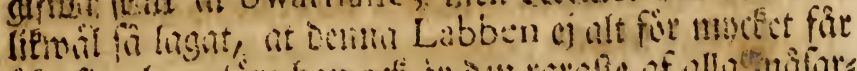

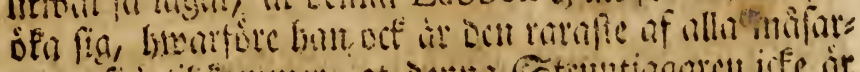

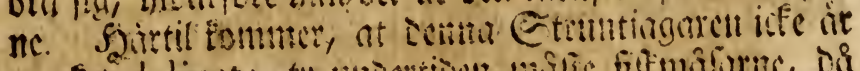

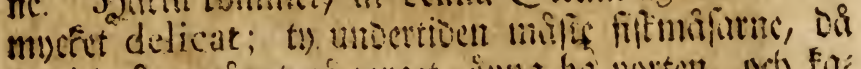

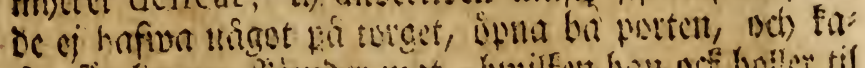

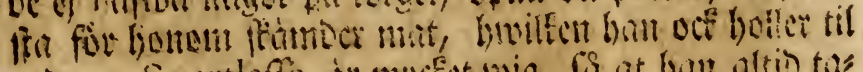

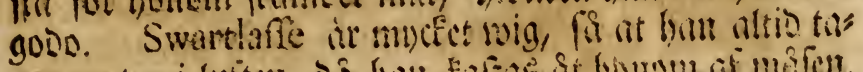

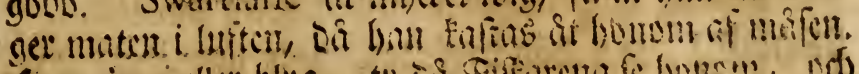

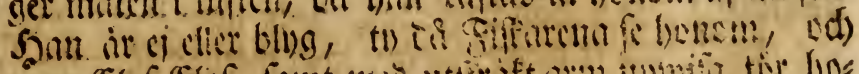

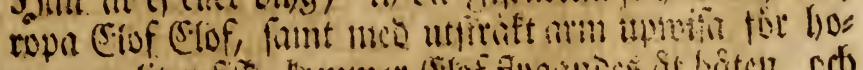

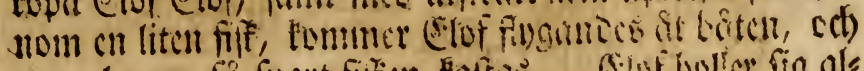

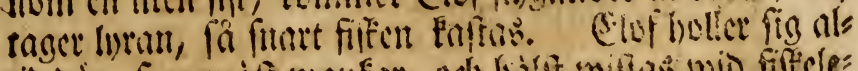

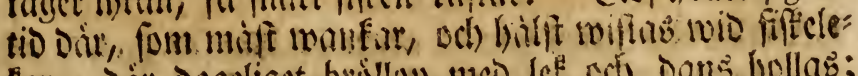

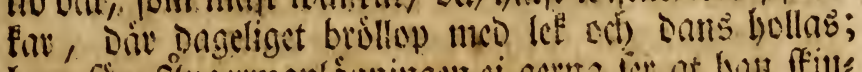

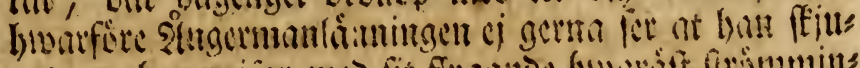

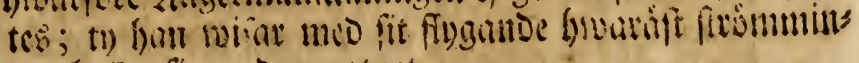
gen botler fig unoer wattict.

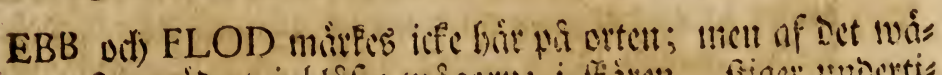

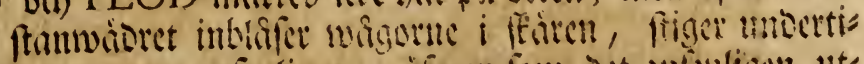
Den watnet anfentigen, afiben font iet winfentigen uts

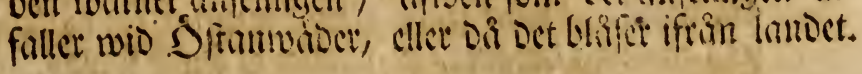

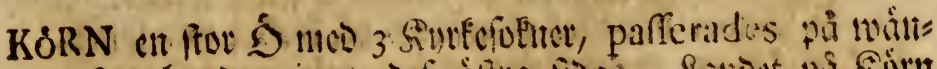

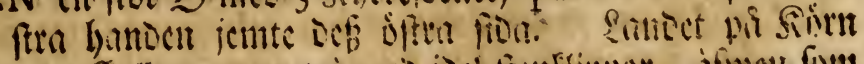
war feallot, uten trit meto iocl frenflipher, afwer foum De andere fiolimar.

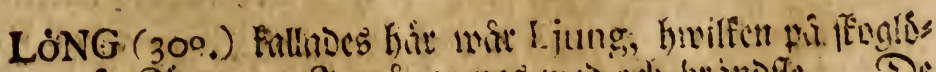

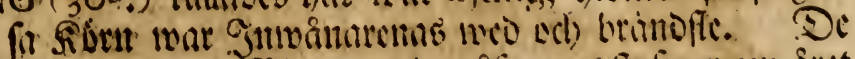

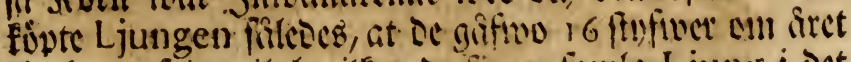

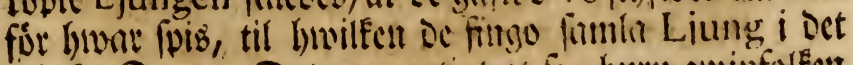
minfin Sorp. Det wure artigt at fo, huru quvinfolfen

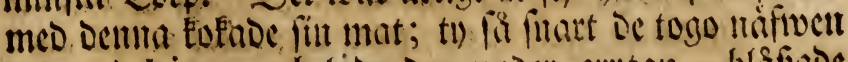

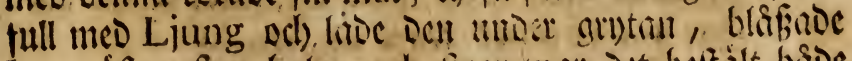

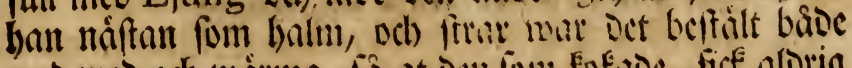

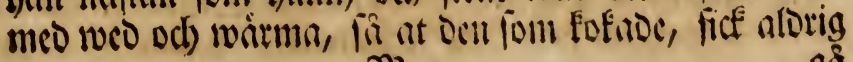
M 4 


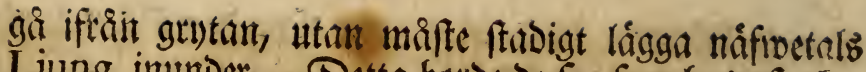
Ljung imunder. Detta borde de ke, fom bog of afta fa litet fesgen.

GRYTORNE woro ufta bår gjorbe fom en cylinder, nteo

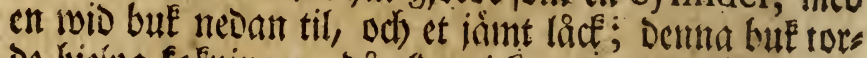

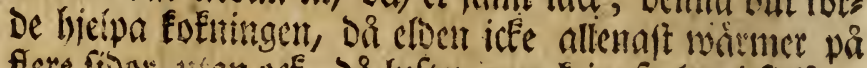
fere fitor, whan uce, da luffer mer Eringfipelar i fielfroa grutan, liafition fom $i$ en olla papini.

BRỏDET, fom băr brufádez, war metendels Tuntrís, tiocet fom et pergament, metr bubbeit fir widot font en

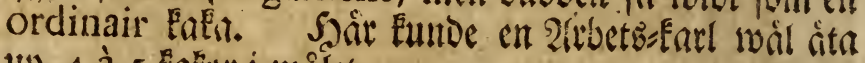
up 4 à 5 fofor $i$ mallet.

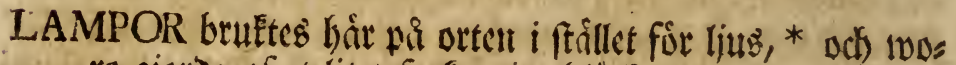
to giorse of et litce furfantigt bläel, til quarters breos,

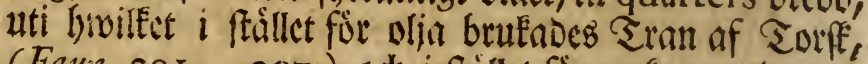
(Faun. 291 - 297.) Dd) i frállet for wefe togo be tor's

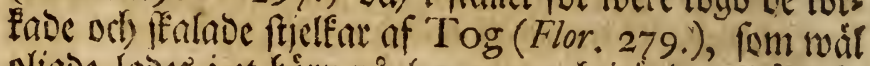

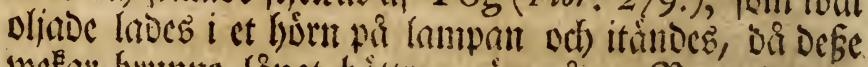

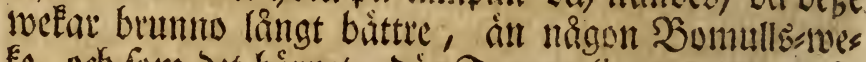
Fe, och fom Det bistnet, Dàr Tranen ligger, serna nâs got bruper, bave folfet bängt inuntoer bet cn Koklóf, forn emutteg oroppet, at traneat ei feulle filliab. Defs fe launpor bängdes merendel' pa frofen wio fipien.

- Kalm Bahus. iss.

TAKEN waro på fífte ftâllen tîfte med Tång ( $Z O$. STERA cller Alga II 37.) til 4 twa rirfingers tjoctle

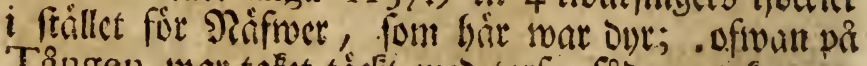
Tången war tafet tấft moo torf; fabana tak moto

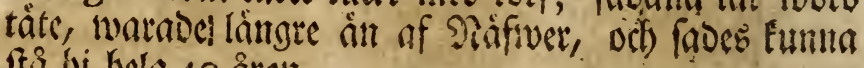
If ai bela 40 âretr.

NATTEN Glcfwo wi i Hôwikenás, fitta gîroen pẩ Sứm,

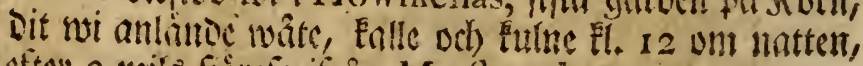
eftei: a mits fiurefa ifrin Marftrand. 


\section{Jul. 17 .}

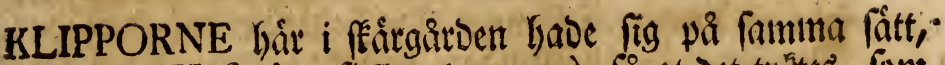

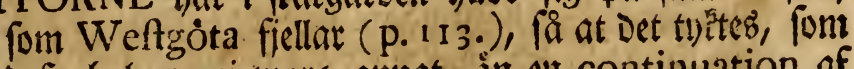
Defe Golmar ci wors numat, ain elt continuation af

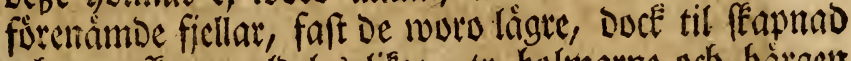
od) egenlfaper aloeleb life; til bolmarne och bargent

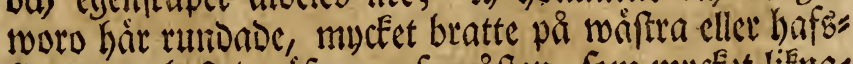
fiDan; De beftedo iffwen of grâfen, fom mucfet lienas

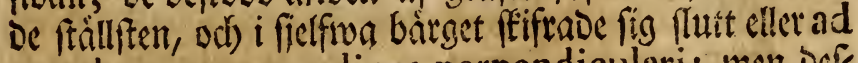
angulum acutum a linea perpendiculari; men DCF

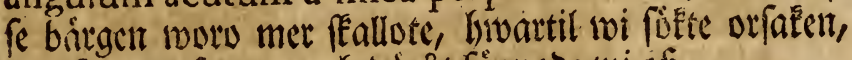
Den famma fummo, oć) Dárât fägnade no of́.

JORD war ingen pi Elipporna, utan be tworo felt renffestgs De, Da be upftego uttit fit willog baf, att watnet fiuts

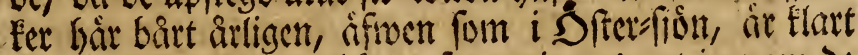
af alla Rofars witnesboro, font minnas, at igenom de inlopy, fom i Deras barnoom funde ingås med ftürffa

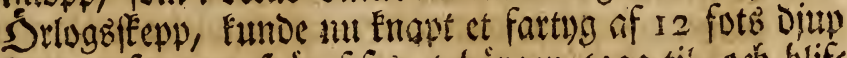

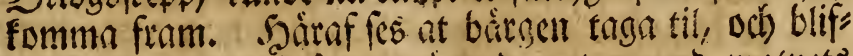
wa hojgre, ci med nigot witrande, utum med matrets aftagande. Ea fitatt borgen begunna Fora un sfivet watnet, fittras de af de rufande wigge, foul pá bem

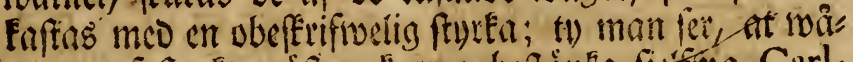
gorne af fraret woiftan funtm beitinfor fivifua Carl-

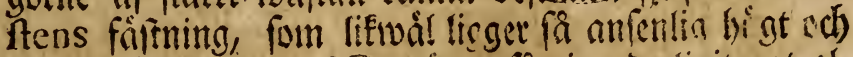

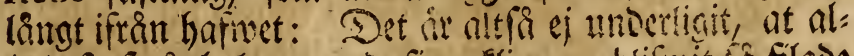

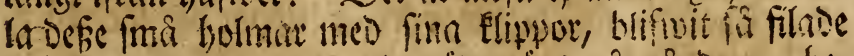

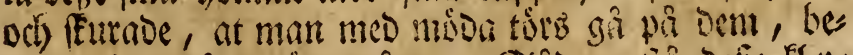

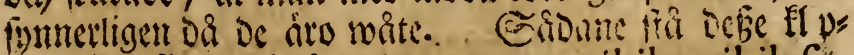
yor, od) Radane blifiua be; tIl ex nihilo nihil fit.

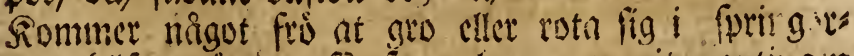

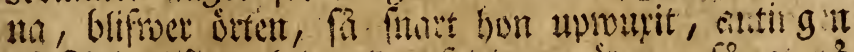

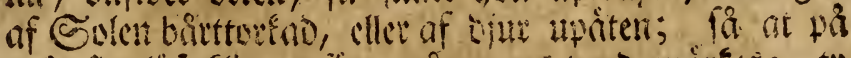

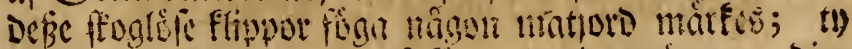
all mylia majte Eommin of formultmade witeser: Ding

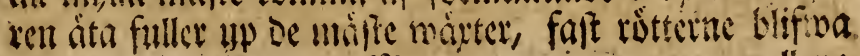
M 5 allena 
allenta qware, foum efter band formultun til mulla; Dode fô ringa, at en orts rot gifiver fnapt fo unyefer nuylta, fom en annoe of fammia llag belofiner til fin fioda:

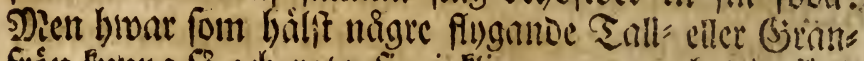
frón funna fa och rota fig i Elipporma, od) Dir fourt

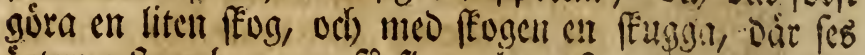

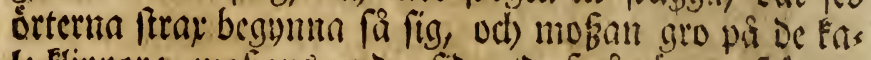
le Elippor; mobans untore fioa, se fimit rotter of orter: na, Eallems ciller Branteng barr fom lemmas orórde af Diuren odh artligen neofallh, ruttna pá jorben, ock

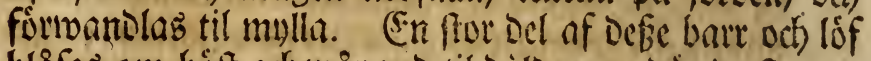

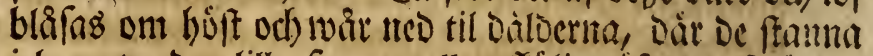

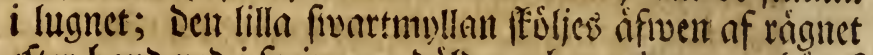
efter hano neo i fpringor, oálocr och) caviteter; bäraf fez De féglisfa Elippor wara bare, tair be féogfulle àro

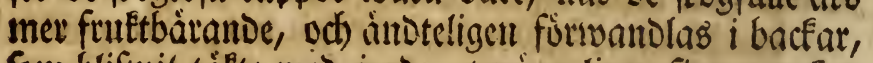
fom blifivit tâlte meo jord, at nappeligen finnes tecfen $i$ Dem ófriget af fourta locerloenes feicf, eller Den tio, oa De nafne upitego utur fit wildoa haf; Daremot hafwa

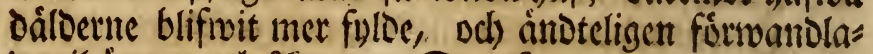
oe til ängar od) áfrar. Den fom ci tror, at fa går

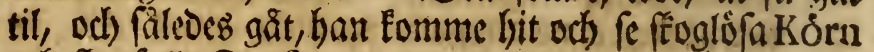
oc) ffogfulla Oroft.

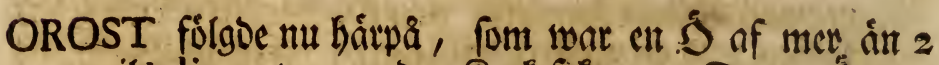
milb diameter, med 7 finterfolnat. Deme S war

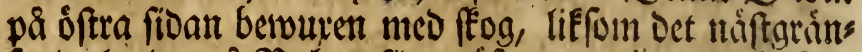

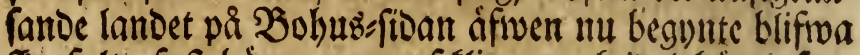
fégfult, faft bảgge woro af flippor ocb) ioel bărg, fom ben forta.

* Swansunds Ssáftgifivaregâro quart 12 ifrån Marftrand.

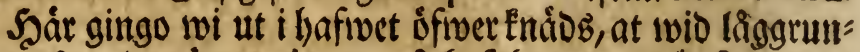
tâ frtanden botanicera pá hafäbotnen; Dár funno wi Zofteram, Ruppian, Soror i botnen, fmá uphogoe 5) Raftegogar, od andra fma Sirit.

RUPPIA. Hort.Cliff.436. BUCCA FERREA-maritima, foliis acutiffimis. Mich. gen. 72. POTAMO. 
MOGETON maritimum, gramineis longioribus foliis, fructu fere umbellato: $R a j$. $\int y n .3 \cdot p$. 134. t.6.f. 1. POTAMOGETON maritimum pufillum alterum, feminibus fingulis longis pediculis infidentibus. Plulk.alm. $305 . t .248 . f .4$. FUCUS tolliculaceus, fœniculi folio longiore. Baub. pin. 365 . Denme òrt àr aldrig formin $i$ Dag funnen pa nagon Sivenff́ botn; lon truper meo fir ftiele nóftan under fanden, upfâtter fima blaber fom cn Subularia, od) băr fina blommor in alis, fom et bet minfta Potamageton gramineum. Radix fimpliciffima, defcendens. Caulis albus, ramofus, articulatus, filj craffitie \& figura, e fingulo geniculo ramos demittens. Folia oppofita, anguftifima, planiufcula, aciculx figura, mollia, bafi membranacea latiufcula, fe invicen amplectentia. Primordia ramorum duorum, fingula duobus foliis conftantia, inter defcripta continentur folia. Spica feffilis, oblonga, inter ramorum primordia. Calyx vel Coralla nulla: fed flos fingulus conftans Antheris 4, feffilibus, xqualibus, fubrotundis, fubdidymis. Piftilla funt Germina $4,5,6$, fubovata, conniventia, ftigmaribus obtufis. Fructus in pedicello elongato, pro fingulo flore ex feminibus $4,5,6$, ovatis, obliquis, terminatis Atigmate plano, orbiculato. Fructus hi finguli infident pedunculis propriis longitudine fructus, hinc fructus, cujusvis floris, quafi umbellatus.

CONCHA fubarenaceo-marina. 2it muflor gifives allmánt pa bafabotten, fiobotten, och i flooer, wet broar o(f) en; at muslor ata fig in uti fielfwa frenar: na wio hafwet, od) Där waja til, at be meo móa fer Dermern funna uttagas, om ide ftenen flas fonoer, mittnar om Pholen odf) Solen PLANCUS conch. 33. Indre utwälia findbotmen, andre lerbotnen, andre mylla, hroarpa wi hár funde fe differencen. Guft Dá wi há̀ fpaferade pa hafśbotnen, fingo wi fe, at botnen pa atfitilliga ftállen war genomborad med 2:ne 
Kål, finalare ån et twât finacr, fort altio froos 2 od 2 jämte bivaraniora, men alorig r cller $3 ;$ wi nisurete Dárjämte ingen upEaftad ioro, och forftoou altfa icfe, bwart jorben efter halen blifwit bartfiro. $28 \mathrm{si}$ figo

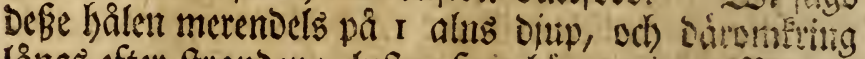
langs efter ftranden; luften fporrbogg ming at fit weta broab flags maffar brufte gora fisbana bal, bein leg

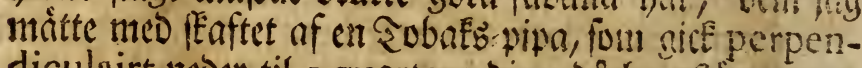
diculairt neber til a quarters Dimp, of bon fiote cmot

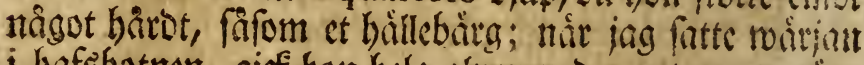
i hafsbotnen, gicf bon bela alman ncod, ttan at mita

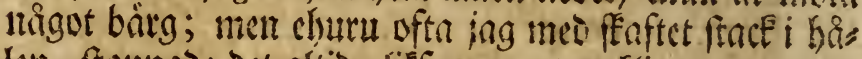
len, ftanmo bet altib, liffom emot ca flippa, utait at nagon mafe wifade fig, ty begunte mi med banderna at geafiwa, hroal fom wi faggo 2:ne bâl, och) nât ini fonmma til botnen pá baren, funto wi en frox mufla, mell ingen mafe; elyutu ofta wi grofno, fa war altio

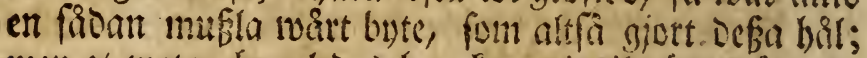
men at meta, bumuleos bon fommit tilwágn oarmed, ciler buru bout tommit fô lantigt neoce unocr fiobotnen, wat cj latt at bearips. silusflan mat cn CONCHA lxvis, convexa, alba, ovata, ftris longitudinalibus inxqualibusoblcuris, cardine interne conjuncto in altera tefta apophyfi alba latiufcula, in altera vero

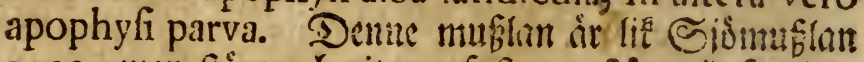
1332, ment forte, lowitare, faftore, ffarr wio finslate fpetfen nágot litet på glint meo cn fubftantia fufca, rugofa, obtufa. Gburu allmin Denne muflan boie war unoer fandbotnen, fägs wi lifwift alorig niggot tecfen til bente wio frumberna, el cller bar iag fumt bente tilfurene i Sincriac. Sen font wilexaminera be

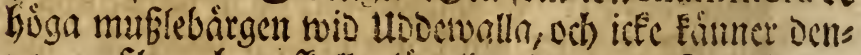
na mubla, han feulle litteligatt tro, at fundiffoben

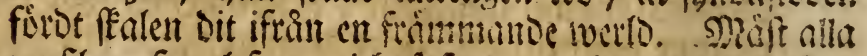

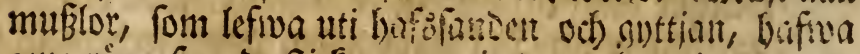

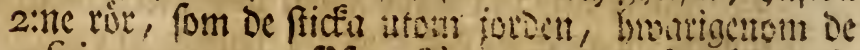
refpirera watnet, fafom Chama * Pholen te Dail, foul lefwer uti lecan unser bofswatnct **, od) Solen, Onyx, 
Onyx, Dactylus Plinii, fom lefwer iti hafafanden, 2 fot diupt ***, brwilfa alla ben frore ReAUMUR bes ferifuit.

* Reaumur act. Paris. I710. P. 573.597.

* Reaum. act. Paris. 1712 p. 163.171 1. 1720

* * Reaum. act, Paris. 17 I2. p. I50. I 58.

LUMBRICUS marinus, ar cm maffe, fom jag for 14 ar

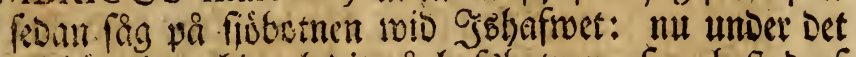

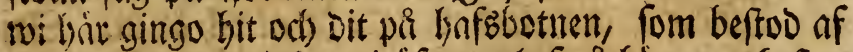

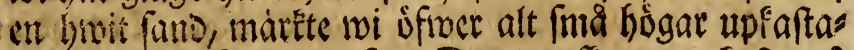
DC, och juft fádane, fom Daggmaff́ane upfafta pa landect. CSSi grofivo, och) fingo àndeligen igen et Djur, fon forticanar fint egen och tyocliga beffrifning, Gnilfent

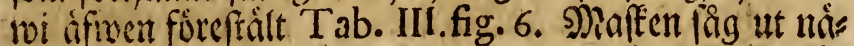
fean foin en Netemaft of I quarters längo, och) lifa tioctlef med en Emanzpenna. Denne maft har midt páet utthángande Vifcus, iftin brilfen bat alt intil nos feet âr fusvoelo i 14 à 15 ringar eller legmenta, af biwils Ea bywarbera beftar af 5 minore fegmentis, och af

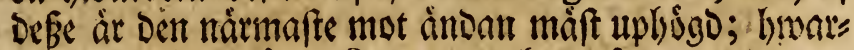

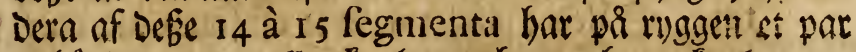
unhiogie puncter eller Enulor, och utur fivar fhyl ct ups ftäende liar. smen andre delen, eller den änden, fom âr

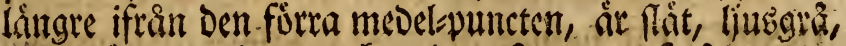
mes piámua loingar od tylor, font meo fma puncter áro beftriboe, allenaft airo 4 ringer cller fegmenta på Denna fioan um férenämoe Vifcus lifa med de förta.

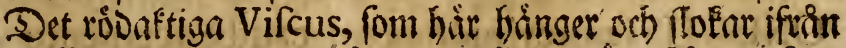

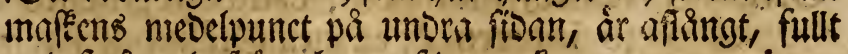
meo fimå gula frón, lywaraf Det tycfeź mara ovarium.

STRANDKÅL, Crambe (Flor. 570.) toôtte fâllfunt pa bolmatne mid franden; men nog umnigt pa fódra fis Dan om Marftrand. Folfet berinttade, at en Sorgas reshultru i Marftrand bave bämtat fádan Ral, fotat

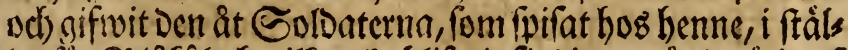

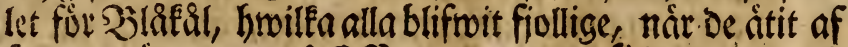
fauma ritt ; men Da Officeratne underfoffte Derom, och funne wäroinnan lifa tofig med gafterna, måfe de 
lemna altfammalls. - Siutbomen botade fig ficlf bas gent Derefter.

HAFS-ÅRTER. PISUM petiolis fupra planiufculis, caule angulato (Flor. 608.). PISUM ftipulis integerrimis. Hort. Cliff. 368. AEt. Fockb. 1743. p. 1:10. Flor. lapp. 272. waitte pi niggra fratllen iblanid

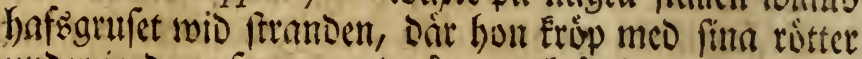

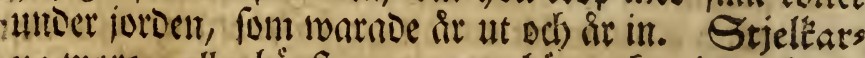
ne woto a cller boggft 2 quarter bogie, Eantige och nas got litet litone. 2slommorite woro blifbrofige. Stis

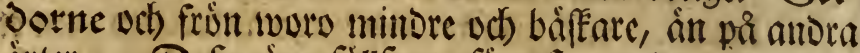

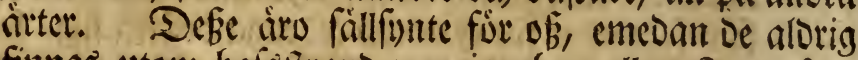
fiunas utom bafsiftranden; iag hat allenaft en gâng feot Dem forrut wio aloraúfiwerlita fanten af Botnifka swifen, Dár De wainte uti Groita fanden; iag har plan=

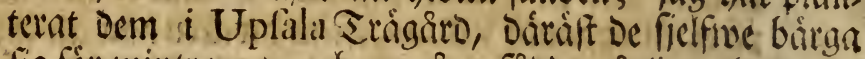

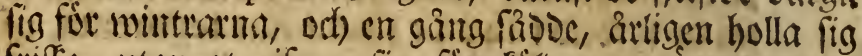
friffe, utton at gifiva fig för Estocir. Pitterne fun=

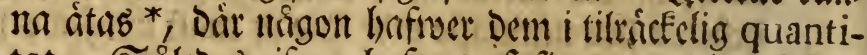
tet. Silleder sifner bafinet ó fima egna djur, fifétar, muElor, Eill och) ârter.

* Flor, lapp. 272.

INSECTER fummos atffitllige i hafivet, itfano bnilfa iag marfte 3 llag, fom alorig tilfurene marit befferefne, näml. 2:me Onifci odt en Scolopender.

ONISCUS femicylindraceus, caudx fegmento ovato oblungo integro, âr un liten (jirafugga; har 7 fútter Hai hivardera fitoan, men Dernted feilies hon ifran de anowa, at bon icfe cir oval, utan lié en balf Cylinder, Dertil mes fögn ltörre, in en lus. Sticten beftär of et enoa aflangt ftucte, fom ár utan fostter.

ONISCUS ftriis transverfis fufcis fant tillita med Detr fürta i bafiwet. SJufwabet âr Dubbelt ftórte àn en luz;

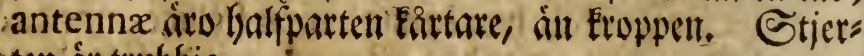
tem âr trubbig. 
SCOLOPENDRA plana pallida: linea longitudinali rufa. Dettri lilla infect, fom âr Enapt et troárfits ger lingt, fans ritt mustet $i$ hafmet. Jonl ár fmal fom en tra, har firarta joon, många bleta fótter, och en blet trowp, hwilfen lângb at ruggen bar en róbaltig linea; fierten beftar ytterit of en rumbaftig led.

SKÅLAR (Faun. Ir.) Forade bair od) Dár up utue matnet

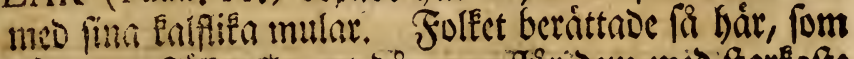

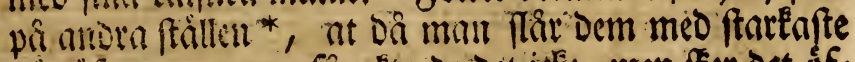
fior iffwer ryggen, fâ afta De Det icfe, men ffer Det of wer noforn, fit oo oe litteligen od) frrat. Pedes anteriores manibus vix fiffis: unguibus 5 , cultratis, digitis interioribus fenfim minoribus. Pedes poferiores retracti verfus caudam: plantis palmatis: unguibus 5 rećtis; digitis exterioribus 1 , 5 . fenfim longioribus; 2. 4. minoribus; 3 minimo. Cauda breviffma, planiulcula, obtufa. Auricule nullix.

* Ir. Gottl. 270.

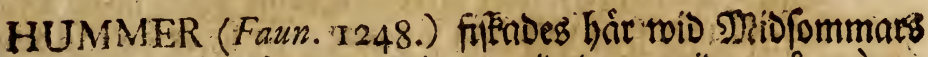
tisen pî 6 famtnars Diup; wid Denna tisen pa 8 à 10 famnars djup: men frain pa bơften pli is à is fams nats Diup; fî at Scinurarne mits pif fommaren bolla fis mismoint intil landet, of feocrmera fafom Solen forfoga fig alt mer od) nect bärt ifran waira frander.

HUMMERTINAN, meo hlvilfell Summarne făngas, ár

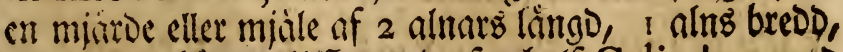
orh I alng hogo, til fapanad of I half Cylinder, med ingingur pa bagge aitoar; Denne beftar af en flat bots tell, pa builfen 2:tte halfrunda bogar uprattaż, pa bos garne linggeb 3 fpichilar cller cofta longitudinales, hivarifirer waifives et garn eller nát, meo ingång pa bigse inour, fâfom par en bama eller micile; på bots nen lisiges et par ffenar, nt timan ma fiunta neb, ods wio hems bities et rep meo on toete eller racfa, fom

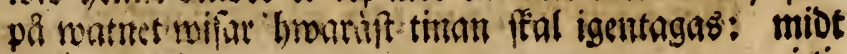


I tinan bontrges en bud fife pa ér thí, bwilten air sent

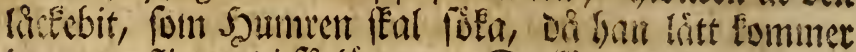
ith, men flipper ci fi latt ut. De fämonos Scumraute

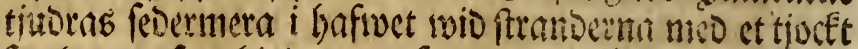
fegelgarn, fombindes om foten, vib) for sem uttognas

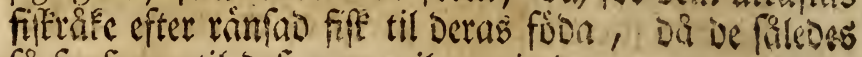
fá foafera, til Des man wil nistta Dem.

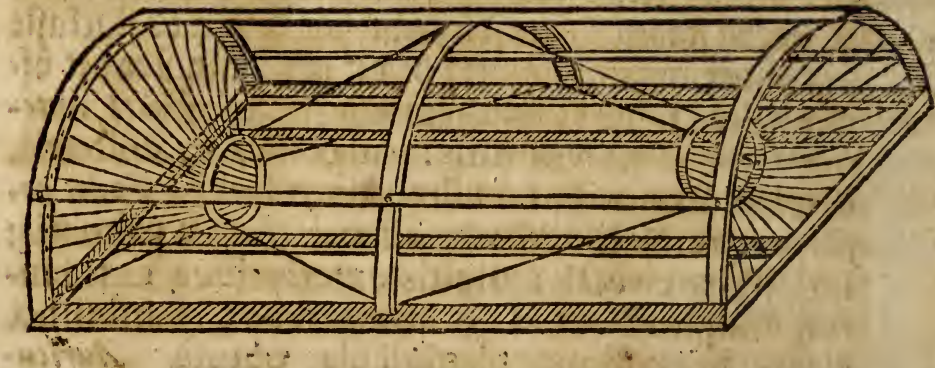

Llii figuren fone jag wilat Det, fom uti timan are af trd givetot wh) utftafat ingangarme; om lafaren lisgger alles

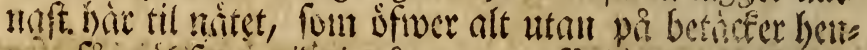

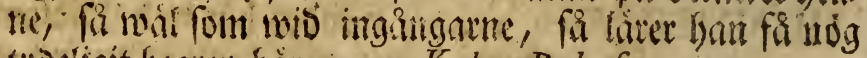
twoeligit begrew boir sm. Kalm. Babuf.

GIRDAR fagos itfeillige pr Oroft med metiga lágenticter,

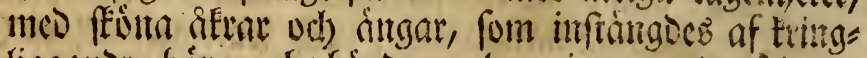
liggande bairg od) bigoer; lymarigenom de frinoes ifrait ftark Esolo od blalt, fomt belsollo langre de wats me Golenter ftrálat.

IIGUSTER (Flor. 4.) toilte hit pi Oroft willot, fom utomi Dentu fentgaro nappeligen ar funnen $i$ Siverige. Silg fang bet federmera i S rigardarma, Dair Det wajte fa frodigt ods fô bärligit, at jag alorig feot des liée;

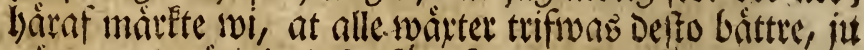
natmare de siro intil fin fúbelfesort. Derfore ffulle iag rion Dem, fom $i$ Siverige wille anlingga LiguterSactar uti fima Srigardar, at De icfe bômta Liguftret ifran Holland eller nigon fiarmare ort ; ; utan bălore 
Gâlore ifrån beß̉e nordiffe lánber, fom båttre paßa fig med waitt climat, Gwaraf man Eam wara fáter at Det

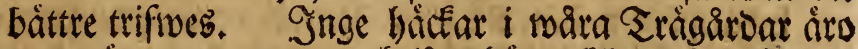

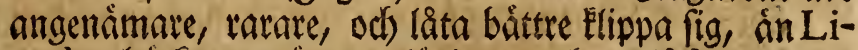

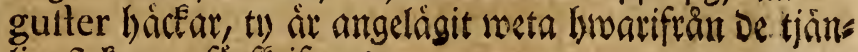
ligaft kuma fürffrifionob.

CONCHA Pectunculus dićta (Faun. 1340.) Eaftade

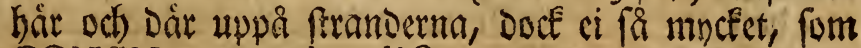
CONCHA Mitulus dicta. (Faun. 1333.)

TREMELLA fubrotundo - finuofa difformis gelatirofa fanz allmànt på Confervis $i$ bafiwet, ofti beftod liffom af et gelée til confiftence of Tremeila (Flor. 1020.) ods til anfeenoe of Tremella IOzI, faft hou

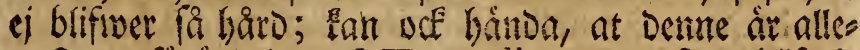
naft en forrintoring of Tremella 1021, fom blifwit minore och lofare of rummet.

TREMELLA hemifphxrica fparfa. Da mi gingo på Elipporna, fom frabigt tooro wite, fis wion watnet upfranltes af wighurten, märlte mi, at fötterne altiemt wille finta, howilfet fom af en ocinoelig hop groma grisn, fom woro liefom fällade pá Dése roista flippor: grynen woro til formen balfrumbe, til confiftencen

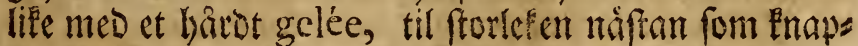
penál Enappar; De mayte immediate ivid Elipportn, och latra toara famma art med de fmá grynt, fom ofta finas yå Confervis marinis.

MANIETTER befiumerligen af MEDUSA (Faun. 1287) Eaftades up pir alla flippor wid hafivet.

FISKANDET fEeDde hầ merendels med Nåt, frol, ellef

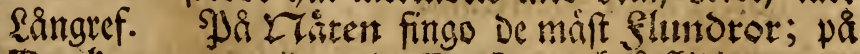
Kroken genoni metmoe Corfe, och attfifillig amman

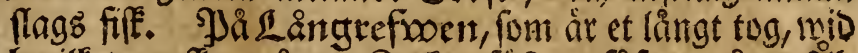

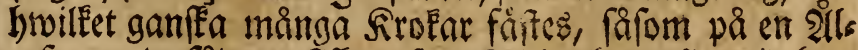
ref, togo de fädana fiker, fom ftadigt bstla fig wid bot:

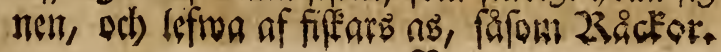


SMÅ BATTAR fäg man merefas af en Sart, fom $i$ bafftams

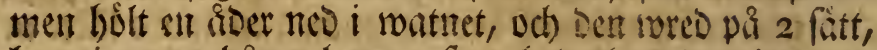
hwarigenem baten lopp, font bade bant oragitu med sider. Denne nu allmintme Eonfere bafiva folfet fiefert lärt af fiffen, fom meielft fut ftiert gove fit mâfta lopp.

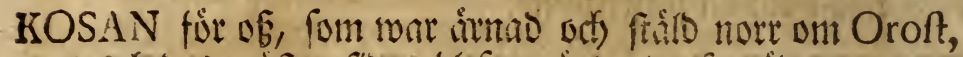
oct) Des waftra fida, blef nu ambrad of wadoret; cmes: Dan et ftarti wáftan waider ef bebagade tilftidia of for Denta gângen fouma lingre utt, ty mâfe wi moa och fülia reveen it Uddewalla.

UDDEWALLA-FJÅL låg rîtt $i$ wiffer fớ Uddewalla fard af 2 quarts lingo ; pa et ftâlle war han fá grund, at ban ei flippte from fartug, fom moro iffere 12 fot Diupa. Smterfita inloppet, eller intom bamnen, dir

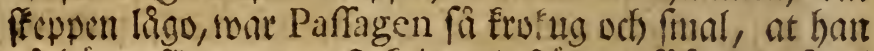

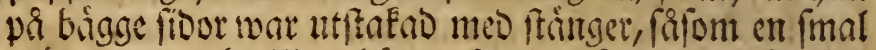

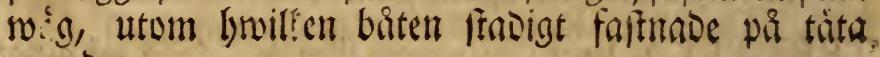
stumber.

BOMMEN wio Uddewalla war om aftonen tiffuten, fom

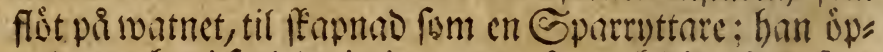

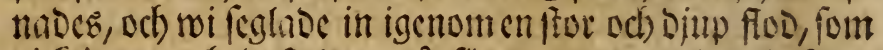

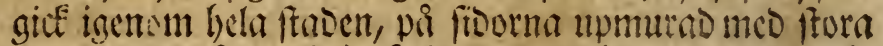

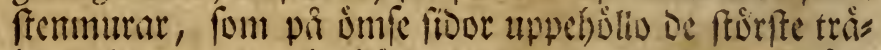
byggningar, uttur bmilfa genom muten woro neigain gar med frentrappor ifrän fieffiwa bufen, at man immediate funte friga ifrån fórfirugan noder til watnet eller baten; fâ at wi ty)fte of Eummit $i$ fjelfiva Venedig, oá wi fommo til Uddewalla.

NATTEN fom of at hrmila $i$ Uddewalla, efter 6 mils fiơrefa ifri̊n Marftrand.

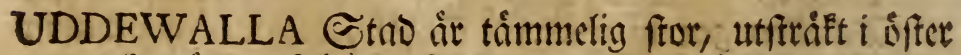
oct) waifter pa bànge fitoor oin en ftor och) fitlla flutande flod, Dir han gâr in $i$ hafivet, getnem en lâng fiol. Soulen aro fom i Gótheborg frora tribuggningar, bes firmertigen at wajira fiton af fiaden; be aro majptedels 
betálte med tegeltak, forrfeode med feina formfer, beflas gne med brider, if froerftrulte med rijot och mâlade med bla eller Gmita Ynutar.

HUSEN hafwa emellan fónfterlufterna ci någon horizontail flo af timmerffactar, efter Det fätt fom allmaint hos of ấ antagit, utan perpendiculairt influggne fractear, bwilta gưra at woiggen emellan fónfferlufters na alorig far bugt, eller gifiver fig ut; Detta fâtt àr bäts tre ân wirt; men fan Docf icfe brutab, Dôn bufen icte

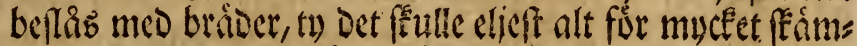

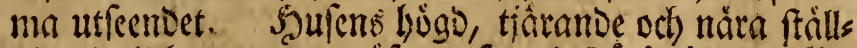
nimg intil lowamondra, affmen font i (jörheborg, fîts ta fraden i frürta fara fêr elosm fonmen, froitligen wio fadan byggnad Ean bämmaE, cinuru Stadoente härigenom blifina muctet tâcfe, lâts te ach angenime at bebo.

TRÅGÅRARNE befïgos, och mi funno uti Siơomin Jóns Kocks Trigianto en Trâl hola of 500 fima frułts

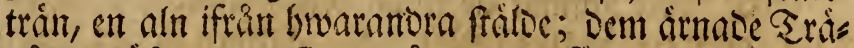
gårosintáftaren omflytta på ryggave fängar, efter Det allmànt bàr antagne Principium, at ju boggre en wákt

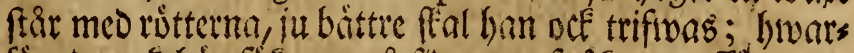

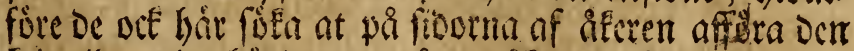
fringliggande bigoen, pa Det kiecten må ligga något bogre an lamoet, bilf fom be bir utrônt, at fiden wäs ler dả frodigate; men lyad trâ angar , bưr man fe fórut, at de ruggabe fängar, pa hyoilfa träben warba ftâloe, torde meo tiden af rágll och) féurar jämna odj planera fig fielfive, oa trídens rütter warioa blots tade.

TOBAKEN, fom bair worr planterad, gof of anlecining at ratt forrfta, hwarfüre Tobafsfängarte allmánt gơ ras pa fiborta mes Djupa perpendiculaire gångar;

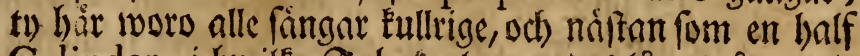

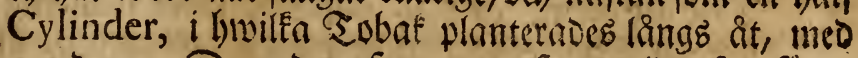
5 raber. De rader, fom rodo fatte midt efter fängs 
ryggen, woto ratt hoge wäter, off ffulle fitatt ga $i$ blomma; men de plantor, fom ftodo i andra rabeit, els ler pa fiborm, woto ligre, od) De, fom frovo i utterfta, eller nederfta raden woro fi fmi, at ie cirmu icfe bes

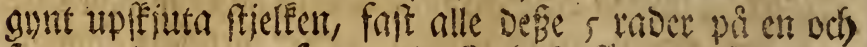
fomma bag woro fatte; De Sobaleforingar, fom lago

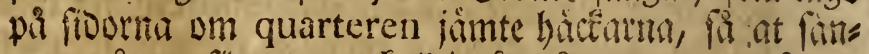

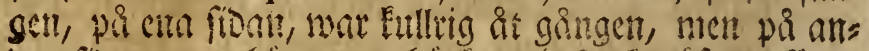
bra fluat wai bog emot bicker, beftudte afwen forra

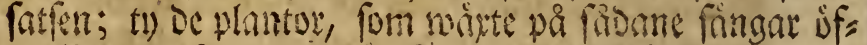
metir eller pa ruggen, dar Golen fanengt babunde emot

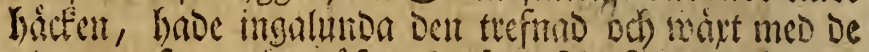
Hlantor, fom wate sfiverfi pa de fria fangar, broilea bade ojupa gangar pa smie fidor; alifa bartotera de Sobatiolnitare ratt finc fängar, fom pa sme fivor bafwa Djupa gängnt.

WINTRARNE bade fa bucrat med Trigåroarta emels lan âten 1739 odb I74I, at Det aimm fowed Därefter, Dori bock famma ai félat warit muck lintorig minter

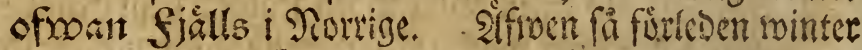
emeilan 1745 . od 1746 . war Den linorigafte vointer $\mathfrak{i}$ Grverige; ment Montpellier fa frate torb, at all fpiritus foll med i Enlan pa Thermometern, emot vas

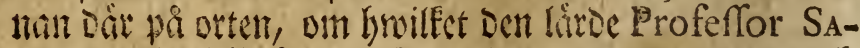
VAGES mig tilffrifwit. Sal, wintren emellan $1735.0 \mathrm{~d}$ ) 1736, ba broe hat i Gwerige, od där jag wiftades i Sollams, war Den linbrignfe, fros SBrimuimat i Ealls tarne uti Noveborago i America, Gwarifuan un bade bref: Sairnf tuctes Det liffom de ftarke wintrarne hwart ar feulle loolla fig grummate pa nagta wiba ftäls len $i$ werloen.

THE hade Ylrobjen WALBorg forfárbigat af en Gromente

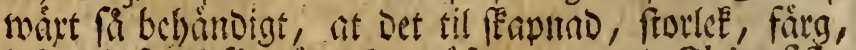
luft od) fina fi nota fom ifiverents meo Chinefinkt Thé de Bou, nt Den, fom iffe wore fommerlin fans

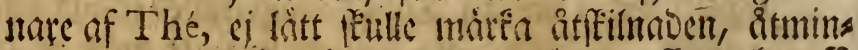
ftone om en blefwe giviole litet woind, ffulle ban fa 
geunt orida betta, fom oet Chinefinka. Tagh hat ouctit itffilligt Thé, fom warit tillagadt, at imitera oct Chincfíka; men mins mig icfe fmafnt något, fom fommit noimore. Inventor baraf fabe, at ban fum: De furffrofin fig buarie bag mainga Seb. af Detta Thé, od at lyan babe fita orfater, broarfute ban idfe nu

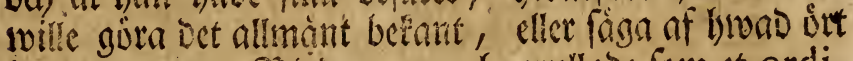
blaben togos. Silaben moro hoprallabe fom et ordinairt Thé de Bou, orb fáranoe icfe gutlt; be utflogo fig i warnet, afwett fom Thé-blad, od bave ungefar famma ffapnat. De noro fi wál afploctade, at wi icfe pa et enva fatlle funte fe, oni folia woro oppofita eller alterna. Set war ej arfitinoigt fö cn Botanices Profeffor, at fråga cn anman nammet pâ en Emenfe ore, da ban fielf habe blaben for fig utflagne

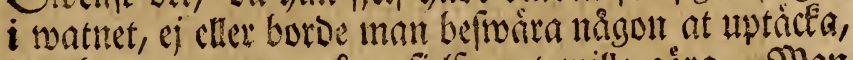
Det bait en amue ging fielfmant mille goira. Nan bir loonon: pris, fom et faonnt Thé paffunnit, od fa natt præparerat, bâlit fom det är tagit of en ort, for ár lambt barfofammare oin Thé trabet, och bos of mas ter ganfer mucfet, ment aloramait pa Carls-barma.

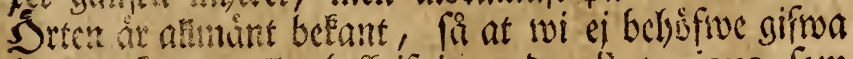
hemes figure eller beferifnimg; Den Botanicus, fom

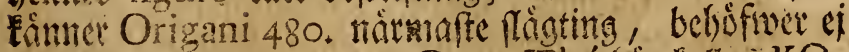
mitare nuin befrifuning. Setta Thé bur Eallos KONUNGS-THE, efter wi bafrom fiefar:Thé, fom fommer iftån China.

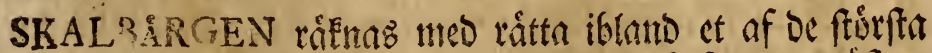
Bohus-Rint under; ty De figga uppa sanoet, miftan hela quarten pa fomliga fätlen, iftän hafivet. Defbe

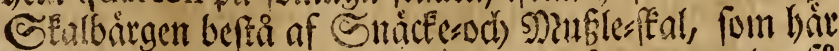
àro famlabe i Den mucenbet, at man fan unowa bet ia

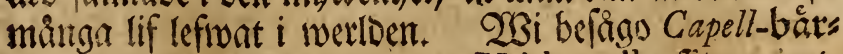
get, fom låg $\frac{x}{2}$ quart utom Uddewalla foore port, Feoan gingo wi til Sammered, fom ling triffan I quart ifrain froden $i$ norooft. YJa brigge ftifllen woro Defe SEalbạtg; befunnerlig och formànligaft nis Sammered. Sar woro bare ab fullrige graftenb stipyor श्र 3 hivilfa 
bwoilfa pa ben froan, fon wette ât ftaden eller floben, Dár inloppet forbom warit, lutade neder åt. Gordent

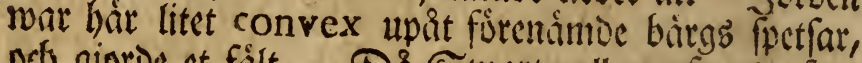

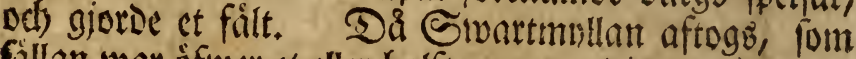
fallan war iffwer et eller halft quarter biut, mitte bets

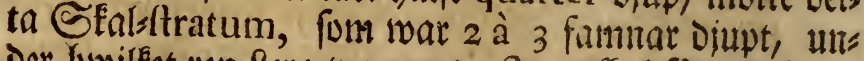

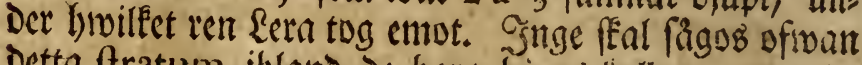
Detta ftratum iblano De bara bargos fullar, utam de singo alle i fä́t ifrän bärgen nedfore under jorben, of ta til fere buferefottb breod. Sfalen lảgo bàr rena,

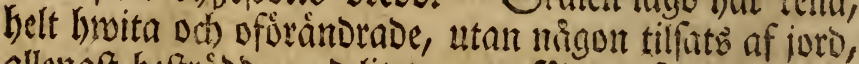
allenaft beftroboe med litet grus, fadant fom upfaftas mid frenterna. Sag füte meo forgfíllighet alla be

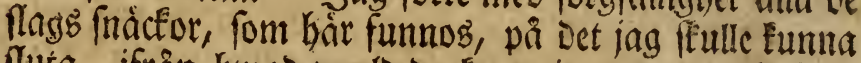
fluta, ifrän biwad werld de fontmit, eller om bafwet âfiven fom lanoet füränorat fina Snbuggare. Sag fial hit fpecificera Dem allefamman, oct) uti $T a b$. V. af: tepha Dem, at De minge bifiwa tyoeligare forr hrwar och en, Doce utefluter jing No. 5. och) 10. uti figuren, fafom för hiwart barm betanic.

3) LEPAS, qux BALANUS Uddevallenfis. Tab.V. fig. I. Dente àr frourre, àn wåre Balani, fom nu allmånt finnas $i$ hafivet, ock beftât of 4 ftörte valvulis erectis, brwilea up ât alt mer och mer gisna ifrä hwarandra, Doce fa, at De emellam gifnimgen áro. ilsups bâftade med tummare feat, hwartvid it at märfa, Det 2:ne ganff́a fimala valvulx fitta emellan de 3 pá ena fidan, fâ at fórft aro 3 frora valvula, fooan I fmalas

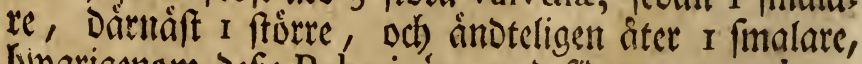
bibarigenom defe Balani âro meo fidouna 6 -valves. Denne ât allmànt befant utumlanos. Figuren ầ gifiven af Seert ARGENVILLE $i$ Dé Concbiologia tab. 30. fig. A. Detta féal ầ hăar Den allmannafte, faftån valvulx, fom nu aro ffild ifrản bwaranora, ciro fints emellan olięe, och) frwàrligen Éámes of en, fom ej ác bemma i Paturkunnigheter.

2. CONCHA oblonga obtufa: fulcis transverfis. Tab. $V$. fig. 2. Deme ât en liten.mufla, aflang, trubbig 
mot bágge åndarna; fealen åro ifsopfoffte mot bet trubs biga ánjoan, utanpå hafiwa de grofiva foror, fom gå

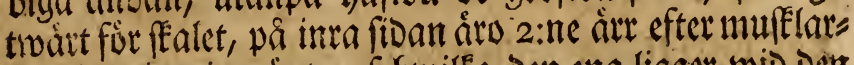
na, fom aro irgriopte, of hiwilfa Den ena ligger wid Den trubbigare atroan, Den andre up emot baffanten. Deme ât maft intil Den fustra allmainnaft, och gớr foürfia quantiteten of finalen. Sag fifulle tro, at PHOLAS, five Concha, intra lapidem cretaceum. Liff.ang.

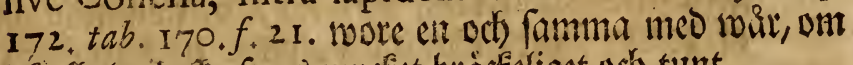

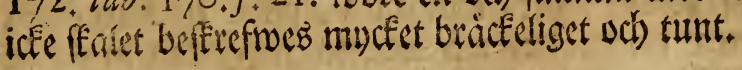

3. CONCHA lævis, altera tantum parte clufilis, apophyfi admodum prominente lataque prædita. $L$ ift.

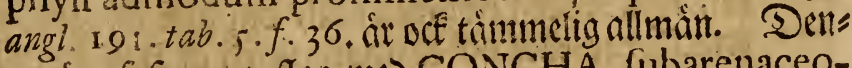
$\mathfrak{n e}$ ar of fanme flag med CONCHA fubarenaceomarina, fon i går beffecefs, Dár lyon låg mco fina twå

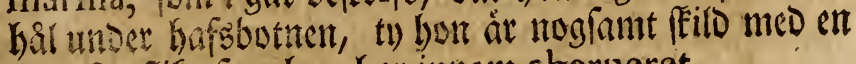
liter fenfifie, fom hon Gar intom cherneret.

4. COCILLEA tefta ovata, fpiris quinque ftriatis fafciatis, aperturx margine poftico dilatato rotundatu, eller KUPUNGE, fom Lift. angl. 162. Tab.3. f. 9. beffrifruit, mindere allman.

5. CON HA Mytulus dicta (Foun. 1333 .) nffiwen bât mins Dre altmont, och faft hon miftat fir bla fárg, bar hoon

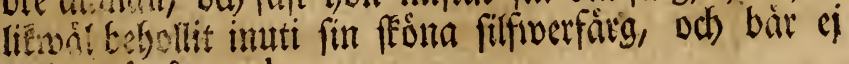
tecten af nigot cherner.

6. COCHLEA fpiris quinque utrinque producta ftris acutis imbricatis. Tab.V.f.6. timmelig nlls mån; Denne har mid bafin et roftrum, gettom bmil: Fet Den ovala opertura evacuerar fig uti en canal; houn har ftrias longitudinales, fom gå helt tâtt ut med biva $\mathfrak{a} \mathfrak{a} \mathfrak{a}$ kanter.

7. CONCHA Pecten dicta friis numerofiffimis.

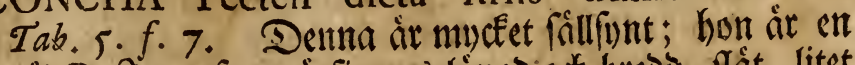
ród Pecten af 4 trwainfingers lángo od breos, flât, litet $\mathfrak{R} 4$ ferynts 


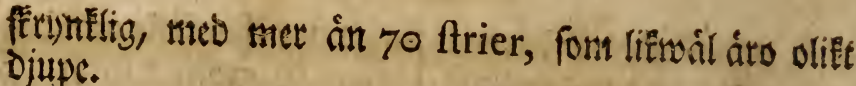

8. COCHLEA fpiris octo oblonga utinque producta lineis duabus elevatis. Tab.V.f.8. Deme àr aldrararaft od f fürft.

9. LEPAS Concha anatifera transverfim fulcata longitudinaliter ftriata, Tab.V.f.9. trimmelig allmain; af Demina finter allenaft valvulx in angulum acutum porrectx, odf undertiden pa fanter fórófte med en laing fmal valvula, fom bar ftrias ad angulum rectum transverfas. Emedan man ei fumnit Bentn hel, ty lenmas i twifiwelsmål, om hon ấ en Concha anatifera, eller off et operculum af Balanus No. I.

10. CONCHA Pectunculus dicta (Faun. 1340.). Sairaf feb, at alle Defe feal án af fäbana hafabjut, fom

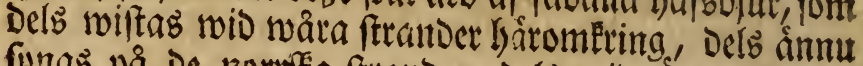

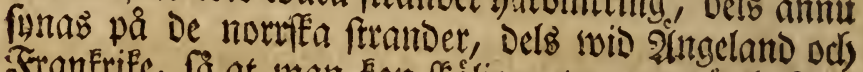

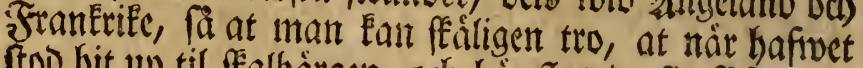
ftod hit up til ffalbairgen, och bat futade fig fafom uti en foitz, befunnerliga fom De maffe ficiten bair utanfos: re Dá legat. under watten, bafiva Defie feal af ftarfa máftanwäbret blifivit Drifte bit uv i wriell. Det ấ ci unoerligit, at nigie benna tiocn Dragit fig til mer foore orter, ty man fer bet famma pả Sollanoffa Sils lent, fom fórut warit i Belt, fècermera hăr pa Sufterna, oć) nu fưr tiden tedan fommit til Lovers Banc.

SKALEN af Defa barg brukas til Falforantureri od til hivits menambe; pa DeÉa Strata ligga nu umbertiden źrear; Dár åklar ligga pá fabana fenlfált, gifwa be twacfer fíd, allenaft Där icfe fommer mycfen torka: pa atrora ftål Ien lagas sandoblocigarna meo Detta srufet, fom Driraf blifwa mucfet fafte, ty fíalet upfuper ivaffian. De ålofte féalen, fom ligga hoggt odb längft ifran hafwet, âro De loffafte och) lywitafte; med deß̧a gnidas gâlfiven, feban de áro rentwaittade, at De máge blifiva bwitare, 
ach) bormerne gnida barmed bảbe wåggat och taf, at govit Dem ljufare.

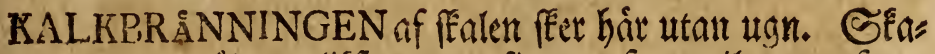
len upgrâtwas liefom utur fina grufiwor, til et par fams nars Diup; De fallas igenom et groft fóll, at hafasłan Den ma lenna bein, ty annor jord âr iffe blandad med Dem. Sernáft lággé̉ tweo och) féal ftratum fupra ftra-

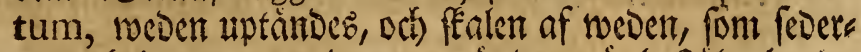
mera brimer et par Dagar; taer Detta âr beftält, begjus tes be brände féfen med watten, fafta baftigt in $i$

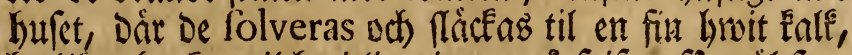

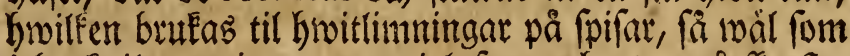
i bruk til nuturning, men i luften och utan pa fererftes

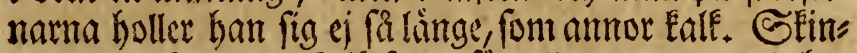
redare brufa Denma Ealf fram fór all annor, at med hoo nom taga baren of fimn frint. Sm Dȩ́a fes Kalm. Babuf. 180 .

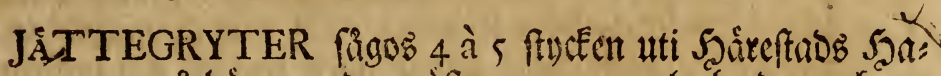

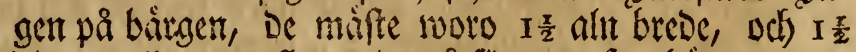
Djupe, alle worto funttande pa fiodrue efter bärget; ges mene man trodDe, at inbygganne fordom brueat Defa bal, til at $i$ Dem mala fim fâd, fôtrain be wifte of quare nar, eller offé at bäruti fista fit furn til grun, men an= Dre beristtade fig funnit i defia has frora fenar, til fáp: nob fom en fanmantrutit glob eller lentiformes.

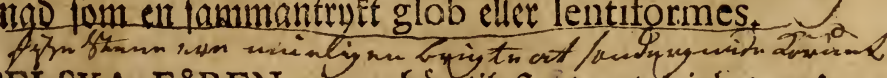

ANGELSKA FÅREN gingo bir til ftort antal $i$ bet med De gemena, och) ffiltbes lätteligen fá wail med Derab tioss $\mathfrak{E}_{\mathfrak{a}}$ firanfar, fom med bet at fcrotum hángie med

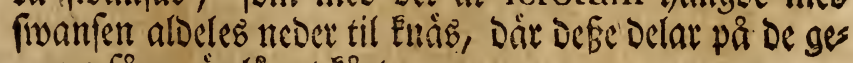
mena fâten àt långt kârtare.

HEDER 190. Waitte frtar utanfise fobra porten af Udde-

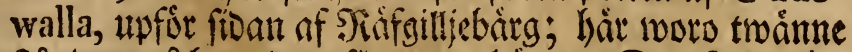
ftând, et pảswarbera fitom om barget. Den fom twak:

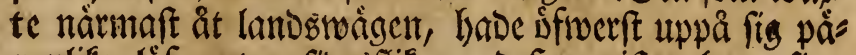
roulife löf, utan fibesfiefar; Debe amiftar boro fina I s

bloms 
blommor bittioa on waren, ofl hafiwa blifwit of Botanifterna nobiliterade med namu af Hedera Poëtica. Sgondoentie bruta blnden at lánga pá branmfar,

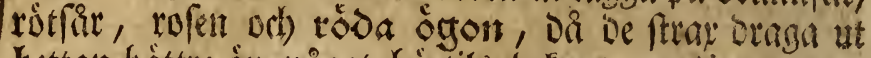
bettan bättre ân ntågot bourtilb beEant medicament;

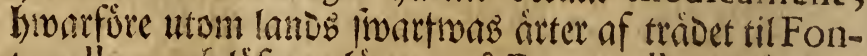

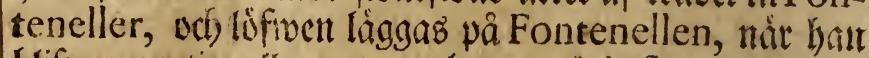
blifwer oartig, eller antager hetta, och) inflammation.

\section{Jul. 19.}

Soag blefwo mi quare i Uddewalla, od) refte ut at befe Surbrumien.

STYNGET, Oeftrus (Faun. Y024.), eller en fluga ftor font

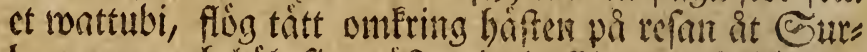

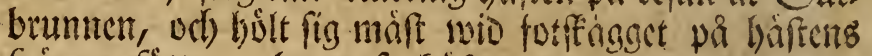
framme fütter, bsoaraf baffen orondes, ftamwade odi) cfomoftaft wille ftarna.

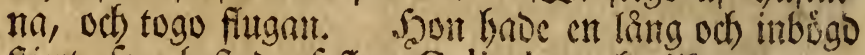
ftiert, fom beftod of flera Cvlindrar, Gmilfe woro uts Dragne utur broaranora, fafom et perfpectiv; men baoe ingen tagg, havarmeo hon funde fira. 万utrous

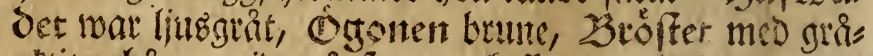
attiga has, midt pa fivart och ffillot, magten gul, Doingarne wattufargade med cn ftor more twarflice,

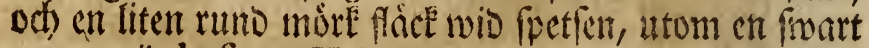
punct wid bafin. Halteres cller, fnapparne unber wins

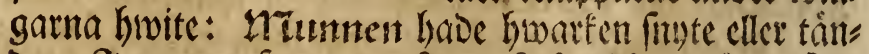
Der: 9 magen futuos många fmia aflanga cigg, fom

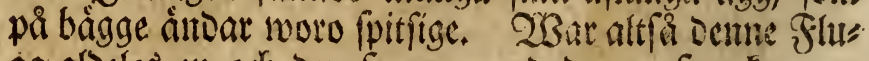
ga aldocles en och) Den famme mes Dem, fom Fomuna

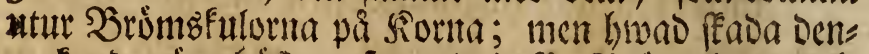

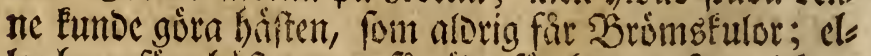

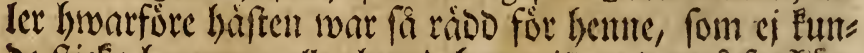
De fricfa bonon; ; cller bwad hon wille neder pif fotefígs get, wet jag icfe âmu, th) wetenlespen om de minfta Eráfenz Politie are ânu til Det minfta fumnig, odj 
mucfet ljus ffulle merlden fa uti âtikilliga fafer, om man bave mera tio od shoga at utrúna Defie trienens hemliga waiga:; Det ât Doce aldeles troligt, at Denna

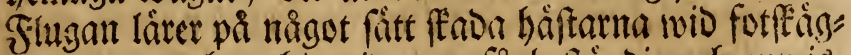
get, emedan bou hartwio war fa beftimbig odf crivis, at bar plantera fima fro; famféflufigtalle eller Sprttt, eller någon annan fotspafion bog bifterme of bente fordrfakas. 2Bi wete icfe åmm, hwarfore forta, fon om fommartiden lopa for Denna Fluga, ftanna ooth ita fifte, fa fract De fást Eommit til watten, at watnet far iffiver forleden. Gag har uptaft for werlocit, at Curbma, fom plagar Sapparnes Sienar, àr en antor fort af Detta lägtet, od) at Sicnarne für Derab flul om fommaren fote bogoerna, faftint Den, fom fenalt beferifuit Rappland Dingit Detta i twifwelsmal, emes

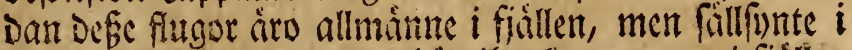
sirpomarten; ment man bort ibogtomma, at $i$ fiállen witab de máfta Ficnarne om fommartiden, o(t) ganfer fa blifiva quare i sappmatect; man bor ocf ihogeom: ma, at flugorne uteomma enbaft och allenaft of Jiss narnes rugg mibt pi fonmmaren; ât altfi nos mándigt, at flere flugor finnas i fiăllen woid en Sioja, fon áger rooo:De Sienar, än wiD cn Sioja i Sappmarken, fom bar ro Sienar; om jag wil rifin fafom et medium 3 flugor for limar Sien, fi fattle wio cn fadan Soja $i$ fiâllen funas 3000 fingor, da 30 funnos i sappmnes fen; men Deremot, om alle Sienorte, fom aro i fiallen, fifulle om fommaten wiftas i sappmatfen, wors nids fafeligit, at ingen fluga fletle fimas i fiällen: SMed et oro, bela fáenen Eonimer Dirvi nn, at mig Glifwer lärot, om Sienfrunget Curbma nigonfin Eommer, fober els

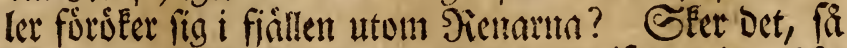
bar jag orietis fatż, men til Def bet wifas mig, fare min fats på faftafte gruno.

HEDER (Flor. 190.) waite wid et birg pa winfter hant

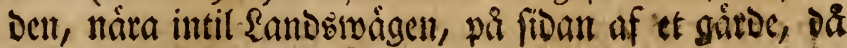
man refte til 3 aggetofta. 
BAGGETOFTA SURBRUNN ligg I quart ifrin Ud-

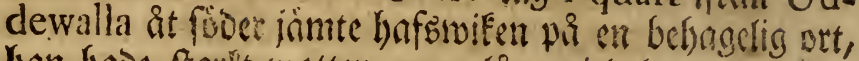

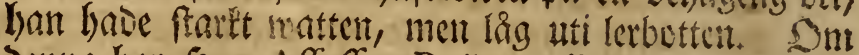
Denna har Sacrt Affeffor Boëtıus ffrifinit en fpeciel Tractat.

CALMUS (277.) wakte willot til ftêrfta muckenket, uti

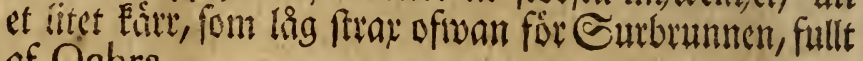
af Ochra.

STRUTHIOPTERIS 84I. maipte i Baaggctofta âtr, iems te wrigen, ganffa ymunig och ftor. Foliola partialiainfima integra, haftata, \& veluti ex tribus foliolis lanceolatis compofita. 2irent, form nyligen woro uts murne, frodo tillifa med de fiolgamle siken i centro, ei balfiparten fá lange fom blaben.

IMPATIENS 722. waite tillifa med PARIS 325. if fitgs gan of Struthiopteris.

HUMLE 81 8. whipte wio gấrbesgåroarne svilot.

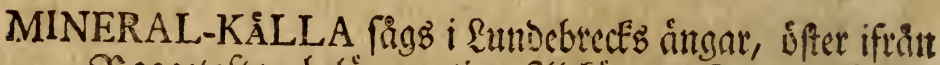
SBnngetofta, belägen uti et 2llekérr. Denne lifmade til fituation ganffa mucfet $253 i E s b e r g b$ Eurbenum; hon babe mudeter ochra blamono med confervis, men watnet war frwagare, än Żaggetuftag.

ALSINE altiffima nemorum $370, B$, waikte nuycet om fring Sîllan.

CAMPANULA maxima I 80 . froo gamfera hog och muce

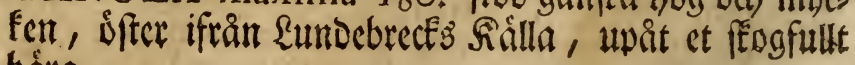
bårg.

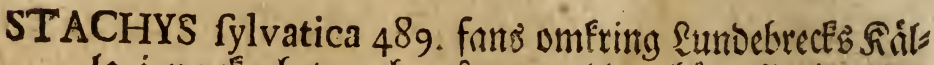

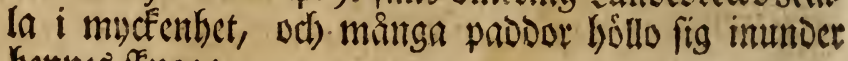
betmes fingga.

PADDOR (Faun. 253.) holla fig pa fínggriée fratlen, bes fonnerligen wid bargberotter, Dâr Denne frintanoe Stachys 


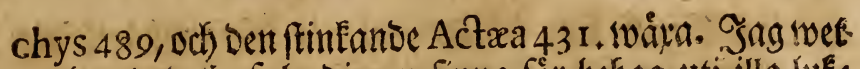
ej, huad Debe fule Dimen fimta for bel)ag uti illa luks tamoe orter, od) jag hat feot; buru bladoorne tringt fig in i bufen, oa Stachys foetida inburits; áfiwen fa uti Ukraine, Där Cotula fetida 703. wajer mer ân pa amora fắllen, finmaz fa mainga Syaboor, at de gî Dfwer alt $i$ hufen; men fa finart Cotula i De lánder

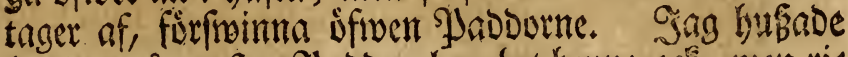
bumberl pa en ftor Jjaboa, ban bet leime oct, men ris

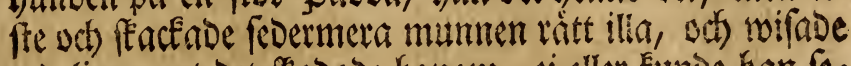
twoeligen, at Det feababe honom, cj eller Eumbe han fe=

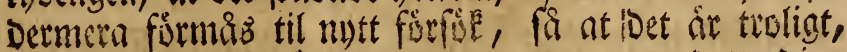
bivao Lister berittar, at un man tnget Decta Djut

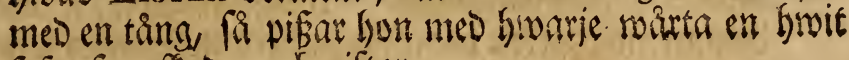
faft, fom kadar odb giftar.

WATTUPROFWEN anftilltes med 3:tie watten, nam ligen med Lundebredis watten N. I. med 23 agges toftas No.2. Dd) med et watten, fom togo uti Siads mamnen Jóns Kocks Trágåro, ttur en Fâlla, fom bost rent matten, men nägot bwitaftigt No. 3. De furthollo fig fon fortier: 2if.

Thea viridi I. fubpurpurafcens. 2. fufco-purpurea. 3: pallida.

Spir. Salis Ammon. 1.2.3. immutata.

Coccinella I, obfcure purpurea: 2. nigro purpurafcens. 3. rubra.

Syr. Violarum 1, cinereo-virefcens. 2, faturatius cinereo-virefcens. 3. carulefcens. Succ. Heliotropii I, 3. violacea, 2, faturatius violacea.

Mercur. Sublimato I+ 2. prxcipitatur grofse granulata. 3. tenuiffime granulata.

ZBar altfa ऊaggetoftz watret fraut of Mineral, sum

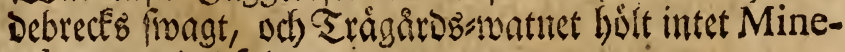
ral, utom jurbfalt.

WÁGGLO̊SS faber mucet faleert fordrifuas med et wios feppeliget medel, at man tnger 3 af Deba torggelón, 
odf) lingser bem $i$ lifftiftan, nair noggon birtbåres. Si credere fas. 2amdra bafwa für et fafert mebel, at fos

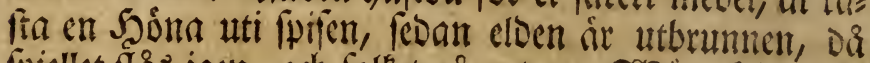

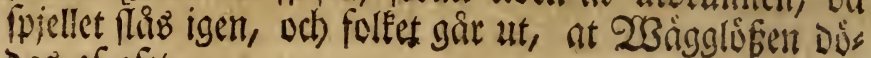
oas af ofet.

FÖRSTOPPNING förfífrader aloralattaft Eppnas, on froèn tages af Saumle, brwilfa lïttrligen Eunta fonmlas, Dâr Saunle naigon tio legat; men emot fürtitoppaning of Urinet recommenderades Semina Nafturtii, ocb) ändetligen emot Fâfeblob, at blooa pa bett jern.

LAXEN berîttabes af âtfilliga, på norra fiban om $U d-$ dewalla, fängns getrom et artigt frigspuf Det at befant, at Sarerne bolla fig gerna $i$ frommar, Dair fom watnet med fit brufande blifwer flept med et bisitt

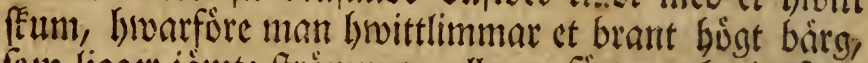
foun ligger jämte frtwommen, eller upfätter et livitt fegel

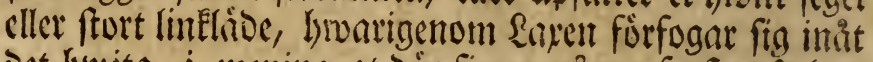

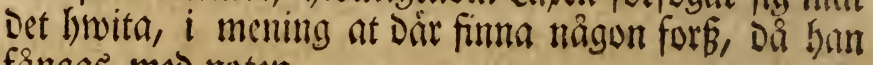
fängas meo noten.

KóTT, fom flagtas om fonmaren, elyaru Det faltaez, Ean

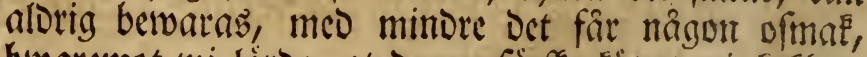
buaremot wi larbe, at Doppa färffa Esttet uti fofgett watten, och fesan bloofaften blifwit wall utframmao, fel Élttet litt berwaras i fin faltning.

LAND'TMANNEN Gáromering bafiwa fin fơrnaim Ita furttenft af fife och feglande, hmarigenum be Delś umeomma, Dels miffá mucfet barta, och) fálebes fürfumma fiit afert, bmillete ei litet bidrager til brift på folt $i$ landet.

SKOGARNE âro mål åmu tilráctellige, boct lófe, at man fan frufta, Det ell ginng ftäberne, buggnaber, fivedior, bute od) utfecepning torde bjelpas at, at utsoa Dem.

NATTEN Rter $i$ Uddewalla. 


\section{Jul. 20.}

RESAN ifrån Uddewalla fúrbi Trollhättan til Hunnebårg.

SONCHUS repens 642, waikte muctet $i$ åfrarna, Dår Det

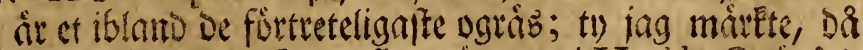

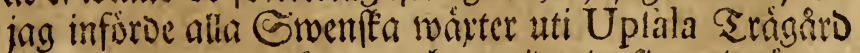
174 I, at inge mer frupo ods) uttriogabe fig med ristters na, ân Derne 642, Qwickroten ros, Scutellaria 499, Stachys paluftris 490, Epilobium 304, odh Egopodium 247. SBlommorne pia Denna Sonchus wainde fig hela Dagen efter Solen, langt färoigne, aur ningun Eoiblomma.

ANGELICA paluftris 234, waikte ritt mucfet i fidlánds te àngar.

* RackNebo. quart. $3 \frac{7}{2}$.

AKRARNE frooo i fara, at Den ftarfa Golbettan, fom nu bela 8 Dagarna badoat efter det fürra muceura och langfanmmo rignet, aloeles ffutle utmergla fäoebsaken, Dirigenum at fäden blefive four frarft odh haftigt orif: wen, ech falledez mognade fürt, àn matningen wäl wore peod.

\section{WÅSTERGÓTLAND.}

RISÅN fiilbe Bohus-\{̨in ifrån Wäftergótland, nár wi Eommit quart $I \frac{x}{2}$ ifrän Siacfnebo.

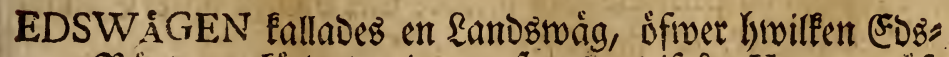
Soinderne Fúrde Det jernet, fom toni ifrä Venern, äfs wen foum Det at Góthehorg, pafferar Troullahttan

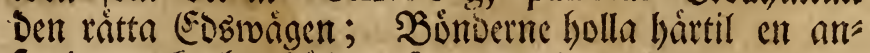
fenlig muctentet bufftar, fom Dageligen til 600:De tals flita worigen. 


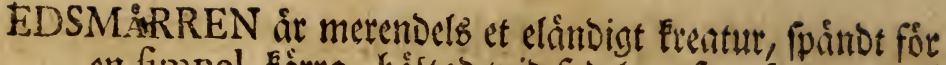
en fimpel Eärra, bâftad swid fadeclen, fon frammantil

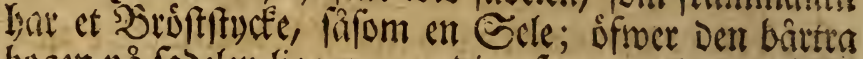
bogen på fadelen ligger en twedja, fom uppeholler fáres: armatna; sfrwer Den fürra bagen ligger ell annor, fon anpeljoller en jerning meo en bafe, fom bâftes $i$ tarts rans arm; jernftángerne lägges längšnt i furs of froce Eictean, at be racta fa lätgt fram, fom ficlfiva marts

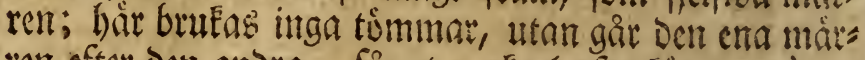
ren efter Den andra, fa at en Earl offa fürer i2 à is

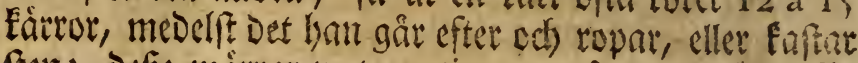
ftent; Defe mairrar weta antigt, it gă utur woiget forr

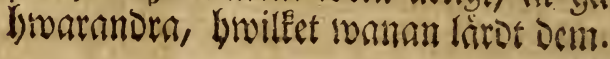

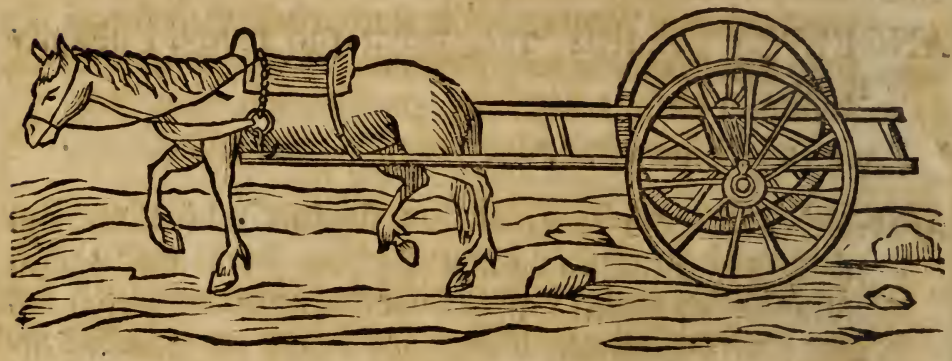

* Almås. quart. $4 \frac{x}{2}$.

SCHOENUS floribus fafciculatis 34 , wajte på họgra handen i myrorta.

PIMPINELLA faxifraga major 246. Mipte pa naigra

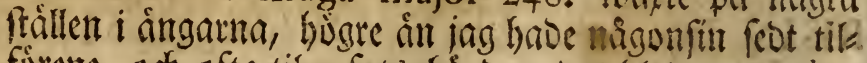
fürete, och ofta til 3 fots higo; Des bino woro du-

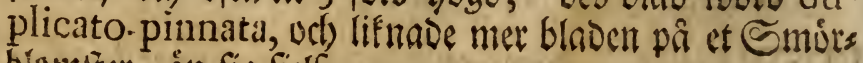
blomiter, sin fig fielf.

W.̈GEN toga af par higgra handen igenom en backig och gammal prog, at Srollgättan.

* Trollhådtan. quart. 4. 
TROLLHATTAN ấ en Del of SiotaEff, dár watnet gơt itfeilliga fall, od) formerar flere fma holmar, wid

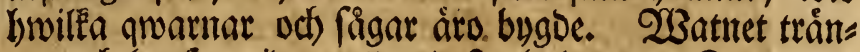
ger od) brufar nis emellan defa bolmar. Fallet wid wâftra fídan emellan (Sullón odf wäftea landet år Det

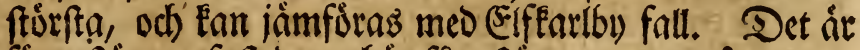
fogga fturre, faft betta loír fätt ftorre nanin, pá en ort, Dár De fällan fe ftora forkar; men for Dem, fom fett De

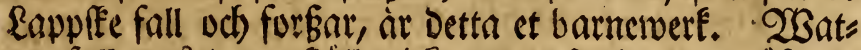
net faller pà Detta ffillet icfe allemaft niD, utan åfiven

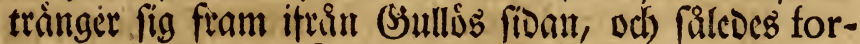
merar en 2ingel $i$ fielfioa fallet: Şăr brutes ofta de ftorlte trän fonder, Dâ De fécla frupa utföre, unoer Det De flottas genum G3otneff. Ofivan för fallen ling en mycfenbet af flottar, Erigftocfar och Biacfar. De trín eller ftoctar, fom frrummen firftat in på Sjullon,

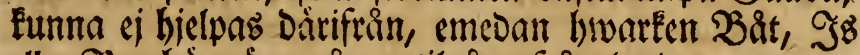

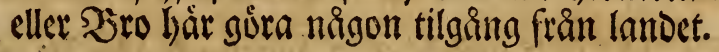

SKRÅDDARE-KLINTEN, fom hinger pa woiftra fitoan utufiver Detta fallet, ât garifea bog, tillita med TJYFBOEN*, eller Det gamla betanta Siofruaresnaftet, fom nu toat unftan igenfollit, liggo bioge til utfeendet nog fafclige.

* OL. MAGN. L.2. c.I9.

CAPRIFOLIUM germanicum 19r, woitte fom ben lámgita Heder i De brante bargsivaiggar, jemte Det bogita fallet, off war nu bepryod meo fina wállultan: De blommor.

ELFGRIPAR fola folfet omEring Stollhåttan Eallats $i$ fors na tioer, odf fommit af Hunnerna, fom fig neofatt omfring Sunnebing od) Trollháttan i Seculo primo;

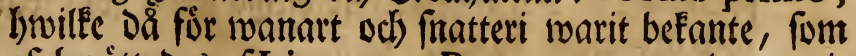
ó berattades af Lieutnant BeLFRAGE; men Denna ti= Den funde mi ej márḱa fádant, faftän bet fullex of fö: refom, fom Den gemenafte forten war nagot mer talan: De, ån bielpfam. 
ANTIQUITETER fimas âtffillige hảr på ortem, bem jag âlla går förbi, emedan be ei hóra til nagot af be 3 provincier, fom Naturems Sacrec of $i$ nảeer anfors trott. 23il umberfafta alleninft Derfíse Antiquariorun omoónmue Dent beráttelfen, fom Lieutenant BELFRAGE lemmade om -2:the frenar, foum fia uti en Sand:

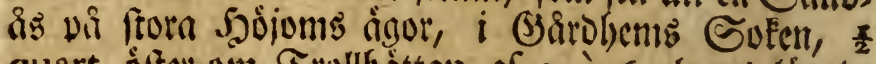
quart oftet om Trullhattant, of 3 à 6 almars lango.

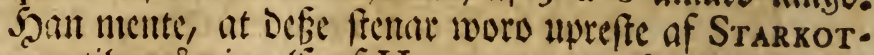

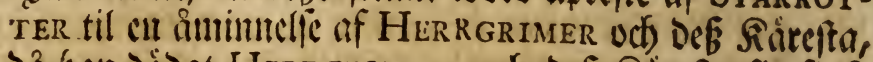

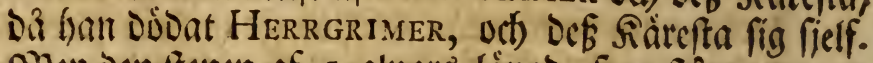
s)en Den fecten of 5 almurb lángo, fom ftâr I quart

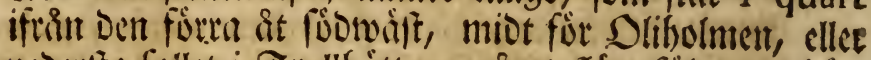

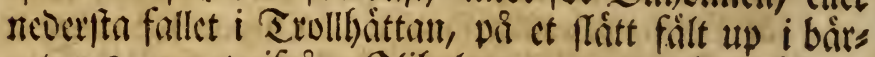
get, $\frac{x}{2}$ quart ifrain Olibolmen, mente ban áfinen

- watia upreft af StarkotTer, dór han baft fin byttar.

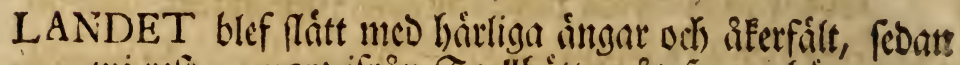
wi teit I quart ifrản Trollhsittant ât sauneboirg.

TUNHEM, 3 quart ifrân Trollhsitton, blef wirt Siatts quarter, feont wi wil lluppit Mintrtrano, Bląeulla

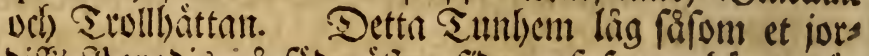

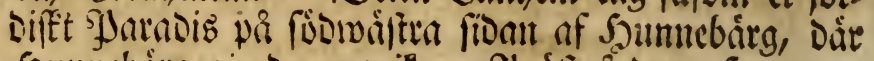

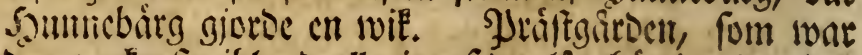
Den waftafte ibland alla ing feot, lẩ há: imvio roten

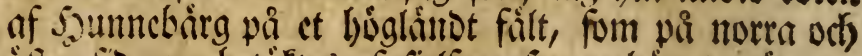

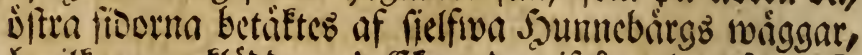

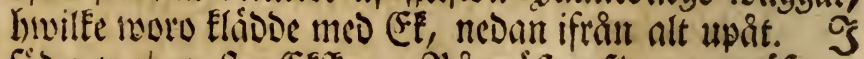

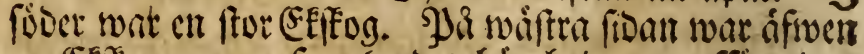
ent EffFeg; ment fom lantoet bár hade en affättning, funde feogen ci finnas of want filtet, utan ipnade en profpect it Den mucfet wiolyftiga fítt, fom roar tâft med aftar sech ingar.

\section{Julii $2 \mathrm{I}$.}

HOLSJÓN ligg i foomêf ifran sunbem; en fallfam fis Dórs 


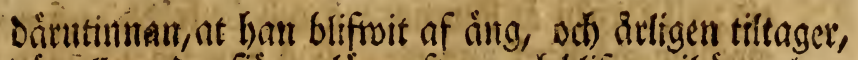
Dár alla anora fivar pläga aftaga ved blifiwa til ang; bait âr reoan I quart lang, men myctet grumb, bat 2:ne inlopp at ofter od) woriftra fioan, od) et enda utlopp at

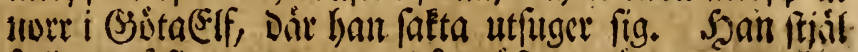

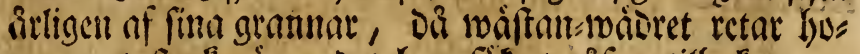
nom, et fructe sing, Det ban fâtert grifioe tilbafa, om ban tumbe fir et fritt utlopp.

HUNNEBARG beftod ifinerft af ct tirgorlunda lintt fólt, Docf bemurit med tríit. $2 B$ äggarne woro perpendicu'aire, flufue od hige fom fivrfommer. Fotelt futtaie utfore, hoptarfad af ras od hionaba ftenar, bes tifte mic allebano fagr Lichenibus leprofis, buars iblano LICHEN leprofus candidus, tuberculis atris 937 , fárgnde Dcm med ftotr brvita flicfore od) $i$ Detta ras frod fullt of EEar, fifom en planterad sunc. ingtat ligo utum rafet, incmot birgsfoten: altom:

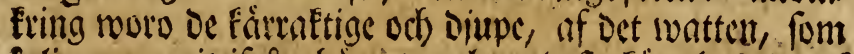
irligen rumnit ifrian boirget, och nt Defe Eeirr beftode af en siup feg lera, mafte jag nogfomit litra, senom bet

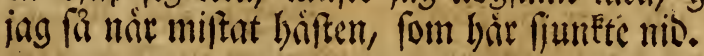

SPRICKHALLER *, eller SAXUM fchiftiforme calcareum cinereum, ir Don ftriart, of hwilfen Saun Itebargs hogid, med bef perpendiculaire waiggat odh deofollite ras beftior. Barget firicfer med perpendiculaire fitiot, fom affítica bowamora ad angulos acutos, âfioen fom en feifimeriten. Etenen belar fig horizontailt, mes mer án quarters tjocfa ffifiver,

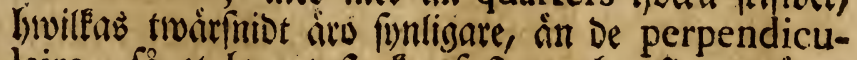
laire, fa at bnort fincle of fenen bar fima twänne fibor, och alle 4 fonterne áro râte, nent ojömt lange, oc) pá llutt affeurte; fa at broarbern bar en angulum acutim odf en obtufam, hworuti Denne ftenart nilts Deles fommer ofioctens med fifincriten. Denne ftent art or mycfet harro at fonderila, od) i brottet har ben ofunliga particlar, meo nigut convex fuperficies. Shat ât ganfe biro; ment mot fislet gifuet ban ingen $\mathfrak{O} 2$ eld; 
eld; til fárgen ár han liugrgrå, och) beftriod med niffan of (unliga mơrÉgra puncter. Dá ban legat nisgon tio i Dagen, blifiver han räfffírgad.

\section{KALM BaHUS 279.}

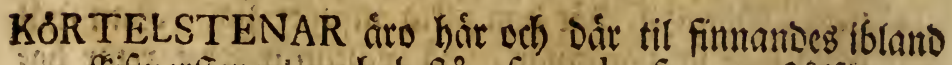

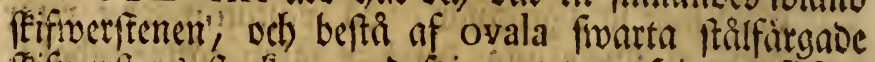

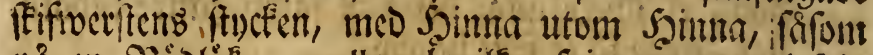

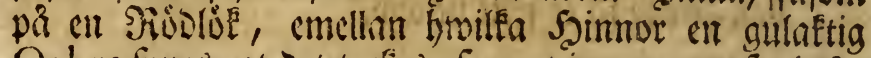
Ochra finess, at Det tnctes, fom et jermontten fipit fig igenom feifweritenen, och) honom coagulerat, bivatuf be oct utumpa sro gulaktige, fom ct ftal.

HUMUS rubicunda ochracea fimnes i muctenhet, fi will ofivampar Saunnebairg, fom wid Dés rot.

SWART KRITA fanb bầ $i$ birget, form ci war anmat

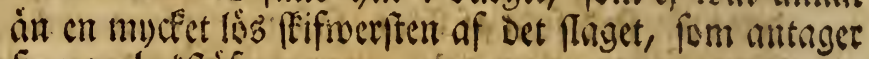
fiwata botfitifner.

SKIFWERSTEN fan băr ali âtffillign Aratis, fom war sanfta morf, och of en bebagelig fivarta; han ioras fade ods rommoe i clden. Sillan fants han fa ftor,

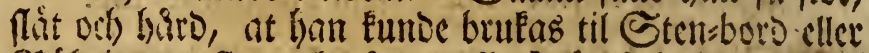

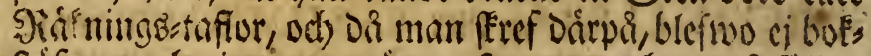

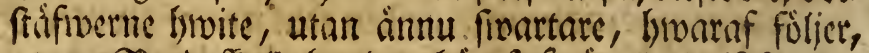
at om Sord feulle berebas baraf, fracirtade De ifrin fig,

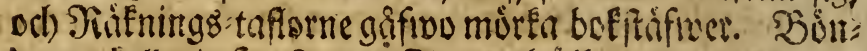

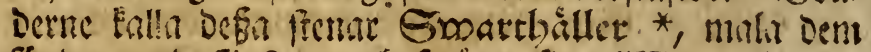

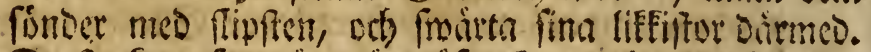

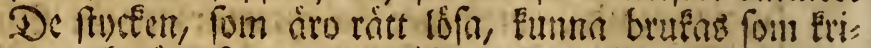

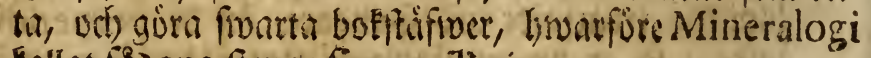
Fallat fidana fienar foosate Zirita.

* Katm Bahts 266.

ALUN helt gedigen figgs ganffa mucten pa finå ftenar ibland rafer; han mar uttwitreo fom en tioct rimtroft uti De langs oul twart lopande fpringor od) fmafade fur. Shír ranr Alun i fưrita quantitet, fom mifit rógoe fig pá loora fidan af Saunebarg mide för Freosturp. 
WAXTERNE på Saumebairgb fito nor noro niagra befints nerlige. Gaig feal uprafna de fortrainfta, forom: Pre. nanthes 645 . Origanum 480 . Orobus caule ramofo 597. Hypericum caule tereti 626. Trifolium lupulinum erectum 617 , od) Defutom HEDERA 190. sfiwerflidigt omfring Sunfem trupandec pá joroen.

SEDUM rupeftre 388 , elieft fálfunt.

GERANIUM lucidum 574 . Den ing endaft tilforene feot pá Gottland.

JUNGERMANNIA bypophylla 930 , frod nu med fina piftiller odf ftamina..

XYLOSTEUM 1 92 , fon hade $i$ fina blad mafear (Vermiculos fubcutaneos), De Dar frópo med trof gángar innom bladent sfre od) undere fiod.

GERANIUM peduncalis uniforis 5.1 . ftoo bát med

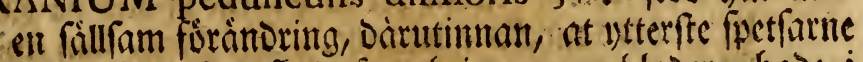
of frielfen, rom feutle frambringa nya blaver, haoc i fíallet liffom grona fitior (Legumina falciculata in petiolo communi leffilia); men oá jag nogere uns Derfof te, woro Defe fitiour ef untmat, án blader, huile fas fitoofanter blifwit tilbafa rullnos, at fntâ gula ma: fín Eunde innom sem ligga betäfte.

CIRC ÆA lutetiana (Flor. 5. ß.) Gar jag alotig fumit tilforene $i$ Siverige, faft min anteceffor Archiater RUDBECE uptät bemne i Eapplans, sct Doctor LECHE $i$ Eftine. Shat woirte bou mide for Freftord uppa fi: Dan of Şunnebárg iâmte Bent geoigna Alun, od foar wól 6 à 8 gingor fisure, in sen lilla allmaimm Circxa. Radix repens; Folia cordata, oblonga, oppofita, acuta, fubdentata, petiolata. Racemus caulem terminans, erectus, longus, laxus, ruber. Flores nutantes. Germen hilpidum. Calyx purpureus, reflexus, Petala ad duas tertias bipartita, in recenti rubicunda, in fenefcenti flore alba. Stamina duo, alba. Stylus ruber: Atigmate bifido. HYPERICUM, androfremum canipoclarenle dictum

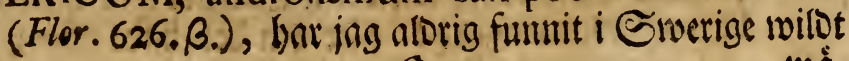




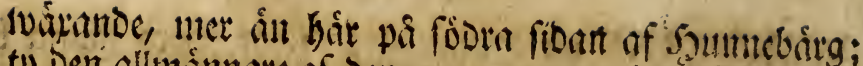
ti) Den allminnare af Denna nummer, bar baibe lubna

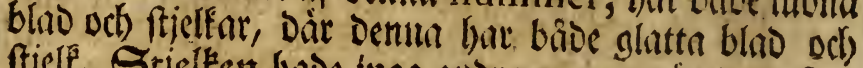
ftisclé. Srjellken bade inga antora grenar, int Dent, fom gjoride foris paniculam, beviffin sef imuceter litet uts fpirtrabe. 23laben bade inga punteter, fom lyfereige: nom Dem, men woil nhara brulta pricfiar i fanten. 3 lommerne woro fiotre, ân pá de sillorir wira Jo-

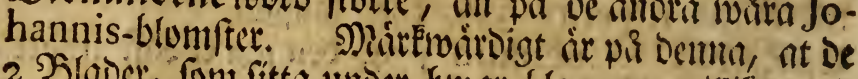
2 Slader, fom fitta under krwar blomma, tillifi meo fielfiva blomfooren, âro fingabe; Sinderne i senma fas

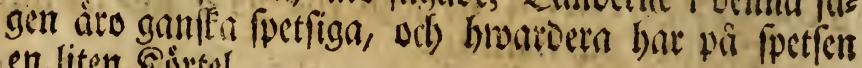
en liten Siurtet.

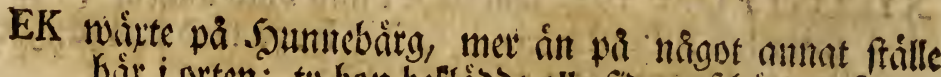
Give $i$ orten; to bour beflidode alla fitoor af birget, fafom

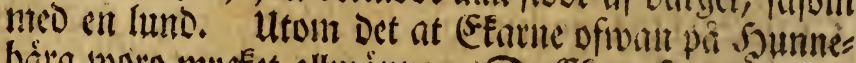
bairg woro mycfet allmainne. De Efar, fom ftodo ph fiborna, woro ef ferdeles ftore, och ännu miltore woro be, fom suarte ofiwan pa barget. Oefe fenare wifrise, ing

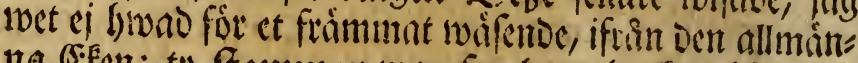
na (E)en; ty ftamunen war fmalate, borken bivitare, o(b) máft betaft med moka cller Hypnis, fa at han til

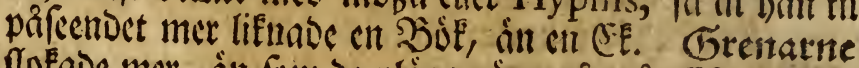

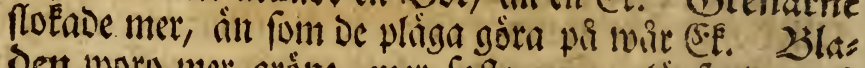

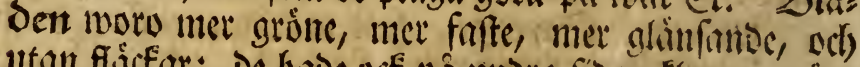

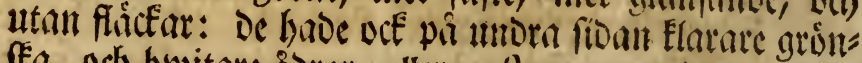
fia, och hwitare sorot, eller coftas, utom bet, at blas

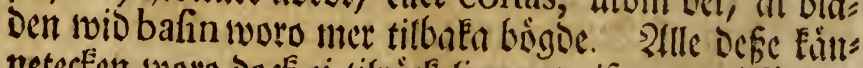

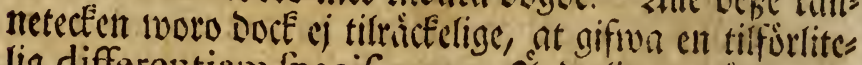
lig differentiam fpecificam. 2(ntoteligen màtfte jag, at Def fruft bnoe inga Bblomfleaft cller pedunculos, broataf ing thoeligen figg, at Denne (EE wor ell different varietet, fom nibrig tifforene of Sncmfin Botanicis blifivit $i$ aft tagen, niml. QUERCUS latifo. lia mas, qua brevi pedunculo eft. Baub. pin. 419 , eller PLA TYPHYLLOS mas. Dalesh. bif. 2 . Jing

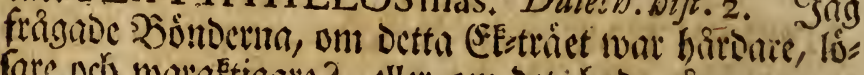
fore oci) waratigne? 
egenffap, int Lett allmäuna $5 \mathfrak{E}$ ent Itten be wifte ci at lemuta mig Diroun Den minfta undoerrittelfe; jag twiflar Docf iffe, med minore en fí ferffilo varietet mânde hafs

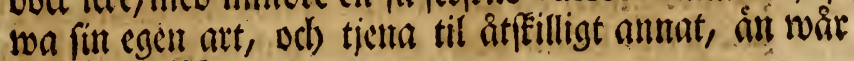
allmámura EE.

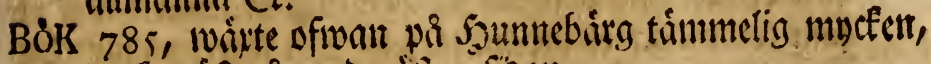
Docé máft pa norbmailtra fioant.

LICHEN 960 , 2(pothefarmes PULMONARTA arbo-

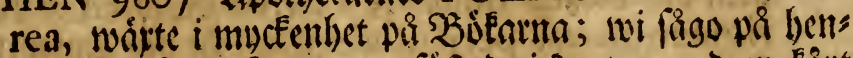

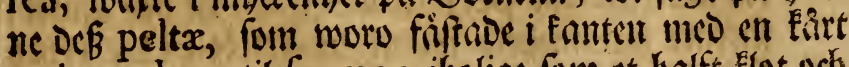
pedunculus, til formen iholige font et balft flot od

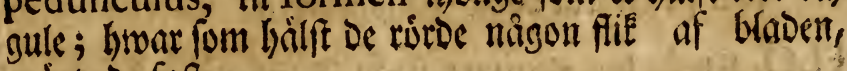
márte de faft.

HYDNUM caulefcens, pileo convexo imbricato,

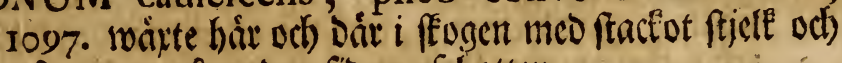
gra taggar po umbra fitoan af batten.

CHANTARELLEN eller AGARICUS caulefcens, lamellis decurrentibus 1049, ch fimamp fom ittes,

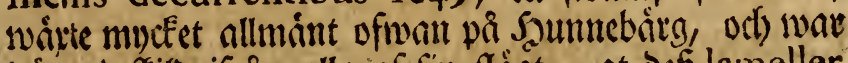
Dármed ffilo ifrän alla of fiir fliggt, at Dé lameller grenade fig, fom ât eljeft owo rnd iblant fivamparne.

AGARICUS cauleficens albus, pileo plicato membranaceo, ftipite nigro 103 \%. Den mintita finamp, wity

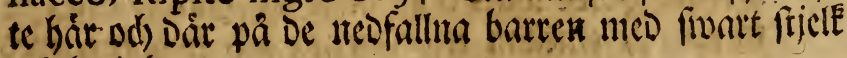
och hiwit batt.

SOP-MOSSA falladeb bair POLYTRICUM vulgare 868.

HUMLE 818. Wátte nilbt, od fróp ibland rafet på roteit af Sunnebitg wid Siammene Silef.

WALLBORG fillabes RHAMNUS 193, fom waite

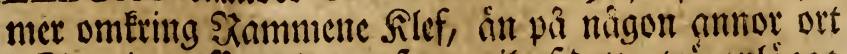
i Siverige, fá nt Den, font wil af Detta tra anlingge

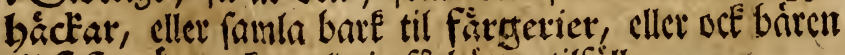
til faftutróna, Earr albrig fá bitttre tilfülle.

CAPRIMULGUS (Faan. 248.) fög up bar ods Dair emets

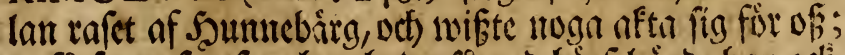

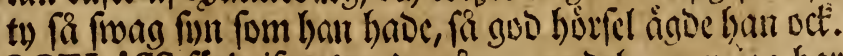

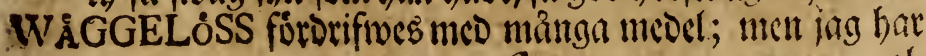

$$
\mathfrak{O}_{4} \div \mathfrak{a l}
$$




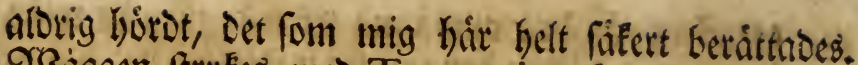

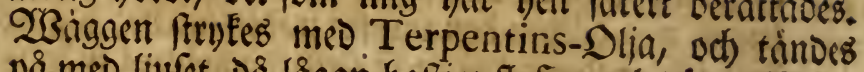
pá meo liufet, Då liagan haftigt flobar, och) Dódar 23 ágs gelófen: Man bor $i$ alt fall bafwa matten til handos, faft Denne läga ei gerna fiweder eller bränner. Den fom wil toâga et fabant fơrfó, fon fáfert Dúdar obys ran, bớr noga Eomma ibog, bailf uti en gammal mak

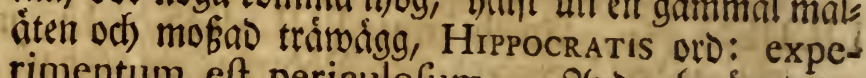
rimentum eft periculofum. 2(nora berattade ct fä́trare hjelpmedel, námligen MENTHA fylveftris, fom ftrait feal disoa 23 inggelifín.

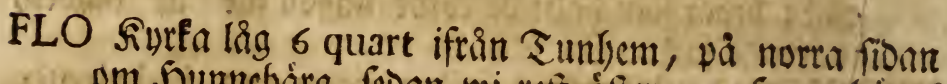

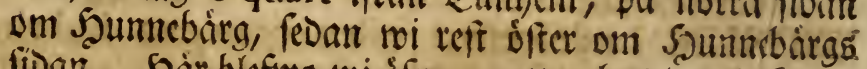
fidan. Saàr blefino wi śfwer nutten boo Dem luxteliga och) narime

\section{Jul. 22.}

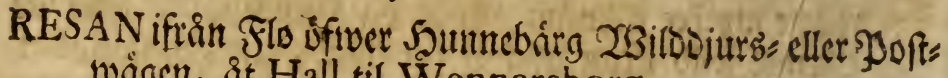
wägen, it Hall til Wennersborg.

RAGNET påftos hela bagen, afruen fom i gäe och i natt;

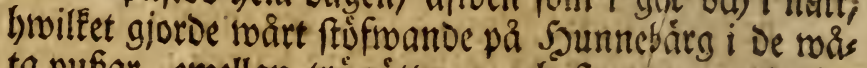
ta pubar, emellan trástótterna odj-frencrme, meo rins nande watten, nog befwárligit, od) icfe (itet nfbrùt Den underfófning, fom eljeft bade kunnat anftallas.

ÅKERFÁLTEN omfring Flo woro Delte i Sacmioro oc Safrejord: Zemjorden war minde, pisides om hoit

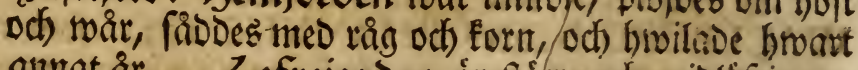
antmat âr. Zुafrejoroen ir fiódre och) wioloffigare, til hivilfen ei gơoflen rádfer; lion plojes allenaft um his:

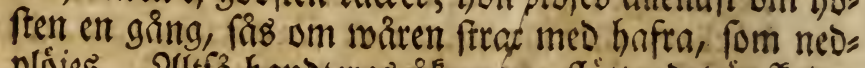

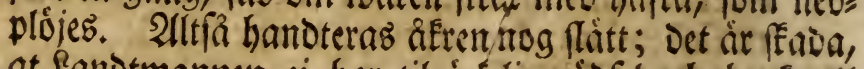
at Ranotmannen ei har tilrectelig goofel oct) bruk, the

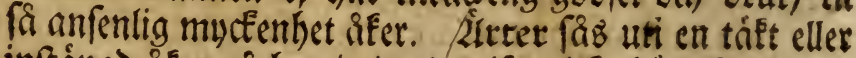

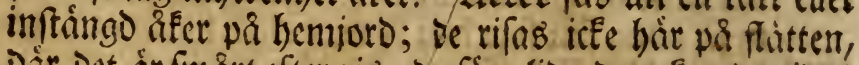
Dár Det ár fivart efter ris, Derfóre lidar de off unvertioeń ganffé mucfet, áfruen fom $i$ âs. 
HUNNEBÅRG fåg ut $i$ Dag bạ på norra fiban, affiveut fom Det wifte fig i gât pa ben föora od) Diftra, thimlis gen omgifivit med ftora flatter, lywarpa fúlgoe Zlbles buftar, feoan fmale fanta angar, fift ras af ucofallne

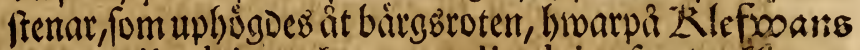
perpendiculaire od) perpendiculairt forave flippor, til fârgen grå, of LICHENIBUS cruftaceis \& teprofis, upftego til en fror hogo. Sfivan uppá war lanoet nagot flatt, Doct ojänt utan bairg meo Grants och Callfeog, fomt Hypnis od) Vacciniis betrift. Det fades bafiun 23 Sioar od atfillign Corp; men ffogen innebrade bet máfta.

DETTERN, en fio, fagb emot norr ifrin Sauntbarg, od) hade communication med Wenern pa fir morra fioa; han mar tämmelig ftor, od) lâg naiftan parallelt mes Wenern.

STRóMMEN, fom oref âtfilliga qwarmar, fill neocr for

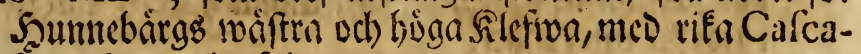
der, ftrat wio Şierpetorpet.

LICHEN foliaceus umbilicatus undique lrvis 970 , mâtte på be perpendiculaire flipporna, fom dorgeligent ftooo wate af Det ftadiga righ, fom Denna Forb ftint: te ifran fig. Şag frod lange i forunoran ufwer Dennid murfgrina mofa, fom aldeles tyftes wara en Ulvar, til Deß jag andeteligen fant af Deß buflor och ifra mio: liga fuperficies, at bet war forenámmoe Lichen, foum fơrandrat fitt lyne of watnet.

HALL ligger wis fobra fidan af WENERN, och gair me fin norra fant ut $i$ fiơn, Det at et bylift bärg meo Sounnebarg, men med fine perpendiculaire Rléfwis hogre, ty Da Sumnebairg war et Bobeffott hogt, wat Sall twâ; och Şalls ras war wio bägsfoten minore biedt, men bwarken funoe Şalls eller Sumnebárg pia nagot fátt til bogoen fomma cmot Slles cller Disise: birg, mucfet mindre meo Sinnekulle. Defie z:ile 
Salls od') Sountrebirg habe inga affôttuingar, fom ic altora 28 siftgóte bárgen, ment ivoro deremot mera brints tc, od) bare pá fiborta. Etentarten mat i Syall mis

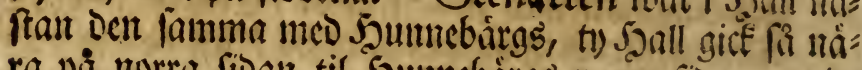

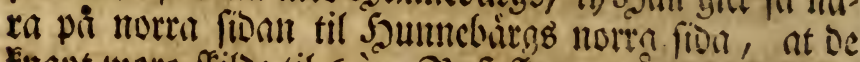

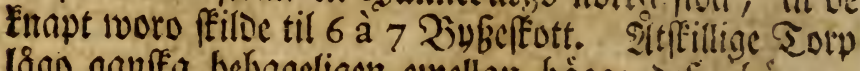

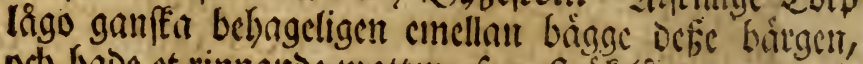

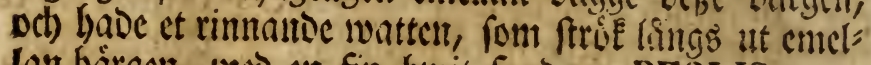
lan bairgen, med en fin bwit fano. PEPLIS 29r,

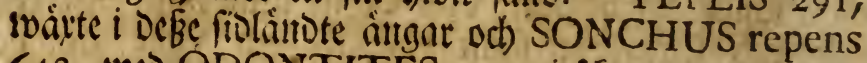
642, MCD ODONTITES 517 , i âfroma, PTARMICA 706, IMPATIENS 722, DCh TROLLIUS 474, i ätgartha. SJall war iffuctwiliet med Talls od) Siramfiog, fafoni saumebonts, men bade uti rafet

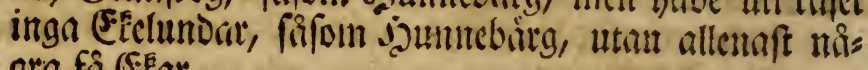

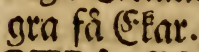

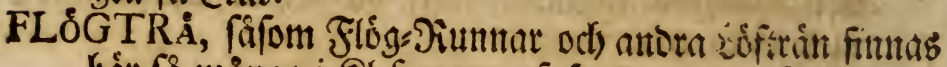

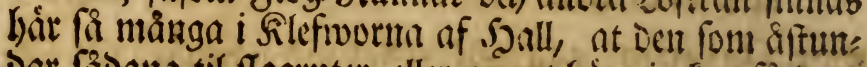

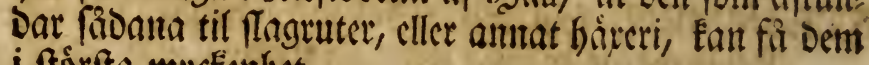
i fośrita muctenthet.

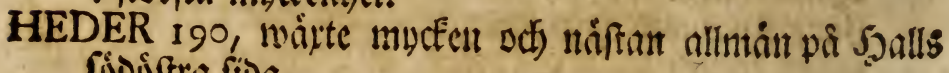
fiosiftra fito.

SEDUM rupeftre 385, wijte musfet $i$ rafet of Szall.

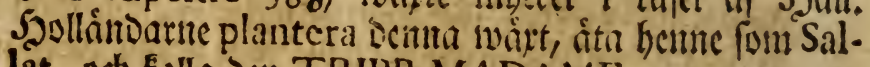
lat, od) Ealla Bert TRIPP-MADAME.

HACKLA àr et oct) fantuma birs mes Sall, til natur, att bogo od utfectide; men feildt ifrian Saulls foora fioa

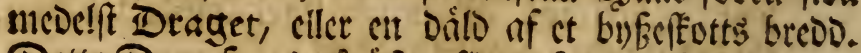
Detta Drag fintes pá siftra fitant, fom Det foroum was rit med en mur of funa feetrat igenftangs; men out bet fiedt af Hunner eller antora, Ienular ing Hiftoricis.

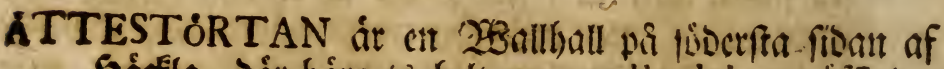

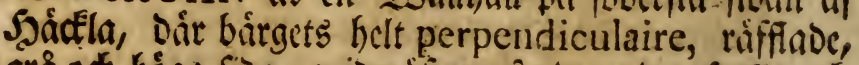

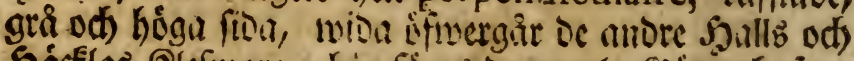

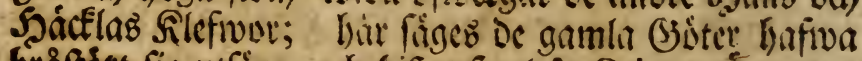
bråfost fig utfore, och bif at fegel it Odens $\$$, utan

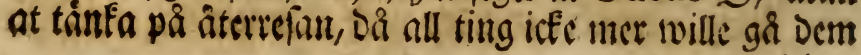


i lag, eller de de blifwit trotte mio werld odj) lefnad.

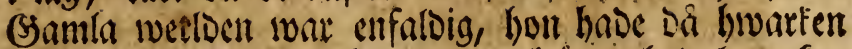
upfunnit Siut eller A renicum, od) annu bade byarfen-

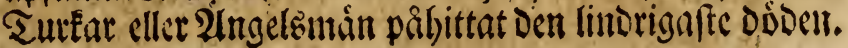

DOMMARE-RINGEN * beftod af 8 ftenar i furtant fatilde; De imtefloto et fílt om 24 ftegs diameter. Defs fe gamla ftenar áro mer din 3 nlmar bogge, 2 aluar bres De, od) I alm tiocfe. (En of Dem war nebfallen od) liffom bopfatt of twoinne längs âtffilde ftenm, lywillet jitg icfe wet, om Eoniten cller sien giort. 2file fitenars ne woro utu grâ of âloecn, fom mängen giort grí pa mimore tio. Dommaresaingen air belinen i fosmanift

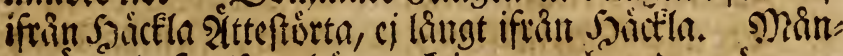

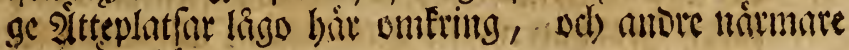
intil श्थteftortian.

- Kalm Bahus 65.

RONNUMS B̈tolig śfwer Sista nersborg it Bohus od) Góteborg), Där Foocr war (mialaft, oit) giove et hogt fall; hon war roitt mail bugo

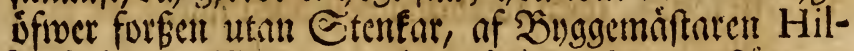

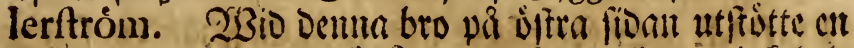
Elippa meo en fant, och formerade en liter winfel cls

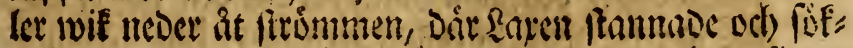
te froila, umber bet ball mippeligen funde arbetin fig up for en brufante forf. Sgar war nrtigt at ifit pit Qitoll, od) babe fe Raren fai i fit netbete, och fämbits med bas.

* Wennersborg. quart. S. ifrian Flo.

$$
\text { Jul. } 23 .
$$

WENNERSBORGS Etad wat ci finturilig frot, ling pa et itimnt filt wio foowififrn fion af Wenern, Dăr lanoct migut litet fietot ut ât fion, fí at Wenern tâlte

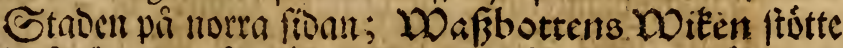
in ait sambet par wiltia fionn, til bela quarts lingoen. Et peist Sorg war birt i Stnden; ods gatorne woro ráte, 
ráte, brede od̆ liure.

Gtaben toltes mu tontri itilta: gande, emedan atféllige nua Buyggmingar Dels hades unoer hánoer, ocls nuligen woro fórfároignoe, och Def fe woro langt práftigare án be ấbre. Sàr i Staden war sandsbofoinge late, men Staden war ej befäft.

CARLS GRAF ling ei lingt ifrin Stnoct, den fiomung

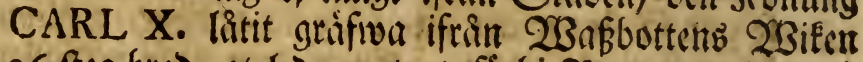
36 fteg breo, at leda watnet forbi Sionmums fall, p\& Det man meo bátar eller flottar Eunde fonmu fióledes neo at Siollhàttant.

GEulle ien thare deffeinen, fom Commercie-Siádet PolHe m projecterat, Fonung CARL XII.approberat, od) Эitffens Şoglofi Etins ber unoerföt, fullfólins, námligen at fá ftilla Transport fortei Srolibattan, fả finge, utom all antmor formon, Wennersborg i)arigenom lángt anfentigare rórelfe, emedan alt bet jern och timmer, fom fommer ifrán Wermeland, pafferar Denna wagen genom (Bjota àt Góteborg, âfwen fom alt antat goos pafferar tie

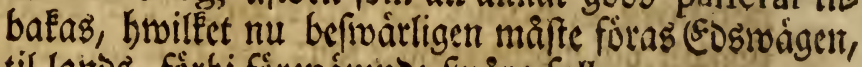
till laitos, fơrbi forcnámmbe fivara fall.

TOBAK war for nảgra och tiugu ár fcoan elt fillfunt dit $i$ Swerige, od) man twiflade, oin oen nigonlin funde $i$ Smerige med bitna planteras, bár utfoummo publique od) private ferifter, forn fóte rifor od) líra Sanotmannen at anlágga fádane unttige plantager; Bonden war owant, rifte pa bufwutoct, web wille ei gerna på benna omóicligheten. Sal. Sanoshofoingen GYLLENBORG fórelipfe Sanotmannen bitr pit orten meo egne anläggningar, at en cller annor Zjonbe blef lucfe: ligen imittab. Na funno wi tilftandet efter onffeat forantrabt; th alt if:an Trollbattan til Tunbem, ods ben Tracten, fom ligger wafter om Sountrebirg, fa wál fom runot omering Sountebsitg, hase fa lucfelige Sobakgplanterare, at ofta ell 30 onve af $\frac{1}{8}$ Semman fumbe fália Sobafs blaber arligen for $100,-200$, ia 300 DIt. S:mt. De woro lycfelige, fon infonmit meo denta nuxtiga tonften, ty hár funoc De anwanda 
all fin gơofel på en liten ấer, utan at fớa den på afs

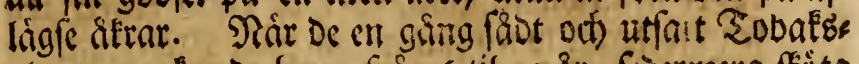
planturna, Eunde barn, frin 6 til ro ki, fisermera ffota alt Det anora meo wattning, renfning, fupande; iuned lertio fick Sonoen meta mimft af et litet Soba blano, än tilforene af ail lim aecr, od) Dertil fict ban penningarne pa et bribe, emeoan atfeillige Tobatzfpinnerier bă woro imrattade, fa at 5 Tobats fourneras af forenámbe Tract. Det beráttades off

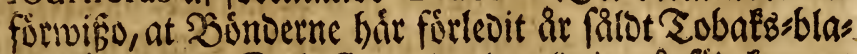
Der for 24000 Dal. Fi:mt, Det De alorig pa fád lunnat fortiena. Saleses twingades de minore af noden at

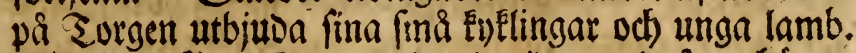
Ja ietta fáttet lunna wi meo tioen unogå at bámta Sobaf: :blader ifran Scolland, 2ingeland och fielfwa Virginien, nâe Bonven ifiveralt fâr fe Denna båtnod, och) antingma modet uti be baffe linoer af Siifet. Doct feulle jug oforgripeligen tioffa, at Denna plantage med ratta borde wara Soorgarent enfeitte, to Den anora aEren blifwer forrummas, form ei fir gòdel, och) gifmet

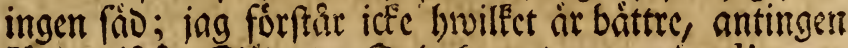
fopa utifran Säo cller Sobat, to) Det ena àr aliment oct) Det anora luxe; jag wet ei eller, antingen mis: bruk of Sorannemin cller Sobak meo ftorre nutta fors

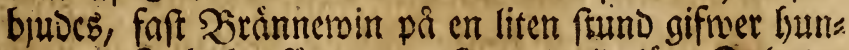
ger, Den Sobaten förtse er. Smedlertio lárer Tobalbe planteringen fielf fätta fima egna tỉmárécn, emedan ba förmanga begunna plantera en wara, faller prifet, om iffe confumtionen fiflle lita fliga getrom of forfío.

ELGAR (Faun. 37.) áro ibland De Djur, fom endaft trifs waz i Ealla länder; hos \&and bhofoingen $i$ Wennersborg J̧⿻上丨 General Major Roos gicte en ung hemtamo (5. Ig, af et ars sloer; han war redan 9 quarter bojg, til färgen mirkaftig, (fulcus), beftroos mes hrwita flicefar; lar och fótter sooro gránftige; hornen woro śnmu (male, utan stenar, ocb) lutone; nǻfebororna afs linga, fom pa en ftarêt lópande băft; fooanfen gans

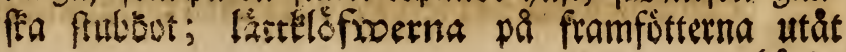




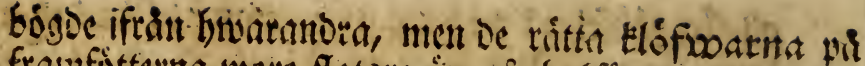
fromfótferna woro flatare ait pá baffotterna. Säts elsfiwarme pí bakfotterta ftoos parallelt tilbata ad angulum rectum ifrin fotterta. Wha ryggen sfiver

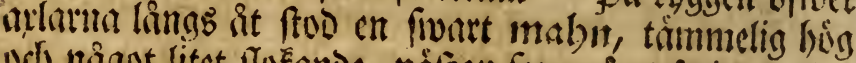

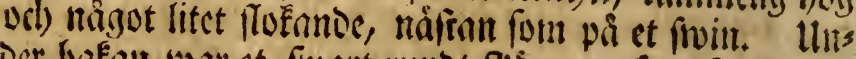
Der bafan war et fiwart numbt fisegg, font feocrmelit friticfer fig ât bafion; Det waitte pil en muto caruncu-

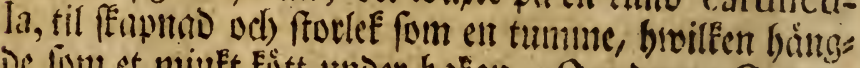
be lom et miuft fott under batan. Dim Denna Caruncula barbx affiven fimes hos bonut, Ean Den aldor literrifie differentia Specifica togas boraf.

WARGLOAR (Faun. 4.) finmo (ifwen bit i Sufsinges Dinmet; Detas Zirapp war robaftig meo hiviti flics Ear, iragent wat hwit, och) Smoantren war ytterft fivant, afinem foun be linga bateat, bivilfar liefom pents flat futa oronch.

FROSSAN botnoes bir fäledes: De tugn hivit Ingefa:

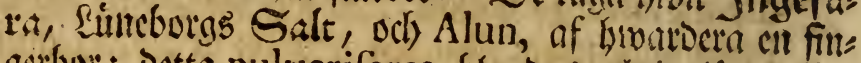
gerbor; Detta pulveriferas, blambas bd) insifives forte int paroxyfimen foumer, odh Patienten fint ci oric: fat hit pí. Detta har hulpit minga, men recom-

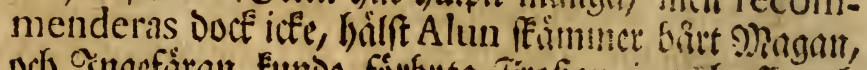

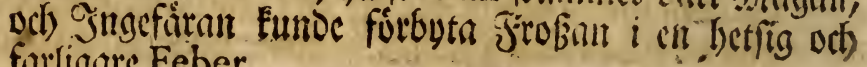
farlignte Feber.

TOBAK waite raitt wacfer, i Wennersborgs Gtno planterad; ty bar war inget letr, fom feroonde batts woitt.

NATTEN blefivo wi ater $i$ Wennersborg.

\section{Julii 24.}

RESAN ftifise if DAL, ferat mi fom baliuaft gentom

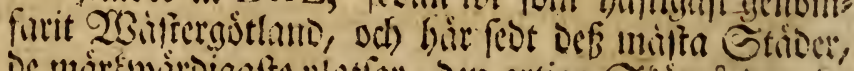

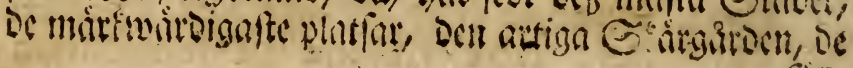


fillfinmme bårg: Kinnekulle, Billingen, Myffebårg, Ollebärg, Haliebärg, Hunnebårg och) Trollhåt-

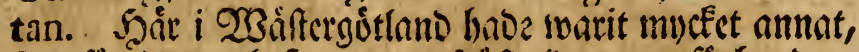
fom forticent at befes; men Shiftetioen war forthanden,

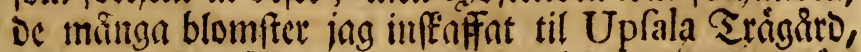
foorade mit upfeende, od) fropper begunte mu nt tríts tha of telor, fom utom Norrige, Damemare, Tuffs

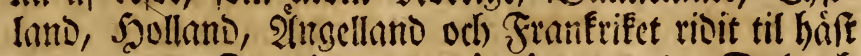
inchom alla Gmeriges provincier, utom Dal ods) 23ermelant. Ty walbe ing hemerian nenom Dal

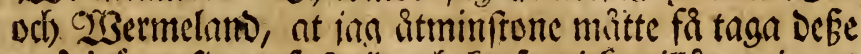
troa $i$ ignafigte, foft tio och) Erafter iffe tillito mig at mida fora umfring.

\section{DAL.}

GRÄNSEN intellan Dal och Oraáfternit!ano woro någre ftenbirig, forn togo til mid Dal, fa finart mi reft sifwer Wennersborgs $\mathfrak{B}$ roen, fom líg sfiwer $2 \mathfrak{B a b b o t t e n z}$

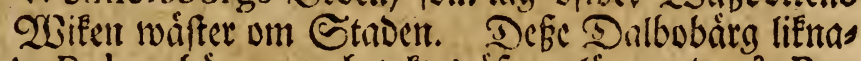

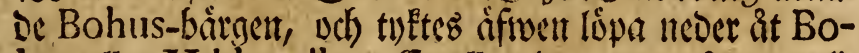
hus cller Uddewalla. Emellan Dem rann ftrax en lis ten baice, uti Wenern, font gioride fifinnoen emellan 23aifteraitland och) Dal. Dals lanto inftinnoe We-

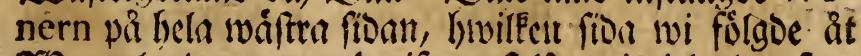
CSGermelant, utatt at besifuna of lasnat in $i$ landet, font

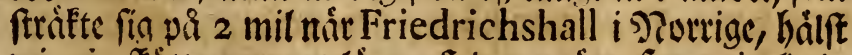

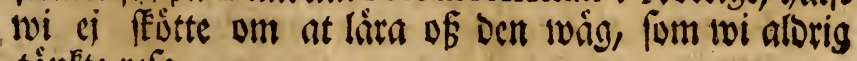
tânfte refa.

WADRET war behaneligit om murgonet, bimmelen flat meo et ongenaint Solfeen; men Sroalorna, fom pläs

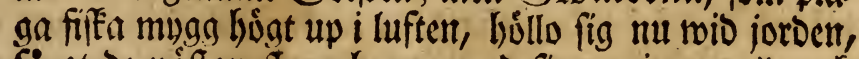
fá at be néfftan togo henne meo fina mingar, * od́ bebodade of det fFur, meo bmillet tri hela Dagen feoers mern blefino iffiverfésigoc.

* It. Sland. 85 . 
* Róshult. quart. $4 \frac{\pi}{2}$.

SONCHUS repens 642. gjorbe pa atffilliga ftillen äfrat: ita bula, odb war et furbateligit ogris fót Eands mannen.

HORNTISTEL fallnieg CARDUUS lanceolatus 654.

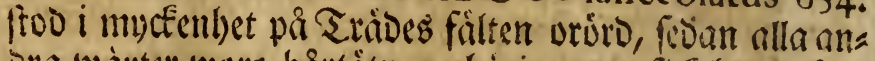
ora maiter woro bartâtne, barigenom fic ban lof at

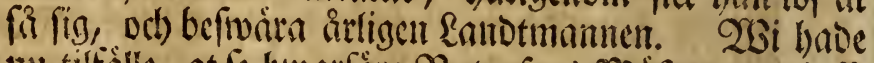

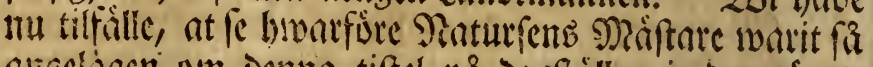
abselingen om Denna tiftel pa be oftällen jorben róres, at ban gifroit Def fro Den formonelr, at Eumna borga

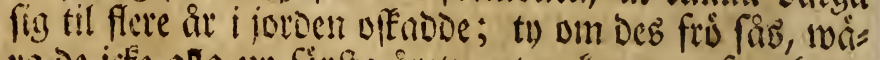
la be icfe alla up forfefa aret; utan fomma efter band lip in til io:De aret; byaraf ffer, at bar Denma tiffel fig

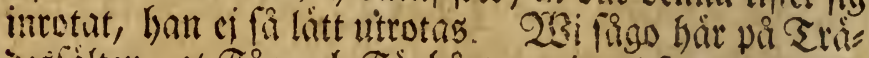
iesfilten, at Fair och Fa birtgnagit altramman?, fa at icfe en enda watt Eumbe bara fro eller fi fig, utom endaft några fa, fom gơmoe fig imellan tiftelenz blas odi) fticlfar, Dem tiftelen med fine buaffe taggar for fivas

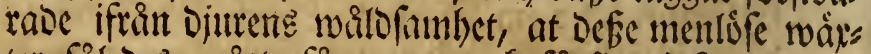
tiit firleocb mâtte fir mogna od) fa fig, befiunnerligen

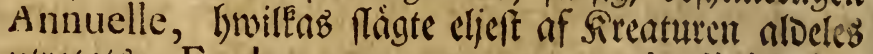
utrotats. En documentum ceonomix divinx!

MJÖLKBLOMSTREN, eller LEDNTODON pedunculis fquamolis 629. habe fintt fig pa nigha lin Dail, fa at man ya langt boll felat troot, at fiftert ftisot full af Raplat. Gag bar albrig fett henme if as Dan mucfenl)et, faft bout meo fine gula blomnor befinis: De Denna tiden alla wrigfidor, to pa furenamoe lindor wat Enapt et chis fitino of anora baitst.

HUSEN pá bonbegaroarne woro merenoclis beflaghe utan pianco (Sranstelior, fon ftodo uprätt jäinte bioaranora, (a) Lsiftade mid waingen, at de längre matte utbarba luften wailsfambeter.

ASPELUNDAR fago kgla Dagen i tang iñmte Byarma, $-2 .:$ 
men befinnerligen ftodo be Gelt nactere wid Frendefors Sivila.

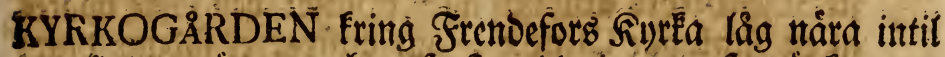
Sanosibigen, od') gaf of anleoning at eftertänéa, ont man Ean eller burr taga mullan utaf Furtogarboarna, at Dermed góda fina afrar eller kâtgarroar? Taturen láret

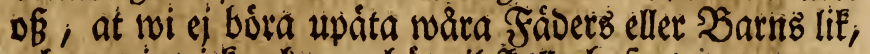

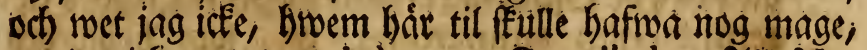
ont Det iffe woro ent inhuman Cannibal. Salle $\mathrm{Na}$ tioner hafiba warit angelaggne, at Derab díba måtte

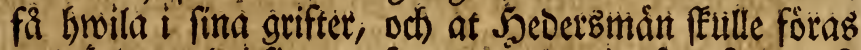
med heder ned i fina grafives at de ci mátte falla rofs biuten til fódo; De formúgnare låta gơra feen=odj faps

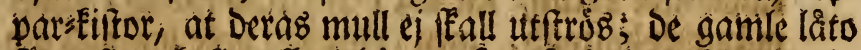
fatta fig och fin affé $i$ hog ar, fom froartigen tunoe rub:

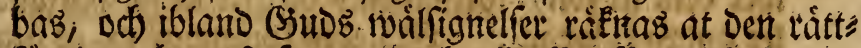
farroigas ben, ftoft, miutl och) affa feal fá ro i fina grifs= ter; Deremot blifwa mifigerningeman af alla Nationer

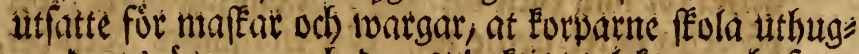
ga Deraz ógon, Ded) De og̉ibaftignz leEamen bafmer

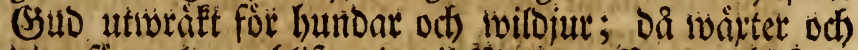

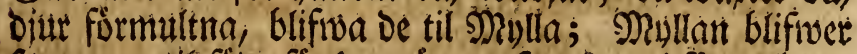
federmetra til fosoo fór be waidter, fom seruti fatt od) ros

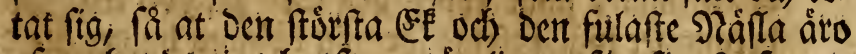
af enabanda ting hopfatte, naimliget af oe finafte fiwarts muthans particlar gentom $\mathfrak{T}$ aturen eller en lapis Philofophorum, fom Slaparen fatt uti livart fró at forbita och fürmandla smollan til fis. egen art. Shấ

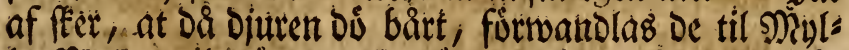

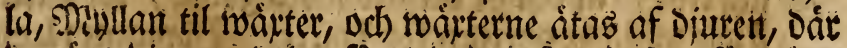

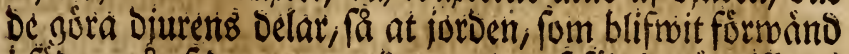

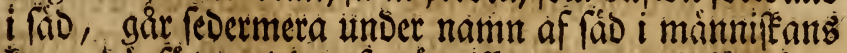

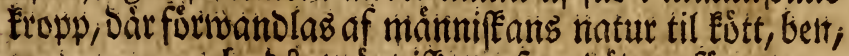

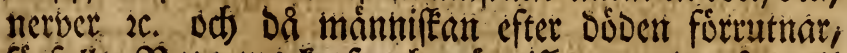

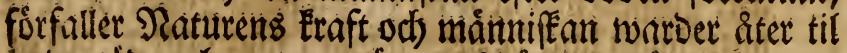
jorb, täban bon tagen ár. Naát nu rórter fomma

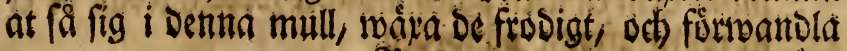




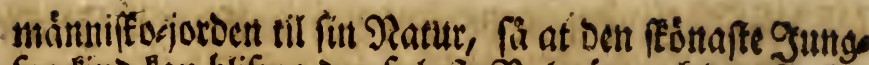
fruefino lan blifwa Den fulafte Bolmórt, och Den ftartar fite Starkotters arm Den lófáfte शate: Den fúrre upátes af en ftintande Cimex, of blifiver et fadant Djur; Derne Cimex âtes feotrmera of foglar och blifiver til fogel, fogelent ittes af nuimmiffan, oct) Dár blifwer en Del af hetme. De gamle Philofophi Pythagorxi, och an $i$ bag de sftindiffe Gymnofophiftx troode en Mecemplycofin animarum; wi, fom bolla bett för fikro, fe cn annor Metempfycolin corporum. D\& jag tager mullen af Sivefogarbarna, tager jag De belar, fom conftituerat och warit fórnantolade af mánnifer

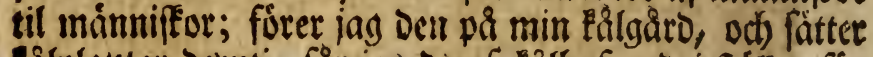

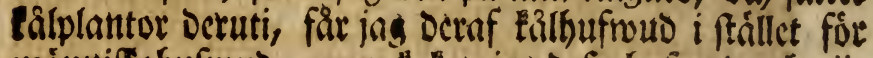
mántiffohufwud, men totat jag Defe hufivud och gits

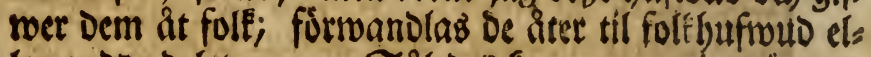
ler nnotu delar zc.: Ssledes foumom twi at áta up

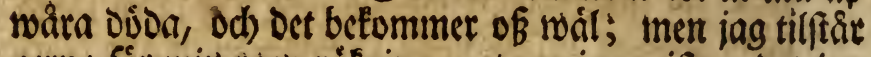
gerna for mint egen rífning, at om jing roifte, Det jag pá Detta fáttet upinte min eller en ammoré Farfabet, at jag icfe gerna ginge pa Den Eílen, utan jag babe Defto

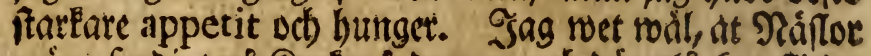

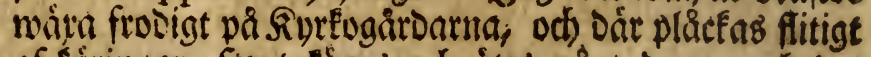

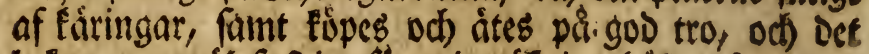
befommer wäl, faft jag fior min ráéning bálore ate betm,

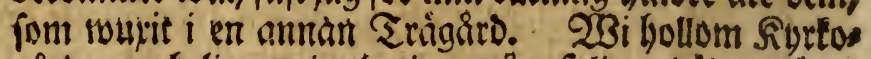

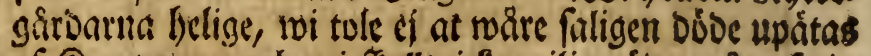

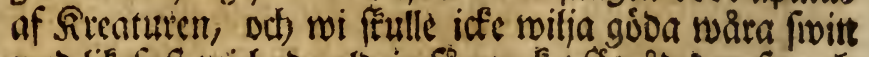
med lie, faft twi bade alorig fa myctet fotrad Deraf; ock jag tror, at om de dermed moro gósoo, soi látteligent

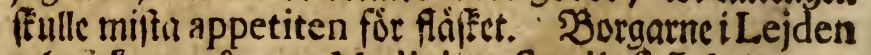
toln icfe en gâng at Medicina Studiofi frola Dee ups stafiva incognito Deras barn, fedan de âto begrafne, at anatomicera Dem hermligen, faft oc lifa incognito

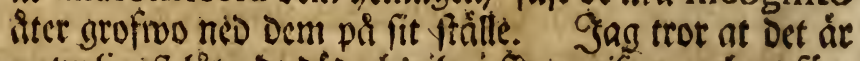

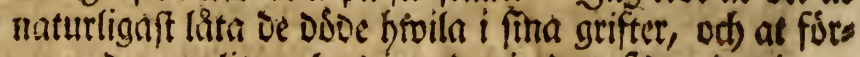
umua Dem en liten plats munder jorden, fedan de ef mer fá twata ofroan pá jorben. 
NEMESIS divina experimentalis demonftrerades of en oluctelig, fom lág pa et ftegel jämte mägen på Ora: landa SeD; Foleet berättade oin bonom, at ban $m a=$

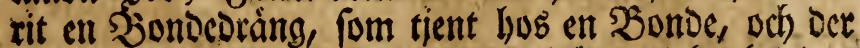
felat frigit in i fin busbondes enda ratt, at husbondent fejermera trånat bart och) DoDt, Da Drángen âftat àn fan, men en of fwerilnimg babe gjort bonom til fader at fin egen ftofootterb barn. Slyctelig Den, fom ef

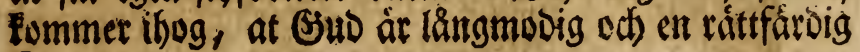
Dommate.

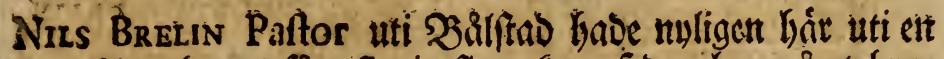
frilla bammi fatt flagit fit anfar, feonn ban pa et brus fanos baf $i$ flere ar blifwit Eaftad af mannga vlycfaits ftormwider. Sans lopp; fom bititilo warit en Ro. man, lírer $2 B e t e n f f a p s=A$ cademien i pinom tio lems na it eftertommande. 50 benna curieufe Magiftern fage wi atffilligt uti Mechaniquen, fom han med egna bänver fơrforbigat, fafom Mulical-Initrumenter, Wagnar, Jlogar zic.

WAGN fagz af Magifter Breins furfartoigas meo 3 hjul, lamt ifinertaft Cour. Basthiulen ivoro 2, men fram: bjulct allendft et, boct mickave icfe wagnen. Sletarna

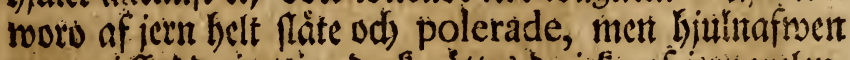

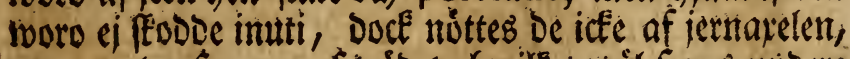

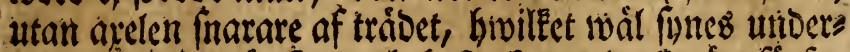
liget, fait bet ár fant od befturtes med allmän fórfos

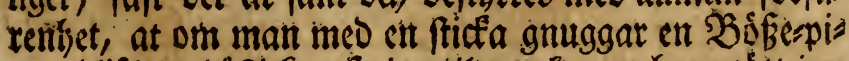
pa, blifwer triaficfan fotig, til et teklt, at lyon nutt jers net, od ide jernet benne, od) Stäftet nister alla Dies taller.

PLOGAR fagos af famma Man förfárbigabe, pa brwilea iftallet fór en Enif eller rift ftodo 3 Enifwar, Doef fials: De fa, at oen ena ftoo frammanfór ben andira pa fire eller trappetwis, Groarigenom torfwen fónoerféars i fies re lemfor, och) faleoes láttare uplaftades och) fonders plogoes; men at Enifroarne gingo trappenisi, fortioits 
brabe at torfiven icté fülle frammas och fáftas mot enife marna wio frärnitgen.

- Act. Stockholm. 1747. trimef. I.

BLAKLINT, Cyanus 7 ro, waiste pá en alecr, fom war fado med Canarie-fró. Detme âfer froo jente fornåtrars na, odf war faso famma Dag med oem1. Canarie-fäs Den wäte tumn odt fa full af Denna Cyano, at aEren ftod belt bla, brwiffet fóll of untoerligit; entedan fnapt

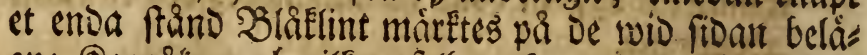
one Gornafrar, Gwilfet ofelbart Eummit Deraf, at Eors net i fina âfrar forrquaft SBlallinter.

RESAN ifrån Rơfshull til Bolftad wa qu quart. 8; Gárifråut refte ni förbi Furforna Grinftad, Järn od) Holm at Mellerud.

ANGARNE ftoov fulle of Arnica 684. Ptarmica 706. Erica 309, od) Pedicularis minor 504.

MAJST ANGER woro upeefte wid de miffe gåroar, rebant fór Midfommarbsnatten; Gár til togż en läng ment gans ffa fintal maft of Stian, hinilfen fealadeb, feoermer bes flitodes hout ringivis alt omfring meo lof oct) alla flags blomfer. Lungoomen bade omering vem wid siofonts maren fina innocente núien, meo füng, Dans och leE, efter fima fürfäbers fitt, til at continuera De gamlas Floralia, ciler at fütruvia fig af fommarens lârta fä́

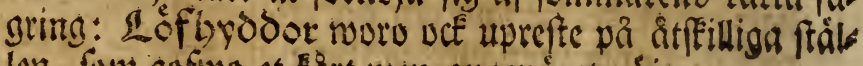
len, foun gofivo et Eart neetr angenamt noije.

* Melterud, quart. 6, ifran Boliftad.

$$
\text { Jul. } 25 .
$$

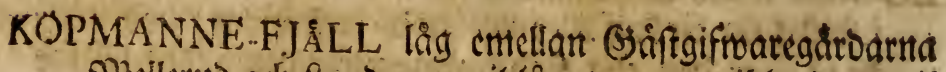
D) 2 ellerub odf Suno, 4 mill lingt, 12 mil breot up tif Sivrrige. Def̧e bárg woro life meo Bohus bárgen til

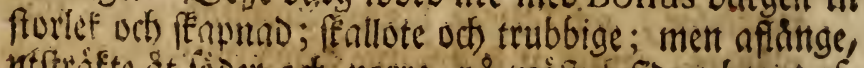

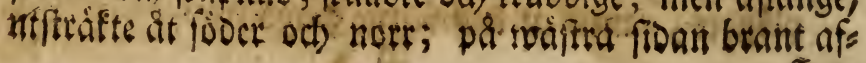


fientre; mett ât offer affluttande. De áro $i$ fig fielfiwa

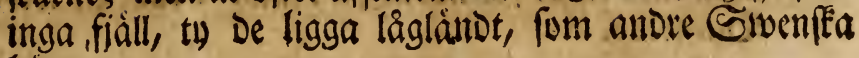
bàrg.

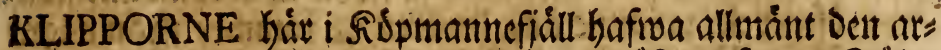
ten, at De blifiva gróne af raignet, âfwen font en ₹ălgs fren, och Dárjemte, at Dâr De fondoerfipructect, hade fprins gorne iboplätts med et ârt, fom ofta fteg hela tumumen bogre ân Elippan; Gnwilfet torde Eommit Deraf, at rảgn, wader od) Sol bade formutt dent lófare Elippam. Def fe irre woro merendels af famma art med ftentent eller of

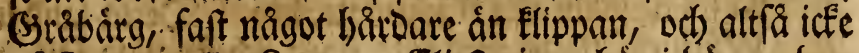
af Quartz eller Spat. Elieft gingo hầ $i$ birgen bre Dare odf) ftotre horizontaile twadrfpringor, fom woro upfisloe meo en hroit Quartz, áfiven fom âbror $i$ en grufiva; Defe fagos bar i oppra Dagen bredare, frürre, bet) pả flere ftällen, àn De jag någonfin tifforeme feot, th Gar war enapt en enda Elippa, fom ei bade flera fis Dana itora ofh lánga Quartz-ftref. Demme Quartzen

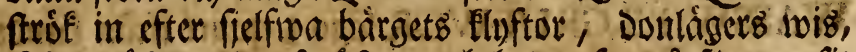
ifrån wáfter neder åt ófter, vch bade ofta på fidortua fit fulband, oct ivid Det famma god och) faft soilgften, fom funde brufas til grutet vci) Dyliett.

ANron SwaB Docirgmáftaren, fom framfor alla Sivenffa gâtt fâ lángt uti malménnningèn, at ingen i Europa med bonom fortifnas Ean, bade of Sfrwertheten blifinit fóroronad at frándigt refa onzering i Swerige til at uys leta Dralmer. Saan, fom til Demua nuttiga ivettenffas pen have upoftat all sin tio, have oct Dertil fedinft all fin lefnad, Da ban buwartern fófte bemwift cller familie; utam af âboga für fciencen oct Publici nutta ftadigt refte, Groarigenom ban oct munnit bet fitunclag ; fom befigar alla. Demie Serr SvaB bade pa intet ftâlle

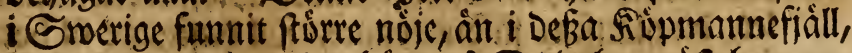
od) De flera bebageliga bárg pá Dal, lywarift ban ups

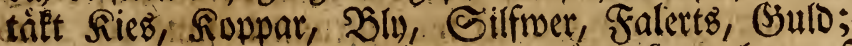

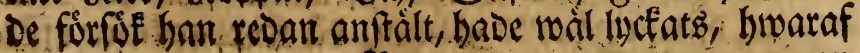


man med tiben fúrmadac pa Denn Tract en wiolyftig Sörigstag.

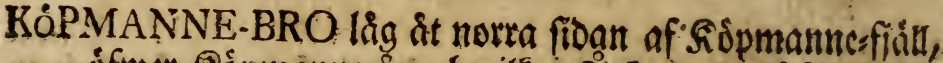
ifwer Ripmanne:ailt, hroilfen $\mathcal{A}$ Eommer iftån nágéa fión i Werneland, fáfom Stora Led, Silen, Led lậng, Lax-ljön, och faller bàr ut $i$ Venern, gifwe: ent beqmonilig Transport ofh fart at ech froin Venern.

WESTGO゚TE-DAL En!lả Den Delen of Dal, fom ligger

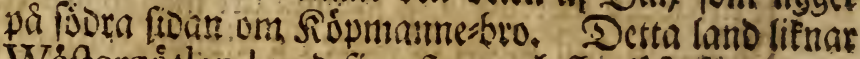
Wảftergótland mei fina ftora oć) fEoglófa fält, wida

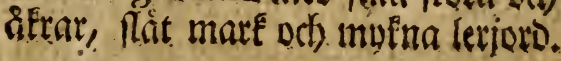

WERMELANDS-DAL ix Den antera häffen af Dals lario, fom figger nort om Sivmmannesbro, och fer alls Deles ut fom Wermeland; ty Det ár mera bärgaltigt, mera leogfullt, fullt af fisiar, och bar mycten fandomo.

WASSWIKS-GRUFWOR lågo $\frac{1}{2}$ quart iftån Ranostuá

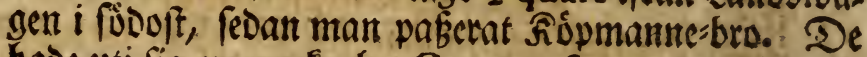
babe uti fig en mycfenfet $Q$ uartz, fom iaar artigt ods fällfant meed nágon broit Ealk blandab, uti Gyvilfen

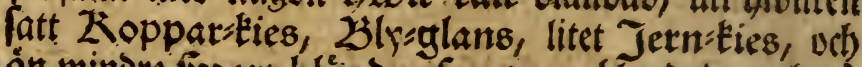
án mindere froart blande, fom woro blamdabe, oct) pa fidorna befládoes adorotna meo đálgften. B̉lanien ffildes ifrản Siefen meo fófring, fesumpning, fiets ning, xoafenting, altfa nog móbofamt; men artigt wat at fe buru Roppar:tiefen fetilDes ifrän Blyzglanfen, fedan sialmen pả fórenámoc fítt loar pulveriferad,

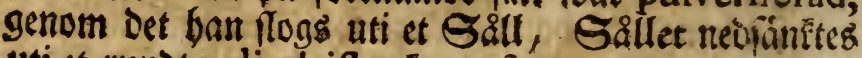
utt et runot cylindrifkt for, fullt meo matten, svat: Itet fprang in under nebfönffningen igentem/ porerna på fallbotnen, och) under intufandet uploftade Dalmsmis: Iet, Da glunfen, fafom tyngre, föll baftigare til botnen,

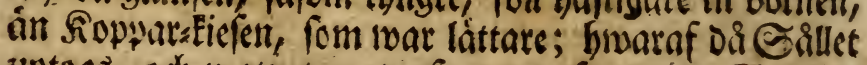
uptong, oct) wotnet wat afuunnet, fans ren Fies ligs ga ofioan pa setra glanfen, Da Sopparticfen affitapae Diss ifrän $\mathfrak{B}$ lyzmalmen. 
SPATUM album lamcllofum undulatum, en clieft fälfunt fren, fägs băr i grufet, allmuint iblano Quartzen.

* Lund. quart. 7 t.

SEDUM petreum 387. giorbe talen i sunt belt knvita, med fita broita blommor.

\section{iNIMMEN en ftor fio lâg pa waftra fitoan om waigen.}

INIMS-WIMMA fallabes en fiffe, fom til mucfentifet fain:

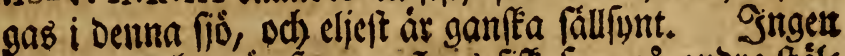

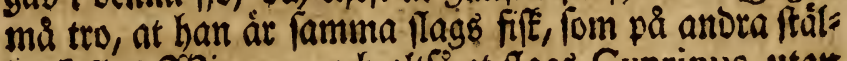
len Hallas 23 imma, octh altfấ et flags Cyprinus, ufatt ban ât en COREGONUS eller Silt af et quarters. längo. Den forra vyggfenan beftár af 12 miuta ftràlar, iblant blwille be 3 fórfta äro bele, men be ane ore grenige; Den forrfta frtålen ár alotamiml, Den ans ora längre, Den treoje lángft; men Den ifte, eller $12:$ te

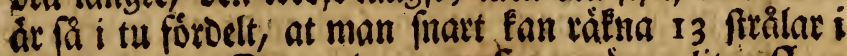
Denna fenen. Den ambra ryggefenan ár en liten flote feira, utan ftrâkar, mindre fet, án på De andora Sits flagen, odb náftan fägad i Eanten. Zbutefenorne bafo wa hwarbera so frrảlar, fom alla áro forbelte, utom Den forfta, fom âr hel. Gumpfenan har 14 ltralat, af bioilfa I fartaft, 2 lángre, 3 lángfi. Esjertent ấ tweetlofo. Gidessftrimman (linea lateralis) ât raf. Uttom Dentra 23 imma finnes i famura fis ocflá ent Gitleja, fom ár fror fom Sil', af z alns längd, helt

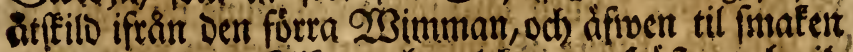
olifa meo ptora Silen; bon leter om Sab́ften, hivile tet gifmer tiltäna, at, Gour afroen ât af Sits fligtet.

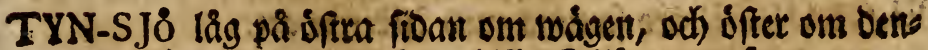
na fió woro nágre Stwabiffe Eiffiver grufivor.

- TORPANE. guart. $5 z$. 
HóET f́ămbes af Det Dagclige rágnet, fom warat alt ifrản Srollbattan, intil Des wi fomma hit, med Dageliga

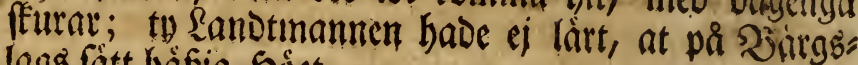
lags fatt hàbia Şoet.

WÅEN låg liffom en Allée emellan de alt ifrån roten up til toppen gicuiga, boga oct grona (Stanat.

* AMÅl. quart." 5. Shár fingo mi brvila, od) blefruo

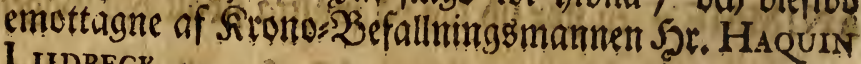
HIT) LIDBECK.

\section{Julii 26 .}

AMALS Gtad ár Den enda pá Kela Dal, beligent rátt wacs 6in tert wio wafter fidan af Venern. Jja norka od) wha foora fiban ár han omgifwen meo nog hoga bairg; pa offra fidaul gár on láng 2 bil af Venern up til ftas

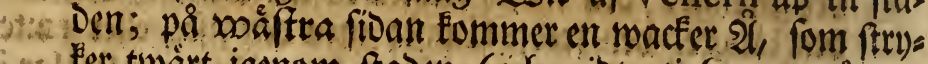
is ler twät igenom fraden (od) midt uti bonom gor $\mathrm{ch}$ *os bugt, friom et Siumftet S), fa at fartugen begrwämligen Whunna upltiga i fielfwa ftaden. Elieft ar ftaden liten,

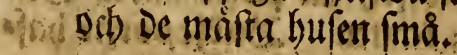

FISK făngas mer i Ảmál, án wio någon annor ftầ wio Wenern; ty begáfwo wi of neder at ftranden, i tan=

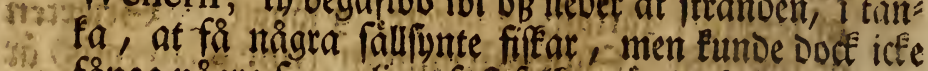
51) fanga några (unnerliga, faft folfet námbe àtfilliga, fom wi ontét fá le, fácom: WIMMER, hroilfa lara mata de famme mi fágo $i$ gar. GUPPOR, fanfée af famma fort, men en varietet. STENSUGARE, torde wara Sten impan (Faun, 279.). GADDSUR, ar, Ean bánoa, Gafterofteus (Faun. 277.). GLI fades wara rors af minfta forten. ELGKUT, OR áro famma meD Zlkufooait.

ALKUFVA war Den endafte wi funde fanga, bwilfen til alla Detar fom ifwerens meo Det Sili, fom fags wio Alingsåsp.132, CYPRINUS nin. (Faun. 331.), fait bart 


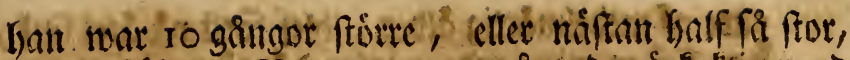

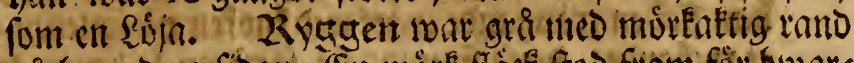
pil lwardera fioan. En mute flice froo finm för bwar= Dera igat. Égontingen war hwitaftig. bufrous Det, eller feallen war náfan genomfinand, oft be frivos mes froarta flicefar; pa froppen tworo inga fiall. zkyggfenan war lang odf fitifg med 7 ftralar. 2brofts fenan bade i à i 2 ftrilar. Hagfenan 8 ftríar. Gumpfentan 9 ftralar, af hmilfa den forfte wat kats taft, och Den anore längft. Etjerten war flyfo med 18 frralar: geltáctet bade 3 ftrálar. Denne litla fife fanz bave i ma brictar och wid frtanden af Wenern; ban mar mucfet frial od) nappade baftigt pa frofi, fom war gjord of en liten tnappenail.

TIPULA fubvirefcens, dorfo fufco, oculis nigris, eller en liten Niugga, ia, fá liten, fom den minfta lops

pa eller gnet, fprang baftigt pa Den' Elappuren, fom lág alt frilla wat wio franter af wagorna. Antenne filiformes, longitudine fere thoracis. Oculi nigri. Thorax gibbus, dorfo fufco, lateribus pallidis. Abdomen anguftum, fubvirefcens. Ale incumbentes.

HóET bárgabes mu, faft bet af langlamma rågnet ef mat wail tortadt. Sorgmâfaren Serr PETTER ABBER lít Derfure faita uti Det famma litet falt, to ban fase fig ofta forfoet och funnit, at sodet federmera bivarfert brinner eller unênar, Débutom blifwer Det bebagetigare for Booffapen. -2Bi larde bàr, at oet el wore nog wail torfa Syoet pa flon eller flaget, utait boj det afmen fots tas i fâter cller fack, at Dar taga en liten warma cller fivettning, lnat of icfe, untnar bet, oct) llâr fig i las Dorna.

SJUKDOMMAR ftailla fig efter orten odf lefinas fattet, liffom irterne efter land och jordmon. $23 i$ fágnade of, at här allenaft fa fe en rar fiufDom, dangt mer ån Den, fom bonum belt od, bollen agde. En fattig?ors 


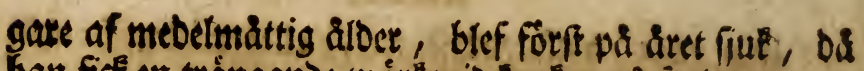

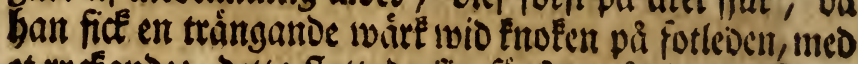

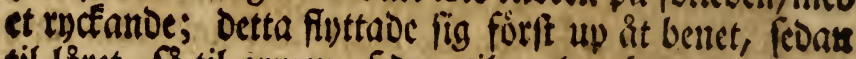
til låret, fâ til armen, feoan til areln, ánoteligen feuls Drorma, alt med et rucfaroc, at mant utan ps tumbe $\mathfrak{k}_{\text {, }}$ buru Mufclarne rnEtes; fift gicf Det ifian feulororna ned i magen, fom blef baro, fipand foin ent trumma, odb) udaffan ỏm, med bjertsánglan, qwaf o(b) méllófa. Detta fom igen biwar 14:De Dag, cller 3:Dic weEa. Paroxyfmen eller fluttningetr ifrän fot finulen til benet, låret, armen, arefin, Fülororna, waraoc fällan ófwee $\frac{3}{4}$ eller boggft $\frac{3}{2}$ timma $i$ fantoer, men $i$ magen ftoo Det lángre med et mumlande, fom twore nágot lefwande i magen. Paroxylmen begyntes befinmerligen om af tonetr, ftumbom off om niorgonen, befinnerligen om Patienten wat allena, ods ferćmbe fig. Deme fiul Dom fommer nármaft sfiveretis med Die varen. Foreft. obf. 804. ellet Volatica fcorbutica. Barth. ate. 2. n. 118 .

\section{Jul. 27.}

SỏNDAGEN firabes fơtr iniobagen uti Åmåls Givtuta, men efter midoagen foro wi ut pá lanoct, at lára of Dak Botaniquen of ganta Jigor ods Ssummor, hrwilla

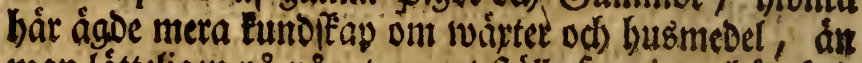
man látteligen på nảgot autat ftálle funnit; bả falo Iadeb

Sinnftágg Nardus Flor. $4 \%$ intara Galium albam 118 . Zjertblomifter Parnafia 252 .

Saltbläcker Menyanthes $16_{3}$. Greck'grás Gentianella 203. EIIgeifiell Epilobium 304.

20ockeblacke Sedum anacapferos 386.

Gtenbáar Rubus repens 4It. Inajblomiter Ulnaria 405. కboitlodk Anemone 450. 
Jeltegrås Ranunculus lanceolatus 458 . Solnojegtâs Ranunculus acris 466 . ITjóltegubbar Ajuga 475 . Stogabumle Brunella 498 . b̉oltemiálla Melampyrum vulgare $5^{13}$. Ekallea Rhinanthus 503. Grangrás Pedicularis 504 . 2lbrabams rot Geranium fylvaticum $\{72$. Joyere Vicia Segetum 60s. ZóEalla Trifolium rubrum 615. irjoleblomfter Leontodon tertium 629. Záftblomfter Arnica 684 .

Dillfan Millefolium 705 .

3areqno Gnaphalium 672 .

Ertlcifirourt Senecio 690.

3orftar Cirfium 6r7.

Skateblomfter Viola tricolor 721 .

Stroppelgras Equifetum aquaticum 836.

Enteltabb Pteris 843 .

Stogoloper Polypodium trifidum. 852 .

MULO-MEDICINA, eller Sażcurer for $\mathfrak{T}$ offapen dro

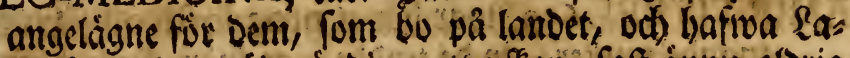

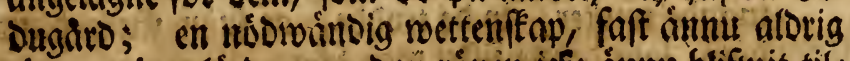
theoretice láro, emedaut runen iefe ânu blifwit tils racfeligen famlade. Somar Nation bar fit egit fátt at bota Doffapen, od) lom ini Gar fingo tilfálle, at tala meo Dem, fom i minga tioer omgats meD \&aougáross od) Boffapssfétfel, uct iblano Dem med fadana, fom bảnuti woro mycfet namnfunnige, fa wil man firt ups rảena Det fornoimfta.

XXNA fallas bär octfamma bog Sior, fom bos Eivinen. Gä Eallag xorent, eller Det, fom exciterar venerem.

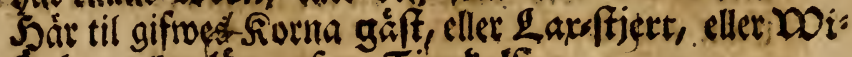
pebo, eller lope of en cjurtalf.

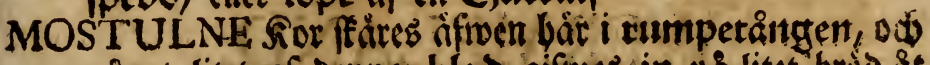
nagot litet of Denna bloo giffees in pa litet broo at Siocn; cller tager Epanfezgeona, fa ftort fom en li ten árt, oç gifnes benne ip.

IBISWA 
IDISLA, eller ttår Sioent ej Gjortar eller ibiflat, fî tages bet, fom en annor sio idiblat, od gifince of Len fiute.

GULSOT botab hos Boffapen, on den ime gula hinnan af magen pả en bơna torfá, pulverileras, $\emptyset$ d) ins

EFTERBÓRDEN bog fior brifmes med en náfwue hơnfes träcf, fom ingifwes i oricta.

WATTUSOT hos far botades meo froólgo fife, fom war tagen utur mager på en gäboa, tortad, pulveriferad, och ingifiven, eller bet, fom bà bólts for àt fecretum, torkas grodstom.

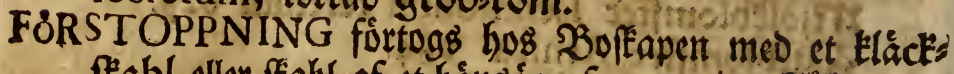
feabl, eller ffabl af et binżágg, fom marit ut tlät. En underligare Cur woar, at rlå of igenom en Enifitio, 3 gångor, od) Det feoan gifwa Son, Docf pa goo tro.

DURCKLOPP hámmaz fympathetice, on matr làter trâcten ifrän fion pá glóogad ₹egelften, at oàr ftetas. BLODSOT botades pá et fálifamt fatt, i Det de togo en Iefivande Scolopendra (Faun, 1263.), Dent famma its weflade uti mätt ler, ach såfroo fion, utan at róra Hrifet nies bara bánderna.

KARRTISTEL 659. war hár et prognofticon for tilfom

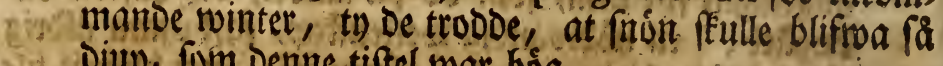

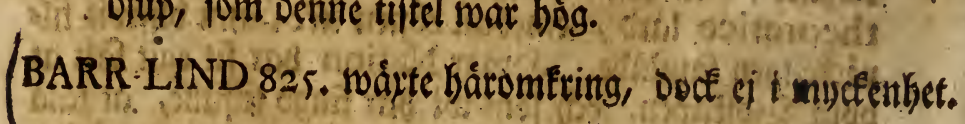

$$
\text { Julii } 28 .
$$

RESAN ifrån Åmal åt Carlftad.

SKILNADEN emellan Dal ofs Wermeland war utfatade med et fors wio wảgen, Dả wi náftan Eonunit 3 quart. 1. ifrån Amål.

\section{WERMELAND.}

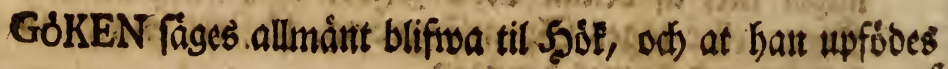
iased 
of en liten foget, fwilfer han pa flutef upater. STwart barn wet, int Ssòten lígger fima cogg uti fma foglars bo,

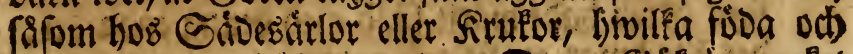
efterfölia Den utelâtte ungen. Denne (siú de muctet lie til forrg, frorlef od) ffapmad med Sparfbüen (Faun. 68.), himarfisre folfet trodt, at han blifwit til şö. Sa, jag blef ocffi fielf beoragen, at jag ainnu ei wet

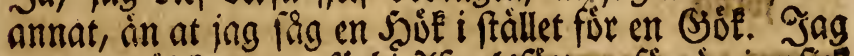
mar ei wäl fommen fórbi 22f fwelşấtter, fơrràn jag fice fe en Fogclunge, fa ftor, fom en Rramsfogel, til fầs gen ierngra, meo bmit mage och hmitt brüft bay hade (om firnen icfe alt fór mudfet beorog mig) en trofig nâf, odh ban feret feärt, fafom en unge of en Siyrto

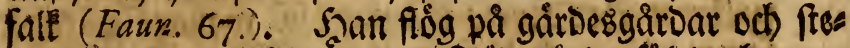

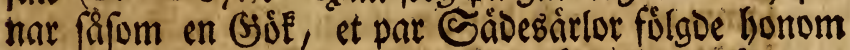
odi) matabe honon! mig mar omejeligit, at fomma bonom fa nâx, at jag turtbe diftinguera fơtterta. Detta gifwer widare inleoning, at nogare efterfuna,

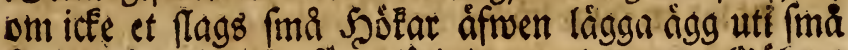
foglars bo, Gnoilfet füranlatit de gamle, at tro ssófens? fotwandling.

* AkWELsåtTteR, quart. 4. ifrån Åmâl.

KULLEN, fom là̃a pâ wänftra banden in wio wágen, (midet fort Sin Siurla, fürrin no fommo fram til ₹ulls

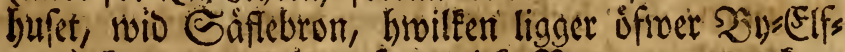

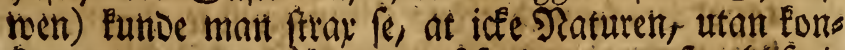
fen anlagt, emedan han wata af farto eller mo, fom blifwit forto ofruan pa en Flippa, Dár bet fringliggande landet, fom ligger lìgre ần Elippan, ei år af fádan fand; Den: the Gulle war ftotre ân andore âtteplatfer, od) Eom i

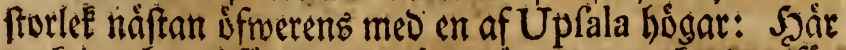
omftring har tilfötene Den bequa amlige martnaten for

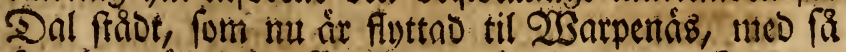
for bequámlighet fơr 23 ermelànningerna, forn Den forre war för Dalbinderna. 
LAPSANA 649, maitte hát wib Bjoftaltog fâ umnigt i

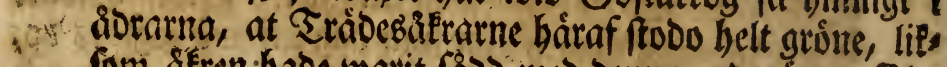

1. fon sifren bade roarit fáos med deuna enda irt; fales 13. Des bar broar ort fit cgit ográb.

SENECIO quX JACÓBEA Senecionis folio incano

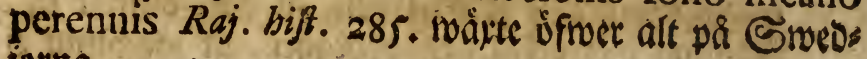
jorna.

ORTE-NAMNEN Găt pir otten toro befinnterliga: ORMKAGE Pteris 843. eller \$ettrams. HWITHUFWUD Eriophorum 44.

BROSJóN fạg til wrigen pá wánftra handen, feodn mat pafterat \$Bo Siurta; Denna fio wallar fig ârligen tits (ammants, DC) blifiver inman East máft all til en fivs lánot âng, fom med tioen ibarber mycfen bảtmio für De Éringliggande Bbyar. De fom efter et par bundrade

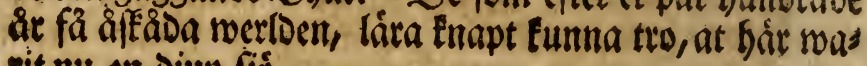
rit nu en Diup fio.

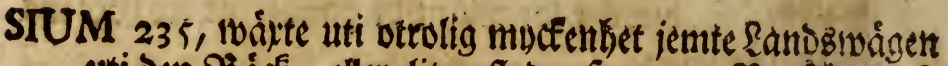
uti ben $\mathfrak{B a ́ c f}$, eller liten floo, fom wat Sotofiounb af lopp.

MASSEWIK, Scetr Olivecronas gårb, låg $\frac{7}{8}$ mill ifrỉit Eandoswagen pạ hojgra hanten, jemte en wif af Venern; badr blefivo wi ofiver natten, od) făgom bufor tapetzerade meo grisna (Srantaquiftar, binilfen enfal:

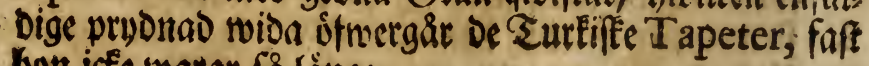
bon iffe roarar fó länge.

\section{Jul. 29.}

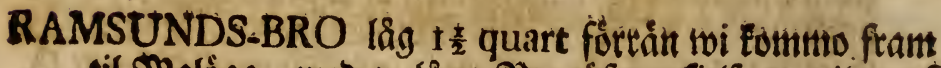
til Malóga, meo culang Bro offwer fielfwia wifen af Venern, fom bär ftact langt inast landet, med en bes bagelig lituation.

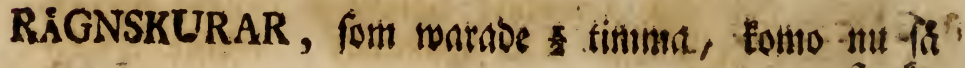
ffarft, 
ftarlt, act med fa if fwerfidoigt matten, at jag náppelic gen $i$ all min lifstio feot Deras lite. Detta rägnet foroe bart en otrolig mocfentet af Det mulna forftams

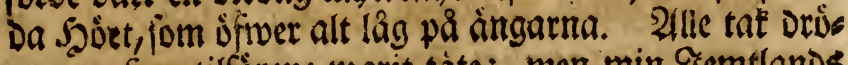
po nu, fom tifforene warit tote; men min Jemtlanos ffintact boilt mig Docf torr, fom gafen.

* Malóga. quart. $7 \frac{7}{2}$.

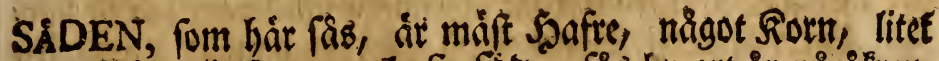

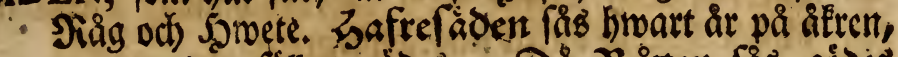

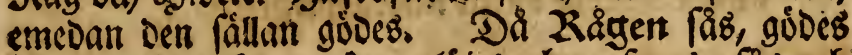
âten, plojes feDan, ater plojes, hroarfwas, fâs och neobarfruas. Kornet fas 2 Baren efter Siágfäbet, ba ấren allenaft en gång plojes, bárfwas, fas, neos barfroab. SJar brutar Zjonden Det máfa bródet af Safremiol, af brvillet bat fa rodl bakar ordinaire fafor, fom tunnbris. Ulnoertiden brugges ocefa af Saafren Doticka, men Snfresmaltet gifwer mocfet litet Dricta.

SLOTTS-BROEN mat mudet lång, fa fallas af några rudera, foin mifradé pa st fátle, Dár fordom fétolat fted et flott.

FRYKEN is en for fio af $8 \mathrm{mils}$ língo mett thog fmal, ligger up 1 gsermelnno. Dente ât allmánt belant for Den fEona Ǵlitterfanden, fom ban faftar til frane. Derna, fa af Sullos rom Gilfroes farig. Samma fand brufas, st ffura $\mathfrak{S e n}$ med, och) dfroen til frej́(and, at proba bolftâfroerna; men ầ nog tung.

ILUGFISKEN, fom finnes i Frnfelt, ár cj antuat, ån GASTEROSTEUS aculeis in dorfo tribus (Faun. 276$.$) .$

NORS.ELFWEN, en fEon od) fitila flod, folgoe wainen bes la 2 quarten, fom ifrian Fryten. 2 ibio fisouma af Denna floo lågo i watnet sintioligt många froctar, qmathollte innom fina sosmmar, bivilfe Boms 
nuar woro fammanfâfte af ihoplânfabe froctar. शits feillige fartug lågo $i$ Denna EIf, at taga om boro Deşa ftocfior, od at forra Dem til Ssitasesif, fom gief ifrär Wennersborg at Bohus of Góthehorg. Det ât fannerligen stroligt, Giwad mucfenthet af timmerffocfar,

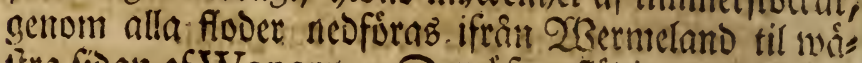
itro fionat af Wenern. Dent ofiverfósige utffepennins

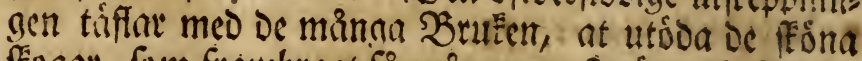

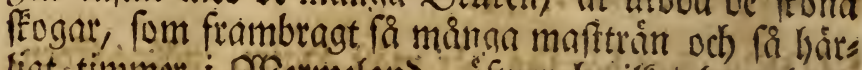

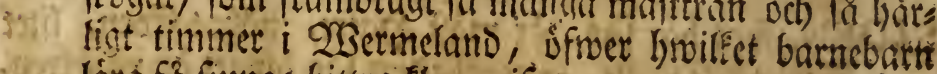
* loria fia fiunga Gittra Elagemifor.

* Litlevor. quart. $6 \frac{x}{2}$.

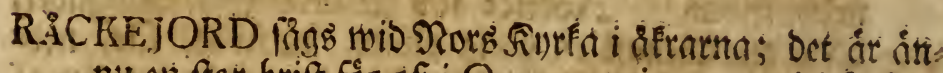
nu etr ftor brift for of i Oeconomien, at wi icfe haf: wom alla jotb:forter nog tilrácfélinen funnige, odf

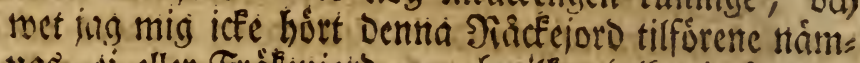

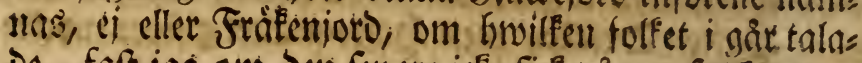
ie, faft ian ont bent fenare icfe fiet nánon fullleontmes lig eller tilfurlitelig anleotning. Silicfeioroen át på

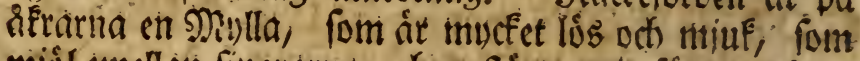
nijol emellan fingrarna; bon ftoter meo fórgen niggot

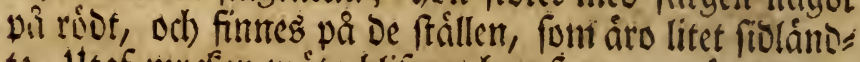
te. It nf mudeen weita blifiver hon fur; men innut mer

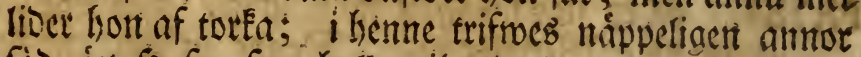

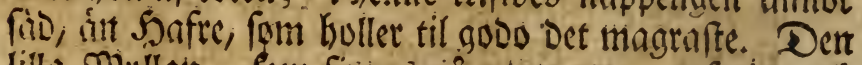
litha milltan, fom fintes pá utmagra, tufiviga och friftiga Sjungheder, ar af famma art; of folfet berâts

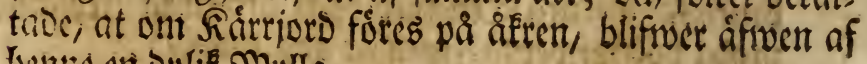
bente ent bylie Nulla.

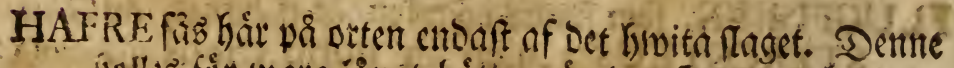

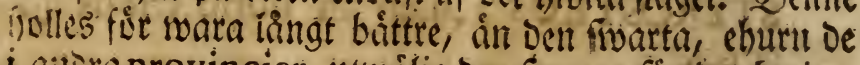
i atcra provincier, utivália ben fivar ta fór bet flwita.

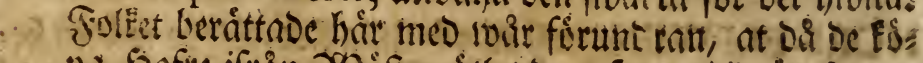
Dit Soufre ifran 23iffergutlano, for a altio ár imart, biffiver Den famma, bót fảos, efter $n$ ägrt generatio-

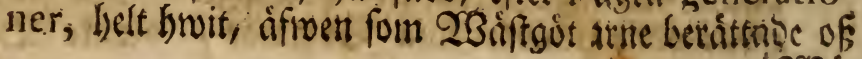


i 23 affergiothno, at om Der fả bivit Safte, blifiver

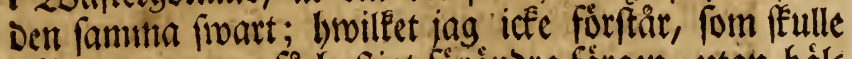
Climatet funma fá baftigt fúraintora fárgen, utan bál= Dece troe jaz, at bet muena miötet, fom blä iftän de

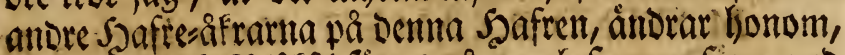
áfmen fom da SBlátal fâttes pa en och famma fang med

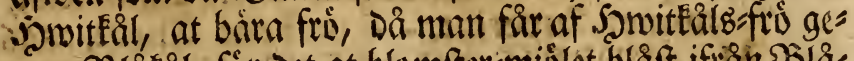

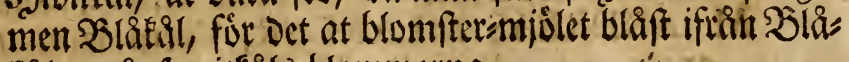

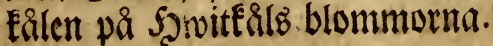

SóRMOEN beguntes ftrax wio silla Nor, Dár nartúnadss platfen Doarpenás. ât anlago. Denna Nio ltiâtite fig hela 3 quarten, och beftod af lós famt diup Saldo Mo, betất med Sall, sjung ud Mijolon rib.

* Carlstad. quart. 6.

CARLSTAD in en mebelmatting ftor Stro, fout ligget thimmeligen horizontelt, nảgot fitet uplougo at -notra fidan; Etorn Sifiven Clara delat fig in mid Staden i z:ne Delar, fom bigge ga ut $i$ Wenern, oth afifitia

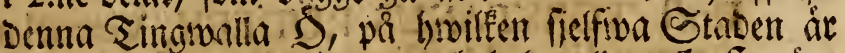

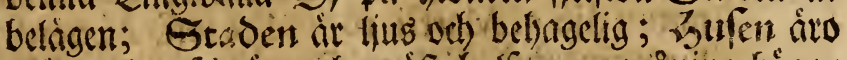
maiftedels af 'tea, od maft halfonnor waiting hojga;

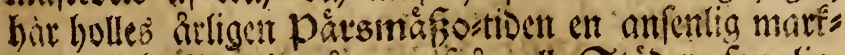
nad, tit hwillen intwanarne frân alle Stíber, fom ligs ga tring Wenern, fegla meo fiuma fartyg. Saâr âr et Superintendents fate, Gymnafium od) Schola; wio Gymnafium arro allemaft 5 Lectores:

I. Theol. Nic. Spak.

2. Log. \&Eth Jon. ULLHOLM.

3. Grrc. \& Hebr. Pet. STROKIERK.

4. Eloq. \& Poếl. Ben. D. Tingrerg.

5. Math. \& Hift Jor. Fr YxelL. STôr fä nnoe ing Hiftorix Naturalis od Phyfices Lectoratet, emedan fom jag márét, at $2 W_{S e r m e l a n d}$ S Studerande i. Uphala aro mer fallime och begifne pa Detta Studium, an naggon ammor Nation. Denna $25 \%$

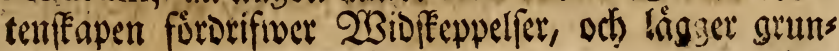
D. 
Dest till all Privat-Oeconomie od) Manufacturer, $p d$ broilta et Siifes wólfä́t ftódjer fig. Uti Synodal bus fet woto de máfta 23 ermeland s Superintendenter ars tigt affeilorade, fafom:

M. SVENO B. CAM压, Vermelandus, primus Superintend. Carolftad. obiit anno xtat. - muner. Superint. - - pcft nat. Chrift, 1666.

M. ANDREAS KILANDER, Vermeland, natus anno - - P. \& P. NyEd. 1656. Superintend. II:dus Carolft, I 666.obiit 1672 . (III:tius deeft.)

Doct. ERLANDUS BROMAN, Vermelandus, per Vermeland. Dal. \& Wik. borealem quartus Superintend. obiit anno 1693, muner. Super. - - xtat fur. 60.

M. BENEDICTUS S.CAMIEN, Vermel, Superintend. Carolft. poft priores quatuor conftitutus a. MDCXCIII. obiit a. Ch. 1704 .

M. JONAS ARNELL, Helfing. nat. 1642. Paftor Holm.168 I. Superint. VI:tus Carolft, I 704 . Obiit 1707.

Doct. TORSTANUS RUDEEN, Vermelandus, natus die 9. Martii 166.I. Profeft. Aboenf. 1692. Superintend. VII:us Carolft. 1708. obiit Lincopix d. 9. Sept. $1>29$.

(DANIEL NORLIND deeft).

Doct. INGEMUND BROEMS, Suderm. nat. $166 \%$. Prot. Pern. 1703. Theol. Prof. Prim. Abox 707. Superint. Carolf. IX. 1718. obiit I72I.

Doct. JOANN. STEUCHIUS, Upf, natus 1676. Prof. Lund. 1708. Upᄃ. 1710 .

(MAGN.AURIVILLIUS, Ūplob.1740.deeft). Doct. NICOLAUS LAGERLO\&, floret.

ILODEN Claras fidor, mio foora fanten af Etaben, Dár watnet afiḱrit Dem, beftodo af moden fin, llar, runo, biwit eller gulblanoad Quartz-fano; i befé fande frander funtes ftora trän begräna ocb fulliggande, mer an til a almars djup under famien, of groiltarma moro 


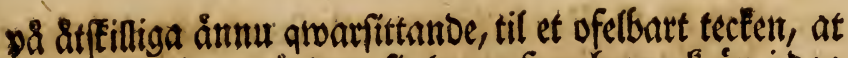
Elfroen antingen anorat fit lopp r. fom bor sof an i bag gưv, faft på andra fiban; eller har off Gár forbom wa: tit en fiugfand, Da Wenerns ittander ftriffe fig bit up; ti) Denne fand â aldeles en och) famma med flugs fanden pa Farsien i Bottland.

TJURBAST Eallades DAPHNE $3 \mathrm{Ir}$, af bimilent bars fen (Cortex Mezerei) hạr faldes wio martinaden af Bónberna, fafom et prafervativ fớ $\mathfrak{b}$ offápert.

KRÁFWETAN curerades bâr i latdet af Gâringar med decoct af cortice Mezerei, uti broilfet simueflutat

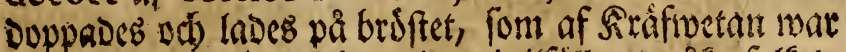

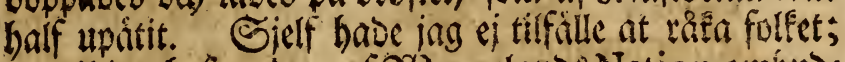
(t) wil jag bafwa bem af 23 ermelands Nation dmibuds

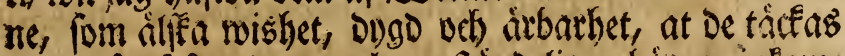

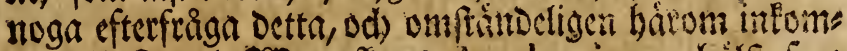
ma til Fungl. 2SeteufE bela Den lároa werloen, intil benna tio, alorig swatit $\mathfrak{i}$

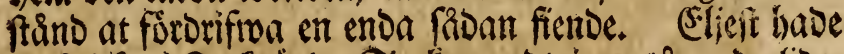
en Flicta i Fruézänoe Eocten undt $i$ en tâ mes olides lig pina, fom angrep henme firfom furthag, buivilfen blef

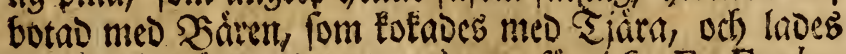
Derpoi, Do wodrten immom $\frac{x}{2}$ timma furtsice. Er. Eurén.

HÁLSO-BRUNNEN liag miot uti ct Forşgata, in $i$

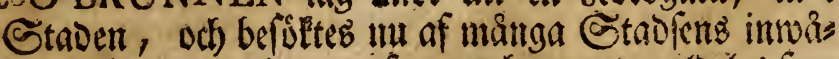
nare; han war ojupt grafivent och nutrad, alo des font en allmàn brum, utan nágot funnerligit aflopp, to wattret miffe Djupt ifräm uporagns. 2 gathet war

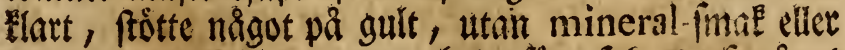
Iuft; Det gullaftiga watnet hade forrorfacats af natgot litet jord-falt eller jernevictriol, fom brattits of on ellet lertwatten; Det hase altia figa nagon frurkande mineralink Eraft, fafom anora furbrumnarz soatten, od)

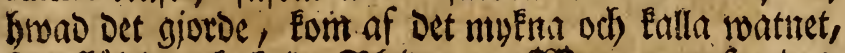

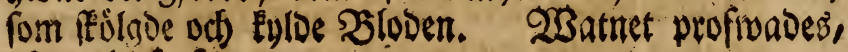
Dá Det blef af

D. 2 Sac- 
Saccbar. Saturni ladtefcens, opaca. Gallar.pulvere flavelcens, minime violacen Spir. Sal. armoniac. immutata.

Ol. Tartar.per deliqu. opalefcens. Spir. vitrioli immutata. Syr.violarum cinereo-carulefcens. Coccionell: pulv. purpurea. Mercur. fublim. fubflavefcens fedimento noti granulato.

MARSWIN år hăr $i$ Smerige et utlándffet diur, form pâ fầ ftallen, fơr ro ffull, föbas od) alfreas i bufert. 233 have băr tilfälle at beffififina dem uti Sar. Doctor LA-

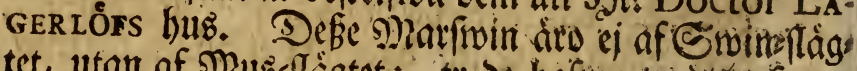
tet, utam of Mus=-lägtet; th be bafwa twoititre fram

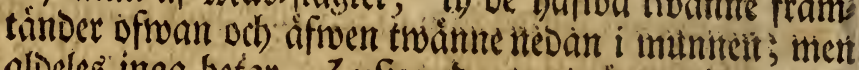
aldeles inga betar. Juf wouber twat tämmeligeth tibctet

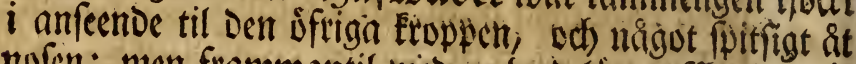
nofen; men frammantil mied en bred lẩpp affíturit, nás

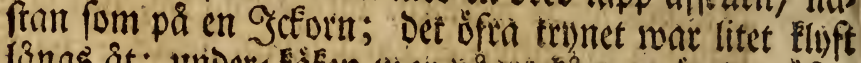

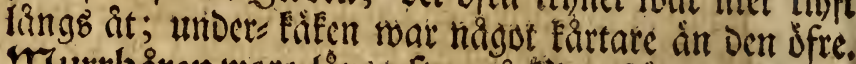

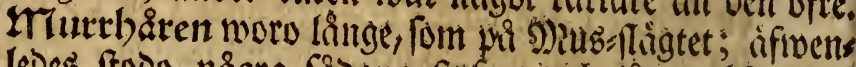

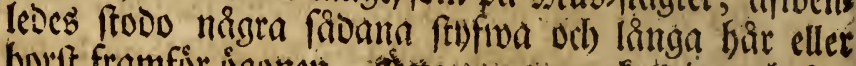

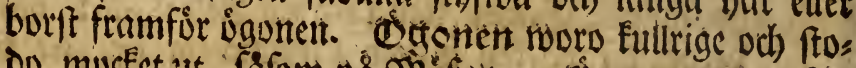
Do mycfet ut, fafom pa siósen. Eironten woro fros re och nafne, meo bare notor, fafom par Mófen; man

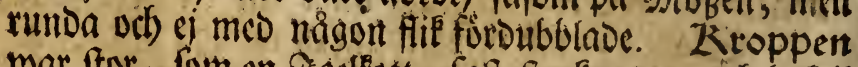
war for, fom en Ggeflett, faft fractugare, oct baftil fulltrao, táft meo tämmeligen ftuffua och långa barr, fom pa nacfen yfoe fig nigot tmer, an pá ifriga Delen af Eroppen. Jalfen niffan aloeles ingen. forterne

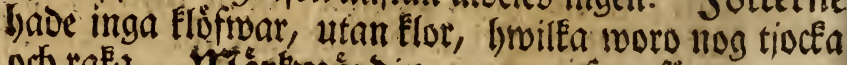

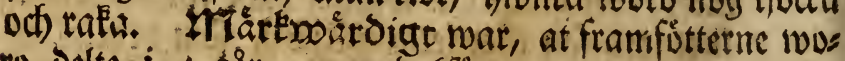
ro Delte $i$, tare, ment baffótterme allenalt i 3 tår. Sxoanfen war nôftan ingen. Sanb lite och gång lifnade Miśbens; Sátgen varierade, fấom pa anore bemtambe Djur. Sam altfá Detta Diur Eallas MUS couda abrupta, palmis tetradactylis, plantis tridacty- 
dactylis, off âr bet famma med MUS, five CUNICULUS Americanus \& Guinenfis. Raj. $\int y n$. 223.

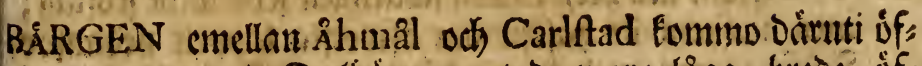
mereng med Dalbärgen, at De woro lága, breve, if:

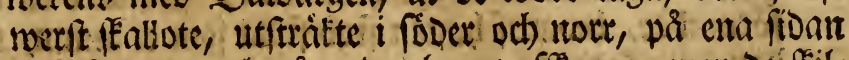
fiatt fulltade odi) pa anora brant affeutue; men de feits

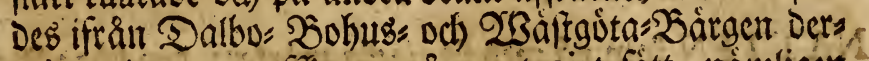
uti, at De noro affêutra pa contrairt fott, namligent at offer, men fmáningem affiuttade át waiftra fio ar ; ooce fom dertue obfervation endaft ar giord wio waigen, fämas bet til at midare befturens of utrơnas af Dem,

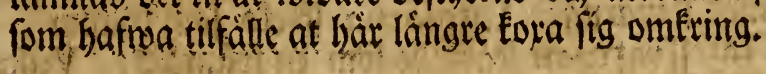

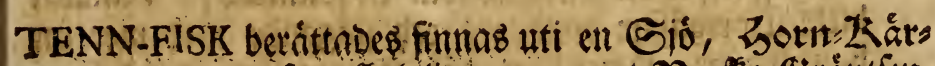
net Eallad, fom ffal ligga up emot গoorfá Brrintefert, oc) at i famma Ejo ej fá finmab mer ân Denne enda fiff. Lector FryXeLt har pa min anmodan tatit bämta bonom dọifirin, och feoermera fficfat Den fams ma til mig, oa ing fant at hanwarGASTEROSTEUS aculeis in dorlo tribus, (Faun. 276.).

LAX-FISKE moro hani $i$ landet 3 befunnerliga: Dejer-fors, Munke-fors obd Skifive i Clara Elf, for brwilla i Arrende fades gifiuas 750 Dir. S:mt. Sill Denne $\mathfrak{a}$ s ren fommer utur Wenern, oft thctes dár fóda od

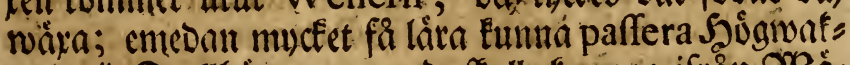

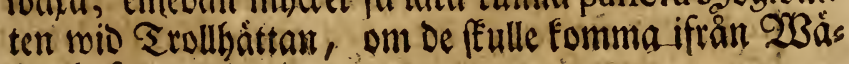
ferthafwet.

\section{RESAN ifrán Carlftad ât Philippftad.}

* PRÁstegårds Scáfigifivaregåro. quart. 5 .

GRINNAR pliga ivara allmàn ro eller oro fár be refande; men alt ifrån Áhmál til Carlftad od oer ifrán til Philippftad war iffe en elloa Srivm pá Samosroágen, fa

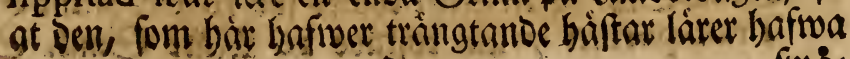


frosightet, at fa bem frart igen, on ban happec nie genlìis.

HiST,AR, fom àro yre, funna froderligen feos utan fooftall; men hair togo De bâften oun bifiverfta läppent nevanfor Seâfebororna med bägge hánderna, fa fratit en faul formáboe, bwaraf hajfen ftoo fitlla form et lamm, mes Dan han fFoddes.

NATTEN fom pa meD fit tiodfa mórfer; Den bogga 3arts fegen fomtes fom en mur Dubbelt boggre'af morfret; R ornablitcet lyfe fom (Gaftselo haitigt od) ofta, utan Din; Bäftarne gniftride, odh flogo clo med feorma einot frenarna; Ligglotra ffreto fom Siaftar, od

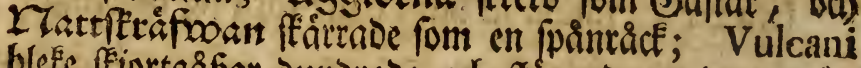

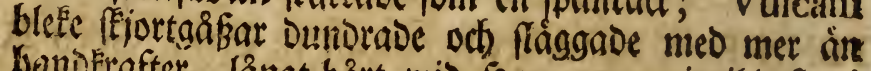
banoernfter, längt bâtt nio Sammarna, intil bes wi fl. I I. om natter fonme fram til Norum, efter s. quarts refa.

\section{Julii $3 \mathrm{I}$.}

Herman Kolthoff, Sirufbs:Patron i Norum, hade haft famma boe meo fine 5 eller 6 Syffort, at haul nio inens: tiltagande furrlorat fin finn; men ehuru Denna olycfan war for Sngfenen allmän, war hon doce iffe arffelig; ty Fadret bade ei haft faumm fivarighet; octh defutan kade Demute $5 B$ tules: Patronen Amaurofin elo

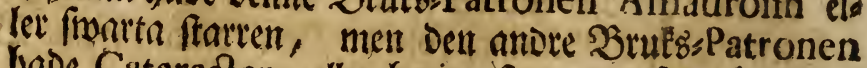
bade Cataractam eller hmita ftarren, fout â of bel annor orfae och) art. Paturen, fom plágar $i$ et mal incoergílla, hivad han $i$ et annat båtttager, bave pà Demma bà ffárpt minnet od) DugDen.

HAMMAREN wiD Norums Brul låg beqmámlig med bchageliga $23 a t t u d r a g$, fom på froorne pruboes mes

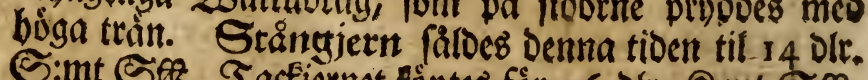

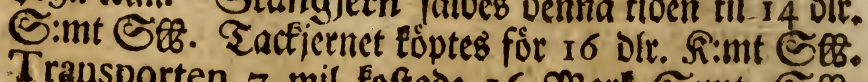
Transporten 7 mil koftade 16 Mart Simt. Ë. 


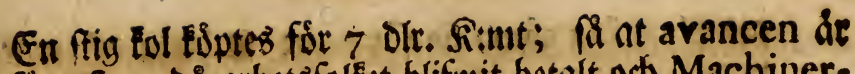
föga ftor, Da arbetbsfolfet blifwit betalt od) Machinerne underbollne.

BÃTAR woro hár artianre bugde àn pá be fläfte ftállett $i$ Stwerige, od beqmámligare at fara igenom flobar; to en bât um 2 I alna längo twar midt på 4 almar bres oć $i_{4}^{\frac{x}{4}}$ aln djup, med 5 bols hógs utom fólen, fon miot undee war Dubbel; Saáten war fpitfad od uporagetr St ânoarna; 10 wrängor niot pa, utom 3 wio hlwars

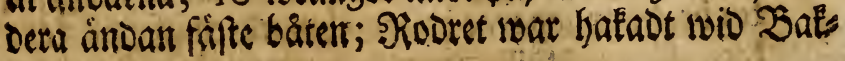
ftammen.

IUREN'(Fl.788.) blifwer Det hügfte triet i Sivetige, odj bet tíflar ofta med EEen $i$ ár odb âlocr. Saerr Affeffor ULR. RUDENSCHÓLD har fumit i f̧inland Gurustrát af 320 âr *. Şấr wid Norum fágom wi en Furus frisce affhuggen, fom war en ibland De frórfta wi nås gomiur feot, th bant wax 33 altiar tang utom featen:

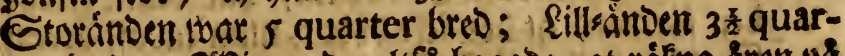
ter bred. 23i mordo altfá hogade, at rátena areen pa

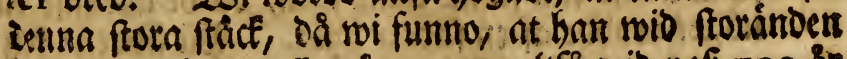
broe 409 vingar eller ar, mar altfa mio pab 100 ar

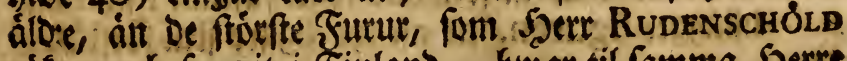
ráfmat od) funuit i Sintano, broar til famma Saerre fieff thef et witne, fom mòtte oह hair pa Norum, od

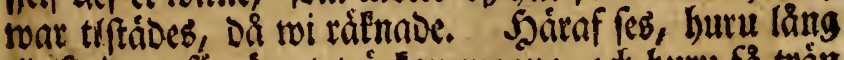
tio fortrab, förrón et trá Ean mognta, od huru få trán

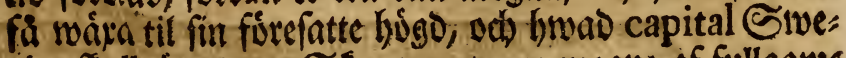
rige fiulleága, um Sfogarne woro mogne af fullgams

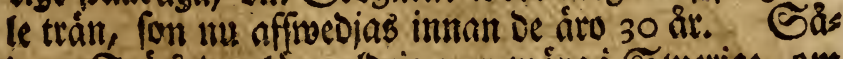
Dane Trágirbar lára aldrig mer wáka $i$ Swerige, om iffe niggon hatal peft atfopar lonbet; to fnart ar ingen winker ellet urå $i$ Siverige, fom ei utfättes for brans Den. Gas buller germa med Dem, form tro, at de måns

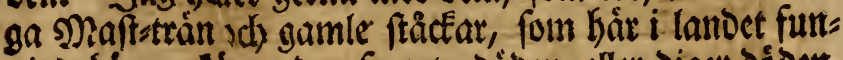
nits, bóra ertánta Den frarta dojden, eller Diger dódent, for fin moder; ts Da Dente ut fopat follet, fingo feogats ue wára fritt, tildeç folfect lumut furófa fig, fa at ins 
3he gen plats fan gúma fig for Dem; the Detta trå war ups

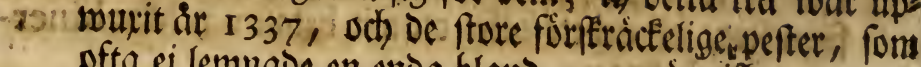
ofta ei lemmade en enda bland 100 mánififor, tyrannilerade emellan 133.0 til 1350 .

$$
\text { * Act. Stockholm.1746: P.rir. }
$$

RÁFFALLA wat anlagd mid Norum, ps ef mucetet lått

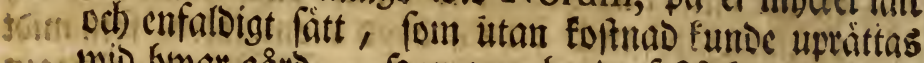
nso wio hyar garto. Soun war bugo af ftactar i furtant,

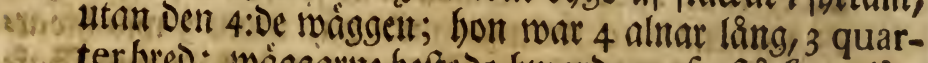

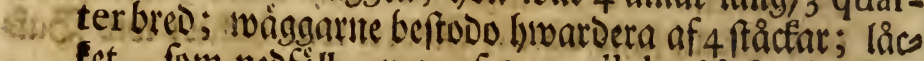
fet, fom neofisll, war of 8 parailela ftactar howfatt, brwilfet upgilorades med en ftång úfiwer en litert galge;

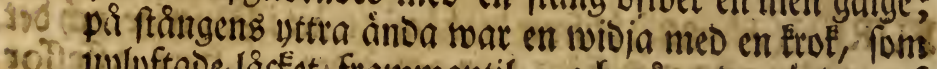

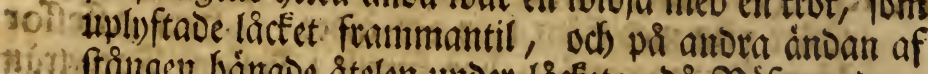

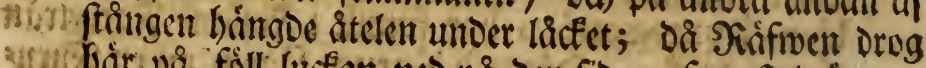

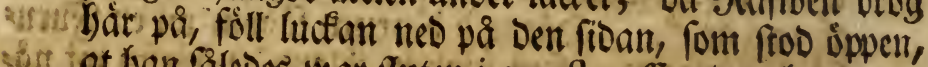
ant int ban falcoes ibar fluten $i$ arreft. Emedan buntoorne

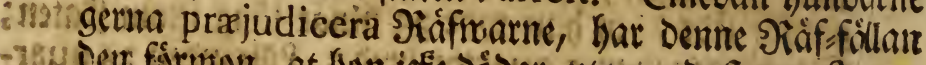

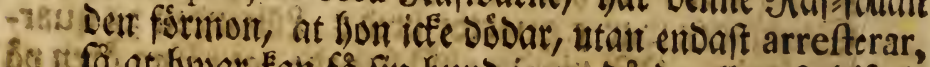

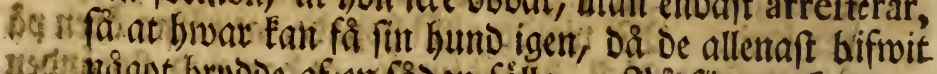

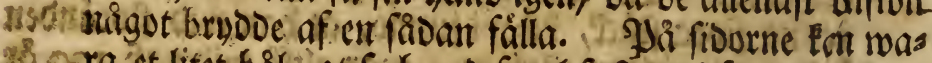

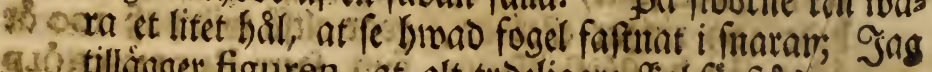

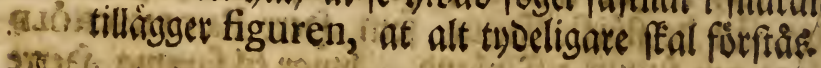

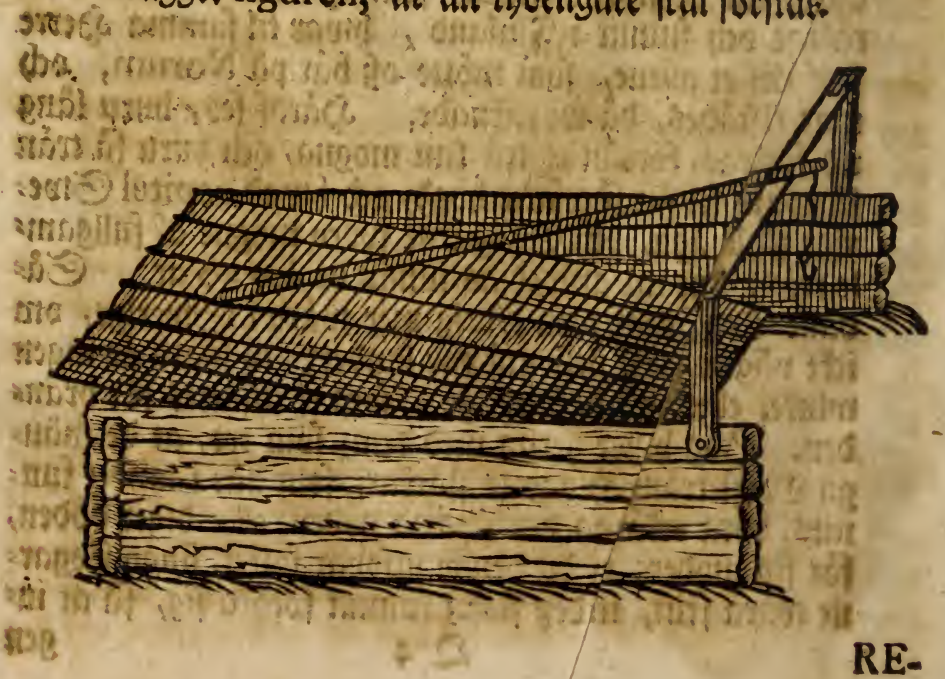


RESAN fortfattes iffin Norum ifiwer álfipen od Norums

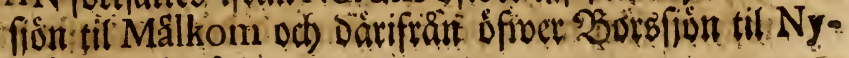
Eds Jureftegåro.

\section{LOBELIA 714, waikte wid alla Stranber.}

PARNASSIA 242, ftoo nu meo fint attiga blomfter. 3252. De nus attlagna blomftren woro alle flamina Eartfare.

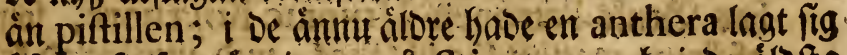
iffwer fielfma byningen pa Stigma, och i de áldofta blommor bade ftamen 2, 3 à 4 lummit úfroer Stigma, od) fedan de miff fit miól, biggt fig ifuian Piftillen ut at fielfroba blomblaber; altifa mifa befe blommor banogripeligen, Jutu Antheræ meo fit miöl foecundera piftillen, fom änu icfe med finq Stigmata fulles tomligen utwurit.

JUNGFRU-RIS Eallaves hạr of 28 ermelánningen SOLL DAGO qux virga aurea 685 .

NAT TEN gaf of Gnila uti NYEDS Jráftegiro, quart. ..

\section{Aug. 1 .}

NYEDS Jotáftegåro war, iblano alla jag feot, máft regulair bugd; En gamfa ftor prubnad, Toin numnits litan. penningar, endaft med gamla Yrósfent regulaira fât, fom áfween genom regulair lefnab hade redon upa pefollit fig med fin Mafa i 46 ars iffechffap.

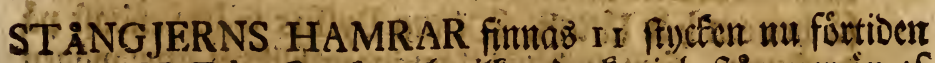
uti NyEds Socfen, hwillent Doct ei beftare mer in of

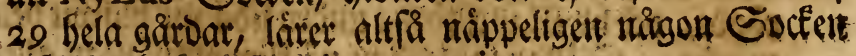
i Silfet hafwa flece i proportiou.

MAGRASTE Gorbmon tom of fore pis refan ât 23 ratte fors, hmarefe cir lós Tallefog watte pa en bed af pure

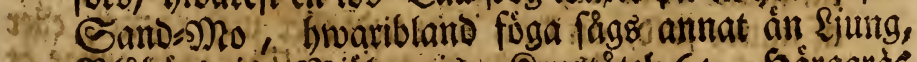

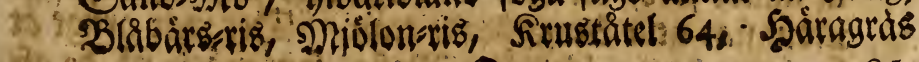
Q 5 s 


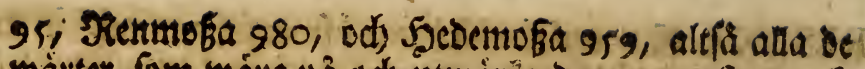

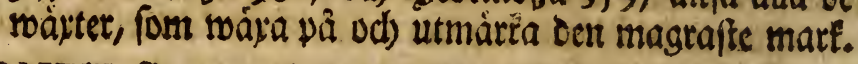

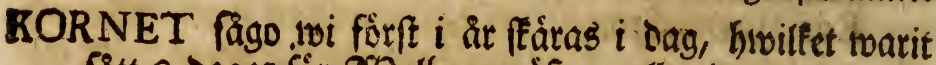
fätt 8 oagar fór 233 alborgmákan, cller Den 23. April. * BRAI IFORS. quart. 8.

ALDERDOMEN meo bes mänga brdatfligheter wiffe fig boo 3 ruE\&sPatronen HINDRIC KoLTHOFE, fom mu af åtens mattbet ligg til fänge, meo utmárgla troup,

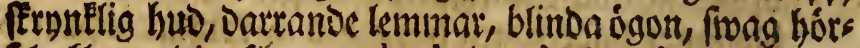
fel, Elenmodigt fíme, calculolus à decubitu, Itranguriofus à calculo. Sá fóra secen of alle genom leÉ, titflighet odi) eftettunta til fEröpligheten.

SURBRUNS-KALLA taig frtat twid Batattfors, foum bate De et godt watter.

KAKELUGNAR Ođ SKÅRSTENAR giutes wio 3 ratt fors belt Cylindrifka, fádana, fom allmánt bär oms fring brufa $a$; oc vunoc siafelugnar taga ci bårt mucs Iet rum, och be rintoe Efárfenarme wore en ftor bes

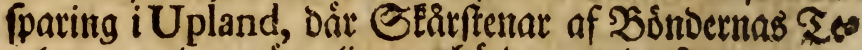
gel upmurade, näppeligent utbároa ef enda åt.

SKOGEN blef mera oifmn af bacfar, od) Jajgoberta mert feogrile od wo najterlige.

VIOL-STENEN (Flor. I 125 .) läg pa bảgge frodor om

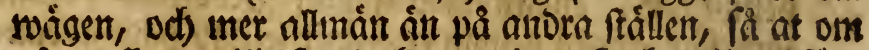

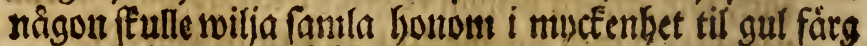
eller Medicine uti febribus ex anthematicis, fan ban

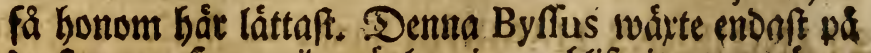
De frenar, fom noid wóglagningen blifwit utur twágent

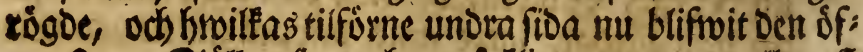
meerfa. Sóillan fants han pá ellipporna, utan allenaft Dár jorden blifivit afrodgo, fom tifforne triet Elippan; to

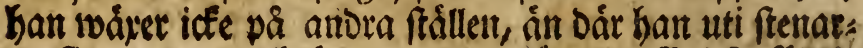

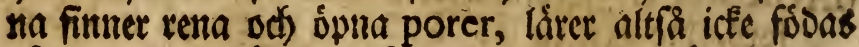
af antror jorb, äl Den, fom nedfaller med rágret, eller utounftat utur Eringftácnde trán. Det war artigt at fe, Guru benna bloorida Nioba, fom wáte yả be afs 
fopade llippor, mifte base fin behageliga fátg orb ffona luft, fa fratt ban begunte waja at Den fidan pa flippan, fon lange legat bar, Dár ban antog en matt, grón och mirkaftig fäg, fa at han fuapt funde fonas; utan wic fade jig, fom frenen marit oren, eller af Solen broino.

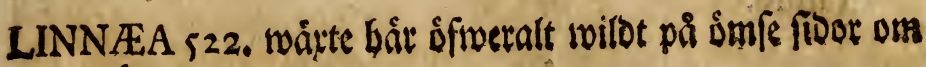
wrigen.

SKOGARNE táfte alt Det, fom fontes under ent roidsfitig horizont, Da wágen lág of foer de higa birgen. Sos la orten beftos af Barrifog, fom upfulte Dailoerna ids bethido bigoerna, at icfe en enda flicf fintes utom Philippitad, fom ei hade Denna gróna uryonad.

LIFFARGAD LERA, fom af roáféan blef ámm ríbare, bróts pa flere ferillen wio wăgen inemot Philipptad. Demna leran ar god til eloftäber, faft bon iefe ár als Deles jorofaft, od bon blefnar mer af broinningen, än fon ronnat. Nicn fürmamligaft brufas hon til ffiut: ler i grufwor; tì bon lăter bardot pacfa fig. Denne liffargade lera àr nog fällunt $i$ Siverige, och belt ht:

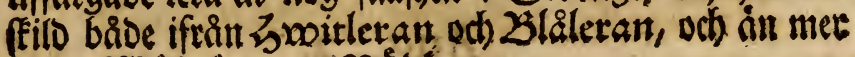
ifrân Wáftgötarnas Róbleta.

MYRA eller en SMofe lag pa rodnftra banden, formán toi fommo fram til Philippitad, och toar nog fullig ellet. uphógo i anfeenoe til mâgen. Jja Gonom ivápte bats ull 45 , Andromeda 335 , ocl Divang $i$ muct Eentet.

WIWANG war et egit od, artigt namu, fom foffet bar allmänt fatt p\& BETULA nana 777 .

Ro̊NN-TRÁNA ftodo hár til ftor muctenbet, odf nu uti bâfta fägring meo fina ruba bát. Siátt behagelige Alleér fór Siramsetoglar.

* Prinupestad. quart. 5.

PHILIPPSTAD af et Juftitiariat ellex sioping fom foit 


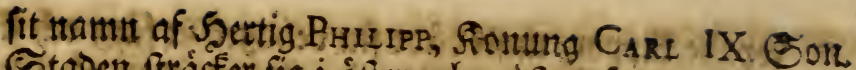

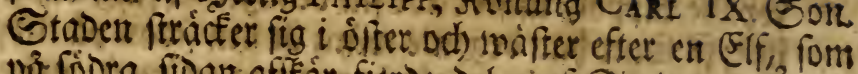

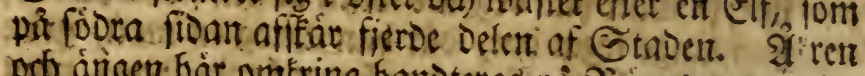

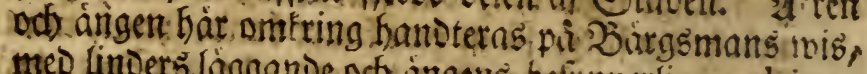
meo limbers lägande ods angens befignerliga cultive-
rande.

\section{Aug. 2 .}

SURBRUNNEN tât wáfter ifrân Gtaden, quart $\frac{2}{2}$, pooct

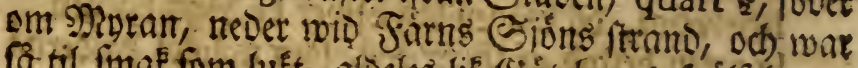

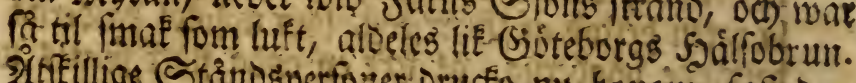
âteitlige Stånosperfoiter oruffo mi honom, faft Dere wio ámm icfe mat någon imrittning. Şan haoe fa farlt aflopy, at ban par 5 Minuter taftade 60 Eanmor eller I fat, altía pá en timuna 12 fat, eller 288 fat om oigntet.

DAGEN gafs til to fố fropp od finne; emedan wi alt iftant Upfala intiles Denna tio intet gifrit of nagor Dag, hivila.

\section{Aug 3 .}

So̊NDAGEN firades i Philippitad.

GókEN have Jar. G. Lundftedt fert ligga fina ágg $i$ Sas

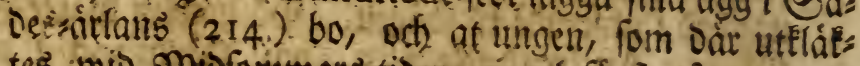
tes roib Mnidfommakstitien, ef got fortza artet, utan bet andra ods fóliander allenaft under leÉtident.

HORN-FISK beráttabes af Sar. Myrman fiumas up i lane Det, bwillen fEulle bafiva et born i pannan; Denma file

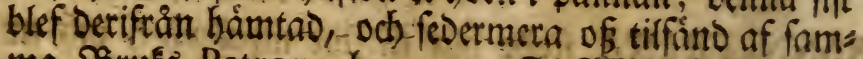
ma Brufs.-Patron; han mar GASTEROSTEUS aculeis in dorfo tribus. (Faun. 276.)

GEDIGEN KOPPAR gafs of af Búrgmátaren Stockenftróm. Denta â fáflam; tis ban or ide pracipiterad; Stufen befitoo til ofrä oclen af hroit Spat, od 
fil theota af en fwart Jeentmalm, liefont ientemalmen itti fielfina generationen pracipiterat $\mathscr{S o p p a r e n ,}$, une Der Det Spaten wätte. Denna ften mar tagen 123 ins terápa Socten ifrin Nerile, utur Sabeltulla Setm grufrua.

GEDIGET SILFWER iftin Noromarfs Serngntima, fom ligger quart 6 ifrain Philippftad at ofter, fingo wi med Den fürta. Eamma grufwa hade blifwit afs

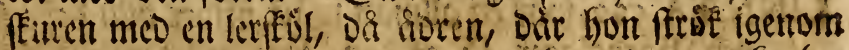
lerféblen, fürmanolat fin Seritemalm uti en mycfenbst seviget Siffrer.

\section{Aug 4.}

RESAN ifrån Philippftad at Nora.

LANDET, fom nu genomrefteg, beftoo af kdga, fort, miot frrifte, aflange ioroboirg, betâfte med tátt, grọn barrfEog, emeillan limilta ligio Diup fulla af Şiơre,

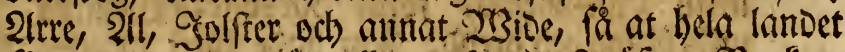

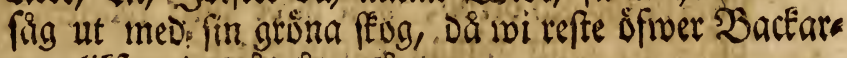
$\mathfrak{n a}$, liffom Derpa giatt róagor.

STENMURAR woro pa atffilliga ftâllent, fơr många \&u tilbafa anlagoe af ftora ftenar; men laár márfte woi, at ftenmurar fring agorme el áro be báfta, och bnoarfore Sonden fâ nơdigt Dem uplägger, náml. emedan tioen ei ffomar Dem, ehuru fafta be tycefas twara; th Den upmus

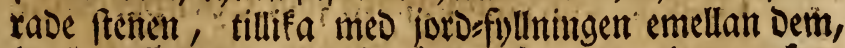
begjutes Britigen meo Sojitringnet; fom um minteren frys

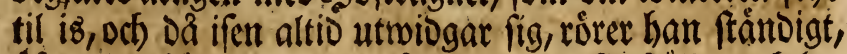
áfmen De frórffe frenar, något litet ur fit ftälle, ods bes finnerligen at fidoorna; om $23 a$ ren fimáltes ifen fơvif

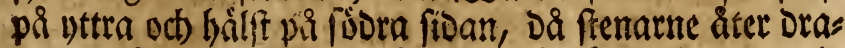
sab ut at, och fâleceb effer fere åt ándteligen ramla oméll, Den ene efter Den andra. Således blifiver en mur, fom med mycfen moior ar hoplago, froirare at reparera, vch pa flutct attróttar §andtmamnen. Dets 
fore fag mall be faffafte ftenmurat, font watit boplags: oe af De fiśrfta Elippor, pả flâfte ftăllen Eulltamlabe,

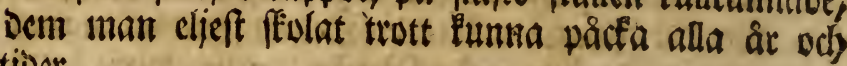
tiber.

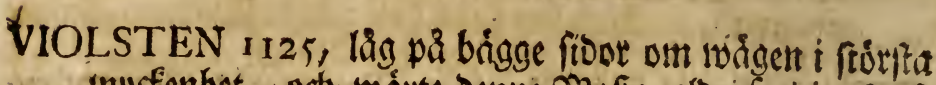

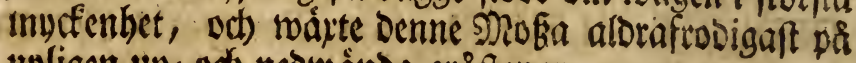
myligen up: oct) nedwa ànda gråftenar.

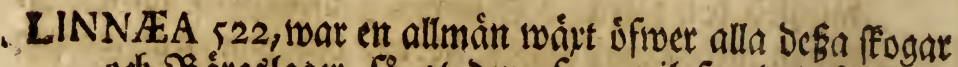
od) Zुárgslager, fin at Den, fom mil famla Deß blaver

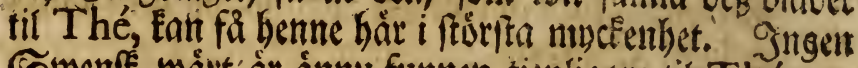
Stwenfé woint' ầ ámu funnen tienligare til Thé; ty

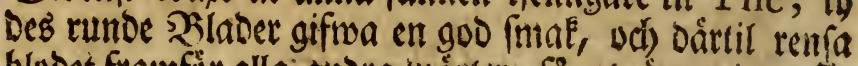
blodet framfios alla andera waitter, fa at dinnu intet fis

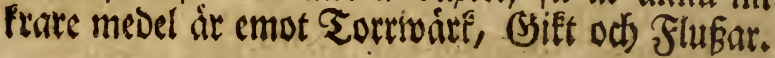

* YNGSHYtTan, quart. 4.

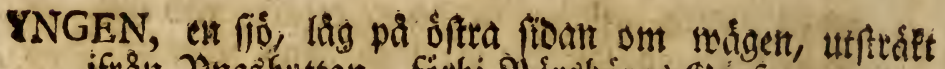

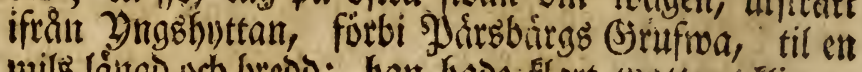
nilis lángo och bredos; ban babe Elaut toatten; Flippor

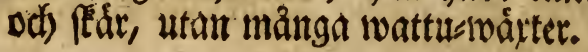

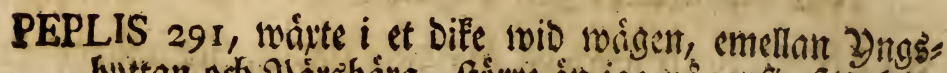

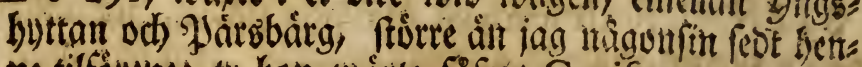
ne tilfortene; th hon wortte frifont Corifpermum ( $\mathrm{Fl}$. 3.) Uti $\frac{x}{2}$ alms bjupt watten, od) flit meo fina iffinets

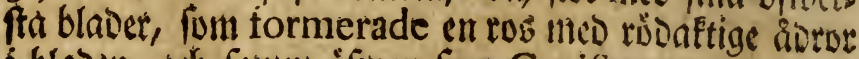
i Glaben, odh) farmm áfiben fom Corifpermum.

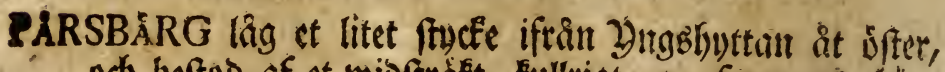

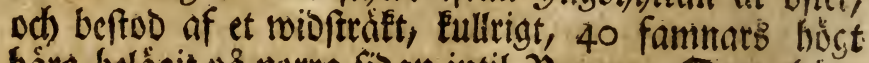

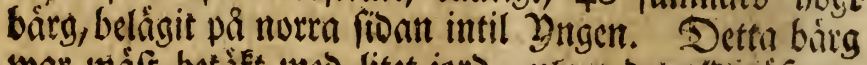

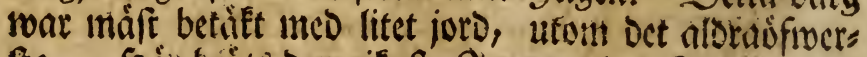
fta. Sgit brótz Den rifafte Sernzmalm, fom utt goos bet taffinde meo Dannemora, Bitsbårg wh Norbärg.

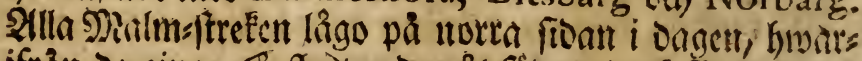
ifrản De singo fir flutt neder at fover, Doce fa, at De pá

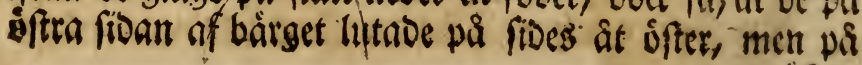


sodfter foban lutade de at wifter, faft ci fa mocet, fom De viftre. Sótiget roar igenomgrafivit med âtfilliga graftooor, aflywilfa månge waro lemnade, Doct mât pa woiftra fioan, men pô ơftre fullfülgdes be flitigare pa Djupet, emedan Der fans Den rif́alte J)?almen, brwaks af ffeot, at någre grufwor bâr blifwit arbetade til 45 fammars Djup neDer om fioplamen, fa at De in alles wos ro 75 famnar djupa. Molmen wants máft mes fétts tembe, och fátlan meo tilmalning, emedan Det baa ej fal ga an, at etter eloningen eller tilmafningen begir":a fenarna ines Ealt matten, pa Det De Dárigenom mäs ge blitwa fprodare, emeoan Denna ftenen blifwer igenom roanct lingt fegare, in utan Det famma. 23 i fagg hos Gruf Fogoen Friberg, meo lomad fit grufman blifivit Drifwen befie fenare aren, od Groas Mralm, Srrabing oct 23 atten bât utuc blifmit uporadit:

นี้. Malmstunor. Gräbertys:tr. Dattubaljor.

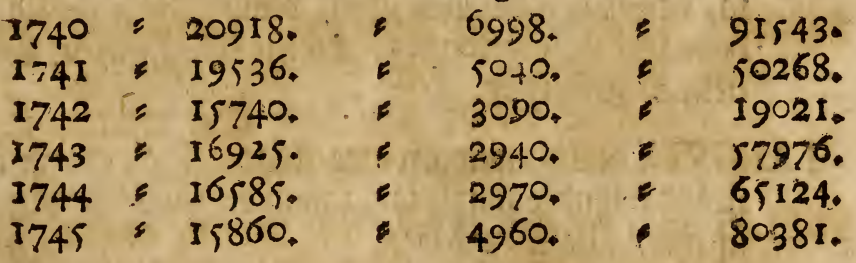

MALMEN frótte máftebels något pâ grơnt, och Den gris ne balten bolts fore at wara gooattig, famt befta af tale; Groita fpringor fagos bait och bân $i$ Slalment of hwit od ren fnle; hair fang Zlingmalm od) Caklg: ften i mucfenbet. Sisoa od grofwa Granatee tooro ei fillifinte. Malmen faldes fällan efter wigt, utat

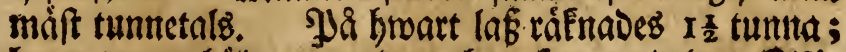
bwar tunna bult 52 puno, bch gaf merendels I SE. jern. En fádan tunna Malm af báfta laget faloes fór 4 Dir, men Den fâmre fór 3 Dir.

WWIT KALK fans $i$ bürget mio 5 famnats Dint, boct ei wio ifiverfte fwetfen af Bärget; Denne hrwite Salt toat flar, groforonis fom et Salt od tiente til en god 
Slüb for jernsmalnen; faft ån Sialf, font uthoertioen

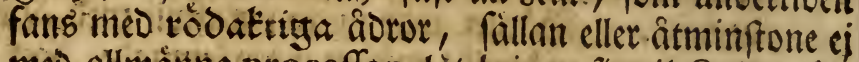

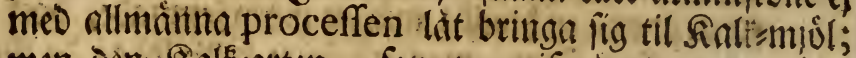
men bern fialesarten, fom loar ifpràngo meo gröna

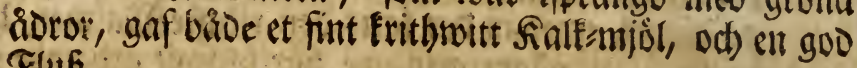
Fluk.

GRỏN KALK wat nog allmăn oméring Malmeet, fí iod fom i f pringorna emellan Malmen.

SANDSTENS-KALK fans på ătffilliga ftálten, form war liffom et medium emellan Cos och Calx; th han bes ftoo of fima Itrifíva, hwita, opacx particlar, bwillen aldrafwaitaift gicf til Raltemiót.

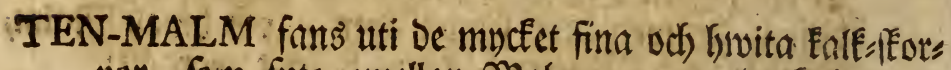
por, fom futo cmellan Malmen; men doef $i$ ringa quantitet; th han befroo allenaft of nagra fima, fivar:

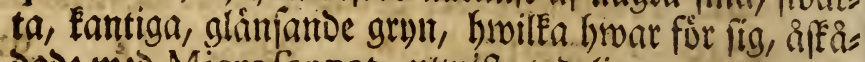
adee med Microfcopet, uttwifte tydeligen, at De woro âtta Zingrauper.

TALGSTENEN war of 2:me fitg: Den ene beftod af frródoe agnlize particlar, fárom en Ollaris, Den anore af fiáll, forfom en Mica.

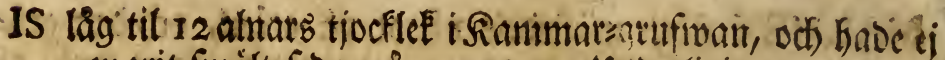

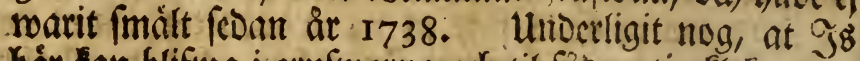

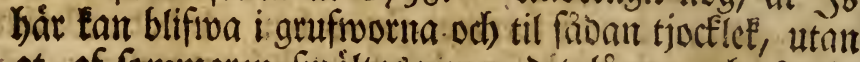
at af fommaren fimáltas; men Det linga ocf) ftarfa Draget, ifrán noera ípningen twaít igenom grufwant, lärer máft bioragit bártil.

WATTEN 1 BLOD formainot har Eommit of Monoculis (Fain. I182.), af Fuco (it. Oel. I10,120,207.) af Byffo (it. Weftg. $\not^{\prime}(4)$; men bar rảabe wi bet 4:De flaget pả Salfbárgen, Dár Yloikarne, at efterapa fint Sórtitorar, forr to feul borat och med Erut firanat Salefifan. Uti caviteterne af Defa fprängoa fiales

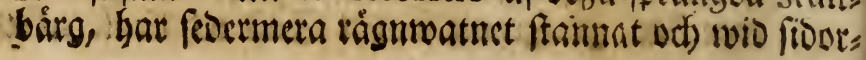


na fatt et blobforgnot od hogrodot miol, bivilfet med fingrnona futto afitufiab. Folfet mente, at watnet utoragit Denna róba färg af Grvita Sinleftenarne, broats til wo Doce ingen anleoning funns.

GRUF-GRABBER betattados af (3) mf follet mara Den

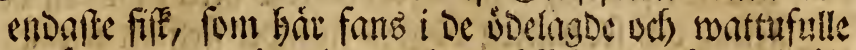
stufioorna; wi unorabe buru fife funnat fommit bit ifiverit pil bórget, til Des ivi fingo fe fifeen bafiva 4 fister ud) rata en Datruto ola eller SALAMANDRA aquatica, (Faur. 256.)

LEMNA monorrhiza 999. tîfte matnet i ie grufivor,

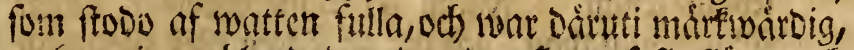
at bon ci warblandato mes andera llag of fit lisgte od bno de minfta blader; under Denna fagoz rontign, flippriga, Elotrumoa, grona of olämt fora grum, af

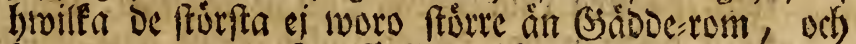

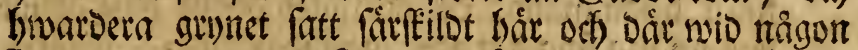
Lemna; men om Debe grun mânde soare en Ulva ef let et fró til naigon Vermis, wet jag ämm iffe.

GERANIUM robertianum 578. wâkte ofweralt $i$ guts

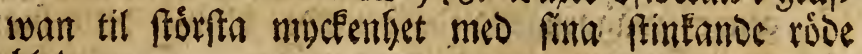
blaber.

HIERACIUM muroram laciniatum minus pilofum C. B. Flor, sv.p. 392, fom år en fortundring af Hieracium 637, fans băk od) Dár i grufworna.

RODING(Faun, 310.) fans uti Siơn Bngen, fom clieft ât fâlliunt utom sapplano od) Wettern; Denne mor ftor fom en for Sie, pa ruggen more, pi fioorta bláa tig, imunder Silfiver=hnit, med branbgula fläcener pa fidorne vef) rógula fenor unver buEen. Eibes:2 is nient war raf; under ógonten babe ban pá De Esttfulla Simbbenen 4 utgrafme puncter, och afwen fa manga minore puncter emellan ifra Eáfent ods sgonen. Den forre Ryuggfenan beftos af a minfa ftralar, af bivils fa de 4 fórte woro bele orf de framfle E⿱rartalt. Sindra Si Singgs 
Fiuggfenan war en flotfena utant frrálar. 23rófts fenan beftod af 14 jitralar. ivaggenan hade 10

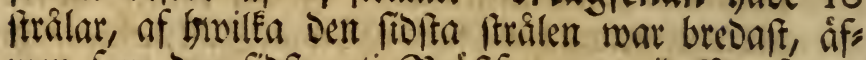
wen fom Den fiofte uti Srofffentam; wid Magfeman war mirktwarbigt at fe ent appendix cartilaginolus feu adipofus, fom bäftades wio vtra bafin af Denna fena, od) feoermera bógdes inumber fielfiva fenan; Den famme war halfparten kistare an fenan odh belt tres fantig, hrwilfet Eánnemîrfe uti differentia fpecifica ingallunda bơr fóngatas. Gumpfertan beftod af 12 ftrálar, iblano hrwilta de 4 forefte woro hela. Srjer: ten beftoo af 19 ftrålar, Htom Dem, fom pa fidorna ftúdja fenan. Fifíten leker merendels 8 Dagar füre

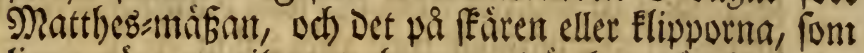
ligga nára up til wattubrunen, Där han gớ fíg balar ftura font et fat, Dermed at han lágger fin rom uti en ring, brwilken ring eller balel ci güres af en, utan flere filear tilfammans; Derfore füers Denne bale af fiféce,

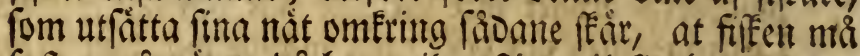
faftna pa nàtet, oả han wil upftiga til fin bale. Les Eetident warar 3 à 8 Dagar, alt fom blailten ár til; th ôr bläften ftarE, blifwer leketioen Eîrtare, emeoan fifeen, genom wägarnas medarbetande, blifwer Defto finatare qwitt fin rom, an uti lugnt waider. Det berâttades at aftlamme fiefare bruka fafta feocermera någon refla

1. Éfruer balen, at rommen ej ma apátas af amnor fipt; - 2 brvilfet är berơmmeligit.

FISKE-REDSIKAPEN, fom $\mathrm{i}$ Swerige brultas, âro allmảnt ifran langa tider befante, och) har Bellonius, uti fit Itinerario, merendeló Dem alla befterifuit; fôfom:

Fot, Sagena, Gallis Traine. Bell, obf. I. c. 74.p. 72 .

flotnet, at oraga uffwer hela fisut.

Stantrinát, at fänga s)iort, 2ibburtar, Sbábsor. boimma af Sarm, at taga Gádoour under lets tident.

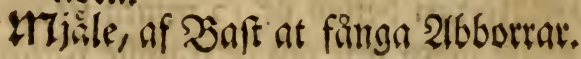


Zbborbaf, Truble huic proximum eft. Bell. ibid. 73 .

Forshaf, Pifcatio cum quadrangulo reticulo. Bell, ibid. 72.

Zatifia, af uprittade fprott for Sorapen.

Drag, lineas per tempeftatem ducere. Bell. ibid. 74 .

Mete, lineis pircatio. Bell.ibid.74.

Langref, ducenti hami longa lerie ex fune. Bell. ibid. 74 .

Ljuftra, pifcatio noctu ad lucen. Bell. ibid. 74 .

ABBOR-HÅFWEN, fom brutas hac i bngeffion, ât firl! font, och minnes jag mig icfe feot bonom tilforne. (5.n jerusting, fom unid pâ Ean hopledno til 2:me baif:circlar, ât of 3 alnats diameter; men pá fioes nhigot brebare, efler $3 \frac{x}{2}$ aln $i$ diameter. Botnen ar rumb od) flat, fom en tolltie af en alns diameter, gjots af tagel, raggs eller froingarbsere, fom fpiralt ar bowfinot, och utom Ering mes en tioce jernaring omgifwent: Salf

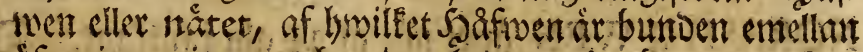
ofra jern =ringen od neora botnen, in formam Coni truncati, ar 9 quarter bjupt. ITafétite aro fa fore, at minfta fingren kan intrabas genom Dem, med 1)tterfta fpetien. Denna Salif ar uplango mco 4 tåg, pa amban af en 4 alnar: lang ftang, ibuilken fíng

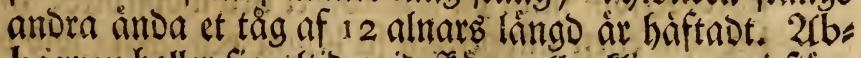
borren boller fig altio wio fereren eller Elipporta i fion. Dit ror Fiffaren, oci) flapper ned Salfwen pa botmen, hiwarpa 2ibborren, fom altio álfar rumoa ringar, begyns

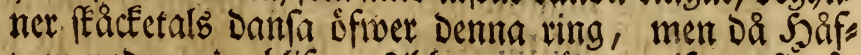
wen uporages, blifwer 2 bborren rído, gifwer fig ât botnen, od) fáledes uptages uti Şâfiben, ofta til an fenlig musfenbet.

\section{ङe figuren pa anbra fibart.}




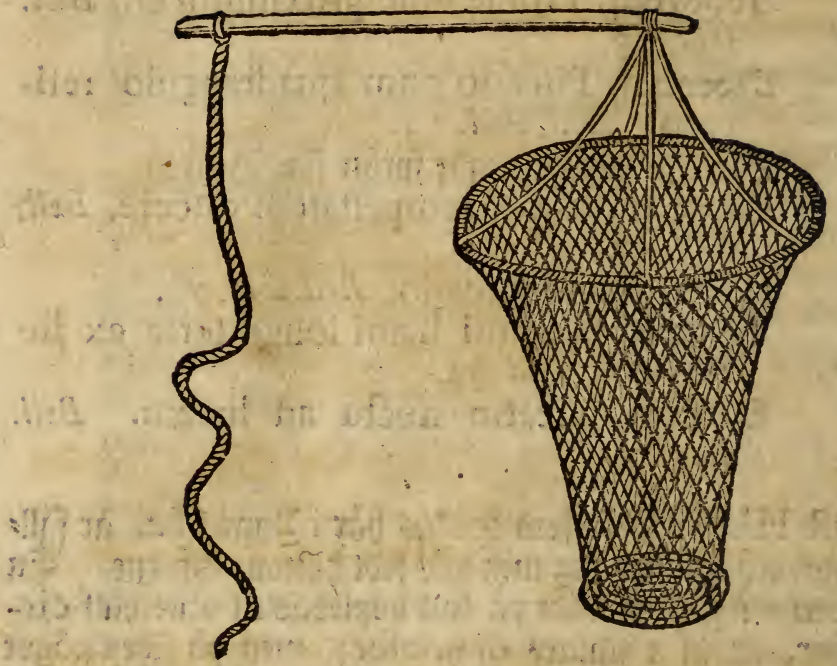

QWIDD (Farn. 33r.) fantraz ocf twib ftranderna meo fórenämoe 2(bborbaff, od) brufas til nga dir man woil meta 21bbotrar eller utfätta sisingref.

NORRSHAFWEN air et nat of $4 \frac{x}{2}$ alm $i$ furfent. Detta träbes lóft pa en tándel, fom bar-wib alla 4 húrnen

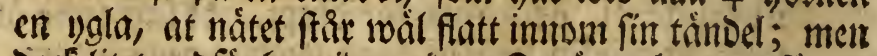

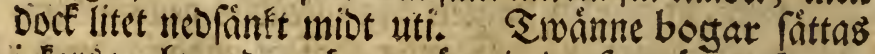

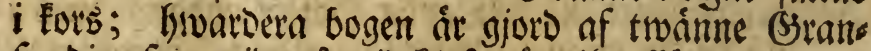

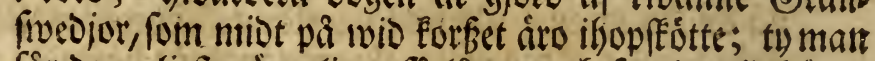

- firr bem clieft mippeligen fá länga och fmala mio bágge

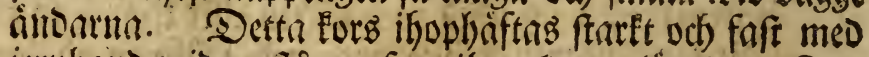
jernband wio en ffing of 5 til 7 almar: lingo. Sors fets átoar infâttas igenom hyor fin ygla, fom ár wio

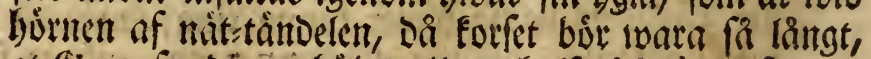

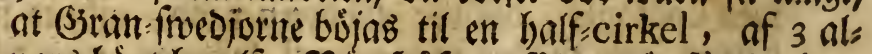

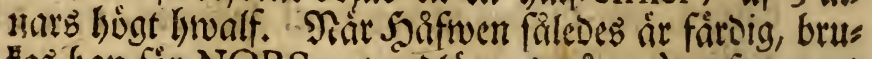
Eaś han fơr NORS, at neolíggaz̉ på 2 à 3 famnarz

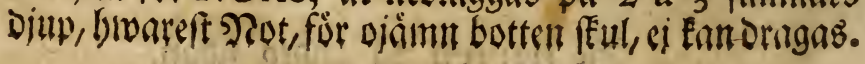




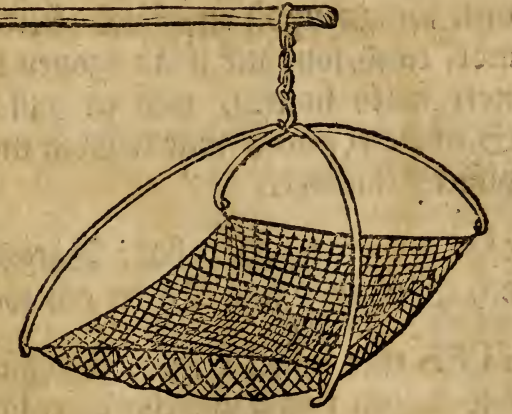

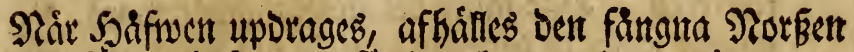

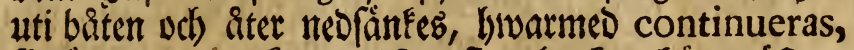

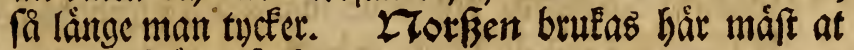

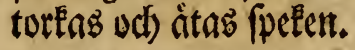

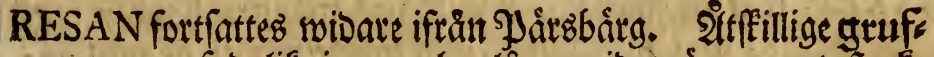
soor af bulié jernemalm lägo mid maigen, et ftude

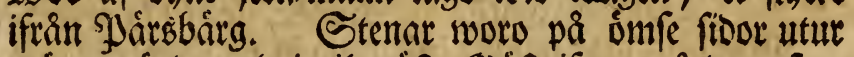

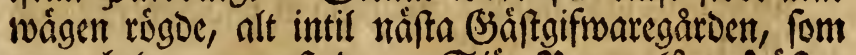
woro helt genomroftade. Siön yngen lảg pả ưftra

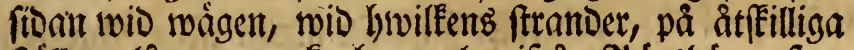
fiállen, låg en nuycfentet malm ifrän Đjársbárg, fom Dit blifwit fóro fijoledes.

RANA manibus tetradactylis fiffis, plantis pentadactylis fubpalmatis, eller et flags fmả Stoggapadoot,

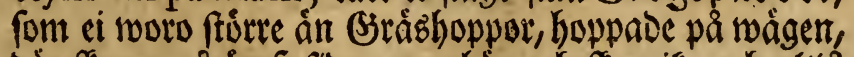
Dár ffogen pa ómfe ftoor war bog od fFogrie, oct) altfá ftadigt utoumftabe et of funliget rảign; th Defe fma Djur áffé fidoant climat med Violftenen II25. DCh) Linnxa 522. De wors muctet liee med Đadoungar

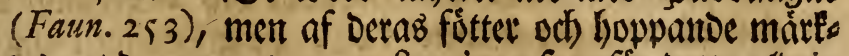
teb, at be worto et ammat fpecies, fom fór Detta alorig blifwit befant; ty ár nodigt at beffrifrwa Det famma. framfotterne hade 4 tår, fom woro aldeles Elófoe

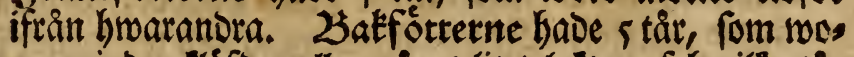

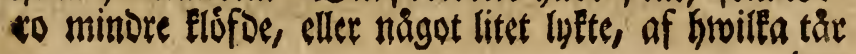


Den fierbe war langft. - Kroppen war inunber grá; men of rwan uppi morffblet (fufco-lividum) med en

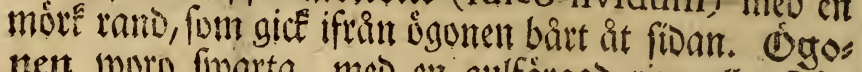
nent woro fivarta, med ell gulfä́rgad ring eller Iris. Gumpen (anus) war trubbig med thâgra bivita blela pancter inunder.

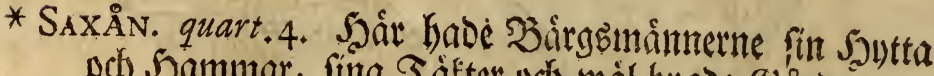

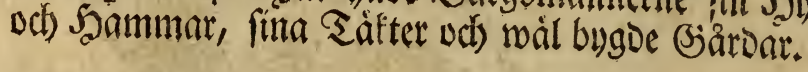

HILSTER eller SALIX 792, ftoo på wainftra handen it:

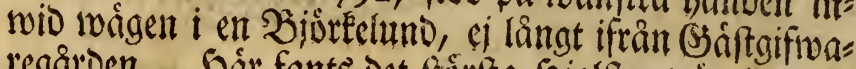

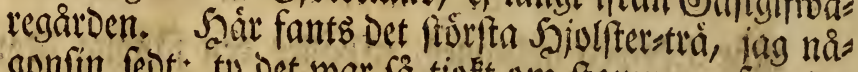
gonfin reot; ti) Det war fá tioft om ftammen, fom Det

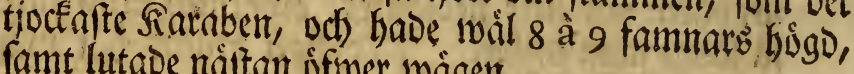
famt lutade náltan óf fimer wigent.

SAXEN war en fmal fio, fom låg på wainftra hanoen wio

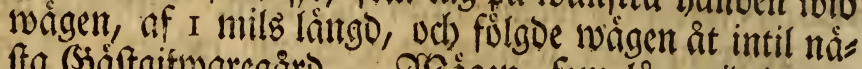

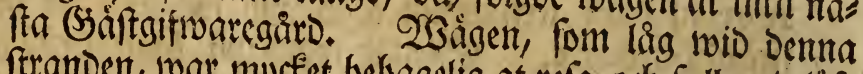
frtanden, wat myctet bebagelig at refa, och) full med lof:
trun.

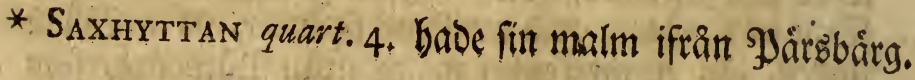

NERIKE od WESTMAN-

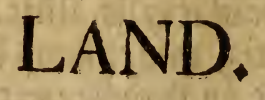

\section{Aug. 5 .}

SWART ELFWEN iffuerteftes. 2̊tffillige bollo Den for ffilmad emellan Wermeland och Weftmanland, faft andre $i$ gär beráttade of, at itffilmaden emellan Wermeland och Nerike twar lagb 2 quart bak om Saxhyttan. Det år unverliget, at Şnbuggarme náp: peligen fielfwe wifte, i hwad land de boode, emedan de woro andelige 23 eftmanlánningar, och werldoslige? Pes rifesboer. 
TORRWARPEN, en läng och fimal fiob, lịg pả foorra fí Dan om waigen, wch war mistet frotig, befinnerligen wid (Sintsyuttan.

VIOLSTEN I 25 . lảg allmaint $i$ ffogen; men fägs par itts tet amuat ftêlle, ân jemte wägen, Dầ frenarne wors omeullrabe, cller océ pá flippor, fom tilförene warit

1 betáfte meo 10ro, men nulfigen afrógde.

* Grythyttan. quart. 2. mar eil ftor by med en gata Ded) torg, emedan han i forma tider warit àmmad til ftad, fom feulle Eallas Goythent. Deume Eocfen bar fortit blifwit imrâttad 1633, 17. April af nảgra byar cller tory, fom tagitó ifrán Nora, Philippftad od) Carisnoga; f foban I 64I, I I Augufti fadot Estrobs

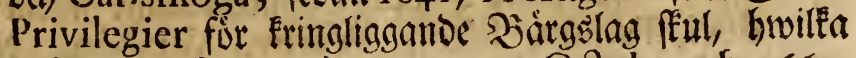
blifinit confirmerade 1649,12 Octob. Ddf I660, 3 Mart; men ei Torgs och Miarfnad s'plats. 1660 , 26 Nov, confirmerades $\mathfrak{B}$ ârgsing friheten, Doct under beffattuing. 1668, is Sept, blefivo Privilegierne allemaft til en tio prolongerade, emeoan be nâftaranfande (miå Stíberme Nora od) Linde ci fun= De befta, utan at doclígga hivaranora, om flare Etás Der fả nấra féulle aulläggab.

RESAN ftáloes i fooce at Loka, fom läg 5 quart ifrån

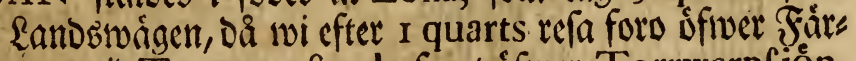
gan wio Torrwarpfund, famt of fiver Torrwarpl jón.

WÁGEN war Den aldorabehageligafte, th Gan betâftę på

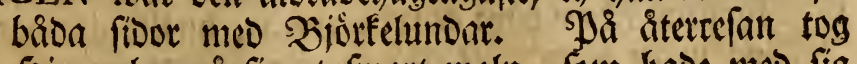
Simmelett pá fíg et fivart molin, fom bade med fig Dunoer, blipt od) Hagstign.

FLOTGRÅS 771. wápte i Torrwarpen twio Gárgan, Dár Det flit med andarna af blaber, och under ytartafte frormen brivt fá wagorna, at watnet ftoo belt fpegels flart, fá lángt Detta gráfet mầte. Det ár mártiwàrs Digt, at De flutande 23 attentwa deter fá lumma Dámpa wågarna, ọch uti mari pacifico wete wi, at fá wiôa $\mathrm{Si}_{4}$

FU- 
FUCUS Sargazo dictus dói fluser, fâ ấ Det ocf et

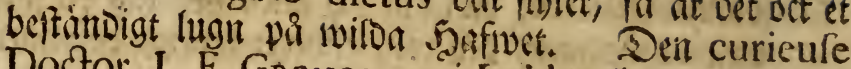
Doctor J. F. GRonovius i Leiden har getrum bref

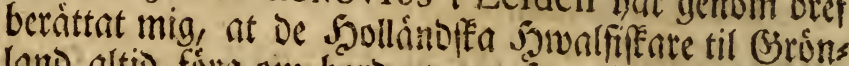
Iano altio fór om boro et par fat slia, hrwilta de i

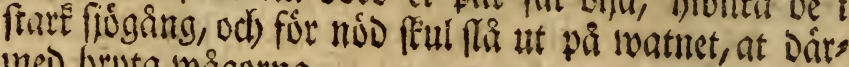
meo bruta wågorma.

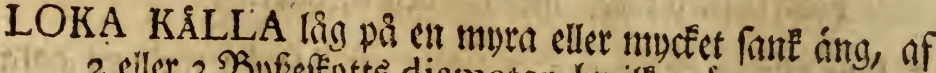
2 cilce 3 Bybeffettz diameter, hwoilfen äng war frings

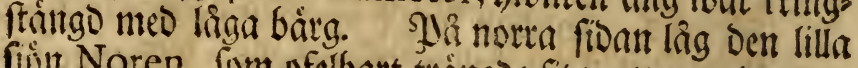
fiunt Noren, fom ofelbart trintgoe fit watten under Dent affign od) houga wägen, at ängen ftabigt blef wất; pẩ foora fiton ligg fiisn Loken, til hrwilfen Elfiven Trófa gice. Stlle wäre Sivenffec Surbumnar, fifom Medevi, Fahlun, Satra, Wårby, Wixbårg, Norrnialm, Gỏtheborg, Fallorne, Ramnlóla, Romaby, Lindahl \&c. nga jernewatten, meo blicffinaf, blâflugtig Şinta och gul Ochra. Zlll ciro tingerade af et urle eller Mon; alle ayera pa ennbanda fätt, neo Det be corroborera of firt martialinka fraft, oct) differera enDaft til fearfictern. Sien Loka Fálla out af bel amman art, och áger lywarten Smâ, Sult, Şin: str cller Ochra, har alffá hel numor Eraft; th Den corroborerar icfe, diluerar meera, och relaxerar mern, giv

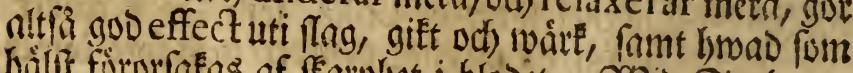

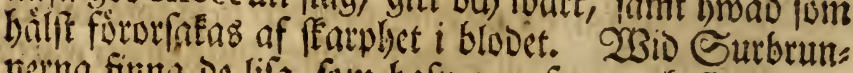
nerna finta de lifa, fom hafwa en fivag ofs flapp mas ga cller fropy, en Leucophlegmatie eller Cachexie,

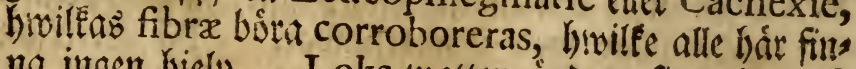
na ingen bjeft. Loka watten änorar fig wio peufs weet ei met än gement watten; fmatar, fom Det behas geligafte och) retrafte Éálleswatter, âr mucfet Ealt, men ej barroorncfit; Bet har ingen finnertig luEt; men bi bet omfarualpaz, Énmes bet nágot litet bitumineuft; Det looller ocfif intet mineral, odf Enan berföre ei med de ordinaira profiven igenÉumas, tu har Det af Medicis blifwit mer fortunoradt, od) Dár ingent Theoric rarit, 


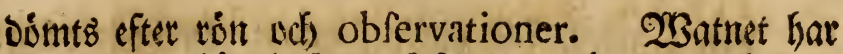
ifrån Sebenhos blifwit of 2llmogen bruft, oct) Dem lärots af niagon hándelfe, od continuerats of en fừ: faren Empirie, Dá \&anotmannen baft för ârlig pläg= foo, fom an $i$ bag warar, at refa bit Sniofommars nats ten, och) Dricfa Detta fésina matnet, at med Dé Ealla watten Esla fin bloo, at meo Det flara matnet affétslja Déf fílta, at meo Det fmidiga watnet lofa Det fuanda. Sag wet ingen, fom intil Denna Dag fänt Detta wats net, odf jag har fielf aloris foreftat Det famma, elburu jag ofta oructit Det, fom warit forrot hărifrån uti bouteiller; ut vidi obftupui; ty jing hat feot ofs wer roo:be Siállor med Loka mattert, utan at weta Dȩ́ wertan; jäg har mången roo:De gång lâféat mint matta fropy med fid ant watten, Da ban watit fivett af Florx tjänft; jag har warit Den, fom nogaft befferifs twit Loka Siállor (Flor. Lap . 4I4.) utan at weta om Loka. De mâfe Lokx fíllor bar jag feot i sapp: marken, o(t) Doir funnit et tecfen, fom náfan alorig

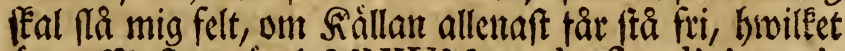
ôt en Miofa, náml. MNIUM caule fimplici geniculis inflexo (Fl.Sv.913.); ty) Dár benne wáter i nås

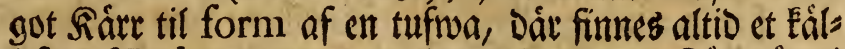
lefeg af Loka matten; med et ord: Loka Sâlla ît ei anmat, án en KALL-KÅLLA. Sall= Fállor áro helt anore Siallor àn Springsfiallor, ty Ralls Fíllorme fits nes allenaft i fiolänte Sátr, fom befta af oy eller Sâtrs mulla; ofta fta fadane Fáur om fammarstiden fulle af Golliumt watten, då liênâl uti Dem finnes et Eállefeg

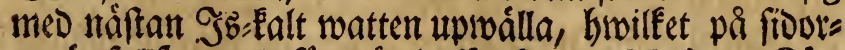

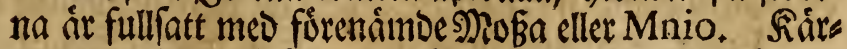
ren taga fura och fetma utaf Det ftabiga watnet, hivars fore all Sairsmylla faut gơras til brännande torf, och alla rótter, fom ligga i Siárren, bafwa muceen fetma; nâx Denna Dy blifwer uttorkas $i$ jorben, fórmandlas bon til feifiboerften; ffifwerfterien, fom ár mober tilften: Eolent, oct) bartgbecket, wifar lwo fetma be fat utue Rárren. Siallesållornes wattin fuger fig Diupt iges Si 5

nom 


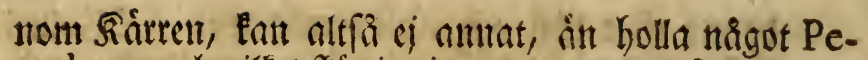
troleum, hwillet févities isentont wattets farwalpnitg uti glafet, Da Det rojjer fig med luften; holler fáleder Loka watten ei antrat, ät et rent falt wattert med Petroleum, hrwillet gêr Det fmidigare och lâttare at brics fa. I Sappmaiten finmas oinnocligt mảnga fádana

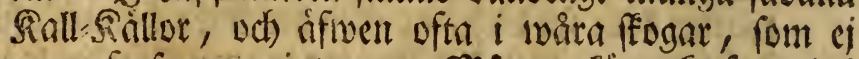
getra frufa um winteren. Mången lór ocf ofta ned i Dem bâse med bâft od) ficoa. Sappen oricfer od) uts màlier Deras watten framfór alt amat, Dá han Det Ean bafma, faft man icte loet at Sall= Sállormas matten, i ruct $t$ pa Gurbruts fiatt, gjort fî bătliga Curer.

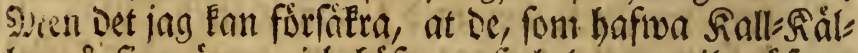
lor pá fina ógor, ci telsófroa refa bela 30 milen sfruer ใ̊ำ efter wattert:

AKERTISTEL eller SERRATULA foliis dentatis fpinofis 662. war $\mathrm{i}$ mucfenbet uphámtad wid Tors pen, Den bionoerne torkat, at af bonom taga Den myé, na och långa ull (Pappus), fom fitter pá frón, med

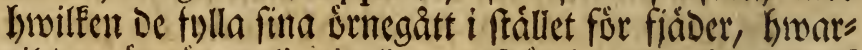

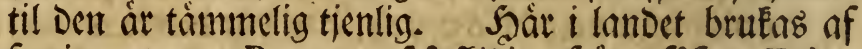
fattiga at taga Pappen af átficlliga frôn, fafoum Eriophorum, Epilobium, Salix, Serratula ; ty) Eanots maunen har ej mânft ânmu De andra, hroilfa alla jag pa fit ftälle har aptefnnt *; men muttan blifiner ringa, t1) en Del walfar fig tilfanman i flimpar, andore finos laE fơnoer til miól.

* min Oratio de Telluris incremento. p.68.69.

WAGEN ifrän Grythyttan, feoan wi lommit tilbala ifrån Loka, foutfattes ât Nora. Şan mar ljuffig at refa, men natten fon pa, od) effer 2 quarts refa fágo ivi $i$ Den móréa untten bela jorobrinet i fogarna bivitt, och liefom meo fino betialt, hivilfet Doce ej ammat war ôn et tiofft och) ganfféa frort hagel, fom băr fallit fór några timumar feonan, under âfecounderet. Efter 4 quarts res fa ifrån Grythyttan, ftante wi uti $23 a r n a ́ s$ sfiver natten. 


\section{Aug. 6.}

WARNAS GARD lig på en UDoc, fom på 3 fioor omgafs med fion Halfwars-Norn, litfom meo en balfmane; Siarben liag nagot uphoggo; men lanoet enellan gărs Den och fión war af láglandte Sindar, fa at Denne gåro bór wăl rientas ibland de baif belägne Emerigez gâroar.

* KÅRINGEBoRN. quart. 5. ifråı Grythyttan. Şǻr war Sytta od) Şammar. Jern = malmen togs ifran Soigborn od) Solmgrufwan. Stangjernet filloes hat til 36 olr. Sip:mt Get.; Transporten til Arboga war 7 olr. Sip:mt. forr lafet; lowart lab beftod af $2 \frac{x}{2}$ @6t.

KALKEN, fom brufadeb bår til fluf, war helt broit, od Dáruti fállfam, at ban war beftrós med particulis longis, fparfis, fubprismaticis, procumbentibus, fá at figuren nata fom ifwerens meo de froarta, ftrobs: Da particlar af Dlânde, fom finne: i Soppar:mnlmen wis nua fiopparbárget, faft Debe particlar woro bwis te odf) Flare.

* Greksåsa. quart. 6. Şár war åfwen en Scutta. Mals men togs ifrån Gtubárgs= od) Tubárge = grufroor: Siòn Greken lảg ej langt Kátifian.

LIF-FÅRGAD LERA, aldeles lié Den, fom grofis wio Philipptad, men något legare, fágs wis twàgen jemte Sllisbåcf.

* Gytorp. quart. s.

MALVA montana feu Alcea rotundifolia laciniata (Flor. 583), waite med róba blommor midt emot SSåroen Sárgsangen, fom fanfe torde fommit utur Srågåroen. Set ár ef utan all grund, hivad gemente man allmänt talar om hennes blumiter, at om en Flic: fa, fom hemligen lemnat tuglarna, luftar pá Deras 


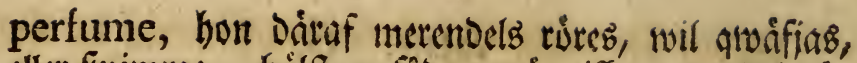

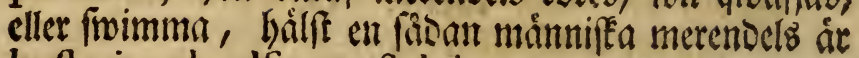
hyfterica ab adfveta, fed denegata venere.

NORA ling quart $\frac{x}{2}$ iftrản wigen på wainftera banden; Det war ell liten ftâ, mco 2 lânga och ráta gator, 3 twa ărs gator, odf) et frort grónt torg. Shufen woro af tra, meo en woining, Docf fingge. Staden war ontgifs wen i Pour, Softer odt Suder af Sion Norn, men pá wáftra fitoan feitbes bau med en floo ifrän landet.

* Bondeby. quart. s. Natten fom pá, od w wi hade árnat

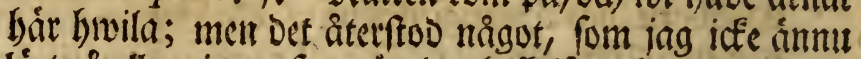

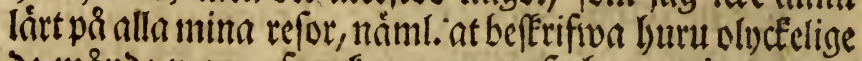
de månoe wara, fom Eemma at refa hoo nationer, de oár bivartern hafiva feoer, lagar, eller moral for ógor nent, och) hauru fult et folk Eumbe blifwa, Dár bet ei tils Gólts, at bemuta refantoe meo beffedighet odh búflige

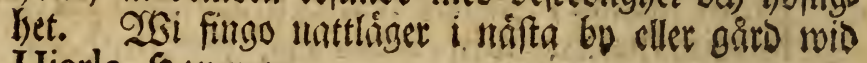
Hjerle Şammar.

\section{Aug. 7 .}

BRYNE woro hár tagne af fin ftállftetr.

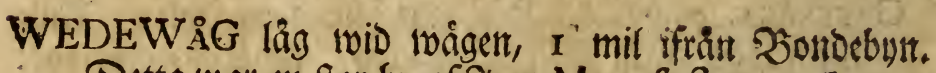
Detta twar en fror by af Jern:Manufactur-were, nucs

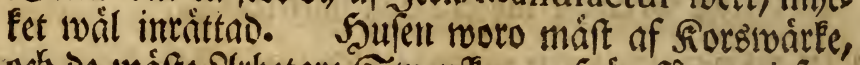

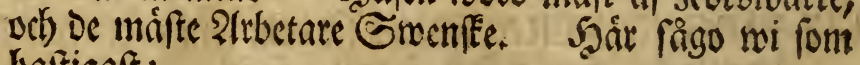
baitigaft:

Metallgjutatespoeteffadent.

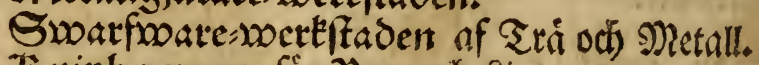
Kiniphammer for Yror od Siat.

Sarfimedian fưr Sakar, Snifruar od) Silingor. Staintg jertits

Spie: imedjan o(t) Solje/nimedjan, font bigge arbes

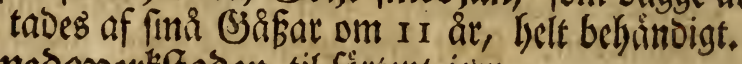
Smedsoetkftaden til füternt jetu. 


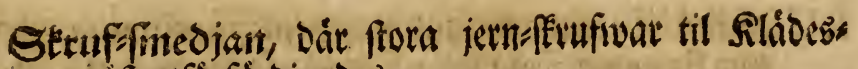
pràiáar fúrfároigadez.

Sortement-fimedjant til inftrumenter af allabanoa llag.

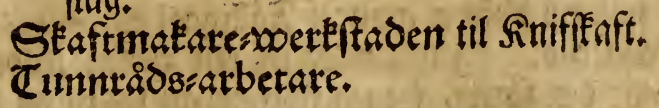

QWARNBACKA war Den Delen of Wedewåg, form liag Difter om Floden. SJâr figg

Znip:bamear.

Sil-fimedjan für filar.

Zniff:imedjan, băr Sinifbladen polerades meb fons Derftustt jermilngg.

* Blixterbo, quart. 8.

RANUNCULUS foliis capillaceis 472 , begynte $\mathfrak{n u}$ atrora gången at blomma i Sáarren, och) utfatte et par runda blad, fom flisto pa ivatnet jemte blommorna.

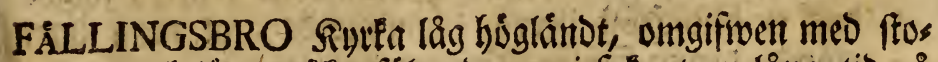

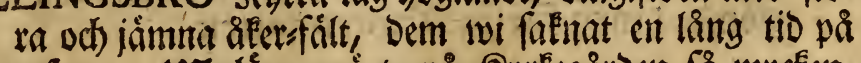

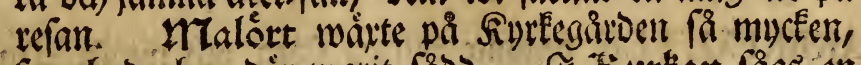
fom bade han Dâr warit fado. I Kurkan figg en (Sraffen med Fadrens od) Eomens namn, fom baigs ge wonrit Paftores, famt derab barnb namm: CAROIUS OlaI Hising. natus 1572, denatus 1642. hwilfen hade in Sinter adh 5 Díttrat. Carolus: Caroli Hising, natus 1603, denatus 1669. hade

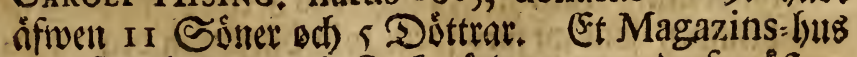
eller fiornboo ftoo pa Furetogárben, nurrao af gråfter, med mer in I famm tiocf mur. Fállingsbro âr en

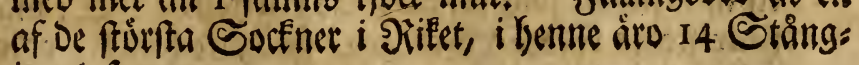
jernỏs Săamrar.

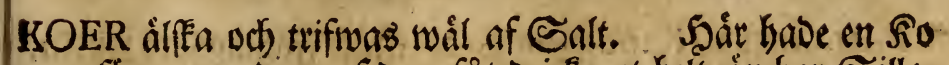
for et par Dagar feban fart Driffa et belt ámbar Eillas fa, bruaruf bout Dog, Då Des tarmar fảgos ihoporngne,

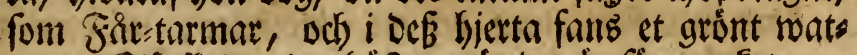
ten. ESa feadar bet băfta, nár Det âd fưr mucfet. 
RUDERA efter en gammal faftning, berittades af J Jro fen STRANG feola fer, pa et búgt bairg wio fenater, I mil ifrản Fällingsbro.

NATTEN bio of Glifiva qroar i Fällingsbro, quart. $3 \frac{x}{2}$

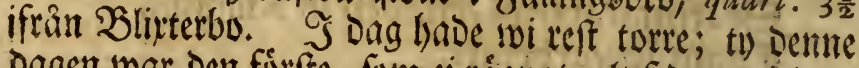
Dasen war Den forfte, fom el rigmat, alt fedan wi lem nade Uddewalla.

\section{Aug. 8 .}

WINTEREN lemutar of fent on 23 anem med fita frofte

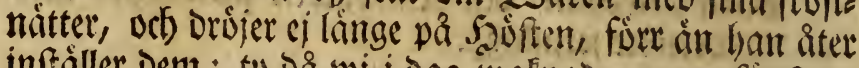
inftaller Dem ; ty oa wi i ong waEnnoc, woro fonftren belt wata of futtighcten, fom fiffat fig pa Ealla glafet, emedan natten warit fall, fa at litet timfrefi lagt fig pa marken; falcoes batwe wi warit frie ifran froftnäs ter, alt ifran Golftandet, til ilutet of Siotmanaben; men elyuru fercets Denna Sommaten tvarit, folgoe Ducf en blid Shift oerpa, fom gionde, at man firat glombe Denna forf ta DD intersitattelt.

ÅKERFALTEN begunte blifiua ftorn o(f) wioftracte at Weftmanland, fom i fio bar fit mafta formont.

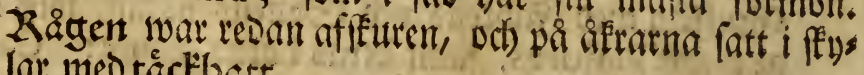
lar med tácklbatt.

STOCKHOLMS W£GEN tog wio nagot fort, ân Då wi Fonimo fram til Fâllingbbto Srog, nûmligen Den famma, fom wo fors forbi wis barttefan Den 14 Junii, (p.9.), at wi altfá mâfe băr efter refa famma woig tillba:

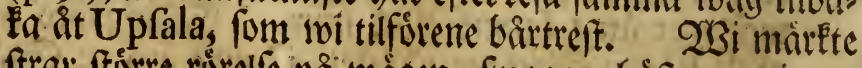
ftrap fórte rúrelfe pa waigen, froagare bajftar, ogiture folf, odf Dubbelt Durare (șiffsifware.

* FállingsBro Krog. quart. 2. ifiån Fêllingšbro fibrta. ALANDS ROT eller ENULA (695.) ftod i Fislgarbars na med fina ftora od) gula blomfter, uti lwilfa wi mårete nígot befivmuerligit, fom ingen Botanicus tils 
fóreme feot. Altie Botanici hafiwa âftumbat fitia Dets ta fpecies ifrán Genus Afteris, emeDan brtenz uto feende ár mycfet olif med be andera Afteres; men ingen Gar funnat fiuna naggon notam effentialem generis, fórr án i ong, oả jag mårfte en Character effentialis, hwarmeo Enula icfe allenoft fifilies ifrän Aftere, utan ock ifrän alla compofitis fyngenefix, Invillem bejtod Dáruti, at De 5 Anthere connatx in cylindrum, hiwardera wio bafin, utgingo med 2 neds âtftiende $\mathfrak{B u r f t a r , ~ f o m ~ w o r o ~ f a ̊ ~ l a ̊ n g e , ~ f o m ~ f i e l f i u a ~ f i - ~}$

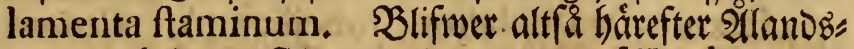
roten et belt 'nut Genus under namn af Enula, eller ráttare, at brufa Plinii ord: INULA.

* Arboga Gtad. quart. 5 .

GRINNEN, fom ftod frrar utan fơr Arboga, wat for of en for raritet, to wi hade iffe haft någon grim pa Eanosivagen, feDan ro lemmade Ånål i Sal, eller wid paß́ pa 30 mils refa; en ganffa ftor tróft fúr trangtaits De bâftar.

SKATORNE, fom futo pa Giárbeşarroarna, woro nu als le plactade om balfen, at bara Dunet fatt qmay, Grwile

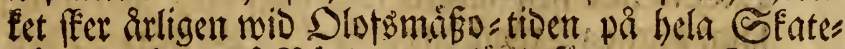

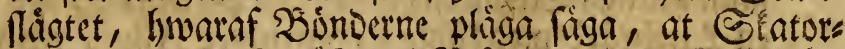

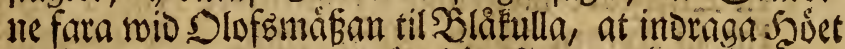

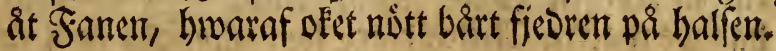

* Kópings Stad. quart. 7. Git hade Bjáftaifivaregărden blifwit finttad ifrán Oftuna efter wair aftefa.

KåLEN haoe til många ftåno hog Đoreften Brovallius blifivit forránornd, od lie fom fiuk ; ia twi brogo up Des rôtter, beffoso be af många sjämma, faffíttande fnylor, broilfa fưrmentes commit af maffar, fom átit fig igenom roten.

NATTEN sfwer Glefrou wi i Kóping, efter en tort odh warm bag. 
COREOPSIS Bidentis, eller HEPATORIUM aquatile, folio non divifo, flore aureis undique petalis radiato. Triumf.obf.65. Den wi fingo wio Bohus Slott (pag. 160.), waitte $i$ Dilen jemte wágen wio

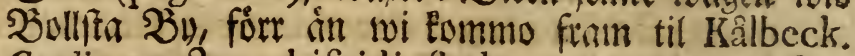
Caulis erectus, hifpidiufculus. Folia oppofita, lanceolata, feffilia, amplexicaulia,. profunde incifo-ferrata, bafi vix coalita aut reflexa. Fiores cernui, five nutantes, pedunculis fulcatis, unifloris infidentes. Involusrum pentaphyllum, reflexum, lanceolatum, fubciliatum. Periantbium e duplici foliorum terie: fingula foliolis 5 . ovatis, coloratis. Corolle radius ex s ad 8 flofculis, lingulatis, ovatis, integerrimis, luteis, fterilibus. Seminum coronæ ex dente quadruplici, retrorfum aculeato.

* KÅLBECK. quart. 6.

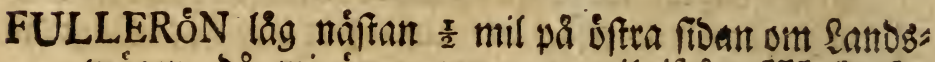
mágen, Da wi ámu woro $\frac{x}{2}$ mil ifrian Wefterås. Denne wactre Sáteg̉găro rvar Şans Excellences

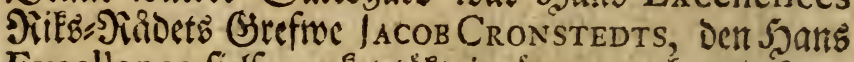
Excellence ficff nucfet tailt inroittat, ofh mo ftora

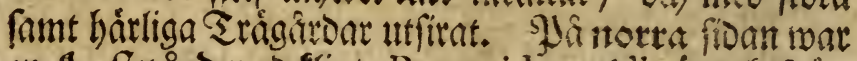

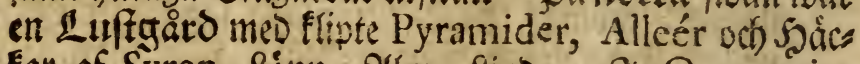
Far af Syren, sinn, Alim, simo. Os Orangeriet bängoe woaffra Childerier, fom utmifuoe De 4 arfents tiber, af Gwilfa Dointeren artigaft fóreftáloe de finis

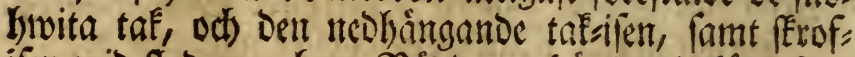
ifen wio floderna, butu Sounderna bána wes, Esora fina flabar med luona mysor, od) wantar, fant fporen i Inín, och rimmen pa de bladlófa trân. T Orange-

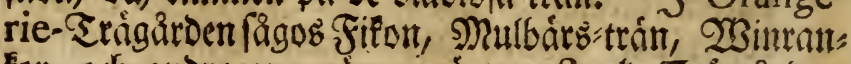

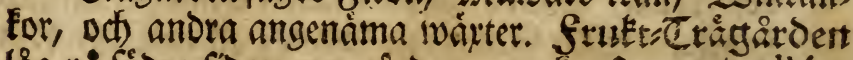

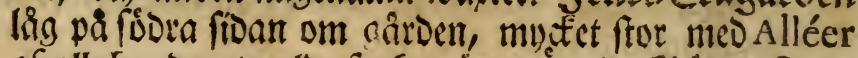

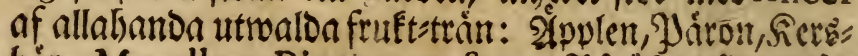
bár, Moreller, Bigarauer \&c. Jyá sfttra fio an lág

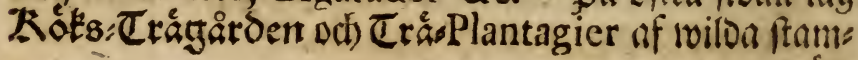




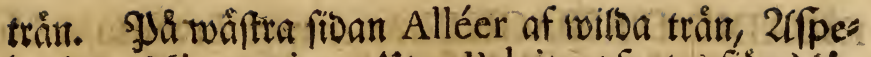
lundar, Minagerier. Utur Palaitzet finntez fiơn Màlaren, fa pa norra, fom foora fioan, et frucfe frân Giarden. Biblothequet låg med en ferffilo byggning

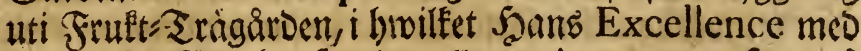
fturita forgfállighet famlat alla De Autores, fom pa Swenffa eller af Smenffa utEommit, nâppeligen en

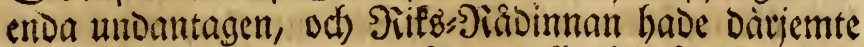
fit egit Bibliotheque, af en gamffa ftor famling med De utwaldafte Sicfebeférifningar.

GOLSKÅDRA Ealladez JACEA nigra 7०9. meo Denna

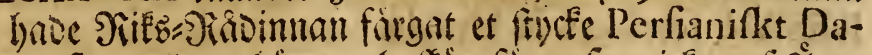
makk, med en boig, gul, fifun fortg, form icke gaf 2ìngs féran efter i nägot mall ; at firicoes intet twifwet âr, meo minore cifwem Dema Centaurea tjenar til fürg.

APIS thorace nigro, lineolis flavis, abdominis fegmentis nigris: margine flavis; primo focundoque ferrugineo. (Faun. 998.) ar en fallfinnt DD is fpa. Syatr i ängen giaf et hail neice $i$ jorden, fom has de nagon genomituctet wallen med en frố; famma ba war helt ruldot, och bogoes i Ero: under jorben, fá at nàr wi upgrofiro Det fanma, war Det en gammal fứrs

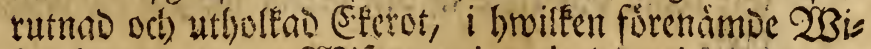
foa boode; Denna $23 i$ pa varierade Daruti, "at magen icfer war frommmitls brun. Caput nigrum macula flava, rotunda, bifida; in fronte macula plava inter antennas, ut \& macula duplex pone oculos. Antenne nigra Maxille fliva. Thorax niger, linea utrinque flava, inter caput \& alas. Punctum flavum fub alis. In tergo thoracis tria paria macularum Alavaruin. Abdominis fegmenta nigra ma gine flavo. Pedes flavi femoribus nigris. Ale absque macula.

\section{Aug. 10.}

SơNDAGEN firades på Fullerún. 
ESCADIE D'ESPAGNIE, âr et mutt inventum Nepotum, eller en Bafelfe of R̈ggehwita, Socfer od) (Si: sthe tronfét; Detta ar en bebagelig ting for Magnates; men i min numn fmafade Det fom tort fromos, af bivils fet wailfignelfen forfwoinner, forrtin bet Eommer $i$ magen. Om Detta ftár $\frac{r}{4}$ Dag uti Ealt rum, uplofes Det til et flem, t) bor Det bewarms uti tort rum, Da Det ar litt, lopt,

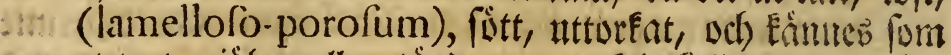
et tort miól emellan tímberna. Slycfelige woro min

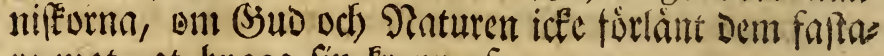
ic mat, at bugga fin fropp af.

RESAN fortfattes at Upfala.

HO-SWAMPEN eller BOLETUS (1087.) begunte tu waik frobigt $i$ angatma. Da Mrible:soerne fatt âta bonom, gafwo de en wormiaftig od) ofmatelig miole, lowilfen unoce lopmandet blef feg, od) uppoifte midt $i$ Érilet, fom habe bon warit fortrollad, bouilfet bode alt Denta fivamp furgiort.

\section{* WESTERas Stad uppebólt of icfe lánge.}

NATTEN tilbragteb I mil ifrån Wenterås, feonn wi blifs wit wate odt Ealle af Den orena waigen och wate wat Dret.

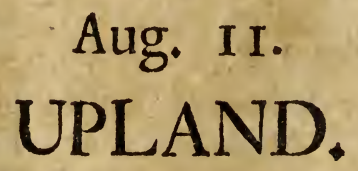

CARDUUS caule crifpo 658 , fom wi bit bela refon mucfet litet feot, begynte blifwa allmân, fâ fuart wi Fommit in $i$ Uplard.

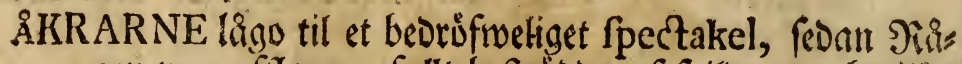
gen war afilagen, fullt beftrioon af fpilda ax, binilfet

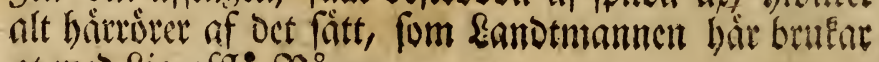
at mes sia afili siagen. 
HöSTEN brave alt ifrån Fallingsbro ftadigt wifat fig fơr waira ígon. SEegen frod wäl grón; men alfrwats fommare àn om fommaren. MTatz ocb 2inctar noro wäl gröme; men utan blomiter, ty) De fürre hàd Fànas

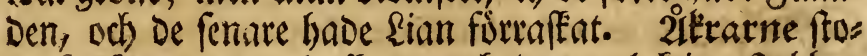
Do fullfatte med gula fFyar, ocb Dent gulaeftiga frubben efter fäden war îprängo med grónt ogrả. Dien fies Do fulle af watten, efter Den wata fommaren, och Den

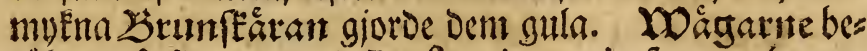
tấftés pa fioorna ineo Perficaria acri, fom nu begun te rudina vel) flofa med apen. Lamotmantiten war ute sfiveralt $i$ fullt arbete: fomlige Bintroc flecgo of fäen med Rian, untoer det Derab sminfole, fom word on bufiwu od armar belt hiwite, fammanbunto bens

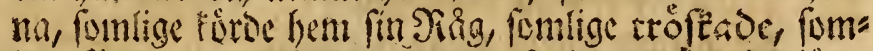
lige fonvergnogo Guthura med fladoen, fomlige jäme

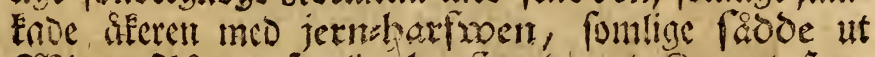

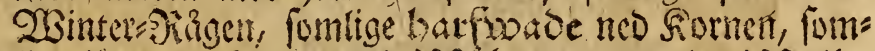

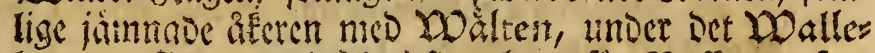

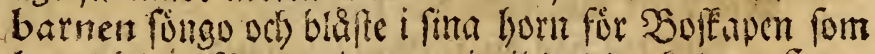

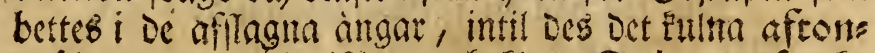

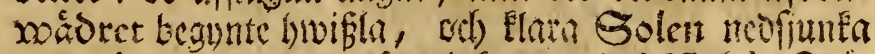
unoer horizonten, od mi fommu in i Uplala Teirs girit.

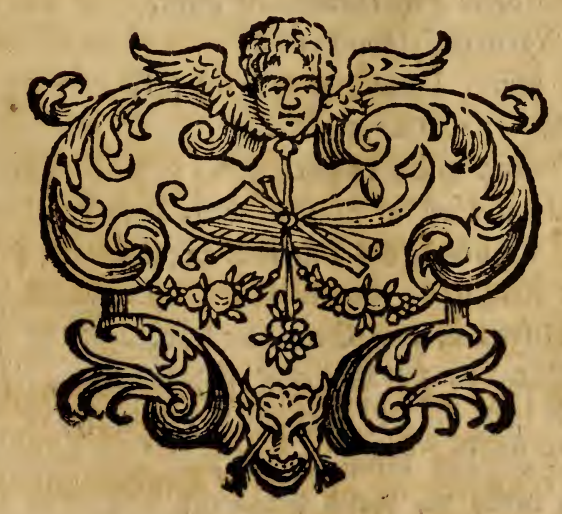




\section{*}

\section{Sil ARCHIATER LINNÆUS,}

5

\& fan nu oct waint Smea fernta Meb frora \&us od) wittra Mån,

Dem bon ei wil mot aniras byta.

E'n lárba rociloen fafnar ån.

Sisar CEL JUS; och $i$ fruftan båfwa

$23 i$ rc , fớr POLHeIMs brwita bảr;

SBBar Enften wil mot åloren ftråfiva,

Eom of fä måtflig ftaba fpar.

I bemt od) pere Ewemfe farbe

f̧a wi ef blott copier baft:

NU werlden fintmer beras wårbe

Fôr påfunos gâfwor, tånfestraft:

LINNEUs detta autal sifer:

En wetenffap $i$ mofrfer gơmb

Med inett och moba ban framfófer;

Şur' En ban bli, $i$ Srvernet glơmo?

Năr ry ftet meb Şans wåroa minue

Şar alla lårba Eamfunb prybt,

Lltanningarnes afunos finme

I noórbmab och forrunoran byot.

Den i Raturens blomfterrife

SBil binna langt od) maigtig bli,

$D$ d) fom LinNaus ei fin life

Pá benne miba rymben fi;

Ekal woigen foref af honom lära,

Deu âr bas jemn odh wall berebs.

Det ăr Franiofen nu en ára,

It blifuxa af den Ewenfia lebo.

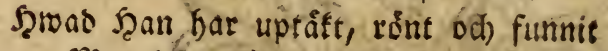

Meo bon, firfset, ineo wett od mat?

toar alla firbas bifall nummit,

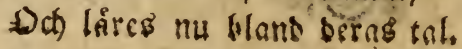


Hp Ewenfa Enillen, fintuden cber Til åbcl tâflan bwar od) cn,

Miundens fritt LiNNAI beber,

\$a bet ban cj må bli alletr.

Stockh. 1747 .

d. 2. Martii,

\section{Hedwig Charlotta Nordenflycht.}

\section{Då \\ D. D. CARL LINNEUS \\ Q̊ttexfom ifrån WäfterGótland,}

Cånbes folljande:

(10)

ir all Europens miba park

SMil at LinNeUs framfar bela,

Rår Tiyfta Jottens fténa marf

Dd) Killa wertben wid, Amfela ;

9lár Themfen, seine od) Sibinn folf

Nies untoran ấ bes lofords tole;

Då har ben nutra werldens unt

De crsigt Ensibctáfte Fjällen,

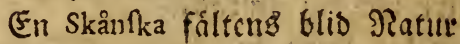

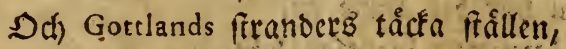

Camt Smålands linber, fruften fett

If wả Linner fora wett.

Når man fring nya werloens fălt,

(Şwars probriad trotiar mer án Gunbra,

Dâr Flora Nayit up fit tâlt)

LiNNEI witterhet mift unbra,

ఇăr Ceylon inffar fe fin praft

Utaf bés hans i oroning braft; 
23air Eivenffa Flora frantiar bår,

Ee'u hon fitt åttartal fótt famla, Soch) Fauna i fin lappmubs fwår, Siring Ewemfta bårgen albrig fantla;

DCh Sala i LINNAeI wårb

Enart trotfat Ganges blomftergåry.

Doc har set matalofía wett,

Sin bwilfet fielf Biaturen talat,

Fi nog af Atlands featter fett;

Det feltes Wermtands fiema balats

D(f). WeftraGóthars wiba lanb,

Ilt mâlas af fâ wå̉big hanb.

Sâlt, Emer, sll, foin åga fít

Det alt fist mäitga mîfe fafna.

En mis̆bets ocl frón Rocroen goik,

Den alorig Sosber fät fe matna,

Dert wå linneus oblat op,

Dd) reft Jzaturents falna hopp.

Mea ufla afuns foin feal fe,

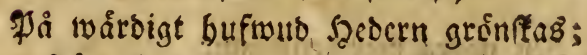

Fi̛r alt du big wil rorgfólt te,

Riun alorig báttre lo̊n big unffers,

भín Dả bu bår bet ămne åx,

Eom åt LinNeus frantfar bát:

2S alfommen hemt wält svithiods liub,

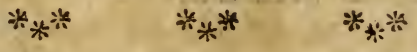
233 âlfommen Ewenfia frillens dra, STBálfommen til Ert blomfter 5us,

Der minfta blab fan witne bára:

sit odf boz of ben fürmon bor,

Som Flora giur $i$ wertben fort. 
Sul şimmel! Sut fon forer alt - SRes ditu solba niefets lagar; dis.netiog

Com $i$ en blick bet alt befalt Eom giort wir fåroa trơtta bagne;

fát ciran fróna lårboms nit, Sd) lvăn Tona arbets flit.

Eá firk i turano folla bar Mäit tio at bumbra tioer fign:

At hon ct wett framialftiat bat:?

Som be ef ågt ach thapt fä åga.

Från Falferbo til Rappeng tiáll

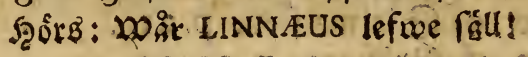

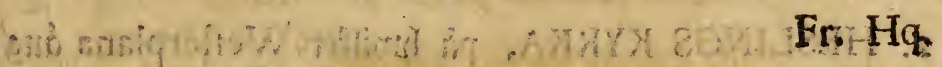

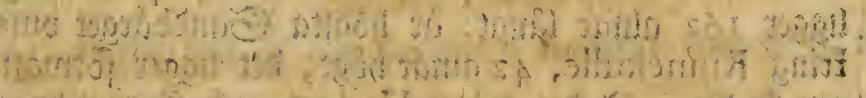

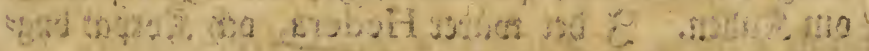

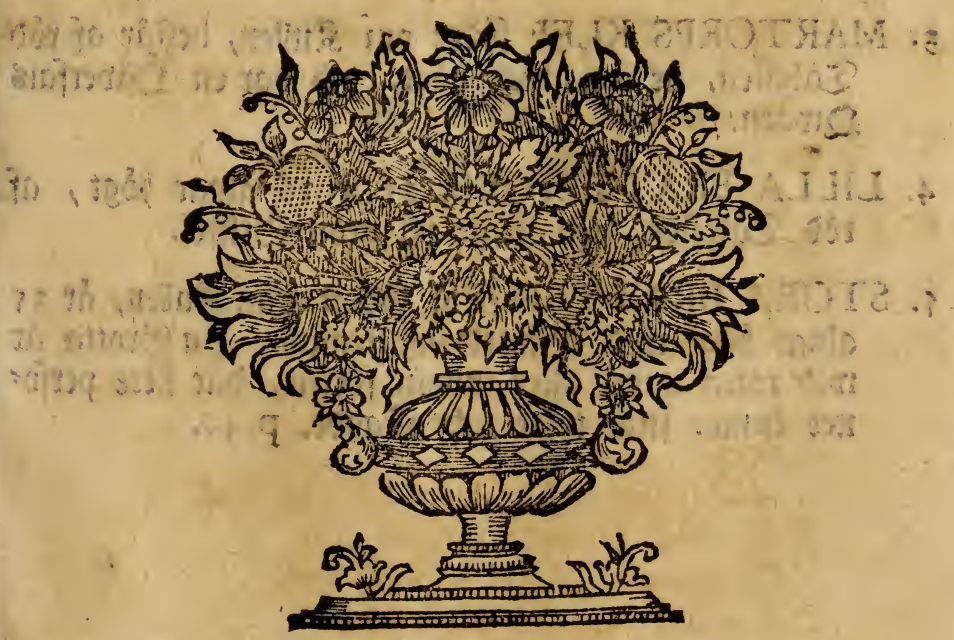

$\mathfrak{S}_{4}, \quad$ Fit, 
$280^{\circ}$

\section{Soinflaring offuer Ropparftycterne.}

\section{Tab. I.}

I. KINNEKULLE i profil pag. 20.22.

a Sandftens Flefivan $p .20$.

b Simltens wallen; Savóebärget p. 2 r.

c Siobftens Silefrona af allwarften, fom inutober bes ftâr af grout Striffell.

d Sorttens Slefiman. p. 37.2I.

e Bacfen af Siullerften. p.2r.

f Sritbirget of Elifmerften. p. 21 ...

g Jaigfullen of (Šrabirg. p. 21.35.

2. HESLINGS KYRKA, pa hiwillet Wefterplana áttg

- ligger 162 alnar liangt; ar louggfta Ganobárget om fring Kinnekulle, 42 aluar hisgt; Det ligger fotmoaift om Sullen. Ş Det wajer Hedera, och Forpar bygs ga Dár.

3. MARTORPS KLEF fovet Sum Sullen, befrär af roo Sälgften, ât 26 alnax bogt, od) bar en Śtiverfals Qwarn.

4. LILLA BRATTEFORS, 25 à 16 nlmat bigt, af

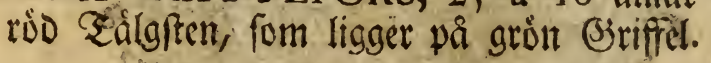

5. STORA BRATTEFORS, fuooft frain Sillen; ir 35 alrar buggt med et ftarft mattenfall. En'Srotta ár wid roten under cller innom forfen, oât flere perfos ner Euma fitta torre oct) offinoor. P. 40 .

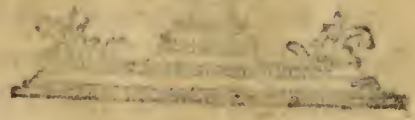




\section{$\% 3 \propto 8 \%$ \\ Tab. II.}

I. Et fructe KEtrá från Skepshwarfivet. p. 150 .

A. ct gummalt majelyol, Doir flugan lagt fina igg.

B. Dar et sammalt maffiol nutaz.

C. Dâx et mutt mafefrot begunnes, fom an upfuldt meo mafénioul.

D. Dát maffílolet givr fin twairbugt, od flutas at upfullab meo mjơl, bwar of puppan tortwando: Iab.

E. Dât bolen flutab med sppet bol trocteffore utat.

2. SKRÅPUKEN af SEepshwarfšflugan, $i$ naturlig fturléf. P. 152.

3. PUPPAN af Den famma $i$ naturlig porfek. p. 152 . 4. SKEPHSHWARFS-FLUGAN $i$ maturlig frorlek. p.

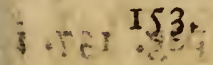


282

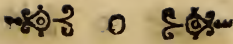

Tab, III.

I. CANCER cantonenis, p. $137+i$ naturlig forcte?.

a Sivggfidan.

b Banfidan.

2. MEDUSA orbiculo fubtus quatuor cavitatibus notato. p. I 72 . pá undre fitoan rrifad i mignateur.

3. MEDUSA orbiculi margine fedecies emarginato p. 172. i mignateur.

a ifra fidon.

b inota fidat.

4. LERNEA cauda duplici tereti, p. izi. i maturlig frorlef.

a Singgftoant.

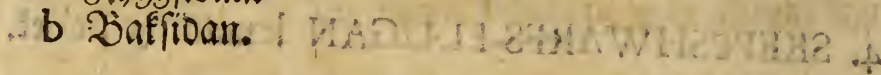

5. BALISTES qux Guaperva Chinenfis, pag. 137. naturlig ptorle.?.

a pit fion peos.

b pâ ringgen fedd.

6. LUMBRICUS marinus. p. 189.

B

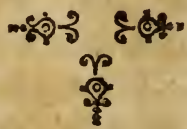




\section{- \\ Tab. IV.}

I. ZOSTERA. p. 166 .

a śfwerfta Delen af fticleen.

b unvera fiton af blavet meo blommor.

c blas utan blomma.

d blá med blommor pa sfra fidan feste.

2. ct frucfe af et blad, fom bår fina blommor inneflutne. i naturlig frorlef.

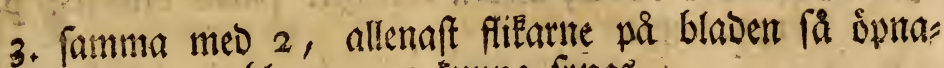
De, at blonmurne fumma funna.

4. Famma med 2. 3, Dâr bladet ât bojgot ifuån blom man.

a ár Spadix meo fine blommor.

$b$ it et fructe of bladet:

5. Anthera:

6. Piftillen.

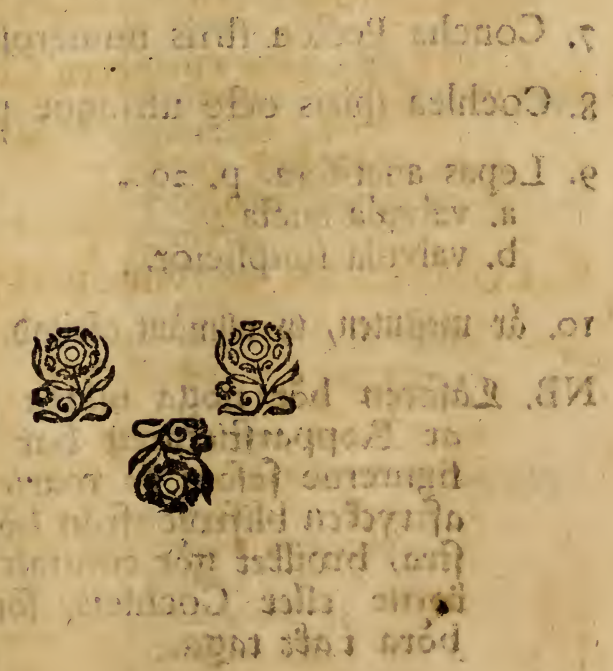

Tab. 


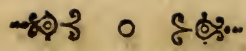 \\ Tab. V.}

Sair aftefras to formàmfte feal, form finnas i Udde. walla Ekalbaitg. P. 197.

LEPAS Balanus uddevallenfis. p. 198.

$b$ en valvula af Ben famma.

2. CONCHA oblonga obtufa fulcis transverfis. p. 198.

3. $\mathrm{CONCHA}$ lrvis, altera tantum parte clufilis. p. 199. 187.

4. COCHLEA Siupunge dicta. pag. 199.169.

5. ât utefluten, th Gon îx allmaint befant.

6. Cochlea Ipiris quinque utrinque producta. pag. 199.

7. Concha Pecten ftriis numerofiffimis, p. 199.

8. Cochlea fpiris octo utri.ıque productav p. 200.

9. Lepas anatifera. p. 200.

a. valvula aucta.

b. valvula fimplicior.

10. år utefluten, ty allmint afritab.

NB. Láfaren bớr noga mitrta noid benuta tafla, at Kopparfticfaren bar uti platen aftagit figurerne fofom de woarir titade, broar af aftrycken blifwoit ifrân bögra fioan til moants frta, bxoilket goer contrair worioning i Snacs Eorne eller Cochleis, fom Zoologi noga bóra i akt taga. 


\section{Siegifter.}

\section{I. \\ Hiftorifta Sater, Orter, Antiquiteter $\mathcal{E} c$.}

1 lingsås 116.

Aranăs I9.

Arb̆oga 9.

Şadfuga på Lindholmen 50.

Berg/ le Regifter effwer Phyfic. ting.

Berga 72.

Billingen 63. \& .70. \&

Blackwell I05.

Blåkulla I82.

Bohus-Fåining 159.

Bohus-kán 158 .

\section{Situation oeraf 163 .}

Borås 109.

Botaniquens upǩomft 5.

Brattefors 40.

כruft, Jäders-25rut 9.

Brunsbo 58.

Bykulle 237 .

Capital-Drăngat II 2.

Carlabylånga 23.

Carls-graf 220.

Carlitad 24I.

Carliten 164 .

Caftell-gåro 162.

Clara-flod 242.

Dal 223.

Diger Doben 247. 248.

Domarefullen $8 \mathrm{I}$.

Domareringen 219.

Dåttern 217.

Eds wågen 207.
Elfgripar 20.

Elfsborgs fFåftning $1477^{\circ}$

Enkóping 4.

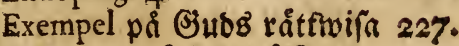

Fabriquer, fe Manufacturer.

Salbygben 72.

Falkoping 79 .

Fjattnallar $\mathbf{x}$.

Frieri 69.

Fryken 239.

Fulleroீen 272.

Fâllingsbro 9.269.

Fraffningar $135 \cdot 147 \cdot 159 \cdot 164$.

Fámflifor 30.

Gamla frwarfmet 149 .

Graf, Grafiten 28.29.

Grindar, rara 245.

Grytthytta 263.

Gullby 162 .

Gymnafier 56. 135.24I.

Gällequift Elott 57.

Göta Elf 158.

Góteborg 135 .

Góteborgs Ren, Situation 135.

Hall 217.

Hamans Gialge 90.

Hellekis 25 .

Hjelmarsrór 81.

Hifingen 158 .

Hofwa 17.

Holfjôn 210.

Hornkärnet 245 .

Hofpital 157. 
Hunneberg 211. \&ะ. 217. Hâlo̊ga 9r.

Håckla 218.

Hojentorp 58.

Ingefätter 66.

Jäders-3rult 9.

Jâttat 25 . 19.

Siraf 25.

Kinnekulle 20. \&e.

Klefwa beb 90. profpecten $\cdot 35 \cdot 36$.

Kolthoff, Z5ruks Patron, 246.

Kongelf 160 .

Krâkan I6r.

Sintfor, Forshem 19.

Hufaby 4I.

Klefwa 9I.

Lidko̊ping 54 .

Mariåıtad 18.

Skara 56.

Wäfterplana 33 .

O̊fterplana 38.

Kàglan 9.

Kóping 7 .

Képmanna-fjall 228 .

Kopinannebro 230.

Kơrn 183.

Laudati, Brelin (Magifter) 227.

Heffelius (Med. D.) 10.

Hof (Lect.Mathef.Scar.) 57. Kalfenius (Epif. Arof.) 5 .

Leche (Med.D.) 142.

Myrman (ZZruff ; Patron)

$$
252 .
$$

Reuterholm (Gubernat.Neric.) 10.

Swab (Anton) 229.

Lecho Elott 5 I.

von Aken (Pharinacop.) II.

Lida flod 54 .

Lidkofing 54.

Lindholmen 49 .

Loka Seâlia 264.

Jiaiftinger $53: 228$.

Manufacturer 0 di) Fabriquer 110.

- \&c. $127 . \& c .120 . \& c$.
Mariehholin 17.

Mariäftad 17.

Marftrand 164:165.

Nilftolpar 56 .

Mofjón 13.

Mulltorp 68.

Măffewik 238.

Mónfeberg 73.85. \&c.

Norfs-Elfinen $2 \hat{j 9}$.

Nora ftas 268.

Norums-23ruf 246.

?ndfel Singarna 85.

Nyeds \$răfrẹgår 249،

Nerike 9.

Odens- Ritlle 89.

Olleftad 105.

Orangerie 8.

Oroft 186.

Orrnås 19.

Oft-Indirka Compagniet 156 .

Pater nofter $\mathbf{I} \delta \mathbf{r}$.

Philippitad 25x.

Fährsberg, DeE Grufivor 254 .

2nuactialfiuare 100 .

O) warnbacfa 260 .

Qninfolfins Siládoraigt 69. 103. 157.

Ronnums bro sfiwer Góta Elf 219 .

Siunftenar $10.43 \cdot 53$. I 44 .

Scara 56.

Scaraborgs Elott 56 .

Sebruánior 103 .

S. Sigfrids Sälla 42.

Giơn, fe VIII. Zkegiftet blano Phyfic. Eatier.

Skjoffde 68.

Elott $\mathrm{x} .5 \mathrm{r}$.

Ethangierns: Samrat II. i ch Sofn 249 .

Stola 45 .

Superintendenter i Wermeland 242.

Sven $i$ SBaynom 100.

Eormocti 241 .

Tengne Eafn 82.

măngas nanm 82. Tigs 
Bärgarter.

Siggare 17.117.

Tiweden 15.

Torn ganféa ftort 54 .

Trallhättan 209.

Tunhem 210.

Tynfje 23 I.

Uddewalla I94.

Upland $\mathbf{r}$.

Des fituation 4 .

Uprala $I$.

Warnăs 267.

Warpenås 24I.

Wafswiks Grufrua 230.

Wedewag 268.

Wenern 4I.

Wenersborg 219.

Wermeland 236 .

fituation Deraf 29 I. 253.
Wermelands-Dal 230.

Weftergótland 16.207 .1

Weftgóta-ग̃al 230.

Weftgóta-Fiållar 130.

Wefterås 5 .

Weftmanland 4 .

fituation beraf 6 .

Yngen 254.

Alleberg 82. \&c.'

Âlleftad I05.

Àmâl 232.

9ire: Portar 5.7 .

शittebacfar, 2itteplater $4 \cdot 41$

81. 88. 91. 144. 219.237.

Sitteftóntan 218 .

Orebro IQ.

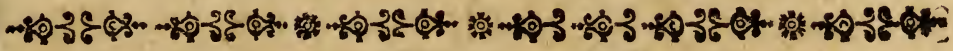

\section{II. \\ Zårtyatter.}

Alun, gesiegen 212.

Alun-Cryftaller 68 .

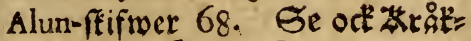
berg, Fifwerfterr.

Sergbect. 89 .

SIlele SI. 92.

Binglants 230.

Cryitaller 141.

Senifors ro6.

Flinta $i$ Limftens $=00$ allen 21 .

IInglans 243.

(sorften $37 \cdot 21.92$.

Granater 255 .

Gråberg 35. II3. II 5. I I6. I65. I 85.2 I I. 228.229 .245 .

e) alle $i$ fóriza ftapel= fen 35 .

Bråftenar, İ์ fa 21.35 .

Humus ochracea 212.

Šcrnikes 230.

Jennmalm 17.252 .253 .254 .
Sorbarter rơoltige 22, 28, 29: $33.37 .63 .71 .83 \cdot 240$.

Soromon, magrafte 249 .

Talffen, grå 2I, 24. 42 . gron $2 \mathrm{r} \cdot 34 \cdot 256$. froit $34.70 .73 \cdot 87$. 230.255 . tơ $21.28 \cdot 33 \cdot 37.70$. 92.

fråligt ftroos 267 ,

Rld̊cfeften 73 .

Roppar, geoiegen 253 .

Sioppar=FeB 230.

Sirita, fuart 212.

Sirritberg 21.28 .35 .

fiórtelftenar 212 .

Refwerften 21.

Rerarter, \&tflillige to."

Per fórwanolas til talf 87:

Rera liffergao 251.267 .

Rimiten $2 \mathrm{I}$, 
Lithomarga 24.

Marmor 73 .

Dilten 2 I. 24.63.68.

Pefiur 4I.

Petrificationer 24. 41.87.88.

Quartz 53.229.230.

æăcficiorb, en jordart 240.

Salt 30. 157 .

faftas bland $b 88^{2} 233$.

Eand $55 \cdot 242$.

Gilitteriand 239.

Eanbmo 24I.

Entibftent $20.21 .33 .70 .7 \mathrm{r}$.

Sanditens Salt 256 .

Silex 73 .

Eilfwer, geoieget 253.
Eilfwermalm 23T.

Efifweriten 2I. 22, 28.35.63.70. 8687212.

Sfifwer fơrbytes til Ralf 87.

Spat 231. 252.

Epriflbållor 21 .

Stalactites 159.

Etemfolfmar 4I.

Ervartbällor 212.

brufas til pwart fairg 212 .

Sellmalm 256.

Tophus 88.

Torf ro.

2lféa beraf grwit od funart 10.

Tálgften 256.

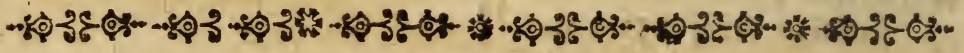

\section{III. \\ Jubemfta Dórter.}

Samnen, oronitgen od) numren, fom fál in parenthefi, daro taghe utur Flora Suecica. Afterifus (*) utrmifar de ftils len, brareft anten talas om nigon wáxtens innuerliga nytta, clfer elieft gởes någon aumårf́ting berwio.

Liguftrum (4) 192.*

Circxa (5) minima 26.92.

B lutetiana. 213.*

Veronica (7) Jpic.minor. 84 .

(8) 2 teenpris 74 .

(10) aquat. fol. obl. 39.

(II) Beccabunga 39.

(12) Pfeudo-chamadrys 107. *

Pinguicula (2I) vulg. 3 I. 60. *

Utricularia (24) 46. 49. *.

Valeriana (30) fyize 6.*30.

Schoenus (34) Rofc fafcic. 208.

(35) $2 \mathrm{gg}$. 108.*

Scirpus (38) Cyperus gramin. 64.

Eriophorum (44) 2ingull 6. 238.

(44) 万arull I I 5.25I.

Nardus (47) Gram. Spart. 39.1 15.
Melica (57) Eergfi.te 23. 31.

Agroftis (6I) Zitypbooen 39.

Aira (63) Tát $=\mathbb{C}_{\text {atel }} 39$.

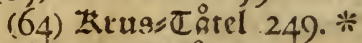

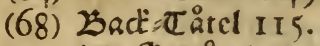

(70) Mryft: Co̊tel 57.I 45.*

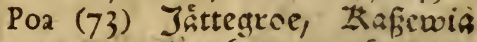
. $44 . * 46.48$. 160 .

Cynofurus (8I) Zzambering 33 . $8 \mathrm{I}$.

Bromus (89) Epairriofia 76.

Feftuca $(90)$ Suxinfwingel 39.

(9I) 2ingfwingel 39 .

(95) fånf foringel $60 \% 62$.

I 15.249 . *

Avena (97) Lanobafre $93 .{ }^{*}$

Arundo (99) vulg. $93 . * 162 . *$

Triticum (I05) Q Owidtor 93. * 207. 


\section{Wixter.}

Afperula (114) oderaba 33. Galium (II6) lut. 6.*39.74. (I I 8)E quadrif.alb.6.*39.74 (I I9) paluft. alb. 39.

Plantago (I28) monsinthos II 5 .

Cornus (131) arbor. 32.*39. 40 . 84.

(132) herbacea 57.83.115. Cufcuta (138) I6.23.43.

Potamogeton (I 39 ) natans 46. Myofotis (14.9) forgăt mig ei. 6.*9. $60 . *$

Lithofpermum (I5I) vulg. 33. (I52) rad. rubra 28. *. Anchufa (I53) Oxtunga 2. Primúla (I62) Aor. rubro 76 . Menyanthes (I63) Trifol, palufir. 39.62.

Lyfimachia (I66) vulg. 39.

Campanula (176) vulg. 3 I. 74.90.

(I79) perfici fol. 74 .

(I80) maxima 27.74 .204$. (I8I) fol.urfice 27.74 . (I83) altiff. hirf. II 5 .

Solanum (I89) Dulcamara 56. *ै 71.

Hedera (190) 33 . 13 I. 201.*203. 213.218 .

Lonicera (I9I) Caprifol. I46.* 209 .

(I92) Xylofeum 3 I. $213 . *$ Rhamnus (I93) catbarr. 23. $3 \mathrm{I}$. $84 . * 215$.

Ribes (I95) Zturtubår 23. Ulmus (219) 23.66.*84. Sanicula $(222,23 . * 27$. Conium (226) I $59 . *$ Heracleum (231) 23.31. 39 . Ligufticum (232) fcotic. $182 . *$ Angelica (233) vulg. 6. * (234) fylv.64.207. Sium (235) 46. 160.238. Phellandrium (238) 6.* 46.47.* 62.

Cicuta ( 239) 6.*46.48.*62.98.* Biftorta (321) 39.64.92. 160.

Sambucus (250) vulg. I6I.
Chærophyllum (243) ろunolok 3.**

Carum (245) 2.49.93. *

Pimpinella (246) $39.208 . *$

FEopodium (247) 62. 207.

Parnaffia (252) 39. I I 5. 249. *.

Linum (255) catharr. 39.

(256) minimum . 41 .

Drofera (257) fol, orbic. 60. *. II 5 .

Allium (263) fylv. Latif. 27 .

Cepa (265) 2Ungl \&E 84.

Anthericum (268) Gram. off 2 r. Convallaria (273) Lilium convallium 6. *

(274) Sigill. Salom. 106. *

(275) fol. vercicill. 75.84 .

Calmus (277) r61. 204.

(279) 200 detetog 184.

(285) Iltog 60. *

Peplis (291) 218.254.*

Rumex (292. $\beta$ ) Herba Britann. 46.

Alisma (300) Plantago aquat. 6 . * 46.47. *

Trientalis (302) 36. II 5.

Epilobium (304) 万immelsgro̊s 6.*74.207.*

Erica (300) vulg. Liung 74. 89. *

II 4 II $5.183 . * 228$. 24.1. 249. *

(310) Tetralix 107. II4.

Daphne (3II) Zzállerbols 243. * Vaccinium (3 I2) Odon. 74 . 107. I1 $5.131 . \%$

(313) 25läbต์ 74. II4. 249. *

(314) Lingon 74.

(315) Tranbår 62.115.

Chryfofplenium (317) Saxifr aw* rea 62 .

Perficaria (3 18. $\beta$ ) Aluitans 46. 


\section{Wäxter.}

Paris (325) folanum quadrif. 3 I. 39.62. 204.

Adoxa (326) Mofchatellina 37. Butomus (328) 46.

Pyrola (33I) minor. 18.20.83. (334) unitiora 36.

Andromeda (335) vulg $25 \mathrm{I}$.

Arbutus (339) Miólonris 1 10.** 241.249 . *

Cucubalus (360. B) marit, rep. I49 164 .

Silene ( 364 ) Ciårblomfter 23. 39.

(366) paniculata alba 39.

(367) caule dicbot. 144.* I46. 164.

Alfine $(370$. B) altilf. nemor 204. Arenaria. $(376)$ maritima $148 . *$ Spergular 377 ) pedunc.dicbot. 56.64

(378) pedunc. fimpl. $147 . *$

Ceraftium (379) villo/o-vifc 39. Lychnis ( 384 fos Cuculi 9. 39 . Oxalis (385) Trifol. acezof. 36 . Sedum (387) petr. alb. 23 I.

(388) rupeftre 146.213. $218 . *$.

1389: acre I80. *

(391) caule er. folit. ann. 49. *

L.ythrum (393) Lyfim./pic purp. 31 . Agrimonia (394, 6. * 39 .

Padus (306) 万kgg 32. *

Cratrgus (399) Sagtorn 23. 31 . Sorbus (400) Rónn 31. 42. * 251. *

Pyrus (402) Malus fylv. 23.31. 67.* 93.*

Mefpilus (403) Chamame fpilus 39. Filipendula (404) vulg. 39 .

(405) Ulmaria 6.*93. Mentha' 481 ) Jylv 216.*

Rofa (406) calyc Jemipinnat. 31 . 40.

Y A.albo 33.52 .

Rubus (409) maximus 135 .

Caltha (473) paluftr 6. *
(4I0) fr.cefio 40.

(413) sijoxtron 115.

Fragaria (4I4) Emultron 17.

Potentilla $(4 \mathrm{r} 5)$ anforina 2 .*

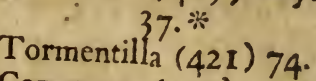

Comarum (422), quinquef. pal. rubr. 6. * 39 .

(426) Iutea 46.

(427) alba 46.

Papaver (428) 2ikerwolmo 2.

Actra (43I) Cbrifopburiana 203 205. *

Tilia (432) $\operatorname{lin} 023.67^{*}$

Ciftus (433) Heliantbemum vulg: 76.

Refeda (439) Luseola I10.*127.* 125.

Aconitum (44I) lycott. 6. *

(442) Stocinbatt 6.* 48. *

Aquilegh (443) vulg. 32.

Stratiotes (444) Alvë paluft. 46. 49. *

Pulfatilla (446) vulg. 62.

Thalictrun (454) minus 26. *

Ranunculus (457) longif. pal.maj. 46.

(458) Flammula $47 . *$ $60 . *$

(459) fol. lin, caule rep. II5.

(466) prat.cr.acr. $6 . * 36$

(472) capillaceus 269 . Helleborus (474) A. globofo 6. I4. 39.64* 92.93 .218$.
Clingrodium (479) $31.197 . *$

480) vulg. 39. 40.74.

Glechoma (483: Hedera serrefore 107.*

Ballota (484) Marrub. nigr. I59. Marrubium (485) alb. vilg. 18. 


\section{Wạxtex.}

Nepeta (486) Mentha cathar. 52. IGeranium (571) uniflor. 39.40.74. Stachys (489) Jylvat. 27.84.204. 205.* $*$

(490) palufir. $93 . * 204$. Brunella (498) vulg. 39 .

Scutellaria (499) fol.cren. 207 . * Rhinanthus $(503 \cdot \beta)$ Crifta galli mas 6 .

Pedicularis ( 504 ) calyc. quinquefd. $39 . * 228$.

(505) calyc. bifid. Kaill=

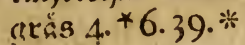

Melampyrum ( 510 ) criftat. $39 \cdot 40$. 62.74 .

(5I2) cerul. coma 62 . (4I3) lut. latifol. 23. 39.62.64. 74. II 5 .

(514) corollis biantibus 23. 39.62.64. 74.

Euphrafia $(5 \mathrm{x} 7)$ rubra 218. Lathrea (518) Squamaria $13 \mathbf{I}$. Scrophularia ( 520 ) nod:f foet. Limnea ( 522 ) 9.36.251.254.* Draba (526) alpina 75 . Subularia (527) 160 .

Lunaria (529) 27 . $213 * 578$.

(572) batr. fol. aconiti $6 . * 64.74 . \quad \therefore$

(574) lucidum $2 \mathrm{r} 3$.

Malva (581) Jylv. fol. fin. 159 .

(583) Alcea 267.

Aftragalus (591) Glycyrrỉize fylv. $23.32 .39 \cdot 40.84$.

Anthyllis (594). Vulneraria ruff. 23.39.74.90.

Orobus (596) rad. tuberofa 39.

(597) caule ramofo 213 .

Lathyrus (599) Jylv. Lut. 39 .

Vicia (603, fipul cren. 40.84.92.

(605) multifler. 2.39 .

Pifum (608) marit. 190.*

Lotus (60), Trifol. cornic 90.

Trifolium (6I ) mont all. \&4.

(61 5) prat.purp.90.93.

(617) lupulin. erect. 213 .

(618) lupul. rep.65.*

(619) Melilotus offic 4.0.

Medicaga (620) legum lunat. 2. *

(621) legum. renifim. $2 . *$

Ononis(622) non fpirofa purp. 25. $39.84 .135 \cdot 162$.

Thlafipi (53 I) incan vaccaria fol. Hypericum (624) caule quadrato 2.*

Ifatis (543) Weide I10.* I25.* 127.**

Brallica (546) perfol. A. lut.91.

Sinapis $(548) 2$ iterfenap $30 . * 72$. Eryfimm (555) fol. int. Lanceol. I60. *

(557) Barbarea 53.62. (558) Alliaria 62.

Cardamine $(560)$ folonif. $26 . * 39$. 76.

(56r) apetala $26 .^{*}$

Dentaria $(565)$ beptaphyll. baceif. 62.

6.*39.

(625) coule ansipiti 6. * *

(627) caule tereti 213 . $\beta$ androf. cumpocl 213 . *

Leontodon (628) bilpidum 14.39. 62.92.

(629) Crepis 224. *

Hypochæris 631) 39.64 .

Hieracium (637) murorum 23. $3 \mathrm{I}$. $39.257 . *$

(638) cichorii folio $32 . * 39.64$.

Raphanus (568) Rapiftr. fi. alb. Sonchus (642) repens 207.* 218. 69.* 107.

Crambe (570) Gtranitåli I 89. | Prenanthes (645) 26.41.213. $\mathfrak{2}$ 


\section{Wåxter.}

Scorzonera (647) latif. 23 . $* * 62 . *$

39.64 .74 .* $92.93 . *$

Lapfana $(649)=38$.

Carduus, (654) lanceolatus 224. *

(656́) acaulis 88.

(657) Girfium 64. 84.

(658) Crispus 274.

(659) paluftris 31. 39 . 236.

Serratula $(6(10)$ tinct. I 10.*

(662) carduus 266 . *

Bidens (664) fol. integris 160 . *

Tanacetum (666) Kienfana 54.74.

Artemifia (667) vulg. I3 1 .

(668) Abrotanum sampe-

fr. 33.42 .

(670; Abfintbiнm 269 .

Gnaphalium (672) 2̌attfotter 74 . Tufilago (680) vulg.20.40.84.

Doronicum (684) Arnica 6.* I 5.* 39.74 .90 .115 .228 .

Solidago (685) Virga aurea 74 . 249.

Senecio (688) facobaa vulg. 160. (689) Jacobsea Senec. facic

II 5.142 .23 I.

A fter (6,34). Gotland. $3 \mathrm{I}$.

(695) Enuia 270.

(6,6) Salicis fol. $39 \cdot 40$

(697) marit. Tripolium 25.* Chryfanthe mum $(7 \infty)$ Bellis major. 39.74 .90 .

Matricaria (7OI) Chamemel. nobil. 107.

Anthemis (7O3) Chamomel. fatid. 205 . $^{*}$

Achillea (706) Ptarmica vulg. II6.

135.218 .228 .

Centaurea (700) Jacea nigra 2.39 . 265 . *

(710) Cyanus 56.72 . 228. * *

Jafione (713) I 8.69. I64.

Lobelia (7I4) I 15.249 .

Viola (716) mart, inod. fylv. 20.*
(7I8) acaul. fol. cord. bifp. 74

Impatiens (722) noli me tangere 26. * 207.218.

Orchis (723) alba bifol. 74

(727) palm.calc. obl. 74 .

(728) palm. prat. Latif. 39 .

(729) palm. prat. macul.74.

Satyrium (733) P Peudo-orchis 14 I Cypripedium (735) Calceolus 13 I. Ophrys (738) fol. ovat. $3 \mathrm{I}$.

Calla (744) Mrifne $15 . * 36$.

Carex (768) Lapplto=ftake 39.

Sparganium (77I) natans 46. $263 . * 4$

Typha (772) 181.*

Alnus (775) vulg. $18.93 . * 181 . *$ B alba 13 .

Betula (776) vuig. $93 .{ }^{*}$ 108.* II5. (777.) nana $25 \mathrm{I}$.

Sagittaria (780) 46.

Myriophyllum! (78I) A. pic. 46.

Quercus (784) vulg. $14.23 .45 \cdot *$ $64 * 67 * 104 * 133 . *$ 149. *

Fagus (785) ¿016 67\% * 108.215." Carpinus (786) Afwenbó r $43 . *$ Corylus (787) bafke! 3 I. $68 . * 74$. Pinus (788) Call, futu $186 . *$ $24.1 .247 . *$

Abies (789) Gran 23. I I4. I 86.* $238 .^{*}$

Salix (792) Jolfter 3.*13r. 262.* Vifcum (816) mifel 31 . $^{*}$

Humulus (818) 万umle 75.84.204. 215.

Populus (819) 21 p 23. 224.

Mercurialis (823) Cynocrambe 23. $39.60 . * 62.64 . \%$

Juniperus (824) vxlg. 23.* 65.* $127 . *$

Taxus 825) Datrlino 19.825. Fraxinus $\left(830\right.$, 2ffe $23 \cdot 67 . * 930^{*}$ Empetrum (832) Ktătbók 107. II5. 


\section{In- och Utlåndfka Ōrter.}

Equifetum (833) arvense 60.63 . (836) Aluviat. frátne 46.48 .*

Ofmunda (84I) Strutbiopteris 15.

$$
33.204^{*}
$$

pteris $(843)$ Ormbunte $6 * 238$. Polypodium (846) Filix mas 93 . (850) ilvenfe $7 \mathrm{I}$.

Afplenium (854) Trichomanes 42 : Acrofticum (856) 42.

Lycopodium (857) caule er, dichot. 36. 115 .

(858) caule er. Jpica glabra ferm. $20.1 \mathrm{I} 5$ (859) triattegrés II 5 Sphagnum (864) Ro somo fra 104.* II 5 .

Polytrichum (868) fropar, 93.* 215 .

Hypnum (872) proliferum I14. * Mnium (913) geniculatum 265.* Jungermannia (930) bypophylla 213 Lichen (937) lepr. cand, tuberc, atr. 29. $* 21 \mathrm{I}$. (942) 5yttelet 147 *
(959) Берemofa $250 . *$

(960) Pulmonaria 215. * (967) fulvus 159.

(971) Deuftus 217.*

(980) Renmofia 10. * 250.*

Lemna (999) Monorrbiza 257.

Fucus (1002) zklot⿳亠口冋! 168 . *

(I009) filiformis repens I68. *

(1010) fcot.edulis 169.* Agaricus (1034) in fol. pini 215. (1049) Chanterelle 215. Boletus (1087) Zoforwamp 274. Hydnuin (1097) imbricat. 215. Phallus ( $\mathrm{IO}^{2}$ ) mtruktor 36 .

Lycoperdon (II16) Tubera 36.* 93.*

Mucus (I 124) ronungsoagg 28. Byflus (II25) Vial-ften 9.250.* * $254 . * 263$. Alga (II37) angufif. vitriar. I66.* 184. * Agroftis (II38).5runbwen 9.39 .

IV.

Tya inbemféa exter, fom ej fté i Flora Suecica.

Cerafus, Kersbo̊rstrå 25.32.38. Cratægus fol.fubr. Jer: 161 . Byffus farinac. flava $53 . *$ I 59 . Potentilla. Quinquefol.fragifer.63. 76.94 . Zoftera 166. 167.

Ulva 169.

Ruppia 186.

Lathyrus narbonenfis 75 . Panicum (pic.altern.remot. lux. I4I Quercus, Platypbyllos mas Dabech. Senecio /ylvatica I I $5.23 \mathrm{I}$. Coreopfis Bidentis 160.272 . Chamemelum marit. I48. 214 . Pifum fylvefire $10 \%$. 
Swenfka O̊rtenamn.

\section{V.

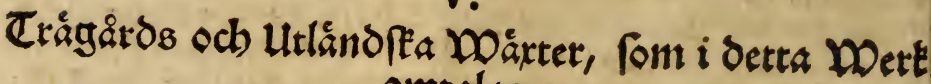 omtalas.}

Caraguana 10.

Linum Siberic. 10. :-

Canarie-fró 12.228.

Hlommuntrá 22.

STalnỏttrá $25 \cdot 52$.

Thé-flominor 107.

Ruta 52.

Rả 270.

Rubia tinct. 12\%.

Potatos $13 \mathrm{I}$.

Rapfat 132.

Rhabarber 16I.

VI.
Sxoenfta ơntenamn, fom i dente Referbeftrifning

216rahamsirot 235.

námnes, ocb icke fta i Flora Succica.

2lmurgråg $\mathbf{r}_{45}$.

Sockeblåcfe 23.4.

Borftar 235 .

Crucifix-ốnt 235.

Efnaz 32.

Elgeftielf 234 .

Eneftabb 235.

FFinnfte̊ng 234.

Folafótter 20.

Grangråz 235.

Gúffinore 62 .

J̧areqmå 235.

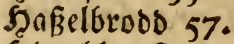

Shiertblomfter 234.

Şoltemiåtla 235 .

फुorntiftel 224.

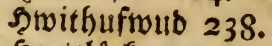

Şwitlåc 234 .

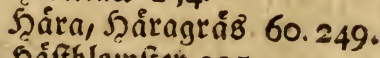

Şifftolomiter 235 .

J̈tegråB 235.

Ternórt 162 .

Tับngefơra 36.

Jogere 235 .

Tungfruris 249.

Tallorás 4 .

Rá̧ensia 44.48.

Rirî̉ 62.
Rrutlaffare I8I.

Siriffot 62.

Eueblommor 36.

fing 183.

S) Rijblomfter 6. 234.

Niólfblomiter 224.235.

Niultagrá8 93.

9)?åra 234.

9\}åafererot 98.

Drmgráz 93 .

Drmenge 238.

Rantslêt 227.

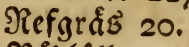

Rỏbtálla 93, 235 .

Saltblåcter 234 .

Efallta 235.

Efateblomfter 235.

Etogefumle $235^{\circ}$

Enarterefmer 16.

S. olmơjegrå 235 .

Sológon 36.

Etenbár $=34$.

Etroppelgrá: 235.

Etátre, ben rátta 46.

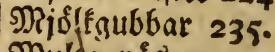

Efogiloper 235 .

Sopmofa 215.

Steḉf̣ráá 234. 
Djur.

Ormt (259) r 46.'

Mabsa $(253) 204$. mitt $\operatorname{nag} 26 \mathrm{x}$.

\section{fiftin.}

Baliftes 137 .

Bårginmulltra $\mathrm{I79}$.

Blennius 182.

Coregonus 23I.

Cyprinus Phoxinus 132. 232 .

Elifulor 232.

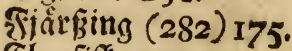

Flugfiff 239 .

Gabofur 232 .

Gadus (294) 5witling 176. (295) Kabbelio 176 . - Langa 177. Eyrblet 177 . Corft I77.

- -178.

Gafteroftets (276) 239.245.252.

Gitppor 232.

Giácba 45 .

STornfiff (276) 252 .

รูwitling (294) 176 .

Ji่ (269) 174 .

Rabbelia (295) I76.

Sint $(221)$ I 76 .

$\operatorname{Pnx}(306) 206.219245$.

Enrblef 177 .

ångn $177 \cdot 178$.

Oftracion I 38.139.

Phoxinus 132.

Bigghwarf 178 .

Pleuronectes (299) 178.

\section{(301) 179 .}

\$igghwarf 178 .

Raja (268) I75.

2nidos (331) 260 .

Råcia (268) 175 .

Rơding (310) $25 \%$

Rispinotta 179.

Sparus 179.
Squalus (269) 174.

Êtenfugare 232.

Tenfife (276) 245 .

Torfé 177. 184

Trachinus (282) 175 .

Trigla (281) 176.

Triumma 232.

2ilfura 182

भinim8:923inm 231.

2rff์. INSECTA:

Bi (1003) 43.

(998) 265 .

Cancer (1244) 173.

(1248) I74. 19I.

exot. 137 .

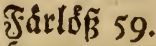

Shumer (1248) r74. Igri

Srabfia (1244) 173.

Monoculus (II82) 14.

(II85) 159.

(II 85 .

$(1083) 84$.

(non dum defer.) 35 .

Minas ( 1116$) 37$.

Oeftrus (1024) 202.

Onifcus 190.

Phalæna (838) 27.

(895) 65 .

(nov.) 140. 141.

Phryganea (nova) 47.

Scolopendra $\left(\mathrm{r}_{2} 63\right)=36$.

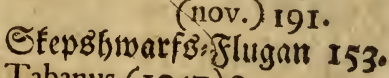

Tabanus (1047)9.

Taftéråta 173 .

Tipula (I 150$) 65.233$.

3ูกำ \&ิ

Btriofă. VERMES,

Afterias (1285) 172.

Cochlea (1298) 27.

(1302) 49 .

(1316) 84 .

(novx) 169.199.200. 


\section{Phyficalir ka Saker.}

Concha (1333) 170.

(1340) 193.

nov. 187 . 198.199.

Lactar af trintes och

Enăderftal 197.

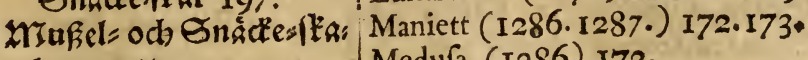

Dentalium 170. lens nytta 200.201.

RoLftroll (1285) I72.

Medura (1286) 172.

(1287) I72, I73.
Patella I71.

(1287) I72. I73.
Patella I71.

Lepas (I349) 170.
nov. I98.200.

Lernea $17 \mathrm{I}$.

Lumbricus (1270) marinus I89.

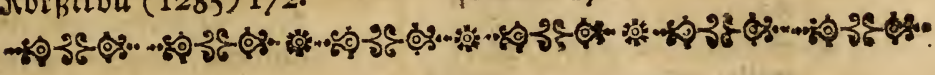

\section{VIII. \\ Phyficalifa Saler.}

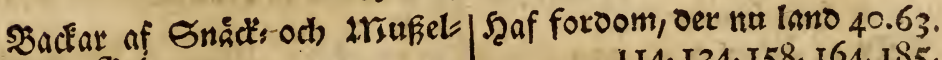

fial 197 .

Benpipor of 6 quart. långo I9.

Berg oraga Dimba til fig 79 .

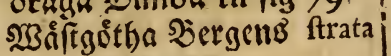

$70.77 \cdot 83$.

bura fiuagen of moln fys nes frọn bơga bergl

på lǻtterna 83

ganfta grónt på norka fi= oan of oe Do siftgótba 85.

Slobfot, fiutoom bos $2 \$$ oftap 38.64 .

Sirattfors fall och grotta 40.

Brunn i bo̊roa Derget I 59.

Canarie-fogel parat fig meosteg:

$$
\text { lita } 1 \text { I. I2. }
$$

Cataractx 86. 209.

(566 od) Slod 183 .

Falbugoens art od) egentzaper 72.76.

Fiff, 'ómfar bemwift 200

flottbiórtat 108.

Flottholmar ros.

ร̌lógtrå 218.

Frofinatt Den forfta 270 .

fुurll of 409. års åloer 247.

Sirotta 48. 51 .

Graberg, ei alla wio Rerpelfen 35.
I I - I34. I58. I64. 185 .

200.

f̧onung

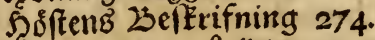

Jord, ingen pঞ Elippor 185 .

Jorbremma 54.

Jorblfott 45.

疋 $i$ fiere át ofmált 256.

Játtar 25.

Țáttegrytor I3I. I47.20r.

Siélla of falt watn 157.

Lithogenefien 78 .

Runver 24.

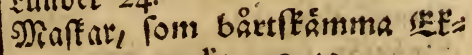
werte I49. $2 c$.

)Ruslor ody Enåcfor fiytt me. ra til fóder 200.

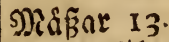

शattens' beftrifning 246.

Drtorn, bwao, och Des mytte 65.

Rågn, tetn oettil 9.71.223.

faller mer pò bergig orter 80.

båfrigt 238 .

Siơat I $3.43 \cdot 44 \cdot 55 \cdot 58 \cdot 108.109$. $217.231 .238 .239 .245 \circ$ 249.254 .

orifwa răgn frơn fig 80. fom àxligen tiltagax 210. 


\section{Mediéá.}

Colen giă neo 2 gẳngox pr̆ en ORg 89.9I

Epatt, fiutoom bos håfat 33 Epringa långs efter Lidkópings torg 54 .

Spritt, fiutoom bos biffar 38 . Stenars formandlande 37.

Strata af Kinnekulle $202 \mathrm{C}$

af Wàitgótha bẹkgen 7077 83.

af Skalbårgen wio Uddewalla I98.

Surbrunnar 56. 112.131. 157 204205.243250 .252 .

Sota brunn 264 .

Sefn til Ki̊gn 9.71.223. til ofreo 108.

Sillánbningar 4323 s.
Srá, bwarfore wika tEaOa de

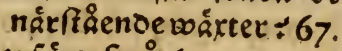

bwarfore fin: 3 . uncer fant 242 .

Deras alloer 247.

Batn fóxwoónot i bloo 14.256.

Ititpe och Eliof faminane De 62.

$i$ Wenern, fomlige at bos= gre, 55 .

Des aftagande ro 185 .

Mintrar ofia $E_{\text {nilla }} i$ Esós oer, $\partial \stackrel{a}{p a ̊ ~ f a m m a ~ t i o ~}$ blisare i norre orter Ig6

Eomma bittion 270 .

भill berbonens brådeligbeter 250. Ifiar 13.

Zittebaffars åloer of Gwats myllan ogrunoaO 89. Srter, ofta foos fór et Cleatur, men forgift for et ans nat 48 .

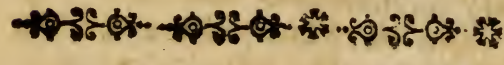

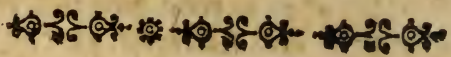

IX.

Medica.

Blobjot 3r.

Srottfålling 144.

Z̉rånfảr, bot Derfơte 202.

Drag eller Fflü ir 8 . bot derfore 254 .

Jrofía 3 I. II 2 .

bot detfóre 180.222.

fulllag, bot derfore 243 .

forrfoppning, bot verfóre 206 . Gutta rofacea 1 I8.

Hofpital 157.

Roppor I45.

Rráfructan, bot dexfore 243.

Lues Venerea 157.

ఇåsblob, bot Dexfóke 206.

Siofell, bot oerfore 202 .
Tiơba \&̊gon, bot 202.

$\Re$ Ift får, bot 202.

Ejuffom, fynnerlig 233.

Cforbjugg 180

Spina ventofa I is.

Etarren, bwit odb: froarta 246. Etenbuggare, gammal mes fri. ER Lungor 35.

Enneni forloranoe 246.

Eăt, bot oerfóre 124 .

Die varen. 234.

Volatica fcorbutica 234.

Vomitus chronicus I80.

Wånt $i$ leder odb asmar 71 . bot for $100_{\text {át }} 254$. 
Oeconomina Saker.

\section{$\mathrm{X}$.}

Regiftex pa bxoarjebanda i Oeconomien, Commercien, ocb Landtbusbollningen.

266อน $=$ รุณ์ 259.

Alun'כirut 68.

Betesmank 80.

SBi 43

Sondfugor 18.

Boffap, oeras fiutoomar 38.64 . præfervativ 243.

23rínile of 万afel 68.

Corf 104.

Liung 183.

3rofb 184. 239.

Såt 194.24\%.

Fabriquer, re Manufacturer.

Tiftande 193.258 .

ఫ๋år 66.

Spantea 58.72

broilla oxter får gerna ata 60.

booilta orter finocliga fỏr Dem 60.

oeras fiutoomar, ods bot

รีåนตนเ $6 \mathrm{I}$. oetfore 145.236 .

F゙a์

ร̌ịg, grôn 3.93.

gul $3.53 \cdot 93$.
ró 146.

Bietter 66.131.162.

Dexas flojinde at fortéom= mis 96.

Betefrall r 35 .

Sirinnar, rara 245 27I.

Bintor 184 .

Girớn fårg 3.93.

Gul fẩn 3.53.93.

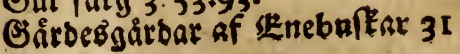

127.

af gărofel 80.89 .

af perpendiculairt fi̊ende ftórar

79. af fien 3I. 89.93.

135.253 .

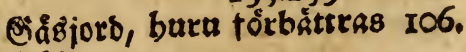
ઉెล̊์ 43 .

ઉ๋ ơb

torf och moßa $13 \mathrm{I}_{4}$

falt 30.

fatt, at belomma den

fión I3.

af KyytKoghroar 225.

Şafre $239 \cdot 240$.

fwarts forrwandlande i bwit, od twoirt orn 240.

Summerfiffe, Ђummertina I9I Soumle, nír ben bơk plåctas 106 bwilten båf 106.

รุน 54.79.104.109.135.194 195

224241 .

af rơo zualtifen 92.

Shwete 30.239 .

ફุñctar borbe anlåggas p\& fals bygoen 80.

รุåf 9

Deras fiutoomar $38 \cdot 202$. 203.

Do af Stätre 46.47.

orter Ptadolige 6.

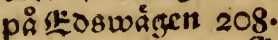

yra lótteligen at f́os 246.

รृญ์ 232.

fóretomna des brinnande och untnande 233 .

Rafelunghar of Jern 250.

Ralt, Kaltbrŏnueri 14.23 .68 .

af Murel od Enátital

20.201

Ror, orter ltabelige 6.98.

at excitera vener. 235 .

mooftuliue, bura botas

235.

Sor 


\section{Oeconoinifka Saker.}

Sor,ther ej ioifh, bwas bot 236 . uat oe ba gulfot, bot 236 at utorifwa efterbiro 236 for forftoppring 236 . for ourtlopp 236.

for bloofot 236 .

Siorn 239.250.

R̃̊̊ 4 . 113 .

Sîfla meo wattutónnor 71 .

Rierror 132.

Siott, brtu oes faltning bebol

Pamper I84. les 206.

Eatibthafic, butu ocn uttotis 93.

Eaxfiffe 206.219 .245 .

Ióftrio planterade wio gåxoar- 7 .

Manufachurer ed Fabriquer IIO. \&c. II7.\&c. I20,\&c.

Miflf, buru den filas 104.

פoribåif 260. ofmatelig 274 .

sjipbruf 126.

Plantager 127.

Slantelafwar 13.

\$logar fynnerliga 227.

Drournftenar II 5 .

รản 116.

besี ogrås 8239.

2Rลกำån 248

श̊̉o fầg 68. 146.

Salt til'gơoning, oes wertan 30.

Edefferi 58. 2t.

Sa)effers nodige Eanoftap odb

Sfepgbnggeri 149. befiefienber 60 .

Etog 9. I5 251 . brift serpå 80.91.134.162. "lóbwirnoigt at llona Den. 247.

Sfurqmarnar 34. Eforficune of Jern 250 . copifal SI. 109. I I6. Etcubrott 33.

Etemmular áro odt obeftånoige. 253.

Ẽtide: Snpeter 38 .

Etringiermibamrat 249.

Swith, bwilta orter de gerna àta 32.36 .93 .168$. Deras fiutoomar 65 . at fóretomm oeras gråf wande $93.2 c$.

Snt of tzafiewia 48 .

$$
\begin{aligned}
& \text { Corf Io9. } \\
& \text { cang is4. }
\end{aligned}
$$

Tcgel:bruE I32.

The inbemftt I06.254.

Thé-fappar af glas 156 .

Tobatsiolatitering 58. I2S. 132.

I95. 220.222 .

Torf brukelig til brânfle 104.

İrăgård 143.195.

23 anil meb 3 bjul 227.

T3nft:toja på biul 72 .

\$ुßarggår 16.

37 cibesproarn 127.

Wåderqroarnar 53.

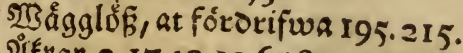
If frar $2 \cdot 17 \cdot 29 \cdot 30.65 \cdot 80,105 \cdot 200$

206.207.252. )ing 4.9.85.90.I I $5.25^{2}$. oerss fliende 97. ifiter 216 .

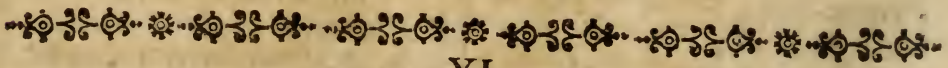
XI.

Órtertas trytta $i$ Oeconomien, Evc.

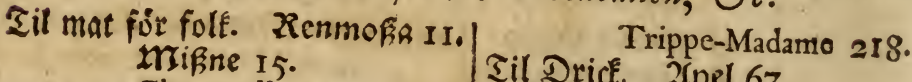
Chanterelle 215 .

Til Drick. 2lpel 67. J ftâfllet fớ Thé. $\ldots . . .197$. 


\section{Ortermas Nytta.}

Ttå nyttiga pd ângat $21 \mathrm{~lm} 66$. Lina 67.

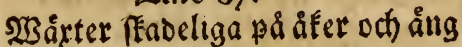

UEE 67

Liung 88.

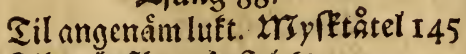

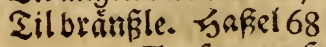

Corfwerz of Sphagnum

$$
103 .
$$

Liund I83.

Til wetar. Docketog IS4.

รil รุåckar. $2 \operatorname{tm} 66$

Liguftrum I92.

Getapel 215 .

Sil Puftyus. Caprifolium 146. Til ritfillige rebrtap od Enic Enrearbeten. 2ifk 67.

2rpel 67.

$2 \lim 67$.

Tilungnsqwaftar. Polytrichumg3 Iil tak. ZKafiewia 48.

$$
\text { coing I84. }
$$

Til Tapeter. fâtit: Erantis: quoiftar 238.

faxuftidtor 33 .

Sil nntta i fanfa furer, froder 2 . zafiewio 44

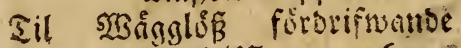
Dill=mzynt2 216.

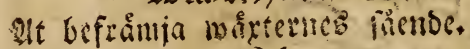
Gorntifel, 224 .

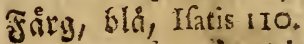

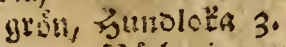
ziótbooippor 93. (i)etnpel 215.

(3) bl, bunslota 3 . Byffus farin. flava

$$
53 .
$$

24pelbate 6793

biottel of 93 .

2 fblbate 93 .

ป̂́:aftio 10.

Luteola 110.

Dristent 250.

Gultist 263.
Fårg, ro̊b, 25yttelet 147 .

Erwatt Miólonzris IIO.

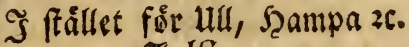

Jolfter 3 .

Lindbart 67.

Iefn til Eall Joromon. Zath

- grés. 4.

til tiltommande SBinter:

tzånetiftel 236.

Til $\mathfrak{B B i b f e p w e l f e r . ~}$

Etogs = Dioler mot

forgiors miólt 20.

Vifcum mot năbeld $3{ }^{T}$.

Prefervativ fur Soffaf. Ciut= bafi 243

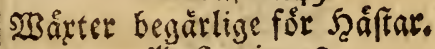

tafifewia 48 .

zlltiof 67.

Linolof 67.

23åxter ffinbelige, eller minbre

nuttige fork saåtar.

Galium lut. 6.

Galium er.quadrif.alb.6.

fărĭgåt mig ei 6.

Litermónia 6.

Hypericum caule quadr.

6.

Ulmaria 6.

caule ancip. 6

Valeriana fylv. 6.

Lilium convall. 6 .

Angelica fat. 6 .

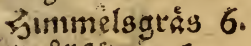

Liviefotter 6.

Drinbunte 6.

Gtorting̊f 6 .

Trollius 6 .

Aconitum 6 .

Anica 6.

Phellandrium 6. 46 .

Alisma 6.47.

Cicuta 99.

233 åter begărlige fór Ror.

Medicago renifiti. 2.

fištine 48. 


\section{Órternas Nytta.}

Ztáferoia 44.48 . zriélóf 67. Lindlof 67.

פgisarter fíndeliga fúr Silor:

Arnica 6.

Ranunculus prat. er. acr.

Caltha 6. 6. .

Aconitum Lappon. 6

Cicuta 6 48.98.

Mercurialis 64 .

Sigillum Salom. I06.

Conium 159.

şaxter benårlige fơ รå:

fơrgrés 60.

Irftiof 67.

Lindlof 67.

23aำter ftabelige fớr gaar:

Myofotis 60.

Gram. Offifragum 60

Iltog 60.

Drofera 60 .

Pinguicula 60.

Mercurialis 60 .

Flammula 60 .
Equifetum 60.

Cicuta 99.

23åxter begårlige fơ Eroin:

Scorzonera 3293.

Tubera 36.93 .

Gura ?iplen 67.

Quidtrot 93 .

Stachys paluftris 93.

Kumin 93.

Kklotång 168.

Not Ening Ejufbomar:

Ortorn p: 经范 65 .

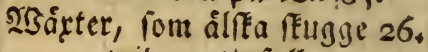
wio mattufall 40.

fom mixa i watn 46. fom trifwas i forer r 15.

fom máa wio fiofirans Der I 15.

Pom frywa ftarf́t med roits terne 207.

fom gifma tilfanna bett mtagrafte jorbmon 249. Wrąteter, fom trifwas bálft i in

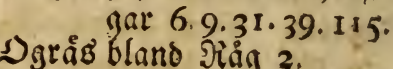

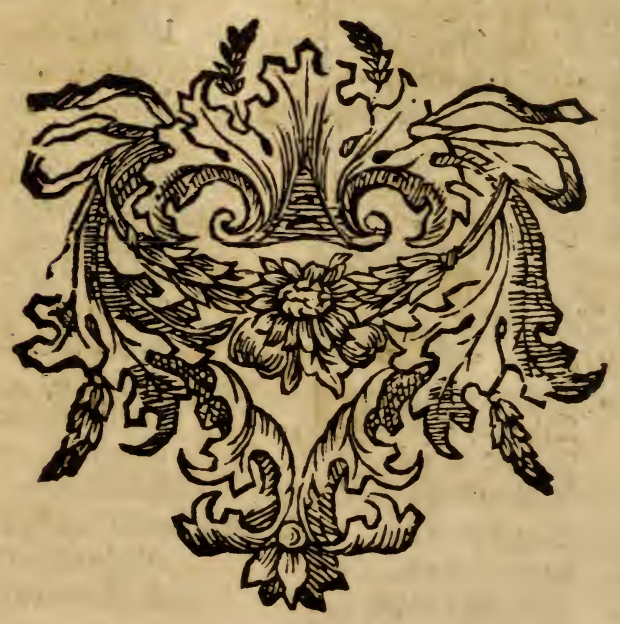




\section{Siáttelfer,}

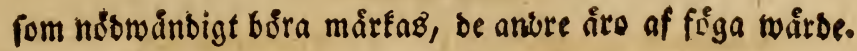

$$
\begin{aligned}
& \text { pág. } 3 \text { linea } 7 \text { (Şundlofa) } 245 \text { lấę } 243 . \\
& \text { - } 7 \text { - } 36 \text { datara datura. } \\
& \text { - } 9 \text { - } 9 \text { (Lychnis) } 368 \\
& \text { - } 23 \text { - } 17 \text { (E) } 184 \\
& \text { - } 25 \text { - } 31 \text { (After) } 622 \\
& \text { - } 27 \text { - } 26 \text { aueta } \\
& \text { - } 31 \text { - } 24 \text { (Mclica) } 56 \\
& \text { - } \quad 25 \text { (Clinopodium) } 497 \text { - } 479 . \\
& \text { - } 29 \text { (After) } 664 \text { - } 696 . \\
& \text { - } 33 \text { - I (Lithofipermum) I53 - 151. } \\
& \text { - } 39 \text { - } 5 \text { (Afragalus) } 592 \text { - 591. } \\
& \text { - } 40 \text { - } 31 \text { (Tuffilago) } 600 \text { - } 680 . \\
& \text { - } 48 \text { - } 30 \text { (Ranunculus) } 459 \text { - } 4.57 . \\
& \text { - } 49 \text { - } 7 \text { (Cochlea) I317 - I302. } \\
& \text { - - Ir (Sedum) } 39 \text { - 391. } \\
& \text { - } 59 \text { - } 6 \text { inloif - inláft. } \\
& \text { - } 60 \text { - } 18 \text { (Anthericum) } 277 \text { - } 268 . \\
& \text { a } \quad 20 \text { (Mercurialis) } 283 \text { - } 823 \text {. } \\
& \text { - - (Flammula) } 258 \text { - } 458 . \\
& \text { - } 74 \text { - } 31 \text { (Orchis) } 529 \text { - } 729 . \\
& \text { - } 135 \text { - } 7 \text { (Rubus) - } 409 \text {. } \\
& \text { - } 160-37 \quad 1 \frac{3}{4} \\
& \text { - I64 - II (Trifolium) } 697 \text { - 6I2. } \\
& \text { - } 166 \text { - } 14 \text { (Zoftera) } 364 \text { - } 1137 . \\
& \text { - } 177 \text { - } 14 \text { Lyrbleck } \\
& \text { - } 178 \text { - } 17 \text { Lânga } \\
& \text { - } 218 \text { - } 23 \text { (Sedum) } 387 \\
& \text { - } 225 \text { - } 12 \text { Grafner } \\
& \text { - } 238 \text { - } 1 \text { Lapfala } \\
& \text { - } 10 \text { (Eriophorum) } 4 \text { - } 44 \text {. } \\
& -249=5 \text { (Parnaffia) } 242 \quad-252 . \\
& \text { - } 256 \text { - } 30 \text { (Byffus) } 114 \text { - } 14 \text {. }
\end{aligned}
$$





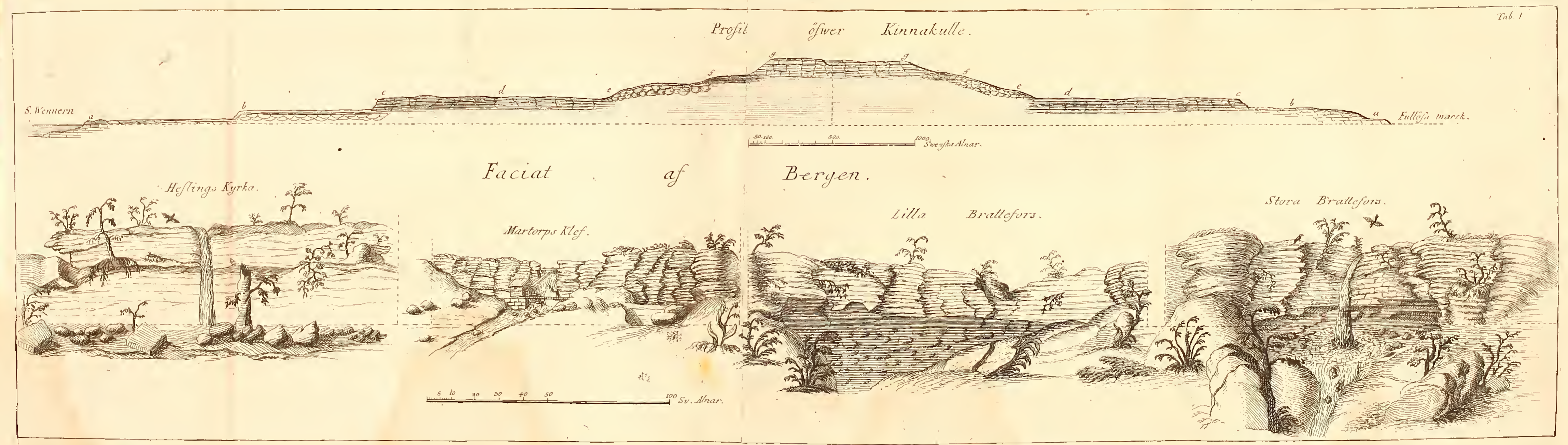




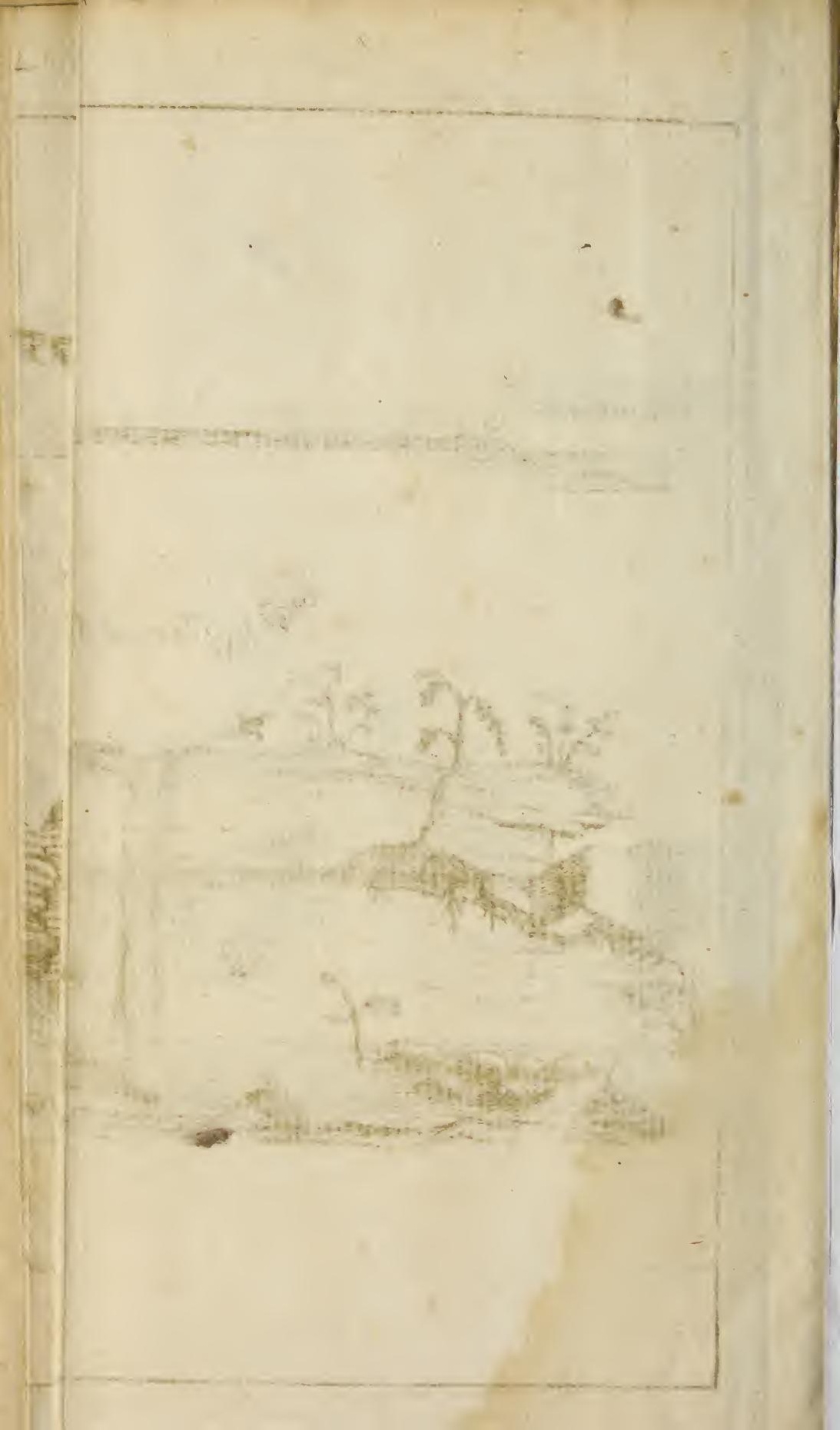




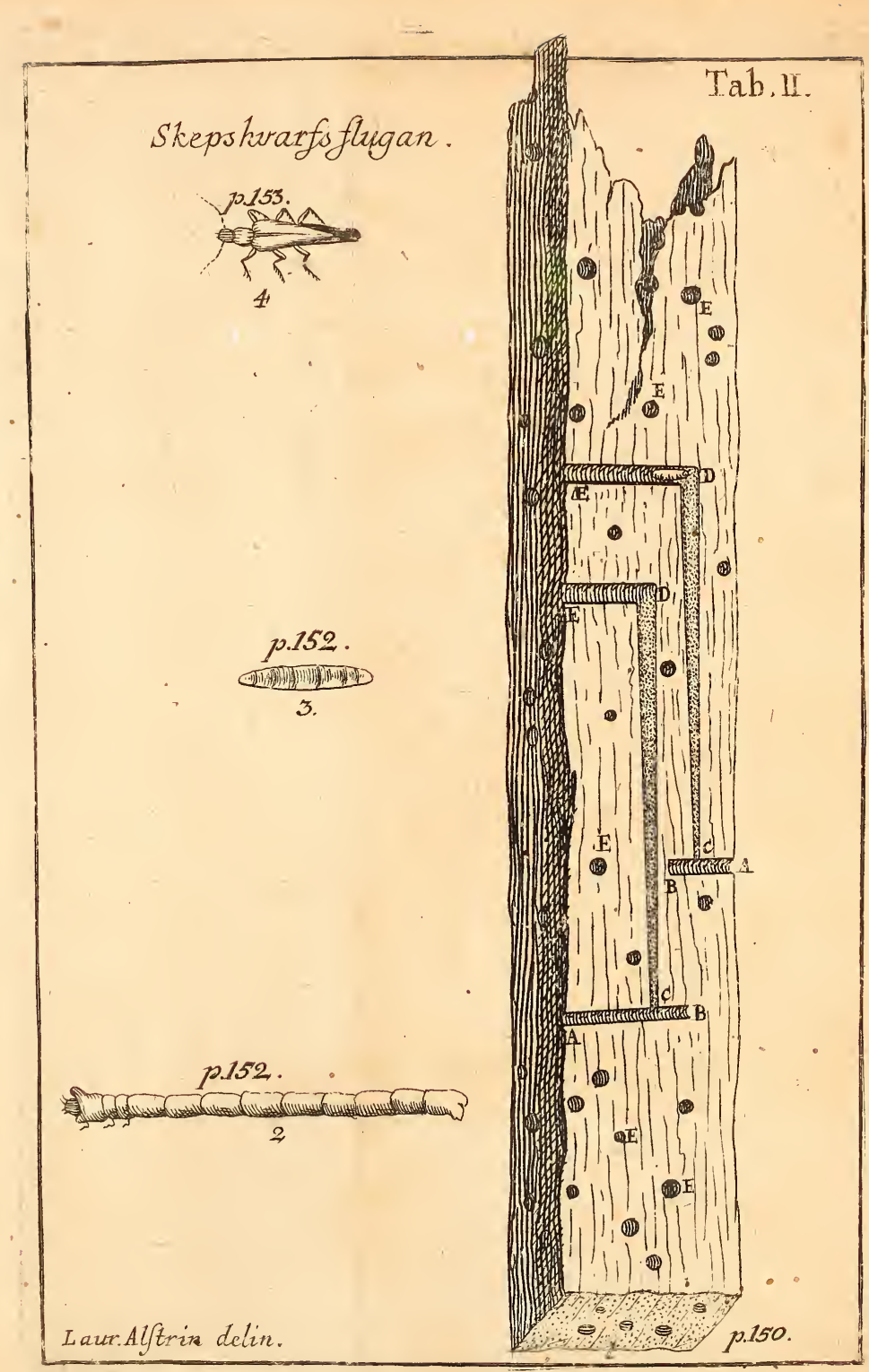





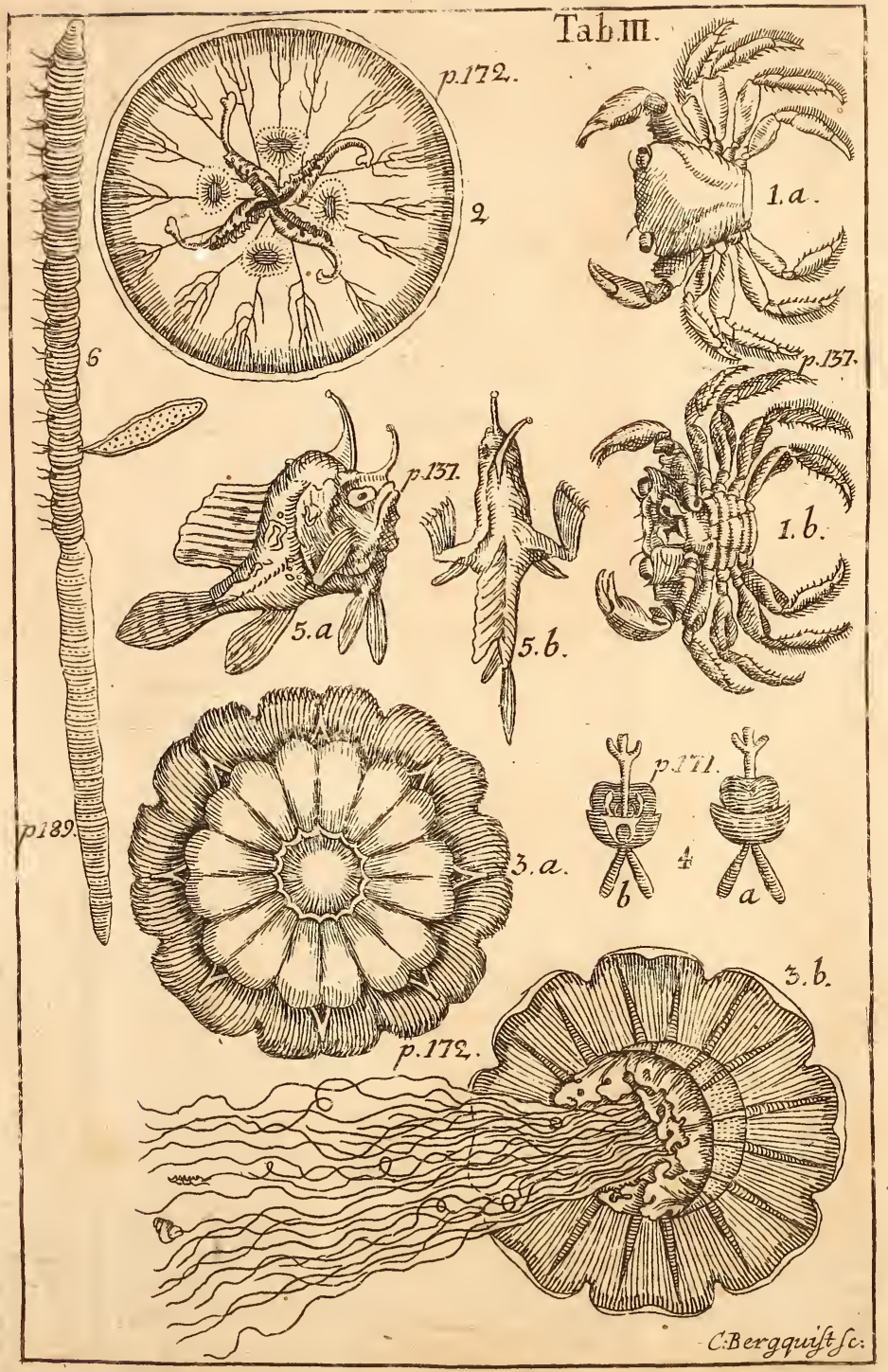





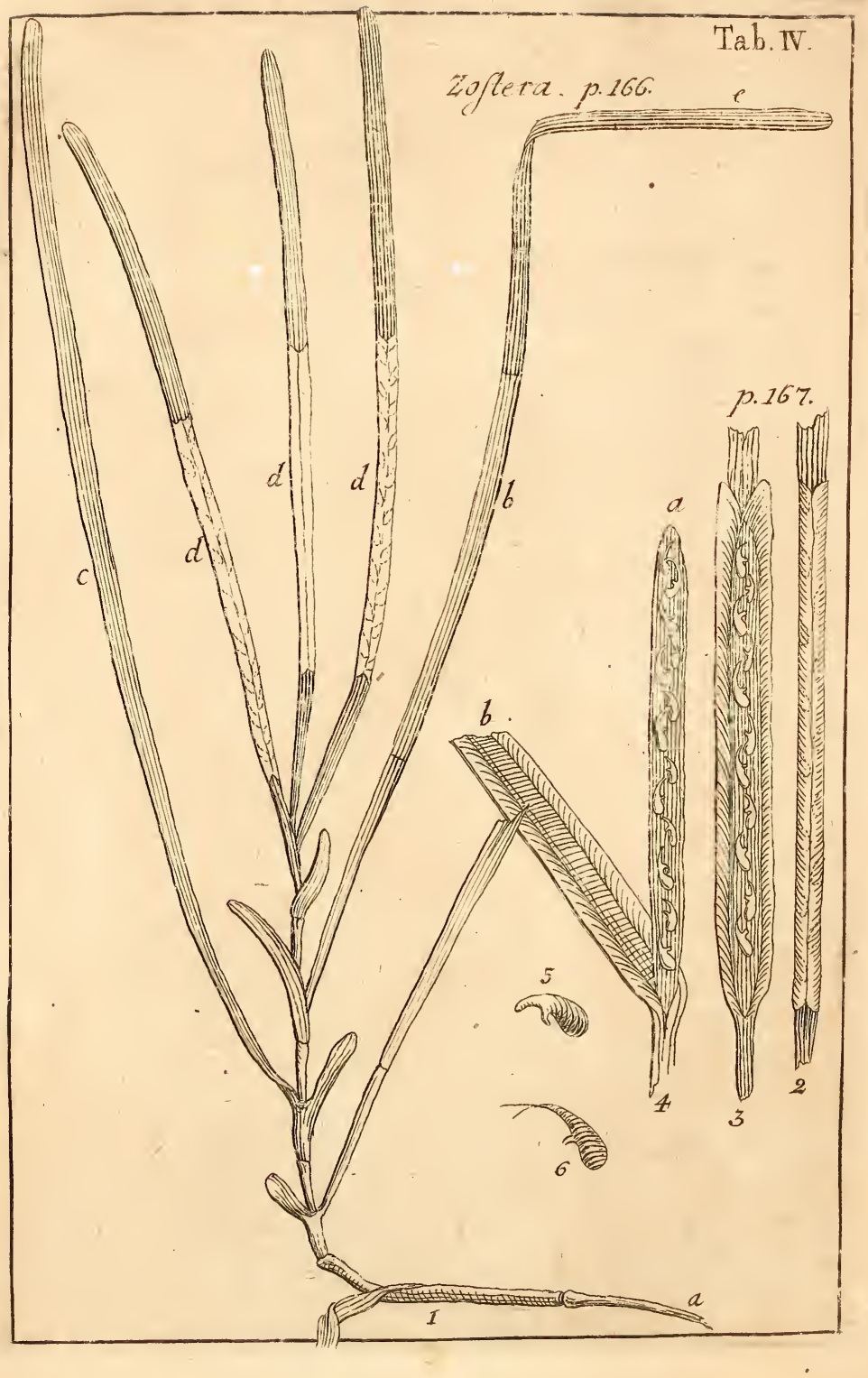





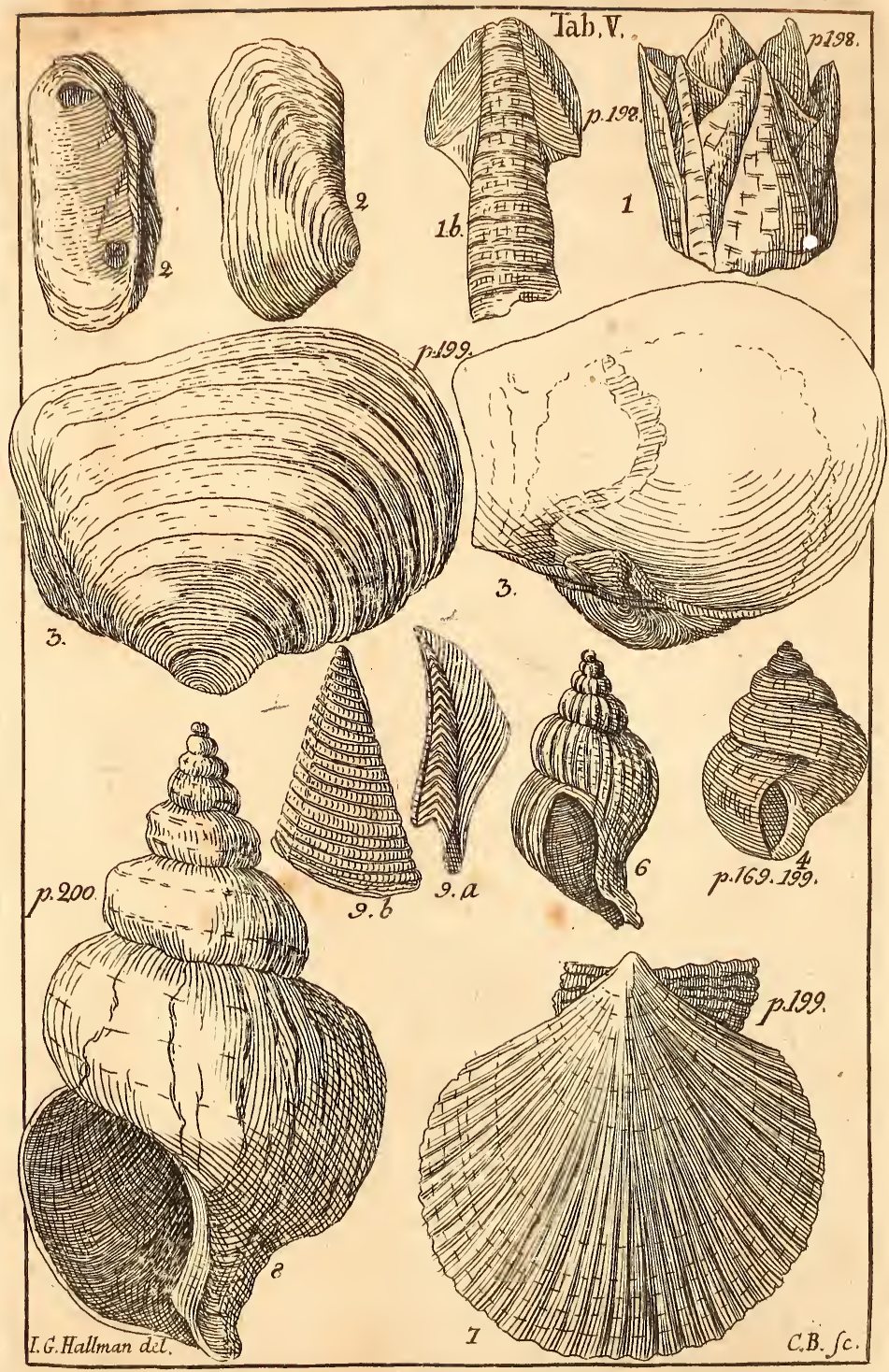




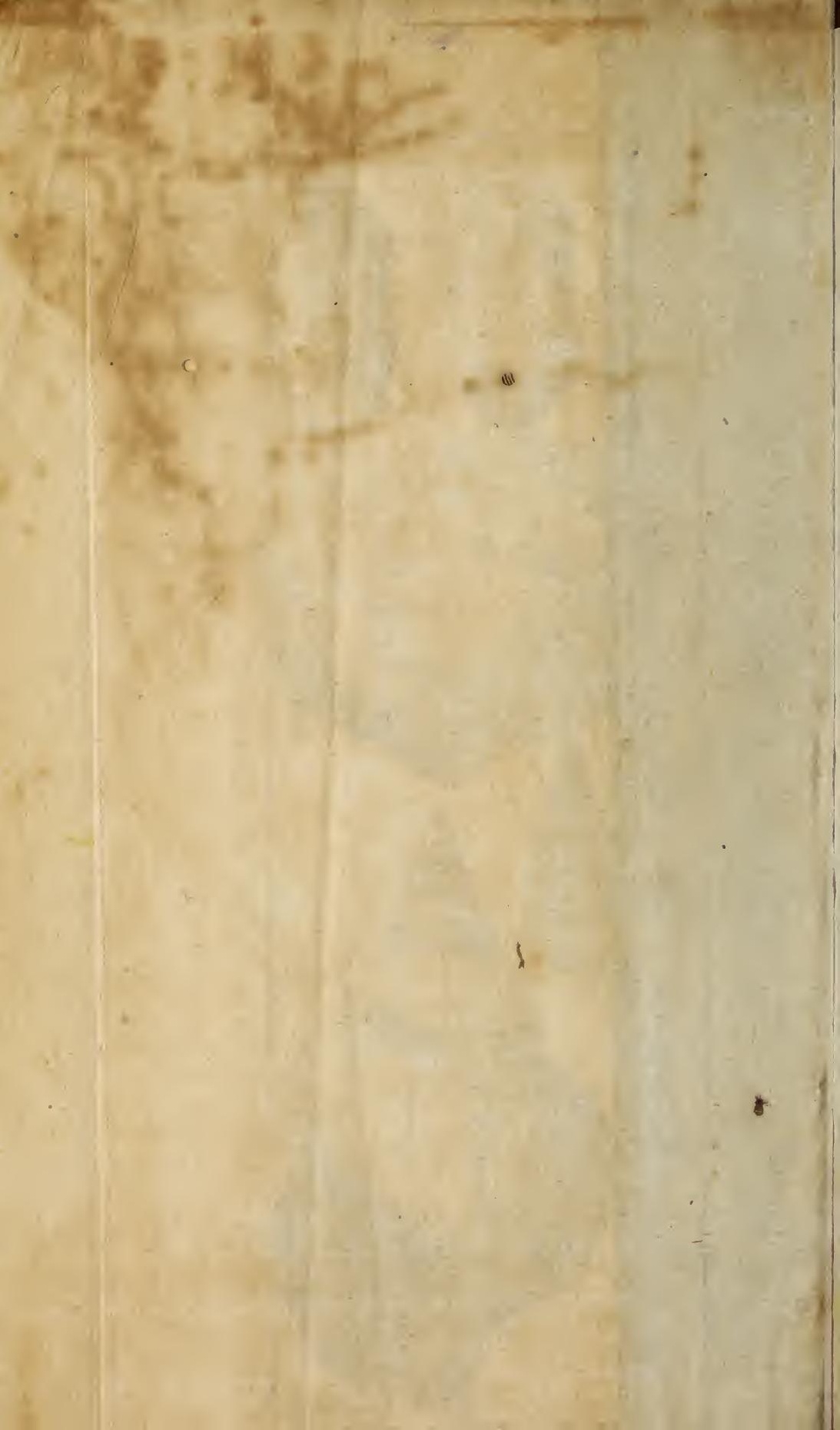





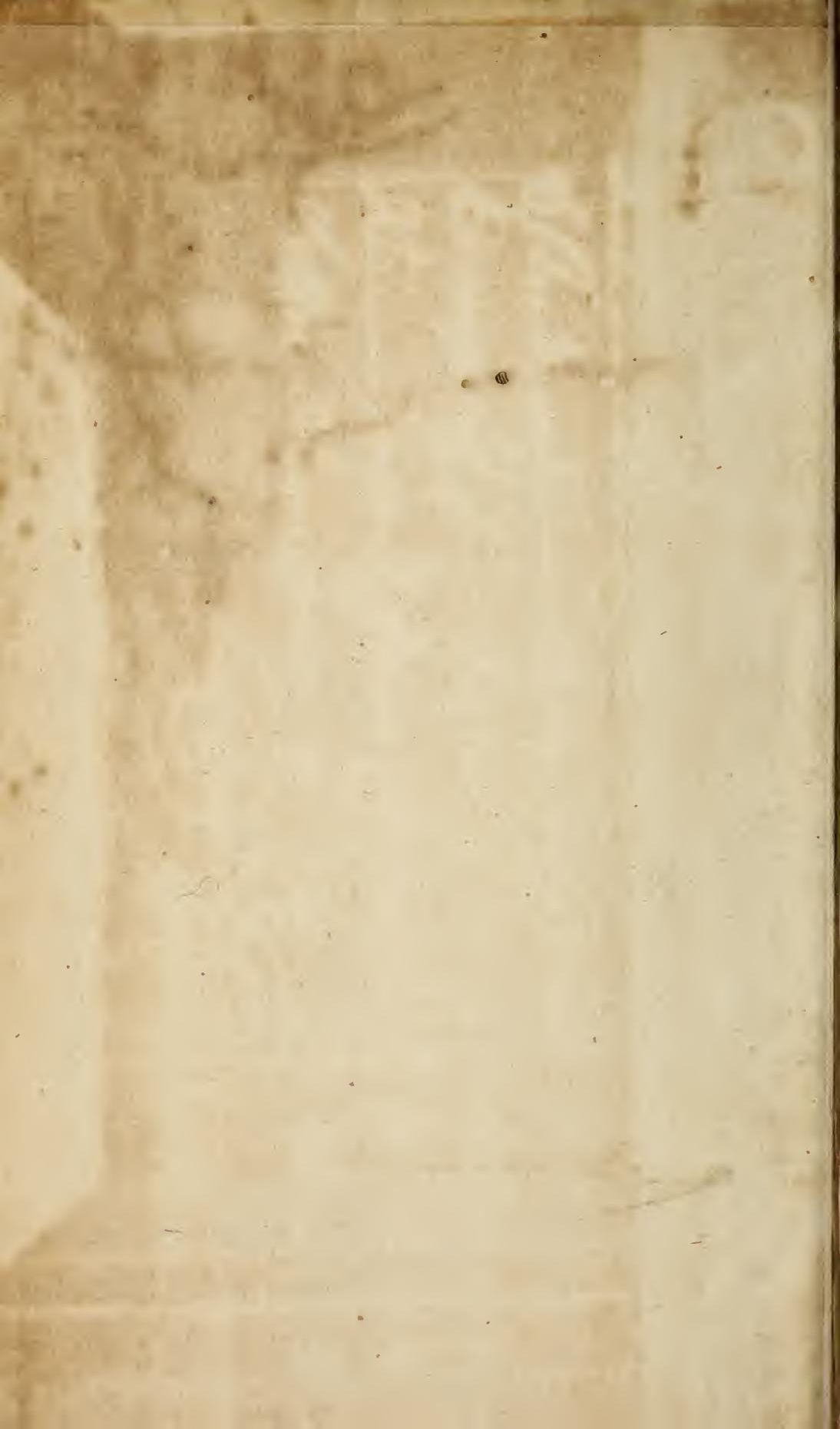




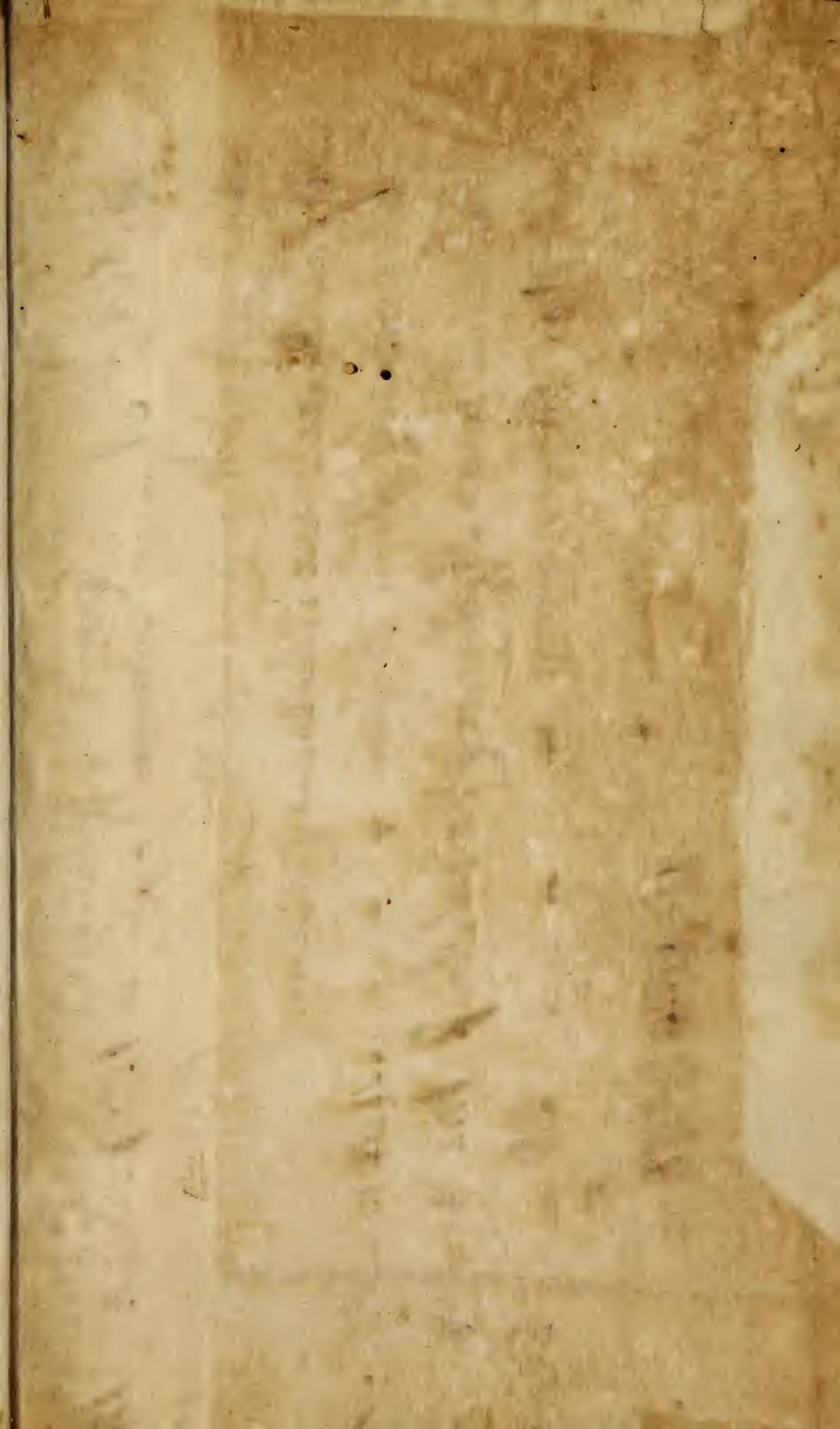


